Thomas Mosiek

\title{
Interne \\ Kundenorientierung des Controlling
}




\section{Thomas Mosiek}

\section{Interne Kundenorientierung des Controlling}

Im Gegensatz $\mathrm{zu}$ dem Anspruch vieler Controller, sich zunehmend als unternehmensinterne Berater in Fragen der Planung, Steuerung und Kontrolle zu profilieren, wird Controlling von seinen internen Adressaten vielfach noch unzureichend als serviceorientierte Dienstleistung wahrgenommen. Controlling im Sinne von Führungsunterstützung - kann sachgerecht nur über eine konsequente Orientierung an den Informationsbedürfnissen und Koordinationserfordernissen des Managements geleistet werden. Ziel der Arbeit ist daher, die potenzielle Kundenorientierung im Controlling einer differenzierten Betrachtung zu unterziehen, um gestaltungsrelevante Tatbestände $\mathrm{zu}$ identifizieren und Anwendungsvoraussetzungen ausgewählter betriebswirtschaftlicher Instrumente darzustellen.

Thomas Mosiek, Dipl.-Kfm., ist Geschäftsführer der BMS Consulting GmbH, einem auf Controlling-Leistungen spezialisierten Beratungsunternehmen. In dieser Funktion berät er zahlreiche privatwirtschaftliche und öffentliche Unternehmen bei der Entwicklung und Einführung von Controlling-Konzeptionen. Der Autor doziert am Centrum für Unternehmensrechung, Münster und an den Verwaltungsund Wirtschaftsakademien Essen, Duisburg und Krefeld. Er war von 1999 bis 2001 wissenschaftlicher Mitarbeiter am Lehrstuhl von Prof. Dr. Berens und verfasste dort seine Dissertation. Zuvor arbeitete Thomas Mosiek als freier Mitarbeiter bei Kienbaum Consultants International. Als jahrgangsbester Absolvent der Wirtschaftswissenschaftlichen Fakultät Düsseldorf wurde er mit dem KonradHenkel-Preis ausgezeichnet. 


\section{Interne Kundenorientierung des Controlling}




\section{Beiträge zum Controlling}

Herausgegeben von Wolfgang Berens

Band 4

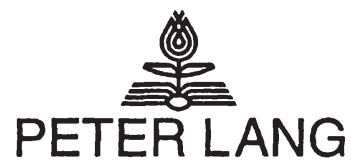

Frankfurt am Main - Berlin · Bern - Bruxelles - New York - Oxford · Wien 
Thomas Mosiek

\section{Interne Kundenorientierung des Controlling}

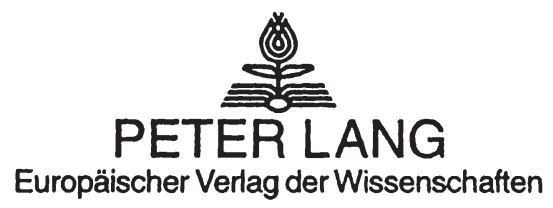


Die Deutsche Bibliothek - CIP-Einheitsaufnahme

Mosiek, Thomas:

Interne Kundenorientierung des Controlling / Thomas Mosiek. Frankfurt am Main ; Berlin ; Bern ; Bruxelles ; New York ;

Oxford; Wien: Lang, 2002

(Beiträge zum Controlling; Bd. 4)

Zugl.: Münster (Westfalen), Univ., Diss., 2001

ISBN3-631-39215-X

Open Access: The online version of this publication is published on www.peterlang.com and www.econstor.eu under the international Creative Commons License CC-BY 4.0. Learn more on how you can use and share this work: http://creativecommons. org/licenses/by/4.0.

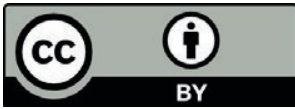

This book is available Open Access thanks to the kind support of ZBW - Leibniz-Informationszentrum Wirtschaft.

Gedruckt auf alterungsbeständigem, säurefreiem Papier.

\author{
D 6 \\ ISSN 1618-825X \\ ISBN3-631-39215-X \\ ISBN 978-3-631-75321-7 (eBook) \\ (C) Peter Lang GmbH \\ Europäischer Verlag der Wissenschaften \\ Frankfurt am Main 2002 \\ Alle Rechte vorbehalten.
}

Das Werk einschließlich aller seiner Teile ist urheberrechtlich geschützt. Jede Verwertung außerhalb der engen Grenzen des Urheberrechtsgesetzes ist ohne Zustimmung des Verlages unzulässig und strafbar. Das gilt insbesondere für

Vervielfältigungen, Übersetzungen, Mikroverfilmungen und die Einspeicherung und Verarbeitung in elektronischen Systemen.

\title{
Printed in Germany 123457
}

www.peterlang.de 


\section{Geleitwort}

Die Controlling-Institution sieht sich in Unternehmen infolge ihres nicht unmittelbar erkennbaren Beitrags zur Wertschöpfung einem zunehmenden Rechtfertigungsdruck ausgesetzt. Dieser macht es erforderlich, den Beitrag des Gemeinkostenbereichs Controlling zur Absicherung der Unternehmensziele zu dokumentieren und optimieren. Grundlage der Optimierungsüberlegungen ist die in größeren Institutionen auf eine Arbeitsteilung zurückgehende Trennung von Controlling- und Managementaufgaben. Demzufolge kann Controlling - im Sinne von Führungsunterstützung - sachgerecht nur über eine konsequente Orientierung an den Informationsbedürfnissen und Koordinationserfordernissen des Managements geleistet werden. Erst wenn das Management über eine methodische und instrumentelle Unterstützung in die Lage versetzt wird, Entscheidungen rational zu fundieren, kann das Controlling seinen Ansprüchen gerecht werden. Die Komplexität der den unternehmerischen Aktivitäten zugrunde liegenden Entscheidungsprobleme, verbunden mit den individuellen Informationsbedürfnissen von Führungskräften, macht daher eine konsequente interne Kundenorientierung erforderlich.

Die Notwendigkeit einer Kundenorientierung im Controlling sowie das diesbezüglich bestehende Theoriedefizit sind Motivation für Herrn MosIEK zur wissenschaftlichen Auseinandersetzung mit der von ihm gewählten Thematik „Interne Kundenorientierung des Controlling“. Im Rahmen dieser thematischen Eingrenzung werden gestaltungsrelevante Zusammenhänge identifiziert und Möglichkeiten eines gezielten Instrumenteneinsatzes geprüft, um hierüber die Kundenorientierung im Controlling einer Operationalisierung zugänglich zu machen. Im Ergebnis eröffnet sich Controllern ein Aktionsrahmen, der es erlaubt, das Leistungsspektrum des Controlling im Rahmen interner KundenLieferanten-Beziehungen zweckgerichtet auf die jeweiligen Adressaten auszurichten und diese somit in die Lage zu versetzen, rational zu führen.

Herm MosIEK kommt der Verdienst zu, sich einer in der theoretischen Auseinandersetzung mit dem Controlling bisher vernachlässigten Themenstellung fundiert angenommen zu haben. Die Ausführungen stellen eine erste geschlossene, Einzelfacetten zu einem Aktionsrahmen verbindende Behandlung der Kundenorientierung im Controlling dar. Herr MosIEK leistet somit wichtige Beiträge für die Weiterentwicklung des Controlling, da er in Abweichung zu ihrer bisher vornehmlich instrumentenzentrierten Sichtweise einen marketingorientierten Handlungsrahmen für das Controlling entwirft, der die eigentliche 
Aufgabe des Controlling der Führungsunterstützung wieder in den Vordergrund stellt. Neben dem Neuigkeitsgrad zeichnet sich seine Arbeit durch einen hohen Systematisierungsgrad aus. Das ausgewogene Verhältnis zwischen theoretischer Grundlegung, Ableitung der Gestaltungsparametersystematik und instrumenteller Absicherung der Gedanken dokumentiert den fundierten wissenschaftlichen Bezug der Arbeit. Die von ihm angestrengten Überlegungen sind zudem für die praktische Ausübung des Controlling von höchster Relevanz.

Durch die sehr gelungene Synopse zwischen Ansätzen aus dem Controlling und dem Marketing eröffnet Herr MosIEK dem Controlling eine neue Perspektive.

Münster, im Januar 2002

Prof. Dr. Wolfgang Berens 


\section{Vorwort}

Die vorliegende Arbeit entstand während meiner Tätigkeit als wissenschaftlicher Mitarbeiter am Lehrstuhl für Betriebswirtschaftslehre, insbesondere Controlling an der Westfälischen Wilhelms-Universität Münster und wurde im Wintersemester 2001/2002 als Dissertationsschrift angenommen. Besonderer Dank gilt meinem akademischen Lehrer und Erstgutachter PROF. DR. WOLFGANG BERENS. Das von ihm geschaffene liberale Arbeitsklima sowie die konsequent verfolgte Integration von Wissenschaft und Praxis an seinem Lehrstuhl haben meine Assistentenzeit zu einem lehrreichen Erlebnis werden lassen. Herrn PROF. DR. DR. H.C. JÖRG BAETGE danke ich recht herzlich für die Übernahme des Zweitgutachtens.

Spezielle Anerkennung gilt Herm DR. ANDREAS HofFJAN für die Zeit, die er sich für konstruktive Kritik und kreative Anregungen trotz seines Forschungsaufenthaltes an der University of Illinois at Urbana-Champaign genommen hat. Für anregende Diskussionen und hilfreiche Hinweise möchte ich ferner den Herren RA Marcus MosieK, DR. WALTER SCHMTTING, DR. ANDREAS SIEMES, DIPL.-KFM. THOMAS TRISKA und DIPL.-KFM. FLORIAN WECKER danken. Für die Entlastung bei zahlreichen technischen Arbeiten sind die Verdienste von Herrn CAND. RER. POL. BJÖRN LUDWIG zu würdigen. Zur Vereinbarkeit von Lehrstuhltätigkeiten, Beratungsaktivitäten und Promotion hat unser Sekretariat mit seiner angenehmen Art wesentlich beigetragen - hierfur meinen herzlichen Dank an Frau GabRIELE PANING und Frau BetTINA KLEINFELDER. Insgesamt möchte ich allen Kollegen am Lehrstuhl für Betriebswirtschaftslehre, insbesondere Controlling furr das außerordentlich gute Arbeitsklima danken.

Für den Gedankenaustausch zur Überprüfung und praktischen Absicherung der Aussagen meiner Arbeit möchte ich dem President der Ericsson Austria AG, Herrn Peter ZeHETNER und der ERICSSON GMBH Deutschland danken.

Abschließend und umfassend gilt meine besondere Anerkennung für Wesentliches was mich heute ausmacht meinen Eltern und meiner Frau Aline.

Düsseldorf, im Januar 2002

Dipl.-Kfm. Thomas Mosiek 
Thomas Mosiek - 978-3-631-75321-7

Downloaded from PubFactory at 01/11/2019 06:13:35AM

via free access 


\section{Inhaltsverzeichnis}

Abbildungsverzeichnis ................................................................................ XIII

Abkürzungsverzeichnis ............................................................................... XVII

$1 \quad$ Einleitung ......................................................................................................................1

1.1 Problemstellung und Zielsetzung ..................................................................1

1.2 Methodik und Vorgehensweise...........................................................................4

2 Theoretische Fundierung der Teilansätze ....................................................7

2.1 Der Controlling Ansatz .....................................................................................7

2.1.1 Controlling als eigenständiger betriebswirtschaftlicher Bereich?....8

2.1.2 Alternative Ansätze für Controlling-Konzeptionen ..........................9

2.1.3 Koordinationsorientierte Controlling-Konzeption ..........................11

2.1.3.1 Konzeptionelle Grundlage..........................................................11

2.1.3.2 Ausgestaltungskontinuum .........................................................13

2.1.3.2.1 Führungsbasiertes vs. fachbereichsbasiertes Verständnis .....14

2.1.3.2.2 Verständnissynthese ..............................................................15

2.1.4 Ausgewählte Entwicklungstendenzen im Controlling ....................19

2.1.4.1 Externe Marktorientierung des Controlling ...................................21

2.1.4.2 Interne Marktorientierung des Controlling ....................................22

2.2 Der Marketing Ansatz ..............................................................................24

2.2.1 Traditioneller Objektbereich ...........................................................25

2.2.2 Ausgewählte Entwicklungstendenzen im Marketing ......................27

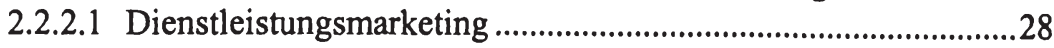

2.2.2.2 Internes Marketing.....................................................................31

2.2.2.2.1 Personalorientiertes internes Marketing ..................................33

2.2.2.2.2 Abteilungsorientiertes bzw. funktionsorientiertes internes Marketing ................................................................................ 34

2.3 Integrationsperspektiven der Teilansätze .................................................36

3 Gestaltungsraum für ein kundenorientiertes Controlling ................41

3.1 Ziele der Kundenorientierung im Controlling und kundenorientierte Führung des Controllerbereichs. 
3.2 Leistungsbezogene Charakteristika des Controlling

3.2.1 Controlling-Leistungen als operative Instrumente zur

Aufgabenerfüllung ... .50

3.2.1.1 Aufgabenzuordnungsaspekte....................................................51

3.2.1.2 Strukturierungsrahmen für die Aufgabenzuordnung ...................51

3.2.1.3 Leistungen als Instrumente der Aufgabenerfüllung.....................58

3.2.1.4 Leistungsspektrum des Controllers ...............................................60

3.2.2 Leistungsbezogene Gestaltungsparameter des kundenorientierten Controlling..............................................................74

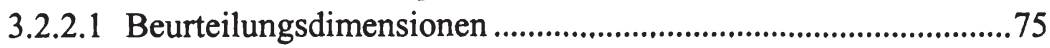

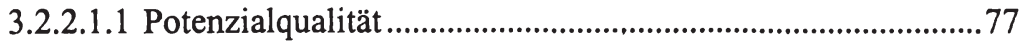

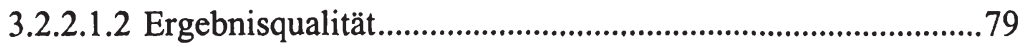

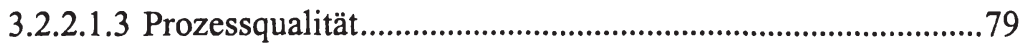

3.2.2.2 Informationsökonomische Leistungseigenschaften ....................82

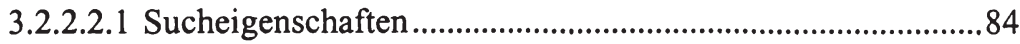

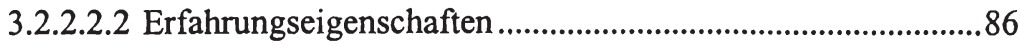

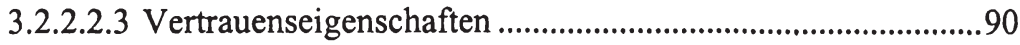

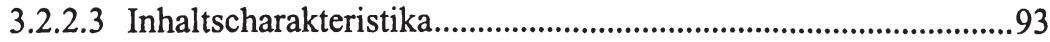

3.2.2.3.1 Unterstützungscharakter..................................................93

3.2.2.3.2 Kontrollcharakter ...............................................................96

3.3 Organisationsbezogene Charakteristika des Controlling...............102

3.3.1 Organisatorische Einbindung und ökonomische Ausrichtung des Controlling als Rahmenbedingungen der Leistungs-

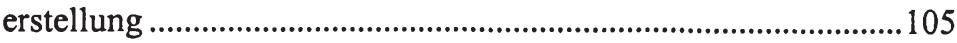

3.3.1.1 Organisatorische Einbindung …..............................................107

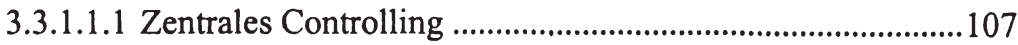

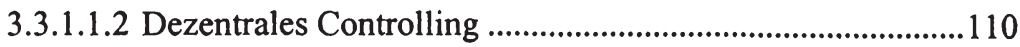

3.3.1.2 Ökonomische Ausrichtung ....................................................115

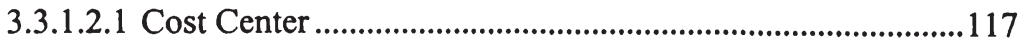

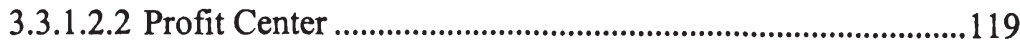

3.3.2 Organisationsbezogene Gestaltungsparameter des kundenorientierten Controlling..............................................................123

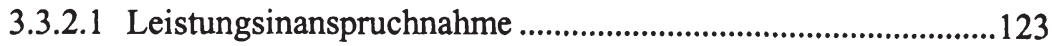

3.3.2.1.1 Obligatorische Leistungen ................................................124

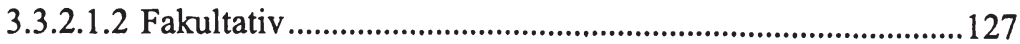

3.3.2.2 Abrufmodalitäten .............................................................128

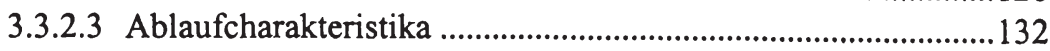

3.3.2.3.1 Sequenzintegrierte Leistungserstellung .............................. 133

3.3.2.3.2 Sequenzunabhängige Leistungserstellung ............................137 
3.3.2.4 Informelle Kontakte und Leistungstradition .............................138

3.3.2.5 Leistungsverrechnung........................................................141

3.4 Kunden- und wettbewerbsbezogene Charakteristika des

Controlling

3.4.1 Kundenbeziehungen im Rahmen der Leistungserstellung ............151

3.4.1.1 Ausrichtung des Controlling (intern vs. extern) .........................151

3.4.1.2 Abgrenzung der internen Controlling-Kunden .........................154

3.4.2 Wettbewerbsbeziehungen im Rahmen der Leistungsverwertung 160

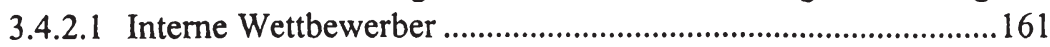

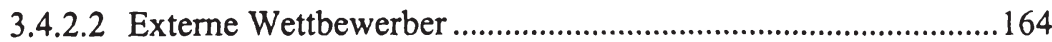

3.4.3 Kunden- und wettbewerbsbezogene Gestaltungsparameter des

kundenorientierten Controlling....................................................169

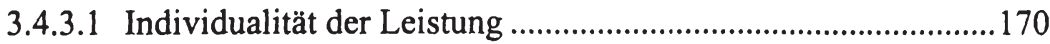

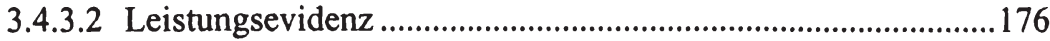

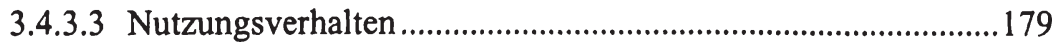

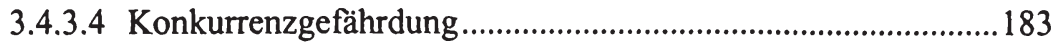

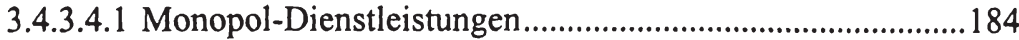

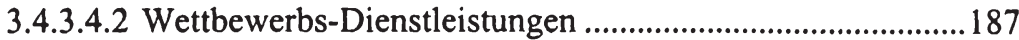

3.5 Implikationen der Gestaltungsparameterbetrachtung für die interne Kundenorientierung des Controlling.

4 Aktionsgrundlagen des kundenorientierten Controlling ................195

4.1 Strategische Controllerbereichs- und Marketingplanung ..............198

4.1.1 Strategische Controllerbereichsplanung ......................................199

4.1.2 Strategische Marketingplanung im Controlling ...........................204

4.1.2.1 Kundenorientierte Strategie.................................................204

4.1.2.1.1 Informationsbedarfsanalyse ...............................................205

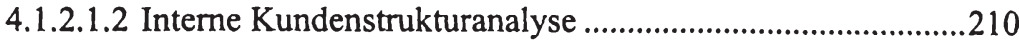

4.1.2.2 Konkurrenzorientierte Strategie ............................................221

4.2 Operative Leistungsanalyse und -planung im Controlling ............225

4.2.1 Benchmarking des Controllerbereichs..........................................226

4.2.1.1 Benchmarkingobjekte im Controlling .....................................222

4.2.1.2 Benchmarkingprozess...........................................................229

4.2.1.3 Benchmarking Dimensionen im Controlling ............................231

4.2.1.3.1 Effizienz als internes Leistungsmaß ..................................2231

4.2.1.3.2 Effektivität als kundenbezogenes Leistungsmaß.................233

4.2.1.4 Best Practice .........................................................................2238

4.2.2 Balanced Scorecard für den Controllerbereich...……753211…….243 
4.2.2.1 Struktur und Ablauf des Performance Measurement .244

4.2.2.2 Spezifikation einer Balanced Scorecard für den Controllerbereich. 245

4.2.3 Target Costing für Controlling-Leistungen ..................................251

4.2.3.1 Target Costing-Methodik ..........................................................251

4.2.3.2 Target Costing-Prozess im Controlling....................................253

4.2.4 Service Level Definitions und Service Level Agreements im

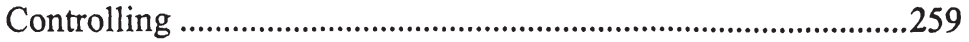

4.2.4.1 Struktur von Service Level Definitions und Service Level

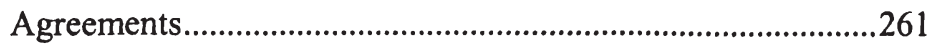

4.2.4.2 Anwendung von Service Level Agreements und Service Level Defintions im Controlling .264 Resumee .275 


\section{Abbildungsverzeichnis:}

Abbildung 1: Schematischer Überblick über die Struktur der Arbeit .................5

Abbildung 2: Kontinuum der Controlling-Verständnisse..................................16

Abbildung 3: Verantwortungsbereiche und Verständniskontinuum .................17

Abbildung 4: Controlling im Wandel....................................................................21

Abbildung 5: Leistungstypologie nach ENGELHARDT, KLEINALTENKAMP

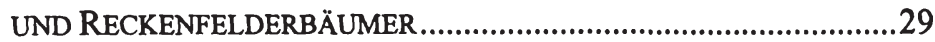

Abbildung 6: Integrationsperspektiven der Teilansätze ...................................39

Abbildung 7: Modifiziertes Marketing Dreieck................................................43

Abbildung 8: Kundenorientierung als Denkfigur bei der Führungsaufgabenwahrnehmung im Controllerbereich

Abbildung 9: Prozess der analytischen und methodischen Fundierung der Aufgabenzuordnung..................................................................53

Abbildung 10: Zusammenfassende Übersicht der Aufgabenzuordnungsas-

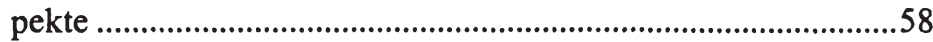

Abbildung 11: Abstraktes Modell eines Controlling-Leistungsraums ...............64

Abbildung 12: Modell eines Controlling-Leistungsraums ................................67

Abbildung 13: Leistungsbündel des Controlling als Gegenstand der Mar-

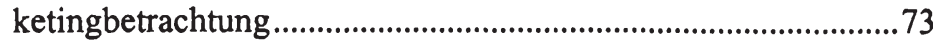

Abbildung 14: Entwicklungstendenzen im Berufsbild des Controllers ..............75

Abbildung 15: Systematisierung beispielhafter Controlling-Leistungen ............81

Abbildung 16: Integrative Leistungserstellung - Leistungsphasen im Con-

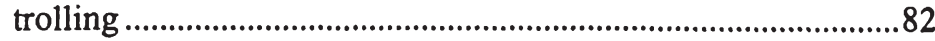

Abbildung 17: Informationsökonomisches Dreieck von Leistungen .................83

Abbildung 18: Beispielhafte Informationsaktivitäten der Transaktionspartner im Controlling ..................................................................89

Abbildung 19: Informationsquellen im Beurteilungsprofil ...............................99

Abbildung 20: Unternehmen mit Controlling-Stellen in Abhängigkeit ............104 Abbildung 21: Prozessallokations-Matrix.......................................................114

Abbildung 22: Erfolgsmaßstäbe für Center-Typen .......................................116 Abbildung 23: Verbesserung der Leistungsinanspruchnahme im Controlling.

Abbildung 24: Bringschuld des Controllers.

Abbildung 25: Informationsfluss und beteiligte Informationssysteme bei der Erstellung der Dienstleistung „Bestandsbewertung von Erzeugnissen"... 
Abbildung 27: Voraussetzungen für die Einzelverrechnung von internen Dienstleistungen

Abbildung 28: Beeinflussbarkeit - Marktbezug - Portfolio des Controlling unter Berücksichtigung alternativer Verrechnungspreisbestimmungsverfahren.

Abbildung 29: Kundenspezifische Berichte nach Hierarchieebenen .................155 Abbildung 30: Adressatenspezifische Controllinganforderungen bzw. Controllerperspektiven bei unterschiedlichen Adressatenkreisen .. 156 Abbildung 31: Informationsangebot, -nachfrage und -bedarf

Abbildung 32: Tätigkeitsbereiche von Unternehmensberatungen mit Controlling-Leistungen.

Abbildung 33: Klassifikation von Reporting-Leistungen.................................172 Abbildung 34: Praktische Bedeutung formaler Reportingstandards ................175 Abbildung 35: Entstehungsmöglichkeiten von Störungen im Berichtswesen .180 Abbildung 36: Arten der Nutzung von Kostenrechnungsinformationen...........181 Abbildung 37: Potenzielle Konkurrenten bei der Einführung einer Balanced Scorecard

Abbildung 38: Abgrenzung zwischen Controlling, interner Beratung und externer Beratung.

Abbildung 39: Parameter-Instrumenten-Matrix

Abbildung 40: Planungsphasen und Instrumente des kundenorientierten Controlling

Abbildung 41: ANSOFF-Matrix für Controlling-Leistungen ...........................200

Abbildung 42: Exemplarisches Kostenrechnungs-Portfolio.............................203

Abbildung 43: Methoden der Informationsbedarfsermittlung.........................206

Abbildung 44: Überschneidungsalternativen von Informationsangebot, -nachfrage und -bedarf.

Abbildung 45: Kriterien der internen Marktsegmentierung im Controlling ....218 Abbildung 46: Kundenbewertung im Controlling ..........................................219 Abbildung 47: Typologisierung konkurrenzgerichteten Verhaltens ................222 Abbildung 48: Analyseschema zur Bewertung des Benchmarking-Potenzials.

Abbildung 49: Überblick über Ansätze zur prozessualen Zufriedenheitsermittlung

Abbildung 50: Kosten und Zeitmerkmale einer Best Practice im Berichtswesen

Abbildung 51: Kundenportfolio zur Entwicklung von Informationsversorgungsstrategien im Controlling. 
Abbildung 52: Berichtsbogen zur Leistungsgruppe Informationsmanage-

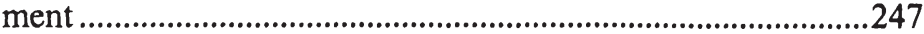

Abbildung 53: Performance-Ergebnisbogen und Performance-Stufen ............248 Abbildung 54: Prozess des Target Costing im Controlling ..............................254 Abbildung 55: Leistungsprofil eines Controllerbereichs................................256 Abbildung 56: SLD „Durchführung des Forecasting“....................................267 Abbildung 57: SLD „Margen- und Abweichungsanalyse“.................................268 Abbildung 58: Ausgewählte Mitwirkungspflichten im Rahmen von SLA's ...269 Abbildung 59: SLA-Struktur 
Thomas Mosiek - 978-3-631-75321-7

Downloaded from PubFactory at 01/11/2019 06:13:35AM

via free access 


\section{Abkürzungsverzeichnis:}

$\begin{array}{ll}\text { allg. } & \text { allgemein } \\ \text { AMA } & \text { American Marketing Association } \\ \text { Aufl. } & \text { Auflage } \\ \text { bspw. } & \text { beispielsweise } \\ \text { BAB } & \text { Betriebsabrechnungsbogen } \\ \text { BDU } & \text { Bundesverband Deutscher Unternehmensberater } \\ \text { BFuP } & \text { Betriebswirtschaftliche Forschung und Praxis } \\ \text { BSC } & \text { Balanced Scorecard } \\ \text { BSF } & \text { Business Support Functions } \\ \text { BU } & \text { Business Unit } \\ \text { ca. } & \text { circa } \\ \text { CIT } & \text { Critical Incident Technique } \\ \text { CMR } & \text { California Management Review } \\ \text { CO } & \text { Controlling } \\ \text { CSF } & \text { Critical Success Factors } \\ \text { DB } & \text { Deckungsbeitrag } \\ \text { DB } & \text { Der Betrieb } \\ \text { DBW } & \text { Die Betriebswirtschaft } \\ \text { Diss. } & \text { Dissertation } \\ \text { DM } & \text { Deutsche Mark } \\ \text { EDD/F } & \text { Controllingbereich der Ericsson GmbH } \\ \text { EDV } & \text { Elektronische Datenverarbeitung } \\ \text { EUR } & \text { Euro } \\ \text { d.h. } & \text { das heißt } \\ \text { e.G. } & \text { eingetragene Genossenschaft } \\ \text { et al. } & \text { et alii } \\ \text { etc. } & \text { et cetera } \\ \text { EWB } & \text { Einzelwertberichtigung } \\ \text { f. } & \text { folgende (Seite) } \\ \text { F\&E } & \text { Forschung und Entwicklung } \\ \text { FEI } & \text { Financial Executives Institute } \\ \text { ff. } & \text { folgende (Seiten) } \\ \text { FI } & \text { Finance } \\ \text { ggf. } & \text { gegebenenfalls } \\ \text { GmbH } & \text { Gesellschaft mit beschränkter Haftung } \\ \text { HdO } & \text { Handwörterbuch der Organisation } \\ \text { HR } & \text { Human Ressources } \\ & \end{array}$




\begin{tabular}{|c|c|}
\hline Hrsg. & Herausgeber \\
\hline http & Hyper Text Transfer Protocol \\
\hline HWB & Handwörterbuch Betriebswirtschaftslehre \\
\hline HWO & Handwörterbuch Organisation \\
\hline IGC & International Group of Controlling \\
\hline Inf. & Information \\
\hline Int. & Interaktion \\
\hline io & industrielle Organisation \\
\hline IS & Information Systems \\
\hline IT & Information Technologie \\
\hline Kap. & Kapitel \\
\hline KKV & komparativer Konkurrenzvorteil \\
\hline $\mathrm{krp}$ & Kostenrechnungspraxis \\
\hline lfd. & laufend \\
\hline LM & Lars Magnus \\
\hline $\mathrm{M} \& \mathrm{~A}$ & Mergers and Akquisitions \\
\hline $\mathrm{MbO}$ & Management by Objektives \\
\hline Mgmt. & Management \\
\hline MIS & Management Informationssystem \\
\hline NIST & National Institute of Standards and Technology \\
\hline Nr. & Nummer \\
\hline NWP & Niederstwertprinzip \\
\hline o.J. & ohne Jahrgang \\
\hline o.V. & ohne Verfasser \\
\hline $\mathrm{pdf} 尺$ & Portable Document Format \\
\hline PWB & Pauschalwertberichtigung \\
\hline PuK & Planungs- und Kontrollrechnung \\
\hline ROI & Return on Investment \\
\hline RW & Rechnungswesen \\
\hline S. & Seite \\
\hline SAP & Systemanalyse und Programmentwicklung \\
\hline SGF & strategisches Geschäftsfeld \\
\hline SLA & Service Level Agreement \\
\hline SLD & Service Level Definition \\
\hline Sp. & Spalte \\
\hline SSC & Shared Service Center \\
\hline TQM & Total Quality Management \\
\hline u. & und \\
\hline u.a. & und andere \\
\hline
\end{tabular}


usw.

WHU

WiSt

WU

www

$\mathrm{Vgl}$.

Vol.

vs.

z.B.

z.T.

$\mathrm{ZfB}$

$\mathrm{zfbf}$

zfo

ZögU

ZVEI und so weiter

Wissenschaftlichen Hochschule für Unternehmensführung

Das Wirtschaftsstudium

Wirtschaftsuniversität

World Wide Web

Vergleiche

Volume

versus

zum Beispiel

zum Teil

Zeitschrift für Betriebswirtschaft

Zeitschrift für betriebswirtschaftliche Forschung

Zeitschrift Führung + Organisation

Zeitschrift für öffentliche und gemeinwirtschaftliche Unternehmen

Zentralverband der Elektrotechnik Elektroindustrie e.V. 
Thomas Mosiek - 978-3-631-75321-7

Downloaded from PubFactory at 01/11/2019 06:13:35AM

via free access 


\section{Einleitung}

\subsection{Problemstellung und Zielsetzung}

Ausgehend von einer historischen Entwicklung, in der sich in vielen Bereichen Marktstrukturen von Verkäufermärkten zu durch ein Angebotsüberschuss gekennzeichneten Käufermärkten entwickelt haben, sehen sich in jüngster Vergangenheit auch Unternehmensteilbereiche mit einer sich ändernden Konkurrenzsituation konfrontiert. Waren es zuerst noch die Unternehmensbereiche, in denen die unmittelbare Leistungserstellung für den Absatzmarkt erfolgte, die sich einer vergleichenden markt- und kundenbezogenen Betrachtung mit anderen Produktionsstätten oder externen Anbietern unterziehen mussten, sind es heute insbesondere Gemeinkostenbereiche, die die Kosten der Erstellung ihres Leistungsangebotes vor den Anforderungen ihrer Kunden rechtfertigen müssen. Im Rahmen derartiger Entwicklungstendenzen sah der Controlling-Bereich' regelmäßig seine Aufgabe darin, zu einer Abbildung bzw. Bewertung der Situationen in den anderen Unternehmensbereichen beizutragen. Die Art und Weise der Erfüllung dieser und anderer Aufgaben hat in der Vergangenheit dazu geführt, dass Controller von denjenigen, denen sie Leistungen erbringen, all zu oft als Erbsenzähler, ${ }^{2}$ Zahlenknechte, Bremser oder auch Gärtner von Zahlenfriedhöfen wahrgenommen wurden. ${ }^{3}$ Ironisch wird in diesem Zusammenhang die Distanz zur Realität, die systematische Erfassung der Irrelevanz, die geistige Durchdringung des Nichts, die Interpretation des Banalen und die Analyse des Unvorhersehbaren zu den Aufgaben des Controllers gezählt. ${ }^{4}$ Stellt man dieser überspitzten Kritik die Rolle eines internen Beraters', die zahlreiche Controller zunehmend anstreben, gegenüber, wird deutlich, dass es grundlegender Umdenkprozesse und gezielter Maßnahmen bedarf, um die Wirksamkeit des Controlling sicherzustellen.

Seit wenigen Jahren erst hat sich daher die Erkenntnis durchgesetzt, dass das Controlling selbst letztlich Bestandteil der oben angesprochen Gemeinkostenbereiche ist und sich daher - bei konsequenter Fortfuihrung dieses Gedankens -

1 Der Begriff Controlling-Bereich wird im Rahmen dieser Arbeit synonym mit dem Begriff des Controllerdienst und dem dem anglo-amerikanischen Sprachraum entstammenden Terminus Controllership verwandt. Gemeint ist stets die institutionelle Ausgestaltung des Controlling. Vgl. Weber (1999), S. 2 und Herzog (1999), S. 3 sowie Kap. 2.1.3.2.

2 Zur geläufigen Verwendung des Begriffs vgl. den Buchtitel von Berens/Hoffjan/Schmitting (1999).

3 Allg. zu Controller-Bildern vgl. Weber/Schäffer (2000), S. 119-122.

4 Vgl. Kraus (2001), S. 304.

s Vgl. hierzu die Kongressdokumentation von Niedereichholz (1999), S, $1 \mathrm{ff}$ 
ebenfalls einer markt- und kundenbezogenen Betrachtungsweise unterziehen muss. Verschärft wird diese Situation durch die zunehmende Konkurrenzgefährdung für Leistungen des Controlling ${ }^{6}$, die durch Outsourcing und eine Liberalisierung unternehmensinterner Märkte begünstigt wird.' Die zentrale Rolle des Controlling im Rahmen der Unternehmensführung und die damit verbundenen vielfältigen Schnittstellen zu anderen Unternehmensteilbereichen erfordern ein gezieltes Bereichsmanagement, ausgehend von einer zukunftsgerichteten Planung über den Leistungserstellungsprozess bis hin zum Rückkopplungsprozess und der Erfolgskontrolle. Insbesondere die fakultative Funktion des internen Rechnungswesens deutet unmittelbar darauf hin, ${ }^{8}$ dass der Betrieb eines darauf aufbauenden umfassenden Controlling nur dann zu rechtfertigen ist, wenn dieses sich konsequent an den Bedürfnissen seiner jeweiligen internen Kunden ausrichtet. Als interner Kunde des Controlling soll zunächst pauschal das Management in allen Funktions- und Objektbereichen eines Unternehmen angesehen werden, bevor im Rahmen einer differenzierten Analyse weitere potenzielle Kunden des Controlling betrachtet werden.

Verhaltenswissenschaftliche Analysen der Vergangenheit betonten drei grundsätzlich alternative Vorgehensweisen, Controlling-Ziele $e^{9}$ in einem Unternehmen durchzusetzen. Zwang als ,rücksichtslose Vorgabe und Durchsetzung von Normen, ohne dabei die Bedürfnisse und Belange anderer zu berücksichtigen"10, ist immer dann eine Alternative, wenn der Controlling-Bereich selbst über die Autorität zur Durchsetzung von Maßnahmen verfügt, oder aber von der Führung gedeckt wird. Bei Manipulation, wird das Verhalten des anderen in meinem Sinne oder im Sinne meines Auftraggebers beeinflusst, und zwar zu fremden Nutzen"". Das für eine dauerhaft angelegte Austauschbeziehung zwischen Controller und seinem internen Kunden notwendige Vertrauen kann nachvollziehbar durch diese beiden Vorgehensweisen nicht geschaffen werden. Vielmehr stellt die Möglichkeit einer Motivation der Leistungsempfänger den einzig sinnvollen Ansatz zur Verfolgung der auf die Unternehmensziele abzustimmenden Controllingziele dar. Die Motivation, Controlling-Leistungen in Anspruch zu nehmen, ergibt sich verhaltenswissenschaftlich aus einem in einer Entschei-

\footnotetext{
Allgemein werden die Begriffe Leistung, Leistungsbündel, Produkt und Leistungsgruppe bezogen auf das Controlling im Sinne unterschiedlicher Aggregationsebenen aufgefasst. Je nach Differenzierungsgrad und Gliederungstiefe des Leistungsangebotes wird auf einen dieser Begriffe rekurriert.

7 Beispielhaft zum Outsourcing von Controlling-Leistungen vgl. Müller/Fickel (1997), S. 109-111.

8 Vgl. Küpper/Weber/Zünd (1990), S. $281 \mathrm{ff}$.

$9 \mathrm{Zu}$ den Zielen des Controlling vgl. Kap. 2.1.3.1.

10 Stamm (1992), S. 259.

11 Stamm (1992), S. 259.
} 
dungssituation empfundenen Mangel und der Erkenntnis, diesen durch zielgerichtete Maßnahmen beseitigen zu können. Grundlegend für den Leistungsempfänger sind in solch einer Situation Transparenz, Verständlichkeit und Nützlichkeit der Leistungen des Controlling. ${ }^{12}$ Transparenz bezieht sich hierbei auf den Inhalt und Umfang der Leistung. Die Verständlichkeit hängt demgegenüber vom Wissensstand und der Interpretationsfähigkeit des Adressaten ab. Darüber hinaus determiniert insbesondere die Nützlichkeit das Ausmaß der Motivation, da die Zweckmäßigkeit der Leistung letztlich ausschlaggebend für die Beseitigung der Mangelsituation ist. ${ }^{13}$ Unterstellt man, dass Motivation ein probates Mittel zur Durchsetzung der Ziele des Controlling ist, wird deutlich, dass der Gewährleistung von Transparenz, Verständlichkeit und Nützlichkeit der Leistungen des Controlling eine gewichtige Rolle zukommt. Da es sich bei diesen drei Anforderungen um subjektiv geprägte Tatbestände handelt, ist der Controller gezwungen, die Präferenzen und das Nutzungsverhalten seiner Kunden zu ergründen, um so seinen Beitrag zur Erfüllung der Unternehmensziele abzusichern. ${ }^{14}$ Hierzu bedarf es von Seiten des Controlling einer konsequenten Kundenorientierung.

Zur Gewährleistung einer umfassenden Kundenorientierung bietet der konventionelle Ansatz des Marketing Lösungsmöglichkeiten für unternehmensexterne Absatzmärkte an. Ebenso wie es im Bereich des externen Marketing notwendig ist, die Marketing-Ziele konsequent an den Zielvorstellungen des Gesamtunternehmens auszurichten, ist es für den Controlling-Bereich unerlässlich, die Bereichsziele den Meta-Zielen unterzuordnen. Ansonsten besteht die Gefahr der Förderung von Bereichsegoismen, die der Zielerreichung des Gesamtunternehmens zuwiderlaufen können. Da aber im Regelfall Analogien zu den Begrifflichkeiten und Vorgehensweisen des externen Marketing für organisationsinterne Austauschprozesse nicht uneingeschränkt bestehen, erscheint eine unkritische Übernahme solcher Lösungsansätze fragwürdig. Der mittlerweile in der wissenschaftlichen Literatur etablierte Ansatz des internen Marketings bemüht sich, den Besonderheiten unternehmensinterner Beziehungen unter Marketinggesichtspunkten durchgängig Rechnung zu tragen. Eine konsequente Anwendung auf einzelne Funktionsbereiche von Unternehmen ist jedoch selten. ${ }^{\text {is }}$

12 Vgl. Stamm (1992), S. 259. Zu Motivationstechniken im Controlling vgl. Biel (1988), S. 40 f.

Bezieht man dies beispielhaft auf Berichte als Controlling-Leistungen, rechtfertigt diese Zweckorientierung erst den Informationscharakter der Leistung, im Gegensatz zur reinen Nachricht. Vgl. Gmünden (1993), Sp. 1725.

14 Zum Zusammenhang von Controlling-Zielen und Unternehmenszielen vgl. Anderson/O'Reilly (1981), S. 491 ff.

15 Eine beispielhafte Anwendung findet sich bei Stauss/Neuhaus (1995), S. $575 \mathrm{ff}$. 
Zielsetzung dieser Arbeit soll daher sein, die Relevanz von MarketingDenkstrukturen und -Instrumenten für das Controlling zu überprüfen, um im Ergebnis einen Aktionsrahmen für ein kundenorientiertes Controlling zu entwickeln, der in Abhängigkeit moderierender Variablen situativ auszufüllen ist. Hierzu bedarf es der Nutzung und controllingspezifischen Modifikation konventioneller Marketing-Techniken. Ergänzend zu Techniken des Marketing gilt es, Möglichkeiten der Anwendung marktorientierter Instrumente des Controlling auf den Controllerbereich selbst zu prüfen. Auf dieser Grundlage kann, unter besonderer Berücksichtigung der subjektiven Wahrnehmung alternativer Kunden, über operationale Leistungen, Qualitäten und Preise eine strukturierte Basis für interne Kunden-Lieferanten-Beziehungen des Controlling geschaffen werden. Dabei besteht die besondere Herausforderung darin, eine konsequente Ausrichtung am Kundennutzen sicherzustellen, ohne das Controlling als bedingungsloses Werkzeug des Kundenwunsches zu verstehen. ${ }^{16}$ Gelingt dies, gilt die Maxime, „,er Kunde ist König“ auch für das Controlling. ${ }^{17}$

\subsection{Methodik und Vorgehensweise}

Der weite Verbreitungsgrad des Controlling in nationalen und internationalen Unternehmen deutet auf eine hohe Praxisrelevanz der dem Controlling zugeschriebenen Aufgaben hin. ${ }^{18}$ Der Dienstleistungscharakter des Controlling macht es erforderlich, die zur Aufgabenerfüllung notwendigen Leistungen, sowie die Modalitäten ihrer Inanspruchnahme an den Bedürfnissen der jeweiligen Adressaten auszurichten. Demgegenüber zeichnet sich die wissenschaftliche Diskussion zum Controlling durch das Fehlen geeigneter Erforschungs- und Systematisierungsansätze der Dienstleistung Controlling aus. Die wenigen Publikationen zur Kundenorientierung im Controlling sind durch teilweise voneinander abweichende Verständnisse gekennzeichnet, da einerseits interne Controlling-Kunden und andererseits externe Unternehmenskunden betrachtet werden. Der hohe Variabilitätsgrad der Ausführungen zum kundenorientierten Controlling ist zudem auf die überwiegend anzutreffenden unternehmensbezogenen Einzelfalldarstellungen zurückzuführen. Die für das Controlling charakteristische Polarität zwischen Praktikerdarstellungen und Theorieansätzen wird in dem betrachteten Bereich weiter dadurch verstärkt, dass weniger controllingnahe Theorien wie die Systemtheorie oder die Entscheidungstheorie Erklä-

\footnotetext{
Vgl. Trilse (2000), S. IV/2

17 Vgl. hierzu den Titel des Editorial von Horváth (1995), S. 183.

18 Empirisch zum Stand des Controlling in deutschen Unternehmen vgl. Amshoff (1991). Speziell zur Kostenrechnungspraxis in der deutschen Industrie vgl. Währisch (1998b).
} 
rungsansätze bereithalten, als vielmehr verhaltenswissenschaftliche, vermeintlich marketingaffine Theorien wie die Neue Institutionenökonomie. ${ }^{19}$ Einen einheitlichen Bezugsrahmen zur internen Kundenorientierung des Controlling gibt es nicht, so dass ein diesbezügliches Theoriedefizit festzustellen ist.

Die Methodik der in dieser Arbeit über einen Forschungsprozess angestrebten Theorieentwicklung orientiert sich an dem Stufenmodell von GROCHLA. ${ }^{20}$ Abbildung 1 zeigt einen schematischen Überblick über die Struktur der Arbeit, welche mit dem Stufenmodell korrespondiert.

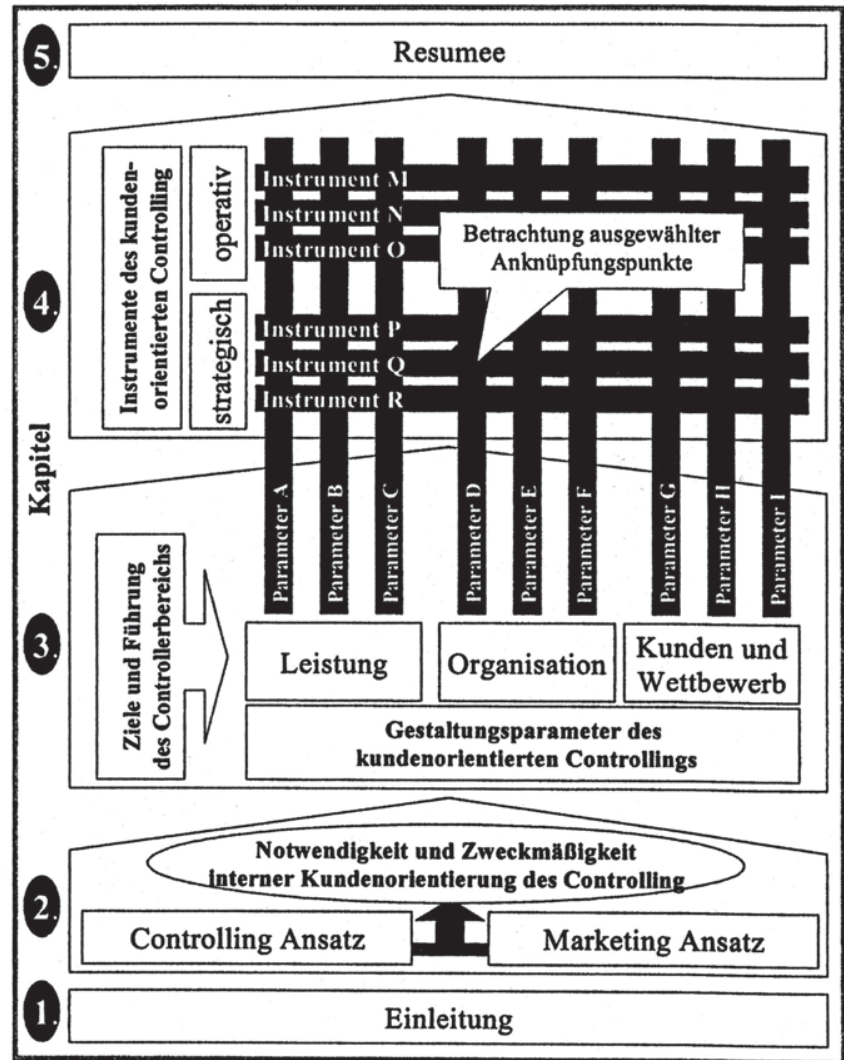

Abbildung 1: Schematischer Überblick über die Struktur der Arbeit

19 Zur Integration der Neuen Institutionenökonomie in das Controlling vgl. Küpper (1997), S. 44 ff.

20 Zum hier und im Folgenden nachvollzogenen Forschungsprozess der Theorieentwicklung vgl. Grochla (1978), S. $61 \mathrm{ff}$. 
Im Rahmen der ersten Stufe werden zur Formulierung und Erfassung des Phänomens Kundenorientierung im Controlling terminologische Aussagen getätigt, um auf ein angemessenes begriffliches Instrumentarium zurückgreifen zu können (Kapitel 2). Hierzu wird der Theorieansatz des Controlling vorgestellt und problemorientiert die Notwendigkeit der internen Kundenorientierung abgeleitet. Im Rahmen der Vorstellung des Marketingansatzes werden primär solche Entwicklungstendenzen betont, die ein hohes Integrationspotenzial im Hinblick auf die kundenorientierte Ausgestaltung des Controlling aufweisen.

In der zweiten Stufe geht es um eine Deskription der Realität zu einer genaueren Abgrenzung des Untersuchungsobjektes, wofür auf die Darstellung typischer Fälle, praktischer Beispiele und statistischer Daten zurückgegriffen wird. Inhaltlich entspricht dies in der Arbeit einer Vorstellung der leistungsbezogenen, organisationsbezogenen sowie kunden- und wettbewerbsbezogen Rahmenbedingungen des kundenorientierten Controlling, da diese als Gestaltungsfundament angesehen werden (Kapitel 3). In Kapitel 3 erfolgt ergänzend die Abbildung der dritten Stufe des Forschungsprozesses, in der explanatorische Aussagen über die Relationen der im Bezugsrahmen enthaltenen Größen getätigt werden. Der Bezugsrahmen als Ordnungsschema für die Realität, muss die wesentlichen, für den Problemkomplex ,interne Kundenorientierung des Controlling" relevanten Größen enthalten. Inhaltlich entspricht dies den aus den Rahmenbedingungen abgeleiteten marketingrelevanten Charakteristika. Diese sind im Sinne von Gestaltungsparametern aufzufassen, denen Hypothesen über handlungsrelevante Zusammenhänge zugrunde liegen.

Die vierte Stufe der Theorieentwicklung hat die Bildung praxeologischer, auf den Entscheidungsrahmen bezogener Aussagen zum Ziel (Kapitel 4.). Hierbei ist der Entscheidungsrahmen als die Variante des gedanklichen Bezugsrahmens aufzufassen, die sich auf praktische Handlungszwecke bezieht. Im Sinne der von einem Entscheidungsrahmen geforderten hohen Informativität und guten entscheidungstechnischen Verwendbarkeit bieten die in Kapitel 4 dargestellten Aktionsgrundlagen konkrete Anhaltspunkte für die operative und strategische Gestaltung der internen Kundenorientierung im Controlling. Die in diesem $\mathrm{Zu}-$ sammenhang vorgestellten Instrumente vermögen in unterschiedlichem Umfang und mit abweichenden Schwerpunkten die marketingrelevanten Gestaltungsparameter abzudecken. Der Forschungsprozess kann als beendet angesehen werden, soweit die vierte Stufe abgeschlossen ist. Inhaltlich wird die Arbeit von einer Einleitung (Kapitel 1) und einem Resumee (Kapitel 5) umschlossen. 


\section{Theoretische Fundierung der Teilansätze}

Sollen auf der Grundlage einer Forschungsarbeit Handlungsempfehlungen gegeben werden, ist es unerlässlich, eine angemessene Durchdringung des gewählten Forschungsgegenstandes vorzunehmen. Zur Systematisierung derartiger Aktivitäten bedarf es eines wissenschaftlichen Rahmens, welcher die Funktion einer „diagnostischen Vorstufe ${ }^{\text {"221 }}$ übernimmt. Bezogen auf die Betrachtung der internen Kundenorientierung des Controlling müssen die aktuellen Stände der Marketing- und Controlling-Forschung auf solche Entwicklungstendenzen, die in der Lage sind, Aussagen über das Erkenntnisobjekt zu machen, überprüft werden. Mittels eines derartigen Bezugsrahmens kann eine ,Inventur des Untersuchungsgegenstandes ${ }^{\text {"222 }}$ betrieben werden. Zur Operationalisierung des Untersuchungsgegenstandes wird eine zielorientiert deduktive Vorgehensweise gewählt. Hierdurch soll der Überbewertung subjektiver Einzelmeinungen begegnet werden. Demgegenüber muss der Bedeutung von Kontextvariablen angemessen Rechnung getragen werden, da Handlungsempfehlungen nur in Abstimmung mit diesen wirksam umgesetzt werden können.

\subsection{Der Controlling Ansatz}

Zielsetzung des folgenden Abschnitts ist eine grundlegende Darstellung des Controlling Ansatzes, um für den weiteren Verlauf der Arbeit ein „konsensfähiges" Verständnis sicherzustellen. ${ }^{23}$ Hingegen ist nicht beabsichtigt, an dieser Stelle eine Basisdiskussion über unterschiedliche Ansätze zu führen oder darüber hinausgehend diese noch um eine weitere, eigene Definition des Autors zu ergänzen. Vielmehr soll über die Verdeutlichung von Gemeinsamkeiten und verbindenden Elemente unterschiedlicher Konzeptionen das bereits postulierte konsensfähige Verständnis sichergestellt werden. Ein solches objektives Verständnis scheint auch größtenteils in der Praxis vorzuherrschen, da die zur wissenschaftlichen Differenzierung verwandten Aspekte regelmäßig im Praxisalltag von integrierenden dominiert werden. Insbesondere auch die Zielsetzung, Teilbereiche dieser Arbeit als konzeptionellen Rahmen für Maßnahmen in der Unternehmenspraxis zu verwenden, erfordert einen pragmatischen Umgang mit den in der Literatur vertretenen Ansätzen.

21 Heinen (1985), S. 134.

22 Amshoff (1993), S. 49.

23 Zur Notwendigkeit eines konsensfahigen Controlling-Verständnisses vgl. Küpper/Weber/Zünd (1990), S. 282. 
Dennoch soll vorab, insbesondere auch zur Legitimation einer wissenschaftlichen Auseinandersetzung mit Controlling-Fragen, die Eigenständigkeit des betriebswirtschaftlichen Bereichs „Controlling” dargelegt werden. Hierdurch soll dem Verdacht begegnet werden, Controlling lediglich als moderne Bezeichnung bekannter Aufgabenbereiche oder als neuen Oberbegriff zu verwenden. ${ }^{24}$ Neben einer solchen wissenschaftstheoretischen Betrachtung soll nachfolgend auf die für die Ausgestaltung des Controlling relevanten Extrempositionen eingegangen werden. Letztlich soll verdeutlicht werden, dass die in der Praxis anzutreffenden Ausprägungen diesbezüglich synthetischen Charakter aufweisen.

Ergebnis dieses Abschnitts ist ein geeigneter Verständnisrahmen, der es dem Leser erlaubt, die im weiteren Verlauf der Arbeit getätigten Aussagen auf Basis eines intersubjektiven und integrationsorientierten Controlling-Begriffs zu deuten.

\subsubsection{Controlling als eigenständiger betriebswirtschaftlicher Bereich?}

KANT stellt bezüglich der Bedeutsamkeit einer eigenen Problemstellung fest: „Wenn man die Erkenntnis als Wissenschaft darstellen will, so muss man zuvor das Unterscheidende, was sie mit keiner anderen gemein hat und was ihr eigentümlich ist, genau bestimmen können, widrigenfalls die Grenzen aller Wissenschaften ineinanderlaufen und keine derselben gründlich abgehandelt werden kann."25

Die Problematik des Controlling-Begriffs ergibt sich diesbezüglich aus der Tatsache, dass Controlling eine Entwicklung der Praxis ist. Die zeitlich versetzte wissenschaftliche Aufbereitung dieses Phänomens führte zu einer Vielfalt von Deutungen, die bis heute Bestand haben. Wesentliche Voraussetzung für eine zielführende wissenschaftliche Auseinandersetzung mit dem Controlling ist ein einheitliches Verständnis des Gegenstandes „Controlling”. ${ }^{26} \mathrm{Zu}$ diesem Zweck erscheint es notwendig, Abgrenzungskriterien benennen zu können, die die Problemstellungen des Controlling von denen anderer betriebswirtschaftlicher Bereiche trennen. Auch wenn - bedingt durch die späte Herausbildung des Controlling im Rahmen der Historie der Betriebswirtschaft - für viele Problemstellungen schon Teilansätze vorliegen, erscheint eine eigenständige Betrach-

\footnotetext{
24 Vgl. Küpper (1997), S. 3 f.

25 Kant (1783), S. 13.

26 Vgl. Küpper (1997), S. 3 sowie Lanter (1996), S. 14 ff.
} 
tung des Bereichs Controlling notwendig, soweit die theoretische Fundierung zu Erkenntnisfortschritten führt.

Im Folgenden sollen unterschiedliche Fundierungsmöglichkeiten aufgezeigt werden und diese in bezug auf ihre Differenzierungswirkung bewertet werden. Auf diese Weise gilt es, die betriebswirtschaftlich eigenständige Problemstellung des Controlling zu verdeutlichen.

\subsubsection{Alternative Ansätze für Controlling-Konzeptionen}

Bei der Abgrenzung unterschiedlicher Controlling-Konzeptionen waren in der Vergangenheit sowohl induktive als auch deduktive Vorgehensweisen zu beobachten. Insbesondere die Tatsache, dass die Entwicklung des Controlling ihren Ausgangspunkt in der Praxis hatte, führte anfänglich zu einer Vielzahl induktiv abgeleiteter Controlling-Konzeptionen. Problematisch in diesem Zusammenhang erweist sich die Vermengung von organisatorischen und funktionalen Aspekten des Controlling. Wissenschaftstheoretisch gesehen, muss jedoch eine Abgrenzung des Gegenstandes Controlling seiner organisatorischen Ausgestaltung vorgelagert sein, da diese situativ differieren kann. ${ }^{27}$ So gesehen ist eine Fundierung des Controlling vornehmlich aus funktionaler Sicht vorzunehmen, da hierdurch die Abgrenzbarkeit und Eigenständigkeit des Verständnisses deutlich wird. Vertreten werden diesbezüglich unterschiedliche deduktive Ansätze, die sich durch ihre Deduktionsbasen unterscheiden.

Als konzeptionsdeterminierend werden allgemein die Controllingziele, -funktionen, -instrumente und -institution angesehen.$^{28}$ Konstituierend für eine Controlling-Konzeption können jedoch nur die Controlling-Ziele sein, da diese kontextunabhängig sind;29 ansonsten wäre eine intersubjektive ControllingKonzeption nicht vorstellbar. ${ }^{30}$ Demgegenüber sind Controlling-Funktionen, Controlling-Instrumente und die Controlling-Institution einer spezifischen Binnen- und Umweltdynamik ausgesetzt und von daher kontextunabhängig nicht bestimmbar. Im Folgenden sollen mit KÜPPER differierende ControllingKonzeptionen unterschieden werden. ${ }^{31}$

27
Zur Aufgabenzuordnung in einer Organisation vgl. Kap 3.2.1.2.
28
Vgl. Hahn (1997), S. 16.
Vgl. Vahs (1994), S. 76 ff.
30
Vgl. Harbert (1982), S. 163 ff.
Vgl. Kupper (1997), S. 7 ff. vergleichbar Weber (1999), S. 20 ff.

Thomas Mosiek - 978-3-631-75321-7 
Die gewinnzielorientierte Controlling-Konzeption stellt die Notwendigkeit in den Mittelpunkt, dem übergeordneten formalen Gewinnziel des Gesamtunternehmens zuwiderlaufende Bereichsziele zu erkennen und auf das Oberziel abzustimmen. ${ }^{32}$ Damit stellt die operativ-taktische Gewinnausrichtung jedoch eine Zielsetzung dar, die bereits von anderen Führungsteilsystemen verfolgt wird. Auch wenn die Gewinnzielausrichtung für das Controlling - unbestreitbar - von besonderer Bedeutung ist, sollte sie nicht als originär differenzierendes Merkmal verwendet werden, weil dadurch der Anwendungsbereich des Controlling unangemessen eingeschränkt würde.

Die informationsorientierte Controlling-Konzeption fokussiert auf die Koordination von Informationsbedarf und Informationsbeschaffung. ${ }^{33}$ Insbesondere die Entwicklung moderner Datenverarbeitungs-, Informations- und Kommunikationstechnologien hat im Bereich des betrieblichen Rechnungswesens zu einem verstärkten Koordinationsbedarf beigetragen. ${ }^{34}$ Darüber hinaus unterstützen Überlegungen, die im späteren Verlauf dieser Arbeit getätigt werden, die Notwendigkeit einer differenzierteren adressatenbezogenen Aufbereitung und Bereitstellung von Informationen..$^{35}$ Auch wenn damit die Problemstellung der informationsorientierten Controlling-Konzeption abgrenzbar und theoretisch fundiert ist, erscheint fraglich, inwieweit es sich hierbei eventuell nur um eine auf die Umweltdynamik zurückzuführende Umbenennung des Rechnungswesens handelt, ohne ein eigentlich neues Erkenntnisobjekt.

Erweitert man die durch die Unternehmensrechnung zu gewährleistende Informationsversorgung um Abstimmungsvorgänge zwischen dieser und der unternehmerischen Planung und Kontrolle, so gelangt man zu einer planungs- und kontrollorientierten Controlling-Konzeption. ${ }^{36}$ Die im Rahmen dieser Konzeption vertretene Ergebnisorientierung korrespondiert in weiten Teilen mit der bereits angesprochenen Gewinnzielorientierung, reicht jedoch durch die Integration strategischer Aspekte darüber hinaus. Ferner werden die im Rahmen der informationsorientierten Controlling-Konzeption angesprochenen Koordinationsvorgänge um solche ergänzt, die zwischen den Teilsystemen Informationsversorgung, Planung und Kontrolle stattfinden. ${ }^{37}$ Bei einem solchen Ansatz wird

\footnotetext{
Zur näheren Erläuterung dieses Ansatzes vgl. Pfohl/Zettelmeyer (1987), S. $150 \mathrm{ff}$.

Vgl. Müller (1974), S. 145 ff.

Zur Verbindung von Controlling und Datenverarbeitungstechnik vgl. These 7.4 von Küper/Weber/Zünd (1990), S. 288 f.

Vgl. hierzu insbesondere Kap. 4.1.2.1.1.

Eine zusammenfassende Darstellung der Konzeption von HoRvÁTH findet sich bei Horváth (1998), S. 142 ff.

Vgl. hierzu die korrespondierende Abbildung von Horvatth (1998), S. 143.
} 
ersichtlich, dass Controlling systemübergreifend zu verstehen ist, und nicht ausschließlich auf systeminterne Koordinationsvorgänge beschränkt werden sollte.

Die sich in den dargestellten Controlling-Konzeptionen abzeichnende Entwicklung deutet darauf hin, dass die dem Controlling zugeschriebenen Koordinationsaufgaben maßgeblich von der Differenzierung des Führungssystems abhängen. Fraglich ist, inwieweit die Abgrenzung weiterer Führungsteilsysteme notwendig ist, um potenzielle Koordinationsdefizite aufzudecken.

\subsubsection{Koordinationsorientierte Controlling-Konzeption}

Auf Basis der planungs- und kontrollorientierten Controlling-Konzeption soll nachfolgend die Reichweite der im Mittelpunkt des Controlling stehenden Koordinationsaktivitäten diskutiert werden, ${ }^{38}$ um abschließend für den weiteren Verlauf dieser Arbeit einen theoretischen Bezugsrahmen zugrundelegen zu können.

\subsubsection{Konzeptionelle Grundlage}

Die bisherigen Erläuterungen deuten darauf hin, dass Controlling grundsätzlich als „eine Komponente der Führung sozialer Systeme”"39 anzusehen ist, welche zur Verbesserung ihrer Funktionsfähigkeit beitragen soll. Im Rahmen einer durch zunehmende Umweltdynamik und steigende Unternehmenskomplexität geprägten Entwicklung, wurden vielfach Spezialisierungsvorteile nicht nur im Leistungssystem, sondern auch im Führungssystem angestrebt. Die hierdurch hervorgerufene Bildung von Führungsteilsystemen erfordert - analog zur Spezialisierung im Leistungssystem - eine zielgerichtete Zusammenführung der Einzelsysteme. Diese als Koordination zu bezeichnenden Abstimmungsmaßnahmen zwischen den Führungsteilsystemen einerseits und des Gesamtsystems auf die Umweltdynamik andererseits, begründen die Notwendigkeit des Controlling. Demnach stellt das kontextunabhängige Ziel der Koordination das konstitutive Merkmal des Controlling dar, welches daher ,erst durch den systematischen Ausbau eines gegliederten Führungssystems entsteht und Gewicht erhält"*0.

38 Die Erkenntnis, dass die Koordination im Mittelpunkt der Konzeption des Controlling steht, hat sich bei der Mehrzahl der Autoren durchgesetzt. Ferner stellt die Herstellung eines Bezugs zu den Unternehmenszielen ein konsensfahiges gemeinsames Merkmal dar. Vgl. beispielhaft Horváth (1998), S. 147.

39 Küpper/Weber/Zund (1990), S. 282.

40 Küpper (1997), S. 16. 
Zur Erreichung dieser Zielsetzung stehen den Aufgabenträgern des Controlling zwei Koordinationsarten zur Verfügung. ${ }^{41}$ Mittels systembildender Maßnahmen sollen Strukturen etabliert werden, die die Abstimmung von Aufgaben erleichtern. Demgegenüber soll durch systemkoppelnde Maßnahmen in einem bestehenden Systemgefüge laufend die Koordination und darüber hinaus dessen Anpassungsfähigkeit an eine dynamische Umwelt sichergestellt werden.

Stellt man dieser Argumentation folgend die systeminterne und die umweltbezogene Koordination in den Mittelpunkt der Betrachtung, stellt sich letztlich die Frage, welche Führungsteilsysteme in diesen Ansatz zu integrieren sind. Nach KÜPPER ist der bisherige Betrachtungsrahmen im Sinne des informations- und planungs- und kontrollorientierten Ansatzes von HORVÁTH um die Führungsteilsysteme Organisation und Personalführung zu ergänzen..$^{42}$ Bei der Darstellung einer derartigen Konzeption des Controlling besteht, bedingt durch die notwendige Kontextunabhängigkeit, oftmals die Gefahr, die Abgrenzung zwischen Führung und Controlling zu verwischen. HORvÀTH bewegt dies letztlich dazu, die Koordinationsfunktion auf Informations-, Planungs- und Kontrollsystem zu beschränken. ${ }^{43}$ Insgesamt erscheint jedoch die Einbeziehung aller Führungsteilsysteme - im Sinne zu koordinierender Elemente - zweckmäßig, da ein derartig verstandener Controlling Ansatz gerade von der „Abstimmungsreichweite" lebt und darüber hinaus keinerlei konkrete Gründe für den Ausschluss einzelner Führungsteilsysteme sprechen. ${ }^{44}$ Theoretisch erlaubt insbesondere auch die Unterscheidung zwischen Primärkoordination, als Koordination des Leistungssystems durch die Führung, und Sekundärkoordination, als Koordination innerhalb des Führungsgesamtsystems, eine Abgrenzung der Aufgabenbereiche zwischen Führung (Primärkoordination) und Controlling (Sekundärkoordination). ${ }^{45}$

Deutlicher wird Praktikern die Unterscheidung zwischen Führung und Controlling regelmäßig erst im Rahmen der institutionellen Ausgestaltung. ${ }^{46}$ Ein Ansatzpunkt, diesen Unterschied auch konzeptionell zum Ausdruck zu bringen,

\footnotetext{
41 Die im Folgenden dargelegten Koordinationsarten gehen zurück auf Horváth (1998), S. 119 ff.

42 Vgl. Küpper (1997), S. 15.

43 Vgl. Horváth (1998), S. 147 sowie S. 149, vergleichbar Schneider (1994), S. 325 und S. 330.

44 So auch Mertes (2000), S. 124, im Gegensatz zu Karlowitsch (1997), S. 9 f.

$45 \mathrm{Vgl}$. Weber (1999), S. 26 ff.

46 Vgl. hierzu Kap. 3.2.1.2 ff.
} 
bietet die Betonung der Servicefunktion des Controlling. ${ }^{47}$ Basis des bisherigen Controlling-Verständnisses war die Koordinationsfunktion. Servicecharakter hat die Koordination regelmäßig dann, wenn durch die Abstimmung der Teilsysteme die Zielerreichung in einem Teilsystem unterstützt wird. ${ }^{48}$ In diesem Zusammenhang wird die unterstützende Wirkung oftmals durch die Bereitstellung geeigneter Koordinationsmethoden oder koordinationsfördernder Informationen erreicht. Die zu Beginn postulierte, und für viele Praktiker signifikante Gewinnzielausrichtung findet regelmäßig bei privatwirtschaftlich organisierten Unternehmen im Controlling eine angemessene Berücksichtigung, sollte jedoch im Sinne einer Übertragbarkeit auf die Gesamtheit sozialer Systeme einen Controlling Ansatz nicht dominieren. ${ }^{49}$

\subsubsection{Ausgestaltungskontinuum}

Neben der bislang vorgenommenen kontextunabhängigen, konzeptionellen Fundierung des Controlling, soll die Betrachtung im Folgenden um Elemente einer kontextabhängigen Wahrnehmung der Controlling Realität erweitert werden, da die Einbindung des Controlling in interne Austauschbeziehungen regelmäßig einer institutionellen Grundlage bedarf. Insbesondere, wenn an späterer Stelle Handlungsempfehlungen für eine effiziente und effektive ${ }^{50}$ Ausgestaltung des Controlling gegeben werden sollen, ist es wesentlich, auf diejenigen Controllingauffassungen einzugehen, die die Wahrnehmung der damit konfrontierten Personen bestimmen. Die regelmäßig theoretisch überhöhte Konzeptionsdiskussion vermag hierzu oftmals keinen Beitrag zu leisten.

Im weiteren sollen auf Basis des zwischen den zwei Extrempositionen „Führungsbasiertes Controlling" und „Fachbereichsbasiertes Controlling“ bestehenden Ausgestaltungskontinuums mögliche Controllingauffassungen diskutiert werden." Eine derartige Diskussion kann weitere Einblicke in die alltägliche Beziehungsdynamik zwischen Controller und Manager geben, welche für eine zielgerichtete Ausgestaltung des Controlling maßgebliche Bedeutung besitzt. Ferner beeinflusst die jeweils kontextabhängig zugrundegelegte Controllingauf-

47 Küpper unterscheidet drei abgeleitete Zwecksetzungen des Controlling. Neben der Anpassungs- und Innovationsfunktion und der Zielausrichtungsfunktion nennt er in diesem Zusammenhang die Servicefunktion. Vgl. Küpper (1997), S. 17-19.

48 Vgl. Küpper (1997), S. 19.

49 Ansonsten bestünde die Gefahr einer Sachzielgefahrdung. $\mathrm{Zu}$ Controlling in offentlichen Institutionen vgl. bspw. Mertes (2000), Junga (2000) sowie Schrijvers (1999).

Zu der Abgrenzung der Begriffe Effizienz und Effektivitat vgl. Kap. 3.

s1 Dieser Vorgehensweise wurde in Anlehnung an Lanter gewahlt. Vgl. Lanter (1996), S, $14 \mathrm{ff}$. 
fassung die Zuordnung und Ausführung von Controlling-Aufgaben durch den Controllerdienst, wodurch gleichsam wesentliche Rahmenbedingungen für eine kundenbezogene Leistungserstellung des Controllerdienstes gesetzt werden..$^{52}$ Die hier mit LANTER gewählte mögliche Führungs- und Fachbereichsbasierung eines Controllingverständnisses erscheint für den weiteren Verlauf der Analyse zweckmäßig, da im Rahmen einer kunden- und dienstleistungsorientierten Betrachtung des Controlling einerseits dem Fachbereich - im Sinne der Serviceeinheit des Controllerdienstes - eine besondere Bedeutung zukommt, andererseits aber auch Controlling von der Führung selbst betrieben werden kann. Soweit diese Aufgabenwahrnehmung für einen spezifischen Controllerdienst nicht grundsätzlich geregelt ist, kann im Rahmen einer marketingorientierten Betrachtung von möglichen Bezugsalternativen ausgegangen werden. Neben den Extrempositionen ist insbesondere auf die Integrativität der Leistungserstellung im Controlling einzugehen, die nur auf Basis einer dauerhaften Interaktion zwischen Führung und Controlling zu gewährleisten ist.

Die hier verwandte Systematisierung von führungs- und fachbereichsbasiertem Controlling weist deutliche Analogien zu anderen in der Literatur verwandten Abgrenzungen auf. So wird die Management-Funktion ${ }^{53}$ des Controlling oftmals der Führungsunterstützungsfunktion ${ }^{54}$ gegenübergestellt. Ferner wird regelmäBig zwischen funktionalem Controlling-Verständnis und institutionellem Verständnis unterschieden. ${ }^{55}$

\subsection{Führungsbasiertes vs. fachbereichsbasiertes Verständnis}

Ein führungsbasiertes Controlling-Verständnis impliziert, dass die Verantwortung für das Controlling institutionell der Führung zuzuordnen ist. ${ }^{56}$ Unter Rückgriff auf den Systemansatz ${ }^{57}$ kommt dem Controlling im Rahmen der regelkreisorientierten Führung die Funktion zu, Führungsprozesse zielgerichtet mittels Planungs-, Informations-, Analyse- und Kontrollaktivitäten zu steuern. Ein solches Controlling-Verständnis korrespondiert idealtypisch mit dem Führungsprinzip des Management by Objectives-Ansatzes (MbO). ${ }^{58}$ Mittels derarti-

52 Vgl. hierzu Kap. 3.2.1.1.

53

Dieser Ansicht sind Ulrich (1985), S. 15 ff. und Deyhle (1990).

54 Standpunkt bspw. von Serfling (1992), S. 16 ff.

s5 Zur Abgrenzung zwischen institutionalem und funktionalem Controlling-Verständnis vgl. Schoch (1993), S. $16 \mathrm{ff}$.

56 Hierzu und im Folgenden vgl. Lanter (1996), S. $19 \mathrm{ff}$.

57 Ausführlich zu dem Sytemansatz vgl. Ulrich (1978), S. $13 \mathrm{ff}$.

58 Zum Gundmodell des Management by Objectives vgl. Bleicher/Meyer (1976).S. 242 , 
ger Steuerungsmechanismen soll neben der unternehmensinternen Koordination insbesondere auch die Anpassungs- und Reaktionsfähigkeit bezogen auf diskontinuierliche Umweltbedingungen gewährleistet werden. Die Integration eines solchen führungsbasierten Controlling-Verständnisses in das Werte- und Normensystem eines Unternehmens kann in unterschiedlichem Umfang erfolgen. Im Extrem reicht das Verständnis bis hin zu einer umfassenden Führungsphilosophie..$^{59}$ Diese zeichnet sich weniger durch operationale Instrumente als vielmehr durch eine dem ,unternehmerischen Agieren vorgelagerte Denk- und Handlungskonzeption ${ }^{\text {"60 }}$ aus.

Im Gegensatz zum führungsbasierten Controlling-Verständnis liegt beim fachbereichsbasierten Verständnis die Verantwortung für das Controlling überwiegend oder sogar ausschließlich bei dem damit betrauten Fachbereich. Controller Funktion, im Sinne eines Aufgabenbündels, sowie Controller Institution stimmen beim fachbereichsbasierten Controlling überein. Institutionelle Basis für ein derartiges Verständnis ist das traditionelle Rechnungswesen. Die Beschränkung auf Strukturierung, Pflege und Bereitstellung rechnungswesenbezogener Daten führt zwangsläufig dazu, dass bezüglich der Unternehmenssteuerung inhaltlich ein geringerer Einfluss als beim führungsbasierten ControllingVerständnis besteht. ${ }^{61}$ Überträgt man dem institutionalisierten Controlling über das traditionelle Rechnungswesen hinaus weitere Systeme, die der Informationserfassung und Dokumentation dienen, sowie die Koordination dieser einzelnen Systeme untereinander, so charakterisiert die Informationssystemorientierung den Controllerbereich. Darüber hinaus determiniert die Vorgehensweise, wie Informationen der betreuten Systeme beschafft, aufbereitet und analysiert werden, das Ausmaß einer aktiven Führungsunterstützung. Die Art und Weise, in der darüber hinaus ergänzende Dienstleistungen im Sinne von Betreuungsund Beratungsaktivitäten erbracht werden, bestimmt letztlich, inwieweit die Grenzen zwischen fachbereichsbasierten und führungsbasierten Verständnis an Trennschärfe verlieren.

\subsection{Verständnissynthese}

Die bis hierhin über die konzeptionelle Fundierung hinaus vorgenommene Darstellung unterschiedlicher Verständnisse des Controlling in der Praxis verdeutlicht, dass institutionellen Aspekten in diesem Zusammenhang eine gewichtige

\footnotetext{
59 Vgl. hierzu auch Deyhle (1989), S. 180 f. 
Rolle zukommt. Wenngleich aus wissenschaftstheoretischer Sicht eine objektivierte kontextunabhängige Konzeption erstrebenswert erscheint, darf die Diskussion des Controlling nicht an der Wahrnehmung der Aufgabenträger des Controlling und ihrer Interaktionspartner vorbeilaufen. Im Folgenden soll daher durch Erläuterung eines die vorher dargestellten Extremverständnisse verbindenden Kontinuums herausgearbeitet werden, inwieweit eine moderne Controlling-Auffassung Elemente beider Seiten beinhaltet. Das Kontinuum in Abbildung 2 erlaubt, den Übergang von einem Extremverständnis zum anderen anhand inhaltlich aufeinander aufbauender Auffassungen nachzuvollziehen.

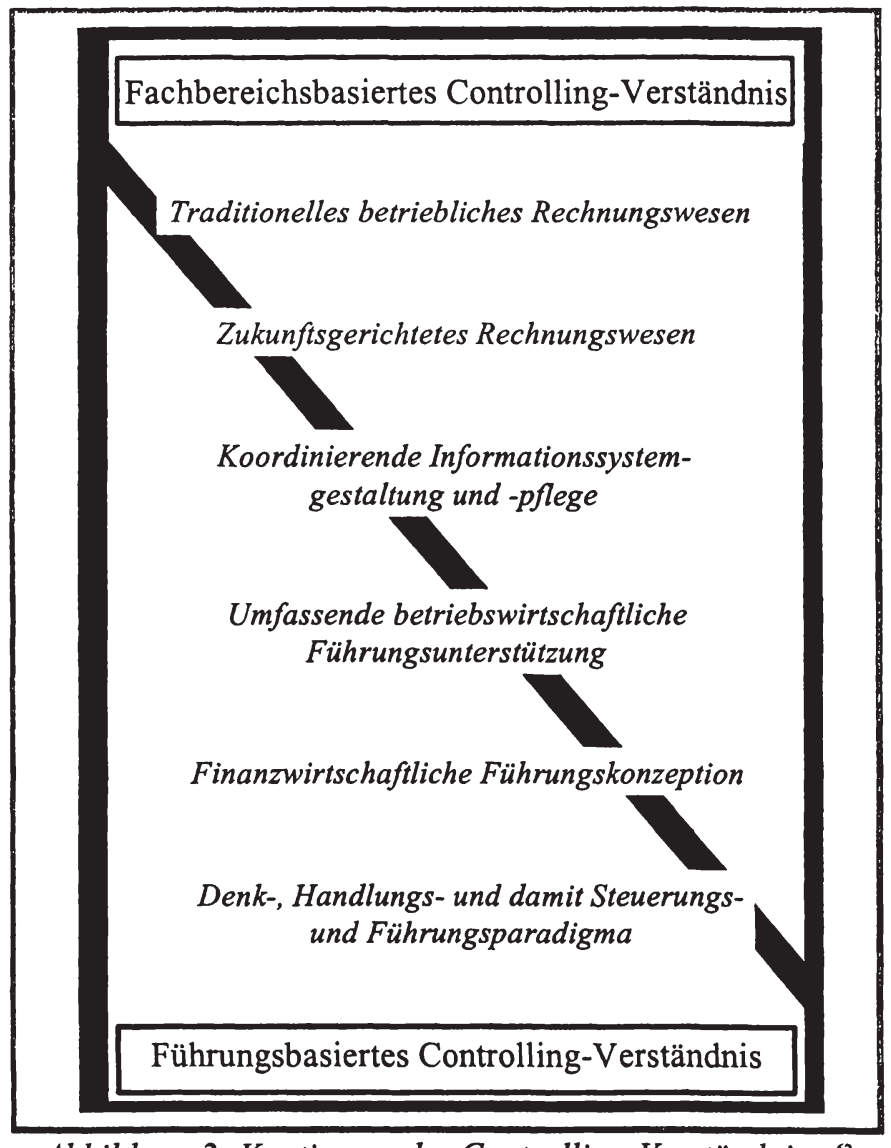

Abbildung 2: Kontinuum der Controlling-Verständnisse $e^{62}$ 
Ein solcher, gleichsam fließender Übergang vermag vielfältige in der Praxis anzutreffende Ausprägungen des Controlling wiederzugeben. Formal lassen sich diese unterschiedlichen Ausprägungen wiederum anhand funktionaler, institutionaler und instrumenteller Aspekte unterscheiden. ${ }^{63}$ Insgesamt reicht das Kontinuum vom traditionellen Rechnungswesen über dessen Anreicherung mit weiteren Informationssystemen und Unterstützungsfunktionen bis hin zu einer zunächst finanzwirtschaftlich dominierten Führungskonzeption, die sich - verallgemeinert - in einem allumfassenden Führungsparadigma wiederfindet. Ergänzt man die Betrachtung um die Aufgabenträger des Controlling und die ihnen jeweils zugeordneten Aufgaben ${ }^{64}$ bzw. Verantwortlichkeiten, so lassen sich, wie in Abbildung 3 dargestellt, grundlegende inhaltliche Entsprechungen zu den Controlling-Auffassungen ableiten.

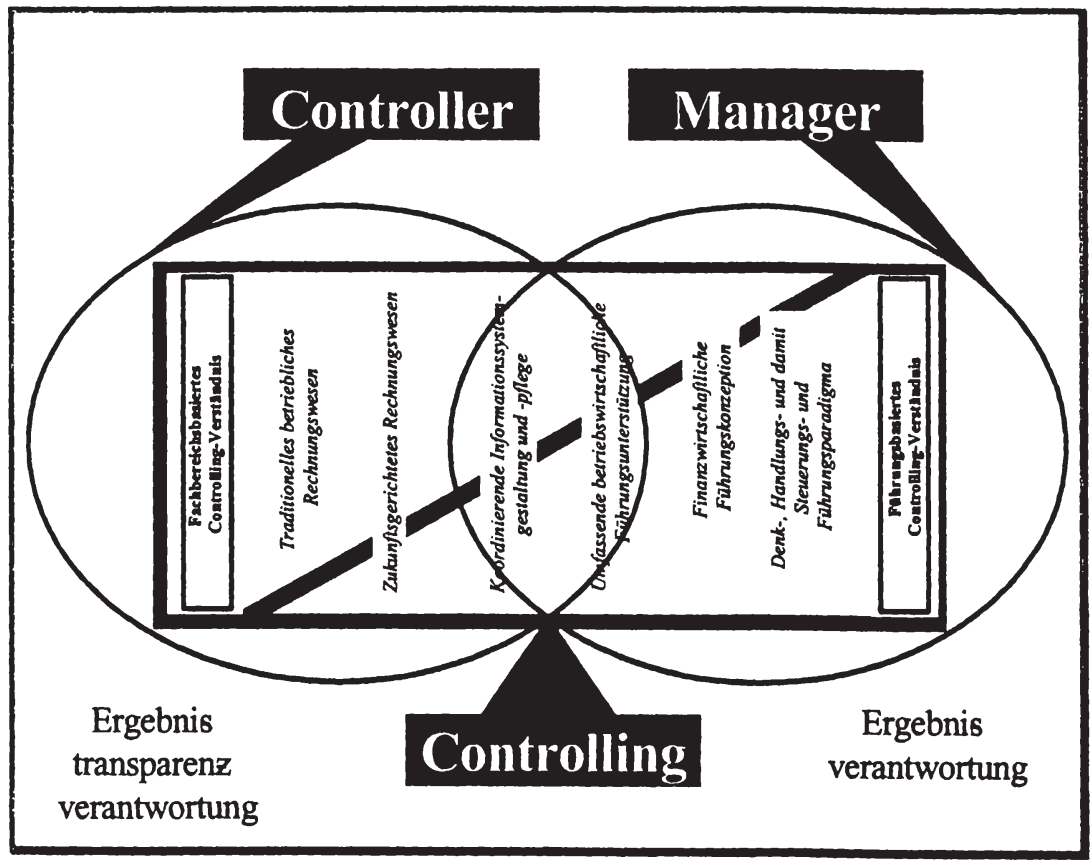

Abbildung 3: Verantwortungsbereiche und Verständniskontinuum ${ }^{65}$

63 Ausführlicher hierzu die Abbildung zur synoptischen Charakterisierung der Controlling-Verstăndnisse nach formalen Aspekten, vgl. Lanter (1996), S. 18.

64 Ausführlicher zu dem Problem der systematischen Aufgabenzuordnung zu Aufgabenträgern des Controlling vgl. Kap. 3.2.1.2.

65 Die Schnittmengendarstellung zwischen Controller und Manager erfolgt in Anlehnung an die Abbildung "Controller und Controlling-Kooperation" von Deyhle/Steigmeier (1993), S. 26 f. 
Kontextabhängig korrespondiert oftmals das Controlling-Verständnis mit den Aufgabenbereichen der Aufgabenträger des Controlling (Manager/Controller). Grenzt das Ergebnis bzw. die Ergebnistransparenz die Verantwortungsbereiche zwischen Manager und Controller noch klar ab, zeigen sich demgegenüber bei der Aufgabenzuordnung und Wahrnehmung in Abhängigkeit des unterstellten Controlling-Verständnisses unterschiedliche Ausgestaltungsmöglichkeiten. Stellt man auf den Service- und Unterstützungscharakter des Controlling ab, so wird deutlich, dass Controlling zwischen Manager und Controller, im Sinne einer Schnittmenge der Verantwortlichkeiten stattfinden sollte. Je nach horizontaler Lage des Verständniskontinuums befinden sich in dieser Schnittmenge mehr führungs- oder fachbereichsbezogene Elemente. ${ }^{66}$ Die Lage des Kontinuums bzw. dessen Überlappung mit der Schnittmenge unterscheidet sich von Unternehmen zu Unternehmen. Darüber hinaus kann im Zeitablauf eine Dynamik beobachtet werden, soweit Aufgabenbereiche und Verständnis einer Veränderung unterliegen.

So gesehen wird deutlich, dass Controlling unabhängig von seiner konzeptionellen Fundierung in der Realität konkreten Wahrnehmungen ausgesetzt ist, die das Verständnis der Beteiligten prägen. Hierbei handelt es sich im Regelfall weder um ein rein fachbereichsbasiertes noch führungsbasiertes Verständnis. Vielmehr verwischen die Grenzen in Abhängigkeit der Aufgabenträger, ihrer spezifischen Fähigkeiten und dem Entwicklungsgrad des Controlling.

Vor diesem Hintergrund soll unter Controlling im weiteren die „Beschaffung, Aufbereitung, (...) Analyse [und Kommunikation] von Daten zur Vorbereitung zielsetzungsgerechter Entscheidungen "677 verstanden werden. Hierdurch wird einerseits deutlich, dass dem Controller unterstützende Aufgaben im Rahmen der zielsetzungsgerechten Entscheidungsfundierung zukommen, die auf Basis institutionalisierter, eventuell fachbereichsgebundener Informationssysteme erfolgen kann. Andererseits kann durch die Analyse, Aufbereitung und Kommunikation dieser Informationen mit unterschiedlicher Intensität auf führungsbezogene Handlungen eingewirkt werden. Insbesondere das Kommunikationsverhalten prägt hierbei die Wahrnehmung des Controlling durch das Management. Über eine stärkere Fokussierung des Controllers auf den Manager als seinen unmittelbaren Leistungsadressaten - im Sinne einer konsequenten Kundenorien-

66 Die horizontale Lage des Verständnis-Kontinuums und damit der Schnittmengeninhalt kann z.B. Ausdruck der Kontextabhängigkeit des Controlling-Verständnisses sein.

67 Berens/Hoffjan/Strack (1995), S. 144. Ebenso auch Rieper/Witte/Berens (1996), S. V. Die Ergänzung in den rechteckigen Klammern erfolgte durch den Verfasser. 
tierung - können durch die Aufgabenteilung bedingte Nachteile kompensiert werden. Die systematische Integration der Bedürfnisse der Führung in die Leistungen des Fachbereichs Controlling vermag zu einer Integration der Fachbereichs- und Führungsperspektive beizutragen. Haben Manager und Controller dieses Verständnis gleichermaßen verinnerlicht, kann das Controlling wirksam seinen systeminternen und systemübergreifenden Koordinationsverpflichtungen nachkommen. Inwieweit aktuelle Entwicklungen Umfang und Effizienz des Controlling beeinflussen, soll im Folgenden übersichtsartig dargestellt werden.

\subsubsection{Ausgewählte Entwicklungstendenzen im Controlling}

Unterstellt man dem Controlling den Anspruch, aktive Führungsunterstützung leisten zu wollen, so sind die derzeitigen Entwicklungstendenzen im Controlling vor dem Hintergrund aktueller Führungsprobleme des Managements zu betrachten..$^{68}$ Die stetig steigende Umweltdynamik erfordert eine Veränderung in der Struktur der gewohnten Führungsteilsysteme. Neben einer Unterstützung der Anpassung einzelner Systeme kommt hierbei dem Controlling insbesondere die Aufgabe zu, die Koordination der Veränderung im Führungsgesamtsystem zu gewährleisten. Die neue Struktur der Führungssysteme geht mit dem Management veränderter Zielgrößen einher. ${ }^{69}$ Mengen-, Zeit- und Qualitätsdaten ergänzen pagatorische und kalkulatorische Rechengrößen. Neue Führungskonzepte wie Teamorientierung und Profit-Center-Lösungen stellen insbesondere in Verbindung mit hybriden Unternehmensformen wie Unternehmenspartnerschaften und virtuellen Unternehmen eine Herausforderung fur das Controlling dar, auf das dieses sowohl mit neuen Leistungen als auch mit eigenen Organisationsanpassungen reagieren muss.

Die Organisation des Controlling zeichnet sich derzeit noch überwiegend durch konservative Stabs- oder Linienlösungen aus. ${ }^{70}$ In jüngster Vergangenheit rücken aber Ansätze wie Selbstorganisation, Center-Organisation und die lernende Organisation immer mehr in den Mittelpunkt der Betrachtung. RegelmäBig wird ihre Anwendbarkeit auf den Controllerdienst diskutiert und für zukünftig als tragfähig erachtet. ${ }^{71}$ Die Auswirkungen solcher Organisationsänderungen

68 Die aktuellen Führungsprobleme werden in Anlehnung an WEBER und SCHĀFFER diskutiert. Vgl. We. ber/Schăffer (2000), S. $134 \mathrm{f}$.

Vgl. Weber/Schäffer (2000), S. 134.

70

Zur empirischen Auswertung von Organisationsplänen vgl. Amshoff (1996), S. 546 ff.

Vgl. Reiß (1997), S. 373 ff. 
auf die Effizienz und Effektivität der Leistungserstellung des Controlling sollen an späterer Stelle diskutiert werden. ${ }^{72}$

Eine Verbindung der organisatorischen Neuausrichtung des Controlling mit neuen Aufgaben und Instrumenten stellt der Ansatz des Lean Controlling dar, welcher insbesondere Verhaltens $-{ }^{73}$ Kultur- und Kommunikationsaspekten eine besondere Bedeutung beimisst. ${ }^{74}$ Der ganzheitliche Ansatz des Lean Controlling stellt die Prozessperspektive in den Mittelpunkt und betont Dezentralisierung und Eigensteuerung als probate Mittel, Flexibilität organisatorisch zu verankern. Eigenverantwortlichkeit bedingt die Notwendigkeit von mehr Controllingaktivitäten in dezentralen Bereichen, was gleichsam eine Umorientierung des Controllers erfordert. Durch die Dezentralisierung entstehen neue Zielgruppen für das Controlling. Lean Controlling führt somit zu einer quantitativen Ausweitung der Zielgruppe (unteres Management), bei gleichzeitiger qualitativer Veränderung des Aufgabenspektrums. ${ }^{75}$ Der hiermit verbundene Trend zum Selbstcontrolling verlangt vom Controller die Fähigkeit, andere in die Lage zu versetzen, selbständig Controlling betreiben zu können. Hierdurch erweitern insbesondere Moderation, Coaching und Implementierung von Konzepten das Spektrum der bisherigen Aufgaben des Controllers. Die Gefahr, dass der Controller sich hierdurch langfristig selbst überflüssig machen könnte, besteht nicht real. Vielmehr fordert Selbstcontrolling "den Controller auf, die Rolle des Spielmachers zu übernehmen und andere die Tore schießen zu lassen"”.

Eingebettet in die bisher beschriebenen Entwicklungstendenzen sollen im Folgenden zwei grundlegende, für den weiteren Verlauf der Arbeit wesentliche Entwicklungen im Controlling dargestellt werden. Zum einen erfordert der $e x-$ terne Marktdruck eine angemessene Berücksichtigung absatzmarktbezogener Sachverhalte, insbesondere aufgrund der Tatsache, dass die Nachfrage bei der Mehrzahl der Unternehmen regelmäßig den zentralen Engpassfaktor darstellt." Ferner ist, wie bereits im Rahmen des Lean Controlling angesprochen, für das Controlling ein zunehmender interner Marktdruck spürbar, der es erforderlich macht, die Bedürfnisse der internen Kunden bei der Erstellung der Leistungen zunehmend zu berücksichtigen. Das Controlling muss zudem selbst immer öfter

\footnotetext{
Ausführlich zur Organisation des Controlling vgl. Kap $3.3 \mathrm{ff}$.

Zur Konzeption des verhaltensorientierten Controlling vgl. Karlowitsch (1997), S. 24 ff.

$\mathrm{Zu}$ Aufbau und Gestaltung eines Lean Controlling vgl. Biel (1996), S. $50 \mathrm{ff}$.

Hierzu und nachfolgend vgl. Reichmann (1996), S. 559.

Biel (1996), S. 54.

Zur Bedeutung des Engpassfaktors Nachfrage für die Begrindung der Marketing-Theorie vgl, Kap. 2.2.

Thomas Mosiek - 978-3-631-75327-7
} 
Rechenschaft über die Wirtschaftlichkeit seiner Leistungserstellung ablegen. Einen Überblick über die im Folgenden zu diskutierenden Quellen des Veränderungsdrucks im Controlling gibt Abbildung 4.

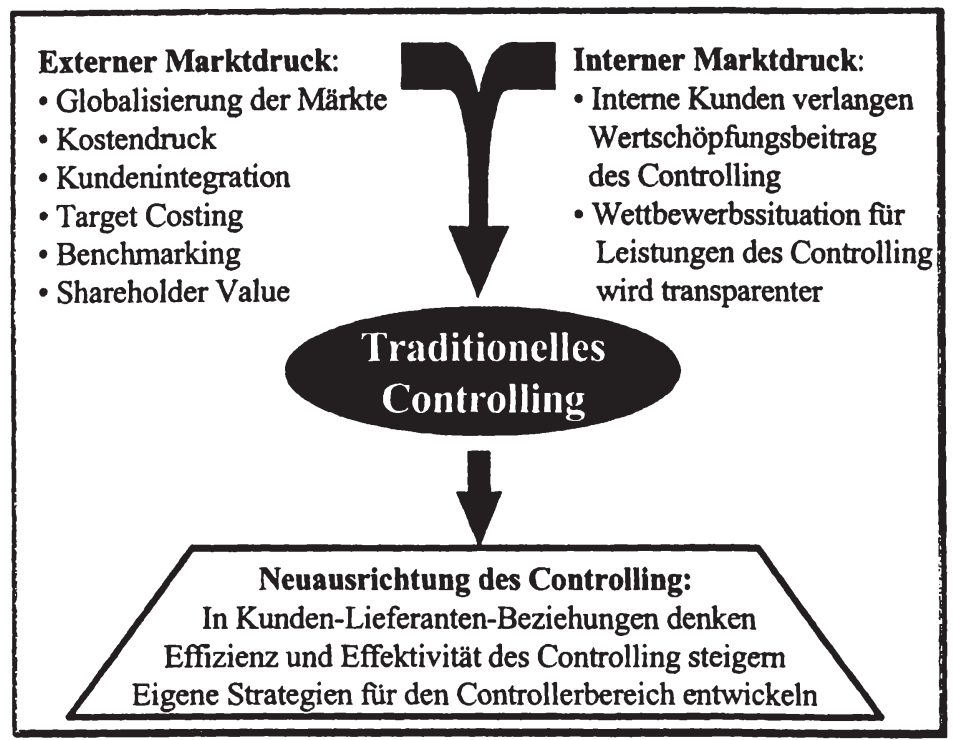

Abbildung 4: Controlling im Wandel ${ }^{8}$

\subsubsection{Externe Marktorientierung des Controlling}

Die vorherrschende Ausrichtung des Controlling auf interne Prozesse hat in der Vergangenheit oftmals dazu gefürt, dass absatzmarktbezogene Sachverhalte nur unzureichend Berücksichtigung in Analysen des Controlling gefunden haben. Insbesondere in Unternehmen mit deutlichem Technologievorsprung fehlte regelmäßig die Notwendigkeit, die Nachfrage als Knappheitsfaktor angemessen abzubilden. Im Rahmen eines sich verschärfenden Wettbewerbsumfeldes wurden als Reaktion zunächst Kostensenkungspotenziale ausgeschöpft, bevor sich letztlich auch im Controllerbereich die Erkenntnis durchsetzte, die nachfragedeterminierten Erlöse auf ihre Gestaltbarkeit hin zu untersuchen. WEBER fordert in diesem Zusammenhang, „den Inside-out durch einen Outside-in-Blick zu ergän$z{ }^{6 / 79}$. Konsequent auf alle marktbezogenen Aktivitäten angewandt heißt dies,

78 Ergänzt auf Grundlage der Darstellung von Scharnagl/Schubert (1999), S. 312 und der Ausfuhrungen von Niedereichholz (1999), S. 9.

79 Weber (1997), S. 16 sowie Homburg (1999), S. 7. 
absatzmarkt-, kapitalmarkt- und wettbewerbsumfeldbezogene Anforderungen durch geeignete Controlling-Maßnahmen zu analysieren und aufzubereiten, damit auf dieser Basis das Management in die Lage versetzt wird, angemessen zu reagieren. Konkret wird bspw. versucht, über Absatzsegmentrechnung, Kundendeckungsbeitragsrechnung, Werbeerfolgskontrolle und Target Costing Kundenanforderungen und -verhalten in das Controlling $\mathrm{zu}$ integrieren. ${ }^{80}$ Ferner wird mit dem Shareholder Value-Konzept $t^{81}$ eine konsequente Kapitalmarktorientierung verfolgt, welche durch transparenzschaffende Instrumente des Risikocontrolling abgesichert werden kann. Benchmarking-Aktivitäten vermögen wettbewerbsbezogen die Performance von Unternehmensbereichen abzubilden und ermöglichen gleichsam Verbesserungspotenziale im Sinne einer Best Practice zu erschließen.

Den generellen Trend zu einer Marktorientierung im Controlling vermögen gleichermaßen moderne Kennzahlensysteme und Performance MeasurementInstrumente wie die Balanced Scorecard zu belegen, ${ }^{82}$ da durch ihre Multidimensionalität absatzmarkt-, kapitalmarkt- und wettbewerbsbezogene Sachverhalte in Verbindung mit klassischen monetären Steuerungsgrößen Abbildung finden. Die hier beschriebene ,externe“ Marktorientierung des Controlling soll im Folgenden von einer ,, internen " Marktorientierung abgegrenzt werden, welche ihren Betrachtungsschwerpunkt auf die internen Kunden-Lieferanten-Beziehungen des Controllings legt. ${ }^{83}$

\subsubsection{Interne Marktorientierung des Controlling}

Zunehmend spielt die interne Ausrichtung des Controlling eine entscheidendere Rolle. Hat man früher die formale Existenz des Controllings auf die ihr zugrundeliegende Organisationsentscheidung zurückgeführt, ${ }^{84}$ rückt heute im Gegensatz zu dieser hierarchischen Betrachtung die Nachfrage nach ControllingLeistungen als konstituierendes Merkmal für die Rechtfertigung der Existenz des Controlling in den Mittelpunkt. Es hat sich die Erkenntnis durchgesetzt, dass die Wechselwirkung von Angebot und Nachfrage gleichermaßen positiv

\footnotetext{
80 Vgl. hierzu auch Abbildung 4. Allg. zur Kundenbeziehung als neue Bezugsgröße des Controlling vgl. Lube (1997), S. $183 \mathrm{ff}$.

81 Zum Gegenstand des Shareholder Value-Konzeptes vgl. Baetge (1997), S. $108 \mathrm{ff}$.

82 Zur Einbettung der Balanced Scorecard in den Prozess der Marktausrichtung des Controlling vgl. Weber/Schäffer (2000), S. $147 \mathrm{ff}$.

83 Ausfilhrlich zu internen Kunden-Lieferanten-Beziehungen vgl. Neuhaus (1996) sowie grundlegend zum Management derartiger Beziehungen Künzel (1999).

84 Vgl. Weber (1997), S. 7.
} 
auf die Bereitstellung des Leistungsangebotes interner Dienstleister auswirkt. ${ }^{85}$ Über bereits früher proklamierte Elemente einer Kundenorientierung im Controlling wie Empfängerorientierung und Bedarfsgerechtigkeit hinaus, wird derzeit versucht, Kundenorientierung in einem umfassenderen Sinn zu implementieren.$^{86}$ Neben einer zielgerichteten Positionierung des Controllerbereichs im Unternehmen und gegenüber seinem potenziellen internen und externen Wettbewerbsumfeld wird versucht, über die Inhalte einzelner Leistungen, die Struktur des Leistungsgesamtangebotes sowie die Umstände der Leistungserbringung interne Kunden- und Marktorientierung zu praktizieren. Regelmäßig bleibt die Umsetzung derartiger Maßnahmen jedoch hinter den Ansprüchen zurück. Insbesondere das Zusammenspiel hierarchie- und marktbezogener Koordination erfordert an dieser Stelle eine genaue Analyse des Unternehmenskontextes sowie der beteiligten Transaktionspartner. Es ist zu berücksichtigen, dass unternehmensintern Rahmenbedingungen für Leistungsinanspruchnahmen gelten müssen, da sensible Leistungsinhalte nicht jedem zwangsläufig zugänglich zu machen sind. Vielmehr bedarf es im Einzelfall einer vorherigen Autorisierung durch die Unternehmensführung. Darüber hinaus ist zu berücksichtigen, dass die Leistungsnachfrage einzelner Mitarbeiter teilweise durch egoistische Motive geprägt sein kann, die oftmals organisationsinternen Auseinandersetzungen um Macht und Prestige entspringen. ${ }^{87}$ Darüber hinaus ist zu klären, inwieweit der Kontrollcharakter einzelner Leistungen die Berücksichtigung von Nachfragerinteressen einschränkt bzw. verbietet. ${ }^{88}$ Hierdurch wird deutlich, dass speziell auch der Charakterisierung von einzelnen Leistungen besondere Aufmerksamkeit geschenkt werden muss, da nur auf Basis einer derartig dezidierten Analyse Möglichkeiten und Grenzen einer kundenorientierten Ausgestaltung ergründet werden können. ${ }^{89}$

Im Rahmen der durch die Unternehmensleitung vorgegebenen Inanspruchnahmemöglichkeiten des Controlling und in dem Bewusstsein, dass neben Unternehmenszielen immer auch Individualziele verfolgt werden, stellt die Konfrontation von Angebot und Nachfrage ein zweckmäßiges Interessenausgleichsverfahren dar, welches einer effizienten Allokation von Controlling-Leistungen

85 Zur Allokation und Verteilung individueller Güter uber den Ausgleich von Angebot und Nachfrage vgl. Thieme (1992), S. $20 \mathrm{ff}$.

86 Vgl. hierzu die Anwendung der Balanced Scorecard auf den Controllerbereich in Kap. 4.2 sowie Gleich/ Haindl (1996), S. 262 ff.

$87 \mathrm{Zu}$ spezifischen Einflußfaktoren auf die Nachfrage von Kosteninformationen vgl. Höffken (1991), S. 85 ff.

88 Vgl. Kap. 3.2.2.3.2.

$89 \mathrm{Zu}$ dem Möglichkeiten einer kundenorientierten Ausgestaltung der Kostenrechnung vgl. Homburg/Weber/ Aust/Karlhaus (1998), S. 39 ff. 
dienlich sein kann. Daher erscheint es notwendig, einen Gestaltungsrahmen für eine gesamthaft nachfrage- bzw. kundenorientierte Ausrichtung des Controllerbereiches zu entwickeln. Hierbei ist es zweckmäßig, Ansätze der Marketingwissenschaft auf ihre Anwendbarkeit hin zu überprüfen, da diesen der dominante Kundenfokus eigen ist.

\subsection{Der Marketing Ansatz}

Der Wandel von Verkäufermärkten zu Käufermärkten kann als maßgeblich für die Bedeutungszunahme des Marketing in Praxis und Wissenschaft angesehen werden..$^{90}$ Induziert durch die Verlagerung des zentralen unternehmerischen Engpasses in den Absatzbereich wird dieser zum Ausgangspunkt unternehmerischer Planung. ${ }^{91}$

Im Kern der Managementphilosophie des Marketing steht die Befriedigung der Bedürfnisse aktueller und potenzieller Kunden..$^{22}$ Durch die Bereitstellung eines geeigneten Leistungsangebotes soll Kundenzufriedenheit generiert werden.93 Über diese kann langfristig eine Bindung des Kunden erreicht werden, ${ }^{94}$ auf deren Basis weitere Transaktionen gesichert werden sollen. Maßgeblich für die angestrebte Zufriedenheit des Kunden ist das Verhältnis zwischen den Erwartungen des Kunden vor dem Leistungsaustausch und den tatsächlichen erlebten Ergebnis der Transaktion. Die Bestimmung der Kundenzufriedenheit kann einerseits anhand intersubjektiv nachvollziehbarer Indikatoren erfolgen, anderseits spielen jedoch schwer operationalisierbare psychologische Faktoren eine gewichtige Rolle.95 Das Konstrukt der „Kundenorientierung“ zeichnet sich in diesem Zusammenhang durch den Anspruch aus, alle marktrelevanten Aktivitäten eines Anbieters unter dem Aspekt der Verbesserung des Kundennutzens zu sehen. ${ }^{96}$ Die Kundenorientierung bestimmt somit maßgeblich den Grad der Kundenzufriedenheit. ${ }^{97}$

\footnotetext{
Vgl. Meffert (1999), S. 5.

Vgl. Gutenberg (1984), S. 104 ff.

So bspw. McCarthy (1964) und Kotler (1999).

Ausführlich zu Bedeutung der Kundenzufriedenheit vgl. Pepels (1998), S. 920 ff. Zur strategischen Bedeutung der Kundenzufriedenheit vgl. Simon/Homburg (1998), S. 17 ff.

94 Zum Kundenbindungspotenzial im Business-to-Business Marketing vgl. Kleinaltenkamp (2000), S. 83 f.

95 Zum Management von Kundenzufriedenheit vgl. Günter/Platzek (1992), S. 109 ff.

96 Vgl. Hanan/Karp (1991), S. 101.

97 Vgl. Backhaus (1997), S. 27.
} 
Die Identifikation und Befriedigung von Kundenbedürfnissen erfordert eine differenzierte Analyse des jeweils relevanten Marktes auf Basis seiner Marktstrukturen und Marktprozesse..$^{98}$ Darauf aufbauend ist der Gesamtmarkt nach geeigneten Abgrenzungsmerkmalen derart zu segmentieren, dass die Teilsegmente untereinander möglichst heterogen, in sich jedoch weitestgehend homogen sind. ${ }^{99}$ Eine erhöhte Transparenz der Leistungsangebote und eine zunehmende Vergleichbarkeit der Kernleistungen führt auf Anbieterseite zu der Notwendigkeit, Differenzierungspotenziale auszuschöpfen. Diese aktive Gestaltungsaufgabe steht im Mittelpunkt des Marketingmanagements. Auf Basis der gesetzten Marketing Ziele sind die Marketingsituationen als die Zustände des jeweiligen Unternehmensumfeldes zu bewerten. Dies ist der Ausgangspunkt für den Einsatz der Marketinginstrumente, welche die Aktionsparameter des Marketingmanagers darstellen, zielgerichtet auf die Gestaltung des Marktumfeldes einzuwirken. ${ }^{100}$

\subsubsection{Traditioneller Objektbereich}

Analog zu der gesellschaftspolitischen Entwicklung des 20. Jahrhunderts unterlagen die Ansprüche, die heute unter dem Begriff des Marketing subsumiert werden, einem erheblichen Wandel. Wurde Marketing zunächst als Distributionsfunktion, dann als zentrale Engpassfunktion und später als Führungsfunktion aufgefasst, hat sich das heutige Verständnis zu dem eines marktorientierten Führungskonzeptes hin gewandelt. ${ }^{101}$ In diesem Sinne definiert MEFFERT Marketing als „Planung, Koordination und Kontrolle aller auf die aktuellen und potenziellen Märkte ausgerichteten Unternehmensaktivitäten. Durch eine dauerhafte Befriedigung der Kundenbedürfnisse sollen die Unternehmensziele im gesamtwirtschaftlichen Güterversorgungsprozess verwirklicht werden." ${ }^{102}$ Demgegenüber wird mit Marketing im öffentlichen Bewusstsein regelmäßig die Unternehmensfunktion assoziiert. Insbesondere aber die Betonung von Marketing als Management von komparativen Konkurrenzvorteilen (KKV) verdeutlicht die Notwendigkeit einer produktspezifischen ,Koordination von Unternehmensfunktionen (z.B. F\&E, Beschaffung, Fertigung, Vertrieb, Finanzierung) im Hin-

98 Marktstrukturen und Marktprozesse determinieren zusammen das Marketingsystem. Vgl. Bruhn (1990), S. $16 \mathrm{ff}$.

99 Vgl. umfassend Freter (1983) sowie uberblicksartig Meffert (2000), S. 181 und Scheuch (1993), S. $274 \mathrm{ff}$

$100 \mathrm{Vgl}$. Bruhn (1990), S. $26 \mathrm{f}$.

$101 \mathrm{Zu}$ den Entwicklungslinien des Marketing vgl. Meffert (1999), S. 6 ff. und Peples (1998), S. 9 ff. Speziell zu Internationalisierungstendenzen vgl. Backhaus/Büschken/Voeth (2000), S. 33 ff.

102 Meffert (2000), S. 8. 
blick auf die Erfordernisse des (Absatz-) Marktes" ${ }^{103}$. Hierdurch wird zum Ausdruck gebracht, dass Quellen für $K K V^{\prime}$ 's in allen Unternehmensfunktionen zu suchen sind.

Sowohl die Einbeziehung aller marktbezogenen Aktivitäten und die umfassende Bedürfnisorientierung, als auch der funktionsübergreifende Charakter des Marketing haben in der Vergangenheit dazu geführt, dass sich Marketing als annähernd allumfassender Ansatz nicht unerheblicher Kritik ausgesetzt sah. ${ }^{104}$ Überspitzt formuliert SCHNEIDER mit Blick auf die Marketing-Management Definition von KOTLER, ${ }^{105}$ dass „Beethovens Skizzen zur 9. Sinfonie oder die Konzipierung des Kommunistischen Manifests demnach ebenso wie eine räuberische Schutzgeld-Erpressung durch Mafiosi ${ }^{* 106}$ mit derartig umfassenden Definitionen vereinbar zu sein scheinen. Letztlich verbirgt sich hinter dieser Äußerung die Kritik, dass die Marketingwissenschaft mit ihrem Selbständigkeitsanspruch, ${ }^{107}$ im Sinne einer Konzeption der Unternehmensführung, zwangsläufig in Konkurrenz zur Betriebswirtschaftslehre an sich tritt. ${ }^{108}$ An dieser Stelle sei auf die Analogien zur Fundierung des Controlling als eigenständiger betriebswirtschaftlicher Bereich hingewiesen. In vergleichbarer Form stellt sich die Abgrenzung zur Unternehmensführung als ein Problem dar. ${ }^{109}$

Insbesondere jedoch die Umweltdynamik in einer globalisierten Gesellschaft, verbunden mit einer heterogenen Entwicklung der Konsumentenwünsche, erfordert - vielleicht mehr denn je - eine umfassende Orientierung aller Unternehmensaktivitäten am Nutzen der Kunden bzw. an erzielbaren Wettbewerbsvorteilen. ${ }^{110}$ Daher soll im Folgenden weniger der theoretischen Auseinandersetzung um spezifische Konzepte des Marketing Aufmerksamkeit geschenkt werden, als vielmehr konkreten praktischen Entwicklungstendenzen, die die Wahrnehmung der Marketing „Praktizierenden“ prägen. Diese sind regelmäßig durch ein dua-

103 Backhaus (1999), S. 8.

104 Hierzu beispielhaft Schneider (1983), S. $197 \mathrm{ff}$.

105 Kotlers Definition entspricht der der AMERICAN MARKeTING AsSOCLATION (AMA): „Marketing ist der Prozess von Planung und Umsetzung der Entwicklung, Preissetzung, Kommunikation und Distribution von Ideen, Gütern und Dienstleistungen zur Ermöglichung von Austauschprozessen, die die individuellen und organisationsbezogenen Zielsetzungen erfuillen." Zitiert nach Brown (1985), S. 1.

106 Schneider (1997), S. 16.

107 Vgl. Schneider (1997), S. 29.

108 SCHNELDER formuliert dies in einer von drei Thesen, in denen er sich mit dem „Image“ der Marketingwissenschaft auseinandersetzt. Vgl. Schneider (1983), S. 198.

109 Naher hierzu Kap. 2.1.1.

$110 \mathrm{Vgl}$. Meffert (1999), S. 26. 
les Führungskonzept geprägt, welches Unternehmensfunktion und Leitkonzept des Managements gleichermaßen beinhaltet."

\subsubsection{Ausgewählte Entwicklungstendenzen im Marketing}

Neben der durch die dargestellte historische Entwicklung der Märkte bedingten Wandlung in der Grundausrichtung des Marketing, lassen sich Tendenzen eines Deepening und Broadening im Marketing beobachten. ${ }^{12}$ Deepening als Vertiefung des Marketing, im Sinne einer Erweiterung der Zielinhalte, führt zu einer Einbeziehung solcher Bereiche, die nicht primär auf ökonomische Ziele ausgerichtet sind.$^{113}$ Ferner hat die Ausweitung des Gegenstandsbereiches des Marketing (Broadening) zu einer Anwendung von Marketing-Gedanken auf nicht erwerbswirtschaftliche Institutionen geführt. ${ }^{114}$ Gemeinsam ist diesen Entwicklungen, dass das Begriffsverständnis nunmehr allgemein ein Management von Austauschbeziehungen abdeckt, ${ }^{115}$ was gleichermaßen unternehmensinterne Transaktionen einschließt.

Eine weitere für die Entwicklung des Marketing maßgebliche Tendenz ist die stärkere Spezialisierung auf Wirtschaftsgütertypen. Nahm Marketing seinen Ausgangspunkt bei den Verbrauchsgütern, entwickelten sich rasch spezifische methodische Instrumentarien für Gebrauchsgüter, Investitionsgüter und schließlich auch Dienstleistungen.

Auf Basis der Gesamtheit derartiger Entwicklungs- und Spezialisierungstendenzen im Marketing sollen nachfolgend zwei ausgewählte Bereiche weiter betrachtet werden. Da es sich bei den im Kern dieser Arbeit stehenden Controlling-Leistungen um interne Dienstleistungen handelt, sollen einerseits die Spezifika eines Marketing für Dienstleistungen herausgearbeitet werden. Andererseits muss den Besonderheiten unternehmensinterner Austauschbeziehungen Rechnung getragen werden, wofür der Ansatz des internen Marketing verantwortlich zeichnet.

\footnotetext{
11 Vgl. Meffert (1999), S. 8.

$112 \mathrm{Zu}$ den Entwicklungen des Deepening und Broadening vgl. Meffert (2000), S. 1276 f. sowie Peples (1998), S. $39 \mathrm{f}$.

113 Hierzu zählen bspw. das gesellschaftsorientierte Marketing oder das Öko-Marketing.

$114 \mathrm{Zu}$ nennen sind hier öffentliche Verwaltungen, Kulturinstitutionen etc.

115 Vgl. Meffert (1999), S. 9.
} 


\subsubsection{Dienstleistungsmarketing}

Die Bedeutungszunahme des tertiären Sektors in modernen Volkswirtschaften hat auch in der Marketingwissenschaft zu einer differenzierteren Auseinandersetzung mit dem Tatbestand der Dienstleistung geführt. ${ }^{116}$ In Bezug auf Untersuchungsgegenstand und Anwendungsspektrum wird im Marketing regelmäßig zwischen Konsumgüter-, Investitionsgüter-, und Dienstleistungsmarketing unterschieden, auch wenn eine genaue Abgrenzung oftmals Schwierigkeiten bereitet. ${ }^{17}$ Zielsetzung des Dienstleistungsmarketings ist einerseits, die Charakteristika des Dienstleistungsbegriffs in Abgrenzung zu anderen Wirtschaftsgütern herauszuarbeiten, um andererseits das heterogene Gesamtspektrum der Dienstleistungen so zweckmäßig strukturieren zu können, dass ein konkreter Anwendungsbezug hergestellt werden kann. Ein gängiges Verfahren zur Abgrenzung des Dienstleistungsbegriffs ist eine Definition auf Basis konstitutiver Merkmale. Als konstitutive Merkmale der Dienstleistung in Abgrenzung zur Sachleistung werden die Potenzial-, Prozess- und Ergebnisorientierung angesehen. „Dienstleistungen sind selbständige, marktfähige Leistungen, die mit der Bereitstellung (z.B. Versicherungsleistung) und/oder dem Einsatz von Leistungsfähigkeiten (z.B. Friseurleistung) verbunden sind (Potenzialorientierung). Interne (z.B. Geschäftsräume, Personal, Ausbildung) und externe Faktoren (also solche die nicht im Einflussbereich des Dienstleisters liegen) werden im Rahmen des Erstellungsprozesses kombiniert (Prozessorientierung). Die Faktorenkombination des Dienstleistungsanbieters wird mit dem Ziel eingesetzt, an den externen Faktoren, an Menschen (z.B. Kunden) oder deren Objekten (z.B. Auto des Kunden) nutzenstiftende Wirkungen (z.B. Inspektion beim Auto) zu erzielen (Ergebnisorientierung). "“18

Die Bemühungen derartig umfassender Definitionen verdeutlichen das Problem, das heterogene Gesamtspektrum der Dienstleistungen treffend wiederzugeben, ohne jedoch die Trennschärfe zum Begriff des Sachgutes zu verlieren. ${ }^{119}$ Daher wird gegenwärtig weniger der Definition, als vielmehr der Klassifikation einzelner Dienstleistungen Aufmerksamkeit geschenkt.

Gütertypologische Abgrenzungen stellen demnach ein wichtiges Klassifikationsinstrument dar, welches in der Lage ist, die bezogen auf den Analysegegen-

\footnotetext{
116 Zum Anteil von Dienstleistungstätigkeiten im produzierenden Sektor vgl. Kleinaltenkamp (1995), S. 163.

117 Vgl. Meffert/Bruhn (2000), S. 6.

118 Meffert/Bruhn (2000), S. 30.
}

119 Die Zweckmäßigkeit einer derartigen Abgrenzung stellen ENGELHARDT ET AL. in Frage. Vgl. Engelhardt Kleinaltenkamp/Reckenfelderbäumer (1993), S. 395 ff. 
stand differenzierungsträchtigsten Merkmale abzubilden. Die Einordnung einer spezifischen Dienstleistung oder Dienstleistungsgruppe wird entlang eines Kontinuums zwischen den zwei Extremausprägungen des jeweiligen Merkmals vorgenommen. ${ }^{120}$ Die Abbildung einer Dienstleistung in dem durch die Merkmale aufgespannten zwei- oder auch mehrdimensionalen Raum verfolgt das Ziel, möglichst homogene Dienstleistungstypen zu identifizieren, um diese einer zielgerichteten Bearbeitung durch das Marketing zugänglich zu machen. Als wegweisend gilt in diesem Zusammenhang die in Abbildung 5 dargestellte Leistungstypologie von ENGELHARDT ET AL. ${ }^{121}$, die aufgrund der regelmäßigen Verbindung von Sach- und Dienstleistungen diese Unterscheidung nicht mehr explizit vorsieht, sondern vielmehr auf den Immaterialitäts- und Integrationsgrad fokussiert. ${ }^{122}$

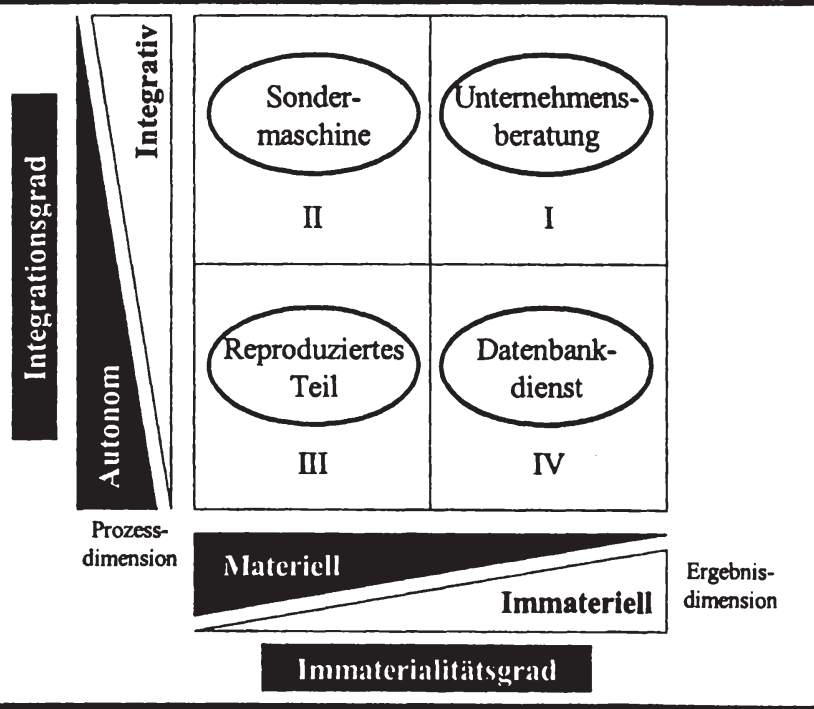

Abbildung 5: Leistungstypologie nach ENGELHARDT, KLEINALTENKAMP UND RECKENFELDERBÄUMER

Bezogen auf die oben erwähnten konstitutiven Merkmale der Dienstleistung stellt die Typologie von ENGELHARDT ET AL. mit der Integrativität ein Element

${ }^{120} \mathrm{Vgl}$. Meffert/Bruhn (2000), S. 33 sowie Meffert (1994), S. $522 \mathrm{ff}$.

121 Engelhardt/Kleinaltenkamp/Reckenfelderbăumer (1992), S. 35.

$122 \mathrm{Zu}$ der Struktur derartiger Leistungsbulndel vgl. Engelhardt/Kleinaltenkamp/Reckenfelderbaumer (1993), S. $395 \mathrm{ff}$. 
des Leistungsprozesses und mit der Immaterialität eine Eigenschaft des Leistungsergebnisses in den Mittelpunkt. Immaterialität und Integrativität haben unterschiedliche Auswirkungen auf das Abnahmeverhalten der Nachfrager. ${ }^{123}$ Beispielsweise führt ein hoher Immaterialitätsgrad zu Beschaffungsunsicherheiten beim Nachfrager aufgrund einer mangelnden Vergleichbarkeit. ${ }^{124}$ Demgegenüber kann ein hoher Integrativitätsgrad zu einer Reduktion von Unsicherheit beim Nachfrager führen, da dieser in den Prozess der Leistungserstellung involviert wird. Die Qualität des Ergebnisses derartiger Leistungen ist jedoch schwer voraussagbar, da diese sowohl von den Bemühungen des Anbieters als auch von denen des Nachfragers abhängt.

Auf Basis der Typologie von ENGELHARDT ET AL. haben sich in der Vergangenheit weitere Konzepte entwickelt, die den Anspruch haben, über eine differenziertere Aufspaltung der Dimensionen weiterführende Aussagen abzuleiten. ${ }^{125}$ Der umfassende Charakter derartiger Systematisierungen, sowie die Differenzierungstendenzen deuten darauf hin, dass der Rückgriff auf eine größere Anzahl von Merkmalen für spezifische Fragestellungen zweckmäßig erscheint.

Insbesondere unter dem Primat marketingbezogener Fragestellungen versuchen institutionenorientierte Abgrenzungen Charakteristika der Erstellung und des Verzehrs von Dienstleistungen abzubilden. ${ }^{126}$ Im Ergebnis vermögen die einoder mehrdimensionalen Systematisierungsansätze jedoch immer nur bestimmte Charakteristika wiederzugeben. Um umfassend mit Marketingmaßnahmen auf die Erstellung und Verwertung von Dienstleistungen einwirken zu können, bedarf es jedoch eines differenzierten Eigenschaftsprofils spezifischer Leistungen oder Leistungsgruppen. Auch wenn dies teilweise branchenbezogen für Dienstleistungsunternehmen angedacht ist, ${ }^{127}$ bleiben die diesbezüglichen Bemühungen für unternehmensinterne Dienstleistungen oder noch spezifischer für Controlling-Leistungen weit hinter den Ansprüchen zurück. ${ }^{128}$

$123 \mathrm{Vgl}$. FlieB (1995), S. 320 f.

124 Bei Unsicherheitsproblemen unterscheidet man bezogen auf den Zeitpunkt des Kaufs zwischen ex-anteUnsicherheit (Beschaffungsunsicherheit) und ex-post-Unsicherheit. Vgl. Backhaus (1999), S. 297. Zur Bedeutung des Unsicherheitsphänomens im Rahmen der Abgrenzung von Geschäftstypen vgl. Backhaus/Aufderheide/Späth (1994).

125 Bspw. zerlegen MEFFERT/BRUHN die Integrationsdimension in die Teildimensionen Individualisienungsgrad und Interaktionsgrad. Vgl. Meffert/Bruhn (1995), S. 31 ff.

126 Vgl. Meffert/Bruhn (1995), S. 35 ff.

127 Vgl. Meffert/Bruhn (1995), S. 48.

128 Einen Ansatz für die Typologisierung unternehmensinterner Leistungen findet sich bei Witt(1988),S. $660 \mathrm{ff}$. 
Im Rahmen der Beschreibung einzelner Elemente des Dienstleistungsmarketing wurde deutlich, dass ausgehend von dem allgemeinen Versuch einer Abgrenzung gegenüber der Sachleistung, zunehmend versucht wird, Dienstleistungstypengruppen zu identifizieren. $\mathrm{Da}$ der Ausgangspunkt hierfür regelmäßig das Gesamtspektrum möglicher Dienstleistungen ist, ist die für zielgerichtete Maßnahmen erforderliche Differenzierungswirkung oftmals gering. Im weiteren Verlauf dieser Arbeit soll daher parallel zu einer inhaltlichen Analyse von Controlling-Leistungen bzw. Leistungsgruppen sowie des Leistungserstellungsprozesses hinterfragt werden, welche spezifischen Systematisierungsansätze in der Lage sind, die jeweiligen Charakteristika der Leistungen des Controlling abzubilden. Da es sich bei dem Gesamtspektrum der Controlling-Leistungen wie noch zu zeigen sein wird - im Sinne geläufiger gütertypologischer Ansätze um eine recht homogene Gruppe handelt, ist die Entwicklung eines spezifischen Systematisierungsansatzes erforderlich.

Inwiefern das Marketing darüber hinaus Empfehlungen für die Optimierung interner Austauschbeziehungen bereithält, soll anhand einer Darstellung des Ansatzes des internen Marketings gezeigt werden. ${ }^{129}$

\subsubsection{Internes Marketing}

Das Charakteristikum einer Dienstleistung, nämlich dass diese als Ergebnis einer Interaktion zwischen Anbieter bzw. anbieterseitig involvierten Mitarbeitern und Kunde ist, hat zu der Erkenntnis geführt, dass die Verankerung von Kundenorientierung im Bewusstsein aller Mitarbeiter als grundlegende Führungsaufgabe zu begreifen ist. Die Erfüllung dieser Aufgabe - im Sinne der Sicherstellung einer dauerhaft hohen Leistungsqualität - erfordert aufgrund der zentralen Rolle der Mitarbeiter eine Absicherung durch geeignete personalpolitische Maßnahmen. ${ }^{130}$ Diese kundenorientierte Beeinflussung des Personals ist ein zentraler Anspruch des internen Marketing. Das Grundverständnis des internen Marketing weist demgegenüber unterschiedliche Ansatzpunkte auf: ${ }^{131}$

1.) Internes Marketing als Maxime der Bedürfnisorientierung im Sinne einer an internen Adressaten ausgerichteten Unternehmensführung (Interne Kundenorientierung)

129 Zum Zusammenspiel von internem und externen Marketing vgl. Schulz (1999), S. 623 ff.

$130 \mathrm{Vgl}$. Stauss (1995), S. 28.

131 Stauss (1991), S. 261. Vgl. fermer Schulz (1992), S $111 \mathrm{ff}$. 
2.) Internes Marketing als Sammelbegriff für die gegenüber internen Austauschpartnern eingesetzten Mittel der Verhaltenssteuerung (Interner Marketinginstrumentaleinsatz)

3.) Internes Marketing als Methode der innerbetrieblichen Implementierung einer im Hinblick auf externe Märkte konzipierten Marketingstrategie (Interne Steuerung zu absatzmarktorientierten Zwecken).

MEFFERT nennt inhaltlich vergleichbare Schwerpunkte des Verständnisses, indem er auf die Maxime der Mitarbeiterorientierung, den internen Methodeneinsatz, sowie die Gestaltung interner Austauschbeziehungen fokussiert. ${ }^{132}$

Die hier erwähnten unterschiedlichen Ansatzpunkte des Verständnisses zeichnen sich alle durch ihre starke Personalorientierung aus. $\mathrm{Zu}$ einer besseren Strukturierung des internen Marketings im Sinne der Problemstellung dieser Arbeit erscheint es zweckmäßig, eine Unterscheidung hinsichtlich der Akteure des innerorganisatorischen Marketings vorzunehmen. ${ }^{133}$ Betreiben Führungskräfte primär Marketingmaßnahmen, um Einstellungen und Verhaltensweisen der Mitarbeiter zu beeinflussen, spricht man von personalorientiertem internen Marketing. Sind interne Dienstleister primär die Akteure des Marketings, spricht man von einem Marketing interner Dienstleistungen. Dieses beinhaltet die Elemente kundenorientiertes Prozessmanagement sowie abteilungs- bzw. funktionsorientiertes Leistungsmarketing. Darüber hinaus existiert eine Form des kooperationsorientierten Marketings, bei dem die Beteiligten einer Unternehmenskooperation Maßnahmen zur internen Effizienzsteigerung oder zur externen Interessenbündelung anstreben (Genossenschaften, Einkaufskooperationen, etc.).

Es ist jedoch an dieser Stelle darauf hinzuweisen, dass die hier angesprochenen Konzepte weniger als Alternativen anzusehen sind, sondern vielmehr sich ergänzende Handlungsbereiche darstellen, die spezifische Problemlösungen in Abhängigkeit von Unternehmenssituation und Marketing-Akteuren anbieten. ${ }^{134}$ Nachfolgend soll im Sinne der Problemstellung zwischen den beiden grundsätzlichen Ansätzen des durch die Unternehmensführung geprägten personalorien-

132 Vgl. Meffert (2000), S. 1120. Diese Aspekte vermögen konsequent eine umfassende Prozessorientierung abzusichern. Vgl. Meffert (2000), S. $26 \mathrm{f}$.

$133 \mathrm{Zu}$ der im Folgenden gewählten Differenzierung vgl. Stauss (1995b), Sp. $1046 \mathrm{ff}$.

134 Stauss (1995b), Sp. 1054. 
tierten internen Marketing und dem durch interne Dienstleister geprägten abteilungs- bzw. funktionsorientierten internem Marketing unterschieden werden. ${ }^{135}$

\subsection{Personalorientiertes internes Marketing}

Ausgehend von den drei dargestellten Ansatzpunkten für das Grundverständnis des internen Marketings wird nachfolgend die interne Steuerungsfunktion zu absatzmarktorientierten Zwecken in den Mittelpunkt der Betrachtung gestellt. ${ }^{136}$ Gegen die anderen beiden alternativen Ansätze spricht, dass zwar eine ausschließlich interne Kundenorientierung im Falle extremer Knappheit der vom Personal erwünschten Ressourcen sinnvoll sein kann, ${ }^{137}$ andererseits jedoch in Konfliktsituationen zwischen interner Mitarbeiterorientierung und externer Kundenorientierung zur Existenzsicherung des Unternehmens den Interessen der externen Kunden Vorrang gewährt werden muss. ${ }^{138}$ Ferner ist ein Transfer der Methoden des externen Marketings zur Verhaltenssteuerung innerbetrieblicher Austauschpartner nur eingeschränkt möglich und sinnvoll, ${ }^{139} \mathrm{da}$, soweit der Arbeitsplatz als mittels Marketinginstrumenten zu vermarktendes Produkt anzusehen ist, ${ }^{140}$ dies die Gefahr einer wenig innovativen Umbenennung personalpolitischer Instrumente in sich birgt. ${ }^{141}$

Somit verfolgt das personalorientierte interne Marketing die Zielsetzung, Mitarbeiter über Marketing Strategien zu informieren, die Bedeutung kundenbezogener Interaktionen zu verdeutlichen und über die Vermittlung notwendiger Fähigkeiten und Fertigkeiten Mitarbeiter in die Lage zu versetzten, Kundenkontaktsituationen zielgerichtet zu bewältigen..$^{142}$

Als Instrumente zur Durchsetzung derartiger Ziele werden im wesentlichen der absatzmarktorientierte Einsatz personalpolitischer Instrumente, der absatzmarktorientierte Einsatz interner Kommunikationsinstrumente sowie der personalorientierte Einsatz externer Marketinginstrumente diskutiert. Insgesamt ergibt sich daraus ein Instrumentenpool, der Maßnahmen wie internes Training,

\footnotetext{
135 In ahnlicher Weise unterscheidet NEUHAUS die mitarbeiterbezogene und abteilungsbezogene Perspektive bei der Analyse interner Kunden-Lieferanten-Beziehungen. Vgl. Neuhaus (1996), S. 10 ff. sowie S. 40 ff.

136 Dies entspricht der Variante 3. Vgl. Kap. 2.2.2.2.

137 Vgl. Variante 1 in Kap. 2.2.2.2.

$138 \mathrm{Vgl}$. Rafig/Ahmend (1992), S. $184 \mathrm{ff}$.

139 Dies ist der Anspruch von Variante 2. Vgl. Kap. 2.2.2.2.

140 Vgl. Flipo (1986), S. 5 ff.

141 Vgl. Stauss (1995b), Sp. 1048.

142 Ausfuthrlich zu diesen Zielen vgl. Stauss (1991), S. 264.
} 
interne und externe Individual- und Massenkommunikation, Personalmanagement, interne Marktforschung und interne Marktsegmentierung beinhaltet. ${ }^{143}$

Der Schwerpunkt eines so verstandenen personalorientierten internen Marketings ist in der Implementierung der Denkhaltung einer konsequenten Kundenund Mitarbeiterorientierung zu sehen. Hierdurch soll eine nachhaltige Absicherung absatzmarktbezogener Unternehmensziele verfolgt werden. ${ }^{144}$ Pointiert geht es um einen „Verkauf der Konzepte nach innen“(145.

\subsection{Abteilungsorientiertes bzw. funktionsorientiertes internes Marketing}

Neben einer unmittelbaren Fokussierung auf den Mitarbeiter als solchen, wurde in jüngster Vergangenheit die Betrachtung auf Abteilungen als Teilbereiche der Gesamtorganisation ausgedehnt. Insbesondere in Fällen, in denen Mitarbeiter keinen unmittelbaren Kundenkontakt mehr haben, stellen andere Abteilungen die einzigen Interaktionspartner dar, denen somit die Kundenrolle zukommt.

War im Rahmen des personalorientierten internen Marketings im wesentlichen die Führung der Aktionsträger des Marketings, werden im Rahmen des abteilungs- bzw. funktionsorientierten ${ }^{146}$ internen Marketings Unternehmensteilbereiche zu Marketingtreibenden. Ein so verstandenes internes Marketing darf nicht als ein Instrument zur Förderung von den Gesamtunternehmensinteressen zuwiderlaufenden Bereichsegoismen fehlinterpretiert werden, vielmehr entspricht eine solche Ausgestaltung konsequent dem Ansatz des Total Quality Management (TQM). ${ }^{147}$ Dieser ist gleichsam als konzeptionelle Basis für das abteilungsorientierte interne Marketing anzusehen. ${ }^{148}$

Auf Grundlage der Entwicklung des Qualitätsverständnisses, welches sich im Laufe der Zeit, ausgehend von einer ergebnisbezogenen Qualitätsprüfung hin zu einer prozessbezogenen Qualitätssicherung gewandelt hat, rückt die Analyse der Netzwerke interner Kunden und Lieferanten immer mehr in den Mittelpunkt

143 Vgl. Stauss/Schulze (1990), S. $149 \mathrm{ff}$.

144 Vgl. Bruhn (1999), S. 22.

145 Meffert (2000), S. 1119.

146 Im Folgenden sollen bezogen auf Marketingaktivităten die Begriffe Funktion und Abteilung synonym verwandt werden.

147 Zur historischen Entwicklung des Total Quality Management sowie der damit einhergehenden Ausweitung des Qualitätsdenkens vgl. Adam (1998), S. 125 ff.

148 Vgl. Neuhaus (1996), S. $40 \mathrm{ff}$. 
der Betrachtung. Bei einer konsequenten Übertragung auf die gesamte unternehmerische Wertschöpfungskette müssen die Qualitätsanforderungen somit auf den gesamten Prozess der Leistungserstellung Anwendung finden, inklusive indirekter und steuernder Arbeitsprozesse. ${ }^{149}$ Insbesondere die Strukturierung sozio-technologischer Systeme in Subsysteme, sowie eine darauf aufbauende Analyse bestehender Interdependenzen charakterisiert umfassende Qualitätskonzepte. ${ }^{150}$ Ein Qualitätsbegriff ohne eine angemessene Berücksichtigung der Anforderungen interner Kunden ist daher heute kaum noch denkbar. ${ }^{151}$

Betrachtet man den Anspruch interner Dienstleister, durch Unterstützung ihrer Kunden einen Beitrag im Sinne der Unternehmensgesamtziele zu leisten, lassen sich auf Basis des TQM-Ansatzes verschiedene Ziele für eine zu entwickelnde interne Marketing Strategie ableiten: ${ }^{152}$

- Schaffung und Nachweis eines spezifischen Nutzens für interne Kunden und das Gesamtunternehmen,

- Stärkung der Wettbewerbssituation interner Dienstleister gegenüber externen Anbietern,

- stärkere Inanspruchnahme der Leistungen durch interne Kunden,

- Verbesserung der Ressourcensituation durch Erlangung angemessener Gegenleistungen,

- effizientere Nutzung der zugewiesenen Ressourcen.

Spezifiziert man derartige Ziele für einen Funktionsbereich, können darauf aufbauend konkrete Aktionen geplant werden. Hierbei ergeben sich verschiedene Parallelen zum externen Marketing. Ein idealtypischer Ablauf ${ }^{133}$ beginnt mit einer internen Marktforschung, um die Erwartungen spezifischer Abnehmergruppen sowie die potenzielle interne und externe Wettbewerbssituation ergründen zu können. Die darauf aufbauende Festlegung der Mission einer Abteilung erleichtert die Konkretisierung von Leistungszielen sowie eines in den Augen der Kunden wahrnehmbaren Leistungsangebotes. Auf Basis der vorgenommenen Analyse des internen Marktes erfolgt die Segmentierung der Kunden, um zu gewährleisten, dass bei der darauf folgenden Anwendung des Marketing-Mixes,

\footnotetext{
149 Vgl. Adam (1998), S. 127.

$150 \mathrm{Vgl}$. Schildknecht (1992), S. $101 \mathrm{f}$.

151 Vgl. Zink (1996), Sp. 2072.

152 Geringfügig modifiziert nach Stauss (1995b), Sp. 1053.

153 Der im Folgenden beschriebene Ablauf orientiert sich an dem für eine Personalabteilung entwickelten Schema von Collins/Payne (1991), S. 261 ff.
} 
die gewählten Teilsegmente möglichst zielsetzungsgerecht reagieren. Als Aktionsparameter stehen hier analog zu unternehmensexternen Märkten Produkt-, Preis, Distributions- und Kommunikationspolitik zur Verfügung. ${ }^{154}$ Darüber hinaus lassen sich Elemente eines kundenorientierten Prozessmanagements integrieren, indem die Leistungsplanung phasenorientiert erfolgt, um so Schnittstellen, Verantwortungsbereiche und Mitwirkungspflichten klar abzugrenzen. Ferner können im Rahmen einer innerbetrieblichen Kontraktpolitik Vereinbarungen über Leistungsinhalt, Leistungsumfang und Leistungsqualität geschlossen werden, welche im Fall einer Zielverfehlung zu fixierten Sanktionen führen können. ${ }^{\text {ss }}$

Ein derart ausgestaltetes internes Marketing vermag insbesondere in Zeiten, in denen sich Servicebereiche einem zunehmenden internen wirtschaftlichen und sozialen Legitimationsdruck ausgesetzt sehen, eine konsequente Ausrichtung der Mitarbeiter an den Bedürfnissen ihrer internen Kunden zu gewährleisten. Darüber hinaus kann das sachlich begründete und subjektiv wahrgenommene Profil eines Funktionsbereichs nachhaltig verbessert werden.

\subsection{Integrationsperspektiven der Teilansätze}

Unterstellt man vor dem Hintergrund der bisherigen Ausführungen ein alle Führungsteilsysteme umfassendes koordinationsorientiertes ControllingVerständnis, so ergibt sich bei der Ausgestaltung des konzeptionellen Rahmens das Problem der unmittelbaren Aufgabenzuordnung und Aufgabenerfüllung. ${ }^{156}$ Grenzen Ergebnis- bzw. Ergebnistransparenzverantwortung die Aufgabenbereiche von Manager und Controller noch scheinbar genau ab, bestimmt im Unternehmensalltag die institutionelle Ausgestaltung grundlegend die Wahrnehmung der Betroffenen. Aus diesem Grund kommt dem Controllership/Controllerdienst/Controllerbereich ${ }^{157}$ eine zentrale Rolle bei dem Betrieb eines wirkungsvollen Unternehmenscontrolling zu. Insbesondere die integrierende Darstellung von Verantwortungsbereichen und Verständniskontinuum ${ }^{158}$ verdeutlicht einerseits die Notwendigkeit einer von allen zu internalisierenden Management Philosophie Controlling, sowie andererseits auch die Bedeutsamkeit der organisatorischen Schaffung eines klar abgegrenzten Controllerbereichs. Mit der Errich-

\footnotetext{
154 Eine weitere Differenzierung der Marketing-Instrumente soll an dieser Stelle nicht vorgenommen werden.

155 Vgl. Stauss (1995b), Sp. 1052.

$156 \mathrm{Vgl}$. hierzu ausfuhrlicher Kap. 3.2.1.2.

157 Die Begriffe werden im Folgenden synonym verwandt.

158 Vgl. Abbildung 3.
} 
tung eines derartigen Bereiches werden Schnittstellen zu vielfältigen Unternehmensteilbereichen aufgebaut, die eines zielsetzungsgerechten Managements bedürfen. Verfolgt der Controllerbereich im Sinne eines internen Dienstleisters die Beschaffung, Aufbereitung, Analyse und Kommunikation von Daten, ${ }^{159}$ um damit seine Kunden in die Lage zu versetzten, zielsetzungsgerechte Entscheidungen treffen zu können, bedarf es hierzu einer kundenorientierten Führung des Controllerbereichs. ${ }^{160}$ Dies kann im Einzelfall bis hin zu einem internen Unternehmertum zur Steigerung der Mitarbeiter- und internen Kundenzufriedenheit führen. ${ }^{161}$ Wem in diesem Zusammenhang der Terminus des internen Unternehmers/Intrapreneur für den Ersteller von Controlling-Leistungen zu abwegig erscheint, der sei an SCHUMPETER erinnert, demzufolge die unternehmerische Funktion darin besteht, „dass sie Dinge in Gang setzt ${ }^{\text {‘162 }}$. In diesem Sinne müssen dem Controller als internem Unternehmer zwangsläufig angemessene Instrumente zur Behauptung auf internen Märkten zur Verfügung stehen. ${ }^{163}$ Insbesondere das bereits erwähnte Ansteigen des internen Marktdrucks für Controller zeigt den konkreten Handlungsbedarf auf. ${ }^{164}$

Bei der Suche nach entsprechenden Lösungsansätzen für derartige Probleme verspricht die Marketingtheorie geeignete Hinweise geben zu können. Im Gegensatz zum Begriff des Marketing-Controlling, der durch die Anwendung von Controlling-Instrumenten und -Techniken auf absatzmarktbezogene Fragestellungen charakterisiert wird, ${ }^{165}$ muss bei der vorliegenden Problemstellung die Integration der Teilansätze im Sinne eines Controlling-Marketing erfolgen. Wie bereits dargestellt, existieren unterschiedlich weit gefasste Verständnisse des Marketingbegriffs, die gleichsam die Grenzen seiner Anwendbarkeit determinieren. Von Seiten der Theorie bedingt daher die Art des unterstellten Verständnisses, inwieweit Marketing als tragfähige Denkfigur für die Lösung von Transaktionsproblemen zwischen Controlling und Controlling-Kunden herangezogen werden kann. Marketing, verstanden als umfassende Führungsphilosophie, schließt die Absicherung absatzmarktbezogener Ziele durch die planmäßige Ausgestaltung unternehmensinterner Austauschprozesse ein. Darüber hinaus

\footnotetext{
$159 \mathrm{Vgl}$. hierzu die in Kap. 2.1.3.2.2 gegebene Controlling-Definition.

160 Vgl. Kap. 3.1 sowie Weber (1999), S. 359 ff.

161 Mitarbeiter sind in diesem Zusammenhang alle Mitglieder der leistungserstellenden Einheit „Controlling“.

162 Schumpeter (1942), S. 12

163 Vgl. Ludwig (1995), S. 133 f.

164 Vgl. Abbildung 4.
}

165 Zur Entwicklung des Marketing-Controlling vgl. Meffert (1982), S. 100 f. Allg. zum Marketing-Controlling vgl. Meffert (2000), S. 1129 ff. oder Küpper (1997), S. 370 ff. Zum Handelscontrolling vgl. Ahlert (1994), S. $48 \mathrm{ff}$. 
kann auf Basis des TQM die Etablierung unternehmensinterner Märkte gerechtfertigt werden, soweit der im Rahmen interner Kunden-LieferantenBeziehungen stattfindende Leistungsaustausch nicht den Unternehmensgesamtzielen zuwiderläuft. Eine derartige Betrachtungsweise im Sinne des funktionsorientierten internen Marketings impliziert gleichsam eine funktionale/institutionale Betrachtungsweise des Controlling (Controllerdienst). Bezieht man an dieser Stelle die im Rahmen des Dienstleistungsmarketings entwickelten Verfahren zur Strukturierung von Dienstleistungen ein, so erlaubt eine Analyse des heterogenen Spektrums der Controlling-Leistungen Aussagen über deren zielgerichtete „Vermarktbarkeit" treffen zu können.

Somit sollte zur Ableitung derartiger Aussagen einerseits eine Analyse der Controlling-Leistungen anhand für die Kundenorientierung erfolgsrelevanter Faktoren erfolgen (Kapitel 3), um darauf aufbauend Empfehlungen für die kundenorientierte Gestaltung von Leistungspotenzial, -prozess und -ergebnis geben zu können (Kapitel 5). Kundenorientierung im Controlling ist somit als Handlungsmaxime zu verstehen, welche das Ziel verfolgt, den internen Kundennutzen des Controllings zu verbessern. ${ }^{166}$ In diesem Zusammenhang ist anzumerken, dass die angestrebten Erkenntnisse keineswegs im Sinne von Normstrategien zu verstehen sind. In Abhängigkeit der jeweiligen Leistung des Controlling, der Transaktionssituation sowie den kontextabhängigen Rahmenbedingungen eines Controllerbereichs sollte die Anwendbarkeit und Praktikabilität überprüft werden.

Wichtig bleibt jedoch für alle Controller die dahinterstehende Denkhaltung, die auch ohne eine konkrete instrumentelle Umsetzung in Entscheidungssituationen ein kundenorientiertes Vorgehen erlaubt. Auf Basis derzeitiger Erfahrungen empfiehlt sich eine umfassende Etablierung des Konzeptes insbesondere bei ergebnisverantwortlichen Serviceeinheiten größerer Unternehmen, die einem realen internen und externen Marktdruck ausgesetzt sind.

Abbildung 6 stellt nochmals zusammenfassend den theoretischen Bezugsrahmen der folgenden Ausarbeitungen dar. Auf Basis der jeweiligen Konzepte sowie regelmäßig anzutreffender Verständnisauffassungen beider Konzeptionen lassen sich Entwicklungstendenzen erkennen, die einerseits - problemorientiert - einen spezifischen Handlungsbedarf im Controlling aufzeigen, bzw. andererseits Strukturierungshilfen für derartige Problemstellungen bereithalten. Konkret heißt dies, dass eine konsequente Kundenorientierung im Controlling zu 
gewährleisten ist, soweit eine interne Marktorientierung auf Basis einer strukturierten Analyse des Leistungsangebotes und einer darauf aufbauenden zielgerichteten Maßnahmenplanung erfolgt. Hierbei kann auf die im Rahmen der externen Marktorientierung des Controlling erworbenen Kenntnisse zurückgegriffen werden, da diese - bezogen nun auf das Controlling als Leistungsersteller vielfach zu einer Absicherung der internen Strategie beitragen können (bspw. Target Costing für Controlling-Leistungen).

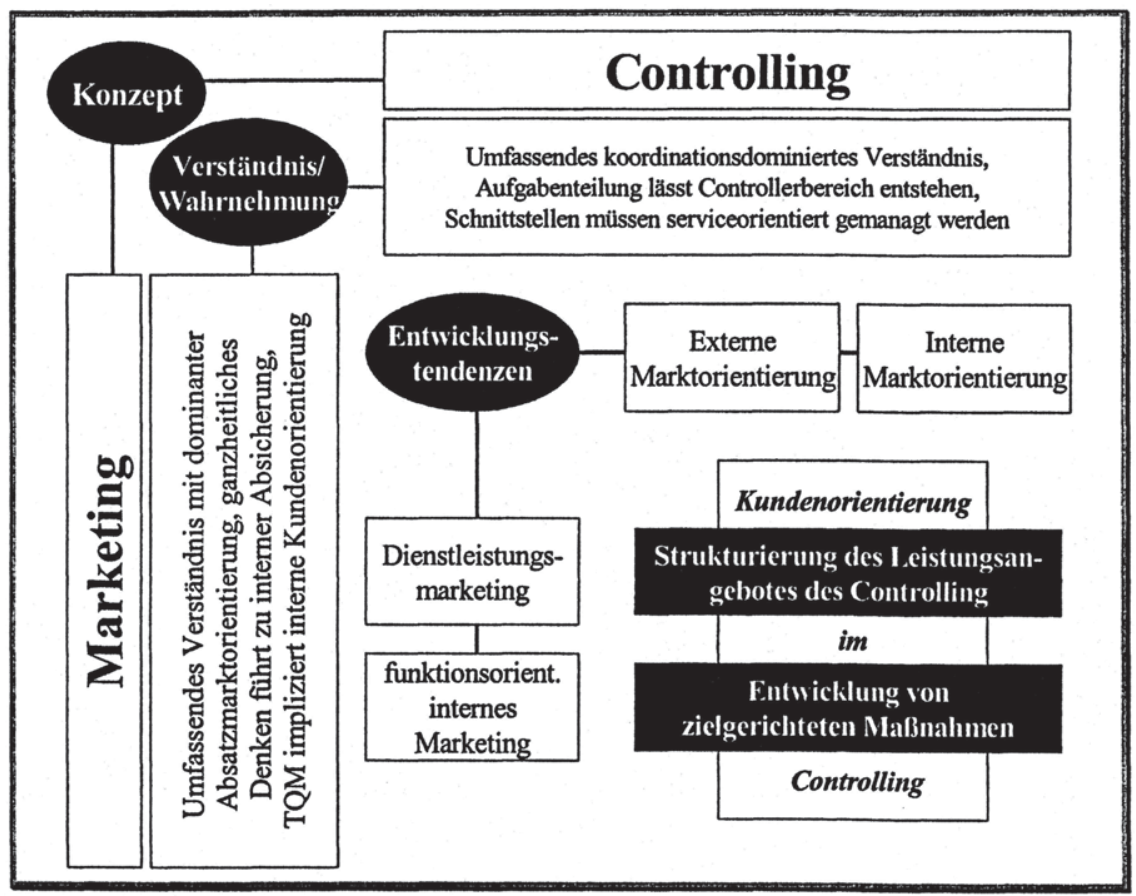

Abbildung 6: Integrationsperspektiven der Teilansätze

Nach der Formulierung und Erfassung des Phänomens Kundenorientierung im Controlling auf Basis terminologischer Aussagen gilt es im Folgenden zunächst eine Deskription der Realität vorzunehmen. Auf Basis einer Darstellung der Rahmenbedingungen des kundenorientierten Controllings können dann Gestaltungsparameter abgeleitet werden, die als Grundlage für einen zielgerichteten Einsatz von Instrumenten aufzufassen sind. Wesentliches Ziel für Maßnahmen zur Steigerung der Effektivität und Effizienz des Controllerbereichs sollte dabei stets die Steigerung der Effektivität und Effizienz der gesamten Unternehmens- 
führung sein. ${ }^{167}$ Dass eine derartige Kausalbeziehung zwischen Bereichs- und Unternehmenszielen regelmäßig auch in der Praxis besteht, zeigen unterschiedliche Untersuchungen. ${ }^{168}$

$167 \mathrm{Zu}$ den Begriffsdefinitionen von Effektivităt und Effizienz vgl. Kap. 3.

$168 \mathrm{Vgl}$. Sathe (1982) sowie Anderson/0'Reilly (1981), S. 491-501. 


\section{Gestaltungsraum für ein kundenorientiertes Controlling}

Unterstellt man, dass das Controlling mittels seiner Unterstützungsfunktion einen Beitrag zur Erreichung der Gesamtunternehmensziele leistet, ergibt sich die Frage nach der Bemessung bzw. Beeinflussbarkeit dieses Beitrags. Da jedoch wie leicht ersichtlich ist - der Beitrag des Controllings am Gesamterfolg der Unternehmung nur partieller Natur ist, ist es erforderlich, die Wirkung des Outputs des Controlling auf andere Art und Weise zu bewerten. Neben vielen durch das Controlling nicht beeinflussbaren Faktoren des Unternehmenserfolges stellt die Ausgestaltung der kundenbezogenen Interaktionen des Controlling eine gestaltbare - für die Wirksamkeit des Controllings maßgebliche - Variable dar. Im Folgenden Kapitel sollen daher auf Basis des theoretischen Fundamentes gestaltungsrelevante Faktoren für eine effiziente und effektive Erstellung und Verwertung von Controlling-Leistungen identifiziert werden. Der Schwerpunkt der Betrachtung liegt gemäß der Problemstellung auf den für die Bedürfnisbefriedigung der internen Kunden maßgeblichen Leistungsmerkmalen. Die Begriffe Effizienz und Effektivität sollen nachfolgend in Anlehnung an angloamerkanische Ausführungen differenziert verstanden werden. ${ }^{169}$ ETZIONI definiert ,efficiency" als ,,... the amount of ressources used to produce a unit of output"; ,effectiveness" meint davon abweichend ,... degree to which [an organization or a part of this] realizes it's goals ${ }^{\text {(1170. }}$. Unter Effizienz soll daher nachfolgend ein internes Leistungsmaß für den Controllerbereich verstanden werden, welches das Verhältnis von Output zu Input wiedergibt. ${ }^{171}$ Demgegenüber stellt die Effektivität unter Rückgriff auf die Marketingtheorie ein externes Leistungsma $\beta$ dar, mit dem die Befriedigung der Erwartungen und Ansprüche der Controlling-Kunden gemessen werden soll. ${ }^{172}$ Effizienz und Effektivität determinieren gleichermaßen das Ausmaß potenzieller Wettbewerbsvorteile. Demgegenüber wird derzeit im Rahmen der Diskussion der Leistungsfähigkeit des Controlling überwiegend auf Effizienzgesichtspunkte Bezug genommen, da diese sich regelmäßig besser operationalisieren lassen. ${ }^{173}$ Aufgrund der Besonderheiten der unternehmensinternen Dienstleistung Controlling, ist es für eine Analyse der Effektivität grund-

169 In der Wissenschaft gibt es eine anhaltende Diskussion zum Begriffsverstăndnis von Effizienz und Effektivität. Vertreten werden sowohl Ansătze, die von einer weitgehenden Übereinstimmung der Begriffe ausgehen, wie auch solche Ansătze, die Effektivität als eine grundsătzliche Voraussetzung fulr Effizienz ansehen. Einen guten Überblick zum Stand der Effizienzforschung und den damit korrespondierenden Begriffsabgrenzungen bietet Benz (1998), S. $48 \mathrm{ff}$.

Etzioni (1964), S. 8. Die Ergannzungen in den eckigen Klammern erfolgen durch den Verfasser.

171 Ohne den speziellen Bezug zum Controlling vgl. Plinke (1995), S. 85.

172 Vgl. Plinke (1995), S. 85.

173 Zur Effizienzforschung im Controlling vgl. Benz (1998). Zu Effizienzkriterien des Controlling im Versicherungsbereich vgl. Hopp (1992), S. $87 \mathrm{ff}$. 
legend erforderlich, zunächst Produkteigenschaften und Marktgegebenheiten für Controlling-Leistungen zu betrachten, bevor Gestaltungsempfehlungen gegeben werden können. Einige Problembereiche, die die Anwendbarkeit des klassischen Marketing-Instrumentariums erschweren und daher einer differenzierteren Analyse bedürfen, sind: ${ }^{174}$

- relative Monopolstellung des innerbetrieblichen Anbieters,

- organisatorische Einbindung und Kompetenzen des Anbieters,

- Enge des relevanten Marktes,

- mangelnde Leistungsevidenz ${ }^{175}$,

- mangelnde Prognostizierbarkeit der Qualität und Quantität des Bedarfs,

- Kapazitätsvorhaltepflichten,

- Breite des Leistungsspektrums und Dienstleistungscharakter der Produkte.

Ohne eine Ermittlung von Gestaltungsparametern, unter denen alle für die kundenorientierte Leistungserstellung und -verwertung erfolgskritischen Tatbestände verstanden werden, können Gestaltungsempfehlungen nur unzureichend gegeben werden. Gestaltungsempfehlungen sind hier gemäß einer im Rahmen des abteilungs- bzw. funktionsorientierten internen Marketings angesprochenen, umfassenden internen Marketing Strategie zu verstehen. Als Strukturierungsrahmen für die Identifikation gestaltungsrelevanter Faktoren soll nachfolgend das Marketing Dreieck verwendet werden. Dieses Analyseinstrument vermag die Interdependenzen zwischen den Bedürfnissen der Nachfrager, dem Problemlösungs-Know how der Konkurrenten sowie dem eigenen Problemlösungs-Know how des Anbieters abzubilden. ${ }^{176} \mathrm{Im}$ Mittelpunkt stehen hierbei die relativen Positionen der beteiligten Akteure. Zielsetzung für den betroffenen Anbieter ist regelmäßig die Erzielung von $K K V^{\prime} s$ in der subjektiven Wahrnehmung der aktuellen und potenziellen Nachfrager. Eine als Rahmenbedingung der Leistungserstellung und Verwertung vorausgesetzte Wettbewerbssituation wird im Rahmen der nachfolgenden Analyse zunächst pauschal unterstellt, bevor differenziert auf das Ausmaß der Wettbewerbs-/Konkurrenzgefährdung ${ }^{177}$ unterschiedlicher Leistungen des Controlling eingegangen wird. Ein Verständ-

174 Die Darstellung der Besonderheiten unternehmensinterner Dienstleistungen erfolgt in Anlehnung an Witt (1985), S. $163 \mathrm{f}$.

175 Unter der Evidenz einer Leistung ist hier das Bewusstsein über deren Bedeutung zu verstehen. Vgl. Witt (1988), S. 670. Eine ausfuhrliche Behandlung der Evidenz erfolgt unter Kap. 3.4.3.2.

176 Vgl. Backhaus (1999), S. 28.

177 Vgl. Kap. 3.4.3.3. 
nis des Wettbewerbs als ,Suchprozess, der darauf gerichtet ist, durch Generierung neuer Lösungen vorhandene Bedürfnisse besser (oder billiger) zu befriedigen, um daraus einen eigenen ökonomischen Vorteil zu ziehen"178, ist für die Folgebetrachtung zielführend, soweit die Zielsetzung einer Verfolgung eigener ökonomischer Vorteile mittels geeigneter Koordinationsinstrumente auf die Erreichung der Gesamtunternehmensziele abgestimmt wird.

Die Durchführung einer Analyse des Controlling auf Basis des Marketing Dreiecks soll sicherstellen, dass alle relevanten Determinanten der Leistungserstellung und -verwertung angemessen berücksichtigt werden. ${ }^{179}$ An dieser Stelle macht die Tatsache, dass es sich bei dem Controlling um eine unternehmensinterne Dienstleistung handelt, eine Modifikation des Grundschemas erforderlich. Abbildung 7 zeigt ein auf diesen Erkenntnissen basierendes modifiziertes Marketing Dreieck.

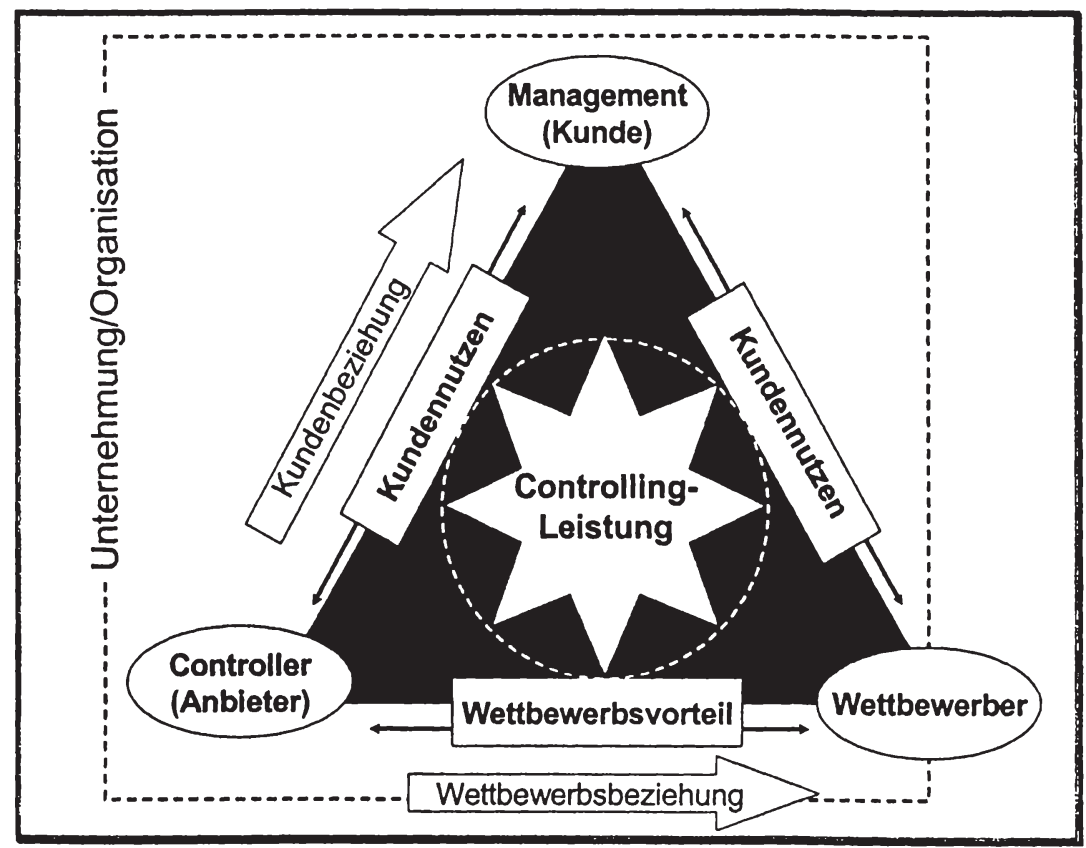

Abbildung 7: Modifiziertes Marketing Dreieck

178 Backhaus (1999), S. 26, ferner vgl. Meffert (2000), S. 6 f.

179 Der Betrachtungsschwerpunkt liegt hierbei auf unternehmensinternen Determinanten, da diese für Gestaltungsfragen im Controlling regelmäBig eine böhere Relevanz aufweisen. So auch für die Kostenrechnung Aust (1999), S. 141. 
Die Unternehmensgrenzen als Schranken der unmittelbaren Leistungsverwertung des Controlling erfordern eine angemessene Berücksichtigung. ${ }^{180}$ Demgegenüber stellen die Unternehmensgrenzen für externe Wettbewerber keine absolute ,Marktzutrittsbarriere" dar, vielmehr ist auch hier das Ausmaß der Konkurrenzgefährdung in Abhängigkeit der jeweiligen Leistungsinhalte zu bestimmen.

Bezogen auf die Betrachtung von Controlling-Leistungen kommt den Marketing Dreieck die Funktion eines Visualisierungs- und Analyseinstrumentes zu. Beabsichtigt man auf Basis dieser Darstellung die Ausgangssituation des Controlling zu analysieren, erscheint eine leistungs-, organisations- sowie kundenund wettbewerbsbezogene Betrachtungsweise des Controlling zweckmäßig, um nachfolgend die Positionierung eines Controllerbereichs vornehmen zu können.

Durch eine derart strukturierte Vorgehensweise zur Entwicklung darauf aufbauender Handlungsempfehlungen kann dem Verdacht begegnet werden, das in Literatur und Praxis etablierte Marketing Konzept über die Grenzen seiner Anwendbarkeit hinaus, blind auf dafür ungeeignete Sachverhalte zu übertragen. Die im Folgenden gewählten Strukturierungskategorien Leistungsbezug, Organisationsbezug sowie Kunden- und Wettbewerbsbezug erheben nicht den Anspruch einer vollständig trennscharfen Abgrenzung einzelner gestaltungsrelevanter Charakteristika des Controllings. Durch ihre Auswahl soll vielmehr die Vollständigkeit der Abbildung aller marketingrelevanten Sachverhalte sichergestellt werden. Ferner würde eine marketingorientierte Analyse idealerweise eine simultane Betrachtung von Leistung, Kunde und Kontext erfordern, da diese drei Betrachtungsebenen sich wechselseitig bedingen. Eine strukturierte Analyse kann jedoch nur bei sukzessiver Darstellung der Betrachtungsebenen erfolgen, so dass sich gewisse Interdependenzen erst im Laufe der Arbeit erschließen lassen.

Bevor jedoch auf Basis der gewählten Strukturierungskriterien auf einzelne Erfolgsdeterminanten eingegangen wird, soll ein Gesamtüberblick über das Führungssystem des Controllerbereichs gegeben werden. In diesem Zusammenhang kann bereits ansatzweise gezeigt werden, wo Verantwortlichkeiten einer gesamthaft kundenorientierten Ausrichtung des Controlling anzusiedeln sind, ohne den Gestaltungsempfehlungen vorzugreifen. Zudem soll analog zu der konzeptionellen Fundierung des Controlling dargestellt werden, inwieweit die

180 Im Rahmen der Analyse werden nur die unmittelbaren unternehmensinternen Kunden betrachtet. 
institutionelle Ausgestaltung die Wahrnehmung der Akteure beeinflusst und prägend für Umsetzungsmaßnahmen ist.

\subsection{Ziele der Kundenorientierung im Controlling und kundenorien- tierte Führung des Controllerbereichs}

Diskutiert man konkrete Maßnahmen zur internen Kundenorientierung des Controlling, oder - allgemeiner noch - Implementierungsmöglichkeiten von Marketing-Strategien für den Unternehmensteilbereich, so stellt sich zunächst die Frage nach den damit verfolgten Zielen. Ausgehend von der Definition der Kundenorientierung, ${ }^{181}$ ist das allgemeine Ziel diesbezüglicher Maßnahmen in einer Verbesserung des Kundennutzens zu sehen. Im Gegensatz zum Old Concept of Marketing, in dem es um Verkaufsaktivitäten bereits spezifizierter Produkte geht, steht bei dem New Concept of Marketing, im Sinne der Kundenorientierung, zunächst die Ergründung von Bedürfnissen und Präferenzen der Nachfrager im Mittelpunkt, um darauf aufbauend Produkte spezifizieren zu können. ${ }^{182}$

Zielsetzung des Controlling muss es daher sein, nicht den Absatz seiner Leistungen zu maximieren, sondern vielmehr das hinter der Bedarfsartikulation stehende Problem des Kunden sachgerecht zu lösen. Kritisch ist eine derartige Zielsetzung immer dann, wenn die artikulierten Kundeninteressen den Gesamtzielen des Unternehmens zuwiderlaufen. Wesentlich für den Controller ist in diesem Zusammenhang, das Bedürfnis seines Kunden bezogen auf den Unternehmenskontext zu objektivieren, ${ }^{183}$ d.h. der Controller muss sicherstellen, sich primär an solchen Bedürfnissen zu orientieren, die mit den Unternehmensgesamtzielen harmonieren, da er sonst seiner Rationalitätssicherungsfunktion nicht gerecht würde. ${ }^{184}$ Ein solches Vorgehen kann grundsätzlich immer dann als auch im Sinne des Kunden angesehen werden, wenn dieser nicht vorsätzlich, sondern nur aufgrund anderer Rationalitätsengpässe, wider den Unternehmenszielen handelt. So betrachtet kann auch Kontrolle und Widerspruch des Controllers in Einzelsituationen als Kundenorientierung verstanden werden, ${ }^{185}$ soweit diese in einem größeren Entscheidungsunterstützungszusammenhang sicherstel-

181 Vgl. Hanan/Karp (1991), S. 101 sowie hier Kap. 2.2.

182 Zum Old und New Concept of Marketing vgl. Bidlingmaier (1973), S. 14.

183 HOFFKEN sieht es demgegenüber als Aufgabe der Unternehmensleitung an, die Inanspruchnahme von Kosteninformationen zu reglementieren. Vgl. Hoffken (1976), S. 91 ff.

184 Zur Rationalitätssicherungsfunktion des Controlling vgl. Weber (1999), S. 39.

$185 \mathrm{Vgl}$. ausfuhrlich hierzu die Ausfuhrungen zum Kontrollcharakter von Controlling-Leistungen in Kap. 3.2.2.3.2. 
len, dass der Kunde langfristig seinen mit den Unternehmenszielen korrespondierenden Individualzielen nachkommen kann. Im Regelfall wird der Kunde ein solches Verhalten des Controllers - eventuell im Gegensatz zu seiner kurzfristigen Wahrnehmung - langfristig und retrospektiv als nutzbringend erachten, womit das Ziel der Kundenorientierung erreicht wäre.

Kundenorientierung kann darüber hinaus aber auch im Controlling als eine Strategie verstanden werden, sich im Unternehmenskontext über den Nachweis eines einzigartigen Nutzens zu behaupten und damit seine Stellung in der Organisation zu stärken. Übersichtsartig lassen sich die Ziele des kundenorientierten Controlling dann wie folgt darstellen: ${ }^{186}$

- Schaffung und Nachweis eines spezifischen Nutzens für ControllingKunden und mittelbar über diese für die Gesamtunternehmung,

- Verbesserung der Inanspruchnahme und Nutzung problemlösungsadäquater Leistungen durch interne Kunden, um so die Existenz des Controllerbereichs zu legitimieren und Kapazitäten sachgerecht auszulasten,

- Verbesserung der Ressourcensituation durch Erlangung angemessener, anreizkonform gestalteter Gegenleistungen,

- effizientere, auf die Kundenpräferenzen ausgerichtete Nutzung der zugewiesenen Ressourcen,

- Stärkung der Wettbewerbsposition gegenüber externen und internen Anbietern über Transparenz und Innovation.

Werden konkrete Maßnahmen auf Basis des Ziels der Nutzenverbesserung angestrebt, erscheint es unmittelbar ersichtlich, dass die Initiative derartiger Aktivitäten eine konkrete Aufgabe der Bereichsführung ist. Bezieht man dies auf den Controllerbereich, so ergibt sich die Notwendigkeit, die Aufgabe einer kundenorientierten Ausgestaltung des Controlling in den Kontext der Führungsaufgaben des Controllerbereichs einzubetten, bzw. darzustellen, inwiefern eine konsequente Kundenorientierung die Wahrnehmung der einzelnen Führungsteilaufgaben im Controlling beeinflusst. Abbildung 8 gibt einen Überblick über die Führungsaufgaben im Controlling.

Die Art der Darstellung impliziert die teilsystemübergreifende Notwendigkeit, Maßnahmen der Kundenorientierung im Controlling abzusichern. Wie bereits im Rahmen der Ausführungen zum TQM dargestellt, stellt die Qualität interner

186 Hier spezifisch für Controlling-Leistungen. Allgemein vgl. Stauss/Neuhaus (1995), S. 586. Vgl. hierzu auch die Ziele des abteilungs- bzw. funktionsorientierten internen Marketings in Kap. 2.2.2.2.2. 
Austauschprozesse eine unmittelbare Voraussetzung für den Unternehmenserfolg dar. Diesbezüglich wird im Rahmen der Grundprinzipien des Malcolm Baldrige National Quality Awards die „Verantwortung der Führungsebene”"187 als unerlässliche Voraussetzung für ein TQM interner Dienstleister genannt. Nachfolgend sollen teilsystemspezifische Anknüpfungspunkte für eine Kundenorientierung im Controlling aufgezeigt werden.

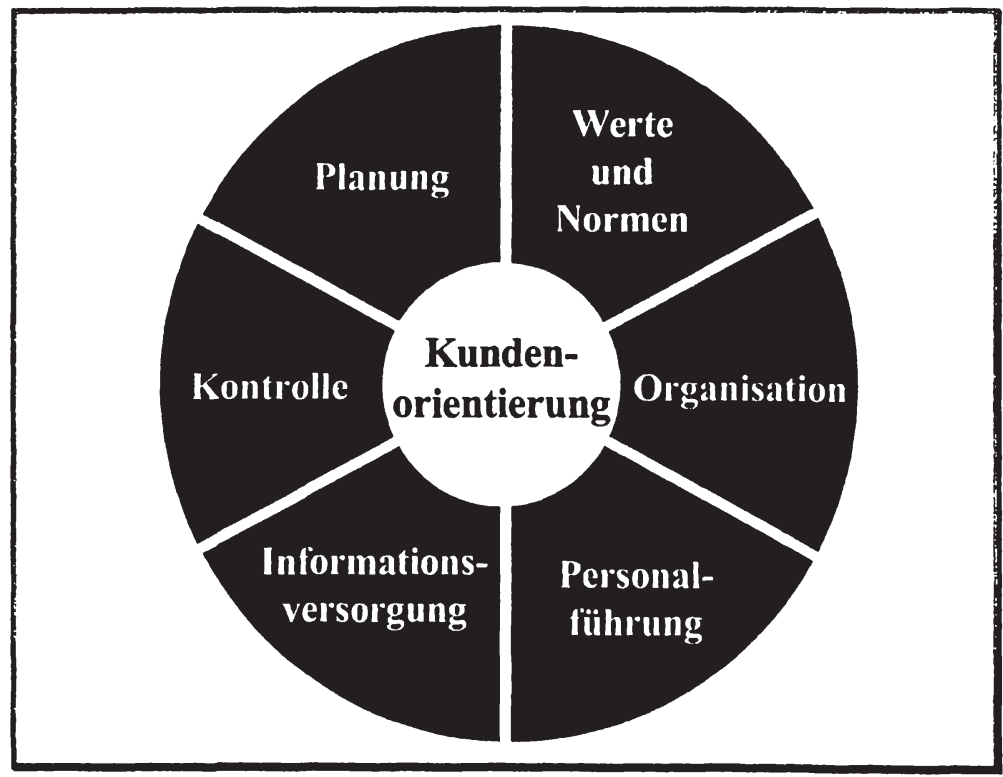

Abbildung 8: Kundenorientierung als Denkfigur bei der Führungsaufgabenwahrnehmung im Controllerbereich ${ }^{188}$

Die Fundierung von Werten und Normen stellt eine Teilaufgabe der Controllerbereichsführung dar. Auch wenn der Spielraum für die Ausgestaltung eines eigenen Leitbildes wesentlich durch Werte und Normen des Gesamtunternehmens eingegrenzt ist, erscheint eine Abgrenzung gegenüber anderen Unternehmensteilbereichen notwendig und sinnvoll, ohne einem Bereichsdenken zu sehr Vorschub zu leisten. Insbesondere über die Definition eines eigenen Leitbildes besteht die Möglichkeit einer Profilierung gegenüber unternehmensinternen Kun-

187 NIST (1993), S. 2 ff. Vgl. ferner George/Weimerskirch (1994), S. 7.

188 In Anlehnung an Weber (1999), S. 360. 
den und Lieferanten. Ferner kann schon die gemeinschaftliche Erarbeitung eines Leitbildes grundlegende Umdenkprozesse auslösen. ${ }^{189}$

Aus der organisatorischen Einbindung des Controlling ergeben sich unmittelbar organisationsspezifische Führungsaufgaben für die Bereichsführung. Insbesondere das Zusammenspiel zentraler und dezentraler Controllingeinheiten lässt zusätzlichen Koordinationsbedarf entstehen. Ferner determiniert die Organisation des Controlling wesentlich die Wettbewerbssituation des internen Dienstleisters Controlling und damit die Möglichkeit, Ansprüche aktueller und potenzieller Kunden zu befriedigen.

Die einfachste Möglichkeit einer anreizkonformen Personalführung im Controllerbereich stellt eine auf Basis einer Performance Messung ${ }^{190}$ ermittelte Prämiengestaltung dar. ${ }^{191} \mathrm{Da}$ die Operationalisierung der Controller-Performance jedoch regelmäßig Schwierigkeiten bereitet, ${ }^{192}$ kommt der Motivation über eine zielgerichtete Karrieregestaltung und eine angemessene Partizipation eine besondere Bedeutung zu. ${ }^{193} \mathrm{Im}$ Rahmen eines vermehrten Einsatzes der Balanced Scorecard zur Steuerung von Unternehmensteilbereichen liegt jedoch die Ausnutzung derselben zu Personalführungszwecken im Controlling nah. Insbesondere die Kundenperspektive der Balanced Scorecard könnte bei einer sachgerechten Implementierung im Controlling anreizkonforme Maßgrößen als Basis für ein Prämiensystem liefern. ${ }^{194}$ Hierdurch erhält die Kundenorientierung eine angemessene Berücksichtigung auch im Personalführungssystem.

Die Ermittlung der Performance des Controller Bereichs hat einen unmittelbaren Bezug zu den Informationsaufgaben der Controlling Führung. Neben der Sicherstellung einer regelmäßigen und bereichsübergreifenden Kommunikation kommt insbesondere der Publikation von Performance-Daten des Controlling eine besondere Bedeutung zu. Hierdurch kann die anderen Unternehmensteilbereichen abverlangte Transparenz selbst - bezogen auf eigene Prozesse und Er-

189 Verschiedene Ausgestaltungsmöglichkeiten von Controllerbereichs-Leitbildern finden sich bei Weber (1999), S. 362 ff. sowie Weber (1997b), S. $180 \mathrm{ff}$.

190 Zum Begriff der Performance Messung bzw. dem Performance Measurement vgl. Gleich (1997), S. $114 \mathrm{ff}$.

191 Zum Verhältnis zwischen intrinsischer und extrinsischer Motivation von Controllern in Abhängigkeit von unterschiedlichen Controller-Aufgaben vgl. Gaulhofer (1987), S. 1125 sowie Pfohl/Zettelmeyer (1987), S. 157.

$192 \mathrm{Zu}$ den Möglichkeiten einer Performance Messung im Controlling vgl. Kap 4.2.2.

193 WEBER benennt in diesem Zusammenhang die technisch-funktionale Kompetenz sowie die Autonomie und Unabhăngigkeit des Controllers als die „Karriereanker”. Vgl. Weber (1999), S. 392.

194 Die konkrete Ausgestaltung einer Balanced Scorecard furr einen Controllerbereich findet sich bei Galgenmuller/Gleich/Pfohl (1999), S. 535 ff., Gleich (1997b), S. 343 ff. sowie Gleich/Haindl (1996), S. $262 \mathrm{ff}$.

Thomas Mosiek - 978-3-631-75321-7 
gebnisse - dokumentiert werden. Eine solche kunden- und lieferantenbezogene Kommunikationspolitik kann wesentlich zu einer Akzeptanz der Leistungen und der damit verbundenen Kosten (soweit diese nutzungsadäquat verrechnet werden) beitragen.

Die zielgerichtete Führung des Controllings bedarf letztlich wechselwirkender Planungs- und Kontrollaktivitäten. Analog zu externen Märkten muss das Leistungsspektrum bezogen auf die Kundenbedürfnisse geplant werden, sowie im Anschluss an den Leistungserstellungsprozess einer Bewertung, inwieweit Einzelleistungen zur Bedürfnisbefriedigung der Kunden bzw. der Gesamtorganisation beigetragen haben, unterzogen werden.

Insgesamt wird aus dieser überblicksartigen Darstellung von Zusammenhängen zwischen Kundenorientierung und Führung des Controlling deutlich, dass letztlich keines der Führungsteilsysteme ohne konsequente Fokussierung der Leistungsadressaten auskommt. Will man die Kundenorientierung konsequent im Führungssystem des Controlling absichern, empfiehlt es sich, auf dafür entwickelte Denkfiguren des Marketing zurückzugreifen und diese auf ihre Anwendbarkeit hin zu überprüfen. Im Folgenden sollen daher auf Basis der vorgestellten Strukturierungskategorien grundlegende Gestaltungsparameter des Controlling beschrieben und analysiert werden. Hat man so zu einer Präzisierung dieser Tatbestände beigetragen sollen im vierten Kapitel - aufbauend auf die hier überblicksartig beschriebene Führung des Controllerbereichs - Gestaltungsempfehlungen für eine marketingstrategische Ausgestaltung gegeben werden.

\subsection{Leistungsbezogene Charakteristika des Controlling}

In der nachfolgenden leistungsbezogenen Betrachtung sollen, wie später auch für die organisations- sowie kunden- und wettbewerbsbezogene Perspektive, zunächst Gegenstand und Rahmenbedingungen - hier der Erstellung von Leistungen im Controlling - dargestellt werden. Dazu bedarf es zunächst einer Betrachtung der im Vorfeld der Leistungserstellung vorzunehmenden Aufgabenzuordnung, bevor auf Leistungsinhalte eingegangen werden kann. Darauf aufbauend kann auf die aus Marketing-Sicht relevanten und damit fur die Kundenorientierung maßgeblichen Gestaltungsparameter der internen Leistungen des Controlling eingegangen werden. 


\subsubsection{Controlling-Leistungen als operative Instrumente zur Aufgaben- erfüllung}

Im Rahmen der Analyse des Gegenstandes der Controlling-Leistungen, sowie die die Inhalte einzelner Leistungen tangierenden Rahmenbedingungen ist eine Betrachtung der Aufgaben des Controlling unerlässlich. Unter Aufgabe ist im Folgenden eine ,dauerhaft wirksame Aufforderung an Handlungsträger, festgelegte Handlungen wahrzunehmen "195 ${ }^{\text {"1u }}$ verstehen. Die Beschreibung des Gesamtspektrums der Controlling-Aufgaben sowie deren Kategorisierung ist Gegenstand unterschiedlichster Untersuchungen. Geläufig sind in diesem Zusammenhang z.B. Analysen von Aufgabenkatalogen der Berufsverbände, Auswertungen von Stellenanzeigen oder empirische Vergleichsuntersuchungen von Unternehmen. ${ }^{196}$ Als erster umfassender Aufgabenkatalog für die amerikanische Controlling Praxis gilt der Definitionsansatz des FINANCIAL EXECUTIVES INSTITUTE. ${ }^{197}$ Derart kasuistische Aufgabenaufzählungen sind geeignet, einen anschaulichen Überblick zu geben.

Wissenschaftstheoretisch sollte die Konkretisierung spezifischer ControllingLeistungen jedoch auf Basis des gewählten konzeptionellen Rahmens und der damit korrespondierenden Ziele erfolgen. Auf Grundlage des gewählten Ansatzes können Controlling Funktionen abgeleitet werden, die ihrerseits wiederum Aufgabenbereiche begründen. ${ }^{198}$ Zur Erfüllung spezifischer Aufgaben stehen dem Aufgabenträger die Leistungen als operative Instrumente zur Verfügung.

Ein derartiger Zusammenhang impliziert, dass insbesondere die Aufgabenzuordnung sowie die davon abhängigen Leistungsinhalte den Spielräumen der jeweiligen institutionellen Ausgestaltung unterliegen. Will man somit die Leistungsinhalte einer dezidierten Betrachtung unterziehen, ist es zunächst zweckmäßig, die für die Aufgabenzuordnung maßgeblichen Aspekte zu betrachten. Erst auf Basis effizient zugeordneter Aufgaben können die Inhalte der zur ihrer Wahrnehmung vorgesehenen Leistungen zweckmäßig abgegrenzt werden. Somit determiniert die Aufgabenzuordnung die Bandbreite des potenziellen Leistungsspektrums eines Controllers, wodurch einer umfassenden Kundenorientierung im Controlling wesentliche Rahmenbedingungen gesetzt werden.

195 Gabler (2000), S. 224.

196 Ein guter Überblick über Erhebungen zum Aufgabenspektrum des Controllers findet sich bei Serfling (1992), S. 36-59 und Horváth (1998), S. 17 ff.

197 Vgl. Financial Executives Institute (1962), S. 289.

198 HORVÁTH betont in diesem Zusammenhang, dass die Klänung der Controlling-Funktion unabdingbar der organisatorischen Institutionalisierung vorgeschaltet sein sollte. Vgl. Horváth $(1978)$, S, 134, $321-7$ 


\subsubsection{Aufgabenzuordnungsaspekte}

Die Aufgabenzuordnung stellt die Grundlage für die Ausgestaltung des Controlling-Leistungsangebotes dar. Eventuelle Mängel im Rahmen der Leistungserstellung und -verwertung können ihren Ursprung bereits in einer unsachgemäßen Aufgabenzuordnung haben. Die potenzielle Einengung des Gestaltungsspielraums durch die Aufgabenzuordnung erfordert bereits an dieser Stelle eine methodische Unterstützung der Vorgehensweise.

Die primär funktionale Betrachtungsweise des Controlling in der Vergangenheit hat zu einer oftmals wenig differenzierten Auseinandersetzung mit der Zuordnungsproblematik von Aufgaben zu Aufgabenträgern geführt. Insbesondere die regelmäßig proklamierte Kontextabhängigkeit der Aufgaben des Controlling darf nicht dazu führen, ${ }^{199}$ das Problem der Aufgabenzuordnung zu vernachlässigen. Vielmehr sollte auch dieser Bereich einer Analyse auf Basis intersubjektiver Strukturierungsmerkmale zugänglich gemacht werden. Neben controllingspezifischen Merkmalen ist hier insbesondere die Einbeziehung organisationstheoretischer und verhaltenswissenschaftlicher Erkenntnisse erforderlich.

Bezogen auf das Erkenntnisziel dieser Arbeit bietet eine strukturierte Analyse der Aufgabenzuordnung einen ersten Ansatzpunkt, Zuständigkeiten für die Erstellung von Controlling-Leistungen abzugrenzen, sowie darauf aufbauend die Ausgestaltung der Leistungsbeziehungen zwischen Controller und Manager zu optimieren. Insbesondere auch die Tatsache, dass - soweit bei der Aufgabenzuordnung Aufgabenträgeralternativen bestehen - diese potenzielle Wettbewerber des Controlling sein können, verdeutlicht die Entscheidungskonsequenzen in diesem Bereich. Da die Aufgabenzuordnung wesentliche Rahmenbedingungen für die Leistungserstellung und -verwertung der Controlling-Leistungen setzt, sind diesbezügliche Interdependenzen offenzulegen. Ferner kann nur über eine begründete Aufgabenzuordnung die Transformation konzeptioneller Ansprüche auf die Ebene der operativen Leistungserstellung gelingen.

\subsubsection{Strukturierungsrahmen für die Aufgabenzuordnung}

$\mathrm{Da}$ die Zuordnung von Aufgaben den Gestaltungsspielraum des Controllerdienstes wesentlich determiniert, werden hierdurch bereits grundlegende Entscheidungen über die Wirksamkeit und damit Wettbewerbsfähigkeit des Controlling getroffen. Allgemein ergibt sich die Notwendigkeit einer Aufgabenzuord-

199 Zur Kontextabhängigkeit der Controlling-Aufgaben ygl. Zünd (1985), S. 31. 
nung aus der arbeitsteiligen Aufgabenwahrnehmung komplexer Organisationsformen. Den durch die Arbeitsteilung hervorgerufenen Spezialisierungsvorteilen stehen Koordinationskosten gegenüber. ${ }^{200}$ Der Controllerdienst ist in diesem Zusammenhang als eine Ausprägung der Spezialisierung anzusehen, da eine strukturelle Differenzierung nach Inhalten vorgenommen wird. ${ }^{201}$ Insbesondere empirische Beobachtungen haben sich in der Vergangenheit der Frage angenommen, welche Inhalte dem Controllerdienst zuzuordnen sind. ${ }^{202}$

Betrachtet man demgegenüber konkrete Entscheidungssituationen in der Praxis, so ergibt sich die Notwendigkeit - sofern man sich bei der Ausgestaltung nicht ausschließlich den empirischen Befunden anschließen will - systematisch kriteriengeleitet vorzugehen. In diesem Zusammenhang werden regelmäßig einzelne, für eine effiziente Aufgabenzuordnung relevante Kriterien diskutiert, wobei eine Strukturierung nach Kosten- und Nutzenbeiträgen oftmals am naheliegensten ist, regelmäßig jedoch, aufgrund der mangelnden Operationalisierbarkeit der Nutzenkomponente, wenig pragmatisch erscheint.

Einen ersten umfassend geschlossenen Strukturierungsrahmen für die Zuordnung von Aufgaben zu Controllern bietet HERzog an. ${ }^{203}$ Mittels Eignungs-, Verhaltens- und Koordinationsaspekten strukturiert er das Problem der Aufgabenzuordnung. Im Folgenden soll in Anlehnung an HERzOG eine inhaltliche Konkretisierung der Aspekte vorgenommen werden, sowie Interdependenzen zwischen der auf Grundlage dieser Aspekte vorzunehmenden Aufgabenzuordnung und den daraus resultierenden Rahmenbedingungen für die Leistungserstellung im Controlling aufgezeigt werden. Die hier verwandten Strukturierungsaspekte können darüber hinaus bereits im Rahmen der Aufgabenanalyse Anwendung finden, so dass bei der Aufgabenbildung Aufgabenkomplexe anforderungskonform abgegrenzt werden können. ${ }^{204}$ Den Prozess der analytischen und methodischen Fundierung von Aufgaben zeigt Abbildung 9.

Im Sinne der Kundenorientierung des Controlling lässt sich die strukturierte Aufgabenzuordnung als ein quasi vorgelagertes Instrument verstehen, über das

200 Bei der Arbeitsteilung lassen sich weiterhin die Ausprägungen Mengenteilung und Artenteilung unterscheiden. Bezogen auf Controlling-Aufgaben ist von einer Artenteilung auszugehen, da nur diese die Realisierung originärer Spezialisierungsvorteile erlaubt. Vgl. Kossbiel (1974), Sp. $257 \mathrm{ff}$. Im Bereich der Spezialisierung wird zwischen personaler, funktionaler und struktureller Spezialisierung unterschieden. Vgl. Reiß (1992), Sp. 2287 ff.

202 Hierzu beispielhaft Stoffel (1995), S. 157 ff. oder Amshoff (1993), S. $308 \mathrm{ff}$.

203 Vgl. Herzog (1999).

204 Insbesondere bei der Abgrenzung des Leistungskataloges erscheint ein solcher Ansatzreleyant vgl-73.1.1.4. 
für den Controllerbereich und die dahinter stehenden Persönlichkeiten ein organisatorisches Fundament geschaffen wird, welches maßgeblich mitbestimmt, inwieweit Leistungen des Controllers überhaupt kundengerecht erstellt werden können. Werden Aufgaben dem Controlling unsachgemäß zugeordnet, so können sowohl Eignungs-, Verhaltens- und Koordinationsaspekte einer kundenorientierten Aufgabenwahrnehmung entgegenstehen.

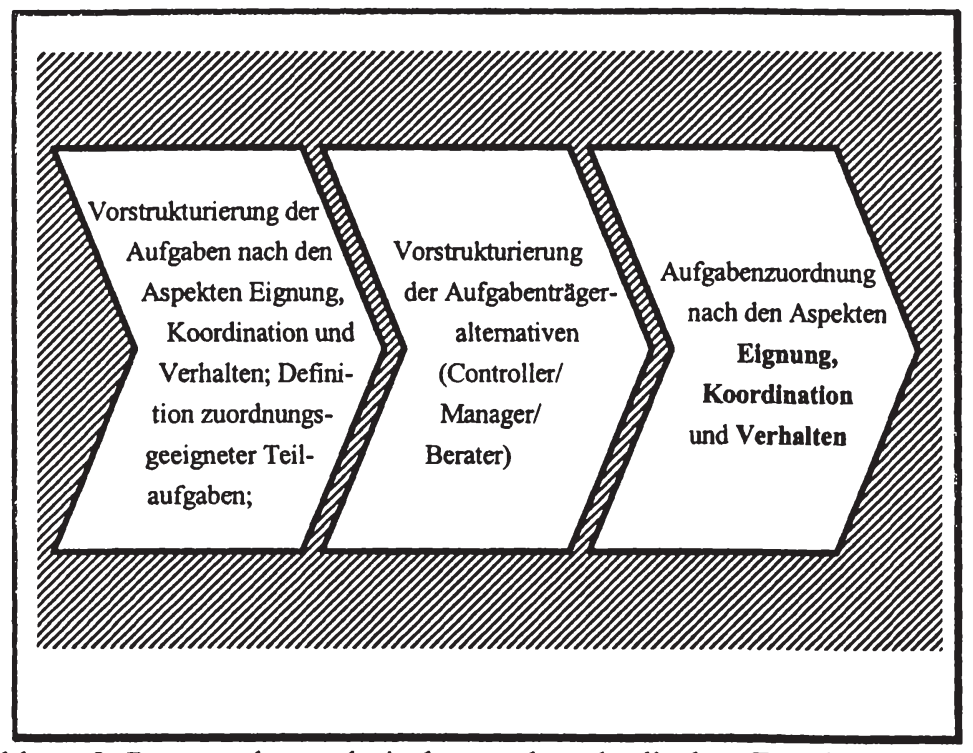

Abbildung 9: Prozess der analytischen und methodischen Fundierung der Aufgabenzuordnung ${ }^{205}$

\section{Eignungsaspekte}

Insbesondere der Dienstleistungscharakter von Controlling-Leistungen macht es erforderlich, im Rahmen der Aufgabenzuordnung auf die Eignung des jeweiligen Aufgabenträgers einzugehen, bzw. bei disponiblen Aufgaben denjenigen Aufgabenträger zu benennen, der für die Erfüllung einer Aufgabe das nötige „Können” besitzt. ${ }^{206}$ Bezogen auf konkrete Aufgaben ist also abzuwägen, ob Manager, Controller oder ein alternativer Aufgabenträger diese wahrnehmen soll. Zur Operationalisierung der Eignung eines Aufgabenträgers führt HERZOG

205 Vgl. Herzog (1999), S. 277.

206 Nach HerzoG geben die Eignungsaspekte das „Konnen” und die Verhaltensaspekte das „Wollen” des Aufgabenträgers wieder. Zusätzlich bedarf die arbeitsteilige Aufgabenbearbeitung organisationsweit der Berilcksichtigung von Koordinationsaspekten. Vgl. Herzog (1999), S. 45. 
die kurzfristige Kapazität, die Qualifikation sowie die Faktorkosten an ${ }^{207}$ Die Kurzfristigkeit der Kapazitätsbetrachtung erscheint aufgrund langfristig am Arbeitsmarkt zu deckender Bedarfe schlüssig. Insbesondere das Problem schwankender Kapazitäten aufgrund periodisch anfallender Tätigkeiten und erzielbare Lernfortschritte im Zeitablauf begründen eine Dynamik, deren Handhabung eine zielgerichtete Ausgestaltung des Leistungsangebots erfordert. ${ }^{208} \mathrm{Je}$ intensiver in diesem Zusammenhang zeitliche und inhaltliche Kundenanforderungen vom Controlling berücksichtigt werden, desto mehr bedarf es einer am Kunden orientierten Kapazitätsplanung. Im Rahmen einer kostenmäßigen Bewertung der Kapazitäten spielen die Faktorkosten alternativer Aufgabenträger eine bedeutende Rolle. Neben langfristig anzusetzenden pagatorischen Kostengrößen sind kurzfristig Opportunitätskosten zu verwenden, die im Fall freier Kapazitäten gleich null sind. ${ }^{209}$ In diesem Zusammenhang ist weiterhin die Faktorpreisrelation von Manager und Controller von herausragender Bedeutung, da diese auch bei vergleichbarem Qualifikationsniveau regelmäßig für eine Aufgabenzuordnung zum Controller sprechen wird. Die hier angesprochene Qualifikation zur Wahrnehmung einer Aufgabe ist grundlegend durch das bei einem Aufgabenträger vorhandene Wissen sowie die ihm zur Verfügung stehenden wissensgenerierenden Fähigkeiten charakterisiert. Eine weitere Unterteilung des vorhandenen Wissens in Fakten-, Beziehungs- und Methodenwissen ist in der Lage, den bei einem Aufgabenträger verfügbaren Wissensbestand zu strukturieren. Neben dem konkreten Bestand an Wissen ist insbesondere die Fähigkeit neues Wissen zu generieren erfolgskritisch. Hierzu stehen dem Aufgabenträger Aktivitäten der Informationsaufnahme, -verarbeitung und -weitergabe zur Verfügung. Letztlich entscheidend für die Generierung neuen Wissens sind somit kommunikative (Informationsaufnahme und -weitergabe) und analytische (Informationsverarbeitung) Fähigkeiten. Bei einer Gesamtbewertung der Qualifikation von Aufgabenträgern ergibt sich aus statischer Sicht eine Überlegenheit von Aufgabenträgern mit exogenen Eignungsvorteilen. Dynamisiert man hingegen die Betrachtung, so werden endogene Eignungsvorteile, also Lerngewinne im Sinne einer Spezialisierung oder Generalisierung immer bedeutender.

\section{Koordinationsaspekte}

Die arbeitsteilige Wahrnehmung von komplexen Aufgaben, welche in der quantitativen und qualitativen Beschränkung eines jeden Aufgabenträgers begründet liegt, erfordert eine zielgerichtete Koordination, um die angestrebten

\footnotetext{
$207 \mathrm{Zu}$ den weiteren Erläuterungen der Eignungsaspekte vgl. Herzog (1999), S. 61 ff.

$208 \mathrm{Zu}$ den Abrufmodalităten der Controlling-Leistungen vgl. Kap. 3.3.2.2. 
Unternehmensziele erreichen zu können. ${ }^{210}$ Eine adäquate Berücksichtigung von Koordinationsaspekten im Rahmen der Zuordnung von Aufgaben zu Controllern erscheint aufgrund der zentralen Koordinationsfunktion des Controlling unerlässlich. ${ }^{211}$ Die Aufgabenträger des Controlling betreffende koordinationsrelevante Sachverhalte sind in diesem Zusammenhang einerseits eine mögliche Effizienzsteigerung durch Realisierung von Controlling-Maßnahmen, andererseits aber auch die zusätzliche Notwendigkeit von Koordinationsmaßnahmen, bedingt durch die Integration eines Controllerdienstes in die Gesamtorganisation..$^{212}$

Zielsetzung der Durchführung von Controlling-Maßnahmen kann die Entlastung des Managements sein, um dieses in die Lage zu versetzen, seine begrenzten Ressourcen effizienter zu Koordinationszwecken einzusetzen. Derartige Unterstützungsaktivitäten bieten regelmäßig großes Potenzial für eine Verbesserung der Kundenorientierung im Controlling, da nur in Abstimmung mit dem Kunden ergründet werden kann, in welchen Bereichen die Entlastung effizient ist. Aufgabenträger des Controlling können aber auch unmittelbar koordinativ tätig werden, indem sie als zentraler Informationsmittler agieren oder, wenn Sie nicht unmittelbar an einer Interaktion teilhaben, diese durch das Bereitstellen geeigneter Normierungsinstrumente unterstützen..$^{213}$ Noch wichtiger in diesem Zusammenhang ist die Rolle des Controllers als Übersetzer, ${ }^{214}$ die es ihm ermöglicht, in Entscheidungssituationen ,als Dolmetscher in der Sprache der monetären Bewertung zu wirken"21s und somit koordinativ tätig zu werden. Aufgabe der Kundenorientierung ist es in diesem Zusammenhang über eine Bedarfsanalyse sicherzustellen, dass in die ,richtige Sprache“ übersetzt wird.

Will man sich im Rahmen der Leistungserstellung des Controllerdienstes diese positiven Koordinationswirkungen zu Nutze machen, ergibt sich die Notwendigkeit, die zusätzlich durch die Einrichtung einer Organisationseinheit „Controlling” entstehenden Abstimmungsnotwendigkeiten zu analysieren. Insbesondere im Rahmen einer wettbewerbsorientierten Betrachtung sind an dieser Stelle zusätzliche organisationsintern entstehende Koordinationskosten den Kosten

\footnotetext{
210 Nach KüPPER gehört die Zielausrichtungsfunktion zu den abgeleiteten Zwecksetzungen des Controlling. Vgl. Küpper (1997), S. 18 f.

211 Zur Diskussion der Koordinationsfunktion des Controlling vgl. 2.1.1.2.

212 Zur näheren Erläutenung der Koordinationsaspekte vgl. Herzog (1999), S. 110 f.

213 Normierung als übergeordnetes Führungsinstrument kann sich hierbei bspw. auf Datenmaterial oder Prozessabläufe beziehen.

214 Hierzu vgl. auch Weber (1999), S. 401 f.

215 Herzog (1999), S. 174.
} 
einer Beschaffung auf externen Märkten gegenüberzustellen. ${ }^{216}$ Ferner sind bei einer Betrachtung durch den Controllerdienst induzierter Koordinationskosten neben der angesprochenen Koordinationskostenanalyse Leistungserstellungsinterdependenzen, Synergien sowie wissensökonomische Reifegrade ${ }^{217}$ unterschiedlicher Leistungen zu analysieren. ${ }^{218}$ Hieraus können Erkenntnisse über eine koordinationskostenminimale Aufgabenzuordnung insbesondere zwischen Manager und Controller abgeleitet werden.

\section{Verhaltensaspekte}

Um eine Geschlossenheit der Aufgabenzuordungsaspekte zu gewährleisten, erfordert die Dominanz sachbezogener Aspekte der letzten beiden Abschnitte eine ergänzende Berücksichtigung verhaltensbezogener Aspekte. ${ }^{219}$ Insbesondere die Annahme der Existenz begrenzter Rationalität und opportunistischen Verhaltens ist in der Lage, Verhaltensbesonderheiten gegenüber einer rein sachbezogenen Betrachtung zu erklären. ${ }^{220}$ Begrenzte Rationalität findet ihren Ursprung in den limitierten Fähigkeiten des jeweiligen Aufgabenträgers. Resultierend daraus können sich Auswirkungen auf das individuelle Verhalten ergeben, zusätzlich aber auch interaktionsbedingt Auswirkungen auf die Gesamtorganisation. Das Spektrum möglicher opportunistischer Verhaltensweisen des Controllers ist wesentlich durch seine Machtposition und die Komplexität seiner Leistungen determiniert. Insbesondere der Zugriff auf sensible Unternehmensdaten und die aus Nachfragersicht schwere Bewertbarkeit der ControllingLeistungsqualität $t^{21}$ eröffnen dem Controller umfangreiche Verhaltensspielräume, die eigennutzorientiert ausgeübt werden können. Die Fragestellung, ob und gegebenenfalls inwieweit eine Marketing-Konzeption für das Controlling diese Tendenzen verstärken könnte, soll an späterer Stelle diskutiert werden.

216 Hierzu allgemein Arrow (1974) oder konkret zu Make or Buy-Entscheidungen für interne Dienstleistungen vgl. Hahn/Hungenberg/Kaufmann (1994), S. 74 ff. und Witt (1988), S. 77 ff.

217 Der wissensökonomische Reifegrad bezieht sich auf die Delegierbarkeit von Aufgaben. Operationalisierungskriterien sind in diesem Zusammenhang die Beschreibbarkeit, die Wiederholungshäufigkeit sowie die verbleibenden Freiheitsspielräume des Aufgabenträgers. Allgemein vgl. Dietl (1993), S. 174 ff. sowie Strobel (1979), S. 29 f. oder bezogen auf das Controlling, Herzog (1999), S. 168.

218 Vgl. Herzog (1999), S. 152 f.

219 Eine grundsätzlich stärkere Berücksichtigung verhaltensbezogener Aspekte im Controlling fordert seit längerem Gaulhofer (1989), S. 144 f. Ein geschlossener konzeptioneller Rahmen findet sich bei Karlowitsch (1997).

220 Allgemein vgl. Williamson (1975), ausfuhrlich zu den Verhaltensaspekten im Controlling vgl. Herzog (1999), S. $176 \mathrm{ff}$.

221 Hierzu ausfuhrlicher Abschnitt 3.1.2.2. 
Möglichkeiten zur Verringerung opportunistischen Verhaltens und begrenzter Rationalität korrespondieren unmittelbar mit der Aufgabenzuordnung. Werden Controlling-Aufgaben durch das Management wahrgenommen, empfiehlt sich die Sicherstellung einer angemessenen Transparenz sowie die Implementierung geeigneter Kontrollmechanismen.

Ferner kann der Controller als kompetenter Sparringspartner des Managers für die Sicherstellung der Rationalität sorgen.222 Ein weiterer, fortschrittlicher Ansatz stellt die Rollenaufteilung dar. Im Gegensatz zur strukturellen Spezialisierung werden hier Aufgaben von mehreren Aufgabenträgern bearbeitet, um die individuellen Einzelperspektiven zu wahren. Hierdurch kann gleichsam eine Kontrollfunktion ausgeübt werden. ${ }^{223}$ Nimmt der Controller die zuzuordnende Aufgabe wahr, ist, neben den bereits erwähnten Maßnahmen, eine ausreichende Neutralität seiner Person zu gewährleisten, was maßgeblich durch die institutionelle Ausgestaltung seiner Stelle beeinflussbar ist. Ferner stellt eine in Abhängigkeit der Organisationsstruktur mögliche Job Rotation ein geeignetes Mittel zur Flexibilisierung opportunismusanfälliger Machtstrukturen dar.

Abbildung 10 stellt zusammenfassend wesentliche Elemente der Aufgabenzuordnungsproblematik dar. Letztlich bestimmen die Aufgabenzuordnungsaspekte und der in Abbildung 9 dargestellte Aufgabenzuordnungsprozess das Potenzial der Kundenorientierung im Controlling. Wird eine Aufgabe auf dieser Basis rational dem Controller zugeordnet, bedeutet dies, dass er einen „Anbietervorteil" gegenüber alternativen Aufgabenträgern hat, den er über eine kundenorientierte Interaktion mit dem Management in einen Beitrag zur Erreichung der Unternehmensziele umwandeln muss.

Die Effektivität der Aufgabenzuordnung kann somit das Ausmaß des Anbietervorteils eines Controllers beeinflussen und damit potenziell den Anteil, den das Controlling zu Nutzensteigerung des Managements beitragen kann.

222 Zum Rollenbild des Controllers vgl. Eschenbach (1997), S. 15 ff., Deyhle/Conrad (1997), S. 409 sowie Controller Verein (0.J.).

$223 \mathrm{Vgl}$. Herzog (1999), S. $239 \mathrm{ff}$. 


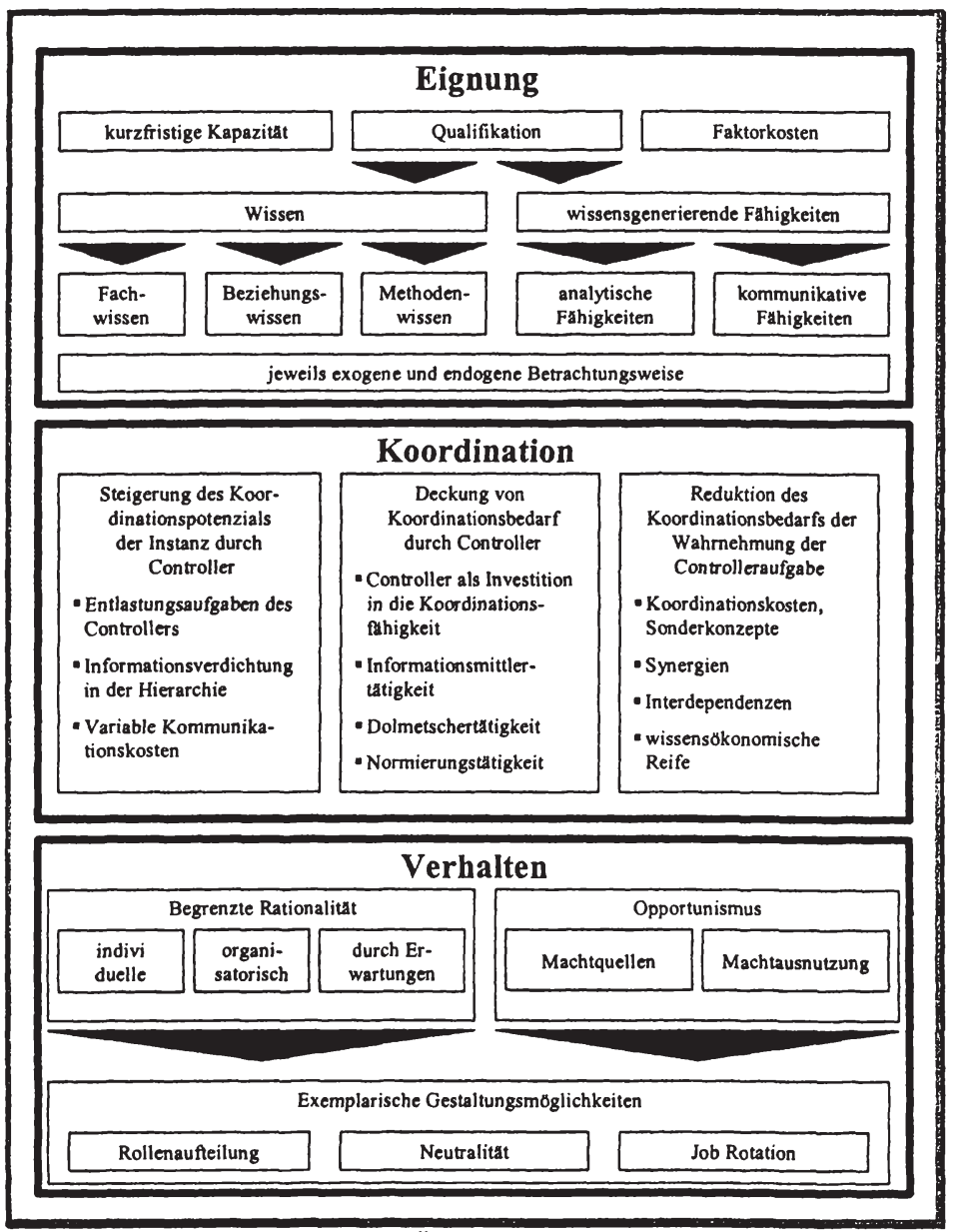

Abbildung 10: Zusammenfassende Übersicht der Aufgabenzuordnungsaspekte ${ }^{224}$

\subsubsection{Leistungen als Instrumente der Aufgabenerfüllung}

Der in den vergangenen Abschnitten vorgestellte Strukturierungsrahmen für die Aufgabenzuordnung ist als methodische Unterstützung zu verstehen, Organisationsentscheidungen analytisch $z u$ fundieren. ${ }^{225}$ Geht man davon aus, dass die in

224 Herzog (1999), S. 279.

225 HERZOG, auf den dieser Strukturierungsrahmen zurückgeht, will dadurch keineswegs intuitive Führungsentscheidungen verhindern, vielmehr sollen diese mittels einer Checkliste unterstlitzt werden, um der Vielzahl von Wirkungszusammenhängen Rechnung zu tragen. Vgl. Herzog (1999), S. 276. 
diesem Zusammenhang getroffenen Organisationsentscheidungen als Datum für den entsprechenden Controller anzusehen sind, so ist unmittelbar ersichtlich, dass hierdurch wesentliche Rahmenbedingungen für die strategische Ausgestaltung seines Leistungsangebotes fixiert werden. Umgekehrt betrachtet besteht an dieser Stelle die Möglichkeit, frühzeitig ein Fundament für potenzielle Wettbewerbsvorteile des Controllerdienstes zu legen. Da diese Gestaltungsmöglichkeiten jedoch nicht unmittelbar in den Entscheidungsspielraum des Controllers fallen, sondern vielmehr originäre Aufgabe der Unternehmensführung/Organisation sind, wurden sie im Vorfeld der Leistungsanalyse angesprochen. Dennoch besteht an einigen Stellen für den Controller vermeintlich die Möglichkeit, Einfluss auf die Aufgabenzuordnung auszuüben. Gerade die Unbestimmtheit des Controlling-Verständnisses führt in vielen Unternehmen dazu, dass der Controller sich partiell Aufgaben über die Festlegung von Untersuchungsschwerpunkten selber suchen kann. ${ }^{226}$ Ferner führt die häufige Variation der Controlling-Organisation regelmäßig zu damit verbundenen unsystematischen Aufgabenverschiebungen, die der Controller mitgestalten kann. Soweit der Controller auf diese Art und Weise Einfluss auf sein Aufgabenspektrum hat, sollte er im Einzelfall nur solche Aufgaben wahrnehmen, bei denen sein Nutzensteigerungspotenzial im Gegensatz zu alternativen Aufgabenträgern signifikant höher ist. Eine derartige Aufgabenselektion sollte auch in seinem persönlichen Interesse liegen, da es seiner organisatorischen Profilierung zuträglich ist.

Ausgehend von den Aufgabenzuordnungsaspekten sollen im Folgenden Aufgabenbereiche des Controlling dargestellt werden, auf deren Basis sich zur Aufgabenerfüllung geeignete Leistungen abgrenzen lassen. Versteht man Controlling - wie bereits eingangs geklärt - als „Beschaffung, Aufbereitung, (...) Analyse [und Kommunikation] von Daten zur Vorbereitung zielsetzungsgerechter Entscheidungen" ${ }^{\text {2227, }}$, so wird unmittelbar der Unterstützungs- und Dienstleistungscharakter des Controlling deutlich. Will man auf die Gestaltung des Controlling einwirken, ist es erforderlich, auf die zur Verfolgung der ControllingZiele einsetzbaren Leistungen einzugehen. Das Ziel des Controlling, Koordinations-, Reaktions- und Adaptionsfähigkeit der Führung zu sichern und zu erhalten, ${ }^{228}$ kann auf Basis der Controllingfunktion in Aufgabenbereiche transformiert werden. Die Controllingfunktion ist als gedankliche Zusammenfassung

${ }^{226} \mathrm{Zu}$ den unterschiedlichen Controlling-Verständnissen vgl. Kap. 2.1.3.2 ff.

227 Berens/Hoffjan/Strack (1995), S. 144. Ebenso auch Rieper/Witte/Berens (1996), S. V. Die Ergänzung in den rechteckigen Klammern erfolgte durch den Verfasser.

228 Vgl. Horváth (1998), S. 144. 
einzelner Controllingaufgaben in einer Organisation aufzufassen ${ }^{229}$ und besteht darin, „die Koordination des Führungsgesamtsystems zur Sicherstellung einer zielgerichteten Lenkung ${ }^{\text {“230 }}$ zu gewährleisten.

Auf Basis der kontextunabhängigen Funktion des Controlling lassen sich Aufgabenbereiche abgrenzen, die in Abhängigkeit der institutionellen Ausgestaltung variieren können. Legt man eine auf Basis des vorgestellten Strukturierungsrahmens methodisch unterstützte Zuordnung von Controlling-Aufgaben zugrunde, besteht die wesentliche Herausforderung der Aufgabenträger - hier beispielhaft des Controllingbereichs - darin, Leistungen bereitzustellen, die korrespondierend mit den Unternehmenszielen in der Lage sind, die Bedürfnisse der Controlling-Kunden zu befriedigen. Über den klassisch-betriebswirtschaftlichen Leistungsbegriff hinaus, der unter einer Leistung „das (gelungene) Er-

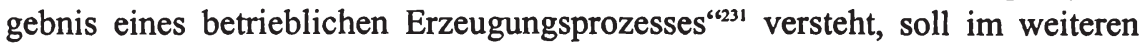
nicht nur das erwähnte Leistungsergebnis sondern vielmehr auch die notwendigen Leistungspotenziale sowie der Leistungserstellungsprozess betrachtet werden. ${ }^{232}$

\subsubsection{Leistungsspektrum des Controllers}

Die Ableitung des Leistungsspektrums erfolgt auf Grundlage der ControllingAufgaben. Trotz der Kontextabhängigkeit der Controlling-Aufgaben kann auf Basis empirischer Untersuchungen ein konsensfähiges Aufgabenspektrum unterstellt werden, ${ }^{233}$ welches sich in vier Aufgabenbereiche unterteilen lässt. Auf Basis abgrenzbarer Planungs-, Kontroll- und Informationsaufgaben sowie das Gesamtspektrum abrundender sonstiger Aufgaben sollen im Folgenden korrespondierende Leistungsbereiche differenzierter betrachtet werden. ${ }^{234}$ Jede Grenzziehung zwischen Leistungsbereichen ist zwangläufig dem Vorwurf der Willkürlichkeit ausgesetzt, soweit im Rahmen der Kategorisierung kein einheitliches Abgrenzungskriterium verwandt wird. Die nachfolgend vorgenommene Differenzierung stellt auf die Abgrenzung für eine Marketingbetrachtung angemessen homogen wahrnehmbarer Leistungsgruppen ab. Zudem muss der Kundenperspektive konsequent Rechnung getragen werden, d.h. Leistungsbe-

\footnotetext{
229 Vgl. Horváth (1998), S. 144.

230 Küpper/Weber/Zünd (1990), S. 283.

231 Gabler (2000), S. 1967.

$232 \mathrm{Vgl}$. hierzu die ausfuhrlicheren Ausführungen in Kap. 3.2.2.1.

$233 \mathrm{Vgl}$. Weber/Weißenberger/Aust (1998), S. $381 \mathrm{ff}$.

234 Die hier vorgenommene Aufgabenstrukturierung orientiert sich an der Aufteilung von Weber (1999), S. 315 ff.
} 
reiche müssen in der Wahrnehmung des Kunden abgrenzbar erscheinen. Insbesondere die Wahrnehmung der Kunden determiniert, inwieweit Leistungen als Einzelleistungen oder vielmehr in Kombination als Leistungsbündel gesehen werden. ${ }^{235}$ Dieser Tatbestand soll im Folgenden angemessen berücksichtigt werden.

Ausgangspunkt einer marketingorientierten Strukturierung der ControllingLeistungen ist letztlich das Problem aktueller und potenzieller Kunden. Da Controlling den Anspruch hat, entscheidungsunterstützend zu agieren, bzw. Entscheidungsprobleme zu lösen, ist es zunächst der Willensbildungs/Entscheidungsprozess der Kunden, der im Mittelpunkt der Transaktion zwischen Controllerdienst und Kunde steht. Aus diesem Grund soll die Abgrenzung aus Kundensicht wahrnehmbarer Controlling-Leistungen zunächst entlang des Entscheidungsprozesses potenzieller Kunden erfolgen. Derartige Entscheidungsprozesse lassen sich unterschiedlich differenziert darstellen, folgen aber regelmäßig gleichen inhaltlichen Abläufen. ${ }^{236}$ Problematisch bei der Isolation von Controlling-Leistungen entlang eines Entscheidungsprozesses potenzieller Kunden ist einerseits die Bestimmung des Entscheidungsobjektes sowie andererseits die Abgrenzung der Controlling-Leistungen von originären Führungsleistungen.

Die Frage nach der Bestimmung des Entscheidungsobjektes lässt sich unter Rückgriff auf die Systemtheorie beantworten. ${ }^{237}$ Betrachtet man den Entscheidungsprozess als ein System, im Sinne einer geordneten „Gesamtheit von Elementen, zwischen denen irgendwelche Beziehungen bestehen oder hergestellt

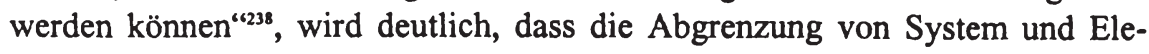
ment in Abhängigkeit des Erkenntnisinteresses variieren kann. Um Redundanzen bei der Abgrenzung von Leistungen des Controllerdienstes zu vermeiden, soll zunächst ein idealtypisch systematischer Entscheidungsprozess ermittelt werden, der aber gleichermaßen Elemente beinhalten kann, die bezogen auf ein anderes Erkenntnisobjekt ein eigenständiges System darstellen können. Lassen

\footnotetext{
235 Aktuelle Tendenzen in der Marketingwissenschaft beschăftigen sich zumehmend mit den Verbundwirkungen von Einzelleistungen, insbesondere solcher zwischen Sach- und Dienstleistungen. Vgl. Engelhardt/Kleinaltenkamp/Reckenfelderbäumer (1993), S. 395 ff.

236 Hierzu beispielhaft der Management-Kreis von SCHUBERT; vgl. Schubert (1972) S. 43 f. sowie der Entscheidungsprozess von Hahn (1991), S. 30.

237 Vgl. Ulrich (1968).

${ }^{238}$ Ulrich (1968), S. 105.
} 
sich derartige Zusammenhänge hierarchisch abbilden, spricht man von Super(Überordnung) bzw. Subsystemen (Unterordnung). ${ }^{239}$

Bezogen auf den abzuleitenden idealtypischen Entscheidungsprozess sollen die für die Durchführung von Planung und Kontrolle notwendigen Zielvorstellungen als Elemente vorgegeben werden. Eine derartige Vorgehensweise ist angebracht, da die Ermittlung des relevanten Ziels als Erkenntnisobjekt eines eigenständigen Entscheidungsprozesses aufzufassen ist, der wiederum ein eigenes System begründet. Auf die Interdependenzen derartig verschachtelter Systeme und die damit verbundene Komplexität soll zunächst nicht weiter eingegangen werden.

Leistungen zur Erfüllung der dem Controllingbereich regelmäßig zugeordneten Planungs- und Kontrollaufgaben können somit entlang eines Entscheidungsprozesses identifiziert werden. Konsequent auf den Unterstützungscharakter der Controlling-Leistungen abzielend, müssen im Rahmen des gesamten Entscheidungsprozesses Teilentscheidungen getroffen werden, die in den originären Verantwortungsbereich des Managements fallen und somit als Daten für den Controller anzusehen sind. Hierdurch erfolgt eine Abgrenzung der ControllingLeistungen von originären Führungsleistungen. Die Integration der Informationsleistungen des Controllerbereichs scheint zunächst auf Basis einer systemtheoretisch geprägten Betrachtung problematisch. Konsequenterweise wäre eine eigenständige Betrachtung der Informationsaufgaben des Controllerbereichs nicht erforderlich, soweit es sich dabei letztlich nur um ein analog strukturiertes System handelt, für das der Controller wie beschrieben die gleichen Leistungen erbringen müsste wie im Rahmen des idealtypischen Systems „Entscheidungsprozess“. Im Folgenden ist jedoch festzuhalten, dass die Informationsleistungen des Controlling integraler Bestandteil der Unterstützungsleistungen (allgemein Planung und Kontrolle) entlang des Entscheidungsprozesses sind und somit im Sinne einer Querschnittsfunktion eigenständig und in Verbindung mit anderen Controlling-Leistungen zu einem in der Wahrnehmung der Kunden differenzierten Nutzenbeitrag führen. Diesem komplexen Tatbestand würde eine Abbildung in Form eines analog organisierten Subsystems nicht gerecht. Informationssystem, Berichtswesen und Kommunikationswesen ${ }^{240}$ ermöglichen erst den zielsetzungsgerechten Ablauf des Entscheidungsprozesses über die Bereitstellung von Transaktionsdaten und Übernahme der Interaktion.

239 Ulrich (1968), S. 107.

240 Ein Zielsystem furr die Gestaltung des innerbetrieblichen Kommunikationswesens findet sich bei Ringle (1982), S. 3 ff. 
Die bis hierhin dargestellten Leistungen vermögen abstrakt breite Teile der Planungs-, Kontroll- und Informationsaufgaben des Controllerbereichs wiederzugeben. Neben der auf Basis von Information und Interaktion geleisteten Entscheidungsprozessunterstützung kommt der Planentstehungskontrolle eine besondere Bedeutung zu. Wie im Rahmen der weiteren Ausführungen noch darzulegen sein wird, muss eine marketingorientierte Leistungsbetrachtung des Controlling auch solche Leistungen einbeziehen, die nicht unmittelbar von allen Kunden freiwillig nachgefragt werden, sondern vielmehr aufgrund der spezifischen Verantwortung des Controllers in Kombination mit anderen Leistungen oder als Bestandteil eines Leistungsbündels integriert erbracht werden müssen. In allen Phasen der Entscheidungsprozessbegleitung sowie im Rahmen der diese fördernden Informations- und Interaktionsaktivitäten besteht eine unabdingbare Leistung des Controllers darin, die Folgen begrenzter kognitiver Fähigkeiten und bedingter Rationalität einzuschränken. ${ }^{241}$ Diese menschlichem Verhalten immanenten Ursachen opportunistischen Verhaltens müssen vom Controller stets berücksichtigt werden und sich gleichermaßen in seinem Leistungsangebot niederschlagen..$^{242}$ Eine Vermengung verhaltenssteuernder Leistungen mit prozess- bzw. informationsbezogenen Leistungen erscheint im Rahmen einer Marketingbetrachtung unzweckmäßig, da sich in der Folge dieser spezielle Betrachtungsbereich einer separaten Analyse und Ausgestaltung entziehen würde. $\mathrm{Zu}$ dem sind gerade die Interdependenzen und Kombinationsaspekte zwischen „hoheitlichen" Leistungen ${ }^{243}$, im Sinne der Opportunismusbeschränkung und reinen Serviceaktivitäten von besonderer Bedeutung.

Die in Abbildung 11 gewählte dreidimensionale Darstellung soll den drei Betrachtungsebenen Information und Interaktion, Entscheidungsprozess und Verhalten gerecht werden und gleichermaßen verdeutlichen, dass alle hier abstrakt abgegrenzten Leistungen als Teilleistungen vielfältig variierbarer Leistungsbündel aufzufassen sind. Die Bedürfnisbefriedigung des Kunden hängt somit maßgeblich von der inhaltlichen Ausgestaltung einzelner Leistungen sowie der Kombination dieser mit anderen Leistungen ab.

241 Vgl. Weber (1999), S. 318 ff. sowie umfassend zu Verhaltensaspekten im Controlling Karlowitsch (1997), S. 24 ff. und Hoffjan (1997).

242 Die Analyse opportunistischen Verhaltens ist Gegenstand der Principal-Agent-Theorie. Dieser Teilansatz der Neuen Institutionen Ökonomie beschäftigt sich mit Auftragsbeziehungen zwischen Auftraggeber und Auftragnehmer. Zielsetzung hierbei ist die Optimierung institutioneller Rahmenbedingungen im Sinne des Prinzipalen. Vgl. Williamson (1985) oder ubersichtsartig Meffert (2000), S. 64 f.

${ }^{243}$ Der teilweise hoheitliche Charakter des Controlling resultiert aus der Tatsache, dass das Controlling aufgrund seiner Kontrollverantwortung von Transaktionspartnern nicht als gleichberechtigter Partner wahrgenommen wird, sondern vielmehr als übergeordnete Instanz. 


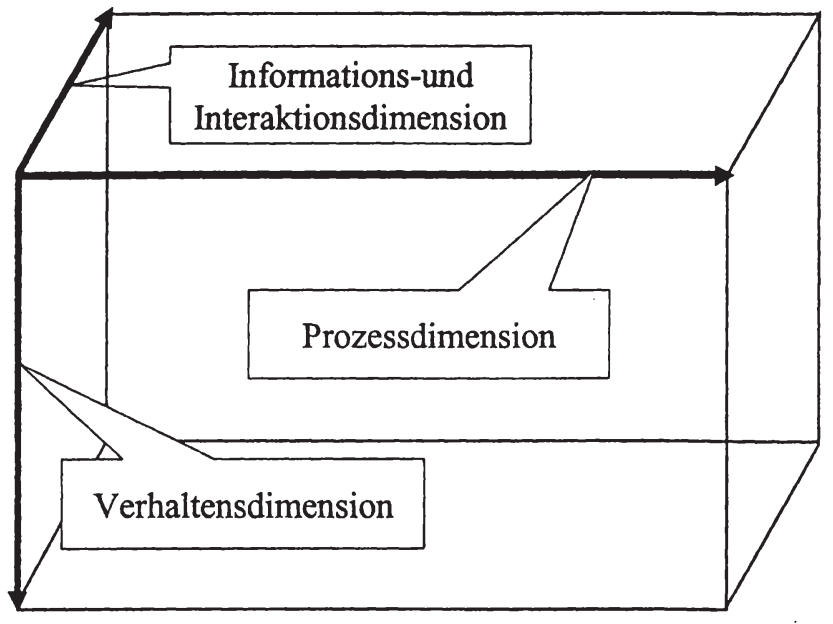

Abbildung 11: Abstraktes Modell eines Controlling-Leistungsraums

In der Wahrnehmung der Kunden erscheinen die prozessbezogenen Leistungen regelmäßig inhaltlich dominant, wohingegen informations- und interaktionsbezogene Leistungen im Sinne einer „Verpackung“ bzw. eines Zusatzservices wahrgenommen werden. Verhaltenssteuernde Leistungen finden einerseits entlang des Entscheidungsprozesses Anwendung, andererseits hängt die Art und Weise ihrer Akzeptanz und Wirkung aufgrund des hohen Aktivierungsgrades der Kunden maßgeblich von der Verpackung/Kommunikation und Interaktion ab. Die Darstellung in Abbildung 11 ermöglicht die parametrische Betrachtung einzelner Leistungen zu Analysezwecken und trägt darüber hinaus dem Gedanken Rechnung, dass im Mittelpunkt von Transaktionen nicht Einzelleistungen, sondern regelmäßig Leistungsbündel stehen. Eine derartige Betrachtungsweise erübrigt auch im wesentlichen die Diskussion, zu welchen Teilen es sich bei dem Leistungsangebot des Controlling um Dienst- oder Sachleistungen handelt. Unternehmensintern wie auf unternehmensexternen Absatzmärkten findet eine zunehmende Vermengung von Sach- und Dienstleitungen statt, ${ }^{244}$ so dass eine derartige Klassifizierung wenig aussagekräftig ist. Vielmehr sollen im weiteren

244 Dies ist oftmals auf die Anreicherung von Sachleistungen durch differenzierungsträchtige Serviceleistungen zurückzufuihren. 
Verlauf - auf Grundlage der Ausführungen zum Dienstleistungsmarketing ${ }^{245}$ marketingrelevante Parameter ermittelt werden, die im Rahmen der Ausgestaltung des Leistungsangebotes Differenzierungs- und Optimierungsmöglichkeiten zulassen.

Aufbauend auf den zunächst übersichtsartig dargestellten Leistungsdimensionen auf hohem Aggregationsniveau sollen nachfolgend konkrete Inhalte zugeordnet werden, um zu zeigen, dass das Gesamtspektrum der üblicherweise vorgegebenen Controlling-Aufgaben durch wenige abstrakte Leistungen als operative Instrumente der Aufgabenerfüllung abzudecken ist. Basis einer derartigen Zuordnung sollen beispielhaft dem Controlling von WEBER zugeschriebene Aufgaben und Teilaufgaben sein. ${ }^{246}$ Bereits jetzt sei darauf hingewiesen, dass in vielen Fällen Leistungen bzw. Leistungsgruppen tätigkeitsorientiert Aufgaben entsprechen. Insbesondere aber die entscheidungsprozessbezogene Betrachtungsweise auf hohem Abstraktionsniveau bedingt, dass strukturell gleiche Leistungen zur Erfüllung inhaltlich unterschiedlicher Aufgaben eingesetzt werden können. Auf diese Art und Weise können Redundanzen im Leistungsspektrum vermieden werden.

Grundlage des Leistungsspektrums bilden Planungs-, Kontroll-, Informationsversorgungsaufgaben sowie sonstige Aufgaben, zu denen bspw. die Beratungsaufgaben gezählt werden. ${ }^{247} \mathrm{Zu}$ den Aufgaben der Planung gehören Planungsmanagement, Planungsunterstützung sowie die Planentstehungskontrolle. Darauf aufbauend ergeben sich nachfolgende Teilaufgaben:

\section{Planungsmanagement}

- Gestaltung des Planungssystems,

- methodische und instrumentelle Unterstützung der Planer,

- Unterstützung des Planungsablaufs durch Übernahme prozessualer Teilaufgaben;

Planungsunterstützung

- Suche und Aufbereitung entscheidungsrelevanter Informationen,

- Generierung von Entscheidungsalternativen,

- monetäre Bewertung von Entscheidungsalternativen,

- Vorabstimmung von Lösungen;

245 Vgl. Kap. 2.2.2.1.

246 Vgl. Weber (1999), S. 315 ff. Die hier aufgefuhrten Aufgaben stellen ein weitestgehend konsensfahiges Aufgabenspektrum dar. Vgl. Herzog (1999), S. $14 \mathrm{ff}$.

$247 \mathrm{Zu}$ den Aufgabeninhalten vgl. im Folgenden Weber (1999), S. 316 ff. 


\section{Planentstehungskontrolle}

- Begrenzung von Opportunismus,

- Einschränkung der Folgen begrenzter kognitiver Fähigkeiten.

Aufgaben kontrollierender Natur sind im wesentlichen die Durchführung von Soll-Ist-Vergleichen und Abweichungsanalysen sowie konsequenterweise das Unterbreiten von Vorschlägen für Anpassungsmaßnahmen.

Im Bereich der Informationsaufgaben werden die Festlegung von Art und Umfang der bereitgestellten Informationen sowie die allgemeine Transparenzverantwortung als Teilaufgaben unterschieden. Transparenzverantwortung beschreibt die Aufgabe des Controllers, Informationssysteme zu gestalten und zu pflegen, Berichte zu generieren und diese adäquat zu kommunizieren. Das Residuum der sonstigen Aufgaben beinhaltet Aktivitäten, wie die zukünftig an Bedeutung gewinnenden internen Beratungstätigkeiten. ${ }^{248}$

Nachfolgend soll gezeigt werden, wie über Einzelleistungen und Leistungsbündel das gesamte Aufgabenspektrum durch den in Abbildung 12 dargestellten Leistungsraum abgedeckt werden kann. Das Aggregationsniveau der Leistungen orientiert sich an den dargestellten Aufgaben. Eine weitergehende Differenzierung erscheint im Sinne der Untersuchung wenig zweckmäßig, da in Folge der Komplexitätszunahme Interdependenzen zwischen Einzelleistungen nicht mehr darstellbar wären. Grundlage für die Analyse ist der Leistungsraum mit einer Prozess-, Informations- und Verhaltensdimension. Angenommen werden kann, dass jeder kundenbezogenen Controlling-Aktivität ein spezifisches Entscheidungsproblem eines Kunden zugrunde liegt und dieses dem Controller kommuniziert wird. Ferner muss eine konkrete Zielvorstellung bestehen, da ansonsten der Zielfindungsprozess im Vorfeld, - im Sinne eines Entscheidungsprozesses - zu durchlaufen wäre.

Somit besteht die erste Leistung des Controllers in der Prozessbetrachtung darin, seinem Kunden bezogen auf sein Entscheidungsproblem Vorschläge zu einem adäquaten Entscheidungssystem und einer damit korrespondierenden Entscheidungsmethodik zu unterbreiten. Die daraufhin vom Kunden zu treffende Systementscheidung ist für den weiteren, in Abbildung 12 dargestellten Prozess als Datum anzusehen.

248 Allgemein zu den Entwicklungstendenzen des internen Consulting ygl. Niedereichholz (2000)

Thomas Mosiek - 978-3-631-75321-7 


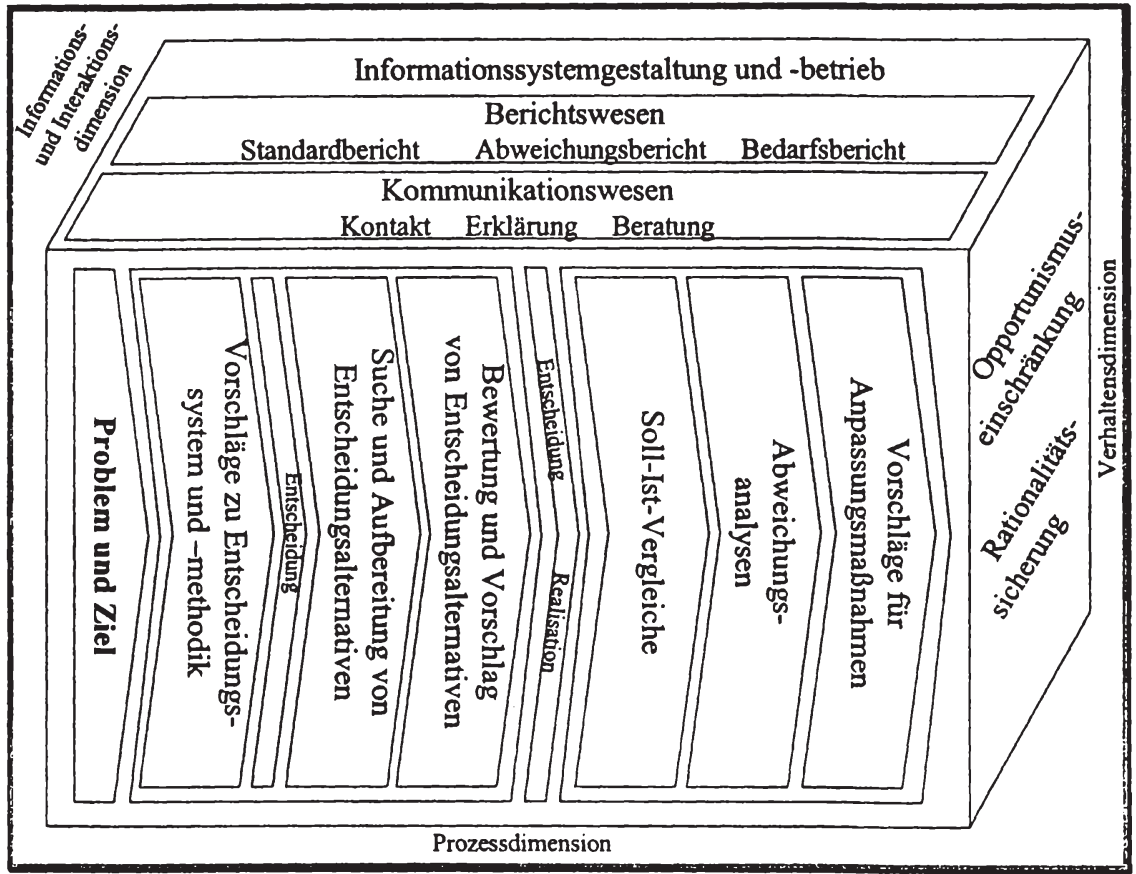

Abbildung 12: Modell eines Controlling-Leistungsraums ${ }^{249}$

Der nächste Prozessschritt, welcher gleichermaßen auch als erster Prozessschritt für solche Entscheidungsprobleme anzusehen ist, die auf Basis eines bestehenden und nicht im Einzelfall zu überprüfenden Entscheidungssystems getroffen werden, ist die Suche und Aufbereitung von Entscheidungsalternativen. Aufbauend auf diese Leistung erfolgt eine Bewertung ${ }^{250}$ sowie gegebenenfalls der Vorschlag einer Entscheidungsalternative, auf deren Basis vom Kunden eine Entscheidung zu treffen ist. Diese stellt ihrerseits ein Datum für den leistungserbringenden Controller dar. Zu einer Beurteilung der Entscheidungsfolgen stehen dem Controller im Rahmen des Entscheidungsprozesses Soll-Ist-Vergleiche und Abweichungsanalysen zur Verfügung. Eine darauf aufbauende Leistung ist der Vorschlag geeigneter Anpassungsmaßnahmen.

249 Die Ableitung der Teilleistungen erfolgt in Anlehnung an die Aufgabengliederung von Weber (1999), S. 315 ff. Zu den Phasen des Entscheidungsprozesses vgl. beispielhaft Hahn (1991), S. 30.

250 Bewertung soll hier in einem umfassenden Sinn verstanden werden, was gleichermaßen eine monetăre Bewertung einschließt. 
Rekurriert man auf die dargestellten Planungs- und Kontrollaufgaben, so wird deutlich, dass die prozessbezogenen Leistungen grundsätzlich weite Teile jener Aufgaben abdecken. Chronologisch angeordnet sind die Aufgaben des Planungsmanagements, der Planungsunterstützung und der Kontrolle vertreten. Insbesondere die Kontrollteilaufgaben finden sich in Form von Leistungen identisch wieder. Eine chronologische Eingliederung der Aufgaben der Planentstehungskontrolle erscheint nicht zweckmäßig, da derartige Leistungen in Verbindung mit potenziell allen Planungs- und Kontrollleistungen erbracht werden können bzw. müssen. Diesem Tatbestand Rechnung tragend, begründen Leistungen zur Erfüllung der Planentstehungskontrolle die Verhaltensdimension. Anspruch der Leistungen dieser Dimension ist, bei allen Prozess- und Informationsaktivitäten sicherzustellen, dass weder begrenzte kognitive Fähigkeiten noch opportunistisches Verhalten dazu führen, dass den Unternehmenszielen zuwiderlaufende Entscheidungen getroffen werden. Diese Leistungen werden im Regelfall nicht separat, sondern in Verbindung mit Prozess- und Informationsleistungen erbracht.

Die Leistungen der Informations- und Interaktionsdimension repräsentieren in weiten Teilen die Informationsaufgaben des Controlling. Im Leistungsraum zählt zu den Informationsleistungen grundlegend die Gestaltung und der Betrieb der Informationssysteme. Angenommen wird hier, dass die Informationssystemverantwortung dem Controllerdienst obliegt, da ansonsten die Informationssystemgestaltung als ein eigenständiger Entscheidungsprozess des Managements aufgefasst werden könnte, in dem der Controller nur entscheidungsunterstützend tätig würde. Gestaltung und der Betrieb der Informationssysteme implizieren, dass die in dem System enthaltenen Informationen inhaltlich potenziell in der Lage sind, die Bedürfnisse der Kunden des Controlling zu befriedigen. Ist die Art der Informationen also potenziell problemlösungsadäquat, hängt es letztlich von den Berichten und ihrer Kommunikation ab, ob Umfang, Bereitstellungsmodalitäten und Interaktionsverhalten den Ansprüchen der Kunden genügen. Ferner leiten sich Inhalt und Umfang der Informationen elementar aus dem Entscheidungsprozess/-schritt ab. Die Festlegung des Informationsumfangs erfolgt als Resultat der Abstimmung zwischen technischen Informationsangebot, problemadäquaten Informationsbedarf und der artikulierten Nachfrage des Managers. ${ }^{251}$ Um die Bedeutung der Kommunikation im Controlling hervorzuheben und zugleich ihrer Marketingrelevanz Ausdruck zu verleihen, bedarf es einer separaten Abbildung des Leistungsbereichs Kommunikationswesen mit den Leistungen Kontakt, Erklärung und Beratung, insbesondere in Abgrenzung 
zum Berichtswesen. Die Kommunikationsleistungen tragen in Verbindung mit Leistungen des Berichtswesens zu dem erheblichen, im Controlling vorhandenen Differenzierungs- und Optimierungspotenzial bei. $\mathrm{Zu}$ den Leistungen des Berichtswesens sollen vorrangig die unterschiedlichen Berichtstypen gezählt werden, sowie die mit ihnen im Rahmen moderner Kommunikations- und Informationstechnologien verbundenen Bereitstellungsmodalitäten. Die hier aufgeführte Struktur der Controlling-Leistungen in der Informations- und Interaktionsdimension deckt grundlegend die vorher mit WEBER ${ }^{252}$ genannten Informationsaufgaben $\mathrm{ab}$.

Insgesamt ergibt sich auf Basis der dargestellten Aufgaben des Controlling ein Leistungsspektrum, welches in dieser Relation weniger Teilleistungen aufweist. Hingegen eröffnet es aber durch seine überwiegend redundanzfreie Struktur die Möglichkeit, über Leistungsbündel spezifische Leistungen des Controlling zu kreieren, über die eine Profilierung in den Augen der Kunden erreicht werden kann. Die Struktur der Dimensionen ermöglicht ferner in Abhängigkeit der Gestaltungsempfehlungen für Teilleistungen auf die Gestaltbarkeit von Leistungsbündeln zu schließen.

Nachfolgend soll ein Beispiel für die Möglichkeit der Darstellung einer im Rahmen der Aufgabenaufzählung genannten Teilaufgabe in Form eines Leistungsbündels gegeben werden. Hinter der im Bereich des Planungsmanagements genannten Teilaufgabe, den Planungsablauf durch Übernahme prozessualer Teilaufgaben zu unterstützen, verbergen sich letztlich Informations- und Dokumentationsaktivitäten, die entlang des Planungsprozesses koordinierend wirken und gleichsam über Konsistenzprüfungen zu einer angemessenen Verhaltenssteuerung beitragen. Eine separate Abbildung dieser Teilaufgabe in Form einer eigenen Leistung im Leistungsraum kann bewusst nicht erfolgen, da die Struktur dies nicht zulässt. Die Verknüpfung prozessualer, informativer und verhaltenssteuernder Komponenten muss konsequenterweise zu einer Verbunddarstellung in Form eines Leistungsbündels aus den angedeuteten Teilleistungen führen. ${ }^{253}$

Zur Veranschaulichung soll abschließend in den hier nach Marketinggesichtspunkten aufgebauten Leistungsraum ein typisches Leistungsbündel des Controllerdienstes eingeordnet werden, um darauf aufbauend zeigen zu können, inwieweit eine derartige Leistungsstrukturierung mögliche Gestaltungsempfehlungen

${ }^{252} \mathrm{Zu}$ einer ausfuihrlichen Darstellung der Informationsaufgaben des Controlling vgl. Weber (1999), S. $339 \mathrm{ff}$.

253 Analog verhält es sich mit der die Koordination planungsprozessbezogener Aktivităten. 
erleichtert. Zur besseren Nachvollziehbarkeit wird ein Leistungsbündel gewählt, welches - belegt durch empirische Studien ${ }^{254}$ - als ein typisches Produkt/typischer Prozess des Controlling angesehen werden kann. Im Folgenden soll die idealtypische Unterstützung einer Investitionsentscheidung durch das Controlling abgebildet werden. Der hohe Standardisierungsgrad erleichtert die objektive Einordnung dieses Produktes, darüber hinaus führt dessen regelmäßig hohe Inanspruchnahme der Controlling-Kapazität zu der Notwendigkeit, Effizienz- und Effektivitätspotenziale auszuschöpfen, ${ }^{255}$ was gleichsam nur auf Basis einer Analyse des Leistungsbündels erfolgen kann.

Beginnend mit der Prozessperspektive kann davon ausgegangen werden, dass das zu lösende Problem in der Durchführung der für die Erreichung der Unternehmensziele optimalen Investition gesehen wird. Zielsetzung der Investitionsentscheidung könnte somit die Maximierung des Marginalbeitrags der Investition zur Steigerung des Unternehmenswertes sein. Die Entscheidungsunterstützung des Controlling beginnt mit dem Vorschlag eines adäquaten Entscheidungssystems bzw. einer angemessenen Entscheidungsmethodik. Bezogen auf die Investitionsentscheidung wäre zu analysieren, auf Basis welchen finanzwirtschaftlichen Vorteilhaftigkeitsmaßes zukünftig entschieden werden soll. ${ }^{256} \mathrm{Mög}$ liche Alternativen wären bspw. ein Barwert, der interne Zinsfuss oder ein Vermögensendwert, welcher auf Basis eines vollständigen Finanzplans ermittelt werden könnte. ${ }^{257}$ Notwendigerweise muss bereits an dieser Stelle die Prozessperspektive um Komponenten der Verhaltens- sowie der Informations- und Interaktionsperspektive ergänzt werden. Die Verhaltenswirkungen eines zu implementierenden Entscheidungssystems implizieren, dass der Controller opportunistischen Verhaltenstendenzen des Managements entgegenwirken muss. So kann beispielsweise die Wiederanlageprämisse des internen Zinssatzes dazu führen, dass diese Entscheidungsmethodik zu unzweckmäßigen Ergebnissen führt, soweit bei Projekten hohe Einzahlungsüberschüsse in den ersten Perioden der Investition anfallen, demgegenüber jedoch keine Wiederanlagemöglichkeit mit vergleichbar hoher Verzinsung besteht. Hierdurch ergibt sich für das Management die Möglichkeit, bereits bei der Auswahl des Entscheidungssystems partiell eigennutzorientiert zu handeln. Gleichermaßen sind Informations- und

\footnotetext{
${ }^{254}$ Vgl. Weber/Weißenberger/Aust (1997), S. 5 ff.

255 In der WHU-Studie „Benchmarking des Controllerbereichs“ werden Standardisierungsgrad und Inanspruchnahme der Controlling-Kapazität als Determinanten fuir das Benchmarking-Potenzial unterschiedlicher Controlling-Leistungen verwandt. Vgl. Weber/Weißenberger/Aust (1997), S. 5. In dieser Arbeit vgl. dazu Kap. 4.2.1.1.
}

256 Vgl. Perridon/Steiner (1999), S. $37 \mathrm{ff}$.

257 Zum vollständigen Finanzplan vgl. Grob (1995). 
Interaktionsaktivitäten in dieser Prozessphase bedeutend, bspw. soweit aufgrund begrenzter Rationalität des Managements (Unkenntnis über die Aussagekraft der unterschiedlichen finanzwirtschaftlichen Vorteilhaftigkeitsmaße) eine diesbezügliche Beratung des Controllers erfolgen muss. Die zu treffende Entscheidung über das Entscheidungssystem obliegt letztlich dem Management.

Wurde ein Entscheidungssystem ausgewählt, kann im nächsten Prozessschritt die Suche und Aufbereitung von Entscheidungsalternativen sehr unterschiedlich ausfallen. Abhängig davon, ob der Kunde des Controllers eine oder mehrere Entscheidungsalternativen vorgebracht hat, hat der Controller eine Aufbereitung dieser vorzunehmen. Die eigenständige Ergänzung zusätzlicher Entscheidungsalternativen hängt maßgeblich davon ab, inwieweit der Controller Investitionsinhalt und -zwecksetzung fachlich beurteilen kann. Demgegenüber kann die Suche rationeller gestaltet werden, soweit es sich bspw. um eine Ersatzinvestition gleichen Typs handelt. ${ }^{258} \mathrm{Da}$ hier zunächst die Suche und Aufbereitung von Entscheidungsalternativen betrachtet wird, welche innerhalb des Controlling vollzogen wird, kommt Verhaltens- und Interaktionsaspekten nur dann eine Bedeutung zu, wenn die zur Beurteilung der Investition notwendigen Informationen unternehmensintern validiert werden müssen. Demgegenüber ist ein Rückgriff auf das Informationssystem des Controlling zu Analysezwecken unerlässlich. Im Rahmen der nächsten Prozessphase gilt es von Seiten des Controllers die Investitionsalternativen zu bewerten, sowie eine geeignete vorzuschlagen. Ist die Investitionsentscheidung zweckmäßig aufbereitet, dominieren in dieser Phase Verhaltens- und Interaktionsaspekte. Als ein Ausdruck opportunistischen Verhaltens kann bspw. die vorsätzliche Überbewertung der Einzahlungsüberschüsse der prognostizierten Zahlungsreihe angesehen werden. Werden derartige Sachverhalte z.B. im Rahmen eines Datenabgleichs mit dem Absatzbereich erkannt, gilt es diese angemessen zu kommunizieren. Auch wenn nicht Vorsatz, sondern vielmehr eine unbeabsichtigt zu optimistische Einschätzung zu der Prognose überhöhter Einzahlungen geführt hat, ist dieser Form begrenzter Rationalität zu begegnen. Insbesondere die Prestigeträchtigkeit vieler größerer Investitionsobjekte begünstigt derartige Verhaltensweisen. ${ }^{259}$ Im Rahmen des Vorschlags einer Investitionsalternative kommt der Kommunikation und Interaktion eine herausragende Bedeutung zu. Die Darstellung des Vorschlages muss sich an dem kundenindividuell zu ermittelnden Informationsbedarf orientieren. Somit muss dem Kunden ermöglicht werden, die für oder ge-

$258 \mathrm{Vgl}$. Weber/Weißenberger/Aust (1997), S. 9.

259 WEBER zitiert in diesem Zusammenhang die sehr sarkastische Einschätzung eines Controllers zu opportunistischem Verhalten bei Investitionsentscheidungen. Vgl. Weber (1999), S. 320. 
gen eine Investitionsentscheidung sprechenden Argumente in angemessener Zeit aufnehmen zu können. Neben der pointierten Darstellung in Form eines Berichtes kann additiv eine Beratung erfolgen, um das Verständnis sicherzustellen. Die nachfolgende Entscheidung über die Realisierung einer Investitionsalternative obliegt wiederum dem Management, da dies als unmittelbare Führungsaufgabe aufzufassen ist.

Ist eine Investition realisiert, gilt es sodann für das Controlling den laufenden Betrieb, bzw. die daraus resultierenden Auswirkungen auf die prognostizierten Ziele zu überwachen. Prozessbezogen stellen Soll-Ist-Vergleiche eine Aktivität kontrollierender Natur dar, die realisationsbegleitend oder auch nach Abschluss des Investitionsvorhabens Bedeutung erlangt. Information und Interaktion kommt in Abhängigkeit der Struktur der zugrundeliegenden Informationssysteme eine unterschiedliche Bedeutung zu. Werden objektbezogene Daten im Rahmen der Systempflege kontinuierlich erfasst, können Soll-Ist-Vergleiche automatisiert erfolgen. Die daraus resultierenden Daten können Kunden aufbereitet oder ungefiltert aus dem DV-System zugänglich gemacht werden.

Demgegenüber erlangt die Interaktion im Rahmen des Prozessschritts Abweichungsanalyse eine weitaus größere Bedeutung, da diesbezügliche Informationen regelmäßig aus unterschiedlichen Unternehmensbereichen beschafft werden müssen. Für die Kommunikation der Ergebnisse der Abweichungsanalyse, wie gleichermaßen für die Vorschläge von Anpassungsmaßnahmen, spielen Verhaltens- und Interaktionsaspekte eine wesentliche Rolle. Bei den zu kommunizierenden Inhalten handelt es sich regelmäßig um komplexe Kausalzusammenhänge, die die Informationsaufnahme- und Verarbeitungskapazität der Kunden intensiv beanspruchen. Neben einer Übermittlung in Form eines Investitionsreports ist daher die persönliche Erläuterung und Beratung von entscheidender Bedeutung. Will der Controller auch langfristig seiner Rolle als kompetenter Berater und Sparringspartner des Managements gerecht werden, ${ }^{260}$ gilt es im Rahmen der Verhaltenssteuerung auch auf Defizite seiner Kunden hinzuweisen bspw. in Form der Unterbreitung einer der ursprünglichen Projektintention zuwiderlaufenden Anpassungsmaßnahme, soweit hierdurch die Zielerreichung der Unternehmensziele verbessert werden kann.

260 Zum Rollenbild des Controllers vgl. Eschenbach (1997), S. 15 ff. sowie Controller Verein (o.J.). Zur Rolle des internen Beraters vgl. Eichler (1999), S. 263. 
Abbildung 13 stellt übersichtsartig anhand des gewählten Beispiels dar, wie Controlling-Leitungen systematisch einer Marketingbetrachtung zugänglich gemacht werden können.

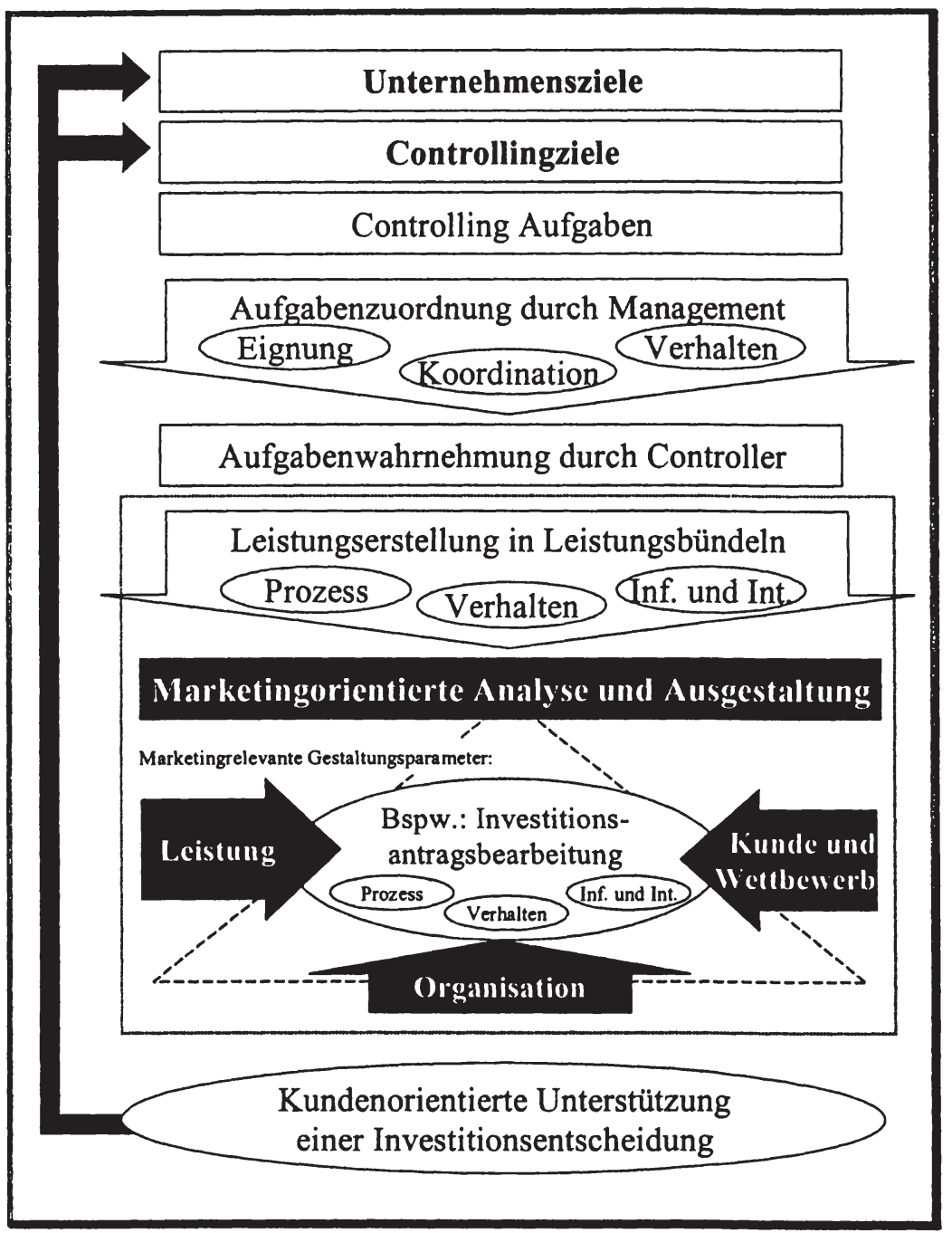

Abbildung 13: Leistungsbündel des Controlling als Gegenstand der Marketingbetrachtung 
Zunächst scheinen die zuletzt angesprochenen verhaltenssteuernden Kontrollaufgaben des Controllers die Grenzen der Kundenorientierung aufzuzeigen. Bei einer umfassenden Betrachtung kann jedoch davon ausgegangen werden, dass die unternehmensbezogenen Individualziele des Managements langfristig auch nur über eine Erfüllung der Gesamtunternehmensziele zu erreichen sind, da ein Großteil der Unternehmen negative Sanktionsmechanismen kennt, denen das Management im Falle dauerhafter persönlicher Vorteilnahme oder Fehlentscheidungen ausgesetzt ist. Demgegenüber ist ein eindeutiger Trend zu erkennen, die Vergütung der Mitarbeiter und des Management über geeignete Anreizmechanismen ${ }^{261}$ an die Erfüllung der Unternehmensziele zu koppeln. ${ }^{262}$ Hierdurch wird auch organisatorisch der Kundenorientierung - im Sinne einer Erfüllung der an die Gesamtunternehmensziele gekoppelten Interessen der internen Kunden - eine angemessene Bedeutung beigemessen.

\subsubsection{Leistungsbezogene Gestaltungsparameter des kundenorientier- ten Controlling}

Im Rahmen der Darstellung leistungsbezogener Gestaltungsparameter des kundenorientierten Controlling sollen die zuvor erörterten Leistungen sowie die aus ihrer Kombination hervorgehenden Leistungsbündel einer differenzierteren $\mathrm{Be}-$ trachtung unterzogen werden. Waren es bislang die Leistungsinhalte, die analysiert wurden, sollen nunmehr die Inhalte anhand von für eine kundenorientierte Gestaltung zugänglichen Merkmalen charakterisiert werden. Über eine Darstellung und Analyse der Beurteilungsdimensionen und Leistungseigenschaften soll gezeigt werden, dass auf Basis der Annahme derartiger Denk-/Strukturierungskategorien relevante Hinweise für die Gestaltung von Kundenbeziehungen gewonnen werden können. Darüber hinaus bietet eine Differenzierung von Leistungen mit Unterstützungs- bzw. Kontrollcharakter im Rahmen der Inhaltscharakteristika einen Anhaltspunkt über das Ausmaß, inwieweit artikulierte Kundenbedürfnisse im Rahmen der Leistungserstellung Berücksichtigung finden können. Ohne den Ausführungen vorgreifen zu wollen, deuten gerade empirische Erhebungen - wie in Abbildung 14 dargestellt - auf einen Wandel im Berufsbild des Controllers hin.

\footnotetext{
261 Zu Motivations- und Anreizsystemen vgl. Küpper (1997), S. 193 ff.

262 Eine Ausnahme hiervon sind Situationen, in denen die Mehrheitsverhaltnisse eines Unternehmens den Konsum von Perquisites zulassen. Hiervon wird dann gesprochen, wenn der Mehrheitsaktionär, bedingt durch das Recht die Unternehmung zu leiten und somit das Management zu stellen, Ressourcen der Unternehmung für persönliche Annehmlichkeiten verwendet, statt diese den bestimmten produktiven Zwecken zukommen zu lassen. Vgl. Hartmann-Wendels/von Hinten (1989), S. $268 \mathrm{ff}$.
} 


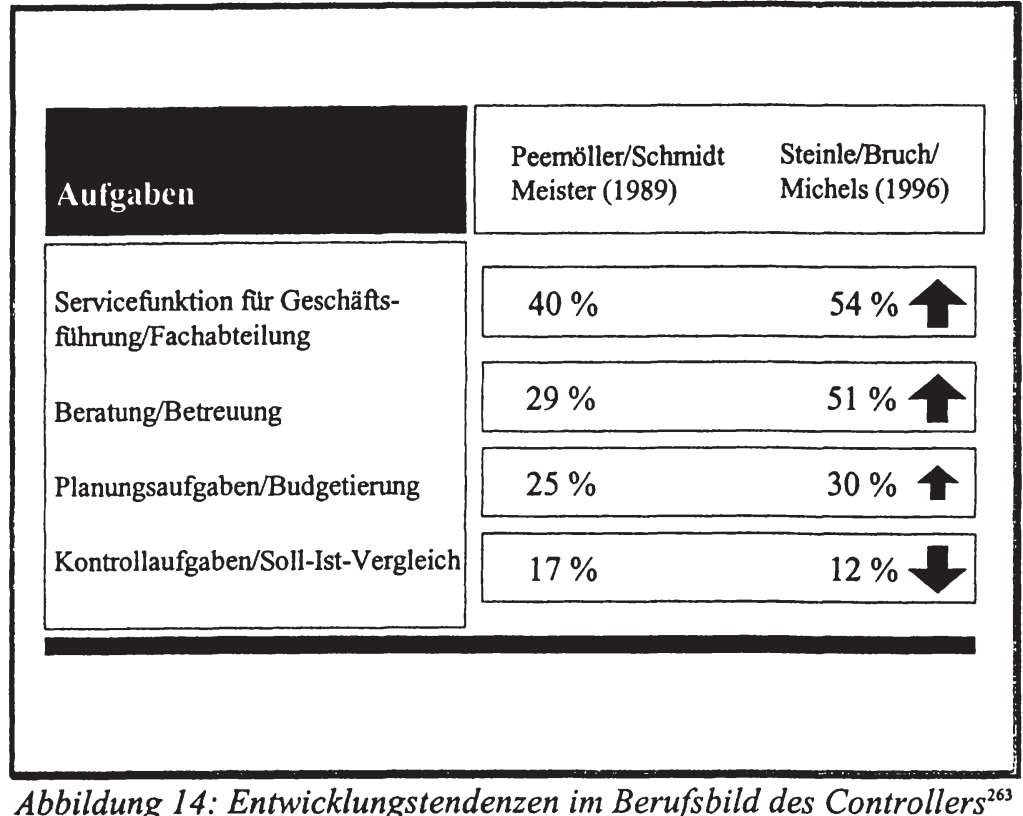

Dieser Wandel ist unter anderem durch eine deutliche Zunahme solcher Aufgaben (Service/Beratung/Betreuung) und daraus ableitbarer Leistungen gekennzeichnet ist, die einer Marketingbetrachtung scheinbar zugänglicher sind als dies frühere Tätigkeitsschwerpunkte (Planung/Kontrolle) waren. Abbildung 14 belegt dies beispielhaft anhand zweier Studien im Zeitablauf.

\subsubsection{Beurteilungsdimensionen}

Wie bereits im Rahmen der Ausführungen zum Dienstleistungsmarketing dargelegt wurde, dienten die Beurteilungsdimensionen ${ }^{264}$ von Leistungen in der Vergangenheit regelmäßig als Ansatzpunkt für Dienstleitungsdefinitionen. ${ }^{265} \mathrm{Im}$ Folgenden sollen - der Argumentation ENGELHARDT ET AL. folgend - ${ }^{266}$ Beurtei-

${ }^{263}$ Steinle/Bruch (1999), S. 52 in Anlehnung an die Erhebungen von Peemöller/Schmidt/Meister (1989), S. 295 ff. und Steinle/Bruch/Michels (1998), S. 443 ff. Zur internationalen Entwicklung des Berufsbildes vgl. Siegel/Sorensen (1999), S. 1 ff.

264 Andere Autoren sprechen in diesem Zusammenhang auch von Qualitätsdimensionen.

${ }^{265}$ Vgl. Kap. 2.2.2.1.

266 ENGELHARDT ET AL. betonen, dass die Beurteilungsdimensionen einer Leistung keine geeigneten Kriterien zur Abgrenzung einer Dienstleistung von einer Sachleistung sind. Vgl. Engelhardt/Kleinaltenkamp/Reckenfelderbäumer (1993), S. $395 \mathrm{ff}$. 
lungsdimensionen weniger als Definitionsmerkmal, sondern vielmehr als Analyseraster für unterschiedliche Typen von Controlling-Leistungen Verwendung finden. In der Literatur werden regelmäßig Potenzial-, Prozess- und Ergebnisqualitäten von Leistungen unterschieden ${ }^{267}$ Hierbei handelt es sich um die für die Beurteilung aus der Kundenperspektive bedeutsamen Qualitätsaspekte.

Die Potenzialqualität eines Anbieters manifestiert sich in den Fähigkeiten und der Bereitschaft, eine Leistung zu erbringen. Die Prozessqualität beschreibt die Eignung des Anbieters, den durch die Integration des externen Faktors initiierten und geprägten Leistungsprozess im Sinne des Kunden zu gestalten. Die Ergebnisqualität zeigt demgegenüber auf, inwieweit das Resultat des Leistungserstellungsprozesses zur Bedürfnisbefriedigung geeignet ist. Betrachtet man beispielhaft die Kostenrechnung als Leistung bzw. besser Leistungsgruppe ${ }^{268}$ des Controlling, determinieren regelmäßig Kompetenz- und Ausstattungsmerkmale ihre Potenzialqualität. ${ }^{269}$ Demgegenüber sind Schnelligkeit und Termintreue als Qualitäten des Bereitstellungsprozesses von Kostenrechnungsinformationen aufzufassen. Sieht man Berichte der Kostenrechnung als Prozessergebnisse, stellen die Genauigkeit oder die Darstellungsform diesbezügliche Ergebnisqualitäten dar.

Bei diesen drei Beurteilungsdimensionen, ist es zur Bewertung der Qualität einer bestimmten Controlling-Leistung notwendig, dimensionsbezogen die aus Nachfragersicht relevanten Nutzenvorteile zu systematisieren. Ferner ist über das Leistungsergebnis hinaus das Nutzungsverhalten der Nachfrager von herausragender Bedeutung, da nur über eine entsprechende Nutzung der regelmäBig vom Controllerbereich zur Verfügung gestellten Informationen eine Zielerreichung auf Gesamtunternehmensebene gewährleistet werden kann.$^{270}$ Aus didaktischen Gründen soll nachfolgend entgegen der sonst üblichen Abfolge zunächst das Leistungspotenzial, dann das Leistungsergebnis und bewusst zuletzt - aufgrund seiner großen Bedeutung - der Leistungsprozess betrachtet werden. ${ }^{271}$

267 Vgl. Meffert/Bruhn (1995), S. 25.

${ }^{268}$ Eine Leistungsgruppe ist in diesem Zusammenhang die Zusammenfassung mehrer inhaltlich zusammengehöriger Leistungen aufzufassen.

${ }^{269} \mathrm{Zu}$ dem Beispiel hier und im Folgenden vgl. Homburg/Weber (1999), S. 584 f. Ausfuhrlich auch bei Aust (1999), S. $105 \mathrm{ff}$.

270 Zur allgemeinen Bedeutung des Nutzungsverhalten vgl. Weiber/Späth (1998), S. 229 ff.

271 Im Regelfall erfolgt die Betrachtung von Prozessqualităten vor den Ergebnisqualităten 


\subsection{Potenzialqualität}

Bei einer Betrachtung der Bedeutung von Potenzialqualitäten für unterschiedliche Controlling-Leistungen muss zunächst beachtet werden, dass die hierfür ausschlaggebenden Potenzial- und Verbrauchsfaktoren stets kontextabhängig sind, da sie nur für einen bestimmten Controllerbereich gelten. Daher muss es im Folgenden weniger um die Beschreibung spezifischer Potenzialqualitäten eines Anbieters gehen, sondern vielmehr um die Art und Weise, wie Controlling-Kunden die Leistungspotenziale eines Anbieters wahrnehmen. In Abhängigkeit dieser Wahrnehmung muss entschieden werden, inwieweit es zweckmäBig ist, Leistungspotenziale bzw. deren Wahrnehmung ${ }^{272}$ als Wettbewerbsparameter einzusetzen. Charakteristisch für Potenziale bzw. Verbrauchsfaktoren ist, dass ohne konkreten Auftrag lediglich sogenannte Vorkombinationen von Leistungen erstellt werden können. ${ }^{273} \mathrm{Zu}$ diesem Zweck ist es notwendig, Vorstellungen über zukünftig nachfragewirksame Leistungen zu entwickeln. ${ }^{274}$ Die in der Vorkombination erbrachten Leistungen stellen in Verbindung mit den verbliebenen Potenzial- und Verbrauchsfaktoren die sogenannten internen Faktoren dar. ${ }^{275}$

Betrachtet man aufbauend auf den vorangegangenen Ausfuihrungen das Leistungsspektrum des Controlling, so ergeben sich zunächst bezogen auf die drei zur Systematisierung verwandten Leistungsdimensionen (Entscheidungsprozess, Information und Interaktion und Verhalten) unterschiedliche Anknüpfungspunkte. Die Fähigkeit und Bereitschaft des Controlling-Anbieters kann sich somit auf inhaltliche Unterstützungsleistungen entlang des Entscheidungsprozesses des Managements beziehen, sowie auf die mit deren Vollzug verbundenen Informations- und Interaktionsleistungen und Verhaltenssteuerungsleistungen. Die Aufspaltung der Potenziale eines Controlling-Anbieters entlang der Dimensionen des Leistungsraums zeigt, dass es aus Kundensicht unterschiedliche Anknüpfungspunkte für die Wahrnehmung der Potenzialqualität gibt. Ohne jedoch auf die Bedeutung der Leistungspotenziale für einzelne Dimensionen des Leistungsraums differenzierter einzugehen, ${ }^{276}$ ergibt sich die Notwendigkeit,

272 Da Potenziale sich regelmäßig einer direkten Bewertung entzichen, kommt bspw. kommunikationspolitischen Maßnahmen in diesem Zusammenhang eine besondere Bedeutung zu.

$273 \mathrm{Vgl}$. Schneider (1995), S. 33 f.

274 Informationsbedarfsanalysen können in diesem Zusammenhang ein geeignetes Instrument sein, auch unabhängig von artikulierten Bedüfnissen zuküftig nachfragerelevante Bedürnisse aufzuspüren.

275 Vgl. Kleinaltenkamp (1997), S. 89.

276 Sehr unterschiedlich stellen sich die Möglichkeiten zur Erstellung von Vorkombinationen dar. Bei der Gestaltung und dem regelmäBigen Betrieb von Informationssystemen kơnnen bspw. recht umfassende Vorkombinationen auf Basis einer Bedarfsvermutung potenzieller Kunden erstellt werden. 
die allgemeine Bedeutung des Leistungspotenzials für die Kundenorientierung eines Controllerbereichs darzustellen.

Die Dominanz der Informationen im Controlling, sowie der Anspruch, Kundenentscheidungen durch eine adäquate Datenbeschaffung, -aufbereitung und analyse zu unterstützen, zeigt zunächst, dass nur über das Vertrauen in die Qualifikation des Anbieters ein Leistungsaustausch zustande kommen kann. Da es sich hier um eine organisationsinterne Austauschbeziehung handelt, unterliegen die Leistungspotenziale des Controlling schon wesentlichen Beschränkungen. Wie im Rahmen der Aufgabenzuordnung angesprochen, ${ }^{277}$ determiniert diese bereits ansatzweise die Leistungsfähigkeit des Controlling. Positiv formuliert kann über eine sachgerechte Zuordnung der Aufgaben entlang der Aspekte Eignung, Koordination und Verhalten das Leistungspotenzial verbessert werden. Insbesondere die im Mittelpunkt der Eignungsaspekte stehende Qualifikation, welche das Wissen und die wissensgenerierenden Fähigkeiten beinhaltet, ${ }^{278}$ hat maßgeblichen Einfluss auf die Fähigkeit, Leistungen bereitstellen zu können, und somit auf das Leistungspotenzial. Die organisatorische Eingliederung und Ausgestaltung des Controllerbereichs und die damit verbundenen Kompetenzen und Abhängigkeiten haben gleichermaßen Einfluss auf das Verhaltenssteuerungspotenzial. Derart objektiv nachvollziehbare Determinanten des Leistungspotenzials dürfen jedoch nicht darüber hinwegtäuschen, dass im Rahmen der Kundenorientierung die Wahrnehmung des Kunden ausschlaggebend ist. So betrachtet, steht regelmäßig eher das Vermarktungs- bzw. Absatzobjekt im Sinne des Leistungsergebnisses im Mittelpunkt der Wahrnehmung des Kunden, da der Kunde aus eben diesem seinen Nutzen bezieht. ${ }^{279}$

Fraglich erscheint in diesem Zusammenhang allein, ob der Kunde das Leistungsergebnis bereits im Vorfeld der Transaktion bewerten kann. ${ }^{280}$ Inwiefern dennoch Leistungspotenziale zum Zweck der Kundenorientierung einer Gestaltung zugänglich gemacht werden können, soll nach der Betrachtung von Ergebnis- und Prozessqualitäten im Rahmen der Analyse von informationsökonomischen Leistungseigenschaften erläutert werden.

\footnotetext{
277 Vgl. Kap. 3.2.1.2.

279 Vgl. Engelhardt/Kleinaltenkamp/Reckenfelderbäumer (1993), S. 399.

280 Aufschluss über derartige Fragen geben die Leistungseigenschaften. Vgl. 3.2.2.2
} 


\subsection{Ergebnisqualität}

Das Leistungsergebnis ist im Sinne eines Resultates anzusehen, auf das der Nachfrager im Rahmen der Integration des externen Faktors bedingten Einfluss hatte. ${ }^{281}$ Regelmäßig handelt es sich hierbei um ein Leistungsbündel. Die Bestandteile des Leistungsergebnisses können sowohl materieller wie immaterieller Natur sein. Dominieren materielle Bestandteile das Leistungsergebnis, so wird dieses in der Wahrnehmung der Nachfrager regelmäßig präsenter erscheinen als das Leistungspotenzial bzw. der Leistungsprozess. Demgegenüber zeichnen sich Controlling-Leistungen - bedingt durch ihren Informationscharakter - oftmals durch Immaterialität aus. Auch der Einsatz von Trägermedien (Datei, Bericht, etc.) zur Übermittlung oder Dokumentation der Ergebnisse darf nicht darüber hinwegtäuschen, dass das intendierte Ergebnis immaterieller Natur ist. Insbesondere Informations- und Interaktionsaktivitäten helfen, die Wahrnehmung des Leistungsergebnisses in den Augen der Nachfrager zu verstärken. Darüber hinaus hängt die Bedeutung des Leistungsergebnisses für den Nachfrager maßgeblich von seinem Einfluss im Rahmen des Leistungserstellungsprozesses ab. Ist das Integrativitätsniveau relativ gering und erfolgt wenig Interaktion im Rahmen des Leistungsprozesses, so wird für den ControllingKunden das Leistungsergebnis Mittelpunkt der Transaktion sein. Beispielsweise interessieren gewisse Adressaten des Controlling im Rahmen des Betriebs einer Kostenrechnung vornehmlich standardisierte Berichte, wie der monatsbezogene Kostenstellenbericht. Wahrgenommen wird hierbei ein konkretes Ergebnis. Steigt demgegenüber bei Controlling-Leistungen das Integrativitätsniveau, wie bei der Entwicklung einer Kostenrechnungskonzeption oder der Erarbeitung einer Strategie, so ist denkbar, dass sich der Kundennutzen vielmehr aus dem Durchleben des Leistungserstellungsprozesses ergibt, ${ }^{282}$ da die dort erworbenen Erkenntnisse nachhaltiger und umfassender sind als ein eventueller Abschlussbericht des Projektes.

Derartige Auswirkungen des im Rahmen des Leistungserstellungsprozesses bestimmten Integrationsgrades auf die Bedeutung des Leistungsergebnisses für den Nachfrager machen eine differenziertere Analyse des Leistungserstellungsprozesses notwendig.

\subsection{Prozessqualität}

Der Leistungserstellungsprozess im Controlling wird, wie bereits im Rahmen des Dienstleistungsmarketing ausgeführt, durch die Integration des externen

281 Vgl. Kleinaltenkamp (1997), S. 90.

282 Vgl. Engelhardt/Kleinaltenkamp/Reckenfelderbăumer (1993), S. 400 
Faktors dominiert. Im Gegensatz zu den internen Faktoren ${ }^{283}$ stellen externe Faktoren materielle oder immaterielle Faktoren des Leistungsnachfragers dar, die in Form von Leistungsbündeln auftreten, ${ }^{284}$ da Informationen regelmäßig mitgeliefert werden. ${ }^{285}$ Das Ausmaß der Integration externer Faktoren kann sehr unterschiedlich sein, was gleichermaßen Auswirkungen auf die optimale Ausgestaltung einer Kunden-Lieferanten-Beziehung hat. Wie bereits dargelegt, prägt der Integrationsgrad die Wahrnehmung der Bedeutung von Leistungsprozess und Leistungsergebnis ganz wesentlich. Aufbauend auf die Leistungstypologie von ENGELHARDT ET AL. entwickelte MEFFERT eine Erweiterung, in der er den Integrationsgrad in die Dimensionen Individualisierungsgrad und Interaktionsgrad zerlegt, um das Ausmaß und die Komponenten der Integration einer differenzierteren Betrachtung zugänglich zu machen. ${ }^{286}$ Für eine Betrachtung der Controlling-Leistungen erscheint eine derartige Typologie prinzipiell ausreichend, da dem von ENGELHARDT ET AL. aufgeführten Grad der Immaterialität letztlich aufgrund des Informationscharakters der Controlling-Leistungen eine geringere Bedeutung zukommt. ${ }^{287}$ In Abbildung 15 sollen beispielhaft verschiedene Leistungen des Controlling anhand der im Leistungsprozess stattfindenden Individualisierung ${ }^{288}$ und Interaktion systematisiert werden.

Eine derartige Durchdringung des Sachverhaltes verdeutlicht, dass die Wahrnehmung des Leistungsprozesses durch die Nachfrager um so bedeutender ist, je größer das Ausmaß der Individualisierung und Interaktion ist, ${ }^{289}$ da hierüber das Ausmaß der Integrativität determiniert wird. Korrespondierend mit den Ausführungen zum Leistungsergebnis zeigt sich, dass der hier beispielhaft eingeordnete Kostenstellenbericht oftmals unabhängig und standardisiert erstellt werden kann, was zu einem insgesamt niedrigen Integrativitätsgrad im Leistungsprozess führt. Folgerichtig wird in der Wahrnehmung der Nachfrager weniger das Prozessempfinden - wie bspw. bei einem Beratungsprojekt - im Mittelpunkt stehen, als vielmehr das Leistungsergebnis.

\footnotetext{
283 Vgl. Kap. 3.2.2.1.1.

${ }^{284}$ CORSTEN spricht in diese Zusammenhang von mehrdimensionalen Verbunden. Vgl. Corsten (1985), S. 129.

285 Vgl. Kleinaltenkamp (1997), S. 90.

286 Vgl. Meffert (1993), S.12.

287 So auch SCHADE für die Leistung der Unternehmensberatung. Vgl. Schade (1997), S. 31.

288 Das Ausmaß der Individualisierung wird nochmals ausfuhrlicher im Rahmen der marketingrelevanten kundenbezogenen Gestaltungsparameter thematisiert. Vgl. Kap. 3.4.3.1.

289 Dies kann sich auch auf die Gestaltung von Informationssystemen beziehen. Vgl. hierzu Gallager (1974), S. 46 ff. und Aust (1999), S. 159. 


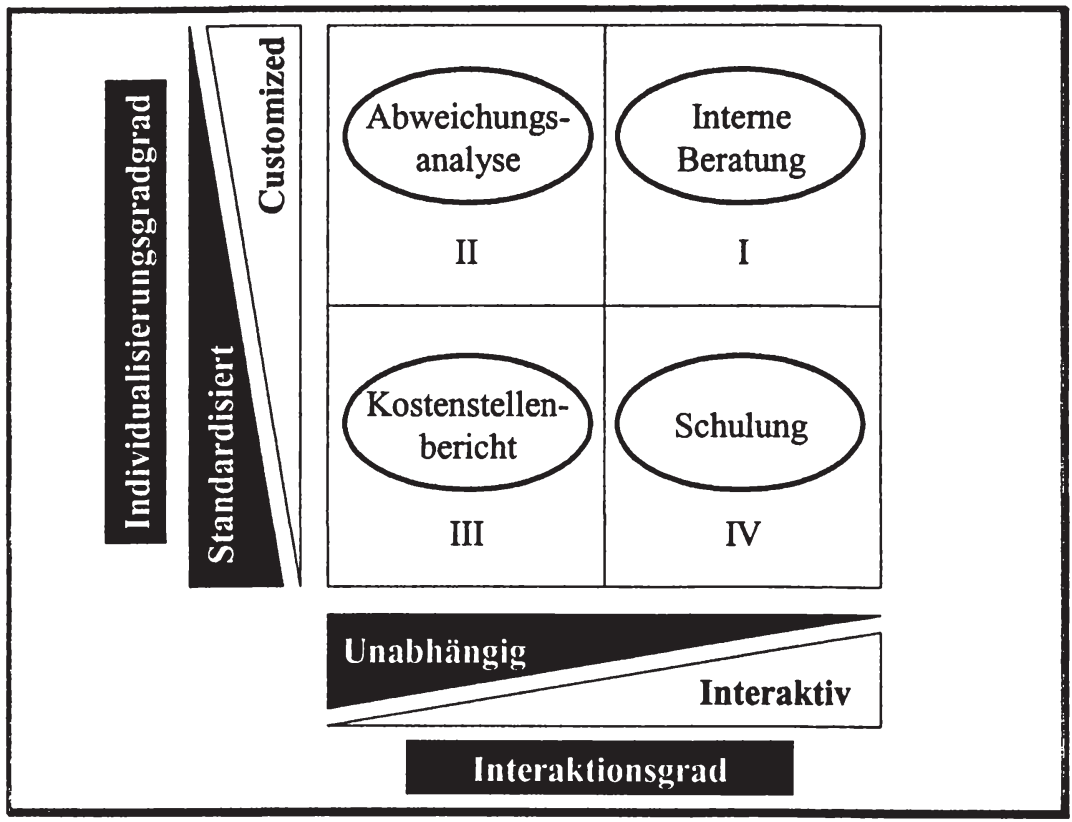

Abbildung 15: Systematisierung beispielhafter Controlling-Leistungen ${ }^{200}$

Leistungspotenzial, Leistungsprozess und Leistungsergebnis stellen wie veranschaulicht wichtige Anknüpfungspunkte für die Leistungsbewertung durch den Kunden des Controlling dar. Unterschiedliche Leistungen des Controlling weisen auch unterschiedliche Bewertungsmöglichkeiten entlang der Beurteilungsdimensionen auf. Daher ist es zweckmäßig, zunächst eine Typologisierung einzelner Leistungen vorzunehmen, um darauf aufbauend zu bewerten, welche der Beurteilungsdimensionen besonders wahrnehmungsrelevant erscheint.

Auf Basis derartiger Ergebnisse und einer fundierten Kenntnis des Produktionsprozesses von Controlling-Leistungen kann eine zielgerichtete Leistungsgestaltung betrieben werden. ${ }^{291}$ Abbildung 16 zeigt zusammenfassend den Prozess der integrativen Leistungserstellung im Controlling.

290 In Anlehnung an MEFFERT'S Erweiterung der Leistungstypologie von ENGELHARDT ET AL., vgl. Meffert (1993), S. 12.

291 Vgl. Schade (1997), S. 32. 


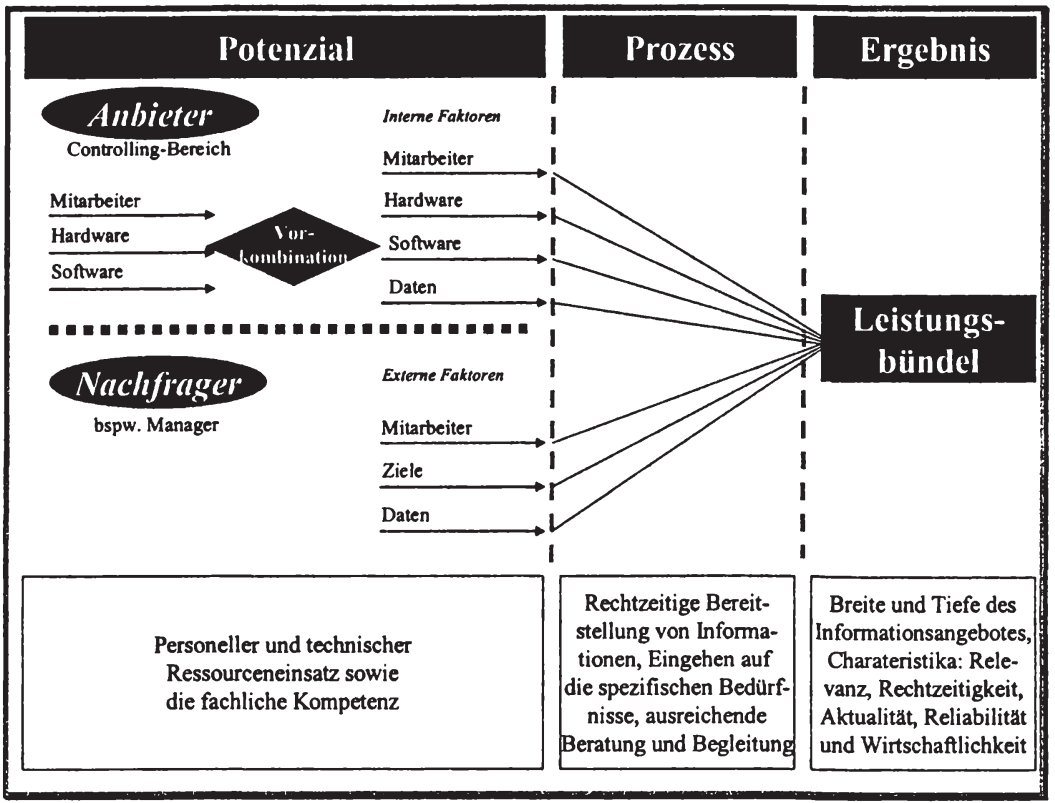

Abbildung 16: Integrative Leistungserstellung - Leistungsphasen im Controlling ${ }^{292}$

Erscheinen Leistungsprozess und Leistungsergebnis zunächst nachvollziehbar wahrnehmungsrelevant, ergeben sich diesbezüglich beim Leistungspotenzial oftmals Schwierigkeiten. Insbesondere die überwiegende Immaterialität der Controlling-Leistungen - bedingt durch den Informationscharakter - führt $\mathrm{zu}$ Unsicherheiten der Nachfrager im Vorfeld einer Leistungstransaktion. Inwieweit die Kommunikation von Leistungspotenzialen zur Unsicherheitsreduktion genutzt werden kann, soll im Rahmen der nachfolgenden Darstellung der informationsökonomischen Leistungseigenschaften gezeigt werden.

\subsubsection{Informationsökonomische Leistungseigenschaften}

Abgesehen von dem Produktionsprozess einer Leistung bestimmen die informationsökonomischen Leistungseigenschaften maßgeblich die Beurteilungsmöglichkeiten einer Leistung. ${ }^{293}$ Die Informationsökonomik als Teilansatz der Neu-

292 Die Darstellung erfolgt in Anlehnung an die allgemeine Darstellung zur integrativen Leistungserstellung von Kleinaltenkamp (1997b), S. 351. Die Erläuterung der Prozessphasen orientiert sich an Tylkowski/Guth/ Spieker (2001), S. 98.

293 Hier und im Folgenden vgl. Meffert/Bruhn (2000), S. 65 ff. 
en Institutionenökonomie beschäftigt sich diesbezüglich mit Analysemöglichkeiten von Informationsasymmetrien und dem Unsicherheitsphänomen. Zur Systematisierung von Leistungseigenschaften unterscheidet NELSON zunächst Suchgüter und Erfahrungsgüter. ${ }^{294}$ Bezug nehmen diese Begrifflichkeiten darauf, ob die Qualität einer Leistung bereits vor dem Kauf (Suchgüter) oder aber erst im Anschluss daran (Erfahrungsgüter) beurteilt werden kann. Die Tatsache, dass ein Gut regelmäßig mehrere Eigenschaften beinhalten kann, führte dazu, dass nicht das Gut als Ganzes klassifiziert wird, sondern vielmehr dieses in einzelne Leistungseigenschaften zerlegt wird. ${ }^{295}$ Eine Erweiterung erfuhr dieses Konzept im Rahmen der Ergänzung von Vertrauenseigenschaften, welche immer dann vorliegen, wenn auch nach dem Kauf die Beurteilung der Leistung nicht zweifelsfrei möglich ist. ${ }^{296}$ Insgesamt lässt sich somit ein Gut, wie in Abbildung 17 gezeigt, auf Basis der Anteile seiner drei informationsökonomischen Leistungseigenschaften in einem Raum darstellen.

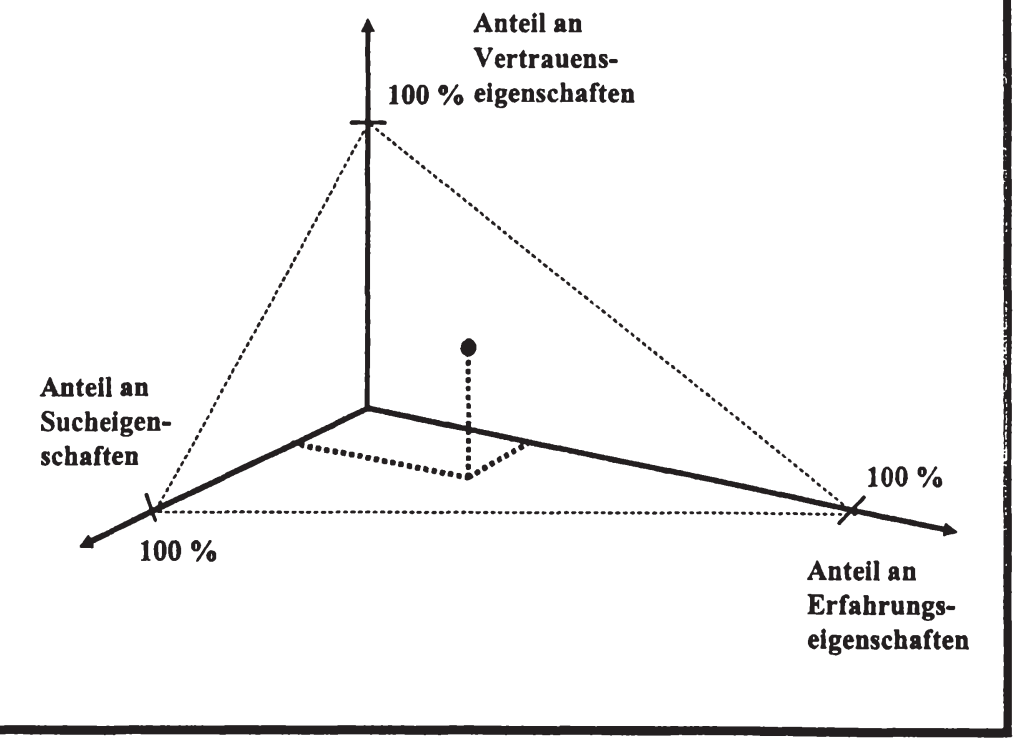

Abbildung 17: Informationsökonomisches Dreieck von Leistungen ${ }^{297}$

\footnotetext{
294 Vgl. Nelson (1970), S. 312.

295 Vgl. Nelson (1974), S. 730.

296 Vgl. Darby/Kami (1973), S. 68-69.

297 Adler (1994), S. 52.
} 
Nachfolgend sollen auf Basis einer genaueren Darstellung der einzelnen informationsökonomischen Eigenschaften Besonderheiten der Leistungen des Controlling dargelegt werden, sowie eigenschaftsspezifische Handlungsempfehlungen abgeleitet werden. Abschließend erfolgt eine Synthese aus Beurteilungsdimensionen und informationsökonomischen Leistungseigenschaften in Form eines Beurteilungsprofils integrativer Controlling-Leistungen.

\subsection{Sucheigenschaften}

Ausweislich der bisherigen Ausführungen zeichnen sich Leistungen des Controlling regelmäßig durch ihren immateriellen Charakter aus, auch wenn Trägermedien zu Kommunikations- und Dokumentationszwecken verwendet werden. Die mangelnde physische Wahrnehmbarkeit führt bei Leistungsabnehmern zu einem Unsicherheitsempfinden. ${ }^{298}$ Dieses Empfinden wird einerseits durch die Anteile der Leistungseigenschaften determiniert, worauf im Folgenden eingegangen werden soll. Andererseits bestimmen Erfahrungshorizont, Lernverhalten und die Wahrnehmungsfähigkeit der Kunden des Controlling, inwieweit die Unsicherheit das Transaktionsverhalten beeinflusst. ${ }^{299}$ Ein weiterer beurteilungskritischer Tatbestand ist die Komplexität einer Leistung, die auch bei materiellen Gütern zu Beurteilungsschwierigkeiten führt.

Sucheigenschaften liegen bei Leistungen des Controlling dann vor, wenn der Kunde im Vorfeld des Vertragsabschlusses die Eigenschaften beurteilen kann. ${ }^{300}$ $\mathrm{Da}$ es sich bei Controlling-Leistungen regelmäßig um unternehmensinterne Dienstleistungen handelt, tritt an die Stelle des Vertragsabschlusses die interne Vereinbarung über einen Leistungsbezug. In Abhängigkeit des Formalisierungsgrades können derartige Vereinbarungen jedoch nahezu Vertragscharakter haben. Ein Beispiel hierfür sind Service Level Agreements, ${ }^{301}$ die zunehmend auch für Controlling-Leistungen geschlossen werden. ${ }^{302}$ Auch bei informellen Leistungsvereinbarungen besteht für den Kunden das Unsicherheitsproblem, da Controlling-Leistungen in der Mehrzahl erst nach der Leistungsvereinbarung

298 Hier und im Folgenden vgl. Woratschek (1996), S. 60.

299

Auf unterschiedliche Kundengruppen des Controlling wird in Kap. 3.4.1.2 eingegangen.

300 MEFFERT/BRUHN weisen darauf hin, dass bei einer engen Auslegung der Definition Dienstleistungen quasi niemals reine Sucheigenschaften aufweisen können, da die Leistung immer erst nach dem Vertragsabschluss entsteht. Leistungen mit hohen Anteilen von Sucheigenschaften lassen die Betrachtung dennoch sinnvoll erscheinen. Vgl. Meffert/Bruhn (2000), S. 65.

301 Vgl. Bölscher (2000), S. 355 ff.

302 Inwieweit Service Level Agreements im Einzelnen als Gestaltungsparameter für die kundenorientierte Ausrichtung des Controlling genutzt werden können zeigt Kap. 4.2.4. 
entstehen. ${ }^{303}$ Insbesondere die Beteiligung des Kunden am Produktionsprozess in Form der Einbeziehung seiner Informationen und - wichtiger noch bei einer kundenorientierten Ausgestaltung des Controlling - seiner Präferenzen bzgl. der Informationsaufbereitung führt zu Beurteilungsschwierigkeiten. Somit hat der Kunde im Rahmen integrativer Leistungserstellung maßgeblichen Einfluss auf die Qualität einer Leistung sowie auf die mit ihrer Erstellung verbundenen Kosten und die dafür benötigte Zeit. ${ }^{304}$ Demgegenüber gibt es im Controlling auch Leistungen, die überwiegend Sucheigenschaften aufweisen, wie bspw. ein kostenstellenbezogener Monatsbericht. ${ }^{305}$ Die hinter einem derartigen Bericht stehende Auswertungssystematik ist für den Leistungsadressaten bei Kenntnis des Kostenrechnungssystems nachvollziehbar, so dass er im Vorfeld der Leistungsanforderung die Eigenschaften einer derartigen Leistung beurteilen kann. Soweit diese Berichte regelmäßig standardisiert erstellt werden, können auch die grafische Aufbereitung und die Bereitstellungsmodalitäten als Sucheigenschaften aufgefasst werden. Dementsprechend ist ein kostenstellenbezogener Monatsbericht als ein Gut mit dominierenden Sucheigenschaften aufzufassen, auch wenn die Inhalte/Zahlen materiell letztlich erst nach der Leistungserstellung vorliegen.

Auf Basis der jeweils dominierenden Leistungseigenschaften hält die Marketingtheorie unterschiedliche Empfehlungen für den Leistungsanbieter bereit, die helfen sollen, die empfundene Unsicherheit in den Augen des Leistungsnachfragers zu reduzieren. Diese Empfehlungen basieren auf der Annahme, dass die empfundene Unsicherheit des Leistungsnachfragers wesentlich das Auswahlverhalten, sowie die diesem zugrundeliegenden Informationsbeschaffungsaktivitäten beeinflussen. ${ }^{306}$ Für das Controlling ist in diesem Zusammenhang wesentlich, dass das Auswahlverhalten nicht ausschließlich auf die Auswahl des Anbieters bezogen wird - hier können sich Probleme bei einer Monopolstellung des Controlling ergeben ${ }^{307}$ - sondern vielmehr auch auf die Leistungsinhalte und den Leistungsumfang. Ersichtlich ist auch, dass das Controlling im Rahmen des informationsökonomischen Dreiecks sehr unterschiedlich einzuordnende Leistungen anbietet. ${ }^{308}$ Hieraus können sich leistungsübergreifende Interdependenzen ergeben.

\footnotetext{
303 Allgemein vgl. Meffert/Bruhn (2000), S. 65.

304 Vgl. Woratschek (1996), S. 61.

${ }^{305} \mathrm{Vgl}$. Weber (1997), S. 14.

306 Vgl. Meffert/Bruhn (2000), S. 66.

307 In diesem Zusammenhang ist ferner relevant, ob die Leistungsinanspruchnahme obligatorisch oder fakultativ ist. Vgl. Kap. 3.3.2.3.
}

308 Vgl. hierzu Abbildung 17. 
Bezogen auf ein spezielles Leistungsergebnis des Controlling, den durch Sucheigenschaften geprägten kostenstellenbezogenen Monatsbericht, wird der Nachfrager regelmäßig versuchen, Informationen über die Beschaffenheit des Leistungsergebnisses zu sammeln (Screening). Da diese sich überwiegend objektiv beurteilen lassen und auch zukünftig eine vergleichbare Ausprägung haben werden (Berichtsinhaltsstruktur, grafische Aufbereitung, Bereitstellungsmodalitäten, etc.), hat der Controllerdienst die Möglichkeit, derartige Informationen zielgerichtet zu kommunizieren (Signaling). ${ }^{309}$ Insbesondere die Möglichkeit der Präsentierbarkeit von Leistungsergebnissen ist als ein Vorteil von sucheigenschaftsdominierten Gütern anzusehen. Aus diesem Grund bieten sich auch bei einem breiteren Leistungsportfolio des Controlling mit unterschiedlichen Produkten zunächst solche mit Sucheigenschaften zur Etablierung und Festigung interner Geschäftsbeziehungen an. Darauf aufbauend können Leistungen angeboten werden, die aufgrund der geschaffenen Vertrauensbeziehung höhere empfundene Qualitätsunsicherheiten aufweisen. ${ }^{310}$ Eine derartige Strategie deutet darauf hin, dass die Qualitätsunsicherheit der Nachfrager im Zeitablauf einer Dynamik unterliegen kann, da sich das subjektive Empfinden der ControllingKunden in Abhängigkeit von Erfahrungshorizont, Lernverhalten und Wahrnehmungsfähigkeit ändert.

Weisen Leistungen mit dominierenden Sucheigenschaften die geringste Qualitätsunsicherheit auf, ergeben sich bei Gütern mit Erfahrungseigenschaften aufgrund des zunächst vorzunehmenden Leistungserstellungsprozesses gravierendere Beurteilungsprobleme aus der Sicht der Nachfrager.

\subsection{Erfahrungseigenschaften}

Charakteristikum der Erfahrungseigenschaften ist, dass die Möglichkeit der Beurteilung erst während oder nach dem Leistungserstellungsprozess gegeben ist. Bezogen auf das Qualitätsunsicherheitsempfinden des Nachfragers ergeben sich hieraus unterschiedliche Implikationen. ${ }^{311} \mathrm{Da}$ Controlling-Leistungen regelmäBig integrativer Natur sind, hat der Nachfrager die Möglichkeit im Rahmen der Integration seiner externen Faktoren Einfluss auf den Leistungserstellungsprozess zu nehmen und somit die Qualität und Kosten des Leistungsergebnisses zu beeinflussen. WORATSCHEK spricht in diesem Zusammenhang von einem

$309 \mathrm{Zu}$ den informationsökonomischen Transaktionsdesigns Screening und Signaling vgl. beispielhaft Fließ (1995), S. $309 \mathrm{ff}$.

$310 \mathrm{Vgl}$. Meffert/Bruhn (2000), S. 66.

311 Nachfolgend vgl. Woratschek (1996), S. 61. 
„Zwang zur Kundennähe ${ }^{\text {(6312 }}$. Dies ist jedoch gerade für das Controlling nicht unmittelbar zutreffend, da nicht allein die Integration eines externen Faktors entscheidet - diese erfolgt auch bei „kundenfernen“ Controlling-Systemen sondern vielmehr die Art der externen Faktoren ausschlaggebend ist. Ohne es an dieser Stelle zu sehr zu vertiefen, ist allgemein von Informationen als externen Faktoren auszugehen, wobei diese wiederum nach ihren Inhalten zu differenzieren sind. Neben der Einbringung der inhaltlichen Informationen, die letztlich aufbereitet werden sollen, sind im Sinne eines kundenorientierten Controlling Informationen über Auswertungs- oder Gestaltungspräferenzen des Kunden als ein externer Faktor anzusehen, über den Kundenzufriedenheit generierbar wird.

Neben den Vorteilen der Integrativität führt die späte Bewertbarkeit der Leistung dazu, dass die Auswahl des Leistungsanbieters in den Augen der Kunden ein besonderes Gewicht erhält. Für Kunden des Controlling stellt sich die Frage, ob, bezogen auf ein bestimmtes Entscheidungsproblem, der Controllerdienst in der Lage ist, effektiv entscheidungsunterstützend zu agieren. Diesbezüglich kommt der Beurteilbarkeit der allgemeinen Fähigkeiten des Controllerdienstes eine wichtige Bedeutung zu. An dieser Stelle schließt sich der Kreis zu den Beurteilungsdimensionen einer Leistung, da es sich hierbei genau um die Bedeutung des Leistungspotenzials handelt. Die Evidenz des Leistungspotenzials eines Controllerdienstes kann somit maßgeblich zur Unsicherheitsreduktion erfahrungseigenschaftsdominierter Leistungen fuihren.

Eine für den Controllerdienst beispielhafte Leistung mit dominierenden Erfahrungseigenschaften könnte eine Abweichungsanalyse sein, soweit der Kunde ex post nachprüfen kann, ${ }^{313}$ ob die genannten Ursachen im Kausalzusammenhang zu der Abweichung stehen. ${ }^{314} \mathrm{Da}$ dies erst nach der Leistungsvereinbarung möglich ist, empfindet der Kunde möglicherweise im Vorfeld eine Unsicherheit über die Fähigkeit und Flexibilität des Controllerdienstes, eine derartige Analyse erbringen zu können (hidden charakteristics). ${ }^{315}$ Das Unsicherheitsempfinden ist auch in solchen Fällen relevant, in denen der Kunde zunächst keine Auswahlmöglichkeit bezüglich des Anbieters hat, da regelmäßig die Selbsterstellung

312 Woratschek (1996), S. 61. Dieser Ausdruck steht auch in der Originalquelle in Anfuhrungszeichen, so dass von einer nicht ganz wörtlichen Intention auszugehen ist.

313 Vgl. Weißenberger (1997), S. 159.

314 WEBER nennt in diesem Zusammenhang firr die Kostenrechnung eine einfache Kostenvergleichsrechnung als Beispiel. Vgl. Weber (1997), S. 14.

315 Allgemein zu den Ausprägungen der Verhaltensunsicherheiten vgl. Fließ (1995) S $396 \mathrm{ff}$ 
der Leistung eine Alternative darstellt. ${ }^{316}$ Hat der Kunde sich für eine Durchführung der Analyse durch den Controllerdienst entschieden, besteht weiterhin eine Unsicherheit darüber, ob der Controller nach der Leistungsvereinbarung im Rahmen des Leistungserstellungsprozesses seinen Verpflichtungen nachkommt (hidden action/ hidden intention). Fokussiert man die Kundenorientierung eines internen Dienstleisters Controlling, so muss eine Verfehlung nicht unbedingt in der Präsentation einer nachvollziehbar falschen Zahl bestehen, sondern vielmehr reicht schon die mangelnde Nachvollziehbarkeit einer richtigen Zahl, um die Erwartungen der Kunden zu enttäuschen. Über die einzelne Leistungsbeziehung hinaus ergibt sich oftmals aus Nachfragersicht das Problem, dass mit bestimmten Transaktionen eine sehr starke Abhängigkeit von einem bestimmten Lieferanten ausgelöst wird. In diesem Zusammenhang spricht man von Faktorspezifität, d.h. der Nachfrager erleidet erhebliche Einbussen, wenn er den Lieferanten wechselt. ${ }^{317}$ Im Bereich des Controlling gilt dies bspw. immer dann, wenn der Controllerdienst für den Kunden spezielle Informationssysteme betreibt. Eine derartige Unsicherheit durch Bindung an Anbieter wird jedoch nur dann empfindungsrelevant, wenn der Kunde vorher die Möglichkeit einer Auswahl gehabt hat.

Wie bereits im Rahmen der Sucheigenschaften dargestellt, stehen Anbieter und Nachfrager verschiedene Aktivitäten zur Verfügung, um zu einer Unsicherheitsreduktion beizutragen. Da diese Aktivitäten bei Gütern mit Erfahrungs- und Vertrauenseigenschaften besonders zweckdienlich sind, sollen sie in Abbildung 18 übersichtsartig dargestellt werden.

Von den dort dargestellten Informationsaktivitäten eignen sich zur aktiven Gestaltung für den Controllerdienst besonders die Kommunikation und damit die Offenlegung der eigenen Potenziale. Insbesondere auch ein strukturierter Produktkatalog ${ }^{3 / 8}$ vermag hier die Leistungsevidenz zu steigern. ${ }^{319}$ Eine weitere Möglichkeit, die eigenen Fähigkeiten zu dokumentieren, stellt die Vorstellung von Referenzkunden dar, die vergleichbare Leistungen des Controllerdienstes in Anspruch genommen haben und in der Folge der Leistungserstellung zufriedengestellt wurden. Eine derartige Vorgehensweise erscheint gerade innerhalb einer Organisation sehr praktikabel und vergleichsweise unaufwendig.

\footnotetext{
316 Dies ist besonderes relevant in Fällen, in denen bei der Aufgabenzuordnung primär auf Basis von Kapazitätsrestriktionen entschieden wurde, die Qualifikationen für eine Leistung jedoch bei Management und Controller vorliegen. Vgl. Kap. 3.2.1.2.

$317 \mathrm{Zu}$ den unterschiedlichen Arten der Faktorspezifităt vgl. Fließ (1995), S. 311.

318 Beispielhaft für denn IT-Bereich vgl. Böni/Britzelmeyer/Schlegel (1999), S. 485 ff.
}

319 Vgl. Weber (1997), S. 7. ff. sowie zur Leistungsevidenz Kap. 3.4.32.2. 


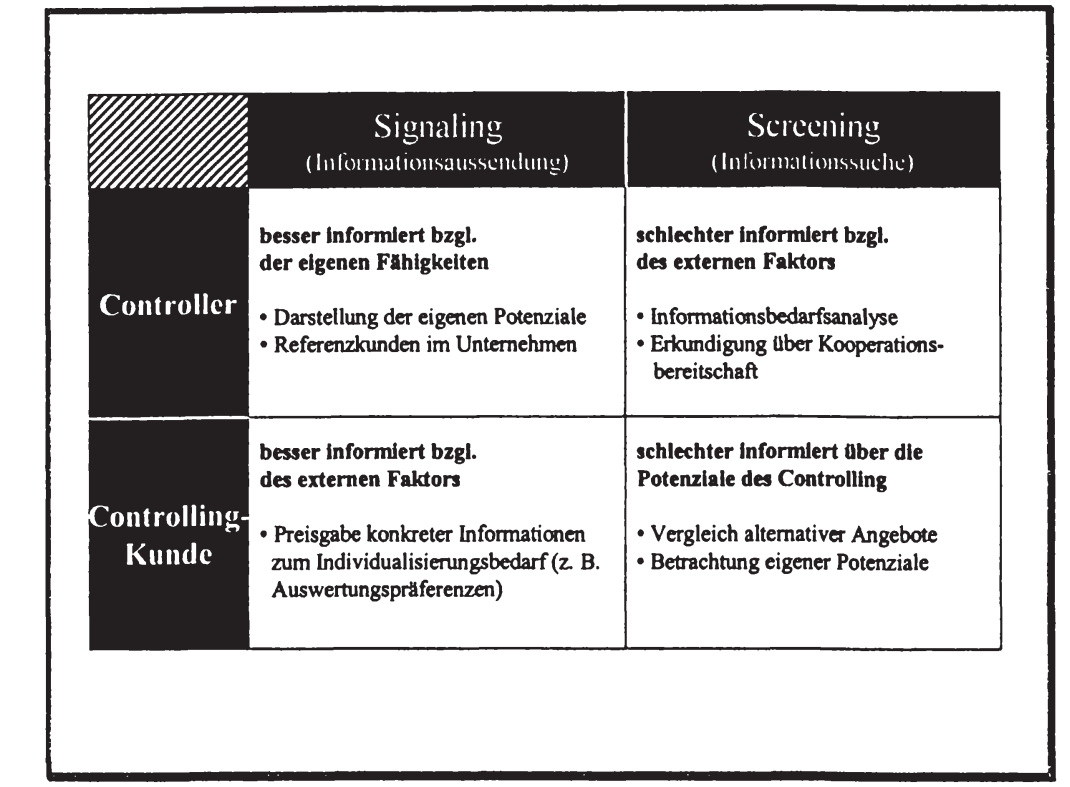

Abbildung 18: Beispielhafte Informationsaktivitäten der Transaktionspartner im Controlling ${ }^{320}$

Über unmittelbare Informationsaktivitäten hinaus stellt auf unternehmensexternen Märkten die Abgabe von Garantien ein probates Mittel zum Abbau der empfundenen Qualitätsunsicherheit dar. ${ }^{321}$ Fraglich erscheint, inwieweit derartige Formen der Selbstbindung organisationsintern einsetzbar sind. Aufgrund der spezifischen Aufgabenzuordnung wird der Controllerdienst im Regelfall auch über den Leistungserstellungsprozess hinaus für die Erfüllung der Kundenwünsche verantwortlich sein, soweit das Leistungsergebnis nicht zufriedenstellend war. Demgegenüber ist für das Controlling die Chance im Rahmen der Abgabe von Garantien darin zu sehen, einen offensiven Umgang hiermit zu betreiben. So kann bspw. bei erklärungsbedürftigen oder schwierig umsetzbaren Leistungen des Controlling vorab eine Beratung oder Umsetzungsbegleitung garantiert werden.

Insgesamt zeigt sich, dass bei erfahrungseigenschaftsdominierten Gütern die Komplexität der unsicherheitsreduzierenden Maßnahmen gegenüber den Such-

320 In Anlehnung an die controllingunabhăngige Darstellung von Meffert/Bruhn (2000), S. 68.

321 Vgl. Jakob (1994), S. 170. 
gütern regelmäßig zunimmt. Einen noch höheren Grad empfundener Unsicherheit weisen Güter mit Vertrauenseigenschaften auf.

\subsection{Vertrauenseigenschaften}

Der für das Controlling charakteristische Umgang mit komplexen unsicherheitsbehafteten Informationen führt regelmäßig dazu, dass das Leistungsergebnis für den Kunden auch im nachhinein nicht beurteilt werden kann. In diesem Zusammenhang spricht man von Gütern mit Vertrauenseigenschaften. Der Kunde kann letztlich nur darauf vertrauen, dass das Leistungsergebnis die vom Anbieter versicherte Qualität hat. Bei komplexen, nicht standardisierbaren Dienstleistungen mit Vertrauenseigenschaften spricht man auch von Kontraktgütern. ${ }^{322}$ Ein mit den Controlling-Leistungen korrespondierendes Kontraktgut ist die Unternehmensberatung. ${ }^{323}$ Die Beratungsleistung existiert noch nicht zum Zeitpunkt des Kontrakts, ist wenig standardisierbar, hochwertig und komplex. Bezogen auf die Aufgabenentwicklung im Controlling, die sich durch eine $\mathrm{Zu}$ nahme interner Beratungsaktivitäten auszeichnet, ${ }^{324}$ deutet dies darauf hin, dass dem Management von Vertrauensgütern Beachtung zu schenken ist. ${ }^{325}$

In Abgrenzung zu Erfahrungsgütern hat der Kunde des Controlling bei Vertrauensgütern auch ex post nicht die Möglichkeit, die optimale Handlungsalternative zu erkennen. Abgesehen von einfach strukturierten und sich häufig wiederholenden Problemstellungen kann davon ausgegangen werden, dass die hohe Umweltdynamik und die Expertenstellung des Controllers dazu führt, dass abweichende Merkmalsausprägungen von Controlling-Kunden nicht erkannt werden können. ${ }^{326}$ Um das sich daraus ergebende Unsicherheitsempfinden des Kunden zu reduzieren, sind vornehmlich vertrauensbildende Maßnahmen zu ergreifen. Neben dem angesprochenen sukzessiven Vertrauensaufbau im Zeitablauf über getätigte Transaktionen von Such- und Erfahrungsgütern kommt der Kommunikation der Reputation des Anbieters eine besondere Bedeutung zu. Die Reputation des Controlling besteht aus der Vertrauenswürdigkeit und der

322 Vgl. Kaas (1992), S. 884 f. sowie Woratschek (1996), S. 63.

323 SCHADE spricht in diesem Zusammenhang von einem geradezu idealtypischen Kontraktgut. Vgl. Schade (1995), S. 27. Zu Leistung und Vermarktung unterschiedlicher Formen der Unternehmensheratung vgl. Miethe (2000), S. $17 \mathrm{ff}$.

324 Vgl. Weber (1999), S. 352, Niedereichholz (1999b), S. 1833 ff. sowie Abbildung 14.

${ }^{325}$ Die komplexe Analyse der Kosten eines Geschäftsbereichs, die nach dessen Aufgabe wegfallen würde, wird von WEBER als ein weiteres beispielhaftes vertrauenseigenschaftsdominiertes Gut des Controlling genannt. Vgl. Weber (1997), S. 14.

So WEIBENBERGER für die Informationsleistung des Rechnungswesens. Vgl. Weißenberger-(19327), S. $158 \mathrm{ff}$. 
Kompetenz. ${ }^{327}$ Wie bereits angemerkt, ist die Kompetenz eine grundlegende Komponente des Leistungspotenzials und in diesem Zusammenhang wahrnehmungsrelevant. Die Vertrauenswürdigkeit bezieht sich demgegenüber auf das Leistungspotenzial und den Leistungsprozess. ${ }^{328}$ Die erfolgreiche Kommunikation der Reputation hängt maßgeblich davon ab, inwieweit Kunden diese Informationen über Fremderfahrungen, d.h. über die Erfahrungen anderer Leistungsabnehmer, validieren können. Ein derartiger Tatbestand führt im umgekehrten Fall dazu, dass die Reputation quasi eine „Geisel“ im Besitz der Nachfrager darstellt, ${ }^{329}$ da die Beschädigung der Reputation für den Anbieter eine ernstzunehmende Sanktion darstellt, insbesondere aufgrund ihres eher langfristigen Charakters. Schwierig stellt sich regelmäßig die Wahrung der Reputation in solchen Fällen dar, in denen der Controllerdienst aufgrund seiner Verhaltenssteuerungsfunktion bewusst gegen die Interessen seiner Kunden verstoßen muss. $\mathrm{Zu}$ unterscheiden sind in diesem Zusammenhang jedoch die kurz- und langfristigen Auswirkungen derartiger opportunismusbegrenzender Maßnahmen. Oftmals kann langfristig die Reputation gerade über derart konsequente Handlungen aufgebaut und abgesichert werden, auch wenn kurzfristig die „Beliebtheit" darunter leidet.

Abschließend soll in Abbildung 19 ein Überblick über die Zusammenhänge zwischen Leistungseigenschaften und Beurteilungsdimensionen gegeben werden. ${ }^{30}$ Hierbei werden die unterschiedlichen Kombinationsmöglichkeiten in Form einer Matrix abgeleitet.

Im Ergebnis entsteht das Beurteilungsprofil einer Leistung, d.h. die für einen bestimmten Leistungstyp besonders wahrnehmungsrelevanten Kombinationen aus Leistungseigenschaften und Beurteilungsdimensionen. Derartige Profile hängen maßgeblich vom Integrationsgrad der Leistungserstellung ab. So verringern sich bspw. mit zunehmender Einbindung des Kunden in den Leistungserstellungsprozess Sucheigenschaften im Leistungsergebnis, da die Einflussnahme des Kunden dieses wesentlich mitbeeinflusst. ${ }^{331}$

\footnotetext{
327 Allgemein vgl. Plötner (1993), S. 43.

$328 \mathrm{Vgl}$. Fließ (1995), S. $313 \mathrm{ff}$.

$329 \mathrm{Vgl}$. Spremann (1988), S. 619 ff.

330 Allgemein furr den Bereich des industriellen Kaufverhaltens vgl. Fließ (1995), S. 330.

331 Vgl. Jakob (1994), S. 177.
} 


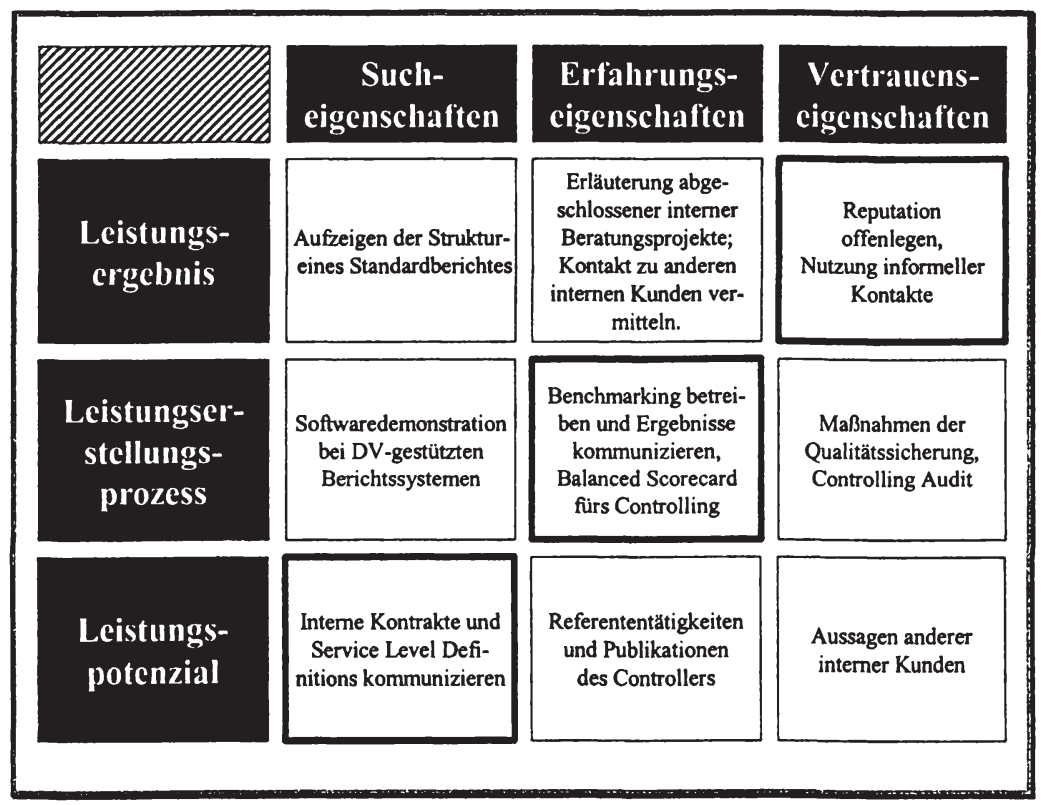

\section{Abbildung 19: Informationsquellen im Beurteilungsprofil integrativer Leistungserstellung ${ }^{332}$}

Die grau schraffierten Felder in Abbildung 19 entsprechen dem Beurteilungsprofil ,integrativer Leistungserstellung “, welche im Controllerbereich regelmäßig vollzogen wird. Demgegenüber sind jedoch die übrigen Felder der Matrix nicht zu vernachlässigen, da, wie bereits angesprochen, der Integrativitätsgrad bei Controlling-Leistungen unterschiedlich ist.

Inhalt der Felder in Abbildung 19 sind nach Beurteilungsdimension und Leistungseigenschaften systematisierte Informationsquellen. Die grau schraffierten Felder deuten dementsprechend auf besonders wesentliche Informationsquellen für Kunden des Controlling hin, welche genutzt werden können, um die Beurteilbarkeit von Leistungen zu verbessern und damit Unsicherheit zu reduzieren. Seitens des Controllerdienstes gilt es daher, Kunden - in Abhängigkeit der auszutauschenden Leistung - entsprechend zielwirksame Informationsquellen zugänglich zu machen.

332 In Anlehnung an die controllingunabhăngige Darstellung von Fließ,(1995), S 330. 


\subsubsection{Inhaltscharakteristika}

Verfolgt man die Zielsetzung, Leistungen und Kundenbeziehungen des Controlling zu analysieren, stellt sich unmittelbar die Frage, welche konkreten Leistungstypen überhaupt einer kundenorientierten Gestaltung zugänglich sind. In diesem Zusammenhang spielt die Zwecksetzung der Leistung, d.h. die Absicht mit der der Controllerdienst eine Leistung erstellt und abgibt, eine wesentliche Rolle. $\mathrm{Zu}$ unterscheiden sind hierbei Leistungen mit vorrangig unterstützendem Charakter (,support/advice services") und solche mit vorrangig kontrollierendem Charakter (,,audit/evaluative services"). ${ }^{333}$ Im Rahmen einer differenzierten Betrachtung sollen nachfolgend die Besonderheiten derartiger Leistungstypen veranschaulicht werden, um - darauf aufbauend - das Ausmaß der kundenbezogenen Gestaltbarkeit beurteilen zu können. Im Mittelpunkt steht die Frage, inwieweit artikulierte oder latente Kundenbedürfnisse in den Leistungserstellungsprozess Eingang finden dürfen, ohne die sachgerechte Aufgabenerfüllung des Controlling zu gefährden.

\subsection{Unterstützungscharakter}

Die konsensfähige Charakterisierung des Controlling als Führungshilfe ${ }^{34}$ deutet unmittelbar daraufhin, dass im Mittelpunkt der Aktivitäten des Controlling die Unterstützung der Unternehmensführung steht. $\mathrm{Zu}$ unterscheiden bleibt in diesem Zusammenhang jedoch einerseits die Service- bzw. Unterstützungsfunktion des Controlling ${ }^{335}$ auf konzeptioneller Ebene, sowie andererseits, auf institutioneller Ebene, die konkrete Erstellung von Leistungen mit in den Augen der Controlling-Kunden wahrnehmbarem Unterstützungscharakter. Auf der konzeptionellen Ebene wird der Servicefunktion des Controlling regelmäßig im Rahmen der abgeleiteten Zwecksetzungen des Controlling Aufmerksamkeit geschenkt. ${ }^{36}$ Konzeptionell gründet sich der unterstützende Charakter des Controlling darauf, dass durch die Übernahme der Koordinationsfunktion die Erfüllung der Führungsaufgaben in den einzelnen Führungsteilsystemen erleichtert wird. Über die Bereitstellung koordinationsfördernder Methoden und die Erhebung und Aufbereitung relevanter Informationen soll konzeptionelle Führungshilfe geleistet werden. ${ }^{37}$

\footnotetext{
333 Vgl. Davis (1992), S. 12 ff. sowie Stauss/Neuhaus (1995), S. 583 f.

$334 \mathrm{Vgl}$. Kupper/Weber/Zund (1990), S. 283

${ }_{335}$ Vgl. Kap. 2.1.3.1.

336 Hier und im Folgenden vgl. Küpper (1997), S. 19

${ }^{337} \mathrm{Vgl}$. hierzu auch die im Rahmen der speziellen Zielsetzungen des Controlling genannte Zielsetzung der Entlastung der Unternehmensfuhrung. Vgl. Harbert (1982), S. 234 f flosiek - 978-3-631-75321-7
} 
Eine derartige Konkretisierung der Servicefunktion auf konzeptioneller Ebene führt regelmäßig dazu, die Grenzen von Konzeption und Institution zu verwischen. Daher kommt der Servicefunktion auf konzeptioneller Ebene auch nur eine spezifizierende Funktion bezogen auf die im Mittelpunkt stehende Koordinationsfunktion des Controlling zu. ${ }^{338}$ Demgegenüber ist die Betrachtung des Unterstützungscharakters einzelner Leistungen des Controlling auf institutioneller Ebene von besonderer Bedeutung, da sich hieraus wesentliche Rückschlüsse auf das Leistungserstellungs- und Kommunikationsverhalten des Controllers ergeben, was gleichsam die Wirksamkeit seines Handelns prägt. Somit kommt gerade bei Zugrundelegung einer koordinationsorientierten ControllingKonzeption der Unterstützungsfunktion auf institutioneller Ebene eine wichtige Rolle zu, da es zur Koordination der Führungsteilsysteme geeigneter Zielsetzungs-, Planungs- und Steuerungsmethoden bedarf. Insbesondere wenn - wie intendiert - die Steuerung ${ }^{339}$ vom Management selbst übernommen wird, ist es gerade der ,Service- oder Lotsendienst ${ }^{\circ 340}$, der den Controller und damit konsequenterweise sein Leistungsspektrum auszeichnet. Bei einer so verstandenen Verantwortlichkeitsverteilung bedarf es einer differenzierteren Betrachtung, inwieweit die dem Controller zur Verfügung stehenden Einzelleistungen rein unterstützend zu verstehen sind, im Sinne von ausschließlich an den Bedürfnissen der Leistungsempfänger ausrichtbar.

Charakteristisch für unternehmensinterne Unterstützungsleistungen ist, dass durch diese die Leistungsadressaten in die Lage versetzt werden, ,ihre Leistung überhaupt bzw. mit verbesserter Qualität zu erbringen" ${ }^{\text {(341 }}$. Im Sinne der Betrachtung des kundenorientierten Controlling zeichnet Leistungen mit Unterstützungscharakter der Tatbestand aus, dass die subjektiven bzw. objektiven Bedürfnisse der Kunden als maßgeblich für das Leistungserstellungsverhalten des Controlling angesehen werden können. ${ }^{342}$ Als externe Faktoren müssen daher neben den inhaltlichen Daten über das zu lösende Entscheidungsproblem des Kunden insbesondere auch Informationen über die Aufbereitungspräferenzen und das Nutzungsverhalten der Kunden einfließen. Soweit es sich hier um idealtypisch reine Unterstützungsleistungen handelt, spielt die Kontrollverantwor-

338 KÛPPER betont in diesem Zusammenhang, dass die Servicefunktion keine eigentlich neue Problemstellung zur Legitimation des Controlling darstellt. Vgl. Küpper (1997), S. 19. ZIENER sieht die Gefahr, dass das Ziel der Führungsunterstützung letztlich alle Stabsaufgaben beinhaltet. Vgl. Ziener (1985), S. 32.

339 Der englische Ausdruck „to control“, zu deutsch „steuern“, bildet den Wortstamm für den Begriff „Controlling“.

340 Blazek/Deyhle (1986), S. 35.

341 Stauss/Neuhaus (1995), S. 584.

${ }^{342}$ Für den Personalbereich vgl. Stauss/Neuhaus (1995), S. 583. 
tung des Controllers zunächst keine Rolle. Konsequent kann die Leistungserstellung an den artikulierten Bedürfnissen der Kunden ausgerichtet werden. Darüber hinaus sind jedoch Situationen denkbar und wahrscheinlich, in denen eine effektive Unterstützung über die artikulierten Bedürfnisse hinausgehende Leistungen erfordert. ${ }^{343}$ In solchen Fällen besteht die Aufgabe des Controllers darin, derartige aktiv Leistungen bereitzustellen, sowie - oftmals genauso wichtig - die Entscheidungsrelevanz der Leistungsinanspruchnahme zu verdeutlichen. Kritischer sind solche Situationen, in denen Leistungen von Kunden nachgefragt werden, die aufgrund der Einschätzung des Controlling bezogen auf das zugrundeliegende Entscheidungsproblem irrelevant sind. In Fällen, in denen dem Controlling diese Informationen zur Verfügung stehen, können sie dem Kunden weitergegeben werden, verbunden mit der Gefahr einer fehlerhaften Anwendung. ${ }^{344}$ Demgegenüber erscheint es konsequent im Sinne der Aufgabenstellung des Controlling, den Kunden über die Entscheidungsirrelevanz aufzuklären, und damit - die Einsicht des Kunden vorausgesetzt - die Information nicht bereitzustellen.

Insoweit wird deutlich, dass auch bei primären Unterstützungsleistungen oftmals eine Bewertung der artikulierten Präferenzen der Nachfrager stattfinden kann, mit dem Ergebnis, sich gegebenenfalls über die Beweggründe der Nachfrageäußerung mit dem Kunden auseinandersetzen zu müssen. Es erscheint ersichtlich, dass - soweit der Controller neben den subjektiven Bedürfnissen seines Kunden diesem einen objektiven Informationsbedarf unterstellt - Zielkonflikte auftreten können. ${ }^{345}$ In derartigen Fällen ist die Grenzziehung zwischen Unterstützung oder Kontrolle/Bewertung nicht mehr trennscharf. Dies darf jedoch nicht darüber hinwegtäuschen, dass die Kundenbedürfnisse Ausgangspunkt der Leistungserstellung bleiben. Die kritische Reflexion über diese Bedürfnisse, verbunden mit der Zielsetzung, den Nutzen des Kunden zu maximieren, stellt üblicherweise auch auf Absatzmärkten eine gängige Vorgehensweise dar. Insbesondere im Bereich komplexer unsicherheitsbehafteter Dienstleistungserstellung werden auch auf unternehmensexternen Märkten im Vorfeld der Leistungserstellung die artikulierten Bedürfnisse der Kunden diskutiert, um eine problemlösungsadäquate Leistung bereitzustellen. Gerade dies kennzeich-

343 Im Folgenden in Anlehnung an die Gedanken von WEBER zum Wechselspiel von Informationsangebot, -nachfrage und objektivem Informationsbedarf. Vgl. Weber (1997), S. 12 sowie Kap. 4.1.2.1.1.

344 Oftmals bedarf es noch nicht einmal einer fehlerhaften Anwendung, da eine Verschlechterung der Entscheidungsqualităt schon durch eine zu große Fulle von Informationen ausgelöst werden kann.

345

Vgl. auch Gmilnden (1993), Sp. 1725 ff. 
net die Integrativität der Leistungsprozesse und wird oftmals als Zusatznutzen empfunden, über den der Anbieter seine Reputation stärken kann.

Betrachtet man die nachfolgend übersichtsartig dargestellten Nutzenkomponenten von Controlling-Leistungen, stellt sich die Frage, inwieweit diese allein über Unterstützungsleistungen erreichbar sind. Situationsbedingt leistet das Controlling: ${ }^{346}$

- Effizienzsteigerung im Tagesgeschäft,

- Frühwarnung bei Fehlentwicklungen,

- Ursachenanalyse von Fehlentwicklungen und

- Bestätigung bei Zielerreichung.

Soweit es zur Absicherung dieser Nutzenkomponenten kontrollierender Aktivitäten bedarf, erscheint diskussionswürdig, ob Kundenorientierung weiterhin als umfassende Handlungsmaxime verstanden werden kann. Insbesondere auch mögliche Wahrnehmungsdifferenzen zwischen Controller und Kunde bezogen auf den Unterstützungs- oder Kontrollcharakter einer Leistung machen eine differenzierte Betrachtung der Kontrollaufgaben des Controllerdienstes, sowie der Rolle des Kunden im Rahmen von Kontrollaktivitäten notwendig.

\subsection{Kontrollcharakter}

Bei einer Beschreibung der Aufgaben des Controlling führt der Begriff der Kontrolle regelmäßig zu Missverständnissen. Ausgehend von den Extremauffassungen, die Controlling einerseits mit Kontrolle gleichsetzen, andererseits weil gerade früher jene Extremauffassung das Verständnis dominierte - einen direkten Zusammenhang von Controlling und Kontrolle abstreiten, ist eine systematische, emotionslose Betrachtung zweckdienlich. ${ }^{347}$

Kontrolle wird in der betriebwirtschaftlichen Literatur überwiegend als Gegenstück der Planung verstanden und daher in einer funktionalen Einheit betrachtet. ${ }^{348}$ Eine derartige Auffassung erscheint insbesondere vor dem Hintergrund der Systembetrachtung angemessen und erlangt darüber hinaus im Rahmen der kundenorientierten Betrachtung des Controlling eine besondere Bedeutung. Ei-

\footnotetext{
346 Abgewandelt nach Berger (1989), S. 185.

347 Vgl. Küpper (1997), S. 176. sowie Horváth (1998), S. 162.

348 Vgl. Hellmich (1970), S. 10 f. und Horváth (1998), S. 167 f. KƯPPER sieht demgegenüber Planung und Kontrolle als singulär betrachtbare Sachverhalte an. Vgl. Kilpper (1997). S. $165 \mathrm{ff}$ 
ne stringent trennbare Wahrnehmung von Planung und Kontrolle bereitet in den Augen der Kunden regelmäßig Schwierigkeiten, insbesondere auch aufgrund des identischen Anbieters derartiger Leistungen. Kontrollen dienen der Unsicherheitsreduktion im Rahmen der Realisierung von Plänen. Im Einzelnen sind Planungsannahmen, Zielwirkungen der ergriffenen Maßnahmen, Mittelverwendung und das Verhalten der Beteiligten zu überwachen. ${ }^{349}$

Zur Typologisierung der Leistungen des Controlling empfiehlt sich zunächst eine Anknüpfung am Entscheidungsunterstützungsprozess des Managements, ${ }^{350}$ da dieser Phasen mit vorrangig kontrollierenden Leistungen seitens des Controlling beinhaltet. In diesem Zusammenhang sind primär die Soll-Ist-Vergleiche sowie die Abweichungsanalysen zu nennen. Insbesondere aber zu einer differenzierteren Betrachtung der Verhaltensdimension im Leistungsraum bedarf es einer Systematisierung der Kontrolle. Inhaltlich gesehen bezieht sich die Kontrolle im Controlling regelmäßig auf institutionelle Aspekte und weniger auf soziale. ${ }^{351}$ Die institutionelle Kontrolle verfolgt auf Basis formalisierter Unternehmensziele einerseits eine aktive Beeinflussung des Mitarbeiterhandelns im Sinne einer Verhaltenssteuerung. Andererseits muss psychologisch bedingtem Fehlverhalten über einen sachgerechten Führungsstil entgegengewirkt werden. ${ }^{352}$ Insbesondere die psychologisch emotionale Komponente erlangt im Rahmen einer kundenbezogenen Betrachtung von Leistungen des Controlling eine besondere Bedeutung.

Ausgehend von der Unterscheidung der Controlling-Leistungen in unterstützende und kontrollierende sind nachfolgend die im Rahmen der institutionellen Kontrolle eingesetzten Leistungen hinsichtlich Kontrollierten, Kontrollträger, Kontrollobjekt und Kontrollprozess zu betrachten. ${ }^{333}$ Das Kontrollobjekt entspricht im Regelfall den Entscheidungsfolgen des in Abbildung 12 dargestellten idealtypischen Entscheidungsprozesses.

Wesentlich ist in diesem Zusammenhang, ob der Kontrollierte selbst Leistungsabnehmer des Controlling ist (Kunde), oder ob die Informationen über den Kon-

349 Vgl. Horváth (1998), S. 162.

$350 \mathrm{Vgl}$. hierzu den Leistungsraum des Controlling in Abbildung 12.

351 Die soziale Kontrolle vollzieht sich in Anlehnung an die Unternehmenskultur sowie die Werte und Normen der Unternehmensmitglieder. Vgl. O'Reilly (1989), S. 12.

352 Vgl. Hoffjan (1997), S. 245.

353 Hierzu und im Folgenden vgl. Hoffjan (1997), S. 245 ff. und S. 417. Der Kontrollrhythmus soll zunăchst nicht betrachtet werden. Soweit es die allgemein zeitliche RegelmäBigkeit des Leistungsaustausches betriff, vgl. Kap. 3.3.2.1. 
trollprozess für einen anderen Kunden bestimmt sind. Vom Kontrollcharakter einer Leistung i.e.S. soll im Folgenden nur dann ausgegangen werden, wenn der Leistungsadressat des Controlling auch selbst Kontrollierter ist. ${ }^{354}$ Ansonsten wäre die Grenzziehung zu Unterstützungsleistungen nicht möglich, da die Kontrollinformationen aus der Kontrolle eines Dritten für den Informationsadressaten des Controlling dann immer nur rein entscheidungsunterstützenden Charakter haben. Ist der Kontrollierte gleichsam Informationsadressat, spielt insbesondere die Machtbasis bzw. die Unabhängigkeit des Controlling eine gravierende Rolle bei der Durchsetzbarkeit kontrollinduzierter Maßnahmen. ${ }^{355}$ Fraglich ist jedoch, ob die Bezeichnung Kunde für den Leistungsadressaten des Controlling angemessen erscheint, soweit dieser selbst die dem Kontrollobjekt zugrundeliegende Entscheidung getroffen hat und somit dafür die Verantwortung trägt.

Als Ergebnis des Kontrollprozesses ergibt sich eine Kontrollinformation, die bspw. Auskunft über das Ausmaß einer Abweichung gibt. Entscheidend für die kundenorientierte Ausgestaltung des Controlling ist in diesem Zusammenhang die psychologische Wahrnehmung derartiger Informationen, bzw. dem vorgelagert, die Empfindungen des Kontrollierten während des Kontrollprozesses. Anzunehmen ist, dass derartige Wahrnehmungen das Verhalten der Kontrollierten maßgeblich prägen und somit Effektivität und Effizienz des Controlling beeinflussen. In diesem Zusammenhang besteht die Herausforderung des kundenorientierten Controlling darin, einerseits die positiven intrinsischen und extrinsischen Motivationseffekte von Kontrollen zu unterstützen, andererseits die negativen Auswirkungen dysfunktionaler Verhaltensweisen zu begrenzen. ${ }^{356}$ Ausprägungen dysfunktionaler Verhaltensweisen sind offener oder verdeckter Widerstand gegen das Kontrollsystem, die Manipulation von Kontrolldaten sowie rigides bürokratisches Verhalten, welches immer dann vorliegt, wenn der Kontrollierte sein Leistungsverhalten einseitig an den Kontrollgrößen ausrichtet und darüber wesentliche andere Einflussgrößen ${ }^{357}$ auf die intendierte Zielvorstellung vernachlässigt. Bezogen auf das Leistungsspektrum des Controlling zeigt sich an dieser Stelle, dass der Kontrollcharakter von Controlling-Leistungen nicht zwangsläufig mit den originär kontrollierenden Phasen der Entscheidungsprozessdimension des Leistungsraums zusammenhängt, sondern gleichsam auch mit vielen verhaltenssteuernden Leistungen des Controlling einhergeht. Immer

${ }^{354}$ Dies ist ein Spezifikum des Controlling. Anders gestaltet es sich bei den tubrigen internen Serviceleistungen. Für den Personalbereich vgl. Stauss/Neuhaus (1995), S. 583.

355 Vgl. Lanter (1995), S. 70 f. sowie zur Organisation des Controlling Kap. 3.3.3.1.

356 Hier und im Folgenden zu den Formen dysfunktionaler Verhaltensweisen vgl. Karlowitsch (1999), S. 25 ff.

357 Regelmäßig handelt es sich bei diesen anderen Einflussgrößen um gleichermaßen erfolgsrelevante Tatbestände, die sich aber oftmals schlechter operationalisieren lassen. 
dann, wenn im Rahmen der Erbringung von Controlling-Leistungen implizit überprüft wird, ob Spielräume für opportunistisches Verhalten der Kunden bestehen, beinhalten diese kontrollierende Elemente. Die Vielzahl derartiger Leistungen deutet darauf hin, dass unabhängig oder gerade bezugnehmend auf das Kontrollempfinden ein Eingehen auf die spezifischen Bedürfnisse der Kunden des Controlling möglich erscheint, ohne die Ziele der Unternehmung zu vernachlässigen.

Letztlich stellt sich die Kontrollsituation als eine spezifische Informationstransaktion dar, in der der Controller in Besitz einer knappen Ressource des Kunden gelangen möchte, um auf dieser Basis weitergehende Bedürfnisse eben dieses Kunden zu erfüllen. Somit ist ein Kontrollprozess stets Bestandteil einer integrativen Leistungserstellung im Gesamtkontext des Entscheidungsunterstützungsprozesses. ${ }^{358}$ Im Sinne des Systemansatzes stellt die Kontrolle damit nie einen isolierbaren Selbstzweck dar. Damit aber stellt sich die Frage, ob eine Kontrolliertenorientierung im Sinne verhaltenswissenschaftlicher Erkenntnisse synonym mit einer Kundenorientierung verstanden werden kann.

Die Verhaltenswissenschaft bietet unterschiedliche Ansätze an, das Unsicherheitsempfinden des Kontrollierten im Sinne beider Parteien zu reduzieren und somit dessen Kooperationsbereitschaft zu erhöhen. ${ }^{359}$ Von diesen sind für Beziehungen, in den der Kontrollierte dem Informationsadressaten entspricht und daher einen tendenziellen Widerspruch zwischen Kontrolle und Unterstützung empfindet, relevant z.B. die Erhöhung der Abweichungstoleranz, die Delegation von Kontrollen im Sinne des Self-Controlling oder eine Reduktion der Kontrollvariablen auf solche, die adressatenseitig wahrnehmungsrelevant sind. Werden derartige Maßnahmen auf die artikulierten Bedürfnisse des Kontrollierten abgestimmt, ohne dadurch die Erhebung der aus Rationalitätssicherungsgründen ${ }^{360}$ notwendigen Kontrollinformationen zu gefährden, kann von einer tendenziellen Kundenorientierung ausgegangen werden. Hierdurch kann die Qualität der Daten um die vermiedenen Verluste dysfunktionaler Verhaltensweisen reduziert werden. Ein derart schizophrener Zusammenhang ${ }^{361}$

\footnotetext{
358 Vgl. Kap. 3.2.2.1.3.

$359 \mathrm{Vgl}$. zu den Ausführungen im Folgenden zusammenfassend Hoffjan (1997), S. $10 \mathrm{ff}$.

360 Zur Bedeutung der Rationalitätssicherungsfunktion im Gesamtkontext des Controlling vgl. Weber (1999), S. $37 \mathrm{ff}$.

361 LANTER zitiert in diesem Zusammenhang das auf WATZLAWICK ET AL. zurlickgehende Phănomen der schizophrenen Doppelbindung, welches bezogen auf das Controlling die Wechselwirkung zwischen Unterstutzung und Kontrolle charakterisiert. Vgl. Lanter (1996) S. 73 sowie Watzlawick et al (1990), S, 195 f.
} 
lässt sich konsequent nur zugunsten der Coachingfunktion ${ }^{362}$ des Controlling auflösen. ${ }^{363}$ Im Mittelpunkt steht hierbei die Lernwirkung der Kontroll- oder Rückkopplungsinformationen, was gleichsam voraussetzt, dass der Kontrollvorgang nicht isoliert, sondern im Kontext des gesamten Prozesses der Entscheidungsunterstützung gesehen wird. Gleiches gilt für Controlling-Leistungen in Entscheidungsprozessphasen, die nicht primär durch Kontrollaktivitäten gekennzeichnet sind, in denen der Controller vielmehr latente Kontrollen zur Opportunismusbegrenzung durchführt. Maßgeblich für die Absicherung der Coachingfunktion im Controlling ist jedoch nicht nur das Rollenverständnis des Controllers und damit verbunden die Kenntnis der Ursachen und Wirkungen dysfunktionaler Verhaltensweisen, sondern gleichermaßen auch die Überzeugung des Managements, die Rolle als Kunde wahrzunehmen und die sich daraus ergebenden Vorteile im Sinne des Gesamtunternehmens zu nutzen. Kundenorientierung im Controlling ist immer dann zum scheitern verurteilt, wenn das Management in seiner Grundeinstellung jede Beratung als Belehrung empfindet und jede Kontrolle als unzweckmäßige Einschränkung des zur Unternehmensführung notwendigen Kreativitätsspielraums. Diesbezüglich ist das gegenseitige Rollenverständnis unabdingbare Voraussetzung für die darauf aufbauende Etablierung einer Kunden-Lieferanten-Beziehung. ${ }^{364}$

Insgesamt erscheint daher die Gesamtbetrachtung, einerseits im Rahmen der internen Geschäftsbeziehung und andererseits im Rahmen des Führungsprozesses, wesentlich. Bezogen auf die interne Geschäftsbeziehung kann auch die Wahrnehmung von Kontrollaktivitäten im Sinne einer Kundenbeziehung erfolgen, da das Gesamtverhältnis zwischen Controller und Manager durch den Unterstützungs-/Servicecharakter des Controlling geprägt ist. Insbesondere Schwierigkeiten bei der inhaltlichen und zeitlichen Abgrenzung einzelner Unterstützungs- von Kontrollaktivitäten bedingen, dass das Management den Controller nicht leistungsabhängig in jeweils unterschiedlichen Rollen wahrnimmt. Diesen Gedanken unterstützt die postulierte Einheit von Planung und Kontrolle. Kontrolle ist in diesem Zusammenhang nicht isoliert $\mathrm{zu}$ betrachten, d.h. die von dem Controlling erbrachten Kontrollleistungen werden von seinen Kunden im Gesamtzusammenhang wahrgenommen, welcher regelmäßig einem Führungsprozess entspricht. Im Rahmen dieses Gesamtprozesses dominiert wiederum die Entscheidungsunterstützungsfunktion die Wahrnehmung der

\footnotetext{
${ }^{362}$ Zur Funktion und Entwicklung des Controllers als Coach seiner Kunden vgl. Berger-Vogel (2000), S. 6 ff.

${ }^{363} \mathrm{Vgl}$. Hoffjan (1997), S. 112.

${ }^{364}$ LANTER schlägt in diesem Zusammenhang vor, einen systematischen Abgleich der Vorverständnisse von Manager und Controller durchzufuhren. Im Einzelnen vol. Lanter (1996) S. 243 ff
} 
Kunden. Aus diesem Grund erscheint die kundenorientierte Grund-/Gesamtausrichtung des Controlling zweckmäßig, ohne dadurch die Effektivität von Kontrollen zu gefährden. Eine derartige Vorgehensweise bietet einen Anknüpfungspunkt dem, ,inhärenten Widerspruch zwischen Hilfestellung und Kontrolle ${ }^{\text {(365 }}$ im Controlling zu begegnen.

Abschließend bleibt festzuhalten, dass Leistungen mit Unterstützungscharakter grundsätzlich einer kundenorientierten Ausgestaltung zugänglich sind. Die Verantwortung des Controllers, Rationalitätssicherung zu betreiben, kann im Einzelfall dazu führen, dass auf Basis der artikulierten Bedürfnisse des Kunden zunächst eine Aufklärung bzw. Beratung betrieben werden muss, bevor gemeinsam über die Bereitstellung einer zielsetzungsadäquaten Leistung entschieden wird. In derartigen Situationen wird quasi unterstellt, dass der Controller den zur Problemlösung objektiv nötigen Informationsbedarf seiner Kunden kennt. Implizit ,kontrolliert" der Controller damit, inwieweit die artikulierten Bedürfnisse dem objektiven Bedarf entsprechen. Eine derartige Vorgehensweise widerspricht nicht der Kundenorientierung, sondern zeigt vielmehr deren speziellen Charakter bezogen auf das Controlling. Auch auf unternehmensexternen Märkten stellt der verantwortungsvolle Umgang mit artikulierten Kundenbedürfnissen keine Ausnahme dar, insbesondere in Märkten, auf denen komplexe Güter gehandelt werden, Geschäftsbeziehungen langfristig angelegt sind und daher eine sehr starke Bindung zwischen Anbieter und Nachfrager besteht. ${ }^{366}$ Erst durch eine solche Vorgehensweise kann die zum Austausch von Vertrauensgütern notwendige Reputation eines Anbieters im Zeitablauf aufgebaut werden.

Gegenüber Aktivitäten mit Unterstützungscharakter zeichnen sich Aktivitäten mit Kontrollcharakter einerseits dadurch aus, dass sie den diesbezüglichen Teilphasen des Entscheidungsprozesses entspringen (Soll-Ist-Vergleich, Abweichungsanalyse), sowie andererseits im Rahmen verhaltenssteuernder Aktivitäten Relevanz erlangen wie bspw. im Rahmen der Opportunismusbegrenzung. Bezüglich der Kontrollierten ist zu prüfen, ob diese den Kunden im Sinne der Kontrollinformationsadressaten entsprechen oder nicht. Soweit sie nicht die unmittelbaren Kunden des Controlling sind, ist deren zielgerichtete Beeinflussung auf Basis der Instrumente des verhaltensorientierten Controllings vorzu-

365 Vgl. Stamm (1991), S. 139.

366 Tendenziell weist daher der Markt fur Controlling-Leistungen mehr Parallelen zu Industriegütermărkten als zu Konsumgütermärkten auf. Eine grundsătzliche Zuordnung zu einem Geschăftstyp im Sinne der Typologie von BACKHAUS erscheint jedoch nicht zweifelsfrei möglich. Vgl. Backhaus (1999) S. 306 
nehmen. ${ }^{367}$ Handelt es sich bei den Kontrollierten demgegenüber um Kunden des Controlling, ist der Kontrollprozess im Gesamtzusammenhang der internen Geschäftsbeziehung zu betrachten, sowie diesbezügliche Wechselwirkungen zwischen Planung und Kontrolle, Unterstützendem und Kontrollierendem zu verdeutlichen. Insbesondere Informations- und Interaktionsaktivitäten im Sinne einer beratenden Plausibilisierung der Zielsetzung und des Informationsnutzens von Kontrollaktivitäten vermögen zu einer Vermeidung dysfunktionaler Verhaltensweisen beizutragen. Besteht gegenseitige Klarheit über das jeweilige Rollenverständnis von Manager und Controller kann die interne Austauschbeziehung im Sinne einer Kunden-Lieferanten-Beziehung betrachtet werden. In einem umfassenden Sinn lassen sich dann Controlling-Leistungen den Service Leistungen zuordnen, von denen man sich in wirtschaftlicher Hinsicht im Rahmen der Etablierung interner Märkte überwiegend nachfolgende Vorteile verspricht: $^{368}$

- Transparenz- und Nutzensteigerung der Gemeinkostenbereiche,

- internes und externes Benchmarking zur Effizienzsteigerung,

- Fundierung von Outsourcing Entscheidungen,

- Kostensensibilisierung interner Kunden.

Gerade über eine derart konsequente Miteinbeziehung des Controllerbereichs selbst in Wirtschaftlichkeitserwägungen kann letztlich Glaubwürdigkeit und das notwendige Vertrauen beim Kunden geschaffen werden, welches Kontrollassoziierung vermeiden hilft und damit eine wirksame Entscheidungsunterstützung für den Kunden absichert.

\subsection{Organisationsbezogene Charakteristika des Controlling}

Anknüpfend an die Diskussion zum funktionalen bzw. institutionellen Verständnis des Controlling sollen im Folgenden speziell die Wirkungen auf die Leistungserstellung des Controlling betrachtet werden, die sich aus der Bildung und Eingliederung eines eigenständigen Controllerbereichs in die Gesamtorganisation ergeben. Lag der Fokus der bisherigen Betrachtung auf den Leistungsinhalten des Controlling, ihrer Bewertbarkeit durch Controlling-Kunden sowie ihrer allgemeinen Zwecksetzung, soll nachfolgend ausschließlich auf die Organisation des Controlling als Rahmenbedingung der Leistungserstellung einge-

367 Vgl. Hoffjan (1997), S. 113 ff.

${ }^{368}$ Vgl. Băssler/Knust/Schindera (1999), S. 268. 
gangen werden. ${ }^{369}$ Anzunehmen ist, dass die Angebotsbereitstellung von Controlling-Leistungen in starkem Umfang von der organisatorischen Eingliederung des Controlling in das Gesamtsystem Unternehmung abhängt und damit die Möglichkeit einer kundenorientierten Ausgestaltung maßgeblich beeinflusst.

Bedingt durch die Vorgehensweise dieser Arbeit wurden bereits einige organisationsrelevante Tatbestände des Controlling erläutert bzw. hypothetisch unterstellt. Im Rahmen der Diskussion der Controlling-Verständnisse wurde gezeigt, dass ein alle Führungsteilsysteme umfassendendes koordinationsorientiertes Controlling-Konzept in der Lage ist, theoretisch ein eigenständiges Erkenntnisobjekt des Controlling abzugrenzen. ${ }^{370}$ Demgegenüber weisen im Rahmen der praktischen Ausgestaltung des Controlling institutionelle Aspekte regelmäßig hohe Wahrnehmungsrelevanz für die betroffenen Unternehmensmitglieder auf. $^{371}$

Aus diesem Grund wurde im Rahmen der Betrachtung des Marketing nach Anhaltspunkten für die Steigerung der Effizienz und Effektivität eines derart existenten Unternehmensteilbereiches "Controlling“ gesucht. Das funktionsorientierte interne Marketing wurde in diesem Zusammenhang als konzeptioneller Bezugsrahmen für die kundenorientierte Ausgestaltung eines fortan unterstellen Controllerbereichs gewählt. Wie Abbildung 20 zeigt, steigt in Abhängigkeit der Unternehmensgröße die Wahrscheinlichkeit für die Existenz spezialisierter Controller-Stellen und damit eines Controllerbereichs.

Eine ausgeprägtere Systemdifferenzierung sowie eine insgesamt stärkere Heterogenität der Umweltbeziehungen führt mit steigender Unternehmensgröße regelmäßig zu der Zweckmäßigkeit der Einrichtung einer institutionalisierten Controlling-Stelle. ${ }^{372}$ Insgesamt weist eine Mehrzahl von $72,3 \%$ der Unternehmen mindestens eine Controlling-Stelle aus, ${ }^{373}$ so dass es gerechtfertigt erscheint, die Wirksamkeit der kundenorientierten Leistungserstellung und -verwertung, sowie die dieser zugrundeliegenden organisatorischen Rahmenbedingungen strukturiert $\mathrm{zu}$ analysieren. Ein erster Bezug zu den organisationsbe-

${ }^{369}$ Die ökonomische Ausrichtung des Controllerbereichs wird in diesem Zusammenhang als ein durch organisatorische Regelungen determinierter Tatbestand begriffen.

370 Vgl. Küpper (1997), S. 13 ff.

371 Insbesondere auch verhaltensrelevante Problemstellungen fallen in diesen Bereich. Vgl. Lanter (1996), S. 62 ff.

372 Vgl. Horváth (1998), S. 802.

${ }^{373}$ Vgl. Küppper/Winckler/Zang (1990), S. 439. 
dingten Einflussfaktoren wurde zu Beginn der Darstellung der leistungsbezogenen Charakteristika im Rahmen der Aufgabenzuordnung hergestellt. ${ }^{374}$

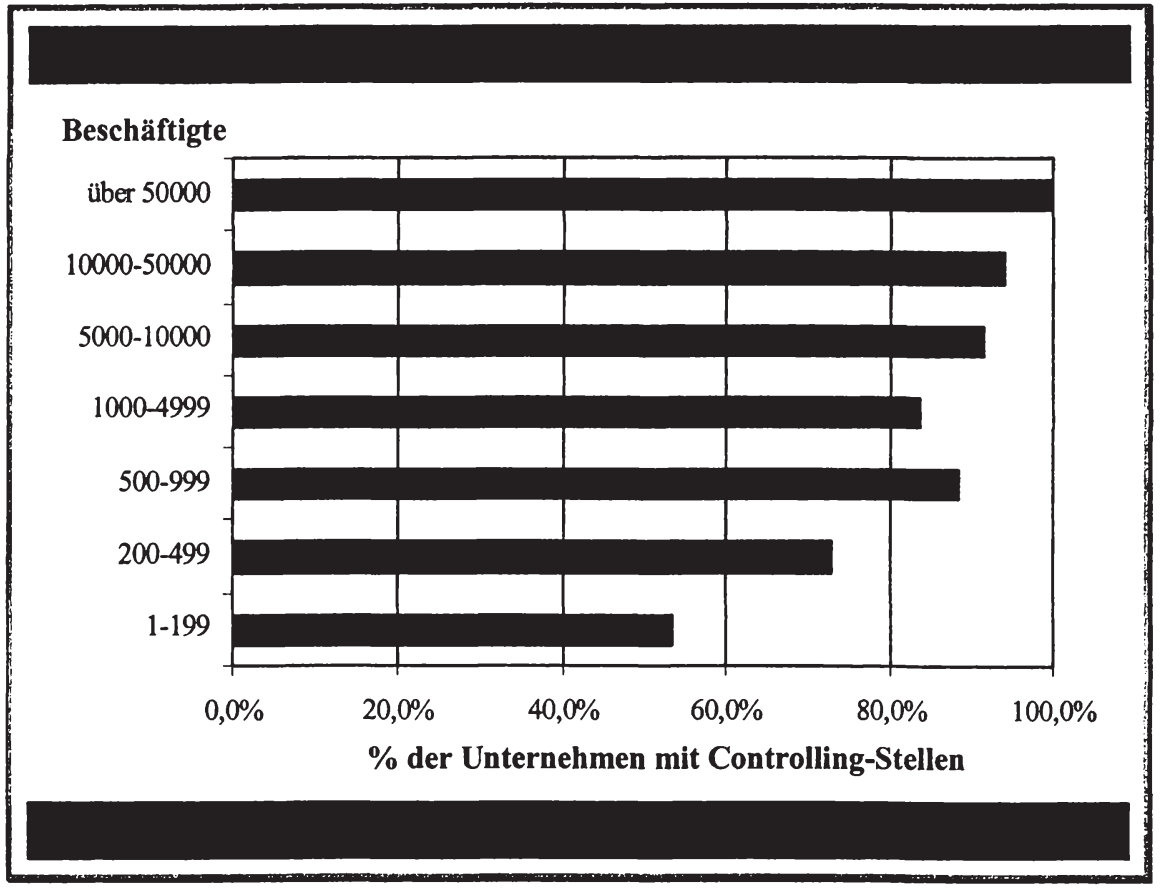

Abbildung 20: Unternehmen mit Controlling-Stellen in Abhängigkeit der Beschäftigtenzahl $l^{375}$

Die folgende Betrachtung fokussiert - soweit Aufgaben systematisch zugeordnet sind - auf die organisatorische Einbindung und ökonomische Ausrichtung des Controlling. Darauf aufbauend gilt es, marketingrelevante Parameter abzuleiten, um über deren Ausgestaltung Anreize zu einer systematischen Nachfrageorientierung zu schaffen. Letztlich sollte die Art der Bereitstellung von ControllingLeistungen das Ergebnis eines auf Effizienz- und Effektivitätsgewinne ausgerichteten internen Wettbewerbs sein. ${ }^{376}$

\footnotetext{
374 Vgl. Kap. 3.2.1.1.

375 Vgl. Küpper/Winckler/Zhang (1990), S. 439. Hierbei handelt es sich um eine Erhebung von 300 Unternehmen, die in Abhängigkeit ihrer Beschäftigtenzahlen klassifiziert wurden.
}

376 Vgl. Währisch (1998), S. 337. 


\subsubsection{Organisatorische Einbindung und ökonomische Ausrichtung des Controlling als Rahmenbedingungen der Leistungserstellung}

Um Anknüpfungspunkte für eine kundenorientierte Gestaltung eines in die Gesamtorganisation integrierten Controlling diskutieren zu können, bedarf es zunächst einer deskriptiven Darstellung der für das Controlling relevanten Instrumente zur Organisationsgestaltung. ${ }^{37}$ Die in der Literatur vorherrschenden Organisationsverständnisse weisen sehr unterschiedliche Schwerpunkte auf, ${ }^{378}$ gemein ist hingegen den meisten die Unterteilung des Organisationsproblems in Regelungen bezüglich der „arbeitsteiligen, funktionsfähigen Teileinheiten“379 und solchen, die sich auf die „raumzeitliche Strukturierung der zur Aufgabenerfüllung erforderlichen Arbeits- und Informationsprozesse ${ }^{\text {“380 }}$ beziehen. Im ersten Fall spricht man allgemein vom Problemfeld der Aufbauorganisation, im zweiten Fall von dem der Ablauforganisation. Aufbau- und Ablauforganisation beeinflussen die Effizienz und Effektivität des Controlling gleichermaßen. Die Ausgestaltung der organisatorischen Regelungen für das Controlling ist stets kontextabhängig. Ohne bezüglich der Kontextfaktoren konkrete Annahmen zu treffen, sollen in Anlehnung an HILL, FEHLBAUM und ULRICH die wesentlichen Instrumente der organisatorischen Systemgestaltung dargestellt werden, ${ }^{381}$ um darauf aufbauend ableiten zu können, welche organisatorischen Instrumente speziell bezogen auf das Leistungserstellungsverhalten des Controlling einer besonderen Analyse bedürfen. Grundlegende Instrumente sind nach $\mathrm{H} L \mathrm{~L}$, FEHLBAUM und ULRICH: ${ }^{382}$

- Zentralisation/Dezentralisation der Aufgaben auf einer Hierarchiestufe,

- Funktionalisierung, im Sinne einer Aufgaben- und Kompetenzzuteilung,

- Delegation von Kompetenzen,

- Partizipation der Mitarbeiter,

- Standardisierung von Problemlösungsprozessen,

- Arbeitszerlegung standardisierter Prozesse.

Bezogen auf diesen Gesamtkanon der Instrumente soll der Schwerpunkt der folgenden Betrachtung zunächst auf einer Diskussion des Zentralisationsgrades

\footnotetext{
377 Vgl. Horváth (1998), S. 800 ff.

378 Ubersichtsartig vgl. Grochla (1980).

379 Kosiol (1980), Sp. 180.

380 Gaitanides (1993), Sp. 192.

381 Vgl. Hill/Fehlbaum/Ulrich (1989), S. 170 ff. sowie diesbezugliche Erläuterungen zur Organisation des Controlling bei Horvatth (1998), S. $803 \mathrm{ff}$.
}

382 Vgl. Hill/Fehlbaum/Ulrich (1989), S. 170 ff. 
des Controlling liegen. Parallel dazu soll die damit korrespondierende Kompetenzverteilung im Sinne der angesprochen Funktionalisierung thematisiert werden. Der Betrachtungsschwerpunkt liegt an dieser Stelle auf den organisationsspezifischen Tatbeständen wie den Entscheidungs- und Weisungsrechten, ${ }^{383}$ weniger auf den bereits erörterten inhaltlichen Aspekten, im Sinne der Aufgabenzuordnungsproblematik. ${ }^{384}$ Zweckmäßigerweise lässt sich die Frage der Delegation im Zusammenhang der Zentralisierungs- bzw. Dezentralisierungsdiskussion betrachten, da die Kompetenzverlagerung eine bestimmte hierarchische Struktur voraussetzt. Die Frage der Partizipation der Mitarbeiter lässt sich auf verschiedenen Betrachtungsebenen ansiedeln. Einerseits betrifft sie die Binnenproblematik im Controlling, d.h. die Beteiligung der Mitglieder des Controllerbereichs an der Führung desselben. Diese Problematik wurde bereits im Rahmen der kundenorientierten Führung des Controllerbereichs unter dem Führungsteilsystem Personalführung diskutiert. ${ }^{385}$ Andererseits betrifft sie die Partizipationsmöglichkeit von Kunden des Controlling, also letztlich nichts anderes als die kundenorientierte Gestaltung von Leistungsprozessen im Sinne einer integrativen Leistungserstellung. Diesbezügliche Grenzen wurden bereits im Rahmen der Leistungszwecksetzung (Unterstützung/Kontrolle) angesprochen. ${ }^{386}$ Darüber hinaus sind Fragen einer derartigen kundenbezogenen Partizipation Mittelpunkt aller marketingorientierten Gestaltungsparameter. Der Problembereich der Prozessstandardisierung und die damit korrespondierende Arbeitszerlegung/-teilung findet einerseits - soweit es die organisatorische Eingliederung betrifft - Berücksichtigung im Rahmen der noch folgenden Betrachtung der $A b$ laufcharakteristika ${ }^{387}$, andererseits betrifft er sowohl Fragen der Aufgabenzuordnung und -wahrnehmung ${ }^{388}$ sowie der Individualität der kundenbezogenen Leistungserstellung ${ }^{389}$.

Im Sinne einer anreizkonformen Leistungsbereitstellung soll parallel zur Diskussion der organisatorischen Einbindung die ökonomische Ausrichtung des Controlling diskutiert werden, da diese die Beanspruchung des Controlling durch seine Kunden, insbesondere bei reinen Unterstützungsleistungen, maßgeblich beeinflusst. Gerade die Wechselwirkung von Position, Kompetenz und

\footnotetext{
383 Vgl. Küpper (1997), S. 441.

384 Vgl. Kap. 3.2.1.1.

385 Vgl. Kap. 3.1.

$386 \mathrm{Vgl}$. hierzu die Inhaltscharakteristika in Kap. 3.2.2.3.

387 Vgl. Kap. 3.3.2.2.

388 Vgl. Kap. 3.2.1.1.

389 Vgl. Kap. 3.4.3.1.
} 
ökonomischer Ausrichtung des Controlling führen oftmals zu Widersprüchen, die eine deutliche Aufgabenwahrnehmung in den Augen aktueller und potenzieller Kunden erschweren. Insbesondere der in vielen Unternehmen vorherrschende Charakter der Controlling-Leistungen als freie Güter führt mutmaßlich zu einer ineffizienten Beanspruchung und Verwendung dieser. ${ }^{390}$

\subsubsection{Organisatorische Einbindung}

Die zentralen Fragen im Rahmen der organisatorischen Einbindung des Controllerbereichs waren in der Vergangenheit einerseits, inwieweit Controlling als Stabs- oder Linieninstanz einzurichten ist. ${ }^{391}$ Andererseits wurden regelmäßig mit der Ausgestaltung verbundene Vor- und Nachteile von zentralem und dezentralem Controlling diskutiert. ${ }^{392}$ Auf dieser Basis sollen im Folgenden die Organisationsformen zentrales Controlling, dezentrales Controlling und Controlling als Shared Service Center unter Berücksichtigung alternativer Weisungsbefugnisse diskutiert werden, um darauf aufbauend Implikationen für die Beziehungsdynamik zwischen Controllern und deren Kunden ableiten zu können. ${ }^{393}$ Diese aufbauorganisatorischen Tatbestände helfen an späterer Stelle, die im Rahmen der Kundenorientierung relevanten ablauforganisatorischen $\mathrm{Ge}$ staltungsparameter zu bestimmen.

\subsection{Zentrales Controlling}

Das Controlling nimmt speziell in seinem institutionellen Verständnis für die Führung eine Servicefunktion wahr ${ }^{394}$ Die Entscheidung, ob Controlling zentral, dezentral oder kombiniert durchgeführt wird, hängt somit maßgeblich von der Struktur der Führungsgesamtorganisation ab. Bis zu einem gewissen Dezentralisierungsgrad bietet die zentrale Wahrnehmung von Serviceaufgaben zunächst Spezialisierungs- und Kapazitätsvorteile. ${ }^{395}$ Ist ein gewisser Dezentralisationsgrad überschritten, werden Serviceaufgaben gleichermaßen dezentralen Einheiten überlassen. Ergänzend wird eine zentrale Koordination dieser Einheiten erforderlich. Derartige Zusammenhänge führen bezogen auf das Controlling zu der Notwendigkeit, einerseits die Auswirkungen einer Einordnung des zentralen

\footnotetext{
390 Vgl. Währisch (1998), S. 332.

391 Vgl. beispielhaft für das Controlling als typische Stabsfunktion Welge (1988), S. 404. Zur Organisation des Controllerbereichs bei HENKEL vgl. Lehner (1989), S. 189 ff.

$392 \mathrm{Zu}$ ausgewählten branchenbezogenen Beispielen vgl. Steinie (1999), S. $34 \mathrm{ff}$.

393 Vgl. Lanter (1996), S. 63.

394 Hierzu und im Folgenden vgl. Horváth (1998), S. 808.

395 Vgl. Newmann (1963), S. 170.
} 
Controlling auf alternativen Führungsebenen zu beschreiben. ${ }^{396}$ Andererseits ist die Beziehung zwischen zentralem und dezentralem Controlling zu betrachten, um daraus resultierende Auswirkungen auf die kundenbezogene Aufgabenwahrnehmung ableiten zu können.

Bei einer zentralen Eingliederung prägt die hierarchische Einordnung des Controllerbereichs maßgeblich, inwieweit der Controller seiner Rolle als interner Dienstleister gerecht werden kann. Ist das Controlling auf der ersten Leitungsebene (Vorstands- bzw. Geschäftfuihrerebene) angesiedelt, führen die damit einhergehenden Entscheidungs- und Weisungskompetenzen regelmäßig dazu, dass die Informations- und Beratungsfunktion des Controllers in den Hintergrund rückt ${ }^{397}$ und der Controller gleichberechtigt mitentscheidet oder sogar dominierend (vor-)entscheidet. ${ }^{398}$ Auch wenn dem Controlling augenscheinlich eine bedeutsame Rolle im Unternehmen eingeräumt wird, führt die damit verbundene Involvierung in die Geschäftspolitik oftmals dazu, dass der Controller weniger als entlastender Sparringspartner angesehen wird, sondern vielmehr als ein Bestandteil der Geschäftsführung mit spezifischen Blickwinkel. Die damit schwindende Unabhängigkeit, verbunden mit dem Bewusstsein der anderen Geschäftsführungsmitglieder, dass das Controlling bereits an anderer Stelle durchgeführt wird, kann zu einem Wirkungsverlust desselben führen. ${ }^{399} \mathrm{Be}-$ trachtet man die spezifische Position des Controllers in Entscheidungsprozessen, so ist festzustellen, dass der Vertreter des Controlling auf der ersten Führungsebene im wesentlichen die Rolle eines Machtpromotors ${ }^{400}$ übernimmt, d.h. er ist in der Lage, seine hierarchische Stellung zur Durchsetzung der Anliegen des Controlling einzusetzen. ${ }^{401}$

Eine Ansiedlung des Controlling auf der zweiten Führungsebene bedingt, dass der Controller nicht dauerhaft in die laufende Geschäftspolitik eingebunden ist und demgegenüber mehr Kapazitäten auf seine Führungsunterstützung im Sinne von Koordination und Innovation verwenden kann. ${ }^{402}$ Die hierdurch geschaffene Distanz zur Führung führt im Sinne der Kundenorientierung zu einer wahr-

396 Vgl. Horváth (1998), S. 809.

397 So auch Küpper (1997), S. 454.

398 Vgl. Steinle (1999), S. 32.

399 Anderer Meinung ist HoffmanN. Er geht davon aus, dass nur eine Ansiedlung auf der ersten Führungsebene die für das Controlling notwendige Neutralität sicherstellt. Vgl. Hoffmann (1968), S. $2181 \mathrm{ff}$.

400 Allgemein zur Rolle von Promotoren und Opponenten im Rahmen von Innovationsprozessen vgl. Witte (1973), S. $16 \mathrm{ff}$.

401 Vgl. Stamm (1991), S. 114.

402 Vgl. Küpper (1997), S. 456. 
nehmbaren Trennung von Führung und Führungsunterstützung, wodurch das Leistungsspektrum des Controllers klarer abgegrenzt erscheint. Zur Erbringung von Leistungen mit verhaltenssteuernden Komponenten ist eine Ansiedlung auf der zweiten Führungsebene regelmäßig noch mit einer angemessenen Kompetenzausstattung und einer hinreichenden Unabhängigkeit verbunden. ${ }^{403}$ Eine Profilierung des Controlling gegenüber anderen, eventuell dem gleichen Geschäftsführungsbereich zugeordneten Funktionen (Finanzen, externes Rechnungswesen, Steuerwesen $)^{404}$ hängt neben der Art der Vertretung im Führungsgremium maßgeblich von der Struktur und Kommunikation des ControllingLeistungsangebotes ab. Ein Controller auf der zweiten Führungsebene hat damit regelmäßig die Rolle eines Fachpromotors ${ }^{405}$ inne, ${ }^{406}$ d.h. er ist durch seine spezifische Qualifikation und eine aktive Informationsvermittlung in der Lage, Wissensbarrieren bei anderen abzubauen und so zu einer Entscheidungsfindung beizutragen. Diese Rolle können jedoch auch Controller niedriger Hierarchiestufen übernehmen. Darüber hinaus charakterisiert aber die Rolle eines Prozesspromotors ${ }^{407}$ auf gerade idealtypische Weise die Position eines Controllers zwischen oberster Führungsebene und nachgeordneten Ebenen. Der Prozessoder auch Koordinationspromotor zeichnet sich durch seine Kenntnis der Entscheidungsfindungsmodalitäten in einem Unternehmen aus und kann dadurch „Einzellösungen zugunsten von Systemlösungen verhindern“408. Im Gegensatz zu Fach- und Machtpromotor, die spezifische Ressourcen in den Entscheidungsprozess einbringen, gelingt es dem Prozess- oder Koordinationspromoter gerade, die Verknüpfung dieser Ressourcen herbeizuführen, was gleichermaßen spezifischer fachlicher und persönlicher Fähigkeiten bedarf. Die hierarchische Position zwischen Entscheidungsgremium und operativen Einheiten begünstigt diesen Tatbestand wesentlich.

Wird das Controlling unterhalb der zweiten Führungsebene angesiedelt, führt dies oft dazu, dass nur noch Teile der Unterstützungsleistungen des Controlling zweckmäßig erbracht werden können. Gewisse Dienstleistungen, wie die Pflege von bereichsbezogenen Informationssystemen, lassen sich bei einer derartigen Eingliederung zwar erbringen, der umfassende Charakter des Controlling, im Sinne der Koordinationsfunktion, kann jedoch keine angemessene Berücksich-

\footnotetext{
${ }^{403} \mathrm{Zu}$ den Leistungen des Controlling im Rahmen der Verhaltenssteuerung vgl. Kap. 3.2.1.4.

404 Vgl. Küpper (1997), S. 456.

405 Vgl. Witte (1973), S. $18 \mathrm{f}$.

406 Vgl. Stamm (1991), S. 114.

407 Zu dem Begriff vgl. Topfer (1984), S. 399.

${ }^{408}$ Fließ (1995), S. 355.
} 
tigung mehr im Rahmen des Leistungsspektrums finden. Darüber hinaus verringern sich die Kompetenzen zur Gewährleistung einer angemessenen Rationalitätssicherung. ${ }^{409}$

Grundsätzlich gesehen erscheint eine kontextunabhängige Empfehlung bezüglich der Eingliederung des zentralen Controlling nicht angebracht zu sein, da erst in Abhängigkeit der zugeordneten Aufgaben über den zu ihrer Erfüllung notwendigen organisatorische Status entschieden werden kann. ${ }^{410}$ Will man jedoch dem Servicecharakter deutlich Ausdruck verleihen, ohne die Kompetenzen unangemessen zu verringern, erscheint aufgrund der genannten Aspekte eine Einordnung des zentralen Controlling auf der zweiten Führungsebene für viele Unternehmen zweckmäßig.

\subsection{Dezentrales Controlling}

Das dezentrale Controlling zeichnet sich dadurch aus, dass es der Linie oder als Stab einer dezentralen Unternehmenseinheit zugeordnet ist. Dezentrale Controlling-Einheiten können demnach in Funktionsbereichen, Sparten, Werken, Regionen oder Tochtergesellschaften eingerichtet werden. ${ }^{411}$ Als problematisch erweisen sich regelmäßig in diesem Zusammenhang potenziell unterschiedliche Unterstellungsmöglichkeiten. Die umfassende Koordinationsaufgabe des Controlling sowie die Fokussierung auf das Gesamtzielsystem der Unternehmung spricht zunächst für einen unmittelbaren Bezug zum Zentralcontrolling. ${ }^{412}$ Demgegenüber sprechen Informationsbeschaffungs- und Akzeptanzprobleme eher für eine Eingliederung in die Hierarchie der dezentralen Einheit.

Eine teilweise Auflösung dieses Widerspruchs kann das „dotted-line-Prinzip“ herbeiführen, welches zwischen fachlicher und disziplinarischer Unterstellung differenziert. In fachlicher Hinsicht bleibt das dezentrale Controlling dem Zentralcontrolling unterstellt, welches somit Aufgaben- und Methoden vorgibt. Demgegenüber untersteht das dezentrale Controlling disziplinarisch der dezentralen Einheit. ${ }^{413}$ Positive Effekte einer derartigen organisatorischen Eingliederung sind: ${ }^{414}$

\footnotetext{
$409 \mathrm{Zu}$ den unterschiedlichen Engpässen rationaler Führung vgl. Weber (1999), S. $39 \mathrm{ff}$.

410 Vgl. Horváth (1998), S. 811.

411 Vgl. Küper (1997), S. 457.

412 Vgl. hier und im Folgenden Horváth (1998), S. 811 ff. sowie vergleichbar Küpper (1997), S. 457 ff.

${ }^{413}$ Unter disziplinarischen Regelungen sind im wesentlichen alle aus dem Arbeitsvertrag resultierenden Tatbestände wie Arbeitszeitregelungen, Entlohnung etc. zu verstehen.
}

414 In Anlehnung an Schüller (1984), S. 210 sowie Lanter (1996), S. 64. 
- Die physische Nähe zu den Controlling-Kunden schafft eine Verbundenheit mit diesen.

- Der Controller wirkt überzeugender aufgrund der unmittelbaren Geschäftskenntnis.

- Unternehmensweit kann eine Abstimmung der Instrumente und Methoden sichergestellt werden.

- Der Zugang zu formellen und insbesondere auch informellen Informationsquellen wird wesentlich erleichtert.

- Der Controller kann sich besser in die Bedürfnisse und Anliegen seiner Kunden hineinversetzen und auf dieser Basis individuellere und bedürfnisgerechtere Leistungen anbieten.

Auch wenn die Vorteile einer derartigen Organisation in vielen Fällen überwiegen, führt die doppelte Zuordnung oftmals zu Identifikationsschwierigkeiten des Controllers und damit zu Akzeptanz- und Neutralitätsproblemen. ${ }^{415}$

Gibt es zentrale und dezentrale Controlling-Einheiten in einem Unternehmen, bedarf es einer klaren Abgrenzung der Zuständigkeiten. Betrachtet man die dem Leistungsangebot des Controlling zugrundeliegenden Aufgaben, so ist im Rahmen der diesbezüglichen Zuordnung nicht nur zwischen den Aufgabenträgeralternativen Management und Controlling abzuwägen, ${ }^{416}$ sondern zugleich auch der Zentralisationsgrad der Aufgabenwahrnehmung festzulegen. Im Falle der Zuordnung einer Aufgabe zum Controlling ist somit zu entscheiden, ob es sich tendenziell um die Gesamtunternehmung betreffende innovative Koordinationsaufgaben handelt, die einer Zentralisation bedürfen, oder eher um regelmäBig wiederkehrende Aufgaben der Informationsversorgung, die Kenntnisse des Umfeldes dezentraler Kunden voraussetzen. ${ }^{417}$

Korrespondierend mit den Aufgabeninhalten und deren Zuordnung ist im Rahmen der Funktionalisierung die zu vollziehende Kompetenzzuordnung zu thematisieren. Linienkompetenzen im Sinne spezifizierter Entscheidungs- und Weisungsrechte können Controllern die notwendige Autorität verleihen, auch in andere Führungsteilsysteme einzugreifen, soweit es die Erfüllung ihrer Koordinationsaufgabe erforderlich macht. Als problematisch erweist sich in diesem Zusammenhang die koordinationsinduzierte Querschnittsfunktion des Control-

\footnotetext{
415 Vgl. zu den negativen Auswirkungen des „dotted-line-Prinzips“ Schuller (1984), S. 210.

${ }^{416}$ Vgl. Kap. 3.2.1.1.

417 Vgl. Horváth, (1998), S. 814.
} 
ling. ${ }^{418}$ Diese führt dazu, dass der zu Koordinationszwecken notwendige Eingriff des Controlling in die Kompetenzen anderer Bereiche zwangsläufig eine Kooperation derselben erfordert, da formale Macht allein regelmäßig nicht zur Erreichung der angestrebten Ziele ausreicht. ${ }^{419}$ Die in diesem Zusammenhang deutlich werdenden Kompetenzüberschneidungen bedingen im Prinzip einen gemeinsamen Verantwortungsbereich beider Transaktionspartner, welcher mit zunehmender Verbreitung partizipativer Managementmethoden (z.B. MbO) wächst. ${ }^{420}$

Stabslösungen im Controlling zeichnet daran anknüpfend die zwangsläufige Notwendigkeit einer Kooperation mit anderen Instanzen aus, da eine unmittelbare Weisungsbefugnis nicht besteht. Das Verständnis des Controlling als Führungsunterstützungsfunktion scheint zunächst mit der Zuordnung von Stabskompetenzen idealtypisch vereinbar. ${ }^{421}$ Kritisiert wird jedoch in diesem Zusammenhang die Nicht-Zulässigkeit der Beschränkung des Controlling auf Serviceund Unstützungsaufgaben. ${ }^{42}$ Der auch in dieser Arbeit zugrundegelegte umfassende koordinationsorientierte Controlling Ansatz impliziert gewisse Entscheidungskompetenzen des Controlling, beispielsweise bezüglich des Informationsoder Planungssystems. ${ }^{423}$ Ferner fallen Fragen der Binnenorganisation des Controlling in den Bereich zuzuordnender Entscheidungs- und Weisungsrechte. Für die kundenorientierte Betrachtung des Controlling ist diese Binnenproblematik weniger relevant, da sie nicht unmittelbar die Wahrnehmung der organisationsinternen Kunden tangiert. Demgegenüber ist die Ausgestaltung des Informations- und Planungssystems mittelbar relevant, da dieses die angebotenen Leistungen des Controlling beeinflusst, bzw. selbst als eine konkrete Leistung aufzufassen ist. Regelmäßig dominiert aber die Wahrnehmung der Kunden die Informations-, Vorschlags- und Beratungsfunktion des Controlling. Darüber hinaus widersprechen Entscheidungs- und Weisungsrechte keineswegs der kundenorientierten Ausgestaltung des Controlling, vielmehr ist durch geeignete Instrumente sicherzustellen, dass die Entscheidungen im Controlling auf Basis einer angemessenen Kenntnis der Kundeninteressen getroffen werden. ${ }^{424}$ So kommt auch die einschlägige Literatur zu dem Schluss, dass eine eindeutige

\footnotetext{
418 Vgl. Pohle (1993), Sp. 666.

420 Vgl. Küpper (1997), S. 453.

421 Vgl. Agthe (1960), S. 52.

422 Vgl. Mann (o.J.), S. $177 \mathrm{ff}$.

423 Vgl. hier und im Folgenden Küpper (1997), S. 454.

${ }^{424} \mathrm{Zu}$ einzelnen Instrumenten vgl. Kap. 4.
}

419 Vgl. hierzu auch die Ausfuhrungen zu den im Rahmen des Kontrollcharakters der Controlling-Leistungen thematisierten dysfunktionalen Verhaltensweisen in Kap. 3.2.2.3.2. 
Trennung zwischen Stab und Linie nicht unbedingt zweckmäßig ist, insbesondere in Zeiten einer zunehmenden Etablierung mehrdimensionaler Organisationsstrukturen. ${ }^{425}$

In Anlehnung an die klassische Aufgabenzentralisierung stellt die Shared Service Center Konzeption einen aktuellen Trend dar, Controllingteilaufgaben organisatorisch zu bündel. Oftmals werden Shared Service Center ergänzend zu anderen Controlling-Einheiten betrieben. Shared Service Center versuchen, konsequent Vorteile zentraler und dezentraler Organisationsstrukturen $\mathrm{zu}$ kombinieren, indem interne Dienstleistungen in überregionalen Organisationseinheiten erbracht werden. Diese weder ausschließlich zentrale noch dezentrale Ausrichtung erlaubt die Ausnutzung von Größenvorteilen insbesondere auch durch die Standardisierung von Prozessen und Technologieplattformen, bei gleichzeitiger Wahrung der internen Kundennähe. Als Kriterien für die Auswahl von Geschäftsprozessen, die sich für eine Eingliederung in Shared Service Center eigenen, lassen sich im wesentlichen die strategische Relevanz sowie die Einzigartigkeit des jeweiligen Geschäftsprozesses anführen. ${ }^{426}$ Demnach bieten sich insbesondere Geschäftsprozesse mit niedriger strategischer Relevanz und wenig Bezug zu einem bestimmten Geschäftsfeld, sowie strategisch hoch relevante Prozesse mit einer einzigartigen Verbindung zu einem bestimmten Geschäftsfeld zu einer Eingliederung in ein Shared Service Center an. Übersichtsartig lassen sich einige strukturell controllingverwandte Geschäftsprozesse wie in Abbildung 21 dargestellt klassifizieren.

Bezogen auf die Leistungserstellung im Controlling ergibt sich demnach die Notwendigkeit, Einzelleistungen auf ihre strategische Relevanz und Geschäftsfeldbezogenheit hin zu überprüfen. Im Anschluss an eine solche Klassifikation kann die Zuordnung von Controlling-Leistungen vorgenommen werden, um darauf aufbauend zu entscheiden, inwieweit der ergänzende Betrieb eines Shared Service Centers wirtschaftlich zweckmäßig erscheint. ${ }^{427}$ Die Vorteile der Leistungserstellung in Form eines Shared Service Centers sind für ControllingLeistungen einerseits in höheren Transaktionsvolumina und andererseits in der Konsolidierung und dem Aufbau von spezifischem Know how zu sehen. Dieses insbesondere zu Beratungszwecken zu nutzende Know how kann gegenüber zentralen Organisationsformen kundenorientierter eingesetzt werden. Um eine

\footnotetext{
${ }^{425}$ Vgl. beispielhaft Kupper (1997), S. 454 sowie Horváth (1998), S. 815.

$426 \mathrm{Vgl}$. hier und im Folgenden Schuurmans/Stoller (1998), S.37.

427 Insbesondere im angloamerikanischen Raum wird das Accounting bei großen Untemehmen zunehmend in Shared Service Center organisiert. Vgl. Gunn/Carberry/Frigo/Behrens (1993), S, $22 \mathrm{ff}$. 
größere Transparenz in die Leistungsbeziehung zwischen Controller und Kunde zu bringen, werden im Rahmen des Betriebs von Shared Service Centern oftmals interne Leistungsvereinbarungen geschlossen. Diese führen regelmäßig zu einer Unsicherheitsreduktion beider Transaktionspartner. ${ }^{428}$

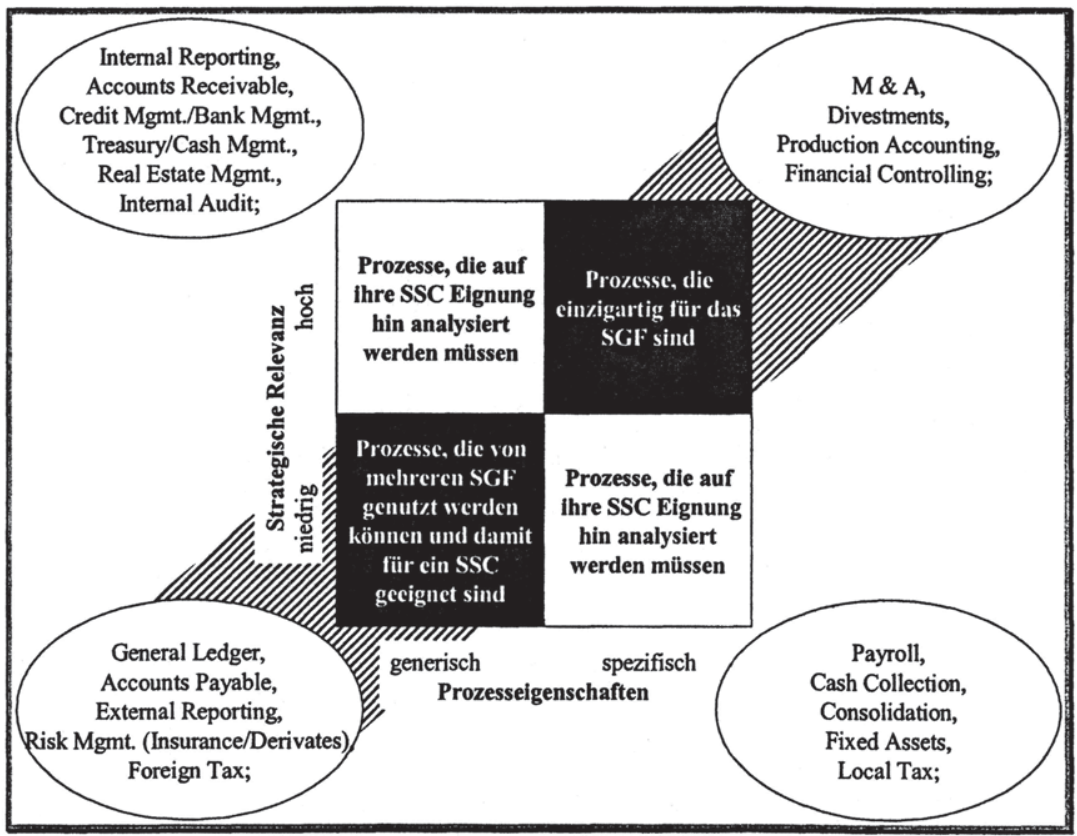

Abbildung 21: Prozessallokations-Matrix ${ }^{429}$

Die hier dargestellten Organisationskonzepte zeigen, dass in der Praxis unterschiedliche Ausprägungen zentraler und dezentraler Organisationsstrukturen des Controlling zu beobachten sind, einschließlich diverser dazwischenliegender Abstufungen. Insbesondere auch die zunehmende Etablierung eines SelfControlling, im Sinne einer Reintegration von Controlling-Aufgaben in das Management bei gleichzeitiger Verlagerung von Koordinationsaufgaben auf niedrigere Hierarchieebenen führt zu der Notwendigkeit organisatorischer Flexibilität. ${ }^{430}$ Daher soll im Rahmen der kundenorientierten Betrachtung des Controlling weniger ein spezifischer Organisationsstatus zugrundegelegt werden,

${ }^{428}$ Zur Bedeutung von Service Level Agreements furr die Gesamtunternehmung vgl. Winckelmann-Ackermann/Bundi (1999), S. 36 ff. Speziell bezogen auf Leistungen des Controlling vgl. Kap. 4.2.4.

429 In Anlehnung an Schuurmans/Stoller (1998), S. 38.

430 Zum Sef-Controlling bzw. Selbscontrolling vgl. Horváth (1995b), S. $262 \mathrm{ff}$. 
sondern vielmehr sollen die sich auf die Leistungsbereitstellung ergebenden organisationsdeterminierten Auswirkungen thematisiert werden. Zur Konkretisierung des Leistungserstellungsverhaltens des Controlling wurde bereits die Zweckmäßigkeit der Betrachtung des Unterstützungs- bzw. Kontrollcharakters der Controlling-Leistungen dargelegt. Ferner sollen später im Sinne der Kompetenzbetrachtung die Leistungsinanspruchnahmemodalitäten analysiert werden, d.h. die Frage ob Leistungen auf Basis organisatorischer Konventionen freiwillig oder obligatorisch in Anspruch genommen werden. ${ }^{431}$ Darüber hinaus stellt die Einbindung des Controlling und seiner Leistungen in den Leistungserstellungsprozess des Kunden (Ablaufcharakteristika) einen wesentlich organisationsdeterminierten Tatbestand dar, da hierfür der Zentralitätsgrad der Controller-Organisation relevant ist. ${ }^{432}$ Ferner wird die Gestaltung der Abrufmodalitäten für Controlling-Leistungen diskutiert, da diesbezügliche Regelungen regelmäBig Gegenstand organisationsinterner Leistungsvereinbarungen/ServiceVerträge sind. Letztlich sind über all diese Erwägungen hinaus die schwer operationalisierbaren informellen Kontakte, sowie die Leistungstradition der Vergangenheit als wesentliche organisationsbedingte Einflussfaktoren anzusehen. ${ }^{433}$

Wurden in diesem Abschnitt überwiegend strukturelle Rahmenbedingungen für die Leistungserstellung und -verwertung des Controlling thematisiert, soll im Folgenden die ökonomische Ausrichtung des Controllerbereichs und die damit verbundenen Ziele und Anreize dargestellt werden. Auf dieser Basis kann im Rahmen der Gestaltungsparameterbetrachtung die Verrechnung von Controlling-Leistungen thematisiert werden.

\subsubsection{2 Ökonomische Ausrichtung}

Betrachtet man die Möglichkeiten einer kundenorientierten Ausgestaltung des Controlling, so stellt sich die Frage, wie eine sachgerechte Inanspruchnahme der Leistungen im Sinne der einzelnen Kunden und des Gesamtunternehmens gewährleistet werden kann. In der bisherigen Betrachtung wurde primär auf Möglichkeiten einer effektiveren Erreichung der Sachziele des Controlling eingegangen. Klärungsbedürftig bleibt daher, welche Formalziele grundsätzlich für den Controlling-Bereich gelten sollen und inwieweit die Verfolgung unterschiedlicher Formalziele einer Nachfragerorientierung dienlich sein kann.

\footnotetext{
431 Vgl. Kap. 3.3.2.1.

432 Allgemein fïr interne Leistungsbeziehungen vgl. Stauss/Neuhaus (1995), S. 583.

433 Vgl. Witt (1988), S. 669.
} 
Auf Basis des dieser Arbeit zugrundegelegten, alle Führungsteilsysteme umfassenden koordinationsorientierten Controlling-Konzeptes besteht eine Aufgabe des Controlling darin, eine Koordination der Organisation mit der Personalführung herbeizuführen. ${ }^{434}$ Betrachtet man nun den Controllerbereich als einen Bestandteil der Organisation, so ergibt sich zunächst die Notwendigkeit - wie in den vorangegangenen Kapiteln vollzogen ${ }^{435}$ - wesentliche Organisationsvariablen des Controllerbereichs zu betrachten. Darüber hinaus erfordert eine wirkungsvolle Ausgestaltung des Controllerbereichs im Sinne der Kundenorientierung die Verknüpfung dieser Organisationsvariablen mit entsprechenden Instrumenten der Personalführung, um Anreiz- und Motivationseffekte zielgerichtet steuern zu können. ${ }^{436}$ Ein Instrument hierzu ist die Bildung von Verantwortlichkeitszentren.

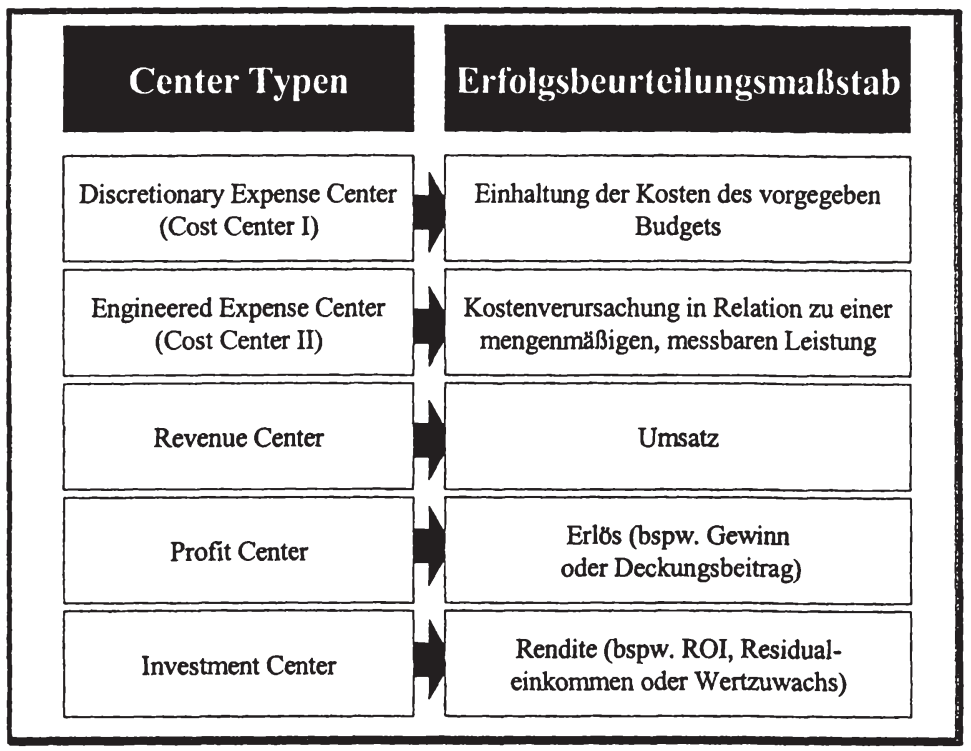

Abbildung 22: Erfolgsmaßstäbe für Center-Typen ${ }^{437}$

Man unterscheidet verschiedene Typen von Verantwortlichkeitszentren (responsibility center), welche auf Basis zugeordneter Aufgaben und Entschei-

\footnotetext{
434 Zum dem hier zugrundegelegten Controlling-Verständnis vgl. Kap. 2.1.3.

$435 \mathrm{Vgl}$. Kap. 3.3.1.1 ff.

436 Allgemein zu den Beziehungen zwischen Organisationsstruktur und Personalfuhrung vgl. Kilpper (1997), S. $284 \mathrm{ff}$.

437 In Anlehung an Herter (1994), ahnlich auch Weilenmann (1989), S. $936 \mathrm{ff}$.
} 
dungskompetenzen differenzierten ökonomischen Erfolgsbeurteilungsmaßstäben unterliegen. ${ }^{438}$ Abbildung 22 stellt übersichtsartig verschiedene CenterTypen, sowie die mit diesen korrespondierenden Erfolgsbeurteilungsmaßstäbe dar.

Bezogen auf das Controlling stellt sich die Frage, welcher Maßstab geeignet ist, einerseits das Leistungserstellungsverhalten der Mitarbeiter im Controlling zielgerichtet $\mathrm{zu}$ beeinflussen und andererseits auch die Leistungsinanspruchnahme und Leistungsnutzung der Kunden des Controlling zu steuern.

\subsection{Cost Center}

Bei dem hier zunächst zu betrachtenden Cost Center lassen sich unterschiedliche Ausprägungen beobachten. ${ }^{439}$ Charakteristisch ist für Cost Center der fehlende Bezug zum Absatzmarkt und die daraus resultierende Schwierigkeit, abbildbare Beziehungen zwischen Kosten und Erlösen herzustellen. ${ }^{40}$ In Ermangelung derartiger Messgrößen werden die verursachten Kosten regelmäßig als Beurteilungskriterium verwandt. Zur Differenzierung der Cost Center unterscheidet man Discretionary Expense Center und Engineered Expense Center. In Discretionary Expense Centern gibt es keine eindeutig messbare LeistungsgröBe, so dass lediglich die Einhaltung eines vorgegebenen Budgets als Wirtschaftlichkeitsmaßstab herangezogen werden kann. ${ }^{441}$ Bei Engineered Expense Centern können Anhaltspunkte für eine Leistungsmessung gefunden werden, so dass die Kostenverursachung in Relation zu den messbaren Leistungen analysiert werden kann.

Charakteristisch für das derzeitige Controlling in der Mehrzahl der Unternehmen ist die ökonomische Ausrichtung in Form eines Cost Centers. ${ }^{42}$ Der fehlende Bezug zum Absatzmarkt spricht zunächst für eine derartige Vorgehensweise. Regelmäßig wird das Controlling als eine Kostenstelle behandelt, der die durch sie direkt beeinflussten Kosten zugerechnet werden. Legt man die Unterscheidung zwischen Discretionary Expense Center und Engineered Expense Center zu Grunde, so ist zu beobachten, dass in vielen Unternehmen keinerlei mengenmäßig messbare Leistungen zur Betrachtung der Wirtschaftlichkeit des

\footnotetext{
438 Allgemein zu den Defiziten der Center-Konzeptionen vgl. Eversheim/Guthenke (1999), S. $18 \mathrm{ff}$.

439 Vgl. Weilenmann (1989), S. $936 \mathrm{ff}$.

440 Hier und nachfolgend vgl. Scherz (1998), S. 32.

41 Vgl. Herter (1994).

442 Bezogen auf die Kostenrechnung vgl. Wahrisch (1998), S. 338
} 
Controllerbereichs erhoben werden ${ }^{43}$ Regelmäßig wird ausschließlich die Budgeteinhaltung bzw. die Kostenminimierung als Indikator einer sachgerechten Mittelverwendung angesehen. Insbesondere die als überwiegend hoheitlich empfundene Stellung des Controlling, verbunden mit einem in der Vergangenheit wenig strukturierten Spektrum von Leistungen, hat zu einer derartigen Vorgehensweise geführt.

Aufbauend auf den von Kunden zunehmend artikulierten Verbesserungsbedarf im Controlling, ${ }^{444}$ bietet eine klarere Struktur des Leistungsspektrums verbunden mit diesbezüglich erhobenen Mengen- und Qualitätsdaten Anhaltspunkte für eine leistungsrelativierte Kostenbetrachtung (Engineered Expense Center). ${ }^{445}$ Zur Absicherung der intendierten Anreiz- und Motivationseffekte ist es erforderlich, Transparenz bezüglich der Beeinflussbarkeit einzelner Kosten zu schaffen. ${ }^{46}$ Darüber hinaus erfordert die Leistungsmessung einen kostenrechnerischen Zusammenhang zwischen Input und Output. Zur Analyse derartiger $\mathrm{Zu}$ sammenhänge für den Controllerbereich eignet sich die Prozesskostenrechnung. Prozesse und Tätigkeiten können hierdurch erfasst, abgebildet und später in Form von Produkten zusammengefasst werden, Aufbauend auf derartige Erhebungen können Leistungskennzahlen für den Controllerbereich erhoben werden, die in Verbindung mit dem Budget Anhaltspunkte für die Bewertung der Wirtschaftlichkeit liefern. ${ }^{47}$ Grundlegender Anspruch des kundenorientierten Controlling ist in diesem Zusammenhang, Leistungsindikatoren der Dimensionen Effizienz und Effektivität gleichermaßen zu berücksichtigen. ${ }^{448}$ Insbesondere die Vernachlässigung der Effektivität - im Sinne der Befriedigung der Kundenbedürfnisse - führt oftmals zu suboptimalen Ergebnissen. ${ }^{499}$

Im Rahmen der marketingrelevanten Gestaltungsparameter des Controlling soll die mit den alternativen Verantwortlichkeitsbereichstypen verbundene Verrechnungsproblematik diskutiert werden. In diesem Zusammenhang ist zu thematisieren, welche Controlling-Leistungen eine anreizkonforme Verrechnung zulas-

443 Beispielhaft hierfur die von WEBER aufgeworfenen Fragen bezilglich der Strukturiertheit des Leistungsangebotes des Controlling. Vgl. Weber (1997), S. 7.

444 Vgl. Kap. 2.1.4.2.

445 Zur Einfuthrung einer Prozesskostenrechnung im Controlling mit korrespondierender Leistungsbetrachtung vgl. Gleich/Brokemper (1998).

446 Hier und nachfolgend vgl. Scherz (1998), S. 33.

$447 \mathrm{Vgl}$. hierzu ausfuhrlich anhand eines Beispiels Kap. 4.2.3.

448 GLEICH/BROKEMPER sprechen in diesem Zusammenhang von formaler und inhaltlicher Qualität des Controllerbereichs. Vgl. Gleich/Brokemper (1998), S. 151.

449 Ein adäquater Lösungsansatz findet sich bei Stadler/Weißenberger (1999). 
sen und auf Basis welcher Größen eine derartige Verrechnung idealtypisch erfolgen sollte. ${ }^{450}$ Inwieweit hierfür die ökonomische Ausrichtung als Profit Center eine geeignetere Grundlage bildet, soll im Folgenden dargestellt werden.

\subsection{Profit Center}

Im Gegensatz zum Cost Center stellen zurechenbare ${ }^{451}$ Erlöse den dominanten Erfolgsbeurteilungsmaßstab eines Profit Centers dar. ${ }^{452}$ Der somit in Verbindung mit den Kosten bestimmbare Erfolg eines Profit Centers ist Ausgangspunkt der ergebnisorientierten Steuerung. Insgesamt müssen daher für die Einrichtung eines Profit Centers im wesentlichen drei Voraussetzungen erfüllt sein:433

- Es muss ein Bezug zum Absatzmarkt bestehen, d.h. die Einheit ist zum Teil eigenverantwortlich für Konzeption, Beschaffung, Erstellung und Absatz ihrer Produkte. ${ }^{454}$

- Die Einheit muss weitestgehend entscheidungsautonom sein, d.h. die Aufgaben der Einheit sollten klar abgrenzbar sein und die Interdependenzen im Rahmen der Aufgabenerfüllung überschaubar sein. ${ }^{455}$

- Im Rahmen der Abrechnung muss eine genaue Zuordnung von Kosten und Erlösen möglich sein. Die Verwendung von Schlüsselgrößen und Verrechnungspreisen erfordert einen hohes Maß an Beanspruchungsgenauigkeit.

Der letzte Punkt der Aufzählung befasst sich im wesentlichen mit der formalen Zuordnungsproblematik, die regelmäßig auf Basis einer differenzierten Kostenanalyse und einer beanspruchungsgerechten Verrechnung für das Controlling gelöst werden kann. Die Anwendung derartiger kostenrechnerischer Instrumente (z.B. Prozesskostenrechnung oder Target Costing) auf den ControllingBereich ist jedoch noch selten. ${ }^{456}$ Der zweite Punkt der Aufzählung behandelt die Entscheidungsautonomie. Im Controlling stellt sich die Frage, ob die Entscheidungsautonomie eine Eigenverantwortlichkeit bezüglich der Bereitstellung aller seiner Produkte einschließt. Auf Basis der Aufgabenzuordnung ergibt sich

\footnotetext{
${ }^{450}$ Vgl. Kap. 3.3.2.5.

451 Die Zurechenbarkeit von Erlöskomponenten stellt eines der konstituierenden Merkmale eines Profit Centers dar. Vgl. Frese (1993), $132 \mathrm{ff}$.

452 Ausführlich zum Begriff des Profit Center vgl. Schweitzer (1992), Sp. $2078 \mathrm{ff}$.

453 Oberblicksartig bei Scherz (1998), S. 34 f.

${ }^{454}$ Modifiziert, aber in Anlehnung an Welge (1975), S. 65.

$455 \mathrm{Vgl}$. Hahn/Laßmann (1993), S. $144 \mathrm{f}$.

456 Beispielhaft Gleich/Brokemper (1998), S. 148.
} 
für das Controlling die Notwendigkeit, bestimmte Leistungen zwangsläufig anbieten zu müssen. Hierunter ist bspw. eine gewisse Informationsgrundversorgung zu verstehen, die auf Basis interner Richtlinien zu gewährleisten ist (,Mussleistungen $\left.{ }^{6}\right){ }^{457}$ Demgegenüber ist ein Großteil der ControllingLeistungen als ,Kannleistungen “ anzusehen, d.h. der Controller kann in Abhängigkeit von Zweckmäßigkeitsgesichtspunkten und Kundenbedürfnissen über deren Angebot entscheiden. Gerade die Innovationsfunktion des Controlling erfordert einen großen Spielraum bezüglich derartiger Leistungen. ${ }^{458}$ Darüber hinaus liegt insbesondere in „Kannleistungen“ ein großes Differenzierungs- und Profilierungspotenzial für das Controlling. Auch fallen gerade kundenindividuelle Einzellösungen regelmäßig in den Bereich der „Kannleistungen“ des Controlling.

Die Notwendigkeit für Profit Center, Interdependenzen im Rahmen der Aufgabenerfüllung überschauen zu können, ergibt sich aus dem Anspruch, den Ressourcenverzehr beanspruchungsgerecht $\mathrm{zu}$ verrechnen. Im Regelfall fallen die Kosten für personelle oder sachliche Ressourcen des Controlling auch in dem organisatorisch ausgewiesenen Bereich an. Darüber hinaus führen die durch die Koordinationsfunktion des Controlling bedingten vielfältigen Leistungsverflechtungen $\mathrm{zu}$ keiner unangemessenen Einschränkung der Eigenverantwortlichkeit. Potenzielle Interessenkonflikte bei der Leistungserstellung des Controlling, welche durch die Existenz unterschiedlicher Kunden bedingt sind, ${ }^{459}$ sind zugunsten einer Orientierung an den langfristigen Unternehmenszielen aufzulösen. An dieser Stelle ist nochmals darauf hinzuweisen, dass eine Nachfragerorientierung des Controlling nicht im Sinne einer unreflektierten Erfüllung der Kundenwünsche zu verstehen ist. Vielmehr sind artikulierte Kundenbedürfnisse vor dem Hintergrund einer langfristigen internen Geschäftsbeziehung und auf Basis bestehender Unternehmensziele zu verstehen. So ist auch im Sinne des Marketing ein kurzfristiges „Liebkindmachen“ aufgrund der langfristig negativen Auswirkungen für den selben Kunden nicht zu rechtfertigen ist. SCHWETZER postuliert in diesem Zusammenhang, das kurzfristige Erfolgsdenken in Profit Centern durch geeignete Koordinationsmaßnahmen an die langfristigen Erfolgspotenziale des Unternehmen zu koppeln. ${ }^{460}$

457 Speziell fulr den Bereich der Kostenrechnung vgl. Währisch (1998), S. 338.

458 Zur Innovationsfunktion des Controlling vgl. Küpper (1997), S. 18.

459 Zur Differenzierung der Kunden des Controlling vgl. Kap. 3.4.1.2.

${ }^{460}$ Allgemein zum kurzfristigen Erfolgsdenken in Profit Centern vgl. Schweitzer(1992) Sp. 2089 
Der regelmäßig für das Controlling diskussionswürdigste Punkt im Rahmen der Abgrenzungskriterien für Profit Center ist jedoch der Absatzmarktbezug. Diesbezüglich ist zunächst zu klären, welches der relevante Markt, die relevanten Marktteilnehmer und das relevante Marktumfeld für das Controlling sind. ${ }^{461}$ In einer engen Abgrenzung wird unter dem Markt der unternehmensexterne Absatzmarkt verstanden, zu dem das Controlling zunächst - bezogen auf seine eigenen Leistungen - keinen unmittelbaren Bezug aufweist. ${ }^{462}$ Legt man ein umfassenderes Verständnis zugrunde, ist der relevante Markt für ControllingLeistungen der unternehmensinterne Markt für die Beschaffung, Aufbereitung, Analyse und Kommunikation von unternehmenszielsetzungsrelevanten Daten. Im Rahmen der Förderung einer zunehmenden Eigenverantwortlichkeit der Mitarbeiter des Controlling erscheint ein auf diesen Markt ausgerichtetes „internes Unternehmertum" angemessen. Es ist die konsequente Fortentwicklung einer Strömung, die sich von dem mit Eigeninitiative verbundenen Leistungsund Erfolgsstreben Innovationen und Effizienzgewinne verspricht. Eine Übertragung unternehmerischer Prinzipien auf unternehmensinterne Bereiche erscheint grundsätzlich immer dann gerechtfertigt, wenn Mitarbeitern - wie im Controlling der Fall - weitgefasste Aufgaben und Verantwortungskomplexe zur eigenverantwortlichen Bearbeitung in einem vorher bestimmten Rahmen anvertraut werden. ${ }^{463}$ Die Verbindung von Markt und Wettbewerb charakterisiert das dominierende gesellschaftliche Verfahren zum Treffen wirtschaftlicher Entscheidungen. ${ }^{464}$ Konstituierendes Merkmal für einen Markt ist zunächst das $\mathrm{Zu}$ sammentreffen von Angebot und Nachfrage. ${ }^{465}$ Soweit in einer solchen Situation Anbieter eigenverantwortlich über ihre Ressourcen verfügen können und Nachfragern alternative Angebote zur Verfügung stehen, ${ }^{466}$ sind regelmäßig gute Marktergebnisse zu erwarten. Die Anwendung anderer Kontroll-, Anreiz und Lenkungsmechanismen erscheint nur dann gerechtfertigt, wenn eine Marktlösung suboptimale Ergebnisse liefert. Beim Austausch interner Leistungen des Controlling soll so die Anwendung von Marktprinzipien, analog denen auf Absatz- und Beschaffungsmärkten, primär eine effiziente Allokation der zur Verfügung stehenden Ressourcen gewährleisten (Allokationsfunktion). Darüber hin-

\footnotetext{
461 Allgemein vgl. Meffert (2000), S. 28 ff.

462 Vgl. Kap. 3.4.1.1.

463 Vgl. Ludwig (1995), S. 127 ff.

464 Vgl. Berg (1992), S. 241.

465 Vgl. Siebke (1992), S. 64.

466 Zur Wettbewerbern des Controlling vgl. Kap. 3.4.2.
} 
aus erfüllt eine gute interne Marktversorgung bezogen auf das Controlling weitere Funktionen: ${ }^{467}$

- Steuerungsfunktion (Ausrichtung des Angebots an den Kundenpräferenzen),

- Innovationsfunktion (Förderung des produkt- und prozessbezogenen technischen Fortschritts),

- Anpassungsfunktion (flexible Reaktion auf Änderungen der Umfeldbedingungen),

- Verteilungsfunktion (Leistungsgerechte Einkommensverteilung),

- Kontrollfunktion (Transparenz des Leistungsaustausches).

Auch wenn derartige Argumente zunächst für eine weitgehende Implementierung interner Märkte sprechen, bereitet eine solche Vorgehensweise für das Controlling teilweise Schwierigkeiten. Die scheinbar hoheitliche Stellung des Controlling und der Kontrollcharakter einiger Leistungen verbunden mit der Pflicht, gewisse Leistungen in Anspruch zu nehmen, führt dazu, dass die Anreizmechanismen interner Märkte für Controlling-Leistungen als nur eingeschränkt effizient beurteilt werden müssen. Auch wenn daher die Bedingungen für die Bildung eines Profit Centers als nicht optimal angesehen werden können, erscheint eine Umsetzung möglich. ${ }^{468}$ Hierbei müssen die positiven Anreizeffekte der internen Marktorientierung potenziellen negativen Auswirkungen eines Kontrollverlustes gegenübergestellt werden. Ferner ist im Einzelfall zu überprüfen, inwieweit in einem Unternehmen überhaupt die Rahmenbedingungen für einen internen Markt gegeben sind (bspw. alternative interne oder externe Angebote), bzw. über welche institutionellen Regelungen derartige Bedingungen geschaffen werden können. ${ }^{49}$ In vielen Fällen ist es überwiegend die mangelnde Transparenz der Leistungen und Leistungserstellungsmodalitäten im Controlling, die zu einer Ablehnung marktorientierter Prinzipien führt. Insbesondere der institutionalisierten Leistungsabgrenzung („Produktdefinition“) und der Leistungsverrechnung kommt diesbezüglich eine besondere Bedeutung zu. ${ }^{40}$ In Cost Centern spielen gemeinschaftlich ausgehandelte Budgetvereinbarungen die zentrale Rolle. Wird ein Service Center demgegenüber als Profit

467 Bei den im Folgenden aufgeführten Funktionen handelt es sich ursprilinglich um die Funktionen des Wettbewerbs im Rahmen einer Volkswirtschaft. Vgl. Berg (1992), S. 241. Eine Ubertragung auf unternehmensinterne Tatbestände erscheint jedoch vertretbar.

468 Für die Kostenrechnung vgl. Währisch (1998), S. 338.

469 Vgl. hierzu den Anspruch von Service Level Agreements in Kap. 4.2.4.

470 Für den IT-Bereich vgl. Kargl (1998), S. 40 ff. 
Center ausgestaltet, müssen im Controlling Kostenumlagen und -verteilungen durch Kunden-Lieferanten-Beziehungen mit einer nutzungsadäquaten Verrechnung interner Leistungen substituiert werden. ${ }^{411}$ Neben der Preiskomponente müssen darüber hinaus insbesondere die Leistungen mittels Service-Verträgen hinreichend fixiert werden (Service Level Agreements), um Qualitäten auch vor dem Hintergrund externer Anbieter intersubjektiv überprüfen zu können. ${ }^{42}$

Zentraler Gestaltungsparameter auch für Controlling-Leistungen bleibt daher die Verrechnungspreisfrage, welche folglich im Rahmen der marketingrelevanten Organisationscharakteristika angemessene Berücksichtigung erfährt.

\subsubsection{Organisationsbezogene Gestaltungsparameter des kundenorien- tierten Controlling}

Nachdem die Organisation des Controlling und unterschiedliche Möglichkeiten einer ökonomischen Ausrichtung beschrieben wurden, sollen Auswirkungen dieser jeweils kontextspezifischen Rahmenbedingungen auf eine kundengerechte Leistungserstellung untersucht werden. Zu diesem Zweck werden, analog zur Leistungsbetrachtung, einzelne Gestaltungsparameter dargestellt, die es im Rahmen der kundenorientierten Leistungserstellung gedanklich zu durchdringen gilt, um darauf aufbauend geeignete Instrumente bewerten und einsetzen zu können.

\subsubsection{Leistungsinanspruchnahme}

Eine zentrale Fragestellung im Rahmen der Betrachtung von Vermarktungsmöglichkeiten innerbetrieblicher Leistungen ist die nach der Auswahlmöglichkeit der internen Kunden. ${ }^{43}$ Bezogen auf das Controlling ist zu klären, ob Leistungen aufgrund organisatorischer oder rechtlicher Konventionen in Anspruch genommen werden müssen (obligatorische Leistungen), oder ob diesbezüglich eine freie Wahlmöglichkeit der Kunden besteht (fakultative Leistungen). ${ }^{47}$ Darüber hinaus ist zu differenzieren, ob es sich um pauschale Konventionen bezüglich der Leistungsabnahmeentscheidung handelt oder aber Möglichkeiten der

\footnotetext{
471 Ein allgemeiner Erfahrungsbericht hierzu findet sich bei Busch/Nolken/Tesch (1999), S. 47 ff.

472 Vgl. Kap. 4.2.4.
}

${ }^{473}$ Die Auswahlmöglichkeit bezieht sich hier zunächst darauf, ob Leistungen in Anspruch genommen werden oder nicht. Die Wahl alternativer Anbieter fuir Controlling-Leistungen wird im Rahmen von Kap. 3.4.2 thematisiert.

474 Vgl. allgemein fur den Bereich der innerbetrieblichen Weiterbildungsleistungen Cramer $(1987)$, S. 8. 
Kunden bestehen, im Einzelnen auf Qualität $t^{75}$ und Quantität der Leistung Einfluss zu nehmen.

\subsection{Obligatorische Leistungen}

Charakteristikum obligatorischer Leistungen ist die Tatsache, dass die internen Kunden des Controlling aufgrund organisatorischer Vorgaben verpflichtet sind, bestimmte Leistungen abzunehmen. Rechtliche Tatbestände können darüber hinaus im Einzelfall zu der Notwendigkeit einer Inanspruchnahme bestimmter Leistungen führen, bspw. dann, wenn das Controlling bestimmte Informationen zur Erstellung eines Jahresabschlusses bereitstellen muss. ${ }^{476}$ Somit beeinflussen die Leistungsinanspruchnahmemöglichkeiten maßgeblich die Ausgestaltung interner Kunden-Lieferanten-Beziehungen des Controlling, da die Verpflichtung zur Leistungsinanspruchnahme regelmäßig der Etablierung interner Märkte widerspricht. Demgegenüber ist ein Vorteil obligatorischer Leistungen darin zu sehen, dass die Leistungsplanung für das Controlling erleichtert wird, da gewisse Mindestabnahmen dieser Leistungen gewährleistet sind. ${ }^{477}$ Beispielhaft wären hier zyklische Planungsleistungen zu nennen. ${ }^{478}$

Die Abgrenzung obligatorischer Leistungen von fakultativen Leistungen auf Basis organisatorischer Vorgaben vorzunehmen, erscheint aus formalen Gründen zweckmäßig. Demgegenüber sollte im Rahmen einer kundenorientierten Betrachtung die Wahrnehmung des Kunden zunächst ausschlaggebend sein. Bezogen auf das Controlling bedeutet dies, dass soweit eine Leistung aus Sicht des Kunden freiwillig in Anspruch genommen wird, da dieser sich vom Leistungsprozess oder Leistungsergebnis einen spezifischen Nutzen im Rahmen seiner Aufgabenerfüllung erwartet, diese auch seitens des Controlling als quasi fakultative Leistung behandelt werden sollte. Der Vorteil ist im wesentlichen darin zu sehen, dass sich das Spektrum intern marktfähiger Leistungen um eben diese quasi fakultativen Leistungen erweitert, da der Kunde diese freiwillig - auch unabhängig von ihrem formal obligatorischen Charakter - nachfragt. Darüber hinaus spielt die Leistungsevidenz in diesem Zusammenhang eine wesentliche

475 Die Qualităt der Leistung schließt hier die unterschiedlichen inhaltlichen Ausgestaltungsmöglichkeiten einer Leistung ein.

$476 \mathrm{Vgl}$. hierzu beispielhaft die in Abbildung 25 dargestellten Aufgaben der Kostenrechnung bei der Erstellung der Dienstleistung „Bestandsbewertung von Erzeugnissen“. Grundlegend zur externen Rechnungslegung vgl. Baetge/Kirsch/Thiele (2001).

477 Ausfuhrlich zur Leistungsplanung im Controlling vgl. Weber (1999), S. 372. Wrrt fuhrt die mangelnde Prognostizierbarkeit des Bedarfs als ein Charakteristikum interner Dienstleister an. Vgl. Witt, (1985), S. 162.

478 Übersichtartig lassen sich derartige Leistungen in Form eines Controllingkalenders darstellen. Vgl. ZVEI (1993), S. 92. 
Rolle. ${ }^{479}$ Eine Leistung ist aus der Perspektive des Nachfragers nicht evident, wenn dieser die Einsatz bzw. Nutzungsmöglichkeiten dieser Leistung nicht kennt. ${ }^{480}$ Bezogen auf das Controlling ist dies ein besonders relevanter Tatbestand, da die Komplexität der Leistungen oftmals zu einer unsachgemäßen Nutzung oder mangelnden Inanspruchnahme verleitet. ${ }^{481}$ Betrachtet man nun formal obligatorische Leistungen aus der Perspektive des Kunden, so kann eine Steigerung der Leistungsevidenz, bspw. durch Erläuterung oder Beratung des Controllers zu einer Wahrnehmungsveränderung des Kunden führen. Nimmt dieser die Leistung dann als für sich nützlich wahr, ist es denkbar, dass er diese obligatorische Leistung zukünftig auch freiwillig in Anspruch nehmen wird, soweit eine Verpflichtung entfiele. Diesen Sachverhalt visualisiert Abbildung 23.

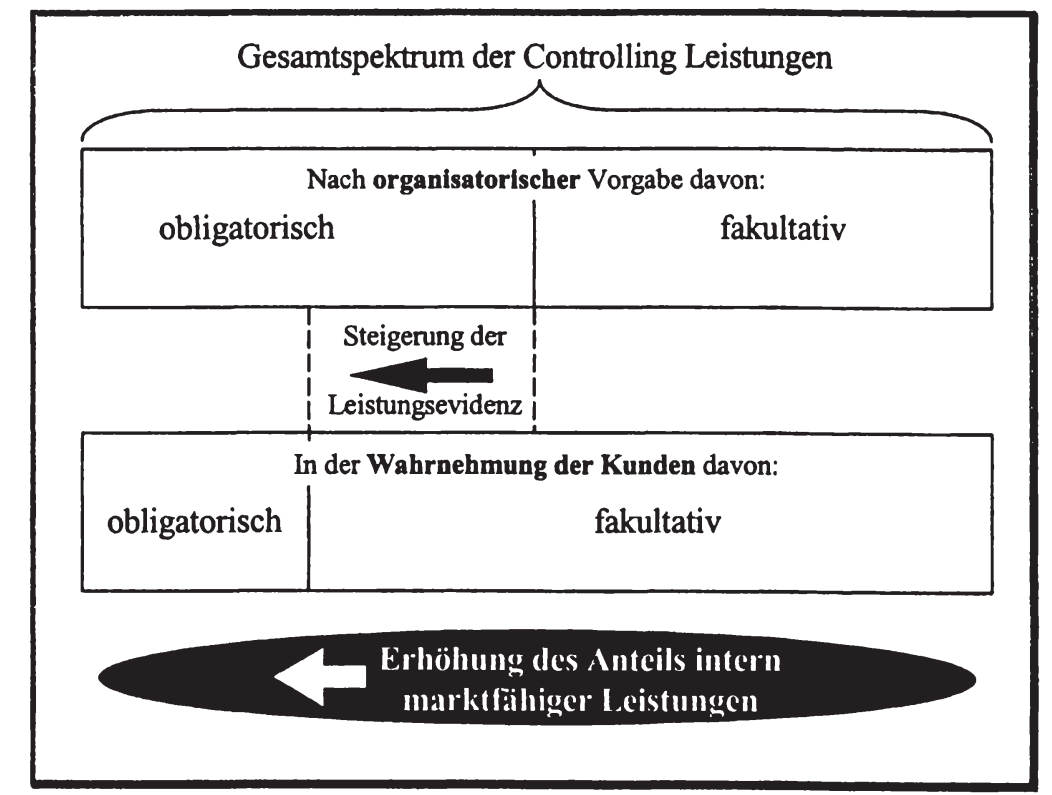

Abbildung 23: Verbesserung der Leistungsinanspruchnahme im Controlling

Eine derartige Betrachtung macht deutlich, dass über eine entsprechende Transparenz des Leistungsangebotes und der Leistungsinhalte die Wahrnehmung der

479 Zur Leistungsevidenz vgl. Witt (1985), S. 167. Ausfuhrlich zur Leistungsevidenz im Controlling vgl. Kap. 3.4.3.2.

480 Vgl. Engelhardt/Schwab (1982), S. $506 \mathrm{ff}$.

481 Beispielhaft zur Nutzung von Kostenrechnungsinformationen vgl. Homburg/Weber/Aust/Karlshaus (1998), S. 37. 
Nachfrager maßgeblich beeinflusst werden kann. ${ }^{482}$ Über den Einsatz geeigneter Instrumente können somit obligatorische Leistungen zugunsten bewusst abgenommener und damit tendenziell marktfähiger Leistungen verringert werden.

Ergänzend sei an dieser Stelle auf die Ausführungen zu den Unterstützungsbzw. Kontrollleistungen hingewiesen. ${ }^{483}$ Insbesondere Kontrollleistungen mit Verhaltenssteuerungsfunktion fallen im Controlling regelmäßig unter die obligatorischen Leistungen. Soweit diese - wie beschrieben - im Kontext eines umfassenden Entscheidungsunterstützungsprozesses gesehen werden, in dem insgesamt der Unterstützungscharakter des Controlling dominiert, ist eine bewusst freiwillige Inanspruchnahme seitens des Kunden denkbar.

Neben der grundsätzlichen Überlegung, ob eine Leistung obligatorisch oder fakultativ ist, ergibt sich oftmals auch bei obligatorischen Leistungen ein bedingter Spielraum bezüglich der quantitativen und qualitativen Leistungsnachfra$g e{ }^{484} \mathrm{Im}$ Controlling spielt die quantitative Nachfrage einer Leistung regelmäßig keine so entscheidende Rolle, da bei obligatorischen Leistungen entweder die Abnahmemenge gleichermaßen fixiert ist (bspw. institutionalisierte Planungsabläufe) oder es sich aber beim Leistungsoutput um reine Informationen handelt (bspw. Standardberichte), die vom Anbieter oder Nachfrager mit wenig Aufwand vervielfältigt werden können. Demgegenüber ist bei integrativ erstellten obligatorischen Leistungen das Leistungsergebnis, allein schon durch die Einbeziehung externer Faktoren des Kunden, variabel ${ }^{485}$ und damit auch formal nicht vorherbestimmbar. Über eine sachgerechte Einbindung des Kunden in den Leistungsprozess kann eine Akzeptanzsteigerung erreicht werden. Nicht eindeutig zu klären ist jedoch, inwieweit den Kunden des Controlling allein durch die Bereitstellung ihrer externen Faktoren der Eindruck einer verpflichtenden Leistungsinanspruchnahme genommen werden kann.

Die Zielsetzung für alle obligatorischen Leistungen sollte vielmehr in einer Steigerung der Leistungs- und damit auch Bedarfsevidenz gesehen werden. Eine Verdeutlichung der Sinnhaftigkeit der Leistungsinanspruchnahme kann im wesentlichen über eine Darstellung der Zusammenhänge zwischen Leistung und Kunden- bzw. Unternehmenszielen erreicht werden. Langfristige Perspektive für das Controlling könnte die weitgehende Zurückdrängung organisatorischer

\footnotetext{
482 Allg. vgl. Witt (1988), S. 660 ff.

${ }^{483}$ Vgl. Kap. 3.2.2.3.

484 Vgl. Cramer (1987), S. 8.

485 Zur Integration externer Faktoren in den Leistungserstellungsprozess des Controlling vgl|Ka Ka 3 3.2.2.1.
} 
Regelungen der Leistungsinanspruchnahme zugunsten einer auf Transparenz beruhenden, bewusst freiwilligen Inanspruchnahme sein. Ersichtlich ist, dass diese Zielsetzung ein entsprechendes Verantwortungsbewusstsein der Mitarbeiter erfordert, nichts anderes wird jedoch bei dem an Bedeutung gewinnenden Self-Controlling vorausgesetzt. ${ }^{486}$

\subsection{Fakultativ}

Auf Basis der Ausführungen zu den obligatorischen Leistungen soll im Folgenden der Gestaltungsspielraum für fakultative Leistungen diskutiert werden. Fakultative Leistungen im Controlling sind solche Leistungen, für die kein Nachfragezwang besteht. ${ }^{487}$ Es hängt im Einzelfall von dem individuellen KostenNutzen-Verhältnis des Nachfragers ab, ob er eine Leistung in Anspruch nimmt oder nicht. Für den Controllerbereich bedeutet dies, dass zum Absatz derartiger Leistungen eine aktive Beeinflussung der Kunden zweckmäßig sein kann. Hintergrund sollte dabei primär die inhaltliche Aufgabenerfüllung des Controllerbereichs sein, erst sekundär die Erreichung der aus der ökonomischen Ausrichtung resultierenden Erfolgsbeurteilungsmaßstäbe. ${ }^{488}$

Wie bereits dargestellt, ist einer der wesentlichen Anknüpfungspunke der kundenorientierten Leistungserstellung die Steigerung der Bedarfsevidenz. Beispielhaft kann dem Kunden veranschaulicht werden, dass zur Fundierung einer bestimmten Entscheidung eine spezifische Auswertung erforderlich ist. Der Kunde, der bis zu diesem Zeitpunkt die Möglichkeit einer derartigen Auswertung vielleicht gar nicht kannte, kann nun auf Basis der erläuternden Ausführungen des Controllers feststellen, dass eine derartige Leistung für ihn nützlich ist. Erst jetzt besteht die Möglichkeit, die Nachfrage zu artikulieren und somit die fakultative Leistung anzufordern. Damit es zu keiner unangemessenen Beanspruchung fakultativer Leistungen kommt, bedarf es eines Regulatives. In Abhängigkeit der Ausprägung interner Marktverhältnisse können Verrechnungspreise mit unterschiedlichen Anreizwirkungen angesetzt werden, ${ }^{489}$ um die Überbeanspruchung des Controllings zu vermeiden. Hintergrund ist die implizite Kosten-Nutzen-Beurteilung des Nachfragers, welche zwangsläufig einer Operationalisierung der Kostenkomponente - hier in Form von Verrechnungs-

\footnotetext{
486 Zum Self-Controlling vgl. ausfuihrlich Horváth (1998), S. 835 ff.

487 Hier und im Folgenden vgl. Cramer (1987), S. 8.

488 Zur ökonomischen Ausrichtung vgl. Kap. 3.3.1.2.
}

489 Allg. zu Verrechnungspreisen vgl. Wagenhofer (1998), S. 23 ff. sowie bezogen auf das Controlling Kap. 3.3.2.5. 
preisen - bedarf. Fakultative Leistungen weisen daher eine tendenziell hohe interne Marktfähigkeit auf, soweit anreizkonforme Verrechnungspreise bestehen. Darüber hinaus wird die Marktfähigkeit wesentlich durch die Möglichkeit, auf alternativen Angebote auszuweichen zu können, determiniert, was im Rahmen der Konkurrenzbetrachtung thematisiert werden wird. ${ }^{490}$

Neben der grundsätzlichen Erläuterung der Leistungsinanspruchnahme stellt sich insbesondere bei fakultativen Leistungen des Controlling die Frage nach der quantitativen und qualitativen Variabilität der Leistungen. Grundsätzlich ist eine umfassende Variabilität bei fakultativen Leistungen gegeben. Bezogen auf den Leistungserstellungsprozess des Controlling ergibt sich diesbezüglich ein in der Praxis nicht unerhebliches Prognoseproblem. ${ }^{491}$ So ist bspw. die Bedarfsplanung von Kosteninformationen, Projektierungshilfen oder Beratungstätigkeiten teilweise mit großer Unsicherheit behaftet, insbesondere aufgrund der von Kunde zu Kunde potenziell unterschiedlichen Qualitäts- bzw. Inhaltsanforderungen ${ }^{492}$ Aus diesem Grund spielen die Abrufmodalitäten für eine zeitgerechte und nachfragerorientierte Bereitstellung von Controlling-Leistungen eine wichtige Rolle.

\subsubsection{Abrufmodalitäten}

Nachdem die Leistungsinanspruchnahmemodalitäten in ihren organisatorischen Kontext eingebunden wurden, soll nunmehr auf die Abrufmodalitäten eingegangen werden. Bei den Abrufmodalitäten handelt es sich um eine Spezifikation der Leistungsinanspruchnahme über die Grundsatzentscheidung "freiwillige oder verpflichtende Inanspruchnahme" hinaus. Bezogen auf das Controlling bestehen in Abhängigkeit des Unternehmenskontextes jeweils spezifische Regelungen bezüglich der Abrufmodalitäten einzelner obligatorischer bzw. fakultativer Leistungen. Im Einzelnen sind hier beispielhaft zu nennen: ${ }^{493}$

- Abrufdauer/Lieferzeit,

- Hierarchische Hemmnisse bei der Nachfrage,

490 Vgl. Kap. 3.4.3.4.

491 Vgl. zu den diesbezilglichen Charakteristika innerbetrieblicher Leistungen Witt (1985), S. 162 ff.

492 WITT nennt Kosteninformationen und Projektierungshilfen explizit als Leistungen bei den Prognoseprobleme auftreten können. Vgl. Witt (1985), S. 164.

493

Im Rahmen einer Untersuchung von unternehmensinternen Dienstleistungstypen hat WrTt einen Katalog vermarktungsrelevanter Eigenschaften erstellt. Mittels einer Faktorenanalyse extrahiert er sieben vermarktungsrelevante Faktoren. Die hier aufgefuhrten Eigenschaften sind Bestandteile des Faktors "Abrufmodalitäten“. Vgl. Witt (1988), S. 664. 
- Abrufbarkeit individuell durch einen einzelnen Mitarbeiter,

- Abrufbar nur durch den Leiter einer Organisationseinheit,

- Formalisiertheit des Abrufs,

- Angebot nur auf Abruf,

- Vorgesetzteneinschaltung ob Abruf oder Angebot erfolgen darf,

- Pflicht der Leistungsabgabe bzw. -anforderung.

Kundenseitig wurde die grundlegende Pflicht der Leistungsanforderung bereits diskutiert. Nachfolgend werden darüber hinaus Spezifikationen bezüglich des Ablaufes und der Binnenorganisation des Controlling vorgenommen. ${ }^{494}$ Analog dazu besteht für gewisse Leistungen eine allgemeine Leistungsabgabepflicht des Controlling, unabhängig von der Artikulation einer Nachfrage. Insbesondere im Bereich von Kontrollleistungen ist dies zu beobachten. Neben derart grundsätzlichen Regelungen stellt sich bei den anderen genannten Sachverhalten die Frage, inwieweit im Sinne einer Kundenorientierung des Controlling, diesbezügliche Regelungen formalisiert werden sollten. Ziel derartiger Regelungen sollte es sein, den Leistungsaustausch transparenter und anreizkonformer zu gestalten. Demgegenüber werden die in diesem Bereich unternehmensintern bestehenden Regeln oftmals als übertrieben formalisiert angesehen. ${ }^{495} \mathrm{Im}$ Gegensatz zu den für unternehmensexterne Märkte geltenden Beschaffungsrichtlinien, die im Sinne einer Absicherung des Unternehmens verstanden werden, fehlt für interne Regelungen meist die Akzeptanz. Von Bedeutung für die Kunden des Controlling ist daher, derartige Regelungen auf solche zu beschränken, die einerseits eine Leistungsplanung im Controlling ermöglichen, andererseits insbesondere die Leistungsinanspruchnahme durch Transparenzund Evidenzsteigerung fördern.

Zielsetzung muss demnach sein, durch entsprechende Leistungsvereinbarungen, ${ }^{496}$ die die Abrufmodalitäten beinhalten, Rahmenbedingungen für die Funktionsfähigkeit interner Märkte für Controlling-Leistungen zu schaffen. Im Idealfall ersetzt das Marktregulativ dann umfassendere formale Regelungen. Soweit die Bedingungen für den Austausch von Leistung und Gegenleistung hinreichend fixiert sind, ist es dann dem individuellen Kosten-Nutzen-Kalkül des einzelnen Mitarbeiters überlassen, ob, wann und in welchem Umfang er Leistungen bezieht. Zuvor ist jedoch die Grundsatzentscheidung zu treffen, welche Leistungen dem internen Marktmechanismus auszusetzen sind. Für intern

\footnotetext{
494 Vgl. Kap. 3.3.2.1.1.

495 Hier und im Folgenden vgl. Witt (1988), S. 670.
}

496 Zur Ausgestaltung von Leistungsvereinbarungen vgl. Kap. 4.2.4. 
marktfähige Leistungen sind dann aus den genannten Items besonderes die Abrufinitiative, die Formalisiertheit des Abrufes sowie die Abrufzeit/Lieferdauer zu spezifizieren.

Die Abruf- bzw. Angebotsinitiative sollte sowohl dem Controller als auch seinem internen Kunden freigestellt werden, da in beiden Sphären Sachverhalte auftreten können, die es erforderlich machen, Entscheidungsunterstützungsaktivitäten anzubieten bzw. anzufordern. Im Rahmen des fortschreitenden Einsatzes interaktiver und integrierter Reporting-Systeme wird, wie in Abbildung 24 dargestellt, insbesondere im Berichtwesen die Bringschuld des Controllers zunehmend in eine Holschuld des Managements transformiert.

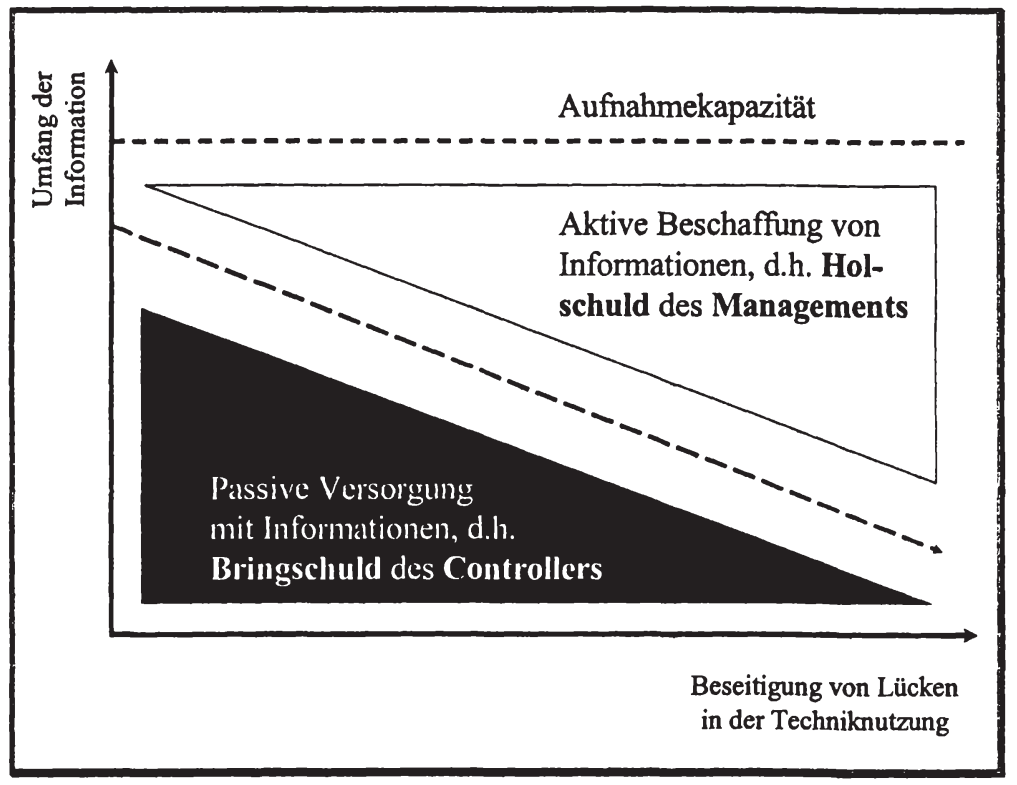

Abbildung 24: Bringschuld des Controllers ${ }^{497}$

Soweit Informationen über Client Server Systeme bereitgestellt werden, kann der Kunde individuelle Informationsanfragen komfortabel durchführen, ohne den Controller im Einzelfall zu beanspruchen. Vorteilhaft an einer derartigen Vorgehensweise sind die realisierbare Zeitersparnis sowie die potenzielle Steigerung der Bedarfsgerechtigkeit. Erfolgskritisch ist in diesem Zusammenhang 
das Nutzungsverhalten der Kunden zu steuern. ${ }^{498}$ Insbesondere der Verzicht auf die persönliche Interaktion bedingt oft eine zu geringe oder unsachgemäße Nutzung der durch das Controlling bereitgestellten Informationen. Insgesamt wird daher die Institutionalisierung einer Durchsprache von Berichten als eine der wesentlichen Anforderungen an das Controlling genannt, die derzeit zu wenig erfüllt wird. ${ }^{49}$

Aus der Perspektive des Marketing ist die in Abbildung 24 dargestellte Wandlung der Bring- in eine Holschuld im Sinne einer Abkehr von der Push-zur Pull-Strategie zu verstehen..$^{500}$ Gemeint ist hiermit die Art der Nachfragerbeeinflussung bei mehrstufigen Vertriebswegen. ${ }^{501}$ Bezogen auf die Informationsbereitstellung des Controlling muss beim Einsatz von Medien wie z.B. dem Intranet die Beeinflussung direkt beim Nachfrager ansetzen, d.h., dieser muss angemessen motiviert werden, die über das Netz abrufbaren Berichte anzufordern und zu nutzen (Pull-Strategie). Demgegenüber verfolgt man vielfach noch die Push-Strategie, im Sinne einer angebotsorientierten Verteilung oftmals zu umfangreicher und wenig zielgruppengerecht strukturierter Informationen. ${ }^{502}$

Die Formalisiertheit des Abrufes von Controlling-Leistungen soll hier weniger unter den bereits angesprochen organisatorischen bzw. hierarchischen Aspekten diskutiert werden. Vielmehr ist für die Kundenorientierung im Controlling die Frage nach notwendigen Mindestformalia wesentlich, die beiden Transaktionspartnern eine überschaubare Leistungsplanung und -abnahme garantieren sollen. Einerseits kann das Controlling durch gewisse formalisierte Regelungen seinen Kapazitätsvorhaltepflichten effizienter nachkommen. ${ }^{503}$ Andererseits kann aus Sicht der Nachfrager die oftmals subjektiv wahrgenommene Unsicherheit bzgl. des Leistungsprozesses und Leistungergebnisses reduziert werden. Im Einzelfall sind daher die Abrufmodalitäten des Controlling zu spezifizieren. Beispielhaft können Vereinbarungen bzgl. nachfolgender Tatbestände getroffen werden: ${ }^{504}$

498 Vgl. auch Kap. 3.4.3.3.

499 Dies ist eines der wesentlichen Ergebnisse eines Benchmarking des Berichtswesens. Vgl. Stadler/Weißenberger (1999), S. 9.

500 Die Pull- bzw. Push-Strategien sind Ausprägungen mehrstufiger Absatzstrategien. Allg. vgl. Kleinaltenkamp/FlieB (1995), S. 1011 f.

$501 \mathrm{Zu}$ den Besonderheiten mehrstufiger Absatzstrategien vgl. Kleinaltenkamp/Fließ (1995), S. $1007 \mathrm{ff}$.

502 Vgl. hierzu die Diskussion um die Bereitstellung von „Zahlenfriedhöfen“, z.B. bei Weber/Schăffer (1999), S. $17 \mathrm{ff}$.

503 Vgl. Witt (1985), S. 163.

504 Dies entspricht im wesentlichen den im Rahmen von Service Level Agreements spezifizierten Tatbeständen. Vgl. beispielhaft Ericsson (2000b), S. $54 \mathrm{ff}$. 
- Anwesenheits-/Bearbeitungszeiten des Controlling,

- Ansprechpartner (in Abhängigkeit des Leistungsinhalts),

- Antwortzeiten bei Anfragen,

- Mitwirkungspflichten der Controlling-Kunden (Informationsbereitstellung),

- Eskalationsregelungen,

- Standard-Leistungsmengen,

- Standard-Qualitäten.

Die nachfolgend in Kap. 4.2.4. zu diskutierenden Service Level Definitions und Service Level Agreements stellen geeignete Instrumente zur Operationalisierung derartiger Faktoren dar. Neben den hier dargestellten Abrufmodalitäten finden Regelungen bzgl. der konkreten Leistungsinhalte sowie ihrer Verrechnung Eingang in derartige Leistungsverträge.

Die hier angesprochenen Bearbeitungs-, Anwesenheits- und Lieferzeiten deuten bereits darauf hin, dass die Leistungen des Controlling in sehr unterschiedlichen Umfang einen zeitlichen Bezug zu den Tätigkeiten ihrer Kunden aufweisen. Daher erscheint es zweckdienlich, die zeitlichen Interdependenzen zwischen den Leistungen des Controlling und den Aufgaben ihrer Kunden zu strukturieren.

\subsubsection{Ablaufcharakteristika}

Im Rahmen der Ablaufcharakteristika soll eine Typologisierung von Controlling-Leistungen in Abhängigkeit ihrer Einbindung in die unternehmerischen Abläufe erfolgen. Aufbauend auf den Überlegungen bezüglich der Leistungsinitiative bedeutet dies für das Controlling, seine Leistungen in Verbindung zu vor- und nachgelagerten Aktivitäten zu betrachten.

Diesbezüglich ist zwischen sequenzintegrierten und sequenzunabhängigen Dienstleistungen des Controlling zu unterscheiden..$^{505}$ Auf Basis einer derartigen Abgrenzung ist zu klären, ob sich Maßnahmen der Kundenorientierung in Abhängigkeit der gewählten Leistungstypen unterscheiden müssen.

505 Vgl. allgemein für interne Dienstleistungen Davis (1992), S. 5 ff. sowie Epelman (1994), S. 1 ff. Speziell zu den Begriffen vgl. Stauss/Neuhaus (1995), S. $585 \mathrm{ff}$. 


\subsection{Sequenzintegrierte Leistungserstellung}

Sequenzintegrierte Dienstleistungen sind dadurch charakterisiert, dass sie Bestandteil eines in einer relativ festen Reihenfolge ablaufenden Prozesses sind..$^{506}$ Das Prozessergebnis hängt daher maßgeblich auch von den erbrachten Leistungen vor- und nachgelagerter Transaktionspartner sowie der spezifischen Kooperation (Schnittstellenmanagement etc.) mit diesen ab. Bezogen auf die Flexibilität der Abrufmodalitäten sequenzintegrierter Leistungen ergeben sich Einschränkungen, da die Einbindung derartiger Leistungen in geregelte Abläufe zeitliche Vorgaben bzw. Abstimmungen erfordert.

Als Beispiel für die Abteilung Personalmanagement wird die verwaltungstechnische Abwicklung der Personalbeschaffung als eine typische sequenzintegrierte Dienstleistung genannt. ${ }^{507}$ Auf Basis einer Bedarfsmeldung und eines spezifischen Anforderungsprofils werden Anzeigen geschaltet, Bewerbungen entgegengenommen, der Auswahlprozess durchgeführt und schließlich eine Einstellung vollzogen. Derartige Prozesse folgen einem nahezu standardisierten $A b$ lauf. Ausgehend von dem dem Controlling zugrundegelegten Leistungsraum, finden sich beispielhaft in der Informations- und Interaktionsdimension Leistungen, die auf die Gestaltung und den Betrieb von Informationssystemen ausgerichtet sind. ${ }^{508}$ In diesen Bereich fällt exemplarisch der Betrieb der Kostenrechnung, sowie darauf aufbauende zielgerichtete Auswertungen. Ein Großteil der im Rahmen der Kostenrechnung erbrachten Dienstleistungen lassen sich wie die in Abbildung 25 dargestellte Leistung - als Workflow-Leistungen bzw. sequenzintegrierte Dienstleistungen ansehen. ${ }^{509}$

In der Grundstruktur erhält die Kostenrechnung Informationen aus vorgelagerten Informationssystemen, die sie in Abhängigkeit ihrer Ausgestaltung verdichtet bzw. aufbereitet, um sie dann nachgelagerten Informationssystemen oder einzelnen Nachfragern zur Verfügung zu stellen. ${ }^{\text {s10 }}$ Für die Dienstleistung ,Bestandsbewertung von Erzeugnissen" stellt sich die Sequenzintegration der Kostenrechnung wie in Abbildung 25 aufgezeigt dar. Eine derartige Einbindung impliziert, dass im Sinne einer kundenorientierten Leistungserstellung insbe-

\footnotetext{
506 Hier und nachfolgend vgl. Davis (1992), S. 9 ff.

507 Diese Betrachtung erfolgt im Rahmen der typologischen Zuordnung von Personaldienstleistungen. Vgl. Stauss/Neuhaus (1995), S. $883 \mathrm{f}$.

508 Vgl. Kap. 3.2.1.4.

${ }^{509} \mathrm{Zu}$ der inhaltlichen Aussage vgl. Wahrisch (1998), S. 333. Zu dem Verhaltnis bzw. der Begriffsidentităt von Workflow und sequenzintegrierten Leistungen vgl. Neuhaus (1996), S. 50.

510 Vgl. hierzu das Grundschema kostenrechnerischer Workflow-Leistumgen van Wrhthisch (1998), S, 333.
} 
sondere dem Schnittstellenmanagement und einer transparenten Prozessgestaltung besondere Bedeutung zukommt. ${ }^{\text {s11 }}$

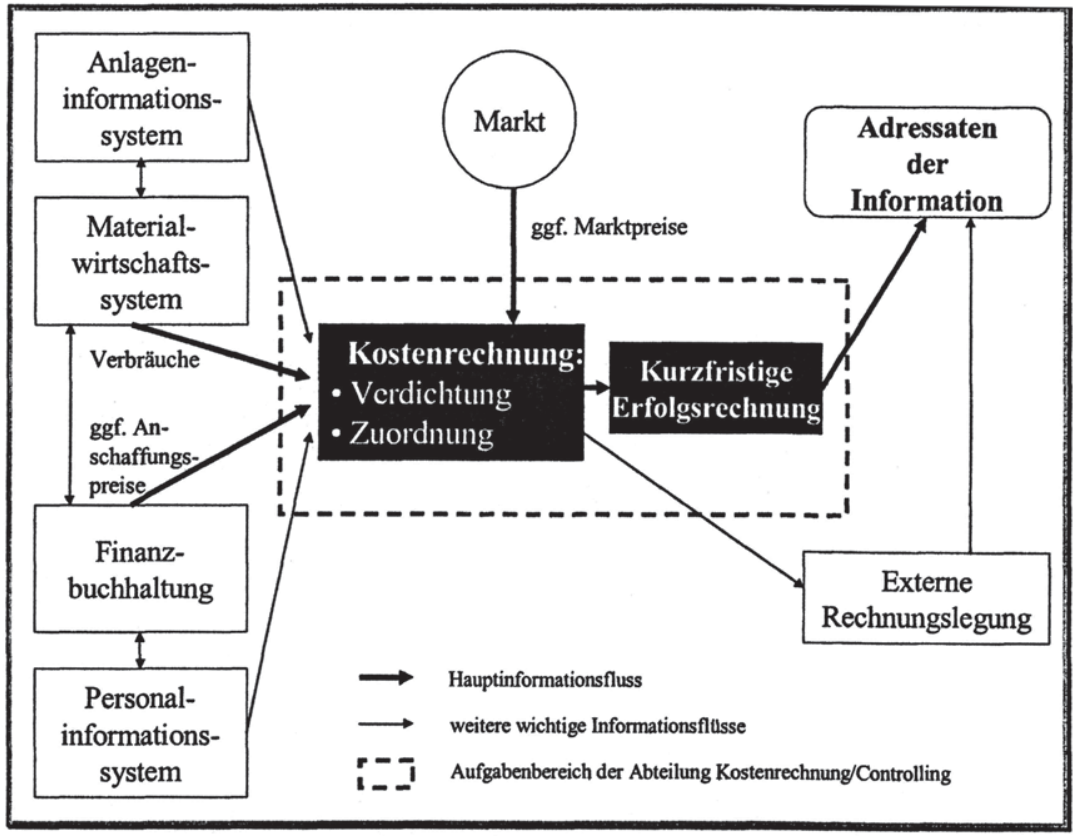

Abbildung 25: Informationsfluss und beteiligte Informationssysteme bei der Erstellung der Dienstleistung „Bestandsbewertung von Erzeugnissen "\$12

Für das interne Marketing von Controlling-Leistungen ist eine Unterscheidung nach der Sequenzabhängigkeit zweckmäßig, da die Anknüpfungspunkte für Maßnahmen sehr unterschiedlich sind. Dem zunächst betrachteten Beispiel der Bestandsbewertung - im Sinne einer Dokumentation - liegt eine systematischtechnische Ursache für die Sequenzabhängigkeit zugrunde. Darüber hinaus gibt es zahlreiche Controlling-Leistungen im Planungs-, Prognose- und Kontrollbereich, die synchron zur Ablauforganisation der Führung und damit sequenzintegriert erfolgen müssen. Diesbezüglich ist es erforderlich, die Ablauforganisation des Controlling in zeitlicher Relation zu der seiner Kunden zu betrachten. ${ }^{513}$ Insbesondere die Erkenntnis, dass die Zeit ein wesentlicher erfolgskritischer

511 Vgl. Stauss/Neuhaus (1995), S. 585.

512 Wahrisch (1998), S. 333.

513 Derartige Betrachtungen nehmen in der Literatur im Vergleich zu Analysen der Aufbauorganisation einen sehr geringen Stellenwert ein. Vgl. Horváth (1998), S. 830 f. 
Faktor der Leistungserstellung des Controlling ist, hat zu einer Bedeutungszunahme der Prozessorientierung auch innerhalb des Controlling geführt. ${ }^{514}$ In dieser Hinsicht gilt es, die Ablauforganisation des Controlling, die die Erstellung spezifischer Leistungen zum Ergebnis hat, den inhaltlichen und zeitlichen Erfordernissen der Kunden anzupassen.

Ein Instrument zu Operationalisierung der zeitlichen Interdependenzen zwischen Führung und Controlling ist der Controlling Kalender. Ein beispielhafter Controlling Kalender ist in Abbildung 26 dargestellt. Über eine jahresbezogene Abstimmung der Controlling-Aktivitäten in Form eines Controlling Kalenders hinaus, versucht man derzeit auch im Controlling der zunehmenden Prozessorientierung der gesamten Unternehmensorganisation Rechnung zu tragen. ${ }^{\text {Is }}$

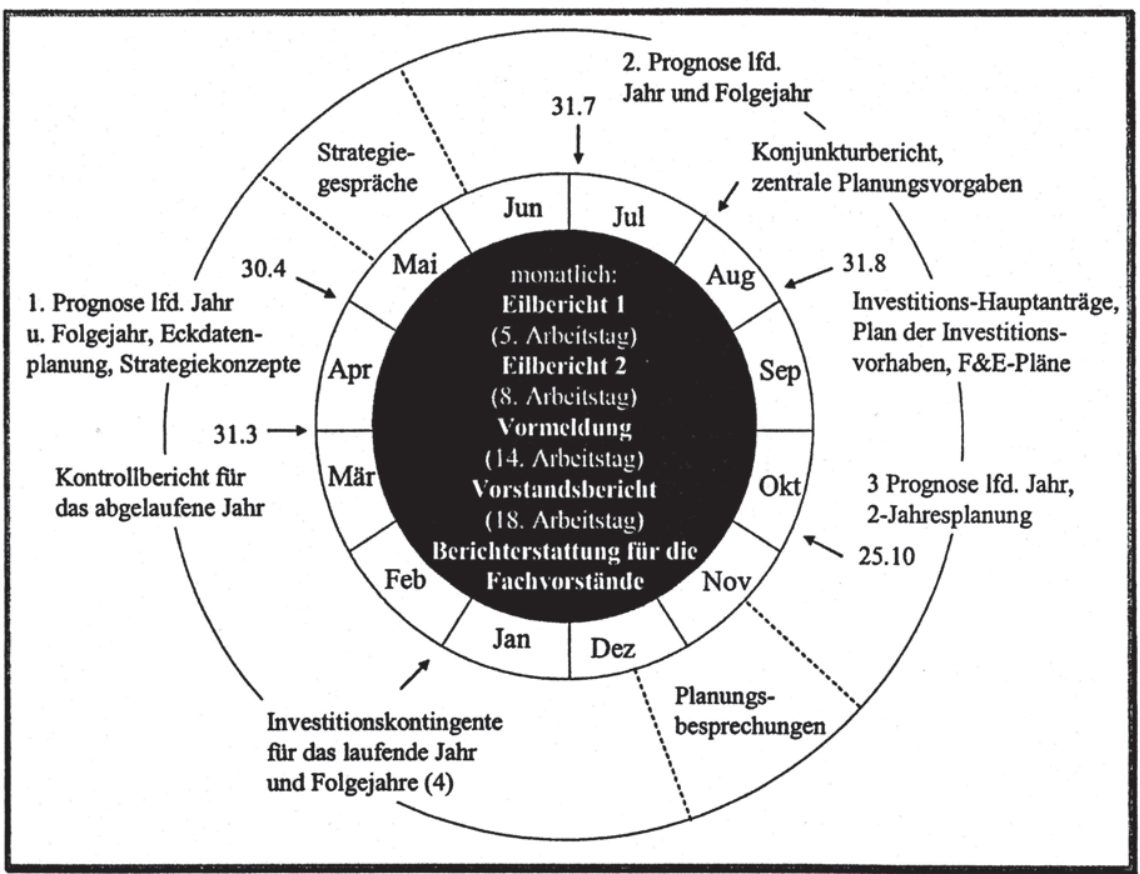

Abbildung 26: Beispiel eines Controlling Kalenders ${ }^{516}$

\footnotetext{
514 Ausführlich zu der Diskussion, inwieweit ein diesbezuglicher Paradigmenwechsel im Controlling stattgefunden hat vgl. Fischer (1996), S. 222 ff.

S15 Hier und im Folgenden vgl. Horváth (1998), S. 831 ff.

S16 ZVEI (1993), S. 92.
} 
Controlling-Leistungen müssen sich inhaltlich entlang der Geschäftsprozesse ihrer Kunden orientieren und zeitlich sequenzintegriert erfolgen. In hierarchischen Systemen vorherrschende vertikale Regelkreise werden somit durch prozessbegleitende horizontale Regelkreise ersetzt. ${ }^{517}$ Ein derartiger Wandel in der Ausrichtung kann wesentliche Auswirkungen auf das Leistungsspektrum des Controlling haben, insbesondere auch aufgrund der Tatsache, dass sich hierdurch gleichsam die Kundenstruktur ändern kann. Kunden des Controlling werden in zunehmenden Maße Prozessteams, weniger die hierarchisch strukturierte Führung. ${ }^{518}$

Insgesamt ergibt sich für die kundenorientierte Bereitstellung von sequenzintegrierten Controlling-Leistungen die Notwendigkeit, die internen Abläufe im Controlling mit den abteilungsexternen Prozessen zu synchronisieren. Regelmäßig erfolgt dies bei Kontrollleistungen, da diese in vielen Fällen entlang des Entscheidungsprozesses des Managements institutionalisiert erfolgen und damit sequenzintegrierten Charakter aufweisen. Darüber hinaus kommt bei anderen Leistungen insbesondere dem Management der vielfältigen technischen, persönlichen und inhaltlichen Schnittstellen eine besondere Bedeutung zu.

Ein Ansatz zur Lösung derartiger Probleme bietet das TQM. Wie bereits dargestellt, ${ }^{519}$ erfordert die Maxime des TQM - im Sinne von „The Next Process Is Your Customer" - auch für interne Dienstleister ein konsequent kundenorientiertes Prozessmanagement. ${ }^{520}$ Diesbezügliche Ziele für den Controllerbereich sind: ${ }^{521}$

- die Ausrichtung der Controlling-Geschäftsprozesse auf die Entscheidungsprozesse des Managements,

- die interne Durchsetzung eines kundenorientierten Denkens,

- der Abbau von Interabteilungskonflikten,

- die klare Festlegung von Verantwortlichkeiten,

- die rationelle Abwicklung von Leistungsprozessen und

- die Verkürzung von Durchlaufzeiten.

\footnotetext{
$\$ 17$ Vgl. Fischer (1996), S. 229.

518 Vgl. Horváth (1998), S. 833 sowie ausfuihrlicher zur Struktur der Kunden des Controlling Kap. 3.4.1.2.

$519 \mathrm{Vgl}$. hierzu die Ausführungen zum abteilungsorientierten internen Marketing in Kap. 2.2.2.2.2. Der TQMAnsatz bildet hierfü einen konzeptionellen Rahmen.

$520 \mathrm{Vgl}$. Stauss/Neuhaus (1995), S. 585.

521 Abgewandelt für das Controlling auf Gundlage der Ausfuhnungen von Stauss: Neuhaus (1995), S. 585.
} 
In Verbindung mit den anderen bereits dargestellten gestaltungsrelevanten Faktoren gilt es daher, die durch den sequenzintegrierten Leistungscharakter bedingten Abstimmungen in Form von Leistungsvereinbarungen zu spezifizieren. Insbesondere der grundsätzliche Ablauf der Leistungserstellung, die hierfür benötigten Teilphasen sowie die Übergabemodalitäten an Schnittstellen sind im Rahmen der Vereinbarungen zu konkretisieren.

\subsection{Sequenzunabhängige Leistungserstellung}

Sequenzunabhängige Dienstleistungen zeichnen sich dadurch aus, dass sie zeitlich unabhängig von organisatorischen Prozessen oder Abläufen erbracht werden können. ${ }^{522}$ Soweit Controlling-Leistungen also nicht in eine Prozesskette einbezogen sind, ergibt sich sowohl für den Anbieter als auch für den Nachfrager die Möglichkeit, individuell Einfluss auf die Abrufmodalitäten zu nehmen. ${ }^{523}$ Darüber hinaus können Quantität und Qualität kontextspezifisch festgelegt werden, da die Leistungen oft wenig standardisiert sind. ${ }^{524}$ Die Initiative zur Leistungserstellung kann sowohl vom Controlling als auch vom Kunden ergriffen werden. Besteht keine ausreichende Evidenz der Leistung, sollte der Controller Maßnahmen im Sinne der dargestellten Pull-Strategie ergreifen. Regelmäßig sollte das auslösende Moment zur Erstellung einer sequenzunabhängigen Leistung die Artikulation eines Kundenbedarfs sein. Insbesondere die Individualität der jeweiligen Bedürfnisse erfordert aufgrund des geringen Standardisierungsgrades eine einzelfallbezogene Leistungserstellung. Beispiele für sequenzunabhängige Leistungen im Controlling sind Sonderberichte und -analysen, die Beantwortung materieller und DV-technischer Einzelfragen, Schulungsmaßnahmen oder Aktivitäten zur Weiterentwicklung der Informationssysteme. ${ }^{25}$ Bei solchen sequenzunabhängigen Leistungen des Controlling besteht grundsätzlich die Gefahr einer zu angebotsorientierten Sichtweise des Controllers. Im Vergleich zu sequenzintegrierten Leistungen kann der fehlende regelmäßige Bezug zu vor- und nachgelagerten Transaktionspartnern zu einem zu sehr produktorientierten Leistungsverständnis führen, ${ }^{526}$ welches oftmals nicht den eigentlichen Bedürfnissen der Nachfrager entspricht. Bezogen auf die noch zu thematisierende Konkurrenzgefährdung ${ }^{\text {s27 }}$ lässt sich an dieser Stelle bereits fest-

522 Der Begriff der sequenzunabhăngigen Dienstleistung orientiert sich an den von EPELMAN dargestellten „offstream services". Vgl. Epelman (1994), S. 1 ff.

$\$ 23$ Vgl. Kap. 3.3.2.2.

524 Vgl. Stauss/Neuhaus (1995), S. 584.

525 Vgl. hierzu die Beispiele zum Service-Leistungsprogramm der Kostenstellenrechnung bei Wăhrisch (1998), S. 335.

$526 \mathrm{Vgl}$. Stauss/Neuhaus (1995), S. 586.

$527 \mathrm{Vgl}$. 3.4.3.4. 
stellen, dass der Druck von externen Anbietern bei sequenzunabhängigen Leistungen tendenziell höher sein wird, da die Integration des Controlling in spezielle Managementprozesse als Marktzutrittsbarriere für externe Anbieter zu verstehen ist. Daher bedarf es gerade bei sequenzunabhängigen ControllingLeistungen einer differenzierten Planung der kundenbezogenen Interaktion.

Letztlich bleibt festzuhalten, dass die für eine kundenorientierte Leistungserstellung zu beachtenden Ziele und Rahmenbedingungen in Abhängigkeit des sequenzintegrierten oder sequenzunabhängigen Charakters einer Dienstleistung variieren. Analog zu den sequenzintegrierten Leistungen stellen aber Leistungsvereinbarungen auch für sequenzunabhängige Leistungen ein adäquates Instrument zur Verbesserung der Kunden-Lieferanten-Beziehung dar. Im Einzelfall gestaltet sich die Spezifikation derartiger Vereinbarungen bei sequenzunabhängigen Leistungen regelmäßig komplexer, da die mangelnde Standardisierung Abgrenzungs- und Prognoseprobleme bedingt.

\subsubsection{Informelle Kontakte und Leistungstradition}

Für das der Strukturierung erfolgskritischer Faktoren zugrundegelegte Marketing Dreieck ergab sich die Notwendigkeit einer Modifikation, da dem Tatbestand, dass es sich bei dem Austausch von Controlling-Leistungen um organisationsinterne Transaktionen handelt, angemessen Rechnung getragen werden muss. ${ }^{528}$ Die Ergänzung der Unternehmensgrenzen als ein wesentlicher Einflussfaktor auf die Vermarktbarkeit bzw. Wettbewerbsfähigkeit von ControllingLeistungen war erforderlich. Die Unternehmensgrenzen sind aber nicht nur im Außenverhältnis im Sinne einer partiellen Marktzutrittsbarriere für externe Anbieter bzw. Marktbegrenzungsbarriere für das Controlling anzusehen, sondern vielmehr kommt auch den gewachsenen Leistungstraditionen innerhalb der Unternehmensgrenzen eine maßgebliche Bedeutung für eine effiziente und effektive Ausgestaltung des Controlling zu.

Über die bis hierhin dargestellten gestaltungsrelevanten Faktoren hinaus spielen daher eine Vielzahl schwer operationalisierbarer informeller Tatbestände eine wesentliche Rolle im Rahmen organisationsinterner Leistungsbeziehungen. Im Einzelnen haben nachfolgende Faktoren einen wesentlichen Einfluss auf die 
informellen Kontakte sowie die Leistungstradition des Controllerbereichs: $:^{529}$

- organisatorische Nähe zwischen Anbieter und Nachfrager,

- Leistungserbringung durch bekanntes Personal,

- Häufigkeit und Regelmäßigkeit der Nachfrage,

- personelle Verflechtung zwischen Anbieter und Nachfrager,

- Know how des Anbieters bzw. des Nachfragers in subjektiver, gegenseitig geschätzter Sicht,

- räumliche Nähe zwischen Anbieter und Nachfrager,

- Zeitraum, in dem es unternehmensintern die Leistung bereits gibt,

- Struktur des Leistungsangebots im Zeitablauf,

- Persönlichkeiten auf Anbieter- und Nachfragerseite.

Insbesondere die bei Controlling-Leistungen dominierenden Erfahrungs- und Vertrauenseigenschaften führen bezüglich der Leistungen zu einer ausgeprägten Bewertungsunsicherheit der Nachfrager. Wie bereits dargestellt, kommt im Rahmen des Abbaus der subjektiv empfundenen Unsicherheit gerade der Kommunikation der eigenen Reputation, der Darstellung positiver Beziehungen zu anderen Kunden oder der Angabe konkreter interner Referenzen eine besondere Bedeutung zu. ${ }^{300}$ Derartige Vorgehensweisen bedürfen aber gerade einer längeren Leistungstradition bzw. ausgeprägter informeller Kontakte, da erst diese den notwendigen Vertrauensaufbau erlauben. Im Einzelfall kann dies dazu führen, dass nicht mehr die Dienstleistung als solche im Mittelpunkt der internen Markttransaktion steht, sondern vielmehr die Beziehung zwischen Anbieter und Nachfrager. ${ }^{\text {s31 }}$ Eine derartige Situation ist fur das Controlling oftmals charakteristisch. Die mangelnde Konkurrenzgefährdung der Vergangenheit hat dazu geführt, dass das Controlling seine Stellung als In-Supplier ausbauen konnte. . $^{532}$ Als Vorteil der In-Supplier Stellung des Controlling ist die Möglichkeit anzusehen, den internen Markt aufgrund der vorhandenen Unternehmenskenntnis zielgerichtet zu segmentieren und auf Basis des bereits erworbenen Vertrauens zu

${ }^{529} \mathrm{Vgl}$. hierzu die empirische Studie von WITT. Im Ergebnis stellt er fest, dass die speziellen unternehmensinternen Beziehungen zwischen Anbieter und Nachfrager und die Tradition einer internen Leistung im Unternehmen zusammen ein erhebliches Erklärungsgewicht bezüglich der Abgrenzung interner Dienstleistungen haben. $\mathrm{Zu}$ den aufgefuhrten Sachverhalten vgl. Witt (1988), S. $664 \mathrm{f}$.

530 Vgl. Kap. 3.2.2.1.

531 Vgl. Witt (1988), S. 669.

$\mathbf{5 3 2}$ Als In-Supplier ist derjenige Lieferant anzusehen, der derzeit in einer Geschaftsbeziehung zu dem betrachteten Kunden steht. Demgegenüber verfolgt der Out-Supplier das Ziel, als konkurrierender Lieferant in die Geschäftsbeziehung einzutreten. Vgl. Engelhardt/Gunter (1983), S. 66. Allgemein zum Supply Management vgl. Günter/Kuhl (1995), S. 399 ff. 
bearbeiten. ${ }^{53}$ Demgegenüber hat sich in der Vergangenheit die mangelnde Verfügbarkeit von Out-Suppliern eher negativ auf die Kundenorientierung und Innovationsfreudigkeit des Controlling ausgewirkt. Darüber hinaus führt die quasi zwanghafte In-Supplier Stellung dazu, dass sich das organisatorische Abhängigkeitsverhältnis auch zunehmend inhaltlich manifestiert und damit oftmals einer effektiven Leistungserstellung entgegenwirkt. Ebenfalls werden durch das Fehlen alternativer Anbieter die potenziellen Verhaltenskonflikte zwischen Manager und Controller, die aus dem empfundenen Widerspruch zwischen Hilfestellung und Kontrolle resultieren, verstärkt. ${ }^{534}$

Betrachtet man die Beziehungen des Controlling zu verschiedenen Kundengruppen vor dem Hintergrund bestehender informeller Kontakte und gewachsener Leistungsbeziehungen, so lässt sich feststellen, dass die In-Demander ${ }^{535}$ Stellung eines bestimmten Kunden sich mitunter negativ auf die Informationsversorgung anderer potenzieller Kunden auswirkt. ${ }^{536}$ Bezogen auf die Kostenrechnung konnte festgestellt werden, dass die Zufriedenheit der Geschäftsführer mit Informationen der Kostenrechnung signifikant höher ist als die der Marketing- bzw. Vertriebsleiter. ${ }^{537}$ Hieraus lässt sich folgern, dass die Struktur der Kostenrechnung regelmäßig eher den Bedürfnissen der Geschäftsführer entspricht. ${ }^{538}$ Darüber hinaus liegt es nahe anzunehmen, dass auch unabhängig von der hierarchischen Stellung der Kunden, die regelmäßig längere Beziehung zur Geschäftsführung ${ }^{53}$ dazu führt, dass bspw. standardisierte Leistungen stärker auf deren Interessen abgestimmt sind. Ein weiterer möglicher Einflussfaktor auf die individuelle Kundenzufriedenheit ist die räumliche Nähe. Bezogen auf die Kostenrechnung weist auch hier die Geschäftsführung regelmäßig einen geringfügig höheren Grad räumlicher Nähe zum Controlling auf, ${ }^{540}$ was vielfach auf gewachsene Strukturen zurückzuführen ist.

533 Vgl. Witt (1985), S. 171.

534 Vgl. Lanter (1996), S. 73 sowie Stamm (1991), S. 139 f.

535 Der Begriff In-Demander wird analog zum Begriff des In-Suppliers für den Nachfrager in einer bestehenden Geschäftsbeziehung verwandt.

$\$ 36$ Vgl. Witt (1988), S. 669.

$537 \mathrm{Vgl}$. Homburg/Weber/Aust/Karlshaus (1998), S. 26.

$538 \mathrm{Vgl}$. Homburg/Weber/Aust/Karlshaus (1998), S. 27.

539 Diese Annahme kann vor dem Hintergrund der erst spăteren Berlicksichtigung konkreter absatzmarktbezogener Sachverhalte im Rahmen der Kostenrechnung getroffen werden.

540 In $82 \%$ der untersuchten Fälle befindet sich die Kostenrechnung im selben Gebäude wie die Geschäftsführung, demgegenüber ist dies bei der Marketing- bzw. Vertriebsabteilung nur in $75 \%$ der Fälle so. Vgl. Homburg/Weber/Aust/Karlshaus (1998), S. 21. 
Insgesamt lässt sich festhalten, dass den informellen Kontakten und der sie bedingenden Leistungstradition ein erheblicher Stellenwert im Rahmen der Bereitstellung von Controlling-Leistungen zukommt. Dieser Sachverhalt impliziert aber gleichermaßen, dass formelle Regelungen, wie Leistungsvereinbarungen, wenig geeignet sind, derartige Gegebenheiten für das Controlling gestaltbar zu machen. Vielmehr spielt hier die Persönlichkeit des Controllers eine bedeutende Rolle. ${ }^{\text {s41 }}$ Zielsetzung für ein dauerhaft kundenorientiertes Controlling muss es daher sein, einerseits bestehende Kontakte positiv auszunutzen. Das geschaffene Vertrauen ist die Grundlage für eine gegenseitige Akzeptanz und damit die Basis für die zukünftige Erstellung und Abnahme komplexer Produkte. ${ }^{542}$ Andererseits muss der Controller in der Lage sein, sein Leistungsspektrum auch unabhängig von Einzelkunden dauerhaft zu hinterfragen, damit er den an ihn gestellten Innovationsanforderungen gerecht wird. ${ }^{543}$ Darüber hinaus ist die Ausgewogenheit der Ansprache aller Kundengruppen unabhängig von bereits bestehenden Traditionen sicherzustellen. In diesem Zusammenhang ist zu berücksichtigen, dass die Evidenz einzelner Leistungen in Abhängigkeit der Kunden sehr unterschiedlich sein kann.

\subsubsection{Leistungsverrechnung}

Ausgehend von dargestellten alternativen Möglichkeiten einer ökonomischen Ausrichtung des Controlling ${ }^{54}$ soll im Folgenden die Frage nach der internen Verrechenbarkeit von Controlling-Leistungen thematisiert werden. In diesem Zusammenhang sollen Implikationen für Verrechnungsmodalitäten aufgezeigt werden, die sich aus den dargestellten Inhaltscharakteristika, Ablaufcharakteristika und den Leistungsinanspruchnahmemöglichkeiten ergeben. Auf dieser Basis ist zu diskutieren, welche Controlling-Leistungen tendenziell eine anreizkonforme Verrechnung zulassen und welche Größen einer derartigen Verrechnung zugrundegelegt werden sollten.

Betrachtet man die Verrechnungssystematik von unternehmensinternen Dienstleistungen, so ergeben sich im wesentlichen drei alternative Verfahren. Eine empirische Untersuchung aus dem Jahr 1998 ergab, dass durchschnittlich ca.

\footnotetext{
541 Ubersichtsartig wurde die Bedeutung der Persönlichkeit des Controllers bereits im Rahmen der Aufgabenzuordnungsaspekte dargestellt. Darliber hinaus kormmt insbesondere dem Wechselspiel von Manager- und Controllerpersőnlichkeit eine bedeutende Rolle zu. Zusammengefasste Erkenntnisses hierzu finden sich bei Lanter (1996), S. 235 ff. Vgl. ferner zur idealen Controller-Persönlichkeit Wagner (1983), S. 95 ff.

542 Vgl. Kap. 3.2.2.1.

543 Vgl. Küpper (1997), S. 17 f.

544 Vgl. Kap. 3.3.1.2.
} 
$67 \%$ aller unternehmensinternen Dienstleistungen über Kostenumlagen verrechnet werden. ${ }^{545} \mathrm{Ca} .19 \%$ der Dienstleistungen werden über differenzierte Verrechnungspreise verteilt. Bei ca. $14 \%$ der Dienstleistungen wird auf eine Verrechnung gänzlich verzichtet. Im Rahmen einer Betrachtung der Ausgestaltungsmöglichkeiten eines kundenorientierten Controlling ergibt sich die Notwendigkeit, Auswirkungen alternativer Verrechnungsverfahren auf eine nachfragerorientierte Leistungserstellung und -verwertung zu betrachten.

Das bezogen auf die Verrechnung unternehmensinterner Leistungen am weitesten verbreitete Verfahren der Kostenumlage verfolgt das Ziel, im Rahmen der innerbetrieblichen Leistungsverrechnung verbrauchende Leistungsabnehmer zu belasten. ${ }^{546}$ Die in diesem Bereich geläufigsten Ausprägungen des Kostenumlageverfahrens sind das Kostenartenverfahren, das Kostenstellenumlageverfahren, das Kostenstellenausgleichsverfahren und das Kostenträgerverfahren. ${ }^{547}$ Gemein ist diesen Verfahren, dass die Umlagemethodik regelmäßig zentral festgelegt wird und daher die Abstimmung zwischen Anbieter und Nachfrager nur eingeschränkt möglich ist. Ferner ist die Berücksichtigung multilateraler Leistungsbeziehungen oftmals nicht möglich, da im Moment der Verrechnung regelmäßig noch nicht alle zu verrechnenden Kostenbestandteile bekannt sind. ${ }^{548}$ Bezogen auf die oft anzutreffende Kostenstelle „Controllerbereich“ wird versucht, die Kosten, die im Rahmen der Leistungserstellung angefallen sind, auf Basis möglichst verursachungsgerechter Schlüsselgrößen umzulegen. Die Praktikabilität dieses Verfahrens hat wesentlich zu seiner weiten Verbreitung in der Praxis beigetragen. Dies darf jedoch nicht darüber hinwegtäuschen, dass für Controlling-Leistungen

- die Lenkungsfunktion der Preise außer Kraft gesetzt wird,

- die Transparenz der Gemeinkosten verloren geht und

- eine Nachfragesteuerung von Seiten des Kunden nicht möglich ist. $^{549}$

${ }^{545}$ Die hier dargestellten Werte beziehen sich ausschließlich auf Dienstleistungen. Im Bereich der unternehmensinternen Sachleistungen ist eine weitere Verbreitung von Verrechnungspreisen zu beobachten. Hier und im Folgenden vgl. Scherz (1998), S. $109 \mathrm{ff}$.

Vgl. Schweitzer/Küpper (1986), S. 180.

547 Zu den Charakteristika der einzelnen Verfahren vgl. Scherz (1998), S. $70 \mathrm{ff}$.

548 Zur Berïcksichtigung multilateraler Leistungsbeziehungen im Rahmen der Kostenumlage vgl. Schweitzer/ Küpper (1986), S. 174 ff.

$549 \mathrm{Vgl}$. Bässler/Knust/Schindera (1999), S. 272 f. 
Die hier aufgezeigten Argumente deuten darauf hin, dass die für Cost-Center typische Form der Kostenumlage wenig geeignet ist, Controlling-Leistungen kundenorientiert $\mathrm{zu}$ verrechnen, da sie unbeabsichtigte Verhaltenswirkungen und eine damit verbundene ineffiziente Inanspruchnahme der Leistungen fördert. Ferner ist eine differenzierte Betrachtung und Verrechnung einzelner Controlling-Leistungen (Standardleistung vs. Individual- oder Projektleistung) nur selten möglich, da die geläufigsten Kostenumlageverfahren die Kostenstelle als Einheit verrechnen. ${ }^{550}$ Die Anwendung von Umlageverfahren scheint daher nur dann gerechtfertigt, wenn der Aufwand für die Anwendung differenzierterer Verrechnungsverfahren im Verhältnis zu deren Zusatznutzen zu hoch ist. $.^{551} \mathrm{Ge}-$ rade aber die Existenz des hierfür notwendigen methodischen Know hows im Controlling wirft die Frage auf, warum Verrechnungspreisverfahren für Controlling-Leistungen noch relativ selten zur Allokation der unternehmensinternen Dienstleistungen angewendet werden.

Insgesamt kommt der Verrechnungspreisproblematik in der betriebswirtschaftlichen Literatur ein großer Stellenwert zu. ${ }^{552}$ Unter Verrechnungspreisen werden Wertansätze für innerbetrieblich erstellte Produkte, Zwischenprodukte und Dienstleistungen verstanden, die von anderen, rechnerisch abgegrenzten Funktionsbereichen bezogen werden. ${ }^{553}$ Verrechnungspreise haben drei grundlegende Funktionen: Die Abrechnungs- und Planungsfunktion, die Lenkungsfunktion und die Erfolgszuweisungsfunktion. ${ }^{54}$ Auf Basis dieser Funktionen erscheint die Anwendung von Verrechnungspreisen zur Effizienzbeurteilung des Controlling diskussionswürdig. Da die Ermittlung von Verrechnungspreisen für andere Organisationseinheiten regelmäßig zu den Aufgaben des Controlling zählt, kann durch einen konsequenten Einbezug der Controlling-Leistungen in die Verrechnungspreisdiskussion die Glaubwürdigkeit und Akzeptanz des Controllerbereichs wirksam gesteigert werden. $\mathrm{Zu}$ einer sachgerechten Ausgestaltung von Verrechnungspreisen für das Controlling ist zunächst zu klären, welche Voraussetzungen für die Einzelverrechnung von unternehmensinternen Dienstleistungen aus Anbieter- und Nachfragersicht gegeben sein sollten. In Abbildung 27 werden die fünf wichtigsten Voraussetzungen in Abhängigkeit ihrer Bedeutung dargestellt.

\footnotetext{
ss0 Eine Ausnahme hiervon ist das Kostenträgerverfahren. Vgl. Scherz (1998), S. 72.

$551 \mathrm{Vgl}$. Bässler/Knust/Schindera (1999), S. 273.

552 Vgl. beispielhaft Horváth (1998), S. 571, Küpper (1997), S. 346 oder Wagenhofer (1998), S. 23.

553 Vgl. Ewert/Wagenhofer (2000), S. 563.

s54 Vgl. Coenenberg (1973), S. 374.
} 


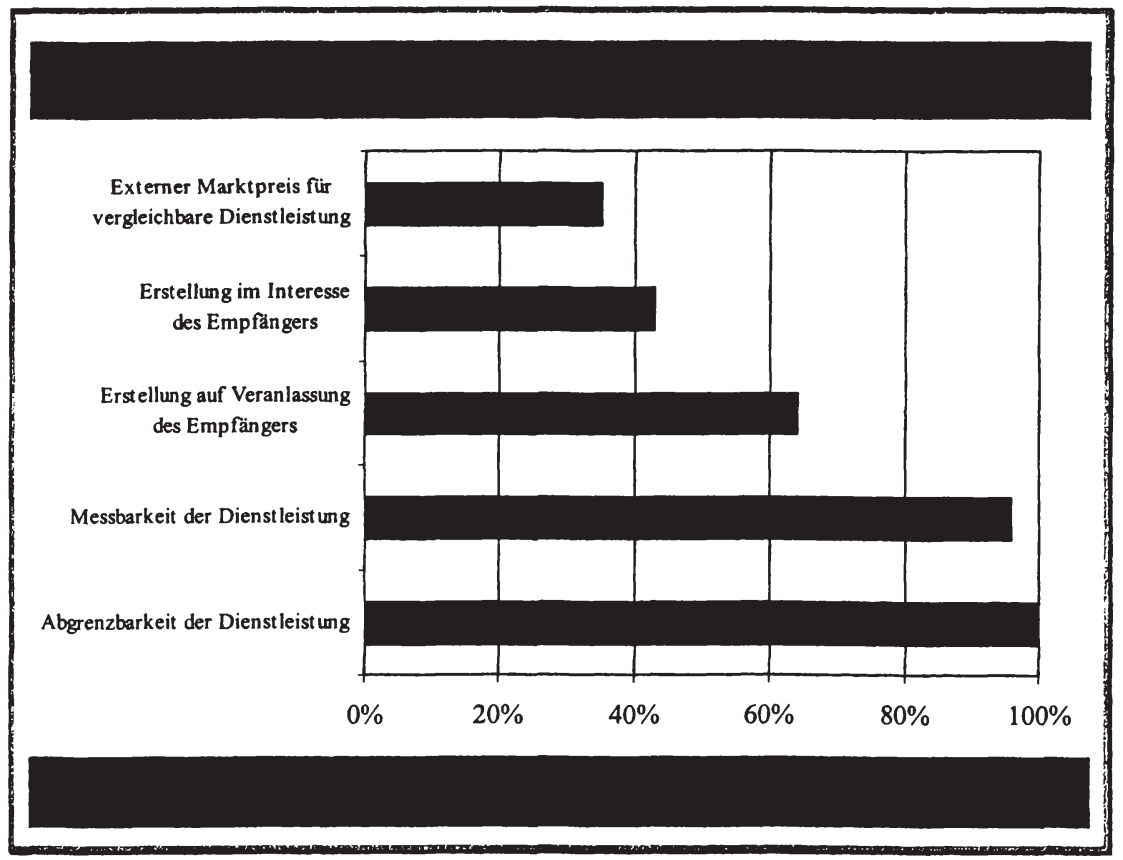

Abbildung 27: Voraussetzungen für die Einzelverrechnung von internen Dienstleistungen ${ }^{5 s}$

Bezogen auf die Leistungen des Controlling wird deutlich, dass der Abgrenzung bzw. Strukturierung des Leistungsspektrums eine außergewöhnliche Rolle zukommt. Der noch relativ seltene Einsatz von Verrechnungspreisen im Controlling darf nicht in dem Sinne fehlverstanden werden, dass eine derartige Abgrenzung nicht gelingen kann. Vielmehr bedarf es des Einsatzes von Instrumenten wie Produkt-Workshops um gemeinschaftlich den Leistungsoutput des Controlling in Form homogener Produkte abzubilden. ${ }^{556}$ Ist eine Controlling-Leistung auf Basis einer derartigen Abgrenzung bestimmt, sind geeignete Verfahren zur Messung des quantitativen und qualitativen Outputs anzuwenden. ${ }^{557} \mathrm{Im} \mathrm{Zu}-$ sammenhang mit dem Ziel einer Nachfragesteuerung durch den Kunden ist die Voraussetzung der Leistungserstellung auf Veranlassung des Empfängers zu

555 Die Darstellung ergibt sich aus den im Text von SCHERZ genannten Prozentwerten. 100\% entspricht einer außerordentlich großen Bedeutung. Vgl. Scherz (1998), S. 113.

556 Zur Bedeutung von Produkten im Rahmen der outputorientierten Führung vgl. Brenner/Horisberger (1998), S.22.

557 Vgl. hierzu beispielhaft Kap. 4.2.2. 
sehen. ${ }^{58}$ Dieser Tatbestand korrespondiert mit den Inhaltscharakteristika und insbesondere auch mit den alternativen Formen der Leistungsinanspruchnah$m e .{ }^{559}$ Die dargestellten Maßnahmen zu Erhöhung des Anteils fakultativer Leistungen können gleichsam als zielführend für eine Steigerung des Verrechenbarkeitspotenzials von Controlling-Leistungen angesehen werden. Im Rahmen dieser Diskussion wurde bereits auf die Bedeutung der Leistungsevidenz hingewiesen, welche im Rahmen der genannten Voraussetzungen für die Verrechnung wesentlichen Einfluss darauf hat, inwieweit der Empfänger die Leistung als in seinem Interesse empfindet. Die Forderung nach der Existenz eines externen Marktpreises hat mit 35\% Nennung keine maßgebliche Bedeutung mehr für die Frage, ob eine interne Leistung überhaupt verrechnet werden kann. ${ }^{360}$ Vielmehr ist die Existenz eines Marktpreises für die Frage nach der Art und Weise der Ableitung geeigneter Verrechnungspreise relevant.

Analog der im Marketing verwandten Prinzipien der Preisbestimmung auf Absatzmärkten wird die Festlegung unternehmensinterner Preise diskutiert. ${ }^{561} \mathrm{Zu}$ unterscheiden sind, auch bezogen auf die Verrechnung von ControllingLeistungen, kostenorientierte Verrechnungspreise, markt- bzw. konkurrenzorientierte Verrechnungspreise sowie nutzen- bzw. nachfragerorientierte Verrechnungspreise. ${ }^{562}$ Grundsätzlich gesehen hängt die Auswahl des Verrechnungspreises von der Art der Leistung, den intendierten Verhaltenswirkungen sowie den Kontextbedingungen des Unternehmens ab.

Kostenorientierte Verrechnungspreise spielen für Controlling-Leistungen derzeit die bedeutendste Rolle, soweit überhaupt eine Verrechnung und keine Kostenumlage erfolgt. Der Begriff kostenorientiert impliziert, dass der kostenrechnerischen Abbildung einzelner Leistungen im Sinne von Produkten eine wichtige Bedeutung zukommt. Insbesondere zur Stärkung der Akzeptanz von Verrechnungspreisen ist die Kostenrechnung ein unverzichtbares Instrument. ${ }^{563}$ Aufgrund des Dienstleistungscharakters der Controlling-Leistungen bietet sich der Einsatz der Prozesskostenrechnung für den Controllerbereich an. ${ }^{564} \mathrm{Mit}$ der

\footnotetext{
$558 \mathrm{Vgl}$. Abbildung 27.

559 Vgl. Kap. 3.3.2.1.

360 Vgl. Scherz (1998), S. 109.

561 Zur Rolle der Preispolitik im Marketing sowie den unterschiedlichen preispolitischen Strategien vgl. Meffert (2000), S. 482 ff. sowie S. 505 ff.

562 Vgl. Meffert (2000), S. $506 \mathrm{ff}$ und Wagenhofer (1998), S. $23 \mathrm{ff}$.

563 WAGENHOFER betont in diesem Zusammenhang die Rechtfertigungsfunktion der Kostenrechnung. Vgl. Wagenhofer (1998), S. 28 f.

564 Hier und im Folgenden vgl. Gleich/Brokemper (1998), S. 151.
} 
Prozesskostenrechnung können die den Produkten zugrundeliegenden Prozesse und Tätigkeiten zeit-, mengen- und kostenmäßig erfasst werden. Im Controllerbereich können auf diese Weise insbesondere für sequenzintegrierte Leistungen aufgrund ihres regelmäßig vorherbestimmten und repetitiven Charakters stabile Verrechnungspreise bestimmt werden. ${ }^{565}$ So kommt bspw. für Leistungen der Kostenrechnung eine derartige Verrechnung in Frage. Wichtig ist in diesem $\mathrm{Zu}$ sammenhang, den Systemverbund der Kostenrechnung zu berücksichtigen, d.h. auch zwangsläufig notwendige Zwischenrechnungen, die nicht direkt von Kunden nachgefragt werden, müssen aufgrund ihrer Funktion für das nachgefragte Endprodukt in dessen Preis einfließen. ${ }^{566}$

Neben der grundsätzlichen Zuordenbarkeit von Kosten zu internen Produkten spielt der beabsichtigte Umfang der Kostenverrechnung eine wesentliche Rolle. Der große Fixkostenanteil des Controllerbereichs führt aufgrund der dominanten Personalkosten regelmäßig dann, wenn keine aussagekräftige Prozesskostenrechnung existiert, zu einer Leistungsverrechnung auf Basis geplanter oder normalisierter Vollkosten. Im Sinne einer marketingorientierten Preispolitik des Controlling können aber auch derartig kostenbasierte Preise zu einer stärkeren Nachfragerorientierung gestaltend eingesetzt werden. Durch wechselnde Gemeinkostenbelastungen im Zeitablauf kann im Sinne der Kapazitätssteuerung Einfluss auf die Abrufmodalitäten einzelner Leistungen des Controlling genommen werden. ${ }^{567}$ Darüber hinaus können dynamische Elemente in die Verrechnungspreise integriert werden, indem bspw. die Preise im Zeitablauf sukzessiv gesenkt werden. ${ }^{568}$ Hierdurch ist der Controllerdienst gezwungen, Effizienzpotenziale auszuschöpfen. Ferner kann in Abhängigkeit der sich aus dem jeweiligen Budget ergebenden Kaufkraft unterschiedlicher Kunden eine Preisgestaltung betrieben werden. ${ }^{569}$ Im Rahmen einer derartigen Diskussion der Preisuntergrenze darf jedoch nicht vernachlässigt werden, dass die kundenspezifische Preisgestaltung von anderen Organisationsmitgliedern als Diskriminierung empfunden werden kann.

\footnotetext{
565 Hier und im Folgenden vgl. Währisch (1998), S. 339 f.

566 So hat bspw. die Kostenstellenrechnung die ergänzende Funktion, die Kalkulationsgenauigkeit bei den Endprodukten $\mathrm{zu}$ erhöhen. Wird die Kostenstellenrechnung nicht separat nachgefragt, müssen ihre Kosten dem Produkt „Kalkulation“ zugerechnet werden. Vgl. Währisch (1998), S. 339.

Allg. furr innerbetriebliche Leistungen vgl. hier und im Folgenden Witt (1985), S. 172.

568 Währisch (1998), S. 340.

569 An dieser Stelle verwischen die Grenzen zwischen einer kostenorientierten und nachfragerorientierten Preisgestaltung.
} 
Bei sequenzunabhängigen fakultativen Leistungen des Controlling mit Unterstützungscharakter, wie bspw. unternehmensinternen Projekten, umfangreichen Einzelanalysen oder Schulungen ist eine kostenbasierte Verrechnung auf Basis von Zeitaufschrieben oftmals gut möglich..$^{570}$ Ein Spielraum des Controllers bei der Verrechnung derartiger Leistungen ist in der einzelfallabhängigen Zuordnung unterschiedlich hoher Einzelkostenbestandteile zu sehen. ${ }^{51}$ Gerade bei den zuletzt genannten Leistungen bietet sich aber auch eine Verteilung auf Basis markt- bzw. konkurrenzorientierter Verrechnungspreise an.

Voraussetzungen für die unternehmensinterne Anwendung von Marktpreisen sind die Existenz eines stabilen externen Marktpreises für vergleichbare Produkte, der externe Marktzugang oder der unternehmensinterne Zwang zur Anwendung von Marktpreisen. Darüber hinaus ist regelmäßig eine Korrektur um Absatz- und Beschaffungskosten notwendig. ${ }^{572}$ Bei sequenzunabhängigen fakultativen Leistungen des Controlling mit Unterstützungscharakter können die Voraussetzungen, soweit die organisatorischen Regelungen getroffen sind, als erfüllt angesehen werden. Über die Anwendung von Marktpreisen kann eine Ausrichtung des Controllerbereichs an der Effizienz und Effektivität externer Anbieter erfolgen. Darüber hinaus erfolgt eine Preissensibilisierung interner Abnehmer. Im Einzelfall können Tagessätze von Unternehmensberatern, Dozenten oder Buchhaltern in Abhängigkeit der zur verrechnenden Leistung als Referenzmaßstab herangezogen werden. Wichtig ist in diesem Zusammenhang die Transparenz der Marktpreise und Leistungen, da unternehmensinterne Abnehmer geneigt sind, die inhaltliche und insbesondere auch die qualitative Vergleichbarkeit interner Leistungen mit Marktleistungen in Frage zu stellen. ${ }^{573}$

Über die Marktorientierung hinaus bieten Verfahren einer nutzen- bzw. nachfragerorientierten Verrechnungspreisgestaltung die Möglichkeit, die individuelle Wertschätzung der einzelnen Leistung in die Preisgestaltung mit einzubeziehen. Wie bereits dargestellt, gibt es auch auf Basis kostenbasierter Verrechnungspreise im Controlling die Möglichkeit, abweichende Budgets der Nachfrager oder Abnahmezeitpunkte zu berücksichtigen. Insbesondere die Kenntnis der Nachfrager und ihrer Präferenzen erlaubt darüber hinaus im Einzelfall eine Preisdifferenzierung, speziell dann, wenn der Controller eine Vorstellung über

\footnotetext{
570 Vgl. Wahrisch (1998), S. 340.

571 Allg. hier und nachfolgend Witt (1998), S. 172.

572 Vgl. Horváth (1998), S. 575 f.
}

573 Dies ist nachvollziehbar, da Marktleistungen regelmaßig ein Differenzierungsstreben zu Grunde liegt. Vgl. Kaldewei (1998), S. 34. Zur Rolle des Benchmarking in diesem Zusammenhang vgl Kap 42.1 .7 
die unterschiedlichen Preiselastizitäten seiner Produkte hat. ${ }^{574}$ So ist anzunehmen, dass Kunden bei transparenten Standardprodukten der Kostenrechnung preissensibler reagieren, als bei komplexen internen Beratungsaktivitäten. Ein Verfahren, welches differenziert den Präferenzen der Kunden bzw. ihrem Zahlungswillen für einzelne Leistungen Rechnung trägt, ist das Target Costing. ${ }^{575}$ Bezogen auf unternehmensinterne Austauschbeziehungen verfolgt das Target Costing die Ziele, die Erstellung marktgerechter Produkte zu fördern sowie die Steuerung der Kostenstrukturen in der Produktplanung zu unterstützen. Im Controlling kann das Instrument dazu eingesetzt werden, Kosten-/Nutzenrelationen einzelner Leistungen zu ermitteln, um dann auf Basis einer Schwachstellenanalyse Prozessverbesserungen und eine Optimierung der Leistungsverrechnung $\mathrm{zu}$ initiieren..$^{57}$

Zusammenfassend lässt sich festhalten, dass Controlling-Leistungen überwiegend noch auf Basis undifferenzierter Umlageverfahren verrechnet werden. Soweit Leistungen unmittelbar vom Kunden beeinflusst werden können ${ }^{577}$ und zudem einen Marktbezug aufweisen, erfordert die Kundenorientierung im Controlling aus Gründen der Motivation und Verhaltenssteuerung von Anbieter und Nachfrager eine spezifische Verrechnung der einzelnen Leistungen. Hierbei darf jedoch die Wirtschaftlichkeit des Verrechnungsaufwandes nicht aus den Augen verloren werden. Bei der Bestimmung geeigneter Verrechnungspreise ist in Abhängigkeit des Erstellungskontextes der jeweiligen Leistung ein kosten-, markt- oder nachfragerorientiertes Verfahren auszuwählen. Die Grenzen nachfrager- bzw. kundenorientierter Preisbestimmungsverfahren zu den anderen genannten verschwimmen bei der Verrechnung unternehmensinterner Leistungen oftmals, da sie auf gewissen Markt-bzw. Kostenannahmen aufbauen.

Abbildung 28 zeigt in Abhängigkeit der Kriterien Kundeneinfluss und Marktbezug eine Zuordnungssystematik für die Verrechnung von ControllingLeistungen. Die Einordnung beispielhafter Leistungen wurde hier tendenziell vorgenommen; im konkreten Fall ist der spezifische Leistungsinhalt sowie der Unternehmenskontext zu beachten.

574 Allg. zur nachfragerorientierten Preisbestimmung vgl. Meffert (2000), S. 512 ff.
575 Zu den Grundlagen des Target Costing vgl. Franz (1993), S. $124 \mathrm{ff}$.
576 Vgl. Gleich/Brokemper (1998), S. 152 sowie darauf bezugnehmend in dieser Arbeit Kap. 4.2.3.
577 Die Beeinflussbarkeit bezieht sich sowohl auf den Leistungsinhalt als auch die Leistungsmenge, 


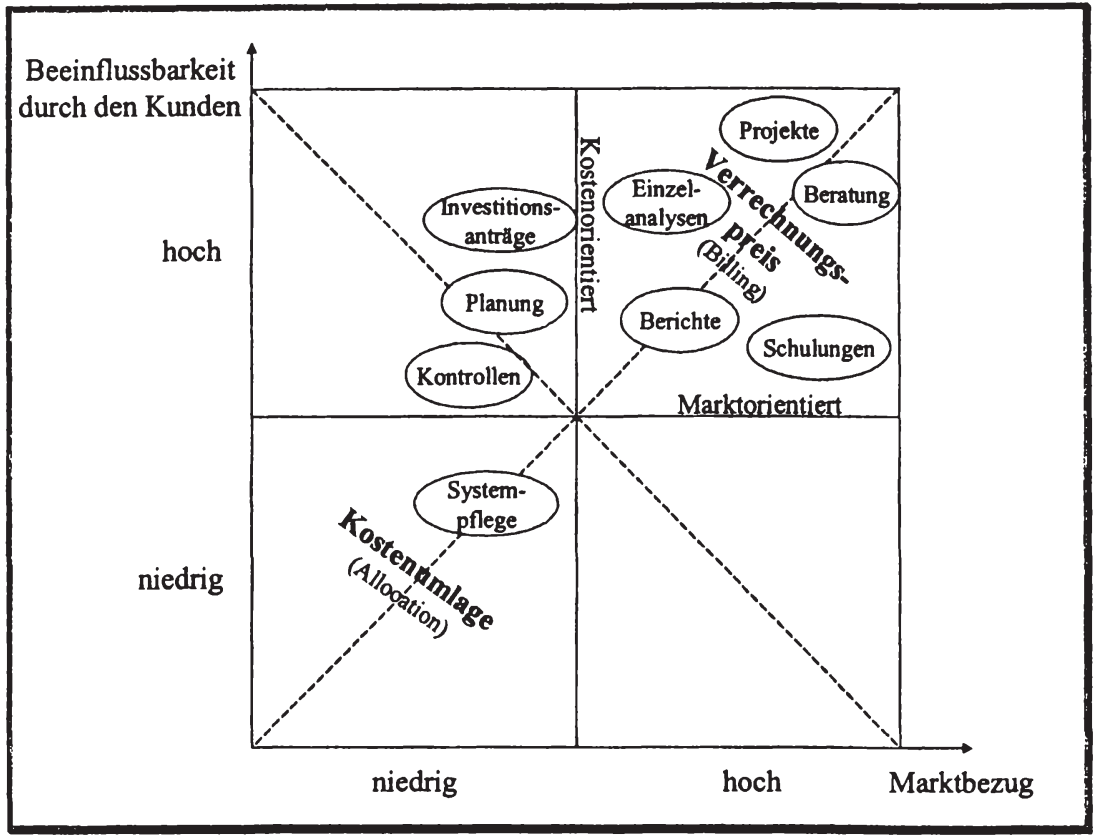

Abbildung 28: Beeinflussbarkeit - Marktbezug - Portfolio des Controlling unter Berücksichtigung alternativer Verrechnungspreisbestimmungsverfahren ${ }^{578}$

Abschließend sei bemerkt, dass bei einer isolierten Betrachtung von Verrechnungspreisen für Controlling-Leistungen regelmäßig nur eine Erfolgskomponente zugrunde gelegt wird - die Kosten. ${ }^{579}$ Daher ist darauf zu achten, dass die Motivation der Mitarbeiter sich nicht einseitig auf Kostensenkungen fokussiert. Vielmehr sollte ein ausgewogenes Verhältnis von Kosten, Qualität und Kundenzufriedenheit für das Controlling maßgeblich sein. Die Gefahr einer Unausgewogenheit besteht regelmäßig immer dann, wenn das Controlling aufgrund des fehlenden Marktzugangs seiner Kunden oder in Ermangelung alternativer Angebote eine Monopolstellung inne hat. ${ }^{580}$ Aus diesem Grund soll nachfolgend die Kunden- und Wettbewerbssituation des Controlling betrachtet werden.

578 Modifiziert auf Grundlage des Portfolios von Bassler/Knust/Schindera (1999), S. 273.

579 Der Kostenbegriff ist hier umfassend zu verstehen. Dies schließt die der Marktpreisbetrachtung zugrundeliegenden Opportunitätskosten ein.

580 Allg. vgl. Kreuter (1999), S. 161. 


\subsection{Kunden- und wettbewerbsbezogene Charakteristika des Control- ling}

Bei der dieser Arbeit zugrundegelegten marketingorientierten Betrachtung des Controlling mag es zunächst verwunderlich erscheinen, dass den kunden- und wettbewerbsbezogenen Charakteristika des Controlling erst an dieser Stelle besonderes Augenmerk geschenkt wird. Zielsetzung der gewählten Vorgehensweise war, zunächst Marketingimplikationen darzustellen, die sich auf Grundlage der allgemeinen Struktur von Controlling-Leistungen und der Existenz eines organisatorisch abgegrenzten Controllerbereichs ergeben. Die hierbei verwandten Denkfiguren des Marketings ließen sich auf Basis der pauschalen Annahme des „Managements als zentraler Kunde des Controlling" nachvollziehen. Wie zu zeigen sein wird, ist die Kenntnis der Leistungen und der Organisation des Controlling eine wesentliche Voraussetzung für die differenziertere Abgrenzung von Kunden und Wettbewerbern des Controlling. Gerade die bereits abgeleiteten gestaltungsrelevanten Sachverhalte können oftmals zu einer diesbezüglichen Segmentierung verwandt werden. $\mathrm{Zu}$ berücksichtigen bleibt aber, dass sich Leistung, Kunde und Organisation gegenseitig bedingen und daher idealerweise einer simultanen Betrachtung bedürfen. Insgesamt erfolgt im Sinne des Marketing durch den gewählten Ablauf eine Konkretisierung der gestaltungsrelevanten Charakteristika, ausgehend von einer strukturellen Leistungsbetrachtung über kontextspezifische Organisationsgegebenheiten bis hin zu der subjektiven Leistungswahrnehmung alternativer Kundengruppen. Wie im Rahmen der instrumentellen Ausgestaltung gezeigt werden wird, steht demgegenüber bei der Planung kundenbezogener Maßnahmen die Ermittlung und Abgrenzung von Kunden und Wettbewerbern am Anfang jeglicher Aktivitäten. ${ }^{51}$

Nachfolgend sollen zunächst die Kunden des Controlling dargestellt und strukturiert werden, um daran anknüpfend alternative Wettbewerber für Leistungen, Kunden-Leistungs-Kombinationen oder die Organisation des Controlling bestimmen zu können. Im Sinne des Marketing Dreiecks, welches als Strukturierungsrahmen zur Ableitung der Gestaltungsparameter verwandt wird, ${ }^{582}$ lassen sich darauf aufbauend Aussagen über Kunden- und Wettbewerbsbeziehungen des Controlling ableiten.

\footnotetext{
581 Vgl. Kap. 4.1.2.

582 Zum Marketing Dreieck vgl. Backhaus (1999), S. 28.
} 


\subsubsection{Kundenbeziehungen im Rahmen der Leistungserstellung}

Betrachtet man die Leistungsbeziehungen des Controlling aus der Perspektive der Marketingtheorie, so erfordert das älteste Axiom des Marketing zunächst, seine Kunden zu kennen. ${ }^{533}$ Erst über eine genaue Kenntnis aktueller und potenzieller Kunden des Controlling kann deren zielgerichtete Ansprache gewährleistet werden. Dies ist die Grundlage für die eigentlich angestrebte Bedürfnisbefriedigung der Kunden. Ein derartiger Zusammenhang impliziert, dass nicht nur die Kenntnis der Kunden und ihrer Bedürfnisse erfolgskritisch ist. Im Rahmen der instrumentellen Ausgestaltung ist insbesondere auch die Art und Weise ihrer Segmentierung von strategischer Bedeutung, ${ }^{584}$ da davon auszugehen ist, dass unterschiedliche Kunden des Controlling auch differenziert auf den Einsatz alternativer Instrumente reagieren ${ }^{585}$ Aus diesem Grund soll zunächst allgemein geklärt werden, welcher Markt als der relevante Markt fur Leistungen des Controllerbereichs anzusehen ist, bevor darauf aufbauend auf einzelne Kunden bzw. geeignete Kriterien zu ihrer Segmentierung eingegangen wird.

\subsubsection{Ausrichtung des Controlling (intern vs. extern)}

Ohne schon differenziert auf einzelne Kundengruppen einzugehen, stellt sich bezogen auf das Controlling die Frage, inwieweit die Informationen des Controlling ausschließlich zu internen Steuerungszwecken verwandt werden, oder aber auch von externen Anspruchsgruppen genutzt werden. ${ }^{586}$ Will der Controller seine einzelnen Leistungen zielgerichtet erbringen, muss er zunächst den für ihn relevanten Mark ${ }^{587}$ abgrenzen, um darauf aufbauend tätig zu werden. Ausgehend von der dieser Arbeit zugrundegelegten Controlling-Definition, lässt sich der relevante Markt für Controlling-Leistungen zunächst als der Markt für die Beschaffung, Aufbereitung, Analyse und Kommunikation von Daten zur Vorbereitung zielsetzungsgerechter Entscheidungen verstehen. ${ }^{58}$ Klärungsbedürftig im Rahmen der Ausrichtung des Controlling bleibt, wer als Entscheidungsträger im Sinne der Definition anzusehen ist. Konsensfähig ist - auch un-

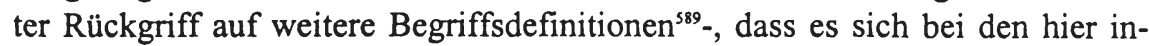

${ }^{583} \mathrm{Vgl}$. Berry/Parasuraman (1999), S. 87.

584 Allg. vgl. Freter (1983), S. 13 ff. sowie zu den Anfängen der Marktsegmentierung Hummel (1954), S. 34 ff.

${ }^{585}$ MEFFERT schreibt in diesem Zusammenhang der Segmentierung einerseits die Funktion der Marktidentifizienung und andererseits die der Befriedigung der Konsumentenbedürfnisse zu. Vgl. Meffert (2000), S. 183.

586 Zum Konzept der Anspruchsgruppen vgl. allg. Dyllick (1992).

${ }^{587} \mathrm{Zu}$ Bedeutung des relevanten Marktes im Rahmen der strategischen Planung von Aktivităten vgl. Abell (1980), S. $17 \mathrm{ff}$.

588 Vgl. hierzu Rieper/Witte/Berens (1996), S. V. sowie die Ausfuhrungen in Kap. 3.3.1.2.2.

589 Überblicksartig vgl. Weber (1999), S. $19 \mathrm{ff}$. 
tendierten Entscheidungen um solche der Unternehmensführung handelt. In marktwirtschaftlichen Systemen sind die Träger der Unternehmensentscheidungen entweder der Eigentümer, sofern dieser selbst im Unternehmen führt, oder ein bestelltes Führungsorgan ${ }^{590}$ In jedem Fall ist die Unternehmensführung als Adressat des Controlling Mitglied der Unternehmensorganisation und damit im Sinne des Marketing ein unternehmensinterner Kunde.

Ferner könnte man aufgrund der hohen Wahrnehmungsrelevanz der Informationsversorgungsfunktion des Controlling (z.B. Reporting), ausgehend von der Struktur des Informationsangebotes, auf die Kunden schließen. Gegenwärtig liegt den durch das Controlling bereitgestellten Informationen überwiegend das auf Planungs-, Steuerungs- und Kontrollzwecke ausgerichtete interne Rechnungswesen $\mathrm{zu}$ Grunde. ${ }^{591}$ Das im angloamerikanischen Raum als Management Accounting bezeichnete interne Rechnungswesen ist wie folgt charakterisiert:

- „Has more than one purpose.

- Is not governed by generally accepted accounting principles.

- Is optional.

- Focuses on segments as well as the whole of an business.

- Has less emphasis on precision.

- Is part of other processes, rather than an end in itself." 592

Wesentlich in diesem Zusammenhang ist aber, dass nicht die Informationsgrundlage - im Sinne einer angebotsorientierten Sichtweise - ausschlaggebend für die Struktur der Kunden ist. Gerade die oft artikulierte Anforderung des Managements an das Controlling, mehr wettbewerbsbezogene Informationen bereitzustellen, ${ }^{593}$ zeigt, dass - unabhängig von der Ausrichtung des zugrundegelegten Informationssystems - der Adressat im Mittelpunkt der Betrachtung stehen muss. Insgesamt rückt die aus der Konzeption des Controlling abgeleitete Führungsunterstützungsfunktion regelmäßig das Management in den Mittelpunkt der durch das Controlling zu erbringenden Service-Aktivitäten. Hierdurch wird gleichsam seine dominant interne Ausrichtung bestimmt.

590 In diesem Zusammenhang spricht man auch von Eigentilmer-Unternehmungen bzw. Geschäftsfuhrer- (Manager-) Unternehmungen. Zu den Trägern der Führungsentscheidungen vgl. Wöhe (2000), S. 102.

591 Vgl. Horvàth (1998), S. 408. Zu den Adressaten der Kostenrechnung vgl. Weber (1990b), S. 124 f. Zu Einsatzfeldern von Informationen der Kosten- und Erlösrechnung vgl. Währisch/Henselek (1997), S. $330 \mathrm{ff}$.

592 Anthony (1970), S. 353.

593 Vgl. Stadler/Weißenberger (1999), S. 9. 
Dennoch ist regelmäßig davon auszugehen, dass durch das Controlling Informationen, in oftmals veränderter Form, unternehmensexternen Anspruchsgruppen zur Verfügung gestellt werden oder auch unverändert in Teile des zu Dokumentationszwecken zu betreibenden externen Rechnungswesens einfließen. ${ }^{594} \mathrm{Bei}-$ spielhaft sind in diesem Zusammenhang als Informationsnutzer Aktionäre, Rating Agencies, die Fachpresse sowie insgesamt die interessierte Öffentlichkeit zu nennen. ${ }^{595}$ Insbesondere auch Finanzanalysten sind im Rahmen der an Bedeutung gewinnenden Kapitalmarktorientierung zunehmend an Segmentinformationen interessiert. ${ }^{596} \mathrm{Da}$ die Funktion des Controlling aber zunächst in der Führungsunterstützung zu sehen ist und Informationen daher auch bezogen auf die Belange der Führung aufzubereiten sind, lassen sich die externen Informationsadressaten zunächst nicht als unmittelbare Kunden ansehen. Im Regelfall erhalten externe Adressaten die Informationen des Controlling in durch andere Unternehmensbereiche gefilterter Form. So ist eine Weiterverwendung der modifizierten Controlling-Daten durch den Public Relation Bereich, den Investor Relation Bereich ${ }^{597}$ oder das Management selbst denkbar. Eine derartige Vorgehensweise deutet jedoch darauf hin, dass durch die Weiterverarbeitung und Filterung der Informationen eine zielgruppen- bzw. bedürfnisgerechte Ansprache seitens des Controlling nicht mehr möglich ist. Da die Gestaltungs- und Interaktionsmöglichkeiten bei diesen externen Adressaten dementsprechend gering sind, ${ }^{598}$ sollten unternehmensexterne Anspruchsgruppen zunächst nicht als Kunden betrachtet werden. Es wäre zu befürchten, dass eine zu undifferenzierte Informationsaufbereitung zu Lasten der primären internen Adressaten des Controlling geht.

Im Folgenden soll daher das Augenmerk auf die internen Kunden des Controlling gerichtet werden, weil hier die im Sinne der Aufgabenerfüllung des Controlling größten Effektivitätssteigerungspotenziale $z u$ sehen sind. Da eine wirkungsvolle Ansprache seitens des Controlling die genaue Kenntnis der jeweiligen internen Kunden erfordert, sollen einzelne Kundengruppen strukturiert betrachtet werden.

594 Vgl. hierzu die beispielhaft dargestellten Zusammenhänge zwischen Kostenrechnung und externem Rechnungswesen im Rahmen der Betrachtung sequenzintegrierter Dienstleistungen in Kap. 3.3.2.3.1. Vgl. Roth (2000), S. $130 \mathrm{f}$.

$596 \mathrm{Vgl}$. Homburg/Weber (1999), S. 591.

$597 \mathrm{Zu}$ den aus dem Shareholder Value-Konzept erwachsenden Anforderungen an Investor Relations vgl. Baetge (1997), S. 112. Eine Diskussion beispielhafter Defizite der Berichterstattung an unternehmensexterne Adressaten findet sich bei Baetge (1996), S. 169.

598 Vgl. Berger (1989), S. 185. 


\subsubsection{Abgrenzung der internen Controlling-Kunden}

Zunächst wurde im Rahmen dieser Arbeit die pauschale Annahme getroffen, dass es sich bei den internen Kunden des Controlling um das Management des Unternehmens handelt. Im Folgenden soll das Management und weitere interne Kundengruppen auf deskriptiver Basis systematisiert werden, sowie ein Ausblick auf Möglichkeiten und Grenzen einer internen Kundensegmentierung gegeben werden. Im Rahmen der in Abschnitt 4 folgenden Vorstellung einzelner Instrumente des kundenorientierten Controlling wird konkret auf Gestaltungsfragen der interne Kundensegmentierung eingegangen..$^{599}$

Ausgehend von dem dem abteilungsbezogenen internen Marketing zugrundeliegenden Ansatz des TQM sind im Sinne der hier unterstellten prozessorientierten Betrachtung alle Abnehmer einer nachfolgenden Prozessstufe konsequent als Kunden zu behandeln ${ }^{600}$ Bezogen auf das Controlling bedeutet dies zunächst die einzelnen Prozesse seiner Leistungserstellung zu identifizieren, ${ }^{601}$ um darauf aufbauend die Schnittstellen zu leistungsabnehmenden Organisationsmitgliedern betrachten zu können. Unterstellt man, dass es sich im Controlling primär um Informationsversorgungsprozesse zur Förderung der Planung, Kontrolle und Koordination handelt, ${ }^{602}$ kommen zunächst alle für die genannten Aufgaben verantwortlichen Organisationsmitglieder in Betracht. Bei einer Abgrenzung dieser potenziellen internen Kunden des Controlling, wird deutlich, dass der Unternehmensorganisation als Rahmen der Leistungserstellung und -verwertung des Controlling eine maßgebliche Bedeutung zukommt.

Betrachtet man Führungskräfte unabhängig von ihren unterschiedlichen Funktions- und Objektbereichen als primär Verantwortliche für eine zielgerichtete Koordination, so lassen sich zunächst entlang der Unternehmenshierarchie unterschiedliche Kundengruppen identifizieren, die es mit spezifischen Produkten zu versorgen gilt. ${ }^{603}$ Abbildung 29 zeigt diesen Tatbestand übersichtsartig für Controlling-Leistungen des Bereichs Berichtswesen.

599 Vgl. Kap. 4.1.2.1.2.

600

601 Exemplarisch zu einer strukturierten Analyse des Berichtsprozesses im Controlling vgl. Stadler/Weißenberger (1999), S. 9.

602 Vgl. hierzu die induktive Analyse von HoRvÁTH auf Basis einer Auswertung von Literatur- und Praxisquellen. Horváth (1998), S. 78.

${ }^{603}$ Hier werden die unterschiedlichen Berichte als Produkte des Controlling aufgefasst 


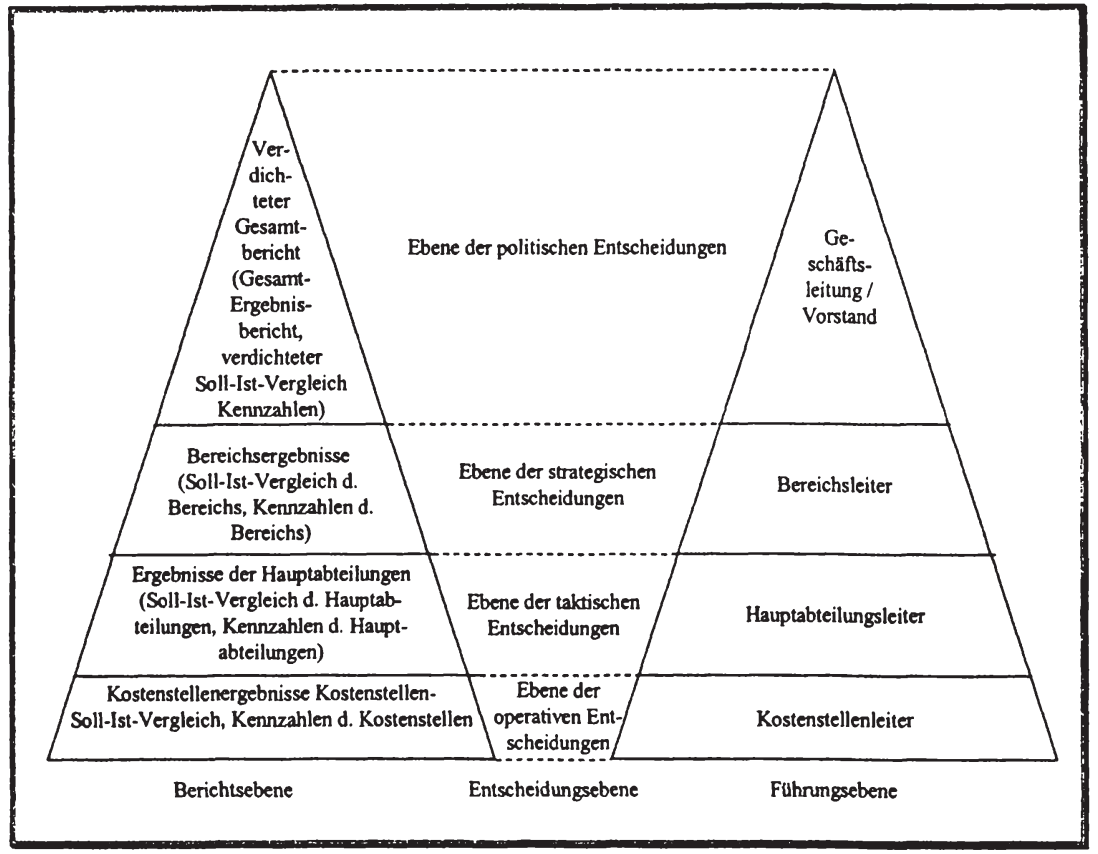

Abbildung 29: Kundenspezifische Berichte nach Hierarchieebenen ${ }^{604}$

Korrespondierend mit den unterschiedlichen in einem Unternehmen bestehenden Führungsebenen lassen sich Kundengruppen des Controlling identifizieren, die in Abhängigkeit der ihnen obliegenden Führungsentscheidungen einen spezifischen Informationsbedarf aufweisen. In Anlehnung an den hier hierarchisch abgeleiteten Informationsbedarf kann das Controlling, wie dargestellt, unterschiedlich strukturierte Berichte generieren und allen Managern der jeweiligen Hierarchieebene zur Verfügung stellen. Wesentlich ist in diesem Zusammenhang, dass nicht nur der Unternehmensleitung Entscheidungshilfen zur Verfügung gestellt werden, sondern allen unternehmerischen Entscheidungsträgern. ${ }^{605}$

Zusätzlich zu der Gesamtunternehmenshierarchie spielt für die Kundenansprache des Controlling die funktionale Gliederung vieler Unternehmen eine besondere Rolle. ${ }^{606}$ Neben Kunden mit General-Management-Verantwortung benötigen unterschiedliche unternehmerische Funktionsbereiche wie Entwicklung,

604 Preißler (2000), S. 119.

605 Vgl. Abbildung 29 sowie Preißler (2000), S. 118.

${ }^{606}$ Für die Kostenrechnung vgl. auch Aust (1999), S. 208 ff. 
Einkauf, Produktion, Vertrieb und Marketing zur Erfüllung ihrer Aufgaben spezifische Informationen ${ }^{607}$ Innerhalb dieser Funktionsbereiche können sich gleichermaßen eigene Hierarchien ergeben, was wiederum eine selektive Zuordnung der Informationen erfordert. Abbildung 30 zeigt exemplarisch für die mittelständische Wirtschaft Informationsleistungen des Controlling in Abhängigkeit von nach Funktionsbereichen abgegrenzten Kundengruppen.

\begin{tabular}{|c|c|c|}
\hline Position & Wesentliche Controllingbausteine & $\begin{array}{l}\text { Dominante } \\
\text { Perspektive }\end{array}$ \\
\hline Geschäftsführer & Bilanz, GuV, Kennzahlensystem, Erfolgsrechnung (allgemein) & Overview \\
\hline $\begin{array}{l}\text { Leiter } \\
\text { Rechnungswesen } \\
\text { kfm. Bereich }\end{array}$ & $\begin{array}{l}\text { Bilanz, GuV, Vollkostenrechnung, Grenzplankostenrechnung, } \\
\text { Parallelkalkulation, Prozeßkostenrechnung, Produkt- und Kundenerfolg, } \\
\text { Erlösschmälerung, Investitionscontrolling }\end{array}$ & $\begin{array}{l}\text { Bereichs- } \\
\text { Overview }\end{array}$ \\
\hline Leiter Vertrieb & Produktkalkulation, Marketingkosten, Personalkosten, Logistikkosten & - Bereichssicht \\
\hline Leiter Fertigung & $\begin{array}{l}\text { Produktkosten, Variantenkosten, Relativkosten, Prozeßkosten, } \\
\text { Instandhaltungs- und Wartungskosten, AfA/Abschreibung, Material- und } \\
\text { Qualitätskosten }\end{array}$ & - Bereichssicht \\
\hline Leiter F\&E & Budgets, Personal- und Sachmittelkosten, Projektzeiten, Projektfortschritt- & - Bereichssicht \\
\hline Leiter Personal & Personalkosten (aufgegliedert), Personalprokuktivität & Bereichssicht \\
\hline Controller & $\begin{array}{l}\text { Vollkostenrechnung, erweiterte Grenzplankostenrechnung, } \\
\text { Einzellkostenrechnung für Sonderfälle, relative Deckungsbeiträge, } \\
\text { Finanzcontrolling mit Planbilanzen, Plan-GuVs, Finanzkennzahlen, } \\
\text { verschiedene Portfoliotypen, Vertriebscontrolling, F\&E-Controlling, }\end{array}$ & $\begin{array}{l}\text { Querschnitts- } \\
\text { sicht und } \\
\text { Overview }\end{array}$ \\
\hline
\end{tabular}

Abbildung 30: Adressatenspezifische Controllinganforderungen bzw. Controllerperspektiven bei unterschiedlichen Adressatenkreisen ${ }^{608}$

Ein gegenläufiger Trend zu der funktions- und hierarchiebezogenen Kundenabgrenzung ist in der zunehmenden Prozessorientierung vieler Unternehmen und der damit verbundenen Abflachung von Hierarchien zu sehen. ${ }^{609}$ Eine derartiger Veränderung der Ablauforganisation erfordert eine unmittelbare Ansprache der

607 Im Rahmen der Koblenzer Studie zur Kostenrechnung wurden der Marketing-/Vertriebsbereich als Funktionalbereich und die Geschäftsfuihrung als oberstes Führungsorgan als exemplarische Kunden der Kostenrechnung analysiert. Vgl. Homburg/Weber/Aust/Karlshaus (1998), S. $21 \mathrm{ff}$.

608 Bei den hier dargestellten Anforderungen handelt es sich um die Durchschnittsmeinung der mittelständischen Wirtschaft, welche im Rahmen einer empirischen Studie erhoben wurde. Vgl. Witt/Witt (1996), S. 46 f. Zur Darstellung vgl. Neuhäuser-Metternich/Witt (2000), S. 202.

609 Vgl. Horváth (1998), S. 830 ff. 
einzelnen Prozessteams durch das Controlling. Soweit Leistungen des Controlling sequenzintegriert zu erbringen sind, stellt dies besondere Anforderungen an das Schnittstellenmanagement zu den Prozessteams. ${ }^{610}$

Neben der Unternehmenshierarchie und den Funktionsbereichen spielen Unternehmensstandorte eine Rolle im Rahmen der Kundendifferenzierung, da sie oftmals abgrenzbare Verantwortungsbereiche verkörpern, zu deren Steuerung spezifische, kontextabhängige Informationen benötigt werden. Zudem beeinflusst die räumliche Nähe des Controlling zu seinen Kunden, inwieweit eine zufriedenstellende Kooperation gewährleistet werden kann. ${ }^{611}$ Korrespondierend mit der Frage einer Kundenabgrenzung nach Werken, Zweigstellen oder Niederlassungen ergibt sich die Frage nach der rechtlichen Selbständigkeit von Unternehmensteilbereichen bzw. die nach der Zugehörigkeit eines Unternehmens zu einem Konzernverbund. Da hier zunächst nur interne Kunden des Controlling betrachtet werden sollten, ist fraglich, inwieweit andere Konzerngesellschaften diesen zuzurechnen sind. Insbesondere die zunehmenden Zentralisierungstendenzen im Controlling verbunden mit der Ausgliederung übergreifender Servi${ }^{c e-C e n t e r}{ }^{612}$ für Controlling-Leistungen spricht für eine gesellschaftsübergreifende Betrachtung der Kunden. In diesem Sinne können Kundengruppen zusätzlich zu den anderen gewählten Kriterien nach der Zugehörigkeit zu unterschiedlichen Konzerngesellschaft unterschieden werden, soweit bspw. dem Konzerncontrolling im Rahmen der Aufgabenzuordnung die Informationsversorgung der Führungskräfte einzelner Verbundunternehmen obliegt.

Gemeinsam ist den hier exemplarisch angesprochenen Abgrenzungskriterien, dass unterstellt wird, die Ausprägungen der Kundengruppen bezüglich Hierarchie, Funktion oder Lage seien charakteristisch für den Informationsbedarf der Adressaten. Im Endeffekt sind daher die hier gewählten Abgrenzungskriterien nur Annäherungsversuche an einen kunden- oder kundengruppenbezogen zu bestimmenden Informationsbedarf.

Der Informationsbedarf stellt aus der Sicht des Controlling-Kunden ein Mangel an solchen Informationen dar, die er zur Erfullung seiner Aufgaben benötigt. ${ }^{613}$ Allgemein betrachtet ist daher aus Perspektive des Marketing allein der Infor-

\footnotetext{
610 Zur sequenzintegrierten bzw. sequenzunabhängigen Leistungserstellung im Controlling vgl. Kap. 3.3.2.3.

611 Das ein möglicher Zusammenhang zwischen räumlicher Năhe und interner Kundenzufriedenheit besteht vermuten Homburg/Weber/Aust/Karlshaus (1998), S. 21 ff.

612 Vgl. Kap. 3.3.1.1.

613 Vgl. auch Garbe (1975), Sp. 1874 ff.
} 
mationsbedarf der Entscheidungsträger für die Abgrenzung unterschiedlicher Kunden von Bedeutung und weniger deren organisatorische Eingliederung. Da die Bestimmung des Informationsbedarfes aber regelmäßig mit großen Unsicherheiten behaftet ist, sind die hier angesprochenen Abgrenzungskriterien als Heuristik $^{6 / 4}$ zu verstehen. Im Rahmen der genauen Ergründung eines kundenspezifischen Informationsbedarfs stellt sich unter Berücksichtigung der Rationalitätssicherungsfunktion die Frage ${ }^{615}$ inwieweit der Controlling-Kunde seinen eigenen Informationsbedarf, d.h. die zur zielsetzungsgerechten Entscheidungsfindung unabdingbaren Informationen überhaupt objektiv bestimmen kann. Berücksichtigt man darüber hinaus, dass das Controlling nicht in jedem Fall in der Lage ist, den subjektiven bzw. objektiven Informationsbedarf seiner Kunden abzudecken, so ergibt sich das in Abbildung 31 gezeigte Bild.

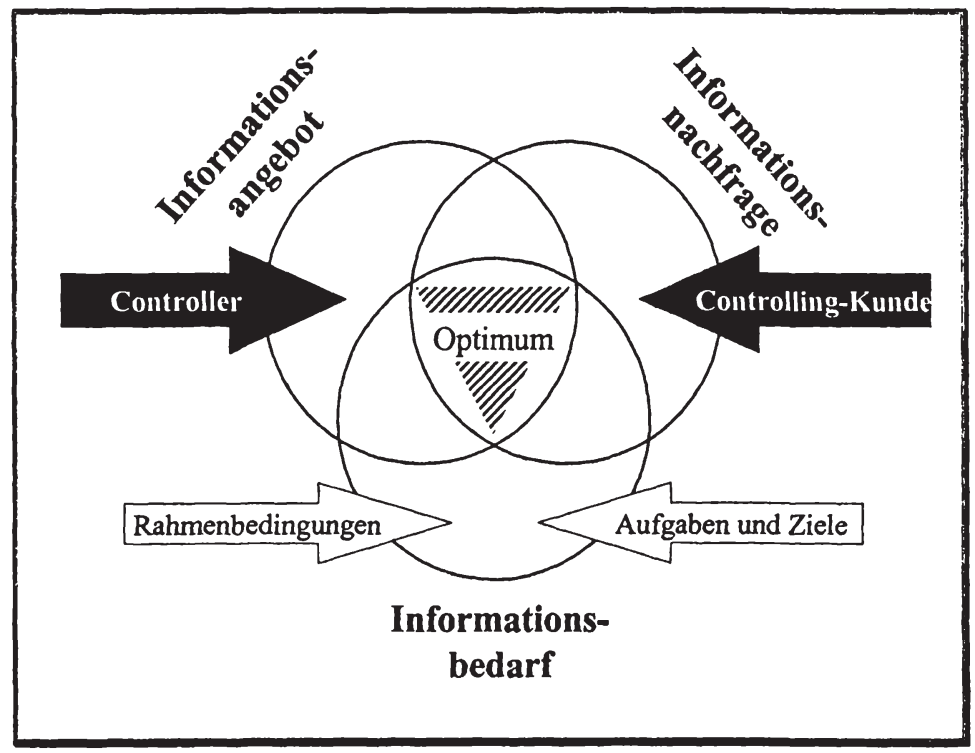

Abbildung 31: Informationsangebot, -nachfrage und-bedarf 616

Ausgehend von einem objektiven Informationsbedarf, der sich kontextabhängig auf Basis der Aufgaben und Ziele des Entscheidungsträgers ergibt, versucht der Controller ein Informationsangebot zu erstellen. Da der Controller den objektiven Informationsbedarf nicht in jedem Fall kennt, bzw. die Informationssysteme

614 Zur Defintion von Heuristiken vgl. Berens (1992), S. 5.
615 Zur Rationalitätssicherungsfunktion des Controlling vgl. Weber (1999), S. 37 ff.
616 In Anlehnung an Berthel (1992), Sp. 875. 
des Controlling diesen nicht immer abdecken, gibt es Bereiche, in denen er unerfüllt bleibt. Maßgeblich für den Leistungsaustausch ist die Ergänzung der durch den jeweiligen Controlling-Kunden årtikulierten Nachfrage. Diese deckt sich nicht in allen Bereichen mit dem objektiven Informationsbedarf, da dieser dem Kunden aufgrund von Rationalitätsengpässen oft nicht bewusst ist. Ferner deckt sich die Nachfrage in solchen Fällen nicht mit dem Angebot, in denen dem Kunden das Gesamtspektrum des Informationsangebotes nicht geläufig ist, bzw. er die Einsatzmöglichkeiten der angebotenen Informationen nicht kennt. Im Einzelnen betrifft dies die Fragen nach der Evidenz einzelner Leistungen ${ }^{617}$ und dem Nutzungsverhalten der Kunden ${ }^{618}$. Welche konkreten Handlungsempfehlungen sich aus der mangelnden Übereinstimmung von Angebot, Nachfrage und Bedarf ergeben, wird zu Beginn der Vorstellung ausgewählter Instrumente des kundenorientierten Controllings ausführlich thematisiert werden. ${ }^{619}$

Insgesamt zeigt sich bei einer Betrachtung der Kunden bzw. Kundengruppen des Controlling, dass es im Sinne einer marketingorientierten Betrachtung erforderlich ist, bei dem unmittelbaren Informationsbedarf des einzelnen Kunden anzusetzen, da die Erfüllung dieses Bedarfes ausschlaggebend für die Nutzung der Informationen und damit gleichsam auch für die Zufriedenheit der Kunden ist. Die Komplexität von Entscheidungssituationen sowie die eigenen Interessen und Präferenzen einzelner Controlling-Kunden führt dazu, dass der artikulierte Informationsbedarf nicht immer mit dem objektiven Informationsbedarf übereinstimmt. Dieser Tatbestand macht es erforderlich, eine interne Marktforschung mittels differenzierter Verfahren zur Analyse des objektiven Informationsbedarfs zu betreiben. ${ }^{620}$ Auf Basis des objektiven Informationsbedarfs gilt es, über den Einsatz entsprechender Instrumente des internen Marketings Informationsangebot und Informationsnachfrage in Einklang zu bringen. Lassen sich darauf aufbauend Kunden mit vergleichbaren Bedürfnissen erkennen, bietet der Ansatz der Kunden- bzw. Marktsegmentierung ein Instrumentarium zur $A b$ grenzung von Kunden- bzw. Marktsegmenten an. Auf dieser Basis können Ansatzpunkte für eine zielgerichtete Ansprache einzelner Kundengruppen identifiziert werden. ${ }^{621}$ Hierdurch soll gewährleistet werden, dass, soweit abgegrenzte Kundengruppen über spezifische Marketinginstrumente angesprochen werden, diese möglichst ähnlich auf den Instrumenteneinsatz reagieren. Für das Control-

\footnotetext{
617 Vgl. Kap. 3.4.3.2.

618 Vgl. Kap. 3.4.3.3.

619 Vgl. Kap. 4.1.2.1.1 sowie Weber (1997), S. 11 ff.

$620 \mathrm{Vgl}$. Wăhrisch (1998), S. 338 sowie ausfuhrlicher zur Vorgehensweise Kap. 4.1.2.1.1.

621 Vgl. Freter (1983), S. 43 ff.
} 
ling stellt sich damit die Frage nach der Ableitung geeigneter Kriterien, welche den unternehmensinternen Markt für Controlling-Leistungen so zu strukturieren vermögen, dass eine effektive Ansprache der Kunden möglich wird. Die Ableitung derartiger Kriterien unter Berücksichtigung der dargestellten Leistungsund Organisationsgegebenheiten des Controlling erfolgt in Kap. 4.1.2.1.

Insgesamt geht es für den Controller im Rahmen der Kundenansprache darum, ein aktives Marketing für seine Funktion zu betreiben. ${ }^{622}$ Er muss bemüht sein, über ein auf die identifizierten Kundensegmente ausgerichteten Marketing-Mix seine Dienstleistungen an die internen Kunden zu „verkaufen“. ${ }^{623}$ Der Marketing-Mix stellt hierbei eine dynamische und situationsabhängige Kombination von Produkt-, Preis-, Distributions- und Kommunikationspolitik dar. ${ }^{624}$ Die Notwendigkeit derartige Maßnahmen zu betreiben ergibt sich aus einer zunehmenden Konkurrenzgefährdung der Controlling-Leistungen.

\subsubsection{Wettbewerbsbeziehungen im Rahmen der Leistungsverwertung}

Das Spektrum der Controlling-Leistungen impliziert, insbesondere aufgrund des wachsenden Anteils von Beratungsleistungen, ${ }^{625}$ dass einzelne Leistungen potenziell sowohl eigenerstellt als auch fremdbezogen werden können. ${ }^{626} \mathrm{Die}$ in Abhängigkeit der einzelnen Leistung oftmalige kurzfristige Irreversibilität einer solchen Beschaffungsentscheidung erfordert eine fundierte Analyse der Kostenund Nutzenkonsequenzen. ${ }^{627}$ Anknüpfend an die im Rahmen der Verrechnung von Controlling-Leistungen getätigten Ausführungen zu unternehmensinternen Märkten ${ }^{628}$ soll nachfolgend die Wettbewerbssituation für ControllingLeistungen diskutiert werden. Wettbewerb soll hierbei in einem umfassenden Sinn als ein Verfahren zum Treffen wirtschaftlicher Entscheidungen verstanden werden, bei dem es um die „Verteilung knapper Mittel auf miteinander konkurrierende Zwecke ${ }^{6629}$ geht. Die Einschränkungen bezüglich der Handlungs- und

622 Vgl. Weber (1994), S. 269.

${ }^{623}$ Vgl. Preißler (2000), S. 119.

${ }^{624}$ Vgl. Bouncken (1995), S. 1.

${ }^{625}$ Vgl. hierzu Peemöller/Schmid/Meister (1989), S. 295 ff. und Steinle/Bruch/Michels (1998), S. 443 ff. sowie Kap. 3.2.2.

626 Allg. zu unternehmensinternen Make or Buy-Entscheidungen vgl. Witt (1988b), S. $77 \mathrm{ff}$.

${ }^{627} \mathrm{Vgl}$. Währisch (1998), S. 335.

${ }^{628} \mathrm{Vgl}$. Kap. 3.3.2.5.

629 Berg (1992), S. 241. 
Wahlfreiheit, ${ }^{630}$ die sich daraus ergeben, dass es sich bei Controlling-Leistungen um unternehmensinterne Dienstleistungen handelt, sollen nicht als Ausschlusskriterien für die Anwendbarkeit einer Wettbewerbsbetrachtung gesehen werden. Vielmehr gilt es im Rahmen der Darstellung potenzieller Wettbewerber auch darum, für Unternehmen, bei denen die Wettbewerbssituation nur hypothetischer Natur ist, durch die gedankliche Durchdringung wettbewerblicher Ordnungsprinzipien, Effizienz- und Effektivitätssteigerungspotenziale aufzudecken.

Die unternehmenspolitische Sensibilität der im Bereich des Controlling generierten Informationen hat in der Vergangenheit überwiegend zu einer unternehmensinternen Leistungserstellung geführt. Eine derartige Vorgehensweise lässt sich als Marktzutrittsbarriere ${ }^{63}$ für externe Wettbewerber verstehen. Im Rahmen einer zielgerichteten Positionierung des Controllerbereichs, bedarf es indes auch bei dem Ausschluss externer Wettbewerber einer Analyse des internen Wettbewerbsumfeldes, sowie einer damit verbundenen Herausarbeitung potenzieller Wettbewerbsvorteile und Schwächen des eigenen Leistungsangebotes. Aktuelle Bestrebungen in Unternehmen, konsequent Strukturkosten auch im Bereich des Controlling zu reduzieren, deuten aber insgesamt auf eine zunehmende Öffnung unternehmensinterner Märkte für externe Anbieter hin. Auch wenn die Abgrenzung interner von externen Wettbewerbern aufgrund komplexer Konzernstrukturen an Bedeutung verliert, soll im Sinne einer deutlichen Strukturierung die Unternehmenszugehörigkeit als Abgrenzungskriterium verwandt werden.

\subsubsection{Interne Wettbewerber}

Aufbauend auf der Diskussion einer strukturierten Aufgabenzuordnung in Unternehmen lassen sich im Controlling die möglichen Aufgabenträgeralternativen als potenzielle interne Wettbewerber begreifen. ${ }^{632}$ Dementsprechend vermag eine erste Analyse der Eignungs-, Koordinations- und Verhaltensaspekte alternativer Aufgabenträger Anhaltspunkte über mögliche unternehmensinterne Wettbewerbsbeziehungen zu geben. Auch wenn Aufgaben regelmäßig langfristig zugeordnet werden, bestehen kurzfristig im Einzelfall Alternativen, soweit andere Unternehmensmitglieder spezifische Qualifikationen bezüglich des an-

\footnotetext{
${ }^{630}$ Die Wahlfreiheit bezliglich alternativer Angebote und die Handlungsfreiheit bezulglich der Ressourcenverwendung werden als Voraussetzung fulr einen funktionierenden Wettbewerb genannt. Vgl. Berg (1992), S. 341.

${ }^{631} \mathrm{Zu}$ unterschiedlichen Ausprägungen von Marktzutrittsbarrieren vgl. Backhaus (1999), S. 69 ff.

${ }^{632}$ Vgl. Herzog (1999), S. 37 sowie Kap. 3.2.1.2.
} 
stehenden Entscheidungsproblems aufweisen. Die in Kap. 3.3.2.4. aufgezeigte große Bedeutung informeller Kontakte in einem Unternehmen relativiert darüber hinaus vielfach die formelle Geltung der Aufgabenzuordnung. Allgemein kann man bezüglich der durch das Controlling zu erbringenden Informationsleistungen drei Anbieteralternativen in einem Unternehmen unterscheiden. Hierbei können die Informationen durch

- die Nachfrager der Informationen selbst,

- intern spezialisierte Anbieter oder durch

- andere Anbieter als die intern spezialisierten

erstellt werden..$^{63}$ Die Alternative, dass Controlling-Informationen durch die Nachfrager selbst generiert werden, besteht grundsätzlich in jedem Unternehmen, unabhängig von spezifischen wettbewerblichen Rahmenbedingungen. Gerade die Tatsache, dass sich Controlling bei dem dieser Arbeit zugrundeliegenden Verständnis in der Schnittmenge zwischen Management/internen Kunden und Controller abspielt, ${ }^{634}$ kann im Einzelfall zu einer Überschneidung einzelner Aktivitäten führen. Wesentlich für die Arbeit des Controllers ist in solchen Fällen zu ergründen, warum controllingspezifische Tätigkeiten von Kunden selbst übernommen werden. Oftmals ist Unzufriedenheit mit der Struktur oder Qualität des Leistungsangebotes des Controlling eine Ursache. Demgegenüber können bewusst im Sinne des Self-Controlling Controlling-Verantwortlichkeiten an derzeitige Kunden delegiert werden, wobei jedoch durch einen zentralen Service sicherzustellen ist, dass die Kunden über alle steuerungsrelevanten Informationen sowie das entsprechende Interpretations-Know how verfügen. Gerade in Verbindung mit leicht bedienbaren Software-Lösungen können Kunden in die Lage versetzt werden, ihren spezifischen Informationsbedarf selbst sachgerecht abzudecken. Dies korrespondiert mit der bereits im Rahmen der Informationsdistribution angesprochenen Wandlung einer Bringschuld in eine Holschuld. Hierdurch können sich Aufgabenschwerpunkte zwischen Controller und seinen derzeitigen Kunden verschieben. Wettbewerbsvorteile solcher dezentralen Lösungen sind in den fundierteren ursachenbezogenen Analysemöglichkeiten der vor Ort tätigen Kunden zu sehen. Demgegenüber sprechen Größen- und Spezialisierungsvorteile für eine zentrale Controlling-Lösung. Fokussiert man jedoch auf die Beratungsfunktion des Controlling, besteht hier weniger eine Wettbewerbssituation zu Kunden, die Teilleistungen eigenständig erstellen, als viel-

${ }^{633} \mathrm{Zu}$ den Differenzierungskriterien und deren Anwendung auf die unternehmensinterne Dienstleistung Kostenrechnung vgl. Währisch (1998), S. 335 f.

${ }^{634}$ Vgl. hierzu das Schnittmengengebilde von Deyhle/Steigmeier (1993), S. 26 f sowie Abbildung 3 
mehr eine Kunden-Lieferanten-Beziehung, da Schulungs- und Beratungsaktivitäten des Controlling ihre Kunden oft erst in die Lage versetzen, zweckmäßige Analysen vornehmen zu können. ${ }^{635}$

Soweit Kunden Informationsleistungen nicht eigenständig erstellen, ist der institutionalisierte Unternehmensteilbereich Controlling als klassische Form des intern spezialisierten Anbieters zu sehen. Eine konkrete interne Wettbewerbssituation entsteht regelmäßig dann, wenn weitere Abteilungen ergänzend zu ihren eigenen Produktprogrammen, aufgrund spezifischen theoretischen Know hows und praktischer Anwendungserfahrungen ohne Rückgriff auf den Zentralanbieter Controlling, Lösungen für andere Fachbereiche anbieten. ${ }^{636}$ Wiederum können informelle Kontakte auf Basis gewachsener persönlicher Beziehungen oder aber auch die hohe Reputation eines Unternehmensteilbereiches zu alternativen Angeboten führen. In Abhängigkeit des zu lösenden Entscheidungsproblems kommen potenziell unterschiedliche Anbieter in Betracht. Darüber hinaus spielt es eine entscheidende Rolle, wie artverwandt Kernleistungen anderer interner Anbieter mit denen des Controlling sind. Insbesondere die Revision ${ }^{637}$, gesonderte Planungs- und Strategieabteilungen sowie interne Consulting-Einheiten sind in diesem Zusammenhang als alternative Anbieter und damit potenzielle Konkurrenten $\mathrm{zu}$ nennen. ${ }^{638}$ Betrachtet man beispielsweise die Aufgaben der internen Revision, so wird deutlich, dass der in diesem Unternehmensteilbereich vorhandene ganzheitliche Überblick über die Strukturen und Abläufe des Unternehmens dazu führen kann, dass die interne Revision als alternativer Anbieter für Beratungsleistungen im Bereich Prozessoptimierung gesehen wird. ${ }^{639}$ Ausschlaggebend für die Bedeutsamkeit der Konkurrenzsituation ist letztlich die Wahrnehmung der jeweiligen unternehmensinternen Kunden. ${ }^{640}$ Will das Controlling sich gegenüber der internen Revision durch eine zukunfts- und umweltbezogene Betrachtungsperspektive abgrenzen, wird die Konkurrenzsituation zu in großen Unternehmen existenten Planungs- und Strategieabteilungen deutlich. ${ }^{641}$ Als Ergebnis einer umfassenden Make or Buy-Entscheidung im Bereich Beratung sind ferner die in vielen größeren Unternehmen gegründeten in-

${ }^{635}$ Zur internen Beraterrolle des Controllers vgl. Weber/Schäffer (1999), S. 32 sowie Weber/Schäffer (1999b), S. 8 ff.

${ }^{636} \mathrm{Vgl}$. Währisch (1998), S. 336.

${ }^{637} \mathrm{Zu}$ Zielen und Aufgaben der internen Revision vgl. Baetge/Roß/Uthoff (1994), S. 9 ff.

638 Vgl. Roth (2000), S. 135.

${ }^{639} \mathrm{Vgl}$. Roth (2000), S. 136. Zu Unterschieden in den Anforderungsprofilen von Controllern und internen Revisoren vgl. Peemöller/Schmid/Meister (1989b), S. 227 ff.

$640 \mathrm{Zu}$ Wahmehmungswirkungen vgl. Meffert (2000), S. $691 \mathrm{ff}$.

641 Vgl. Roth (2000), S. 136. 
ternen Consulting-Einheiten (Inhouse Consultants) zu sehen. ${ }^{642}$ Die Tatsache, dass die Beratungsaufgaben auch im Controlling eine wachsende Bedeutung erlangen, macht deutlich, dass beratende Tätigkeiten in den Bereichen Systemeinführung, Umstrukturierung, Neuorganisation und Strategie zunehmend einer erhöhten Transparenz ausgesetzt sind. Im Ergebnis geht es für das Controlling darum, auf Basis zugeordneter Aufgaben und einer konsequenten Betrachtung der eigenen Stärken und Schwächen Wettbewerbsvorteile in den Augen der Kunden zu realisieren, und somit die Stellung des Controlling über einen konkreten Beitrag zur Erfüllung der Unternehmensziele zu stärken. Hierbei kann es jedoch nicht darum gehen, andere Unternehmensteilbereiche „auszuschalten“, vielmehr soll die vergleichende Betrachtung Handlungs- und Optimierungspotenziale offenlegen.

Neben den genannten potenziellen internen Konkurrenten des Controlling bieten eine Vielzahl unternehmensexterner Gruppierungen Dienstleistungen an, die sich in Teilbereichen mit dem Leistungsangebot des Controlling überschneiden.

\subsubsection{Externe Wettbewerber}

Insbesondere in jüngster Vergangenheit haben Entwicklungen wie Reengineering, Outsourcing und Lean Management zu einer konsequenten Strukturkostenreduktion geführt. In diesem Zusammenhang müssen sich auch ControllingAktivitäten verstärkt einer an externen Anbietern ausgerichteten Leistungsbeurteilung unterziehen, insbesondere dann, wenn Controlling nicht unmittelbar eine Kernkompetenz des Unternehmens darstellt. Im Einzelfall kann dies ein Outsourcing von Leistungen zur Folge haben. Im Rahmen der in solchen Fällen zu treffenden Make or Buy-Entscheidung stehen externe Anbieter dem internen Controlling-Bereich unmittelbar als Konkurrenten gegenüber. Eine Studie von AMSHOFF zeigt, dass $29 \%$ von 320 befragten Unternehmen ControllingAufgaben an unternehmensexterne Anbieter übertragen. ${ }^{643}$ Insbesondere bei standardisierten Leistungen ist eine Qualitäts- und Preisbeurteilung intersubjektiv durchführbar. MÜLLER/FICKEL schlagen in diesem Zusammenhang vier grundlegende Entscheidungskriterien zum Outsourcing von Controlling-Aufgaben vor: $:^{644}$

${ }^{642}$ Zum Stand des internen Consultings in Deutschland vgl. Hoyer (2000), S. 56 ff. Ein allgemeines Entscheidungsmodell zur Optimierung von Make or Buy-Entscheidungen findet sich bei Hahn/Hungenberg/Kaufmann (1994), S. $75 \mathrm{ff}$.

643 Insbesondere bei kleineren Unternehmen ist dies der Fall. Das ausgelagerte Aufgabenvolumen ist jedoch oftmals noch gering. Vgl. Amshoff (1993), S. 340 f. sowie Reiß/Höge (1995), S. 1723.

644 Vgl. Müller/Fickel (1997), S. $109 \mathrm{ff}$. 
- kein Bestandteil des Kerngeschäfts,

- zu geringer Umfang, um Spezialwissen aufbauen und auslasten zu können,

- keine Gefährdung des Geschäfts durch Überlassung von Spezialwissen,

- zeitliche und inhaltliche Abgrenzbarkeit der Aufgabe.

Sicherlich erscheint das Outsourcing auch dann gerechtfertigt, wenn eine langfristige Kooperation mit dem externen Anbieter angestrebt wird und somit das Kriterium der zeitlichen Abgrenzbarkeit nicht erfüllt ist. Konkret kommen als externe Wettbewerber für Controlling-Leistungen Dienstleister wie die DATEV e.G., Unternehmensberater ${ }^{645}$, DV-Berater, Wirtschaftsprüfer ${ }^{646}$ oder aber auch Management Trainer in Betracht.

In jüngster Vergangenheit werden, insbesondere vor dem Hintergrund der Betrachtung des Controlling als interne Dienst- $b z w$. Beratungsleistung, verstärkt Unternehmensberater als Wettbewerber für Controlling-Leistungen angesehen. Daher soll im Folgenden die Konkurrenzsituation zu Beratern beispielhaft einer ausführlichen Betrachtung unterzogen werden. Auch wenn Beratungsunternehmen sich oftmals schwerpunktmäßig mit der Erstellung und Implementierung von Konzepten befassen, erscheint der Übergang zu ,alltäglichen”, regelmäßig intern erstellten Controlling-Leistungen fließend. Bezogen auf die große Gruppe der Unternehmensberater ergab eine Recherche des Verfassers im Mai 2001, dass ca. 58\% ${ }^{647}$ der in der Datenbank des Bundesverbandes Deutscher Unternehmensberater (BDU) ${ }^{648}$ registrierten Beratungen Produkte im Bereich Controlling anbieten. ${ }^{649}$ Berücksichtigt man, dass in der Grundgesamtheit der Erhebung auch Beratungsunternehmen enthalten sind, die ausschließlich Personalberatung durchführen, so ergäbe sich für den Teilbereich der Managementbera-

645 Umfassend zum Marketing fur Leistungen der Unternehmensberatung vgl. Miethe (2000).

$646 \mathrm{Zu}$ Entwicklungstendenzen in der Wirtschaftsprilfung vgl. Baetge (1997b), S. V ff. Eine MarketingKonzeption fur Wirtschaftsprüfer findet sich bei Schmitz (1997), S. $246 \mathrm{ff}$. Zur potenziellen Konkurrenz von Unternehmensberatern und Wirtschaftspruffern vgl. Backhaus/Spăth (1992), S. 761 ff. sowie Backhaus (1990), S. $680 \mathrm{ff}$.

647 Dies entspricht 186 von 321 Beratungen.

648 Der BDU ist der derzeit etablierteste Wirtschafts- und Berufsverband der Management- und Personalberater in Deutschland.

649 Grundlage dieser Erhebung ist eine Auswertung der nach Tätigkeitsschwerpunkten der Mitgliedsunternehmen strukturierten Datenbank des BDU. Es wurde bei der Auswertung keine Differenzierung nach der Branchenausrichtung der Beratungen vorgenommen. Vgl. http:/www.bdu.de/berateraus-wahldatenbank/ default.htm. 
tungen $^{650}$ ein noch deutlicheres Bild. Differenziert man die Gruppe der Unternehmensberatungen, die Controlling-Leistungen anbieten, ${ }^{651}$ weiter nach den Controllerbereich betreffenden Produkten/Produktgruppen, ${ }^{652}$ so ergibt das in Abbildung 32 dargestellte Bild.

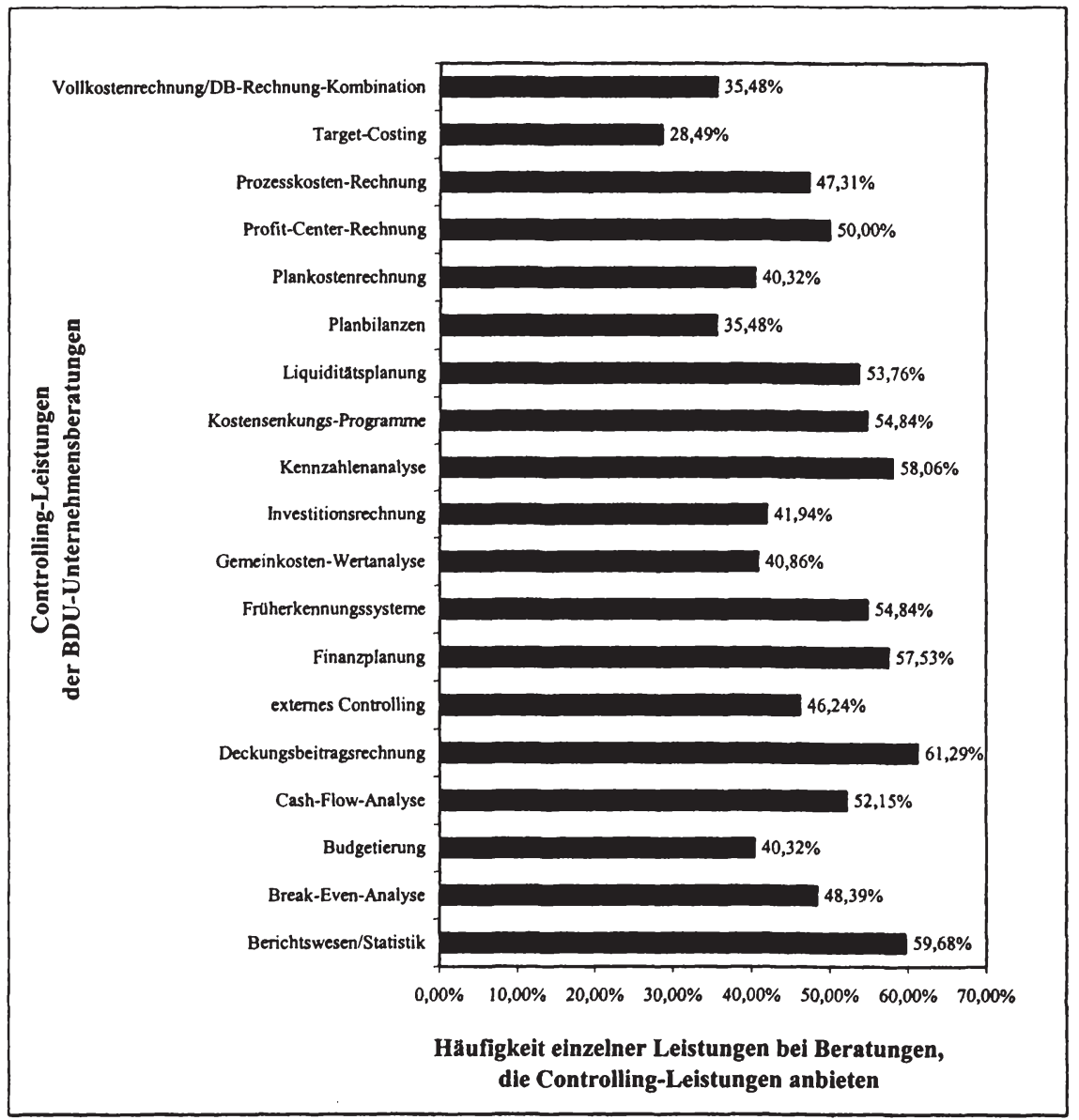

Abbildung 32: Tätigkeitsbereiche von Unternehmensberatungen mit Controlling-Leistungen

${ }^{650}$ Eigentlich sind nur diese als unmittelbare Konkurrenten des Controlling anzusehen.

${ }^{651}$ Diese Gruppe wird im Folgenden als Grundgesamtheit betrachtet.

652 Nach der Systematisierung der BDU Beraterdatenbank sind dies Tatigkeitsschwerpunkte 
Das hier aufgezeigte Leistungsspektrum der Unternehmensberatungen im Bereich Controlling deutet auf vielfältige Überschneidungen zu den Tätigkeitsbereichen des unternehmensinternen Controlling hin. Fraglich erscheint in diesem Zusammenhang, welche Faktoren im Einzelfall für die Erteilung eines Auftrags an das Controlling oder einen externen Berater sprechen. Oftmals spielen unabhängig von der spezifischen Qualifikation der Anbieter unternehmenspolitische Gründe eine entscheidende Rolle. ${ }^{653}$ So werden beispielsweise externe Berater bei Umstrukturierungsprojekten oftmals vorgezogen, da die unternehmensinterne Akzeptanz markterfahrener Berater höher ist und zudem eventuell resultierende Kündigungen unternehmensexternen Beratern scheinbar besser angelastet werden können.

Betrachtet man über derartige unternehmenspolitische Gründe hinausgehende Argumente für die Wahl eines Anbieters, so wird deutlich, dass aus der Perspektive der Kunden regelmäßig wenige kontextunabhängige Faktoren genannt werden. Verhaltensweisen, die von Kunden als potenzielle Wettbewerbsvorteile von Unternehmensberatern gesehen werden, sind: ${ }^{654}$

- Consultants stellen das Kundeninteresse vor das Eigeninteresse,

- vertreten eine unabhängige Position,

- fokussieren auf managementrelevante Themen,

- vertreten einen umfassenden Problemlösungsansatz,

- bieten individuelle Lösungsansätze,

- gestalten die Arbeitsatmosphäre offen und hierarchiefrei, widersprechen gegebenenfalls auch und

- versuchen Manager in die Lage zu versetzen, vergleichbare Probleme zukünftig eigenständig zu lösen.

Auch wenn diese Charakteristika sicherlich den Idealtypus eines Unternehmensberaters beschreiben, besteht die Möglichkeit, hieraus Anhaltspunkte zur Steigerung der Wettbewerbsfähigkeit von Controllern abzuleiten.

653 Vgl. Roth (2000), S. 136.

${ }^{654}$ SCHÄFFER/WEBER sprechen in diesem Zusammenhang von Bereichen, in denen Controller von Consultants lemen können. Im Rahmen der Argumentation des Verfassers stellen dies Wettbewerbsvorteile der Unternehmensberater dar. Vgl. Schäffer/Weber (1999), S. 21 ff. Zur Philosophie einer ganzheitlich-evolutionären Unternehmensberatung vgl. Schrädler (1996), S. 173fff.omas Mosiek - 978-3-631-75321-7 
Demgegenüber erweisen sich in Abhängigkeit des unterstellten Entscheidungsproblems nachfolgende Faktoren als Erfolgsfaktoren des Controllers im internen Markt:.5s

- Innenkenntnis der Kultur, Regularien und Abläufe,

- kein Know how-Abfluss, bessere Geheimhaltung,

- offenerer Umgang als mit Externen durch Vertrauen,

- kürzere Vor- und Anlaufzeiten durch Insiderwissen,

- Verfügbarkeit auf Zuruf,

- meist beteiligungsorientierter Beratungsansatz,

- kennen den Betriebsrat und seine Präferenzen,

- sehen den Schwerpunkt ihrer Beratung in der Umsetzung, auch der Projekte, die vorher von Externen durchgeführt wurden.

Die hier aufgezählten Faktoren zeigen, dass es bei sich inhaltlich entsprechenden Leistungsangeboten zahlreiche Anknüpfungspunkte für den Controller gibt, sein spezifisches Problemlösungspotenzial deutlicher zu kommunizieren und die Kunden von der Qualität seiner Leistung zu überzeugen. Wichtig ist zunächst zu erkennen, wer bezogen auf welche Leistung als Konkurrent anzusehen ist und erst auf dieser Basis seine relative Wettbewerbsposition zu bestimmen. Bei einer Analyse der Wettbewerbssituation ist jedoch zu bedenken, dass bspw. bei den hier aufgelisteten Erfolgsfaktoren des Controllers im internen Markt einige gleichermaßen für das interne Consulting gelten und daher zu Differenzierungszwecken nicht gegenüber jedem Wettbewerber verwandt werden können.

Ein aus der Perspektive der Kunden oftmals herangezogenes Differenzierungsmerkmal sind die Kosten für den Einsatz eines Controllers bzw. eines externen Anbieters. Da die Verrechnung der Leistungen des Unternehmensteilbereichs Controlling in vielen Unternehmen noch nicht differenziert vorgenommen wird, stellt der Einsatz externer Berater regelmäßig die teurere Alternative dar. Im Einzelfall sind auch in Abhängigkeit der Erfahrung des jeweils eingesetzten Beraters Stundensätze von

- 100 EUR bei durchschnittlichen deutschen Beratungen,

- 250 EUR bei renommierten deutschen Unternehmensberatungen und

- 250 - 375 EUR bei international renommierten Consulting-Firmen

${ }^{655}$ Die Aufzăhlung erfolgt in Anlehnung an die Erfolgsfaktoren von Inhouse Consultants. Vgl. Niedereichholz (2000), S. 16. 
zu entrichten. ${ }^{656}$ Die vermeintlich hohen Kosten werden jedoch bei vielen Unternehmen durch das zusätzlich erworbene externe Know how und in zunehmenden Umfang auch durch die kurzfristige und unverbindliche Möglichkeit, Kapazitäten aufbauen zu können überkompensiert. Darüber hinaus bieten einige externe Beratungen neuerdings Modelle an, die ihr Honorar an die objektiv erzielten Einsparungen koppeln. ${ }^{657}$ Durch eine derartige Erfolgsbeteiligung kann im Sinne der Principal-Agent-Theorie ${ }^{658}$ ein Harmonisierung der Zielvorstellungen erreicht werden. Um gegenüber derartigen Entwicklungen konkurrenzfähig zu bleiben, gilt es für das Controlling, anreizkonforme Verrechnungspreise zu etablieren und die Wettbewerbsfähigkeit dieser Preise zu kommunizieren. Insbesondere bei internen Beratungsprojekten, bei denen dass spezifische Know how des Controlling gefragt ist, kann auch bei einer sachgerechten Leistungsverrechnung von kostenbasierten Wettbewerbsvorteilen des Controlling ausgegangen werden. Ursache hierfür sind das Entfallen von Rüstzeiten für die Einarbeitung und die Tatsache, dass die Komplexität derartiger Problemstellungen regelmäßig hoch qualifizierte und damit vergleichsweise teure externe Berater erfordert.

\subsubsection{Kunden- und wettbewerbsbezogene Gestaltungsparameter des kundenorientierten Controlling}

Aufbauend auf der Beschreibung der Kundenbeziehungen des Controlling im Rahmen der Leistungserbringung und der Wettbewerbsbeziehungen im Rahmen der Leistungsverwertung soll im Folgenden auf diesbezüglich relevante Gestaltungsparameter eingegangen werden.

Anknüpfend an die potenzielle Konkurrenzsituation im Controlling ist zu differenzieren, in welchem Umfang einzelne Leistungen einer Konkurrenzgefährdung durch weitere interne oder externe Anbieter ausgesetzt sind. In diesem $\mathrm{Zu}$ sammenhang sollen Monopol-Dienstleistungen und Wettbewerbs-Dienstleistungen unterschieden werden.

Die Existenz alternativer Kunden des Controlling macht es im Rahmen einer Marketingbetrachtung erforderlich, auf Möglichkeiten und Auswirkungen einer Leistungsindividualisierung einzugehen. Ferner ist die beiläufig schon erwähnte Leistungsevidenz ein Tatbestand, der subjektiv, aus der Perspektive einzelner

\footnotetext{
${ }^{656} \mathrm{Vgl}$. Trilse (2000), S. bu/2.

${ }^{657}$ Hier und im Folgenden vgl. Müler/Fickel (1997), S. 109 ff.

658 Allg. dazu vgl. Williamson (1985).
} 
Kunden, zu betrachten ist. Ferner determinieren sowohl das Ausmaß der Leistungsindividualisierung als auch die Leistungsevidenz das kundenspezifische Nutzungsverhalten der durch das Controlling bereitgestellten Informationen.

\subsubsection{Individualität der Leistung}

Auf Grundlage der Betrachtung alternativer Kunden des Controlling stellt sich die Frage, in welchem Umfang der Controller im Einzelfall auf die artikulierten subjektiven Bedürfnisse, bzw. auf den objektiv unterstellten Informationsbedarf seiner Kunden eingehen kann und muss. Die postulierte Kundenorientierung spricht zunächst für eine umfassende Leistungsindividualisierung, da grundsätzlich von sehr heterogenen unternehmensinternen Informationsbedürfnissen auszugehen ist. ${ }^{659}$ Demgegenüber deuten die zunehmenden Wirtschaftlichkeitserfordernisse im Controlling auf die Notwendigkeit einer differenzierten Kosten-Nutzen-Betrachtung der Individualisierungswirkungen bei einzelnen Leistungen hin. Bei vielen Leistungen des Controlling ist ein Mindestmaß an Leistungsindividualisierung aufgrund der zwangsläufigen Integration externer Faktoren in den Leistungserstellungsprozess gegeben. ${ }^{600}$ Auf Grundlage der dem Controlling zugeschrieben Planungs-, Kontroll-, Informationsversorgungsund Beratungsaufgaben ${ }^{61}$ werden insbesondere im Bereich Informationsversorgung die Konsequenzen von Individualisierungs- bzw. Standardisierungsmaßnahmen diskutiert. ${ }^{62}$ Demgegenüber spricht die Sequenzintegration vieler Planungs- und Kontrollaktivitäten für eine inhaltlich und zeitlich standardisierte Vorgehensweise. Der Projektcharakter von Beratungsaktivitäten lässt demgegenüber nur in Teilbereichen den Einsatz standardisierter Instrumente zu.

Aus diesen Gründen sollen nachfolgend primär Auswirkungen der Leistungsindividualisierung im Aufgabenbereich Informationsversorgung betrachtet werden. Hierbei soll der Schwerpunkt auf dem Reporting liegen, da sich ein Großteil der Controller-Arbeit in Form von Reports manifestiert, ${ }^{63}$ wodurch ihnen eine besondere Bedeutung bei der internen Profilierung des Controlling zu-

\footnotetext{
659 Allg. zum Trend der wachsenden Individualisierung des Konsumentenverhaltens vgl. Meffert (2000), S. 107 ff.

660 Vgl. Kap. 3.2.2.1.

661 Vgl. Weber (1999), S. 315 ff.

662 So z.B. Weber/Weißenberger/Aust (1997), S. 5 ff.

663 WrTt bezeichnet in diesem Zusammenhang das Reporting als Kernstück des Controlling. Vgl. NeuhäuserMetternich/Witt (2000), S. 290. 
kommt. Insgesamt ist das Reporting somit als zentraler Träger des internen Kundennutzens anzusehen. ${ }^{664}$

Im Rahmen der grundlegenden Ausführungen zum Dienstleistungsmarketing wurde bereits die Leistungstypologie von ENGELHARDT ET AL. ${ }^{665}$ vorgestellt, welche Leistungen in Abhängigkeit von ihrem Immaterialitätsgrad und Integrationsgrad $\mathrm{zu}$ klassifizieren vermag. ${ }^{666} \mathrm{Zu}$ einer differenzierteren Betrachtung der Controlling-Leistungen wird nachfolgend die auf den Gedanken von ENGELHARDT ET AL. aufbauende Leistungstypologie von MEFFERT/BRUHN ${ }^{667}$ herangezogen. ${ }^{668}$ In dieser Typologie wird die Dimension Integrativität in die Komponenten Interaktion und Individualität der Leistungen zerlegt und stellt somit eine im Ergebnis dreidimensionale Erweiterung der Typologie von ENGELHARDT ET AL. dar. Bezogen auf das Controlling stellt die Immaterialität einer Leistung kein signifikantes Differenzierungsmerkmal dar, ${ }^{699}$ da es sich im Kern der Controlling-Leistungen regelmäßig um Informationen handelt. Betrachtet man daher im Folgenden nur die Erweiterung von MEFFERT/BRUHN, lassen sich Reporting-Leistungen in Abhängigkeit ihres Interaktions- und Individualisierungsgrades systematisieren. Die Interaktion hat bereits im Rahmen der Diskussion des Leistungsspektrums eine angemessene Behandlung erfahren. ${ }^{670}$ Demgegenüber ist das Ausma $\beta$ der Individualisierung ein unmittelbar kundenbezogener Sachverhalt. Hierbei soll die Individualisierung zunächst auf die Ebene der Leistungsinhalte bezogen werden, später dann auch auf die Ebene der formalen Gestaltung. Zielsetzung furr den Controller ist im Reporting in Abhängigkeit der unterstellten Entscheidungssituation alle entscheidungsrelevanten Tatbestände angemessen aufbereitet zu berichten. Klassifiziert man beispielhafte Reporting-Leistungen im Sinne der ausgewählten Typologie, so ergibt sich das in Abbildung 33 dargestellte Bild.

In Abhängigkeit des individuellen Informationsbedarfs der jeweiligen Nachfrager lassen sich Leistungen in unterschiedlichem Umfang standardisieren. Da regelmäßig von sehr differenzierten Informationsbedürfnissen der Nachfrager auszugehen ist, sind standardisierungsbedingte Aufwandseinsparungen im

\footnotetext{
${ }^{664}$ Vgl. Stadler/Weißenberger (1999), S. 5.

665 Vgl. Engelhardt/Kleinaltenkamp/Reckenfelderbäumer (1993), S. $395 \mathrm{ff}$.

666 Vgl. Kap. 2.2.2.1

$667 \mathrm{Vgl}$. Meffert/Bruhn (1995), S. $31 \mathrm{ff}$.

$668 \mathrm{Vgl}$. Abbildung 15 in Kap. 3.2.2.1.3.

669 Zum gleichen Ergebnis gelangt SCHADE furr Leistungen der Unternehmensberatung. Vgl. Schade (1995), S. 31.

670 Vgl. Kap. 3.2.2.1.3.
} 
Controlling dem eventuell standardisierungsbedingten Informationsverlust gegenüberzustellen.

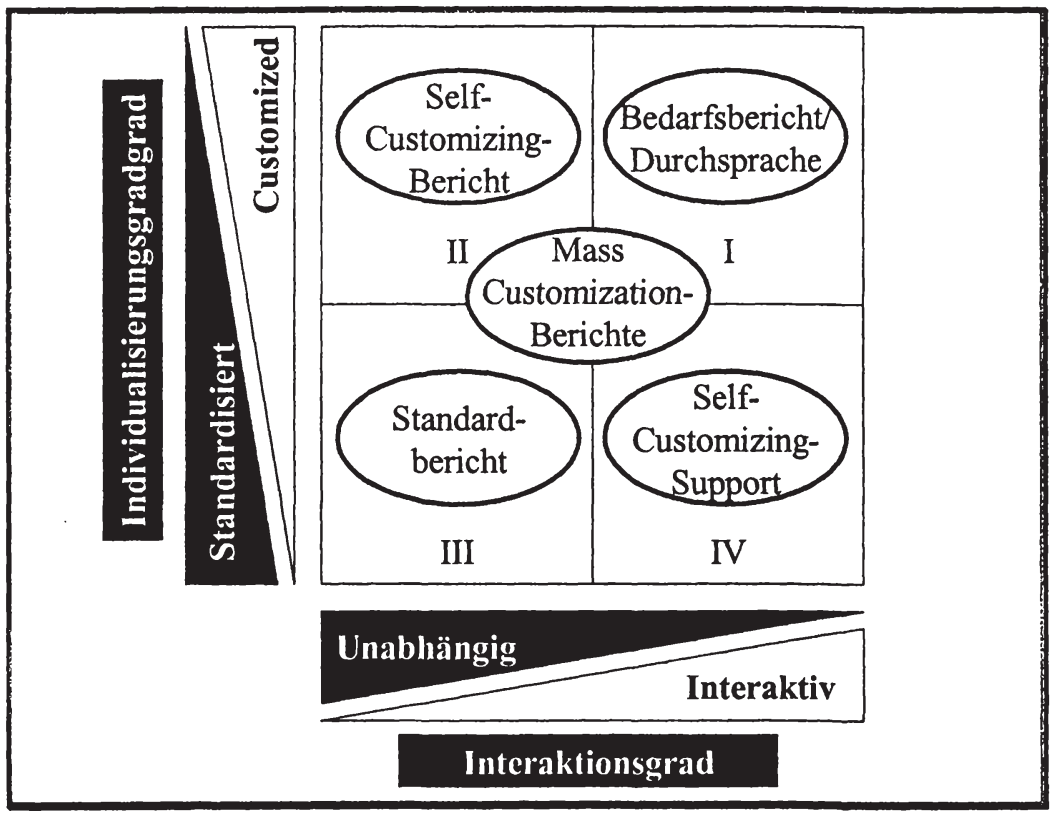

Abbildung 33: Klassifikation von Reporting-Leistungen ${ }^{671}$

Bedarfsberichte und deren institutionalisierte Durchsprache sind regelmäßig nicht durch standardisierte Leistungen ersetzbar. Demgegenüber bedürfen Standardberichte, wie die kostenstellenbezogenen Monatsberichte, bei entsprechender Aufbereitung nicht zwangsläufig individualisierter Elemente. Korrespondierend mit dem Individualisierungsmehraufwand bei Bedarfsberichten ist das Ausmaß der Interaktion zu sehen, da die Bedarfsspezifikation einen umfangreichen Informationsaustausch im Sinne einer Interaktion erfordert. Eine Möglichkeit, Kundenorientierung im Controlling über die spezifisch empfängerorientierte Leistungsindividualisierung konsequent umzusetzen und dabei Kosten und Kapazitätsgesichtspunkten gleichermaßen Rechnung zu tragen, stellt das Verfahren des Mass Customization dar. ${ }^{672}$ Im Sinne eines Baukasten-Systems können individuelle oder standardisierte Berichtsmodule miteinander kombiniert werden, so dass - auch bei einer ausschließlichen Verwendung von Stan-

671 Die zugrundeliegende Leistungstypologie entspricht der von Meffert (1993), S. 12.

672 Hier und im Folgenden vgl. Steinle/Thiem/Rohden (2000), S. $284 \mathrm{f}$. 
dardmodulen - individuelle Berichte generiert werden können. Das Standardisierungspotenzial hängt hierbei maßgeblich von den jeweiligen Berichtsinhalten bzw. -zwecken ab. So schreibt man bspw. dem Gemeinkosten-Controlling weitergehende Standardisierungsmöglichkeiten als dem Beteiligungscontrolling zu. ${ }^{673}$ Betrachtet man den Interaktionsgrad im Rahmen des Mass Customization im Controlling, so liegt zunächst die Verantwortung für die Berichtzusammenstellung beim Controller, der hierbei jedoch auf die Artikulation von Bedarfsinformationen seines Kunden angewiesen ist. Demgegenüber liegt beim SelfCustomization die Verantwortung für die sachgerechte Inanspruchnahme und Kombination der Informationen beim Empfänger selbst. ${ }^{674}$ Insbesondere zeitgemäße Intranet-Anwendungen bieten die technische Basis für derart zentrale Reportbibliotheken, aus denen jeder Kunde seinen Bedarf selbst befriedigen kann. ${ }^{675}$ Bedingt durch die geringere Interaktion zwischen Controller und Controlling-Kunde muss sichergestellt werden, dass die Anwender des SelfCustomizing über das entsprechende Anwendungs- und Interpretations-Know how verfügen. In diesem Zusammenhang erscheint es zweckmäßig, einen teilweise standardisierbaren Support einzurichten, der über eine intensive Interaktion mit den Kunden die Nutzung der generierten Informationen zu fördern vermag. Wesentlich sind in diesem Zusammenhang Schulungsveranstaltungen zu Beginn der Systemeinführung. Ferner obliegt es dem Controller im Sinne der in Kap 3.3.2.2. bereits angesprochen Pull-Strategie, motivierend auf seine Kunden einzuwirken, damit die Leistungen zunächst in Anspruch genommen und anschließend sachgerecht genutzt werden. ${ }^{676}$

Neben den hier dargestellten Vorgehensweisen einer an die Kundenbedürfnisse angelehnten inhaltlichen Leistungsindividualisierung kommt insbesondere im Bereich des Reporting der formalen Standardisierung eine wesentliche Bedeutung zu. War die inhaltliche Individualisierung von Berichten - soweit dies mit vertretbarem Aufwand möglich war - einer Standardisierung vorzuziehen, weist die formale Standardisierung von Berichten regelmäßig überwiegend Vorteile auf. ${ }^{677}$ Die Komplexität der in Controllerberichten zu übermittelnden Sachver-

${ }^{673} \mathrm{Vgl}$. Steinle/Thiem/Rohden (2000), S. 284.

674 Allg. vgl. ReiB/Beck (1995), S. 26. Zu den daraus erwachsenden Auswirkungen auf das Verhältnis von Bring- und Holschuld vgl. Kap. 3.3.2.2.

${ }^{675}$ Dieses Konzept findet bspw. bei der Software PrimaverA Anwendung. Im Intra- und Internet werden Berichtsmodule bereitgestellt, die der Anwender zu bedarfsspezifischen Berichten kombinieren kann.

676 Zum Nutzungsverhalten von Controlling-Leistungen vgl. Kap. 3.4.3.3.

677 Nachteile der formalen Standardisierung sind im wesentlichen in der Vernachlässigung von Besonderheiten und in der mőglicherweise zwanghaften Ausfullung von vorgegeben Strukturen zu sehen. Vgl. NeuhăuserMetternich/Witt (2000), S. 312. 
halte macht es erforderlich, den Adressaten so weit wie möglich an die formale Struktur und Gestaltung eines Berichtes zu gewöhnen, da hierüber die Akzep$\operatorname{tanz}$ und damit das Nutzungsverhalten gefördert werden kann. ${ }^{678}$ Weitere Vorteile von formalen Reportingstandards sind: ${ }^{679}$

- Übersichtlichkeit im Objektvergleich,

- Übersichtlichkeit im Zeitvergleich,

- verbesserte Managementakzeptanz der Controllerfunktion aufgrund nachvollziehbarer, wiedererkennbarer und damit „lesbarerer“ Standards,

- übergreifendes Reporting mit dadurch verringerten Verstänälichkeitsund Einarbeitungsproblemen und

- Übertragbarkeit des Reportingstandards auf neue Mitarbeiter, damit die Flexibilität des Controllingsystems steigt.

Insgesamt können über die Etablierung von Reportingstandards positive Wirkungen auf den Umfang von Berichten ausgehen und damit auf die Beanspruchung der Verarbeitungskapazität des Managements, welche den diesbezüglich zentralen Engpassfaktor darstellt. ${ }^{680}$ Über die Gewöhnung des Managements an die Reportstruktur und -gestaltung können langfristig mehr Informationen übermittelt werden, ohne die Verarbeitungskapazität zusätzlich zu beanspruchen. ${ }^{681}$ Dies darf jedoch nicht darüber hinwegtäuschen, dass lediglich die für den Kunden relevanten Informationen zu berichten sind und nicht die Summe des technisch Möglichen. ${ }^{62}$ Dass die Verbreitung von Reportingstandards und deren Verinnerlichung durch Controller noch nicht selbstverständlich sind, zeigt Abbildung 34. Dargestellt werden wesentliche formale Reportingstandards sowie die diesen von Managern bzw. Controllern beigemessene Bedeutung.

Unter dem Gesichtspunkt der Kundenorientierung wird deutlich, dass noch nicht allen Controllern die Bedürfnisse ihrer Kunden in Bezug auf die Berichtsgestaltung geläufig sind. Insbesondere auch im Bereich der Kommunikation von Berichten können Defizite festgestellt werden. ${ }^{683}$ So wird der Institutionali-

678 Vgl. hierzu die Ausfulhrungen von WITT zu Lerneffekten, die sich bei standardisierten Berichten einstellen. Witt (1992), S. 247.

679 Vgl. Neuhäuser-Metternich/Witt (2000), S. 311.

680 Ausfuihrlich zum Vergleich von Berichtsumfangen vgl. Stadler/Weißenberger (1999), S. 7 sowie Weber/ Wießenberger/Aust (1997), S. $10 \mathrm{ff}$.

681 Vgl. Neuhäuser-Metternich/Witt (2000), S. 311.

682 Vgl. hierzu die oftmals diesbeziglich geăußerte Kritik, z.B. bei Weber/Schäffer (1999), S. 17 ff.

683 Die im Folgenden dargestellten Sachverhalte zeichnen sich durch eine signifikante Differenz in der von Managern und Controllern beigemessenen Bedeutung aus. Kgl. Abbildung 34 978-3-631-75321-7 
sierung eines „Controller's jour fixe“ bzw. von Reportingkonferenzen managementseitig eine weitaus größere Bedeutung beigemessen als dies bei den Controllern der Fall ist. Darüber hinaus scheint die Bedeutung einer sachgerechten Visualisierung im Reporting von Controllern unterschätzt zu werden. ${ }^{64}$ Insgesamt führt die Unkenntnis über derartige Sachverhalte oft - ausgehend von der eigenen Einschätzung der Controller - zu einer zu angebotsorientierten formalen Gestaltung und Kommunikation von Berichten.

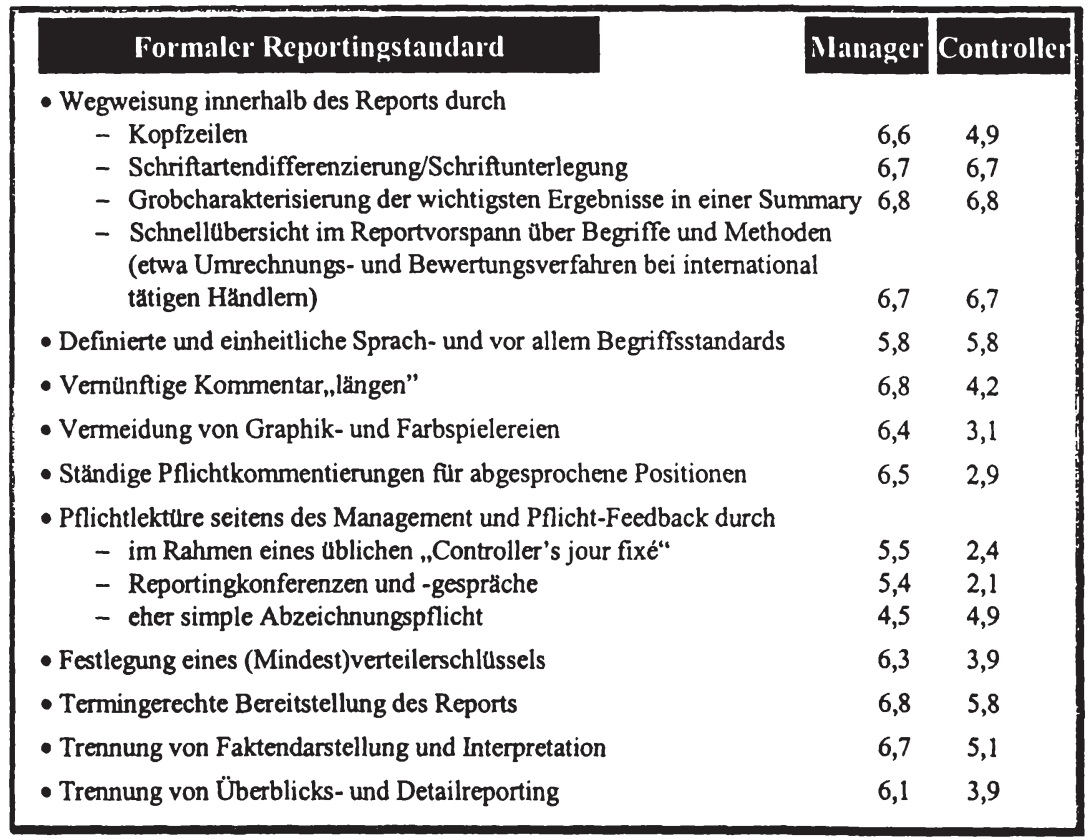

Abbildung 34: Praktische Bedeutung formaler Reportingstandards ${ }^{685}$

Zusammenfassend ist im Sinne der Ansprache alternativer Kunden des Controlling zwischen einer inhaltlichen und formalen Leistungsindividualisierung $\mathrm{zu}$ unterscheiden. Die inhaltliche Individualisierung dient im wesentlichen einer bedürfnisadäquaten Informationsbereitstellung, da auch bei gleichen Problemstellungen in Abhängigkeit des Empfängers unterschiedliche Informationen re-

684 Grundlegend zu der Verwendung von Graphiken in Berichten vgl. Berens/Darius/Schmitting (1997).

685 Die Abbildung basiert auf einer empirischen Erhebung von WrTT. Die zugeordneten Werte zeigen die von Managern bzw. Controllern einzelnen Reportingstandards beigemessene Bedeutung. Die Bewertung erfolgte mittels einer 7er Ratingskala mit dem Höchstwert 7 (=sehr große Bedeutung). Vgl. Witt (1992), S. 248 sowie Neuhäuser-Metternich/Witt (2000), S. 313. 
levant sein können. ${ }^{686}$ Ferner kann über eine inhaltliche Individualisierung die Menge der bereitgestellten Informationen reduziert werden und somit die Informationsverarbeitungskapazität des Managements entlastet werden. In gleicher Weise vermögen formale Leistungsstandards zu einer Gewöhnung und damit effektiveren Verarbeitungskapazitätsbeanspruchung beizutragen. Allgemein führen auf Basis von Bedarfsanalysen durchgeführte Maßnahmen der Individualisierung zu einer Fragmentierung ${ }^{687}$ der unternehmensinternen Märkte des Controlling. Dies erschwert eine wirtschaftliche Bearbeitung. ${ }^{688}$ Durch Konzepte wie Mass Customization oder Self-Customization können die Vorteile von Individualisierung und Standardisierung bedingt kombiniert werden. Die hierbei vorgenommene Modularisierung der Leistungen erscheint insbesondere aufgrund des Informationscharakters der Controlling-Leistungen anwendbar.

$\mathrm{Zu}$ beachten bleibt stets, inwieweit die damit verbunden Anforderungen an die Mitwirkung der Kunden deren Nutzungsverhalten beeinflussen. Eine maßgebliche Bedeutung für die Inanspruchnahme und Nutzung einer Leistung hat in diesem Zusammenhang auch ihre spezifische Evidenz.

\subsubsection{Leistungsevidenz}

Wesentlichste Voraussetzung für die Inanspruchnahme einer innerbetrieblichen Leistung ist deren Evidenz. Eine Leistung ist aus der Perspektive des Nachfragers dann evident, wenn dieser die Einsatz- bzw. Nutzungsmöglichkeiten dieser Leistung kennt. ${ }^{699}$ Charakteristikum unternehmensinterner Dienstleistungen ist, dass die aus der Perspektive der Nachfrager subjektiv wahrgenommene Leistungsevidenz regelmäßig niedriger ist als bei unternehmensexternen Leistungen. Insbesondere die Komplexität der durch das Controlling angebotenen Leistungen führt $\mathrm{zu}$ einer mangelnden Leistungsevidenz und damit zu einer regelmäßig zu geringen oder unsachgemäßen Beanspruchung einzelner Leistungen. Zielsetzung von Marketingmaßnahmen im Controlling muss es daher sein, über zielgerichtete Aktivitäten die Bedarfs- und Leistungsevidenz bei seinen Kunden zu erhöhen, bzw. Scheinevidenz abzubauen. ${ }^{690}$ Im Controlling können sich Evidenzmängel auf

686

687

${ }^{688}$ Für den Bereich der Kostenrechnung vgl. Währisch (1998), S 339.

689 Vgl. Engelhardt/Schwab (1982), S. 506 ff.

690 Von Scheinevidenz spricht man dann, wenn der Nachfrager aufgrund einer falschen Problemeinschätzung eine nicht zielsetzungsadäquate Leistung abfragt. Vgl. Engelhardt/Schwab (1982), S. 507. sowie Witt (1985), S. 167. 
- die Leistungsart bzw. das Sortiment des Controllers,

- die Quantität und den der Zeitpunkt der potenziell nachfragbaren Leistungen sowie

- auf den Anbieter selbst

beziehen. ${ }^{691}$ Die Aufzählung zeigt, dass im Rahmen der Evidenzbetrachtung grundsätzlich zwischen der Leistung und dem Controller als Anbieter zu differenzieren ist, auch wenn von einer positiven Korrelation beider Bezugsobjekte der Evidenz auszugehen ist. ${ }^{692}$ Unterstellt man, dass der Controller als Anbieter im Unternehmen bekannt ist, wird unter Zugrundelegung der Unterscheidung von Leistungspotenzial, -prozess und -ergebnis deutlich, dass der Controller gerade bezüglich seines Leistungspotenzials eine aktive Kommunikation betreiben muss, um die oftmals nachfragerseitig empfundene Unsicherheit zu reduzieren. ${ }^{693}$ Eine Operationalisierung des Leistungspotenzials, im Sinne der Problemlösungsfähigkeit und -willigkeit des Controllers, kann in Form von Service Level Agreements erfolgen. An dieser Stelle wird der Übergang vom Leistungspotenzial und der Zusicherung eines Leistungsversprechens zu dem konkreten Leistungsergebnis fließend. Service Level Agreements erleichtern die Strukturierung des Leistungsangebotes im Controlling sowie eine inhaltliche Spezifikation der einzelnen Leistungen. ${ }^{64}$ Ferner können Evidenzmängel bezüglich der Quantität und des Zeitpunktes der Leistungserstellung über eine Konkretisierung der Abrufmodalitäten in Form von Leistungsvereinbarungen reduziert werden. ${ }^{695}$ Bedenkt man in diesem Zusammenhang, dass das auslösende Moment zur Erstellung einer sequenzunabhängigen Controlling-Leistung regelmäBig die Artikulation eines Kundenbedarfs ist, ${ }^{696}$ so wird deutlich, dass die Leistungsevidenz auch einen Einfluss auf die Kapazitätssteuerung im Controlling hat.

Neben der hier thematisierten Leistungs- bzw. Anbieterevidenz spielt die Evidenz des Bedarfes an sich eine gewichtige Rolle, d.h. die Frage inwieweit eine Kunde des Controlling überhaupt eine Entscheidungsunterstützungsnotwendig-

691 In Anlehnung an die allg. Ausfuhrungen von Witt (1985), S. 167.

692 Vgl. Witt (1985), S. 168.

$693 \mathrm{Vgl}$. hierzu die Ausfuhrungen in Kap. 3.2.2.1 und Kap. 3.2.2.2.

694 Allg. vgl. Winkelmann-Ackermann/Bundi (1999), S. $36 \mathrm{ff}$.

${ }^{695} \mathrm{Zu}$ Bedeutung strukturierter Abrufmodalităten im Rahmen einer kundenorientierten Ausrichtung des Controlling vgl. Kap. 3.3.2.2.

696 Zur Unterscheidung sequenzunabhängiger Controlling-Leistungen von sequenzintegrierten Leistungen vgl. Kap. 3.3.2.3. 
keit erkennt. ${ }^{67}$ Die Inhalte einzelner Leistungen des Controlling, insbesondere die an Bedeutung gewinnenden Beratungsaktivitäten, führen oftmals erst dazu, dass der Kunde die Notwendigkeit der Inanspruchnahme weiterer durch das Controlling bereitzustellender Leistungen erkennt. Somit kann die Bedarfsevidenz insbesondere auf Basis regelmäßiger Beratungen gesteigert werden. Wie die Unterscheidung obligatorischer von fakultativen Dienstleistungen des Controlling gezeigt hat, erfolgt die Leistungsinanspruchnahme nicht in allen Fällen freiwillig. ${ }^{698}$ Im Rahmen der diesbezüglichen Diskussion konnte aber bereits gezeigt werden, dass die Freiwilligkeit der Leistungsinanspruchnahme in vielen Fällen mit der Leistungsevidenz korrespondiert. Denn nur soweit der Nutzen einer Leistung bekannt und nachvollziehbar ist, kann eine Nachfrage auch potenziell unabhängig von organisatorischen $\mathrm{Zwängen} \mathrm{erfolgen.} \mathrm{In} \mathrm{diesem} \mathrm{Zu}$ sammenhang kann daher eine Evidenzsteigerung zu einer Ausweitung des Anteils freiwillig nachgefragter Leistungen des Controlling führen. Aus Abbildung 27 wurde deutlich, dass es gerade die Freiwilligkeit - im Sinne einer Leistungserstellung im Interesse und auf Veranlassung des Leistungsempfängers - ist, die eine anreizkonforme Bereitstellung von Controlling-Leistungen über Verrechnungspreise begünstigt. ${ }^{699}$ Somit trägt eine Steigerung der Leistungsevidenz zu einer Etablierung unternehmensinterner Marktverhältnisse bei.

Grundsätzlich ist davon auszugehen, dass sich die Wettbewerbssituation des Controllers verbessert, je evidenter seine Einzelleistungen bzw. die Struktur seines Leistungsgesamtangebotes sind. Darüber hinaus muss der Controller die Tatsache ausnutzen, dass der Kreis seiner potenziellen Kunden im Unternehmen verhältnismäßig überschaubar ist und er durch seine Unternehmenszugehörigkeit die Möglichkeit einer unmittelbaren Ansprache seiner Kunden hat. Im Sinne eines Personal-Selling sollte der Controller eine kunden- bzw. marktsegmentspezifische Evidenzförderung betreiben..$^{700}$ Wichtig ist hierbei zu beachten, dass aufgrund der informellen Kontakte und der gewachsenen Leistungstradition nicht nur Kunden angesprochen werden, bei denen bereits eine hohe Leistungsevidenz vorherrscht. Vielmehr ist das größte Potenzial bei solchen Kunden zu vermuten, die Evidenzmängel bezüglich des Anbieters und seiner Leistungen aufweisen. Kommt es zu einem Leistungsaustausch zwischen Controller und Manager, spielt über die Transaktion hinaus das Nutzungsver-

\footnotetext{
697 Kriterien zur Operationanalisierung der Bedarfsevidenz finden sich bei Witt (1988), S. 664.

698 Vgl. Kap. 3.3.2.1.

699 Vgl. Kap. 3.3.2.5.

700 Vgl. Witt (1985), S. 168.
} 
halten des Managements eine zentrale Rolle für die Wirksamkeit des Controlling.

\subsubsection{Nutzungsverhalten}

Da die Berücksichtigung von Gedanken des Marketing im Rahmen der Ausrichtung eines unternehmensinternen Controllingbereichs noch verhältnismäßig jung ist und unstrukturiert erfolgt, besteht grundsätzlich - wie auch bei der Evolution der Marketingtheorie - die Gefahr, den Marketingprozess zunächst auf das Ziel der Absatzhandlung als Endpunkt einer Transaktion auszurichten. ${ }^{701}$ Demgegenüber rücken neue Paradigmen der Marketingtheorie den auf Dauer angelegten Charakter einer Geschäftsbeziehung, im Sinne eines Relationship Marketing ${ }^{702}$ in den Mittelpunkt der Betrachtung. Dieser mit dem Investitionsgütermarketing korrespondierende Ansatz erweist sich gerade auch bezogen auf organisationsinterne Austauschbeziehungen als ein tragfähiger theoretischer Rahmen. Insbesondere die Dauer der Geschäftsbeziehung, sowie die innere Verpflichtung gegenüber dem Kunden, charakterisiert die Ausgangslage des Controllerbereichs treffend. Das gegenseitig zu schaffende Vertrauen ist die Ausgangsbasis für die Erreichung der für beide Transaktionspartner identischen Unternehmensziele. ${ }^{703}$ Auf Grundlage dieser Gedanken ist die Betrachtungsperspektive des Controllers über den Leistungsabsatz hinaus auf das Nutzungsverhalten seiner Kunden zu erweitern, da erst über eine sachgerechte Nutzung der Leistung ein Beitrag des Controlling zu der Erreichung der Gesamtunternehmensziele geleistet werden kann. Ferner gibt insbesondere die Art der Nutzung von Informationen Auskunft über die wirklichen Bedürfnisse der Kunden. Klärungsbedürftig bezüglich der Nutzung einzelner Controlling-Leistungen bleibt daher, inwieweit der Kunde über die Inanspruchnahme einer Leistung hinaus zu deren sachgerechter Nutzung motiviert werden kann bzw. grundsätzlich, welcher Art die Nutzung einer Leistung ist.

Betrachtet man die Ausführungen der beiden vorherigen Kapitel, so wird deutlich, dass sowohl die Leistungsindividualisierung als auch die Leistungsevidenz einen maßgeblichen Einfluss auf die Nutzung der durch das Controlling übermittelten Informationen haben. Eine Analyse des Informationsübermittlungsprozesses gibt erste Anhaltspunkte für mögliche Nutzungsbarrieren. Abbildung

$701 \mathrm{Zu}$ den unterschiedlichen Entwicklungsphasen der Marketingtheorie vgl. Meffert (2000), S. $19 \mathrm{ff}$.

702 Vgl. Gummesson (1996), S. 8 sowie Backhaus (1997), S. 19 ff.

${ }^{703} \mathrm{Zu}$ dem großen Anteil an Vertrauenseigenschaften von Controlling-Leistungen vgl.Kap 3.2 .2 .2 .3$. 
35 zeigt Entstehungsmöglichkeiten für Störungen im Berichtswesen, die letztlich als Quellen für eine unsachgemäße Nutzung anzusehen sind.

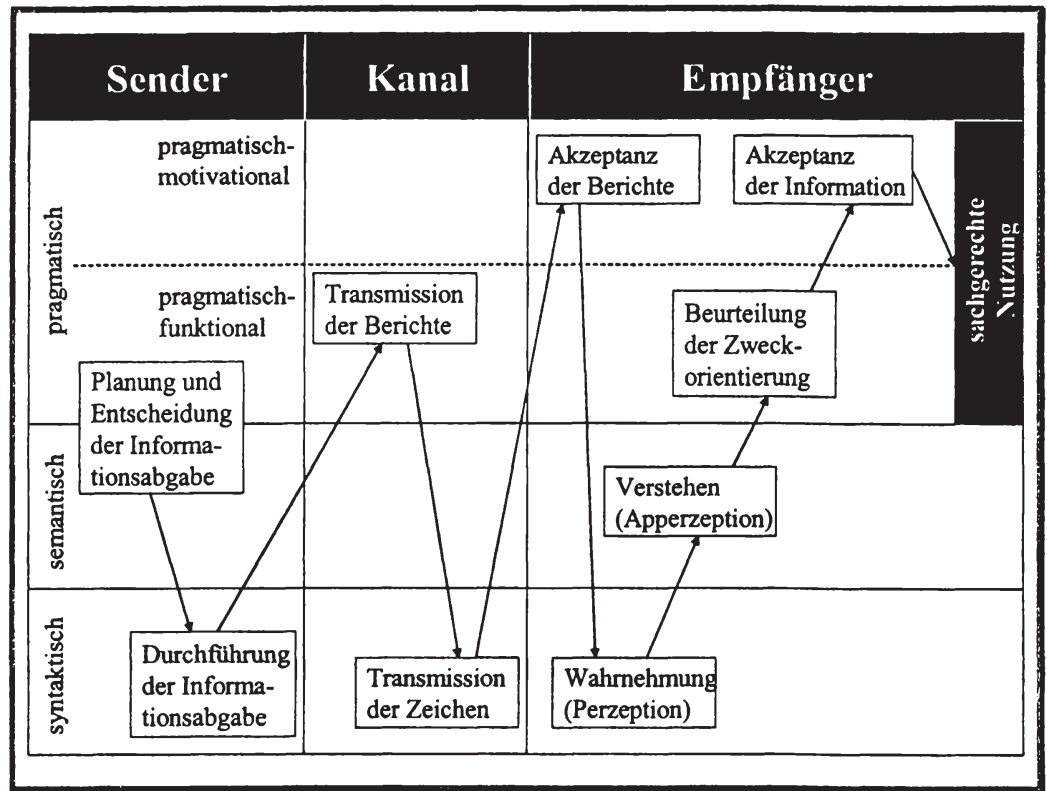

Abbildung 35: Entstehungsmöglichkeiten von Störungen im Berichtswesen ${ }^{704}$

Aufgabe des Controllers im Rahmen eines derartigen Berichtsprozesses ist neben der Sicherstellung technischer Rahmenbedingungen der Informationsübermittlung (syntaktische Aspekte) und einer klaren inhaltlichen Begriffsabgrenzung (semantische Aspekte), insbesondere eine angemessene Zweck- und Empfängerorientierung zu gewährleisten. ${ }^{05}$ Die Nutzung der Information erfolgt nur dann sachgerecht, wenn dem Kunden die Zwecksetzung, d.h. der Beitrag der Information zur Lösung eines bestimmten Entscheidungsproblems ${ }^{706}$ deutlich wird. An dieser Stelle wird wiederum die Bedeutung institutionalisierter berichtsbegleitender Erläuterungs- bzw. Beratungsaktivitäten offenbar. Ist die

704 Die Darstellung von KoCH wird hier um die Komponente der Leistungsnutzung ergänzt. Vgl. Koch (1994), S. 99.

705 Vgl. Horváth (1998), S. 602 f.

706 Der Terminus des Entscheidungsproblems soll hier und im Folgenden in einem sehr umfassenden Sinn verwandt werden. Auch Informationen über Situationen ohne unmittelbaren Handlungszwang (regelmäßiger Geschäftsbetrieb) werfen abstrakte Entscheidungsprobleme auf, auch wenn die regelmäßig implizite Entscheidung lautet, keine Maßnahme zu ergreifen. Vgl. hierzu die vierte Nutzungsmöglichkeit von Berger (1989), S. $185 \mathrm{f}$. 
Zweckorientierung und Akzeptanz der Information gewährleistet, kann bei einem rational handelnden Empfänger von einer zielsetzungsadäquaten Nutzung der Information - im Sinne einer instrumentellen Entscheidungsunterstützung ausgegangen werden. Dass das Nutzungsverhalten regelmäßig vielschichtiger als erwartet ist, zeigt Abbildung 36 bezogen auf Kostenrechnungsinformationen. ${ }^{707}$

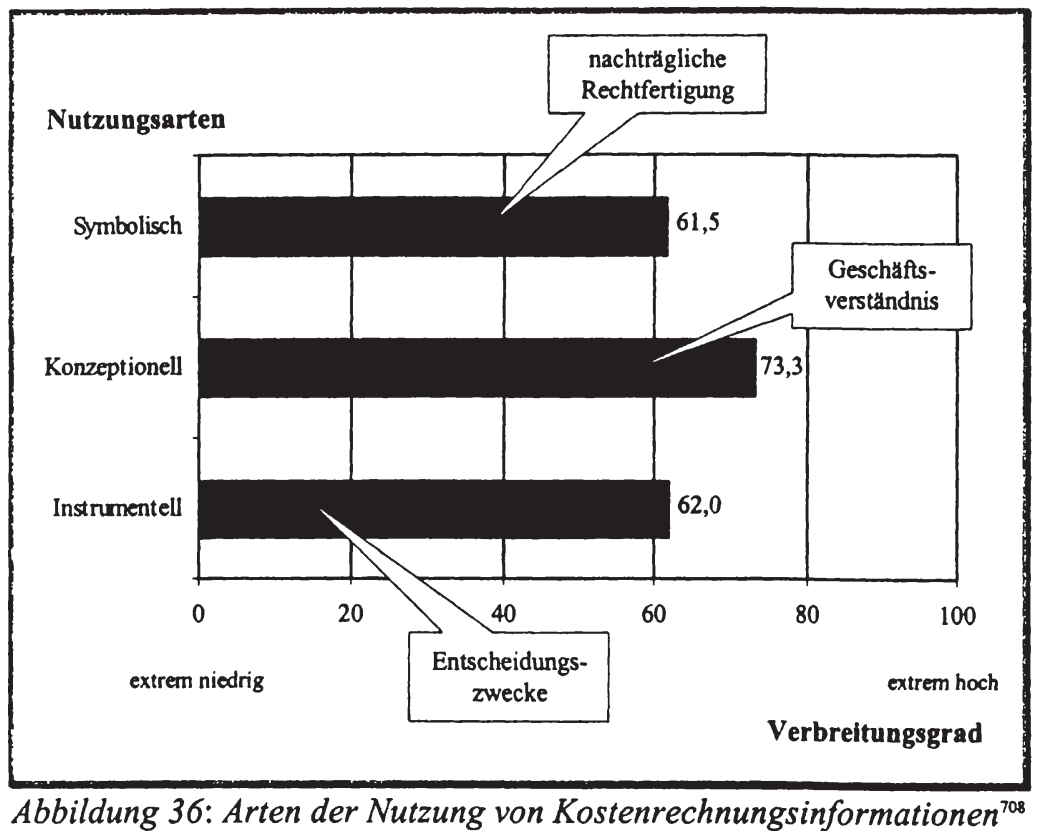

Die regelmäßig der Kostenrechnung zugeschriebene konkrete Entscheidungsunterstützungsfunktion, ${ }^{709}$ im Sinne der Bereitstellung handlungsauslösender Informationen (instrumentelle Nutzung), rangiert im Nutzungsverhalten der Kunden demnach gleichauf mit einer eher symbolischen Art der Nutzung. ${ }^{70}$ Eine symbolische Nutzung der Informationen liegt immer dann vor, wenn die Ent-

707 Eine theoretische Analyse der Einsatzfelder von Informationen der Kosten- und Erlösrechnung findet sich bei Währisch/Henselek (1997), S. 330 ff.

708 Die hier dargestellten Nutzungsarten sind ein Ergebnis der Koblenzer Studie zur Kostenrechnung. Zielsetzung der Studie war die Analyse mb̈glicher Defizite der Kostenrechnung. Die Auswertung erfolgte auf einer Datenbasis von 360 Fragebögen. Vgl. Homburg/Weber/Aust/Karlshaus (1998), S. 37 f.

709 Beispielhaft zu Zielen und Aufgaben der Kostenrechnung vgl. Preißler (2000), S. 139 ff.

710 Hier und im Folgenden vgl. Homburg/Weber/Aust/Karlshaus (1998), S. 36 ff. Die Ableitung der alternativen Nutzungsarten erfolgt in Anlehnung an die allg. Darstellung von Menon/Varadarajan $(1992), 5,53 \mathrm{ff}$. 
scheidung bereits getroffen wurde und die Informationen der Kostenrechnung lediglich zur nachträglichen Rechtfertigung vor anderen Organisationsmitgliedern eingesetzt werden. Werden Informationen der Kostenrechnung zur Förderung des allgemeinen Geschäftsverständnisses genutzt, ohne unmittelbar Entscheidungen auszulösen, spricht man von einer konzeptionellen Nutzung. Im Rahmen der hier zitierten empirischen Erhebung dominiert die konzeptionelle Nutzung von Kostenrechnungsinformationen alle anderen Nutzungsmöglichkeiten. Die entgegen der klassischen Auffassung für Kunden geringere Bedeutung der Entscheidungsunterstützungsfunktion der Kostenrechnung darf nicht als Bedeutungsverlust dieses Instrumentes aufgefasst werden. ${ }^{71}$ Vielmehr ist im Sinne der Kundenorientierung des Controlling das Nutzungsverhalten der Kunden im Rahmen der Leistungserstellung $z u$ antizipieren, so dass das Leistungsergebnis eine möglichst effektive Nutzung in der vom Kunden beabsichtigten Weise zulässt. Analog zu dem hier beschriebenen Beispiel der Kostenrechnung gilt es für den Controller, das Nutzungsverhalten alternativer Leistungen ${ }^{712} \mathrm{zu}$ ergründen, um Inhalt und Struktur auf die Informationsverwendung abstimmen zu können.

Bedenkt man jedoch, dass Controller-Service im Sinne einer konsequenten Ausrichtung am Kundennutzen zu verstehen ist, nicht jedoch als ein bedingungsloses Werkzeug des Kundenwunsches, ${ }^{713}$ so wird deutlich, dass die Nutzungsabsichten der Kunden nicht in jedem Fall alleinentscheidend für die Leistungsbereitstellung sein dürfen. Analog zu den getätigten Ausführungen zu Unterstützungs- und Kontrollleistungen des Controllings ist auch bezogen auf das Nutzungsverhalten zu analysieren, inwieweit opportunistische Absichten dieses beeinflussen. Die Rationalitätssicherungsfunktion des Controlling erfordert gerade bei der symbolischen Nutzung von Informationen abzuwägen, inwieweit ein intuitiv geführtes Unternehmen einen kritischen Counterpart benötigt. ${ }^{714}$ Eine Betrachtung der Leistungsnutzung ist in diesem Zusammenhang oftmals zielführender als eine Beschäftigung mit dem artikulierten Informationsbedarf.

Unabhängig von dem Inhalt der zur Verfügung gestellten Informationen können die spezifischen formalen Eigenschaften des benutzten Informationsträgers den Nutzen des Kunden beeinflussen. Werden Informationsträger verwendet, an denen der Kunde nachträglich - bedingt durch die spezifischen Trägereigenschaf-

\footnotetext{
711 Vgl. Homburg/Weber/Aust/Karlshaus (1998), S. 38 f.

712 Vorstellbar sind hier insbesondere alternative Berichtstypen.

713 Vgl. Trilse (2000), S. II/2

714 Vgl. Weber (1999), S. 39.
} 
ten - Modifikationen vornehmen kann (bspw. excel®-Tabelle), hat dies unter Umständen einen erhöhten Nutzwert für ihn. Der gleiche Informationsträger kann dann zielgerichtet zu einer selektiven Weitergabe von Informationen verwendet werden. Andererseits können unmodifizierbare Informationsträger (pdf $\AA$-Dokumente/Papierkopien) Inhalte eventuell zielsetzungsgerechter im Sinne des Informationsbereitstellers auch über den unmittelbaren Adressaten hinaus wiedergeben. Dies macht deutlich, dass in vielen Fällen der Kreis der Informationsnutzer über den vom Controller zunächst wahrgenommenen Kundenkreis hinaus gehen kann. ${ }^{715}$

Insgesamt kommt im Rahmen einer marketingorientierten Betrachtung des Controlling der Kenntnis des Nutzungsverhaltens alternativer Kunden eine auBerordentliche Bedeutung zu. Erst die Kenntnis des Nutzungsverhaltens ermöglicht es dem Controller, auf die Nutzungsabsicht des Kunden abgestimmte Informationen bereitzustellen. Ferner kann in Fällen einer opportunistischen Nutzung von Informationen über entsprechende Beratungsaktivitäten oder auf Basis organisatorischer Autorität versucht werden, das Nutzungsverhalten zu beeinflussen. Allgemein sollte daher auf der Ebene der organisatorischen Ausgestaltung des Controlling die Durchsetzung der Informationsverwendung als eine zentrale Aufgabe des Controllers angesehen werden, da letztlich erst eine sachgerechte Nutzung von Informationen die Koordinationswirkung des Controlling begründet. ${ }^{716}$

\subsubsection{Konkurrenzgefährdung}

Die in Kap. 3.4.2 getätigten Ausführungen haben gezeigt, dass es für das Controlling als unternehmensinternen Anbieter sowohl interne als auch externe Konkurrenten gibt. Über die pauschale Betrachtung einzelner Konkurrenten hinaus, soll im Folgenden die Konkurrenzsituation leistungsabhängig betrachtet werden. Wie auf unternehmensexternen Absatzmärkten konkurrieren in Unternehmen alternative Anbieter mit ihren Leistungen um die Nachfrage der Kunden, d.h. letztlich stehen zunächst die alternativen Leistungen in Konkurrenz zueinander, dann erst die dahinterstehenden Anbieter. ${ }^{717}$ Dieser Argumentation

715 Für den Bereich der Kostenrechnung vgl. Währisch (1998), S 339.

716 In der Literatur wird diskutiert, inwieweit die Informationsversorgungsfunktion des Controlling als Interpretation der Servicefunktion die Durchsetzung der Informationsverwendung einschließt. Vgl. Küpper (1997), S. 19.

717 Eine Einschränkung dieser Aussage ergibt sich dann, wenn bspw. die informellen Kontakte oder die Leistungstradition in einem Unternehmen sehr ausgeprägt sind. Ferner ist die Reputation des Anbieters bei unsi-

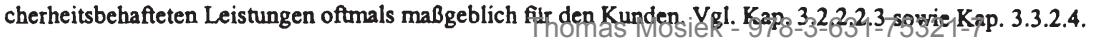


folgend, sollen Leistungen bezüglich des Ausmaßes ihrer Konkurrenzgefährdung betrachtet werden. Eine Konkurrenzgefährdung besteht grundsätzlich immer dann, wenn zwei Leistungen alternativer Anbieter in den Augen der Nachfrager als substituierbar wahrgenommen werden. ${ }^{78}$ An dieser Stelle wird wiederum die große Bedeutung des Nutzungsverhaltens einzelner Kunden deutlich, da die wahrgenommene Substituierbarkeit letztlich von dem vom Nachfrager in einer bestimmten Verwendungssituation gewünschten Nutzen abhängt. ${ }^{719}$ Wohlwissend, dass das Ausmaß der Konkurrenzgefährdung in der Praxis sehr differenzierte Ausprägungen aufweist, sollen im Folgenden Monopol-Dienstleistungen und Wettbewerbs-Dienstleistungen unterschieden werden.

\subsection{Monopol-Dienstleistungen}

Die Unterscheidung von Monopol-Dienstleitungen und Wettbewerbs-Dienstleistungen hat für das Controlling insofern eine Bedeutung, als dass die tendenzielle Zuordnung einzelner Leistungen Aussagen über die notwendige interne Vermarktungsintensität zulässt. ${ }^{70}$

Monopol-Dienstleistungen zeichnen sich diesbezüglich durch einen geringen Konkurrenzdruck aus, welcher im Einzelfall davon abhängt, ob

- ein alternatives Angebot besteht,

- die Leistungen des Controlling aus Sicht der Nachfrager substituierbar sind,

- die Entscheidung über den Anbieter grundsätzlicher Art ist oder fallweise getroffen werden kann,

- interne Nachfrager den Anbieter frei wählen können und ob

- eine leistungsentsprechende Verrechnung oder Umlage erfolgt. ${ }^{21}$

Bei den hier genannten Kriterien handelt es sich sowohl um solche, die durch die allgemeinen internen Wettbewerbsrahmenbedingungen beeinflusst werden als auch um solche, die sich erst aus der Zwecksetzung der einzelnen Leistung

718 Vgl. Meffert (2000), S. 43 f.

719 Wesentlich ist der Bezug zu einer konkreten Verwendungssituation des Nachfragers. Vgl. Bauer (1989), S. 123.

720 CRAMER nennt im wesentlichen drei Einflussfaktoren auf die Vermarktungsintensităt interner Dienstleistungen. Neben der Konkurrenzgefährdung wird die Notwendigkeit der Dienstleistung (Leistungsevidenz) und der Einfluss der Nachfrager auf das Dienstleistungsergebnis (Integrativität) genannt. Vgl. Cramer (1987), S. 6.

721 In Anlehnung an Cramer (1987), S. 7. 
ergeben. Monopol-Dienstleistungen zeichnet bezüglich des internen Wettbewerbsumfeldes die Tatsache aus, dass wesentliche Einschränkungen in Form von rechtlichen Zwängen, Organisationsrichtlinien oder Infrastrukturzwängen vorherrschen. ${ }^{722}$ Bei Monopol-Dienstleistungen handelt es sich daher um solche Leistungen, bei denen der Kunde keine Auswahl bezüglich des Lieferanten hat, d.h. das Controlling ist zwingend als Anbieter vorgesehen. Dies grenzt Monopol-Dienstleistungen auch gegenüber den obligatorischen Dienstleistungen ab, bei denen zwar die Inanspruchnahme auf Basis organisatorischer Zwänge vorgeschrieben ist, nicht jedoch der liefernde Anbieter. ${ }^{723}$ Wenn im Folgenden weiter von Monopol-Dienstleistungen die Rede ist, wird implizit ein Kontinuum zwischen Monopol- und Wettbewerbsdienstleistungen unterstellt, so dass eine Monpol-Dienstleistung nur schwerpunktmäßig und nicht ausschließlich diesbezügliche Charakteristika aufweist. ${ }^{224}$ Ferner ist darauf hinzuweisen, dass letztlich das Streben aller Anbieter darauf ausgerichtet ist, über die spezifische $\mathrm{Be}$ darfsadäquanz ihrer Problemlösung temporär in die Position eines Monopolisten zu gelangen, so dass Monopol-Dienstleistungen des Controlling im Falle bestehenden Wettbewerbs im Sinne einer überlegenen Lösung zu verstehen sind. ${ }^{725}$

Grundsätzlich stellt sich bezogen auf das Controlling die Frage, welche Tatbestände eine Leistung, wenn diese nicht per Organisationsentscheid zur Monopol-Dienstleistung gemacht wurde, faktisch so erscheinen lassen. Ferner ist zu analysieren, inwieweit man die bestehende Bindung der Kunden bei MonopolDienstleistungen positiv - im Sinne der Unternehmensziele - nutzen kann.

Auf Basis der informationsökonomischen Leistungseigenschaften ist davon auszugehen, dass mit wachsendem Anteil von Vertrauenseigenschaften bei Controlling-Leistungen die Bindungsstärke an den In-Supplier, und damit heutzutage regelmäßig noch an das Controlling, zunimmt. Die Reputation des Controllers ist diesbezüglich im Sinne einer wahrnehmbaren Potenzialqualität zu verstehen. Gerade auch bei Entscheidungen grundsätzlicher Art, die zu einer langfristigen Bindung an einen Anbieter führen, ${ }^{726}$ ist die Reputation für Kunden oftmals der einzige Anhaltspunkt für eine Leistungsbeurteilung. Der Vertrau-

722 Vgl. Günther (1999), S. 29.

${ }^{723} \mathrm{Zu}$ den obligatorischen Leistungen vgl. Kap. 3.3.2.1.1.

${ }^{724}$ Dies ist in Analogie zu dem durch Such-, Erfahrungs- und Vertrauenseigenschaften aufgespannten Raum in Abbildung 17 zu sehen.

${ }^{725}$ Allg. zum Wettbewerbsprozess vgl. Berg (1992), S. $241 \mathrm{ff}$.

726 Dies ist vergleichbar zum „Lock-in-Effekt" im Systemgeschăt zu verstehen. Vgl. Backhaus/Aufderheide/ Späth (1994), S. 63. 
ensvorsprung des Controlling ist im Sinne einer Marktzutrittsbarriere für neue Anbieter zu verstehen, wodurch das Controlling zeitweise zum Alleinanbieter werden kann. Rückt man den langfristigen Charakter einer Geschäftsbeziehung bei einzelnen Leistungen in den Mittelpunkt, so zeigt sich, dass die Initialentscheidung eines Kunden das Controlling temporär zu einem Monopolisten machen kann, soweit alternative Folgeentscheidungen des gleichen Kunden aufgrund der nun bestehenden Systembindung nur noch eingeschränkt möglich sind. ${ }^{727}$ Hat sich bspw. ein Organisationsmitglied für den Betrieb seiner Kostenrechnung durch das Controlling entschieden, führt dies dazu, dass gewisse Auswertungen zwangsläufig von dem gleichen Anbieter vorgenommen werden müssen. Zudem führt die Komplexität eines Kostenrechnungssystems regelmäßig dazu, dass ein Anbieterwechsel nur mit erheblichem Aufwand durchzuführen ist. ${ }^{728}$

Betrachtet man grundsätzlich die mit der Leistungsinanspruchnahme des Controlling verbundene Kostenbelastung der Kunden, so wird die Monopolstellung des Controlling insbesondere bei solchen Leistungen gefördert, bei denen pauschale Umlagen einer anreizkonformen Allokation entgegenwirken. Für die Kunden besteht bei derartigen Leistungen keine Notwendigkeit auf andere Anbieter auszuweichen, da die Grenzkosten der Inanspruchnahme des In-Suppliers gleich null sind. Betrachtet man die zeitliche Integration der ControllingLeistungen in die Entscheidungsprozesse der Kunden, so ist davon auszugehen, dass der Druck von externen Anbietern bei sequenzunabhängigen Leistungen tendenziell höher sein wird, als bei sequenzintegrierten Leistungen, da die derzeitige Integration des Controlling in komplexe Managementprozesse als Marktzutrittsbarriere zu verstehen ist. Die Integration des Controlling führt bei bestimmten Leistungen auch dazu, dass der im Einzelfall von Kunden gewünschte Individualisierungsrad durch externe Anbieter aufgrund der mangelnden Kunden- bzw. Bedarfskenntnis nicht erreicht werden kann. Dies korrespondiert mit der Evidenzbetrachtung, denn nur soweit externe Anbieter die Möglichkeit haben, sich über den Bedarf der umworbenen internen Kunden zu informieren (Bedarfsevidenz) und ihr Angebot mit dem des Controlling zu vergleichen (Leistungsevidenz), kann Wettbewerb wirksam funktionieren. In diesen Zusammenhang stellen für das Controlling insbesondere die informellen Kontakte und die bestehende Leistungstradition wettbewerbsbeschränkende Tatbestände dar, da Einzelleistungen nicht auf Basis objektiver Auswahlkriterien beschafft werden, sondern vielmehr auf Grundlage persönlicher Vertraut-

727 Vgl. zu Initialkauf- und Folgekaufentscheidung Backhaus (1999), S. 579.

728 Zur Wechselkostenproblematik vgl. Backhaus (1999), S. 594 f. 
heit. ${ }^{729}$ Betrachtet man den Leistungsaustausch aus dieser Perspektive, so verschwimmt gleichermaßen die Grenze zwischen dem Monopolcharakter einer Leistung und dem des Anbieters.

Insgesamt ergibt sich daher für das Controlling bei Monopol-Dienstleistungen die Notwendigkeit, die Ursache des Monopolcharakters einzelner Leistungen zu überprüfen. Soweit Organisationsrichtlinien, bspw. zur Gewährleistung einer sachgerechten Aufbereitung sensibler Daten oder zur unternehmensspezifischen Moderation von Planungsprozessen, eine Leistungserstellung durch das Controlling vorschreiben, ist sicherzustellen, dass über Wettbewerbssurrogate ${ }^{730}$ die Leistungsfähigkeit und -willigkeit des Controllers sowie die daraus resultierende Zufriedenheit seiner Kunden einer Beurteilung zugänglich gemacht werden kann. ${ }^{731}$ Dies ist insbesondere aufgrund des auch im Controllerbereich anzutreffenden opportunistischen Verhaltens erforderlich. Wird eine ControllingLeistung nicht per Beschluss, sondern vielmehr auf Basis struktureller Bedingungen (bspw. mangelnde Evidenz, Verrechnungsmodalitäten, etc.) zur Monopol-Dienstleistung, ist zu analysieren, inwieweit die internen Wettbewerbsrahmenbedingungen eine Liberalisierung zulassen, ohne die Verfolgung der Unternehmensziele zu gefährden. Soweit Controlling-Leistungen bei uneingeschränktem Wettbewerb Monopolcharakter erlangen, ist dies regelmäßig auf die spezifischen Wettbewerbsvorteile zurückzuführen, die letztlich die Existenz eines eigenständigen Unternehmensteilbereiches Controlling rechtfertigen. ${ }^{732}$

\subsection{Wettbewerbs-Dienstleistungen}

In Abgrenzung zu Monopol-Dienstleistungen zeichnet Wettbewerbs-Dienstleistungen ihre potenzielle Marktfähigkeit aus. ${ }^{733}$ Abbildung 37 zeigt übersichtsartig potenzielle Konkurrenten des Controllers bei der Einführung einer Balanced Scorecard ${ }^{734}$. Über die potenzielle inhaltliche Marktfähigkeit der Leistung hinaus müssen in dem betrachteten Unternehmen die Regeln des Marktes bezogen auf eben diese Leistungen in mehr oder minder großem Umfang gelten. Besteht also für eine Controlling-Leistung ein vergleichbares Angebot eines alternativen Lieferanten und wird dieses aus Perspektive der Kunden als Substi-

\footnotetext{
$729 \mathrm{Vgl}$. Witt (1988), S. 669.

730 Benchmarking ist in diesem Zusammenhang als Wettbewerbssurrogat zu verstehen.

$731 \mathrm{Vgl}$. hierzu Weber/Weißenberger/Aust (1997), S. 3 ff.

732 Vgl. hierzu die Aufzählung der Wettbewerbsvorteile des Controllers in Kap. 3.4.2.2.

733 Vgl. Ginther (1999), S. 29.

$734 \mathrm{Zu}$ den Aufgaben und der Struktur einer Balanced Scorecard vgl Kap. 4.2.2
} 
tut angesehen, muss das Controlling Maßnahmen ergreifen, um seinen Leistungsabsatz sicherzustellen und damit seine organisatorische Stellung zu legitimieren. Die in Abbildung 37 vorgenommene Visualisierung der Stärken und Schwächen einzelner Anbieter vermag dem Controller Anhaltspunkte für seine Angebotsstrategie zu geben. ${ }^{735}$

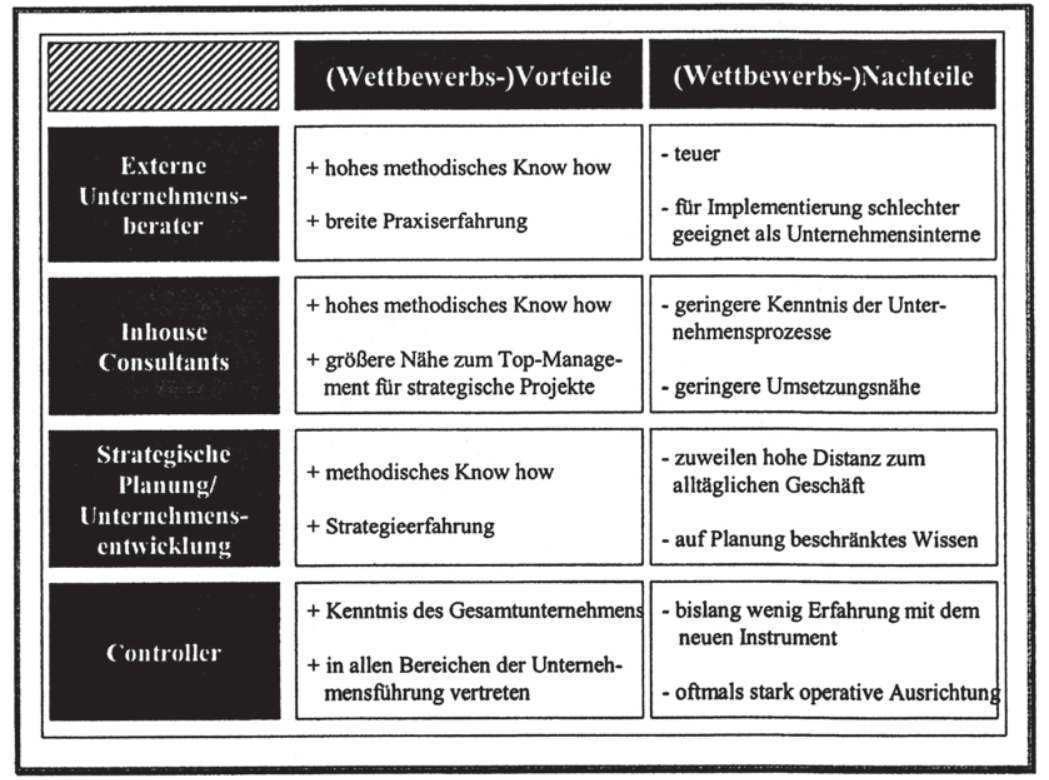

Abbildung 37: Potenzielle Konkurrenten bei der Einführung einer Balanced Scorecard ${ }^{736}$

Neben der pauschalen Klassifikation einer Leistung als WettbewerbsDienstleistung auf Basis der Konkurrenzbetrachtung können einzelne Gestaltungsparameter Auskunft über das Ausmaß der Konkurrenzgefährdung geben. Bezogen auf die Abrufmodalitäten steigt der Konkurrenzdruck immer dann, wenn Leistungen fallweise abgerufen werden, ${ }^{737}$ d.h. wenn keine dauerhafte Bindung an einen Anbieter vorliegt. ${ }^{738}$ Insbesondere sequenzunabhängige Dienstleistungen des Controlling sind daher einer konkreten Konkurrenzge-

\footnotetext{
735 Vgl. hierzu auch die Ausfuhrnungen zu alternativen Formen konkurrenzgerichteten Verhalts in Kap. 4.1.2.2.

736 Weber/Schäffer (2000), S. 79.

737 Vgl. Cramer (1987), S. 7.

738 Inwieweit die Bindungsstärke an einen Anbieter zur Segmentierung von Kunden verwandt werden kann zeigt Kap. 4.1.2.1.1. 
fährdung ausgesetzt. Regelmäßig bedingt darüber hinaus die Art der Verrechnung einzelner Leistungen, inwieweit die Wettbewerbssituation von Controller und seinen Kunden als solche wahrgenommen wird. Wie bereits im Rahmen der Ausführungen zu den alternativen Verrechnungsmöglichkeiten angedeutet, sind die Existenz eines stabilen externen Marktpreises für vergleichbare Produkte, der externe Marktzugang oder der unternehmensinterne Zwang zur Anwendung von Marktpreisen Voraussetzungen für den wettbewerbsorientierten Einsatz von Marktpreisen. ${ }^{79}$ Erst über eine konsequente Anwendung von Marktpreisen kann eine Ausrichtung des Controllerbereichs an der Effizienz und Effektivität externer Anbieter erfolgen.

$\mathrm{Da}$ in vielen Unternehmen die Implementierung von Marktpreisen gerade im Controllingbereich noch wenig ausgeprägt ist, können allgemeine Überlegungen zum Outsourcing analog verstanden werden. ${ }^{740}$ Im Einzelfall heißt das, auch wenn eine spezielle Leistung des Controlling nicht verrechnet wird und daher keinem direkten Marktregulativ ausgesetzt ist, können Überlegungen hinsichtlich eines langfristigen Outsourcing diese Leistung im Sinne einer Wettbewerbsbetrachtung erscheinen lassen. Im Rahmen einer grundsätzlichen Make or Buy-Entscheidung können den beiden Beschaffungsalternativen Kosten- und Erlöskomponenten zugerechnet werden, so dass eine transparente Entscheidung zugunsten einer Alternative möglich wird. ${ }^{71}$

Auch wenn die Überlegungen zum Outsourcing von Controlling-Aufgaben in vielen Unternehmen noch wenig fortgeschritten sind, kann insbesondere bei Projektleistungen von einer konkreten Konkurrenzgefährdung ausgegangen werden. Die in Abbildung 32 aufgezählten Leistungen der Unternehmensberatungen des BDU machen deutlich, dass insbesondere auch um die dem Controller zugeschriebene Beraterrolle ${ }^{7 / 2}$ eine zunehmende Konkurrenz entbrennt. Ferner führen Rationalisierungsbestrebungen klassischer Unternehmen, sowie die von Beginn an angestrebte Flexibilität und Schlankheit junger Unternehmen auch im Bereich dauerhafter Informationssystempflegeaufgaben dazu, dass diese zunehmend an externe Dienstleister vergeben werden (z.B. DATEV) $)^{743}$. Darüber hinaus gilt es für das Controlling, stets auch alternative interne Aufgaben-

739 Vgl. Horváth (1998), S. 575 f.

$740 \mathrm{Zu}$ unternehmensinternen Make or Buy-Entscheidungen vgl. Witt (1988b), S. 77 ff. sowie allg. Hahn/Hungenberg/Kaufmann (1994), S. 74 ff.

741 Zwei Beispiele zum Outsourcing von Controlling-Aufgaben finden sich bei Müller/Fickel (1997), S. $110 \mathrm{f}$.

742 Zur Bedeutungszunahme der Beraterfunktion des Controllers vgl. Abbildung 14.

${ }^{743}$ Vgl. Gleich (1997b), S. 359. 
träger zu beobachten und eine diesbezügliche Profilierung zu betreiben. Abbildung 38 zeigt exemplarisch einen Vorschlag zur Visualisierung der Leistungsschwerpunkte alternativer Anbieter von Controlling-Leistungen. Hierbei ist die Zuordnung einzelner Leistungen jeweils kontextabhängig vorzunehmen.

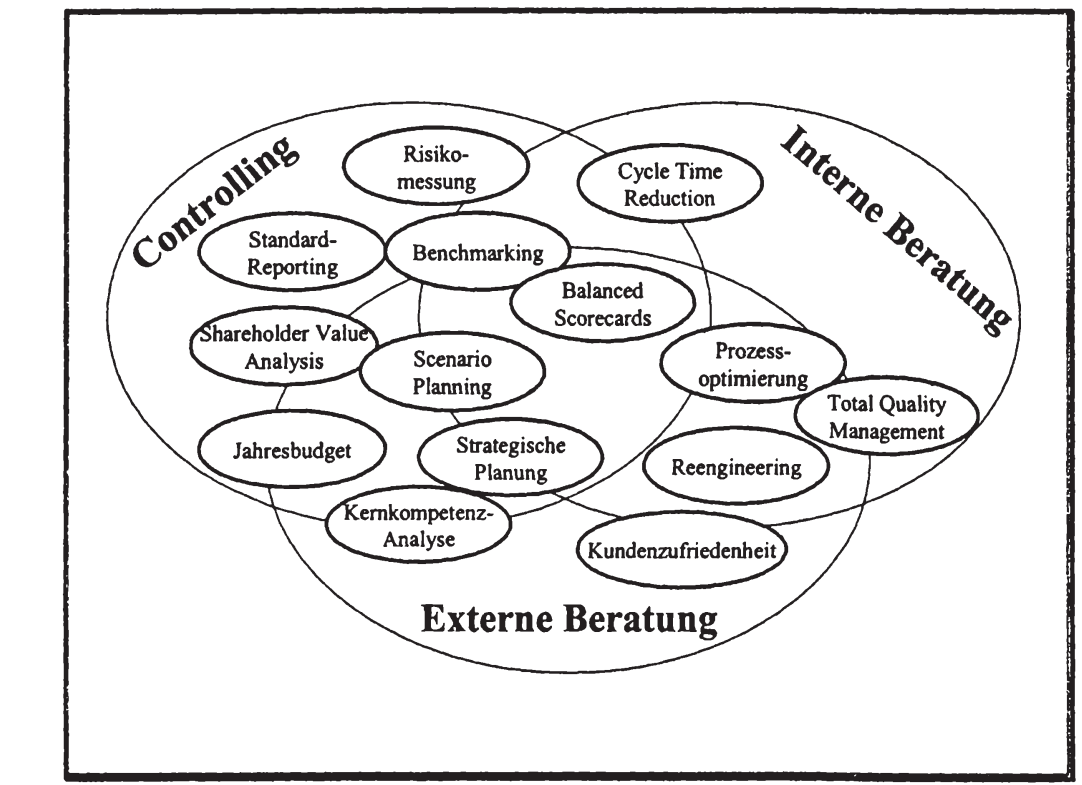

Abbildung 38: Abgrenzung zwischen Controlling, interner Beratung und externer Beratung ${ }^{34}$

Insgesamt ist die Zunahme der Wettbewerbs-Dienstleistungen des Controlling keinesfalls nur als Bedrohung für die Daseinsberechtigung des Controlling anzusehen. Vielmehr ist es für den Controller erforderlich, seine Rolle bezogen auf die Absicherung eines unternehmensweiten Controllings zu überdenken. Zunächst gilt es für ihn, das eigene Leistungsspektrum durch nachfolgend zu beschreibende Maßnahmen wettbewerbsfähig zu gestalten und darüber hinaus dessen Akzeptanz über eine angemessene Kommunikation sicherzustellen. Werden dennoch Controlling-Aufgaben an externe Anbieter vergeben, kommt dem Controller als Koordinator aller Controlling-Aktivitäten die Rolle eines Vermittlers bzw. Händlers $\mathrm{zu}^{7{ }^{74}}$ d.h. er trägt dafür Sorge, dass die Integration

744 Die Darstellung erfolgt in Anlehnung an Roth (2000), S. 137.

745 Allg. zu Maßnahmen innerbetrieblicher Anbieter zur Verbesserung ihrer Marktposition vgl. Witt (1988b), S. $81 \mathrm{ff}$. 
der externen Controlling-Leistungen in die Controlling-Konzeption des Unternehmens gelingt. ${ }^{746}$

\subsection{Implikationen der Gestaltungsparameterbetrachtung für die in- terne Kundenorientierung des Controlling}

Im Rahmen der einführenden Erläuterungen zu Kapitel 3 wurde deutlich, dass ein institutionalisierter Controllingbereich einer gezielten Führung bedarf, um mit einer eigenständigen Organisationsstrategie seinen Beitrag zur Erreichung der Gesamtunternehmensziele abzusichern. Über die Definition eines eigenen Leitbildes, auf Grundlage der Werte und Normen des Gesamtunternehmens, besteht für das Controlling die Möglichkeit einer Profilierung gegenüber anderen Unternehmensteilbereichen und externen Anbietern. Betrachtet man diesbezüglich exemplarisch das Leitbild der International Group of Controlling (IGC) $)^{747}$, wonach Controller begleitenden betriebswirtschaftlichen Service für das Management zur zielorientierten Planung und Steuerung leisten, ${ }^{748}$ wird deutlich, dass die Servicefunktion des Controlling eine konsequent kundenorientierte Ausrichtung des Controllerbereichs erfordert. Darüber hinaus schreibt die IGC dem Controller die Rolle eines internen betriebswirtschaftlichen Beraters ${ }^{79}$ aller Entscheidungsträger zu. Daraus ergibt sich, dass die Serviceleistungen des Controlling einerseits unterschiedliche Zielgruppen betreffen, andererseits aber auch aufgrund des Beratungscharakters von anderen Anbietern erbracht werden können. Diese Ausgangssituation macht es erforderlich, sich Gedanken über die Erfolgsfaktoren des Controlling im internen Markt zu machen.

Zielsetzung der Darstellung eines Gestaltungsraumes für ein kundenorientiertes Controlling war daher die Ableitung für eine Marketingbetrachtung des Controlling wesentlicher Gestaltungsparameter. Zur Strukturierung des Gestaltungsraums wurde ein modifiziertes Marketing Dreieck gewählt, welches zu einer leistungs-, organisations- sowie kunden- und wettbewerbsbezogenen Betrachtung geführt hat. Auf Basis dieser Strukturierung wurden für jede Betrachtungsperspektive zunächst die für das Controlling relevanten Rahmenbedingungen beschrieben. Darauf aufbauend wurden aus Perspektive des Marketing er-

\footnotetext{
746 Hierzu gehört bspw. auch das Controlling von Beratereinsätzen, welches sinnvoll nur intern durchgefuhrt werden kann.

747 Die IGC ist ein Verband verschiedener Einrichtungen die Controllerausbildung betreiben.

748 Vgl. Weber (1999), S. 368.

$149 \mathrm{Vgl}$. hierzu die in Abbildung 14 dargestellte Aufgabenverlagerung im Berufsbild des Controllers.
} 
folgs- bzw. gestaltungsrelevante Parameter extrahiert und differenzierter betrachtet. Im Einzelnen waren dies

bezogen auf die Leistungen des Controlling

- die Beurteilungsdimensionen,

- die informationsökonomischen Leistungseigenschaften und

- die Inhaltscharakteristika;

bezogen auf die Organisation des Controlling

- die Leistungsinanspruchnahme,

- die Abrufmodalitäten,

- die Ablaufcharakteristika,

- die informellen Kontakte und die Leistungstradition sowie

- die Leistungsverrechnung und

bezogen auf die Kunden und Wettbewerber des Controlling

- die Individualität,

- die Leistungsevidenz,

- das Nutzungsverhalten und

- die Konkurrenzgefährdung.

Betrachtet man die Ausprägung einer konkreten Leistung des Controlling wie bspw. eines Berichtes, einer Abweichungsanalyse, eines Planungsvorgangs oder eines Beratungsprojektes bezüglich der einzelnen Parameter, so wird eine strukturierte Beurteilung für den Nachfrager relevanter Tatbestände möglich. Neben der Beurteilung einzelner Leistungen vermag eine derart strukturierte Darstellung gezielte Ansatzpunkte für die Optimierung der Leistungsinhalte und des Leistungsaustauschs zu geben. Entgegen der in der Vergangenheit oftmals zu beobachtenden angebotsorientierten Bereitstellung von Leistungen im Controlling, ${ }^{750}$ müssen so zukünftig Informationsbedürfnisse und Inanspruchnahmepräferenzen ergründet werden, um über eine sachgerechte Nutzung der Leistungen den Unternehmenszielbeitrag des Controlling sicherzustellen.

Wesentlich im Rahmen der hier getätigten Gestaltungsparameterbetrachtung ist zunächst, sich die dahinterstehende Denkfigur anzueignen. In Abhängigkeit der Unternehmensgröße und dem regelmäßig damit korrespondierenden Leistungsumfang des Controlling ${ }^{751}$ wird es aber gerade für größere Unternehmen immer entscheidender, inwieweit sich die Denkhaltung der Kundenorientierung in

$750 \mathrm{Vgl}$. Währisch (1988), S.331.

751 Die Unternehmensgröße zählt regelmäßig zu den Einflussfaktoren auf den Leistungsumfang des Controlling. Vgl. Steinle (1999), S. 29 f. sowie Horváth (1998), S. 802 
konkrete Maßnahmen transformieren lässt. Im Einzelnen heißt das, die hinter der Gestaltungsparameterbetrachtung stehenden Gedanken in Form von regelmäßig anzuwendenden Instrumenten zu institutionalisieren, um hierdurch sowohl strukturell wie auch prozessual die Zufriedenheit der Kunden des Controlling sicherzustellen. Abbildung 39 zeigt, wie die Erkenntnisse der Gestaltungsparameterbetrachtung systematisch im Rahmen der Ausgestaltung von Instrumenten berücksichtigt werden können.

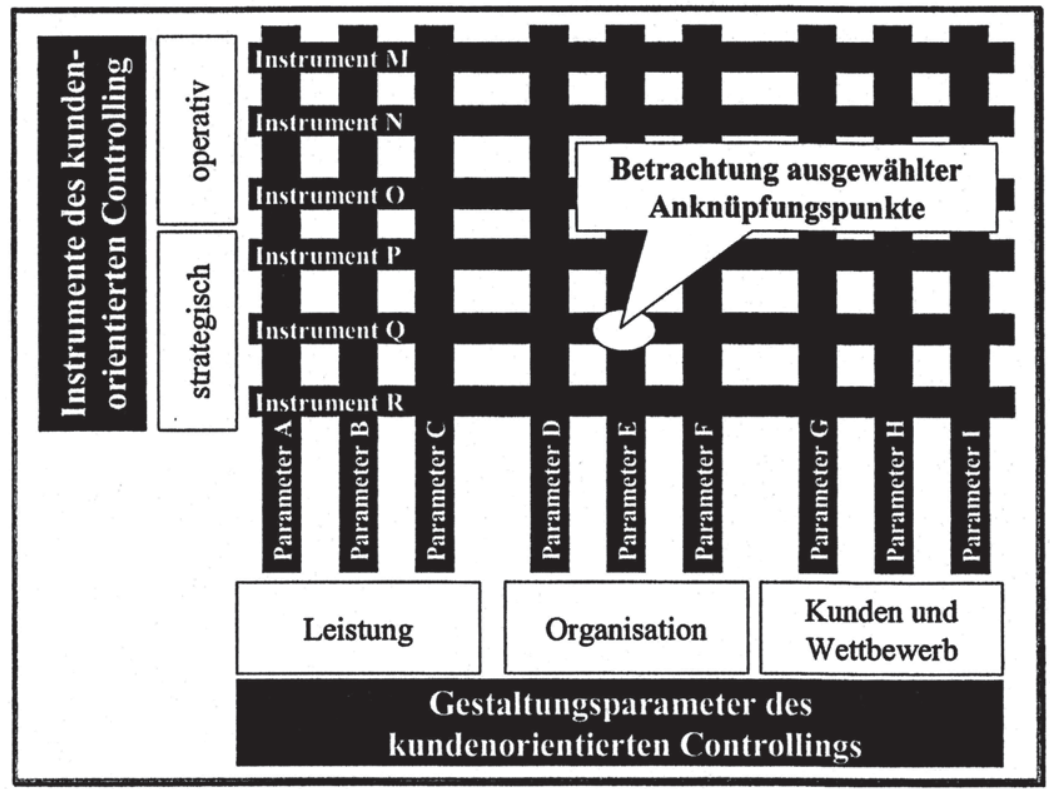

Abbildung 39: Parameter-Instrumenten-Matrix

Neben aus dem Bereich des Marketing bekannten Instrumenten sind es hier insbesondere Instrumente des marktorientierten Controlling selbst, die bei einer sachgerechten Anwendung auf den Controllerbereich zu einer Steigerung der Kundenzufriedenheit beitragen können. Im Folgenden sollen daher beispielhafte Instrumente zur Absicherung einer internen Kundenorientierung im Controlling vorgestellt werden. Hierbei decken die einzelnen Instrumente die extrahierten Gestaltungsparameter in unterschiedlichem Umfang ab, so dass im Rahmen der Kombination einzelner Instrumente auf eine ausgewogene Abdeckung aller für die Kundenorientierung im Controlling gestaltungsrelevanten Parameter zu achten ist. 
Thomas Mosiek - 978-3-631-75321-7

Downloaded from PubFactory at 01/11/2019 06:13:35AM

via free access 


\section{Aktionsgrundlagen des kundenorientierten Controlling}

Aufbauend auf die in Kap. 3 vorgenommene Deskription der Realität und Ableitung explanatorischer Aussagen über gestaltungsrelevante Zusammenhänge, sollen nachfolgend für praktische Handlungszwecke entscheidungstechnisch verwertbare Instrumente vorgestellt werden. ${ }^{72}$ Die vorgenommene konzeptionelle Durchdringung unternehmensinterner Dienstleistungen des Controlling ist für deren zielgerichtete Ausgestaltung unerlässlich, da sich zusehens analog zu den Entwicklungen auf externen Absatzmärkten unternehmensintern ein Wandel in der Dienstleistungskultur vollzieht. ${ }^{733}$ Im Rahmen der engpassinduzierten Abkehr von einer produktionsorientierten Betrachtungsweise hin zu einer absatz- bzw. kundenorientierten Betrachtung, werden die unternehmensinternen Angebote des Controlling nicht mehr ausschließlich als Vorgabe betrachtet. ${ }^{754}$ Vielmehr ist der Ausgleich von unternehmensinternem Angebot und Nachfrage als Prozess der Interessenskoordination zu betrachten, der bezogen auf die Unternehmens- und Bereichsziele geplant werden muss. Auf Grundlage der in dem vergangenen Abschnitt dargelegten potenziellen Marktfähigkeit von Controlling-Leistungen sollen im Folgenden Maßnahmen für das Controlling abgeleitet werden, mit der Zielsetzung, Leistungen auf die individuellen Bedürfnisse der internen Kunden auszurichten, sowie eine marktorientierte Unternehmensführung auch intern abzusichern. ${ }^{755}$ Ferner soll mit dem Übergang von einem angebotsorientierten zu einem nachfrageorientierten Verhalten die Akzeptanz und Entwicklungsfähigkeit des Controlling sichergestellt werden.

Wie im Rahmen der abschließenden Ausführungen zu Kap. 3 angemerkt, sind dazu die auf den internen Markt gerichteten Gestaltungsparameter des Controlling einer regelmäßigen Analyse und Planung durch geeignete Instrumente zugänglich zu machen. Kurzfristig erscheint die isolierte Anwendung singulärer Instrumente möglich. Langfristig sollte im Sinne des abteilungsorientierten internen Marketings eine Integration der einzelnen Instrumente in Form einer internen Marketingstrategie erfolgen. ${ }^{756}$ Bei den hier betrachteten Instrumenten geht es weniger um die Betrachtung homogener Teilgruppen von Marketingak-

752 Dies entspricht der vierten Stufe des Forschungsprozesses der Theorieentwicklung. Vgl. Grochla (1978), S. $61 \mathrm{ff}$.

753 Allg. zu den Herausforderungen marktorientierter Unternehmensfuhrung vgl. Meffert (1999), S. 12 ff. sowie Backhaus/Büschken/Voeth (2000), S. $23 \mathrm{ff}$.

754 WrTt zeigt in diesem Zusammenhang die Parallelen zum Wandel des Old Concept of Marketing hin zu einem den Engpass Absatz fokussierenden New Concept of Marketing auf. Vgl. Witt (1985), S. 164.

755 Vgl. Währisch (1998), S. 331 sowie Weber (1997), S. 15.

756 Vgl. Kap. 2.2.2.2.2. 
tivitäten im Sinne von Produkt-, Distributions-, Kontrahierungs- und Kommunikationspolitik, wie dies im Rahmen des Marketing-Mix erfolgt. ${ }^{757}$ Vielmehr soll dem Gedanken Rechnung getragen werden, dass Kundenorientierung sich nicht in konkreten Ausprägungen des Marketing-Mix dokumentiert, ${ }^{758}$ sondern vielmehr im Sinne einer Grundhaltung gegenüber Leistungsempfängern zu verstehen ist.

Zur Operationalisierung dieser erforderlichen Meta-Betrachtung ${ }^{759}$ soll nachfolgend der Versuch unternommen werden, beispielhafte Instrumente chronologisch im Sinne eines Planungsprozesses zu ordnen. Ausgehend von Instrumenten zur Planung der Controllerbereichsstrategie und der daraus abzuleitenden internen Marketingstrategie für das Controlling erfolgt die Darstellung ausgewählter Instrumente zur operativen Umsetzung und Absicherung einer Strategie. Im Rahmen der hier unter den operativen Gesichtspunkten Leistungsanalyse und -beurteilung betrachteten Instrumente wird auf die spezifische Anwendung von Benchmarking und der Balanced Scorecard im Controllerbereich eingegangen. Zur Leistungs- und Kostenplanung im Controlling werden Target Costing und Service Level Agreements vorgestellt. Bei den hier ausgewählten Instrumenten handelt es sich um beispielhafte Anwendungen, über die die Grundhaltung „Kundenorientierung " abgesichert und darauf aufbauend die Integration von Kundenanforderungen in das Leistungsangebot verbessert werden kann.

Nicht beabsichtigt ist, einen allumfassenden Überblick über die Instrumente des kundenorientierten Controllings zu geben. Vielmehr soll durch Einzelbeispiele die Übertragung weiterer, aus anderen Bereichen bereits bekannter Instrumente, auf das Controlling angeregt werden. Abbildung 40 zeigt die entlang des Planungsprozesses eingeordneten Instrumente. Bei der Gegenüberstellung von Instrumenten und Planungsebenen wird deutlich, dass eine eindeutige Zuordnung Schwierigkeiten bereitet. Die Anordnung der Instrumente erfolgt weitestgehend prozessual. Letztlich bestimmt aber der individuelle Kontext, so bspw. die Tatsache, ob ein Instrument erstmalig eingesetzt wird oder laufend Anwendung findet, dessen Position. Insbesondere die umfassenden Aufgaben der Balanced Scorecard, ausgehend von der Strategieentwicklung, über die Strategiedurchsetzung bis hin zur Strategiekontrolle erschweren eine punktuelle Eingliederung

757 Vgl. Meffert (2000), S. 972.

758 Vgl. zu diesem Gedanken Kühn (1991), S. 100 ff.

759 Vgl. zur Betrachtung der Kundenorientierung als Meta-Ebene Albers/Eggert (1988), $S_{3}$ 10 $f_{5321-7}$ 
in den Planungsprozess. ${ }^{700}$ Bei der hier vorgenommenen Einordnung der Balanced Scorecard wird deren operative Eignung - im Sinne der Absicherung der Strategiedurchsetzung - fokussiert. Gleichermaßen liegt der Betrachtungsschwerpunkt der Instrumente Benchmarking und Target Costing auf dem Ergründen und Beheben spezifischer operativer Leistungsdefizite. ${ }^{761}$

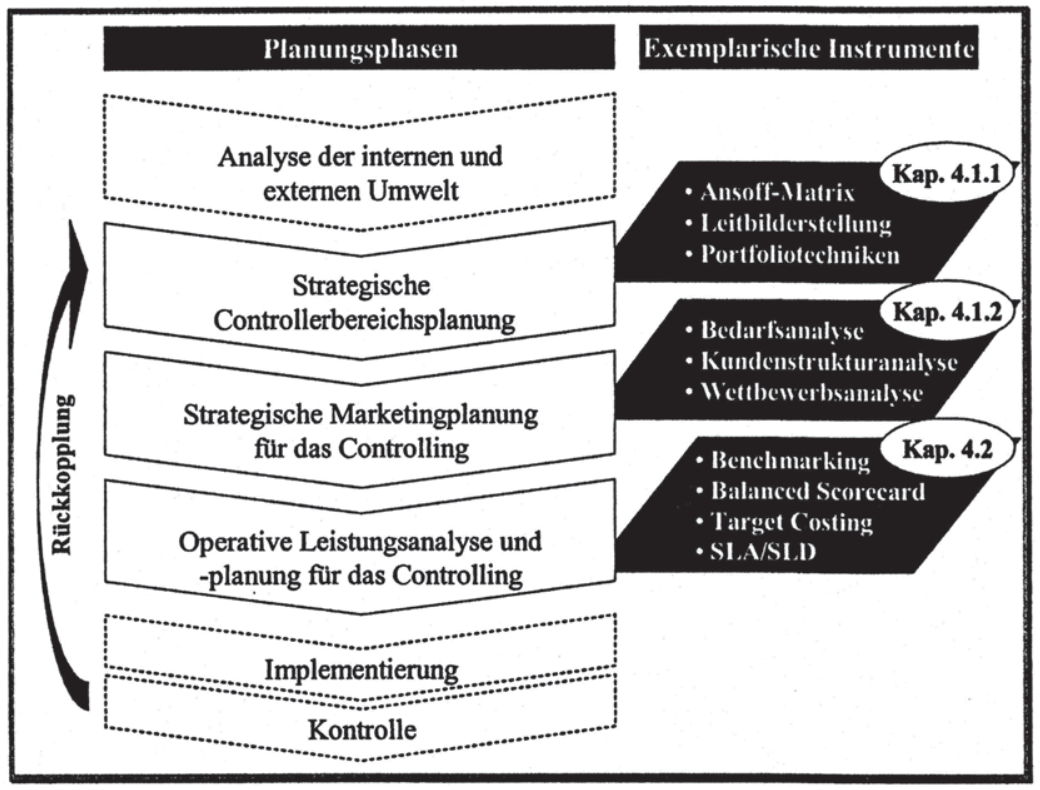

Abbildung 40: Planungsphasen und Instrumente des kundenorientierten Controlling ${ }^{362}$

Wesentlich bei dem Einsatz der einzelnen Instrumente bleibt deren spezifische Anpassung auf die Belange des organisationsinternen Dienstleisters Controlling. In Abgrenzung zu der üblichen Anwendung der Instrumente, ist dem konkreten Organisationsumfeld und den daraus erwachsenen Rahmenbedingungen für die Leistungserstellung und -verwertung des Controlling Rechnung zu tra-

760 Allg. zur Balanced Scorecard vgl. Kaplan/Norton (1996). Zur Aufgabe der Balanced Scorecard im Rahmen der Neuausrichtung des Controlling vgl. Weber/Schäffer (2000), S. $111 \mathrm{ff}$.

761 Auch wenn Target Costing regelmäBig als ein Instrument des strategischen Kostenmanagements gilt, erscheint diese Vorgehensweise aufgrund der gewählten Betrachtungsschwerpunkte angemessen. Insbesondere auch die nachfolgend zu postulierende RegelmaBigkeit des Einsatzes der ausgewthlten Instrumente unterstultzt diese Argumentation. Vgl. zum Target Costing Franz (1993), S. 124 ff.

762 Die Abbildung des Planungsprozesses ohne die Zuordnung von Instrumenten erfolgt in Anlehnung an die allg. Darstellung von Meffert (2000), S. 234. 
gen. ${ }^{763}$ Die Kenntnis der in Kap. 3 abgeleiteten Gestaltungsparameter ist hierfür elementar. Erwähnung sollen die Gestaltungsparameter im Rahmen der Instrumentenbetrachtung immer dann finden, wenn diese einer sachgerechten Ausgestaltung und Anwendung der Instrumente dienlich sind. ${ }^{764}$ Zur Plausibilisierung der folgenden Ausführungen sollen, soweit möglich, konkrete Anwendungsbeispiele aus Literatur und Praxis einbezogen werden.

\subsection{Strategische Controllerbereichs- und Marketingplanung}

Im Rahmen einer konkreten Analyse der Leistungen des Controlling wurde gezeigt, dass Planungsaufgaben regelmäßig zum Aufgabenspektrum und damit auch zum Dienstleistungsangebot des Controllers gehören. ${ }^{75}$ Zielsetzungen der folgenden Ausführungen ist, die im Controlling vorhandene Planungskompetenz zum Zwecke der Kundenorientierung auf den Controllerbereich selbst als Planungsobjekt anzuwenden. Über eine derartige Vorgehensweise kann einerseits eine zielgerichtete Positionierung des Controllerbereichs innerhalb des Unternehmens betrieben werden, andererseits wird die Glaubwürdigkeit des Controlling in den Augen seiner Kunden gestärkt, da ,nach außen postulierte Prinzipien" " 766 - im Sinne der von anderen geforderten Planungsaktivitäten selbst gelebt werden. Einteilen lassen sich die strategischen Planungsinstrumente in solche, die sich auf die grundsätzliche Entwicklung des Unternehmensteilbereiches Controlling beziehen (strategische Controllerbereichsplanung) und solche, die spezifisch auf das interne Marketing des Controlling (strategische Marketingplanung) fokussieren. ${ }^{767}$

Nachfolgend sollen ausgewählte Instrumente dieser Planungsebenen vorgestellt werden, da die Umsetzung der Kundenanforderungen einer Absicherung auf beiden Ebenen bedarf. Wesentlich für die Wirksamkeit der darzustellenden Maßnahmen ist, dass Stellenbeschreibungen und die Erwartungshaltung der Vorgesetzten die für die Planungsaktivitäten notwendigen Freiheitsspielräume nicht unangemessen einschränken.

\footnotetext{
${ }^{763}$ Speziell zu einer Organisationsstrategie vgl. Witt (1985), S. $173 \mathrm{f}$.

764 Nicht beabsichtigt ist den Instrumenten differenziert alle Gestaltungsparameter gegenüberzustellen.

765 Vgl. Kap. 3.2.1.4.

766 Weber (1999), S. 370.

767 Zur Planung von Unternehmens- und Marketingstrategien vgl. Meffert (2000), S. 233 ff. sowie Kleinaltenkamp/Fließ (1995), S. 951 ff. 


\subsubsection{Strategische Controllerbereichsplanung}

Im Sinne der Kundenorientierung des Controlling gilt es, die interne Kundenzufriedenheit in Planung, Kontrolle und Informationsversorgung (Systembildung) sowie in der Gewährleistung deren koordinierten Zusammenwirkens (Systemkoppelung) abzusichern. ${ }^{788}$ Daher obliegt es dem Controller auf Basis der ihm zugeordneten Aufgaben und einer Einschätzung seiner eigenen Potenziale, zukunftsgerichtet sein Leistungsangebot zu planen, um über die Bereitstellung bedarfsentsprechender Produkte seine organisatorische Stellung legitimieren zu können. Aufgabe strategischer Planungsinstrumente ist in diesem Zusammenhang sicherzustellen, dass alle erfolgsrelevanten Aspekte berücksichtigt werden und die Summe der strategischen Dimensionen ausgeleuchtet wird. ${ }^{769}$ Im Ergebnis gilt es, Transparenz bezüglich aller Handlungsalternativen zu schaffen und ein konkretes Selbstverständnis für die künftige Positionierung im Unternehmen zu entwickeln. ${ }^{770}$

Wie bereits bei den grundlegenden Ausführungen zu einer kundenorientierten Führung des Controllerbereichs angedeutet, ${ }^{711}$ spielt im Rahmen werte- und normenbezogener Führungsaufgaben die Leitbilderstellung diesbezüglich eine wesentliche Rolle. ${ }^{772}$ Grundlegend im Rahmen eines solchen Leitbildes ist, dem Problemlösungspotenzial des Controllings und der Adressatenorientierung auch wenn interne Abhängigkeiten bestehen - konsequent Ausdruck zu verleihen. ${ }^{773}$ Auf Basis eines zu kommunizierenden Leitbildes gilt es für den Controller, den derzeitigen Stand sowie die zukünftige Entwicklung seines Leistungsangebotes zu bewerten. Charakteristisch für viele Controllerbereiche ist derzeit noch, dass wenig Klarheit über

- die Erneuerungsrate der Controller-Produkte,

- die Veränderung von Leistungsinhalt, -umfang und -häufigkeit,

- die Zeitbudgetbeanspruchung einzelner Leistungen sowie

- die Struktur und Effizienz der Leistungserstellungsprozesse

${ }^{768}$ Zu Systembildung und Systemkoppelung als Aufgaben des Controlling vgl. Horvàth (1998), S. 146.

${ }^{769} \mathrm{Vgl}$. Niedereichholz (2000), S. 17. Allg. zu den Koordinationsinstrumenten innerhalb der strategischen Planung vgl. Kupper (1997), S. 82.

770 Vgl. Niedereichholz (2000), S. 19.

771 Vgl. Kap. 3.1.

772 Verschiedene Ausgestaltungsmöglichkeiten von Controllerbereich-Leitbildern finden sich bei Weber (1999), S. $362 \mathrm{ff}$.

773 Ein praktisches Beispiel für die Vision eines Controllerbereichs aus der Bankenwirtschaft findet sich bei Trilse (2001), S. 106. 
besteht. ${ }^{774}$ Ein Instrument zur Strukturierung zukünftiger Handlungsoptionen im Controlling ist die ANSOFF-Matrix. ${ }^{775}$ Durch eine Gegenüberstellung gegenwärtiger und zukünftiger Produkte und Kunden lassen sich Handlungsoptionen klassifizieren. Der Vorteil dieses Analyseinstrumentes bezogen auf das Controlling ist insbesondere in dem systematischen Einbezug alternativer Kunden zu sehen, da eine derartige Betrachtung oft vernachlässigt wird. Abbildung 41 zeigt eine ANSOFF-Matrix mit exemplarischen Controlling-Produkten.

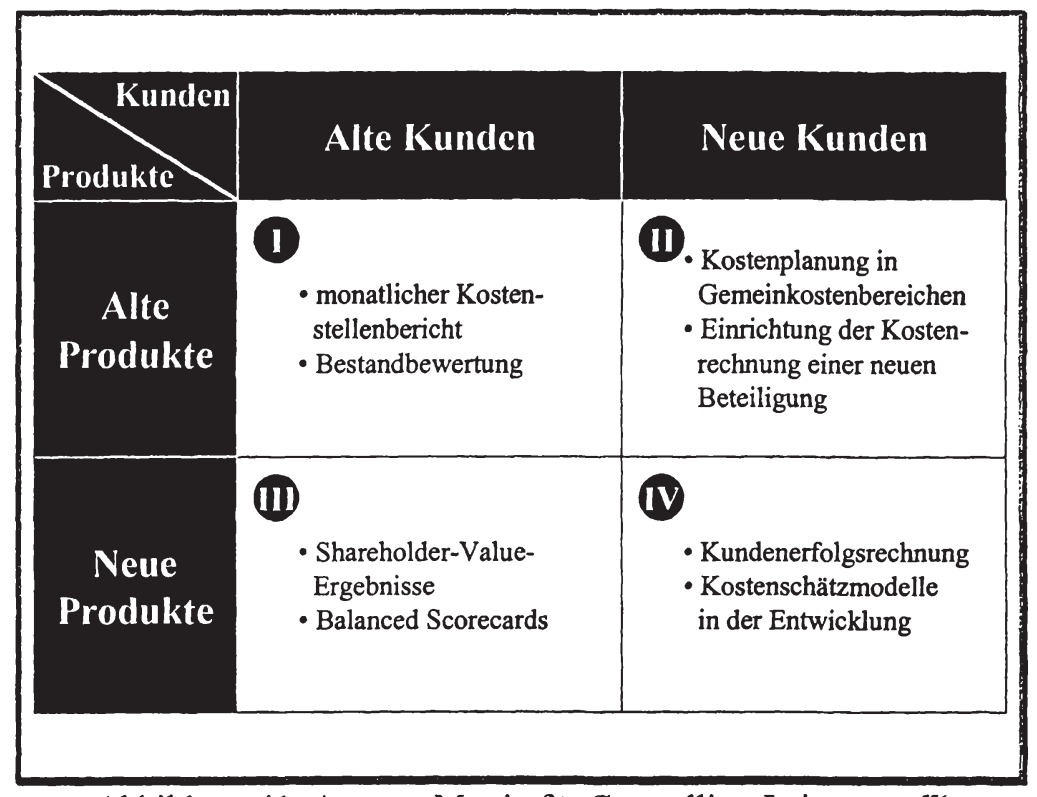

Abbildung 41: ANSOFF-Matrix für Controlling-Leistungen ${ }^{776}$

Aus dieser Matrix ergeben sich idealtypische Marktfeldstrategien wie Marktdurchdringung (I), Markterschließung (II), Produktentwicklung (III) und Diversifikation (IV), ${ }^{77}$ aus denen sich wiederum unterschiedliche Implikationen für ein Marketing des Controllerbereichs ergeben. Auch wenn die der ANSOFFMatrix zugrundegelegte Struktur recht grober Natur ist, führt die Systematik der

\footnotetext{
774 In Anlehung an die von WEBER/SCHÄFFER aufgeworfenen Fragen zur Neuausrichtung des Controlling, vgl. Weber/Schäffer (2000), S. $150 \mathrm{f}$.

775 Allg. zur ANSOFF-Matrix vgl. Ansoff (1966), S. 132.

776 Homburg/Weber (1999), S. 592.

$777 \mathrm{Vgl}$. Ansoff (1966), S. $130 \mathrm{ff}$.
} 
Problemdurchdringung regelmäßig zu kreativen Anstößen. ${ }^{78}$ Insbesondere die Verknüpfung der Marktfeldstrategien mit der Gestaltungsparameterbetrachtung liefert konkrete Anhaltspunkte für die Ausgestaltung der einzelnen Strategiealternativen. So sollte bspw. die bestehende Reputation des Controllers auf derzeitigen Märkten gezielt zu einer Etablierung innovativer Leistungen eingesetzt werden (III). Insbesondere der hohe Anteil von Vertrauenseigenschaften innovativer Leistungen erfordert für eine interne Auftragserteilung eine gewachsene Geschäftsbeziehung mit einer entsprechenden positiven Leistungstradition. Demgegenüber kann über eine Verbesserung der Leistungsevidenz bei Kontrollleistungen oder allgemein über eine klarere Fassung der Abrufmodalitäten eine gezielte Marktdurchdringung betrieben werden (I). Insbesondere über eine Kenntnis des Nutzungsverhaltens können individuelle Kundenbedürfnisse noch gezielter befriedigt werden. Soweit neue Märkte mit fakultativen Leistungen bearbeitet werden sollen, muss der Controller sorgfältig das Ausmaß der Konkurrenzgefährdung einzelner Leistungen beurteilen (IV/IV).

Die hier im Rahmen der strategischen Bereichsplanung diskutierte Strukturierung von Produkt-Markt-Beziehungen des Controlling dient einer langfristigen Absicherung der Erfolgspotenziale. Neben den Potenzialen stellt sich aber immer öfter - insbesondere vor dem Hintergrund einer Konkurrenzbetrachtung die Frage nach den dahinterstehenden Fähigkeitspotenzialen des Controllerbereichs. Unter Fähigkeitspotenzialen des Controlling ist im Rahmen einer nachfragerorientierten Betrachtung vornehmlich die Problemlösungskompetenz des Controllers zu verstehen. ${ }^{779}$ Betrachtet man die Problemlösungskompetenz des Controllers als Technologie, ${ }^{780}$ so können für alternative Leistungen bzw. Technologien des Controlling mittels einer Technologie-Portfolio-Analyse zukünftige Handlungsnotwendigkeiten abgeleitet werden. ${ }^{781}$

In der Technologie-Portfolio-Analyse werden zur Bewertung der Fähigkeitspotenziale eines Controllerbereichs einzelne Leistungen bezüglich ihrer Technologieattraktivität und der diesbezüglichen Ressourcenstärke des Controlling abgebildet. Die Attraktivität einer Leistung zeichnet sich im wesentlichen durch das Nutzenpotenzial aus, ${ }^{782}$ d.h. bspw. inwieweit eine Leistung des Controllings

778 Vgl. Weber/Schäffer (2000), S. 152.

779 Vgl. hierzu auch die Eignungsaspekte im Rahmen der Zuordnung von Aufgaben zu Controllern in Kap. 3.2.1.1. Vgl. ferner Herzog (1999), S. 45 ff.

780 So für die Kostenrechnung Weber (1999), S. 371.

${ }^{781}$ Hier und im Folgenden zum Technologie-Portfolio-Management vgl. Pfeiffer/Metze/Schneider/Amler (1991), S. 78.

782 Vgl. Homburg/Weber (1999), S. 589. 
zur Erfüllung der Unternehmensziele beiträgt. Im Rahmen einer Klassifikation können so Leistungen des Controlling bspw. nach ihrem Beitrag zur Absicherung der Wettbewerbsfähigkeit des Gesamtunternehmens abgebildet werden. Wesentlich im Sinne des kundenorientierten Controllings ist an dieser Stelle das Nutzungsverhalten der Kunden mit einzubeziehen, da auch durch vermeintlich attraktive Leistungen im Falle einer unsachgemäßen Nutzung durch die Kunden des Controlling kein Beitrag zur Erfüllung der Unternehmensziele geleistet wird.

Neben der Attraktivität gilt es für die abzubildenden Leistungen die Ressourcenstärke des Controllings als Anbieter zu bewerten. Zu der Ressourcenstärke zählen im wesentlichen die im Rahmen der Beurteilungsdimensionen diskutierten Potenzialqualitäten einer Leistung. ${ }^{783}$ Hier sind neben der technischen Ausstattung des Controllerbereichs insbesondere die personellen Fähigkeiten und Erfahrungen zu nennen. Wie im Rahmen der Technologieattraktivität angesprochen, ist die Nutzung der Leistung letztendlich erfolgskritisch, d.h. auch bezogen auf die Ressourcenstärke spielt nicht allein die angebotsorientierte Sicht eine Rolle, sondern gleichsam auch die Wahrnehmung des Nachfragers. ${ }^{784} \mathrm{Da}-$ her zählen zu der Ressourcenstärke des Controlling gleichermaßen die Fähigkeiten des Controllers, seine Leistungen zu kommunizieren, Abrufmodalitäten adressatengerecht auszugestalten und somit die Leistungsinanspruchnahme und -nutzung zu fördern. Abbildung 42 zeigt beispielhaft die Einordnung von Kostenrechnungsleistungen in ein Technologieportfolio.

Die vorgenommene Abbildung von Leistungen sollte stets kontextabhängig vorgenommen werden. ${ }^{785}$ Bei der Interpretation des Technologieportfolios ist analog zur ANSOFF-Matrix zu verfahren, d.h. es ist weniger die abschließend genaue Einordnung von Leistungen zu thematisieren als vielmehr die im Rahmen der Klassifikation entstehenden Diskussionsprozesse. Über derartige Erkenntnisgewinne bei der Erstellung des Portfolios hinaus ist langfristig für alle Controlling-Leistungen - im Sinne einer Normstrategie - eine Positionierung entlang des Gleichgewichtspfads anzustreben. ${ }^{786}$

\footnotetext{
783 Vgl. Kap. 3.2.2.1.1.

784 Zur angebotsorientierten Qualitätspräfung der Kostenrechnung vgl. Tylkowski/Guth/Spieker (2001), S. 96 ff.

785 Dies bedingt gerade bei Controlling-Leistungen die Führungssystemabhängigkeit der Attraktivität sowie die Personengebundenheit der Ressourcenstärke.
}

786 Vgl. grundsätzlich Pfeiffer/Metze/Schneider/Amler (1991), S. 78 ff. 


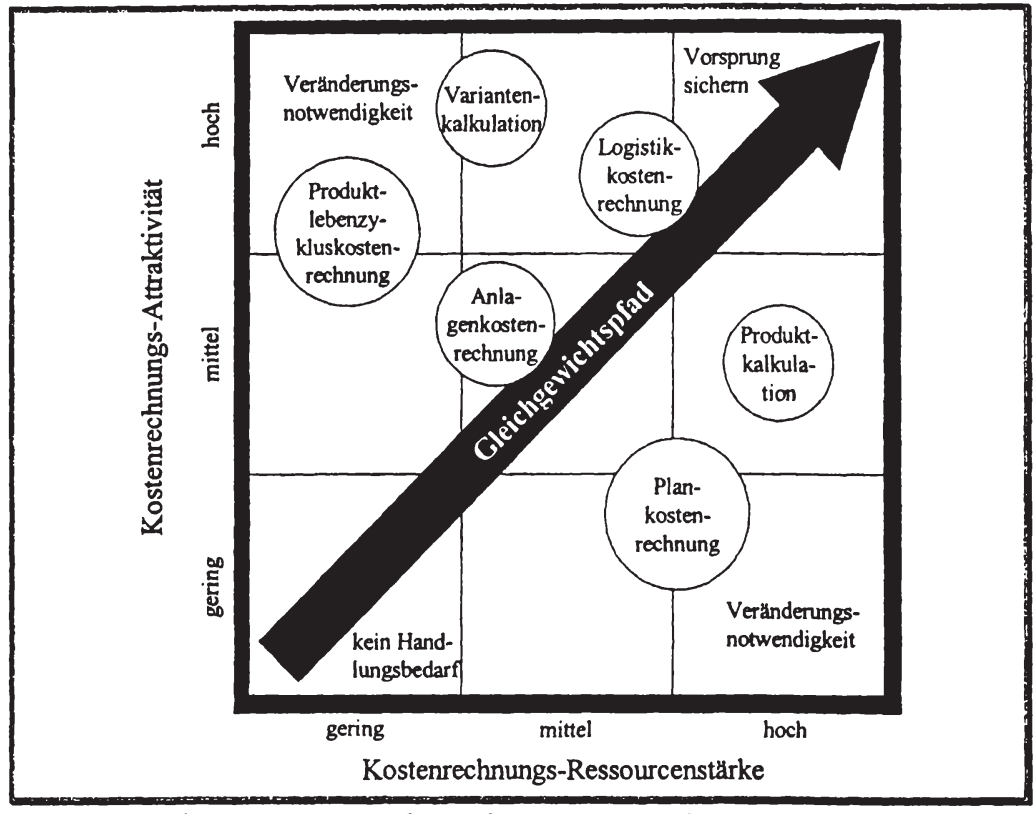

Abbildung 42: Exemplarisches Kostenrechnungs-Portfolio ${ }^{787}$

Bezogen auf Abbildung 42 heißt dies exemplarisch für die Plankostenrechnung, soweit diese z.B. aufgrund zunehmender Anlagenautomatisierung an Bedeutung verloren hat, den auf sie entfallenden Ressourcenverzehr zukünftig deutlich zu verringern. ${ }^{788}$ Wesentlich im Rahmen einer nachfragerorientierten Betrachtung des Controlling ist, bei der Einordnung von Leistungen in das TechnologiePortfolio auch Komponenten der kundenorientierten Leistungsgestaltung und erbringung - im Sinne der abgeleiteten Gestaltungsparameter - mit einzubeziehen. Letztlich haben diese Faktoren Einfluss auf die Bewertung einer Leistung, da das hinter der Attraktivität stehende Nutzenpotenzial jeweils adressatenabhängig bzw. organisationsabhängig zu bewerten ist.

Die hier vorgestellten Instrumente der strategischen Bereichsplanung vermögen für das Controlling einen ersten Überblick über die allgemeine Ausgangssituation, Zielvorstellungen und einen daraus erwachsenden Handlungsbedarf zu

787 In Anlehnung an die Darstellung von Weber (1999), S. 375.

788 Vgl. Weber (1999), S. 376. 
geben. ${ }^{789}$ Grundlegend ist für das Controlling in diesem Zusammenhang die Handlungsoptionen nicht einseitig im Sinne von Wachstumsstrategien zu verstehen, wie dies die meisten Instrumente der strategischen Planung implizieren. Vielmehr gilt es auch über eine konsequente Aufgabenkritik Möglichkeiten eines Downsizing zu prüfen, um darüber einen Beitrag zur Gemeinkostenreduktion zu leisten. Im Folgenden sollen zur Spezifikation der hier im Rahmen der Controllerbereichsplanung bereits angesprochenen Kunden und Märkte Aspekte einer strategischen Marketingplanung im Controlling vorgestellt werden.

\subsubsection{Strategische Marketingplanung im Controlling}

Aufbauend auf den Ausführungen zur strategischen Controllerbereichsplanung sollen im Rahmen der strategischen Marketingplanung insbesondere Maßnahmen zur Bewertung und Gestaltung der Kunden- und Konkurrenzsituation thematisiert werden. Da sich die Ausführungen auf ausgewählte Instrumente beschränken sollen, liegt der Fokus im Folgenden einerseits auf der Art und Weise der internen Kundenerfassung und -bearbeitung, sowie andererseits auf dem Verhalten gegenüber alternativen Marktteilnehmern ${ }^{700}$ Grundlage hierfür bilden die in Kap. 3 beschriebenen Rahmenbedingungen des Controlling. ${ }^{791}$

\subsubsection{Kundenorientierte Strategie}

Analog zu dem auf unternehmensexterne Märkte ausgerichteten Absatzmarketing stellt sich für das Controlling als interner Dienstleister die grundsätzliche Frage, ${ }^{792}$ ob die Gesamtheit unternehmensinterner Kunden differenziert oder undifferenziert $\mathrm{zu}$ bearbeiten ist. ${ }^{793}$ Bei einer undifferenzierten internen Marktbearbeitung werden die durchschnittlichen Erwartungen der Zielgruppe ermittelt und ein daran ausgerichtetes Standardprodukt angeboten. ${ }^{794}$ Demgegenüber zeichnet sich eine differenzierte Marktbearbeitung durch eine zielgruppenspezifische Produktgestaltung und eine individualisierte Kommunikation aus. Im Folgenden soll der Fokus auf strategischen Optionen einer differenzierten Marktbearbeitung liegen, da die Entscheidungsunterstützungsfunktion des

789 Ein Portfolio zur Bestimmung der Controllingattraktivität ausgewählter Geschäftsprozesse findet sich bei Otto (1999), S. 106. Zu weiteren Portfolios für unternehmensinterne Leistungen vgl. Witt (1989), S. 156 ff.

$790 \mathrm{Zu}$ den einzelnen Funktionen der strategischen Marketingplanung vgl. übersichtsartig Meffert (2000), S. 234 sowie ausfuihrlich S. $267 \mathrm{ff}$.

791 Vgl. Kap. 3.4.1 und Kap. 3.4.2.

792 Allg. zu den Dimensionen einer strategischen Marketingkonzeption vgl. Fließ (1995), S. 951 ff.

793 Vgl. Kotler (1967), S. 111.

794 Hier und im Folgenden vgl. Meffert (2000), S. 268 f. 
Controlling eine genaue Kenntnis und Befriedigung der Bedürfnisse einzelner Kunden bedingt. Dessen ungeachtet zeigen die im Rahmen der Leistungsindividualisierung getätigten Ausführungen, ${ }^{795}$ dass mit Verfahren wie MassCustomization oder Self-Customization im Controllerbereich auch hybride Lösungen denkbar sind, die insbesondere unter Kostengesichtspunkten Beachtung finden sollten. ${ }^{796}$

Eine differenzierte Marktbearbeitung setzt zunächst die genaue Kenntnis der Bedürfnisse alternativer Kunden voraus und erfordert darauf aufbauend eine Segmentierung der aktuellen und potenziellen Nachfrager. ${ }^{79}$

\subsection{Informationsbedarfsanalyse}

Zielsetzung der Controlling-Bedarfsanalyse ist die Ermittlung aller aus Kundensicht relevanten, potenziell durch das Controlling abzudeckenden Bedürfnisse, um diese später in ein entsprechendes Leistungsprofil umsetzten zu können. Der Bedarf stellt somit eines der wesentlichen Segmentierungskriterien für eine zielgerichtete Ansprache der Kunden des Controlling dar. ${ }^{798}$ Die Bedarfsanalyse ist im Sinne einer internen Marktforschung zu verstehen.

Da es sich im Kern der Controlling-Leistungen um Informationen handelt, ist zunächst der Informationsbedarf der jeweiligen Nachfrager zu ermitteln. Die grundlegenden Merkmale des Informationsbedarfs sind: ${ }^{799}$

- Art,

- Verdichtungsgrad,

- Aktualität,

- Darstellungsform,

- Bedeutung,

- Termindringlichkeit,

- Qualität,

- Verwendungszweck.
- Menge,

- Häufigkeit,

- Genauigkeit,

- Zuverlässigkeit,

- Messbarkeit,

- Kosten,

- Sicherheit,

$795 \mathrm{Vgl}$. Kap. 3.4.3.1.

$796 \mathrm{Zu}$ Mass-Customization und Self-Customization im Controllerbereich vgl. Steinle/Thiem/Rohden (2000), S. $284 \mathrm{f}$.

797 Vgl. Meffert (2000), S. 269.

798 Aufgrund der Bedeutung und Komplexităt des Informationsbedarfs wird dieser eigens vor den in Kap. 4.1.2.1.2 zu diskutierenden Segmentierungskriterien dargestellt.

799 Zu den Merkmalen und deren Bedeutung im Einzelnen vgl. Horváth (1998), S. 348 ff. 
Wie hier dargestellt, ist der Informationsbedarf in einem umfassenden Sinn zu verstehen, d.h. nicht nur der Informationsinhalt ist relevant, sondern gleichermaßen Präferenzen bzgl. der Informationsdarstellung und -verwendung. ${ }^{800}$ In Abhängigkeit der zugrundegelegten Entscheidungsprobleme des Nachfragers ist daher der Informationsbedarf vom Controller mit geeigneten Instrumenten zu spezifizieren. Deutlich wird, dass der Informationsbedarf der Kunden des Controlling insbesondere in Abhängigkeit des unterstellten Entscheidungs- bzw. Planungshorizontes variiert. ${ }^{801}$ Aus diesem Grund ist bei Instrumenten der Informationsbedarfsermittlung zwischen solchen zu unterscheiden, die sich auf Informationen zur operativen und taktischen Planung beziehen, und solchen, die den Informationsbedarf für strategische Entscheidungen ermitteln können. ${ }^{802}$ Abbildung 43 gibt einen Überblick über wesentliche Instrumente zur Ermittlung des Informationsbedarfs für operative und taktische Entscheidungen.

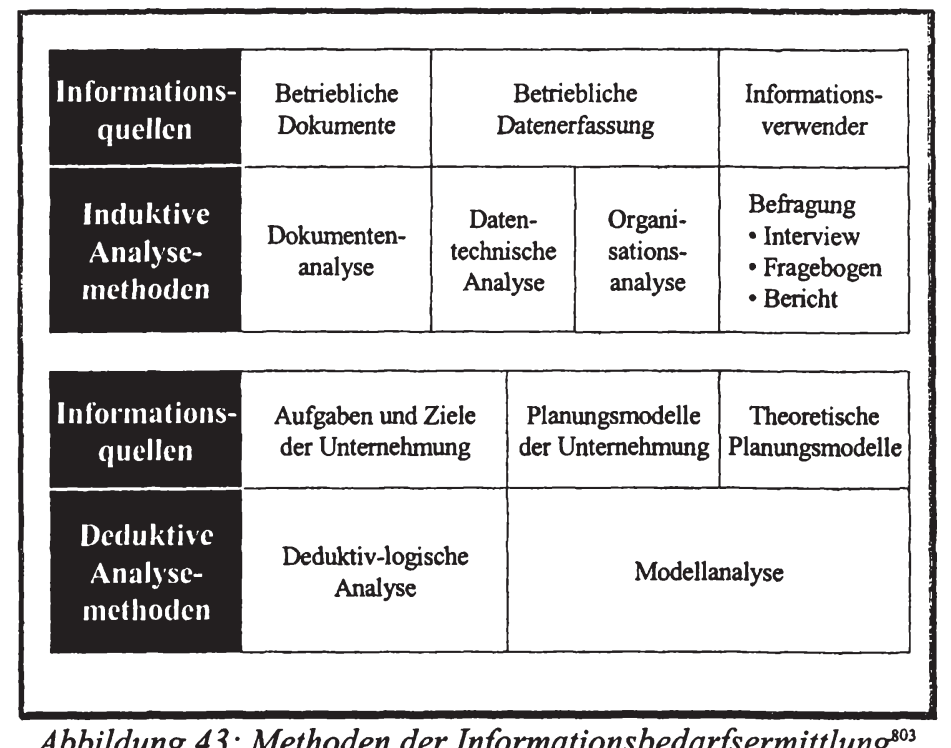

Abbildung 43: Methoden der Informationsbedarfsermittlung ${ }^{803}$

Eine konsequent nachfragerorientierte Ausrichtung des Controlling impliziert zunächst eine induktive Analyse des Informationsbedarfs auf Basis einer Befra-

800 Vgl. für das Beispiel Kostenrechnung Währisch (1998), S. 338.

${ }^{801}$ Vgl. Müßig (1993), S. 288.

802 Vgl. beispielhaft zur Informationsbedarfsermittlung für die operative und taktische Planung Horvàth (1998), S. 350 ff. sowie zur strategischen Planung S. 357 ff.

803 Küpper (1997), S. 141. 
gung der Empfänger. Im Sinne des Marketing ist ein Vorteil dieser Vorgehensweise darin zu sehen, dass dem Kunden unmittelbar die Möglichkeit gegeben wird, seine Bedürfnisse und Bedenken zu artikulieren, was insbesondere organisationsintern, aufgrund mangelnden Kunden-Feedbacks, regelmäßig ein Problem darstellt. ${ }^{804}$ Über Interviews, Fragebögen und Berichte ${ }^{805}$ lässt sich der subjektive Informationsbedarf ableiten. Im Sinne einer sachgerechten Verfolgung der Unternehmensziele besteht jedoch die Gefahr, dass der Kunde des Controllers nicht alle zur Erfüllung seiner Aufgabe notwendigen Informationen eigenständig nachfragt. Die Rationalitätssicherungsfunktion des Controllers erfordert in diesem Zusammenhang, neben dem subjektiven Informationsbedarf seiner Kunden einen objektiv problemlösungsadäquaten Informationsbedarf zu bestimmen. Dieser ergibt sich kontextabhängig auf Basis der Aufgaben und Ziele des Entscheidungsträgers. Zur Operationalisierung dieses Informationsbedarfes eigenen sich Dokumenten-, Daten- und Organisationsanalysen. ${ }^{806}$ Problem dieser Analyseformen ist stets der ausgeprägte Gegenwarts- und Organisationsbezug. Eine sinnvolle Ergänzung dieser Instrumente sind deduktive Informationsbedarfsermittlungsverfahren, welche ausgehend von den Unternehmenszielen deduktiv-logisch oder über Modellanalysen einen objektiven Informationsbedarf zu ergründen versuchen. Analog wird bei der Ermittlung der Informationsbedürfnisse für strategische Entscheidungen, beispielsweise im Rahmen der Critical Success Factors-Analyse (CSF) vorgegangen. ${ }^{807} \mathrm{Da}$ aber auch im Controlling selbst von Kapazitäts- und Rationalitätsengpässen auszugehen ist, ergibt sich neben der subjektiven Informationsnachfrage und dem objektiven Informationsbedarf, ein oftmals mit diesen Mengen nicht deckungsgleiches Informationsangebot des Controllerdienstes.

Im Rahmen einer internen Marketingstrategie für das Controlling stellt sich unmittelbar die Frage nach Ursachen und Lösungsmöglichkeiten für die mangelnde Übereinstimmung der Informationsmengen. In Analogie zu Abbildung $31^{808}$ sollen daher an dieser Stelle differenziert die möglichen Überschneidungen von Informationsangebot, -nachfrage und -bedarf betrachtet werden, um in Anlehnung an die Gestaltungsparameterbetrachtung strategische Optionen diskutieren zu können. Eine Übersicht über die alternativen Überscheidungsmöglichkeiten findet sich in Abbildung 44.

\footnotetext{
804 Allg. zum Kunden-Feedback vgl. Günter (1997), S. 225 ff.

805 Ausfulhrlicher zur Berichtsmethode vgl. MüBig (1993), S. 287 f.

806 Vgl. Horvàth (1998), S. 351.

807 Zur CSF-Analyse vgl. Rockart (1979), S. 81 ff.

808 Vgl. Kap. 3.4.1.2.
} 
Ausgehend von Feld Nr.1, welches die Optimalsituation des Informationsaustausches zwischen Controller und Kunden widerspiegelt, zeichnet sich die Situation in Feld Nr. 2 dadurch aus, dass vom Controlling eine Leistung angeboten und vom Kunden nachgefragt wird, ohne dass die Inanspruchnahme der Leistung bezogen auf das zu lösende Entscheidungsproblem zielführend ist. Maßgeblich ist für den Controller in solchen Situationen, derartige Leistungen überhaupt $z u$ erkennen, da der Leistungsaustausch oberflächlich zu funktionieren scheint. ${ }^{809}$ Werden solche Leistungen identifiziert, kann der Controller über eine anreizkonforme Gestaltung von Verrechnungspreisen, soweit diese organisatorisch durchsetzbar sind, den Kunden dazu bewegen, für ihn irrelevante Leistungen künftig nicht mehr in Anspruch zu nehmen.

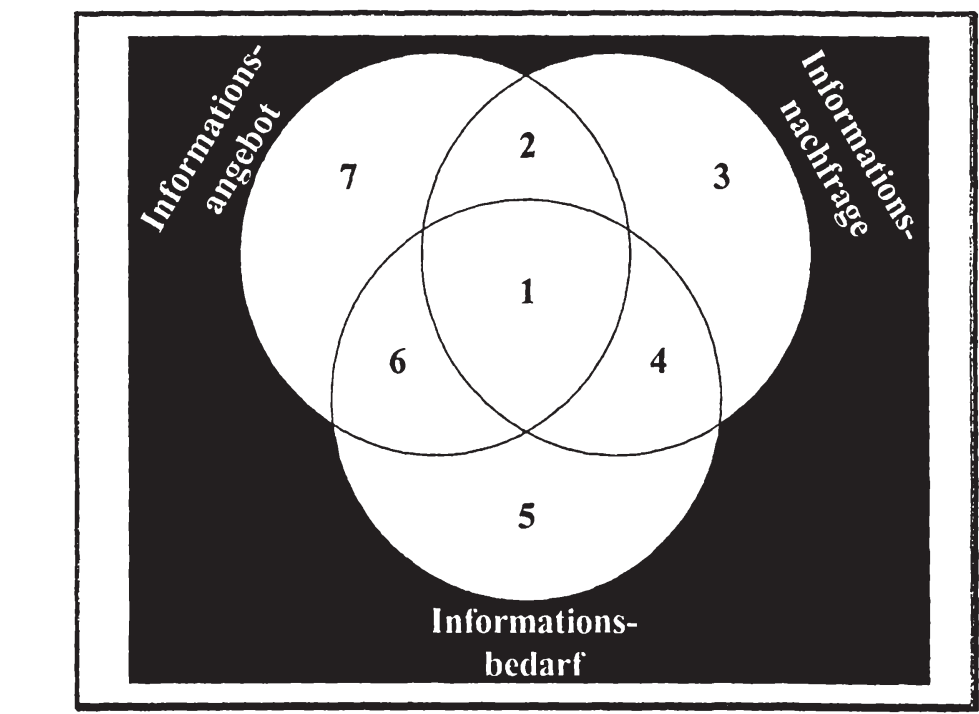

Abbildung 44: Überschneidungsalternativen von Informationsangebot, nachfrage und -bedarf ${ }^{10}$

In Feld Nr. 3 ist regelmäßig die Beratung des Controllers gefragt, da hier die mangelnde Leistungsevidenz oder das fehlverstandene Nutzenpotenzial einer Leistung eine Nachfrage auslöst, die weder zweckmäßig ist noch befriedigt werden kann.

${ }^{809} \mathrm{Zu}$ einigen der Handlungsempfehlungen im Folgenden vgl. Weber (1997), S. 11-13.

810 Weber (1997), S. 12. 
Inhaltlich problematischer ist der Fall Nr. 4, bei dem ein konkreter Informationsbedarf besteht und auch kundenseitig artikuliert wird, diesem jedoch kein Angebot gegenübersteht. Ursache hierfür kann sein, dass der Controller den objektiven und subjektiven Informationsbedarf aufgrund eigener Rationalitätsengpässe oder einer mangelnden internen Marktforschung nicht kennt oder aber die Informationssysteme des Controlling diesen aus Kapazitäts- oder Kostengründen nicht abdecken können. Im Sinne des Marketing ist zur Vermeidung derartiger Situationen darauf zu achten, dass die Artikulation der Kunden, ihrer Bedürfnisse und Zufriedenheit mit dem Leistungsangebot des Controllers institutionalisiert wird. ${ }^{811}$

Feld Nr. 5 kennzeichnet einen konkreter Informationsbedarf, der derzeit nicht durch ein Angebot des Controlling befriedigt werden kann. Zudem wurde der Informationsbedarf nicht vom Management erkannt, so dass diesbezüglich keinerlei Nachfrage artikuliert wird. In derartigen Situationen geht es für den Controller darum, seiner Innovationsfunktion gerecht zu werden und mit neuen Leistungen auf Änderungen der Unternehmensstruktur und des Unternehmensumfeldes zu reagieren. ${ }^{812}$ Oftmals kann auch eine Analyse der potenziellen Konkurrenten und ihrer Leistungsangebote zu konkreten Anregungen führen.

Feld Nr. 6 zeichnet eine Übereinstimmung von Informationsangebot und bedarf aus, eine Nachfrage nach diesen Leistungen wird jedoch nicht artikuliert. Diese Situation bietet regelmäßig zahlreiche Ansatzpunkte für Marketingmaßnahmen im Controlling, da es im Sinne der Unternehmensziele ist, derartige Produkte gezielt zu vermarkten. $\mathrm{Zu}$ analysieren sind hier insbesondere die Ursachen, warum Leistungen des Controlling nicht nachgefragt werden. Dies kann einerseits daran liegen, dass keine ausreichende Transparenz bezüglich des Leistungsangebotes oder des Einsatzes einzelner Leistungen besteht. ${ }^{813}$ In diesem Zusammenhang spielt insbesondere auch der hohe Anteil von Vertrauenseigenschaften der Leistungen des Controlling eine Rolle. ${ }^{814}$ Andererseits ist darauf zu achten, dass die ökonomische Ausrichtung des Controllerbereichs, verbunden mit eventuell von Kunden als zu hoch angesehenen Verrechnungspreisen, nicht zu einem Ausbleiben der Nachfrage führt. Soweit Marketingmaßnahmen keinen Erfolg zeigen, ist unter Umständen im Sinne einer Absicherung

${ }^{811}$ Vgl. hierzu Kap. 4.2.1 sowie zur Operationalisienung der Kundenzufriedenheit Stadler/Weißenberger (1999), S. $8 \mathrm{ff}$.

812 Zur Anpassungs- und Innovationsfunktion des Controlling vgl. Küpper (1997), S. 17 f.

${ }^{813}$ Zur Strukturierung des Controlling-Leistungsangebotes vgl. die Ausfuhrungen zu Service Level Agreements in Kap. 4.2.4.

${ }^{814} \mathrm{Vgl.} \mathrm{Kap.} \mathrm{3.2.2.2.3.}$ 
der Unternehmensziele zu überlegen, inwieweit solche Leistungen obligatorischen Charakter haben sollten. Insgesamt aber sind Leistungen des Feldes Nr. 6 - besonders auch durch flankierende kommunikationspolitische Maßnahmen - in der Gunst der Nachfrager regelmäßig aufwertbar.

Wesentlich bei Leistungen des Feldes Nr. 7 ist, sich als Controller konsequent selbst einer Leistungsbeurteilung durch Kunden oder einem Vergleich mit anderen Anbietern zu unterziehen, um zu erkennen, dass angebotene Leistungen weder freiwillig nachgefragt werden, noch im Sinne der Problemstellung des Kunden zielführend sind. $\mathrm{Zu}$ einem Leistungsaustausch kommt es bei solchen Leistungen auch nur dann, wenn diese obligatorischen Charakter haben. Daher gilt es für den Controller, alle obligatorischen Leistungen regelmäßig einer Überprüfung zu unterziehen und zu bewerten, inwieweit obligatorische Leistungen über eine Steigerung ihrer Evidenz langfristig einer - dann auch erfolgenden freiwilligen Nachfrage ausgesetzt werden können. ${ }^{815}$

Auf Basis der überblicksartig dargestellten Instrumente der Informationsbedarfsanalyse konnte deren unterschiedliche Eignung zur Abgrenzung eines $o b$ jektiven bzw. subjektiven Informationsbedarfs festgestellt werden. Für das Controlling gilt es darauf aufbauend, Strategien der Kundenansprache zu entwickeln, über die sein Informationsangebot mit Nachfrage und Bedarf der Kunden in Einklang gebracht werden kann.

Im Rahmen der Beschreibung alternativer Kunden des Controlling wurde bereits angedeutet, dass Kunden primär nach ihren spezifischen Bedürfnissen abzugrenzen sind. Die Komplexität der Bedürfnisermittlung und die hohe strategische Relevanz organisationsbedingter Sachverhalte lassen es jedoch ratsam erscheinen, weitere operationale Abgrenzungskriterien für Kunden zu betrachten, die für deren unmittelbare Ansprache zielführend sein können.

\subsection{Interne Kundenstrukturanalyse}

Für eine kundenorientierte Ausrichtung des Controllerbereichs ist es unverzichtbar, zunächst alle aktuellen und potenziellen Kunden zu kennen. Die Ausführungen in Kap. 3.4.1.2. konnten diesbezüglich einen ersten Überblick im Sinne einer deskriptiven Systematisierung geben. Diskutiert man darüber hinausgehend strategische Handlungsoptionen, so stellt sich die Frage, nach welchen Kriterien sich alternative Kunden zusammenfassen lassen, damit eine

$815 \mathrm{Vgl}$. hierzu die Ausfuihrungen zu den obligatorischen Leistungen in Kap. 3.3.2.1.1 
Marktbearbeitung im Controlling sowohl bedarfsgerecht als auch wirtschaftlich erfolgen kann. ${ }^{816}$ Der Ansatz der Kunden- bzw. Marktsegmentierung bietet diesbezüglich ein adäquates Instrumentarium ${ }^{8 n^{817}}$ und ist in einem umfassenden Sinn als integriertes Konzept der Markterfassung und -bearbeitung zu verstehen. ${ }^{818}$ Beabsichtigt ist zu gewährleisten, dass - soweit abgegrenzte Kundengruppen über spezifische Marketinginstrumente angesprochen werden - diese auch möglichst ähnlich auf den Instrumenteneinsatz reagieren. Dazu bedarf es der Auswahl geeigneter Segmentierungskriterien ,zur Aufteilung eines Gesamtmarktes in bezüglich seiner Marktreaktion intern homogene [und] extern heterogene Marktsegmente ${ }^{\text {“819. }}$.

Die im vorangegangenen Abschnitt getätigten Ausführungen zum Informationsbedarf zeigen, dass gerade die Bedürfnisse der Kunden einen konkreten Ansatzpunkt für eine Zusammenfassung zu homogenen Gruppen bieten. So können beispielhaft bezogen auf Kostenrechnungsinformationen Benutzertypen identifiziert werden, die aufgrund ihres vergleichbaren Bedarfes mit einem einheitlichen Bericht zufriedengestellt werden können. ${ }^{820}$ Wie an diesem Beispiel deutlich wird, erfolgt bei einer Segmentierung nach dem Informationsbedarf primär eine zielgerichtete Ansprache der Kunden über die sachliche Leistungsgestaltung, im Sinne produktspezifischer Merkmale. Will der Controller im Rahmen der Segmentierung das gesamte Spektrum verhaltensrelevanter Merkmale abdecken, stellt sich die Frage nach der Ableitung weiterer geeigneter Kriterien, bezüglich derer sich die Reaktion der Kunden unterscheidet. ${ }^{21}$ Über das externe Marketing hinaus sind im Controlling insbesondere auch organisationsbedingte Sachverhalte einzubeziehen. Die identifizierten Kundensegmente gilt es sodann mit einer dynamischen Kombination von Produkt-, Preis-, Distributions- und Kommunikationspolitik zu bearbeiten. ${ }^{822}$ Über eine derart individualisierte Bedürfnisbefriedigung soll den Kunden der optimale Nutzen ge-

\footnotetext{
816 Alle später beschriebenen Segmentierungskriterien müssen grundlegenden Anforderungen genügen. Einzelne Anfordenungen sind z.B. die Verhaltensrelevanz, die Messbarkeit, die Zugänglichkeit, die Wirtschaftlichkeit und die zeitliche Stabilităt. Ausfuhrlich vgl. Backhaus (1999), S. 210 f.

817 Vgl. Freter (1983), S. 43 ff.

818 Vgl. Scheuch (1993), S. 274 ff. sowie Meffert (2000), S. 181.

819 Meffert (2000), S. 186.

$820 \mathrm{Vgl}$. Währisch (1998), S. 338 sowie Abbildung 29.

821 Daher spricht man bei Segmentierungskriterien auch von Reaktionskoeffizienten.

822 Allg. zum Marketing Mix und seiner Ausgestaltung vgl. beispielhaft Scheuch (1993), S. 314 ff. oder Pepels (1998), S. $319 \mathrm{ff}$.
} 
stiftet werden und diese somit in die Lage versetzt werden, ihren Beitrag zur Verwirklichung der Unternehmensziele leisten zu können. ${ }^{823}$

In der Marketingliteratur werden Kriterien zur Marktsegmentierung regelmäßig in geographische, soziodemographische, psychographische ${ }^{82}$ und verhaltensorientierte Kriterien unterteilt. ${ }^{825}$ Grundsätzlich sei aber darauf hingewiesen, dass eine unreflektierte Übernahme einzelner absatzmarktorientierter Segmentierungskriterien nicht erfolgen darf. Dennoch können die hier aufgeführten Gruppierungskriterien zu einer Systematisierung spezifischer, für das Controlling relevanter Segmentierungskriterien verwandt werden.

Die geographische Segmentierung von Kunden des Controlling, bspw. nach Kontinenten, Ländern oder Regionen erscheint immer dann angemessen, wenn das Controlling als zentraler Dienstleister spezifische, vom jeweiligen Unternehmensumfeld abhängige Leistungen erbringt. Insbesondere wenn durch die geographische Lage Controlling-Leistungen anderen rechtlichen Auflagen unterliegen, ist eine Differenzierung mitunter zwingend. ${ }^{826}$ Die geographische Lage bedingt regelmäßig auch die räumliche Nähe zwischen Controller und seinem Kunden, welche, wie in Kap. 3.4.1.2. gezeigt wurde, Einfluss auf den Informationsaustausch und Leistungsfluss haben kann. ${ }^{827}$

Bei der Betrachtung soziodemographischer Segmentierungskriterien muss auf besondere Art und Weise dem Organisationskontext des Controllerbereichs und dem seiner Kunden Rechnung getragen werden. Werden im Absatzmarketing unter den sozioökonomischen Merkmalen Beruf, Ausbildung und Einkommen subsumiert und unter den demographischen Merkmalen Geschlecht, Alter, Familienstand, Haushaltsgröße etc., ${ }^{828}$ so sind diese Merkmale organisationsintern umzuinterpretieren. Die soziodemographischen Segmentierungskriterien sind letztlich die Merkmale, die die Person des Kunden in seinem gesellschaftlichen und ökonomischen Kontext auszeichnen. Bezogen auf die in eine Organisation eingebundenen Kunden des Controlling sind dies demnach die hierarchische Stellung in der Unternehmung, die Zugehörigkeit zu einem Fach-oder Funkti-

\footnotetext{
${ }^{823}$ Für den Bankenbereich vgl. Knauber/Kuloglu/Podrazil (1997), S. 301.

$824 \mathrm{Vgl}$. Bouncken (1995), S. 1.

${ }^{825}$ Vgl. Freter (1983), S. 46 oder Meffert (2000), S. $186 \mathrm{ff}$.

${ }^{826}$ Die rechtlichen Rahmenbedingungen werden hier als geographisch determiniert angesehen, können gleichermaßen aber unter die soziodemographischen Kriterien gefasst werden, da sie das gesellschaftliche Umfeld der Kunden betreffen.

${ }^{827}$ Für die Kostenrechnung vgl. Homburg/Weber/Aust/Karlshaus (1998), S. 21.

828 Vgl. Freter (1983), S. 46.
} 
onsbereich sowie dessen Stellung und Entwicklung in der Organisation. ${ }^{829}$ Ferner sind bestehende informelle Kontakte von Bedeutung. Betrachtet man darüber hinaus differenzierter die Strukturen abnehmender Organisationseinheiten, so lassen sich auch bezogen auf Controlling-Leistungen Buying Center ${ }^{30}$ identifizieren.

Ein Buying Center umfasst alle am Kaufprozess beteiligten Personen. ${ }^{831}$ Bei multipersonalen Beschaffungsentscheidungen gilt es, die Rollen der am Kaufprozess Beteiligten zu analysieren, um über eine zielgerichtete Ansprache einzelner Akteure den Prozess gestalten zu können. ${ }^{832}$ Gerade die Komplexität der Controlling-Leistungen in Verbindung mit dem daraus resultierenden Unsicherheitsempfinden der Nachfrager, führt oftmals zu einer Beteiligung mehrerer Vertreter des Kunden. Da es sich bei den Kunden regelmäßig um Organisationseinheiten mit gegebenen Strukturen handelt, nehmen die am Beschaffungsprozess Beteiligten oftmals spezifische Rollen ein. An dieser Stelle wird bereits der fließende Übergang zu den psychographischen Segmentierungskriterien deutlich, da diese Persönlichkeitsmerkmale einschließen. Betrachtet man die typischen Rollen der am Buying Center Beteiligten, erscheint eine Übertragung dieses Konzeptes auf Kundenbeziehungen des Controlling angemessen.

Nachfolgend soll beispielhaft die Beschaffungsentscheidung für das potenziell durch den Controller anzubietende Produkt „Einführung einer Balanced Scorecard $^{\text {" } 833}$ betrachtet werden. Bei diesem Beschaffungsvorgang ist regelmäßig die Geschäftsführung als derjenige Part anzusehen, der die endgültige „Kaufentscheidung" trifft und somit die Rolle des Deciders inne hat. ${ }^{834}$ Soweit die Geschäftsführung den Vorbereitungsprozess im Sinne einer Lieferantenauswahl zwischen Berater und Controller delegiert, übernimmt beispielsweise ein $\mathrm{Ge}$ schäftsführungsassistent die Rolle des Buyers. Hat ein bestimmter Geschäftsführer zuerst die Notwendigkeit zusätzlicher nichtmonetärer Steuerungsgrößen erkannt und die Balanced Scorecard gefordert, gilt dieser bezogen auf den Beschaffungsprozesses als Initiator. Dem Geschäftsführungsassistent oder einer Sekretärin kann die Rolle des Gate Keepers zukommen, da dieser den Informa-

\footnotetext{
829 Vgl. auch Abbildung 46.

${ }^{830} \mathrm{Vgl}$. Webster/Wind (1972), S. 77.

831 Allg. zum Buying Center vgl. Backhaus (1999), S. 58.

832 Vgl. FlieB (1995), S. 339.

${ }^{833} \mathrm{Zu}$ der Konkurrenzsituation im Controlling bezogen auf dieses Produkt vgl. Abbildung 37.

834 Die hier exemplarisch zugeordneten Rollen entsprechen den des Rollenkonzepts von Webster/Wind (1972), S. 35 und S. $78 \mathrm{ff}$.
} 
tionsfluss in das Buying Center kontrolliert und filtert. ${ }^{835}$ Charakteristisch für Leistungen des Controlling ist oftmals auch, dass es einen vom Buyer und Decider abweichenden User gibt, der das zu beschaffende Gut anwendet, oder aber auch die Arbeit damit verweigern kann, was gerade im Bereich der für das Controlling ausschlaggebenden Informationsnutzung schwer festzustellen ist. Bezogen auf das Beispiel können die Bereichsleiter für die konkrete Strategieumsetzung mit der Balanced Scorecard oder die Pflege ihrer Kennzahlen verantwortlich gemacht werden. Die Kenntnis der Nutzer spielt für den Controller eine außerordentlich wichtige Rolle, da erst die sachgerechte Nutzung der Leistungen den Controlling-Beitrag zur Erfüllung der Unternehmensziele absichert. $^{836}$

Die Betrachtung von Buying Centern der Controlling-Kunden ist somit im Sinne einer Mikrosegmentierung zu verstehen, ${ }^{837}$ da die Kenntnis der einzelnen Personen deren Ansprache mittels gezielter „Verkaufsargumente“ erlaubt. ${ }^{838}$ Die Möglichkeit zur Mikrosegmentierung ist gleichermaßen als ein Wettbewerbsvorteil des Controlling gegenüber externen Anbietern anzusehen, da nur ein interner Anbieter die notwendige Organisationskenntnis haben kann. Das Beispiel hat gezeigt, dass zur Strukturierung von Kunden sowohl die im Unternehmenskontext zu sehenden soziodemographischen Kriterien relevant sind, als auch die zu den psychographischen Kriterien gehörenden Persönlichkeitsmerkmale, zu denen die soziale Orientierung und die Risikoneigung zählen. ${ }^{839}$

Neben den allgemeinen Persönlichkeitsmerkmalen zählen zu den psychographischen Segmentierungskriterien produktbezogene Merkmale wie: ${ }^{: 40}$

- die Wahrnehmung der Leistung durch den Kunden des Controllers,

- seine Motive der Leistungsinanspruchnahme,

- seine spezifische Einstellung zum Controlling und

- die Nutzenvorstellungen des Kunden.

Es wird deutlich, dass die hier aufgezählten potenziellen Segmentierungsmerkmale mit einigen der genannten gestaltungsrelevanten Parameter korrespondie-

\footnotetext{
835 Zur Erläuterung der Rollen vgl. Fließ (1995), S. 347 ff.

836 Vgl. Kap. 3.4.3.3.

837 Zur Mikrosegmentierung vgl. Engelhardt/Günter (1983), S. 90 ff.

838 Hier und nachfolgend vgl. Witt (1985), S. 171.

$839 \mathrm{Vgl}$. Meffert (2000), S. 199 ff.

840 Die Aufzahlung ausgewählter Merkmale erfolgt in Anlehnung an Freter (1983), S. 46.
} 
ren. Insbesondere die Wahrnehmung der Leistung hängt maßgeblich mit den Beurteilungsdimensionen, ${ }^{841}$ den informationsökonomischen Leistungseigenschaften $^{842}$ und der Leistungseviden $z^{843}$ zusammen. Die Kenntnis der Nutzenvorstellung der Kunden hängt wesentlich davon ab, inwieweit es im Rahmen der Informationsbedarfsanalysen gelingt, über die subjektiv artikulierten Bedürfnisse auf die Informationsnutzung des Managements zu schließen. Bei den Motiven der Leistungsinanspruchnahme ist zunächst aufgrund des Organisationskontextes zu beachten, dass nicht die persönlichen Interessen der Person des Kunden ausschlaggebend sind, sondern vielmehr die mit der Erfüllung seiner Aufgaben verbundenen Zwänge. Im Sinne obligatorischer und fakultativer Leistungen ist daher zwischen freiwilligen Kunden und Zwangskunden ${ }^{844} \mathrm{zu}$ unterscheiden. Hierbei sind Zwangskunden aufgrund unternehmensinterner Vorgaben verpflichtet, die Leistungen des Controlling in Anspruch zu nehmen. Bedingt durch den verpflichtenden Charakter der Transaktion kommt einer Sicherstellung der Leistungsnutzung eine besondere Bedeutung zu, da die Gefahr einer mangelnden bzw. unsachgemäßen Nutzung besteht. Im Vergleich zu Zwangskunden fragen freiwillige Kunden die Leistungen des Controlling selbständig nach, um hierdurch ihre Entscheidungsgrundlage zu verbessern. Neben dem Zeitpunkt der Inanspruchnahme bestimmen die freiwilligen Abnehmer auch das jeweilige Produkt und dessen Charakteristika. Bei einer segmentspezifischen Bearbeitung dieser Kundengruppen besteht die Herausforderung darin, Zwangskunden zu freiwilligen Kunden zu machen. ${ }^{845}$ Durch eine so veränderbare Motivation der Leistungsinanspruchnahme ergeben sich unmittelbar Rückwirkungen auch auf die Nutzung der durch das Controlling zur Verfügung gestellten Informationen. Ferner erweitert sich möglicherweise das Spektrum der in Anspruch genommenen Produkte über die zwangsweise abgenommenen hinaus. Strategisch betrachtet kann hierdurch einerseits ein Segmentwechsel ausgelöst werden, andererseits kann die Marktdurchdringung gesteigert werden..$^{846}$

Neben den psychographischen Kriterien weisen insbesondere auch die verhaltensorientierten Segmentierungskriterien konkrete Anknüpfungspunkte zu den

\footnotetext{
841 Vgl. Kap. 3.2.2.1.

842 Vgl. Kap. 3.2.2.2.

${ }^{843}$ Vgl. Kap. 3.4.3.2.

844 Zur Erläuterung dieser Gruppen vgl. im Folgenden Berger (1989), S. 185.

845 Vgl. Berger (1989), S. 185.

$846 \mathrm{Vgl}$. hierzu auch die Ausfulhrungen zur strategischen Bereichsplanung in Kap. 4.1.1 sowie Ansoff (1966), S. 132.
} 
betrachteten Gestaltungsparametern auf. Basis der verhaltensorientierten Marktsegmentierung ist das beobachtbare Beschaffungsverhalten der Kunden des Controlling, also das Ergebnis konkreter Transaktionen. ${ }^{847}$ Daher lassen sich die Kriterien zur verhaltensorientierten Marktsegmentierung im wesentlichen auch nach den auf die Kaufentscheidung einwirkenden Instrumentalbereichen des Marketing strukturieren. So können Kunden des Controlling nach ihrem Preisverhalten, ihrem Informations- und Kommunikationsverhalten, ihrem Auswahlverhalten bezüglich der Distributionswege und ihrem grundsätzlichen Produktauswahlverhalten segmentiert werden. ${ }^{848}$

Die Verwendung des Preisverhaltens als Segmentierungskriterium für Controlling-Kunden setzt ein differenziertes System von Verrechnungspreisen voraus. Ferner muss die ökonomische Ausrichtung des Controlling im Einzelfall Spielräume bei der Verrechnungspreisgestaltung zulassen. Denkbar erscheint dann, das auf unterschiedliche Budgets zurückzuführende Preisreaktionsverhalten alternativer Kunden im Sinne einer Preisdifferenzierungsstrategie auszunutzen. ${ }^{849}$ Insbesondere bei automatisch generierbaren Informationsprodukten mit unwesentlichen Grenzkosten ist von einem Preisgestaltungsspielraum des Controllers auszugehen.

Eine Segmentierung nach dem Informations- und Kommunikationsverhalten fokussiert, wie alternative Kunden auf direkte oder indirekte Kommunikation des Controllings reagieren, bzw. darauf, welche Informationskanäle Kunden nutzen. Der regelmäßige Vorwurf, dass auf Abnehmerseite eine zu geringe Angebotskenntnis des Controlling vorherrscht, ${ }^{850}$ verdeutlicht die dominante Rolle kommunikationspolitischer Maßnahmen. Ein Wettbewerbsvorteil gegenüber externen Anbietern ist wiederum darin zu sehen, dass der Controller bezogen auf seine Arbeitsschwerpunkte noch die Rolle des In-Suppliers inne hat und somit Instrumente der direkten Kommunikation im Sinne eines Personal-Selling einsetzen kann. ${ }^{851}$ Alternativ gibt es aber auch zahlreiche Medien zur indirekten Kommunikation der Leistungen des Controlling, wie bspw. das Intranet, Verteiler oder Firmenzeitschriften.

\footnotetext{
${ }^{847}$ Allg. hier und im Folgenden vgl. Meffert (2000), S. 208.

848 Übersichtsartig vgl. Freter (1983), S. 46.

849 Allg. zur Preis- und Konditionenpolitik vgl. Backhaus (1999), S. 429 ff.

${ }^{850} \mathrm{Vgl}$. Steinle/Thiem/Rohden (2000), S. 281.

851 Allg. furr unternehmensinterne Dienstleistungen vgl. Witt (1985), S. 171.
} 
Analog zu der Segmentierung von Kunden des Controlling nach ihrem Informationsverhalten lassen sich Kunden nach der Inanspruchnahme alternativer Distributionswege segmentieren. In einem umfassenden Sinn sind hier insbesondere auch alternative Trägermedien als Distributionswege aufzufassen. Der Informationscharakter der Controlling-Leistungen ermöglicht unterschiedliche Formen des Zugriffs; so können Leistungen bspw. versandt werden, direkt aus Datenbanken abgerufen oder im Intranet bereitgestellt werden. Andere Leistungen wie Beratungs- oder Schulungsaktivitäten können hingegen nur in Anwesenheit des Kunden erbracht werden. Alternative Distributionswege erfordern daher in unterschiedlichem Umfang eine Mitwirkung des Kunden und weisen daher eine hohe Verhaltens- und Reaktionsrelevanz auf.

Die hier aufgeführten Beispiele deuten bereits an, dass die Grenzen zwischen sachlicher Leistung und Distributionsweg in den Augen der Kunden verschwimmen können, da bspw. ein bestimmter Inhalt auf einem abweichenden Trägermedium mitunter nachfragerseitig als ein anderes Produkt wahrgenommen wird.

Als letzte Untergruppe der verhaltensorientierten Segmentierungskriterien sind Kriterien der Produktwahl zu nennen. ${ }^{852}$ Im Einzelnen zählt hierzu die Tatsache, ob ein Unternehmensbereich überhaupt Leistungen abnimmt oder nicht. Ferner kann im Controlling beurteilt werden, in welchem Rhythmus Leistungen abgenommen werden, wie ausgeprägt die Bindung an einzelne Leistungen ${ }^{853} \mathrm{bzw}$. wie hoch die Wechselbereitschaft bestimmter Kunden ist. ${ }^{854}$ Von grundlegender Bedeutung für das Controlling ist ferner seine Kunden nach dem aktuellen und potenziellen Volumen der Inanspruchnahme zu differenzieren, ${ }^{85 s}$ so dass im Sinne einer $A B C$-Analyse Aussagen über die Bedeutung von Kunden möglich werden. ${ }^{856}$ Hierbei darf jedoch die Bedeutung nicht im Sinne einer Wertigkeit verstanden werden, vielmehr ist darauf zu achten, dass der Ressourceneinsatz in einem angemessenen Verhältnis zum Leistungsoutput bleibt. Abbildung 45 zeigt zusammenfassend mögliche Kriterien der internen Marktsegmentierung im Controlling.

\footnotetext{
852 Übersichtsartig vgl. Freter (1983), S. 46.

$853 \mathrm{Vgl}$. Abbildung 46.

$854 \mathrm{Vgl}$. Meffert (2000), S. $208 \mathrm{ff}$.

$855 \mathrm{Vgl}$. hierzu die in Abbildung 46 vorgenommene Klassifikation nach der potenziellen Nachfrage.

856 Zur ABC-Analyse von Kunden vgl. Holtl (1999), S. 26 f.
} 


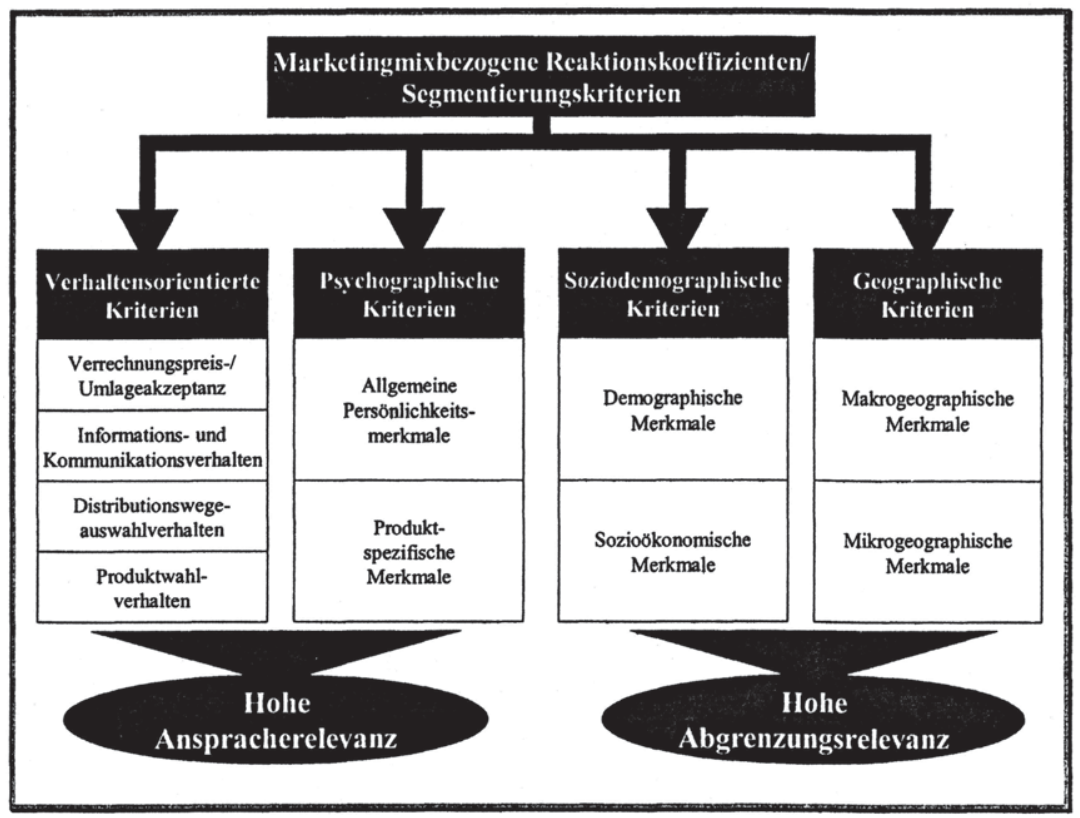

Abbildung 45: Kriterien der internen Marktsegmentierung im Controllings57

Insgesamt bleibt - bezogenen auf die Segmentierungsmerkmale - festzuhalten, ${ }^{858}$ dass sich insbesondere die geographischen und soziodemographischen Merkmale zu einer Abgrenzung der Kunden des Controlling eignen. Die psychographischen und verhaltensorientierten Kriterien bieten demgegenüber konkrete Anhaltspunkte für die Ansprache der Kunden über eine Ausgestaltung der einzelnen Marketinginstrumente. Erstrebenswert für eine Erfassung und Bearbeitung alternativer Kundensegmente des Controlling ist daher regelmäßig eine Kombination mehrerer in der Abbildung 45 systematisierten Kriterien.

Neben der Benennung reaktionsrelevanter Abgrenzungskriterien stellt sich für den Controller insbesondere auch die Frage nach der Wirtschaftlichkeit der Bearbeitung alternativer Marktsegmente. Im Einzelnen heißt dies, dass die segmentspezifischen Marketingstrategien den damit verbundenen Mehraufwand rechtfertigen müssen. ${ }^{859}$

857 Modifiziert in Anlehnung an Freter (1983), S. 46.

${ }^{858}$ Allg. zu den kriteriengruppenbezogenen Tendenzaussagen vgl. Meffert (2000), S. 211.

859 Zur Bedeutung der Wirtschaftlichkeit, bzw. Okonomität im Rahmen der Anforderungen an Segmentierungskriterien vgl. Scheuch (1993), S. 285 f. 
Wichtig ist, insbesondere in Abhängigkeit der ökonomischen Ausrichtung des Controllerbereichs, den richtigen Maßstab für die Bewertung von Marktsegmenten anzulegen, d.h. es müssen über die Bereichsinteressen des Controlling hinaus, langfristige Auswirkungen auf die Unternehmensziele betrachtet werden. Hierzu können bspw. die in einem Marktsegment zum Einsatz kommenden Leistungen betrachtet werden. Hat man diese Leistungen in Form des in Abbildung 42 dargestellten Technologieportfolios bewertet, lassen sich auf Basis der Technologieattraktivität Aussagen über die Bedeutung einer Leistung für das Gesamtunternehmen ableiten. ${ }^{860}$

Einen möglichen Ansatz zur strategischen Bewertung von internen Kunden/Marktsegmenten aus Perspektive des Controlling gibt Abbildung 46 wieder. Das dort aufgezeigte Bewertungsverfahren ist eine Kombination bereits dargestellter Segmentierungskriterien und ist im Sinne eines Stufenverfahrens zu verstehen.

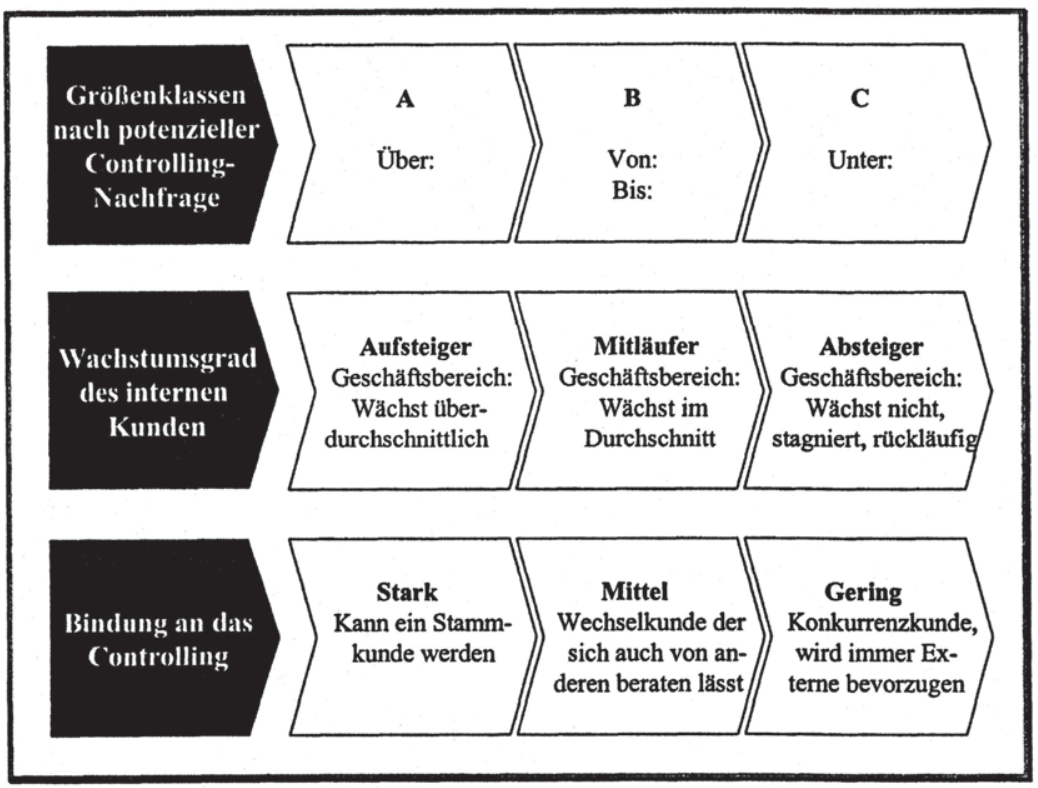

Abbildung 46: Kundenbewertung im Controlling ${ }^{801}$

860 Zur Operationalisierung der Technologieattraktivität von Controlling-Leistungen kann z.B. der Beitrag eines Instrumentes zur unternehmensweiten Kostensenkung herangezogen werden. Für Leistungen der Kostenrechnung vgl. Weber (1999), S. $371 \mathrm{ff}$.

861 In Anlehnung die Darstellung fur das interne Consulting von Niedereichholz (2000), S. 20 
Ausgehend von einer Zuordnung zu abgrenzbaren Größenklassen (bspw. nach Ressourcenbeanspruchung des Controlling) sind Wachstums- und Bindungsgrade zu bestimmen. ${ }^{862}$ Im Ergebnis lassen sich alternative Kundengruppen in ein Portfolio einordnen. Als Dimensionen eines derartigen Portfolios lassen sich bspw. Wachstums- und Bindungsgrad verwenden. Die Größe der Darstellung einzelner Kunden könnte in Abhängigkeit der potenziellen ControllingNachfrage erfolgen. Auf dieser Basis lassen sich Aussagen über die Ausgeglichenheit der Kundenstruktur des Controlling und über eine zukünftig sinnvolle Ressourcenallokation ableiten.

Soweit sich mehrere Kunden des Controlling in einem Segment befinden und diese die gleiche Leistung als problemlösungsadäquat ansehen, kann die Bearbeitung einzelner Kundensegmente des Controlling standardisiert erfolgen, z.B. bei Berichten oder regelmäßig wiederkehrenden Planungsverfahren. Im Rahmen der Ausführungen zur Standardisierung wurde aber ebenfalls deutlich ${ }^{863}$ dass in vielen Fällen Leistungen des Controlling nur für einen Kunden bestimmt sind, wie z.B. Sonderauswertungen oder Beratungen. Die Zusammenfassung mit weiteren Kunden wäre im Sinne einer optimalen Ansprache wenig zweckmäßig. In diesem Zusammenhang spricht man von einem Segment-of-One-Approach. ${ }^{864}$ Die damit auf einen einzigen Kunden bezogenen Marketingaktivitäten werden unter dem Begriff des One-to-One-Marketing subsumiert. ${ }^{865}$ Diese Situation eines quasi bilateralen Monopols stellt für das Controlling keine Seltenheit dar.

Die Ausführungen zu den für das Controlling potenziell relevanten Marktsegmentierungskriterien haben gezeigt, dass es eine Vielzahl von Anknüpfungspunkten für die Erfassung und Bearbeitung von internen Kunden gibt. Im Einzelfall ist jedoch durch den Controller zu entscheiden, inwieweit eine derart differenzierte Analyse und Bearbeitung unter wirtschaftlichen Gesichtspunkten zu rechtfertigen ist. ${ }^{866}$ Selbst wenn die aufgezeigten Kriterien nicht unmittelbar zu einer Segmentierung verwandt werden, ist die Vergegenwärtigung ihrer Reaktions- bzw. Verhaltensrelevanz für den Controller unerlässlich. Darüber hinaus können, wie in Abbildung 46 gezeigt, ausgewählte Kriterien zur Planung abnehmergerichteter Strategien verwandt werden. Hierzu gilt es, die zukünftige

${ }^{862}$ Zur Anwendung des Bewertungsverfahren auf Klienten des internen Consulting vgl. Niedereichholz (2000), S. $20 \mathrm{f}$.

${ }^{863}$ Vgl. Kap. 3.4.3.1.

864 Vgl. Meffert/Bruhn (1995), S. 189.

${ }^{865} \mathrm{Zu}$ der Extremform des One-to-One-Marketing vgl. Kotler/Bliemel (1999), S. 1122.

${ }^{866}$ Zur Wirtschaftlichkeit von Segmentierungskriterien vgl. Meffert (2000), S. 187. 
Bedeutung alternativer Kundengruppen zu bestimmen sowie sich der zu deren Problemlösung einsetzbaren Wettbewerbsvorteile des Controlling bewusst $\mathrm{zu}$ werden.

Je größer der Anteil der Controlling-Leistungen ist, die einer potenziellen Wettbewerbsgefährdung ausgesetzt sind, ${ }^{867}$ um so eher gilt es, sich neben einer abnehmergerichteten Strategie Gedanken über eine konkurrenzorientierte Strategie zu machen.

\subsubsection{Konkurrenzorientierte Strategie}

Im Rahmen der Diskussion konkurrenzorientierter Strategiealternativen ${ }^{868}$ des Controlling darf nicht vernachlässigt werden, dass es sich bei dem Controlling in einer Vielzahl der Unternehmen zunächst noch um einen monopolistischen internen Anbieter handelt, dessen Leistungen einer verhältnismäßig geringen Konkurrenzgefährdung ausgesetzt sind. Dennoch besteht für jeden Controller die Notwendigkeit, sich Gedanken über Anbieter potenziell vergleichbarer Leistungen $^{869}$ - die auch aus Nachfragerperspektive als Substitute wahrgenommen werden - zu machen.

Eine Wettbewerbsbetrachtung impliziert nicht zwangsläufig, sich einer unmittelbaren Konfrontation mit Konkurrenten auszusetzen. Vielmehr sollte der Anspruch des Controllers im Rahmen der Analyse konkurrenzorientierter Strategien sein, Entwicklungen des Wettbewerbs aufmerksam zu beobachten und zu versuchen, an positiven Auswirkungen des Wettbewerbs, auch wenn er diesem faktisch nicht in jedem Fall ausgesetzt ist, zu partizipieren. Der Controller muss sich insbesondere mit der Innovationsfunktion des Wettbewerbs auseinandersetzen. ${ }^{870}$ Soweit andere Anbieter von Controlling-Leistungen dieser konkreten Innovationskonkurrenz ausgesetzt sind, muss der Controller - auch wenn er selbst einem geringerem Konkurrenzdruck ausgesetzt ist - versuchen, an dem wettbewerbsinduzierten Fortschritt zu partizipieren. Diesbezüglich lassen sich für den Controllerbereich anhand der in Abbildung 47 dargestellten Typologisierung konkurrenzgerichteten Verhaltens interessante Handlungsoptionen ableiten.

\footnotetext{
$867 \mathrm{Vgl}$ Kap. 3.4.3.4.

${ }^{868}$ Umfassend zur Konkurrenzanalyse vgl. Kleinaltenkamp (2000), S. 84 ff.

${ }^{869} \mathrm{Vgl}$. hierzu die in Abbildung 32 aufgelisteten Leistungen von Unternehmensberatungen die ControllingLeistungen anbieten.

$870 \mathrm{Zu}$ den einzelnen Funktionen des Wettbewerbs vgl. Berg (1992), S. 241. Spezifisch zu der Aufgabe des
} Wettbewerbs als Suchprozess und Entdeckungsverfahren vgl. Hayek (1968), S. 249 ff, 


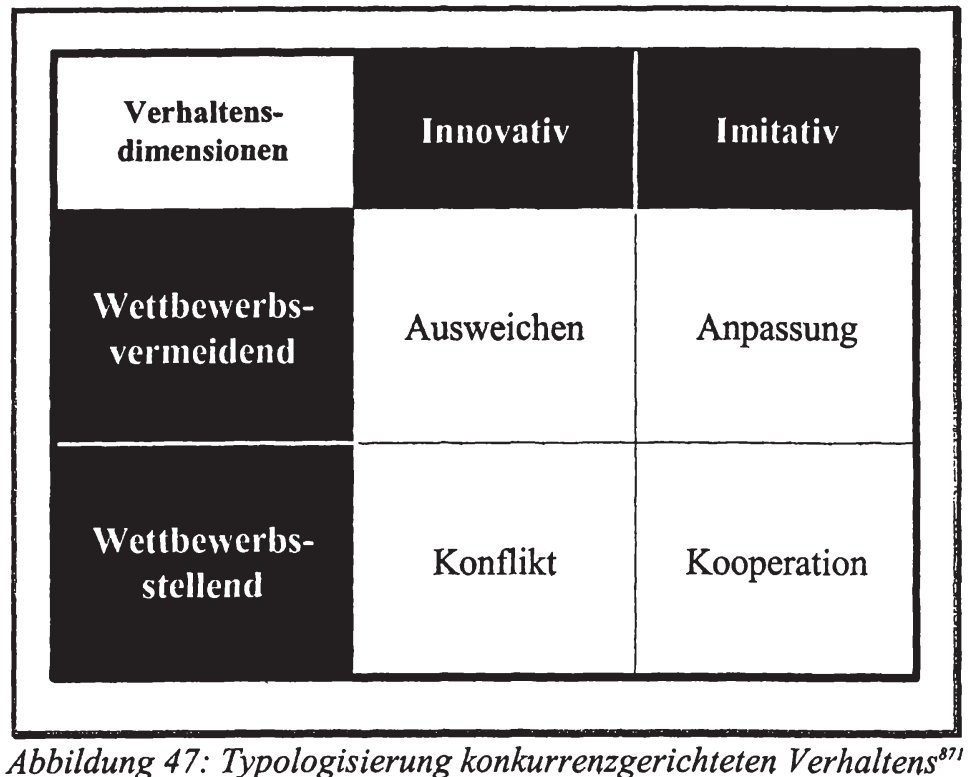

Der geringe Konkurrenzdruck auf Controlling-Leistungen hat in der Vergangenheit eher zu einem passiven, wettbewerbsvermeidenden Verhalten der Controller geführt. Die Tatsache, dass das unternehmensexterne Wettbewerbsumfeld für Controlling-Leistungen zu keiner signifikanten Schwächung der Stellung des Controllings im Unternehmen beitragen konnte, führte regelmäßig zu einem angepassten Verhalten. Im Gegensatz zu einem antizipativen Verhalten wurden bspw. neue betriebswirtschaftliche Instrumente erst in das Leistungsangebot aufgenommen, sobald diese einen gewissen Verbreitungsgrad aufwiesen. ${ }^{872}$

Eine zukünftig erfolgversprechende Strategie für das Controlling stellt demgegenüber die Ausweichstrategie dar. Ein Ausweichen ist grundsätzlich immer dann möglich, wenn man in einem abgeschirmten Marktsegment agiert, ${ }^{873}$ wie dies in der Vergangenheit aufgrund der Organisationsbarriere im Controlling der Fall war. Geht man prospektiv von einer zunehmenden Durchlässigkeit dieser Barriere und einer steigenden Konkurrenz innerhalb von Unternehmen aus, kann eine Ausweichstrategie nur dann erfolgreich sein, wenn über innovative,

${ }^{871}$ Meffert (2000), S. 284.

872 Zur Antizipationsnotwendigkeit bei wettbewerbsstellendem Verhalten vgl. Ansoff (1976), S. $129 \mathrm{ff}$.

873 Allg. im Folgenden zu Ausweich- und Anpassungsstrategien vgl. Meffert (2000), S, 286-288. 
individualisierte Leistungen neue Marktzutrittsbarrieren für Konkurrenten aufgebaut werden können. Insbesondere auch kundenspezifische Marketingaktivitäten können in diesem Zusammenhang differenzierend wirken. Ferner führt die In-Supplier Stellung des Controllers dazu, ${ }^{874}$ dass die bei einigen Leistungen im Laufe der Zeit aufgebauten Spezialisierungs- und Erfahrungseffekte als auch zukünftig wirksame Marktzutrittsbarrieren aufzufassen sind.

Verlässt man die wettbewerbsvermeidenden Strategiealternativen und betrachtet aktive, wettbewerbsstellende Strategien, so ist die auf Innovationen beruhende Konfliktstrategie differenziert zu bewerten. Soweit andere Organisationsmitglieder als potenzielle Konkurrenten betrachtet werden, kann eine offene Konfrontation mit diesen zu erheblichen Nachteilen für die Gesamtorganisation führen. Insbesondere auch der mögliche Schaden für die Reputation des Controlling verbietet regelmäßig eine solche Vorgehensweise. ${ }^{875}$ Zulässig erscheinen lediglich gezielte Kommunikationsaktivitäten, soweit die eigene Problemlösung als zielführender im Sinne der Unternehmensziele angesehen wird. ${ }^{876}$ Gegenüber externen Wettbewerbern erscheint eine auf gezielten Vergleichen aufbauende Propagierung eigener Stärken wesentlich unkritischer. Neben der komparativen Betonung von Nutzenvorteilen ist insbesondere eine vergleichende Betrachtung der mit alternativen Anbietern verbundenen Kosten statthaft. Soweit angemessene Verrechnungspreise verwandt werden, können hierdurch Kostenvorteile des Controlling dokumentiert werden.

Insgesamt wird stets vorausgesetzt, dass das Controlling selbst an einer Erstellung der Leistung interessiert ist und über die dazu notwendigen Kapazitäten verfügt. Demgegenüber gibt es aber viele Situationen, in denen Controller aus Kapazitätsgründen bewusst Aufgaben durch externe Berater ausführen lassen, obwohl sie diese potenziell selbst wahrnehmen könnten. ${ }^{877}$ Derartige Situationen eignen sich insbesondere für kooperative Strategien im Controlling.

Kooperationen unterscheiden sich grundsätzlich durch den Grad der Zusammenarbeit und die damit korrespondierende Art der Bindung. ${ }^{878}$ Zum einen lassen sich Projekte identifizieren, in denen Controller mit Beratern zusammen

\footnotetext{
874 Zur In-Supplier Stellung innerbetrieblicher Anbieter vgl. Witt (1985), S. 171.

875 Vgl. hierzu die Darstellung der außerordentlich großen Bedeutung der Reputation im Rahmen der Vermarktung von Gütern mit Vertrauenseigenschaften in Kap. 3.2.2.2.3.

877 Dies ist oftmals auch der Grund für die Einrichtung interner Consulting Einheiten. Zum Stand des internen Consulting vgl. Hoyer (2000), S. 56 ff.

${ }^{878} \mathrm{Vgl}$. Meffert (2000), S. 284.
} 
spezifische Projektteile bearbeiten, da sie die Organisationskenntnis und das Controlling-Hintergrundwissen haben. Zum anderen wird es für Controller zunehmend bedeutender, die Koordination von Beratungsprojekten zu übernehmen. ${ }^{879}$ Im Einzelnen zählen hierzu nachfolgende Aufgaben: ${ }^{880}$

- Beratung im Vorfeld von Veränderungsvorhaben,

- Aufbau und Pflege von Fachkompetenz zur Beurteilung externer Beraterleistungen,

- Unterstützung bei der objektiven Auswahl externer Berater,

- Mitwirkung beim Abschluss von Beraterverträgen,

- Pflege von aktuellen Informationen über Beratereinsätze.

Insbesondere dann, wenn der Controller selbst die Verantwortung für den Beratereinsatz behält, ${ }^{881}$ ist eine Kooperation zwingend notwendig, da nur so eine Erfüllung der Kundenanforderungen möglich ist. Im Sinne der Kundenorientierung des Controlling ist die Aufgabe des Controllers bei Kooperationen speziell darin zu sehen, auf Grundlage seiner ausgeprägteren Kundenkenntnis den Auftrag des Beraters so zu definieren, ${ }^{82}$ dass eine Erfüllung der spezifischen internen Kundenbedürfnisse möglich wird.

Insgesamt wurde im Rahmen der dargelegten Optionen konkurrenzgerichteten Verhaltens deutlich, dass dieses nicht zwangsläufig mit einer offenen Konfrontation verbunden sein muss. Vielmehr ist durch den Controller im Einzelfall zu bewerten, in welchem Umfang seine Leistungen einer Konkurrenzgefährdung ausgesetzt sind ${ }^{883}$ und inwieweit es daher erforderlich ist, auf das Verhalten der Konkurrenten zu reagieren bzw. dieses zu antizipieren. Wichtig ist unabhängig von dem konkreten Konkurrenzdruck die wettbewerbsinduzierten Innovationen auch in das eigene Leistungsangebot einzubeziehen. Bei fehlenden Kapazitäten oder mangelndem methodischen Know how kann ein wichtiger Beitrag des Controlling durch eine sachgerechte Koordination des externen Beratereinsatzes geleistet werden.

879 Zum Controlling von Unternehmensberatungsleistungen vgl. Witt (1986), S. $129 \mathrm{ff}$.

${ }^{880}$ Hierbei handelt es sich um eine selektive Auswahl von Aufgaben, die einer internen Beartungseinheit bei BASF zugeschrieben wurden. Vgl. Heuck (2000), S. 90.

881 Vgl. Trilse (2001), S. 109.

882 Vgl. Roth (2000), S. 130.

${ }^{883}$ Vgl. Kap. 3.4.3.4. 
Einen abschließend zu erwähnenden, noch verhältnismäßig seltenen Fall im Angebotsverhalten des Controlling stellt das eine Profit Center Ausrichtung begünstigende Konkurrieren mit Beratern auf unternehmensexternen Märkten dar. Eine derartige Vorgehensweise ist zwiespältig zu beurteilen. Einerseits kann zwar gegenüber internen Kunden in der Qualitätsdiskussion die Marktfähigkeit des Controlling-Leistungsangebotes dokumentiert werden, ${ }^{884}$ andererseits besteht durch die zunehmende Vergleichbarkeit mit Beratern die Gefahr eines Profilverlustes, ${ }^{885}$ wodurch die spezifischen, auf der Rolle des Controllers basierenden Wettbewerbsvorteile verwässert würden.

\subsection{Operative Leistungsanalyse und -planung im Controlling}

Auf Grundlage der Ausführungen zur strategischen Controllerbereichsplanung und der strategischen Marketingplanung im Controlling sollen nunmehr solche Instrumente dargestellt werden, die in der Lage sind, eine kundenorientierte Leistungsanalyse und -planung im Controlling zu gewährleisten. Im Einzelnen sollen Benchmarking, die Balanced Scorecard, Target Costing und Service Level Agreements vorgestellt werden. Hierbei handelt es sich um Instrumente, die sowohl im Controlling als auch im Marketing regelmäßig Anwendung finden, jedoch oftmals unterschiedliche Betrachtungsschwerpunkte aufweisen. Liegt der Schwerpunkt der vorzustellenden Instrumente im Controlling oftmals auf dem Prozess- und Kostenmanagement, fokussiert das Marketing stärker die Innovations- und Kundenintegrationsfunktion. ${ }^{886}$ Im Folgenden soll der Betrachtungsschwerpunkt bei diesen Instrumenten - trotz der teilweise strategischen Ausrichtung ${ }^{887}$ primär auf deren Eignung zur Übertragung einer strategischen Position in Maßnahmen zur Strukturgestaltung liegen. ${ }^{888}$ Zielsetzung hierbei ist letztlich die Beseitigung operativer Leistungsdefizite im Controlling durch eine konsequente Ausrichtung an den Nachfragerbedürfnissen und den Unternehmenszielen. ${ }^{89}$

884 Vgl. Steinle/Thiem/Rohden (2000), S. 283.

$885 \mathrm{Vgl}$. Trilse (2Q01), S. 109.

$886 \mathrm{Vgl}$. hierzu auch die Einordnung der Instrumente in die Gliederungen von Marketing- und ControllingLehrbüchern. Beispielhaft Horváth (1998), Küpper (1997), Weber (1999), Steinle/Bruch (1999), Meffert (2000), Meffert/Bruhn (2000), Scheuch (1993) und Pepels (1998).

887 Zur Problematik der Klassifikation dieser Instrumente als operativ vgl. auch Kap. 4.

888 Vgl. Weber (1999), S. 55.

889 Hieraus folgt deren Eingliederung in den Bereich der operativen Planung. 


\subsubsection{Benchmarking des Controllerbereichs}

Ursprünglicher Fokus des Instrumentes Benchmarking waren elementare Wertschöpfungsprozesse. ${ }^{800}$ Über einen nach außen gerichteten Vergleich mit Unternehmen, die den zu vergleichenden Prozess als Kernkompetenz betrachten, sollen Anstöße zur Ausschöpfung von Effizienzpotenzialen gegeben werden. Neben elementaren Wertschöpfungsprozessen wird Benchmarking zunehmend als Instrument zur Analyse von Gemeinkostenbereichen eingesetzt. In diesen Bereichen ist der große Vorteil des Benchmarking darin zu sehen, dass aufgrund des oftmals fehlenden Konkurrenzdrucks erstmalig Referenzmaßstäbe zur Beurteilung der eigenen Leistungsfähigkeit bestehen. ${ }^{891} \mathrm{Da}$ das Controlling oftmals für die Durchführung von Benchmarking-Aktivitäten in anderen Unternehmensteilbereichen verantwortlich ist ${ }^{892}$ und bezogen auf die eigene Leistungsfähigkeit regelmäßig Transparenzdefizite aufweist, erscheint es konsequent, dieses Instrument auch auf das Leistungsangebot des Controllerbereichs anzuwenden. ${ }^{893}$ Im Sinne einer kundenorientierten Betrachtung des Controlling ist ein wesentlicher Vorteil des Benchmarking gegenüber wertanalytischen Verfahren ${ }^{84}$ darin zu sehen, dass auch nichtmonetäre Sachverhalte, wie bspw. die Kundenzufriedenheit, über ordinal skalierte Größen in den Vergleich einbezogen werden können. ${ }^{895}$

Grundsätzlich kann beim Benchmarking zwischen internen, generischen und wettbewerbsorientierten Benchmarking unterschieden werden. ${ }^{896}$ Hierbei erfolgt die Differenzierung im wesentlichen anhand der Vergleichspartner, die entweder Unternehmensteilbereichen desselben Unternehmens, vergleichbaren Unternehmensteilbereichen branchenfremder Unternehmen oder aber direkten Konkurrenzunternehmen entstammen können. Im Einzelfall ist hier nach der Praktikabilität der Durchführung, der Vergleichbarkeit der Prozesse, der Sensibilität der preisgegeben Informationen und dem erwarteten Innovationspotenzial zu entscheiden, welches der geeignetste Partner ist. Über eine einmalige

$890 \mathrm{Zu}$ den Ursprïngen des Benchmarking bei der Firma XeroX vgl. Camp (1989), S. 6 ff.

891 Ohne Bezug zu Gemeinkostenbereichen vgl. Meffert (2000), S. 391.

${ }^{892}$ Beispielhaft zum Verbreitungsgrad des Benchmarking und zur organisatorischen Verantwortung fur Kostenmanagementinstrumente vgl. Franz (1997), S. $481 \mathrm{ff}$.

893

Ein Anwendungsbeispiel zum Benchmarking in Marketing und Vertrieb findet sich bei Feind-Just (1999), S. $188 \mathrm{ff}$.

894 Hier sind beispielhaft das Zero Base Budgeting oder die Gemeinkostenwertanalyse zu nennen. Allg. zu den Begriffen vgl. Steinle (1999b), S. $326 \mathrm{ff}$.

$895 \mathrm{Vgl}$. Weber/Weißenberger/Aust (1997), S. 4.

896 Vgl. Camp (1989), S. 60 ff. Bei HORVÁTH wird eine Differenzierung von Benchmarkingformen hinsichtlich

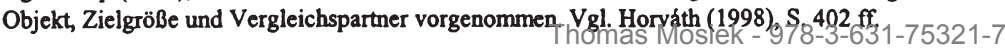


Anwendung hinaus ist Benchmarking heute als kontinuierlicher Prozess zu verstehen, da die unternehmensinterne und -externe Dynamik eine dauerhafte Aktualisierung der gewonnenen Daten erfordert. ${ }^{897}$ Im Ergebnis wird eine Best Practice angestrebt, d.h. ein idealtypisch gestalteter Prozess, der für die am Benchmarkingprojekt Beteiligten als Referenzobjekt für ihre unternehmensindividuellen Prozesse angesehen werden kann.

Im Folgenden soll der Ablauf des Benchmarkingprozesses im Controlling dargestellt werden. Ferner sollen anhand zweier, beispielhafter generischer Benchmarkingprojekte wesentliche Erkenntnisse für eine kundenorientierte Ausgestaltung des Controlling diskutiert werden. ${ }^{898}$

\subsubsection{Benchmarkingobjekte im Controlling}

$\mathrm{Zu}$ Beginn eines jeden Benchmarkingprojekts gilt es, das zu analysierende Benchmarkingobjekt zu identifizieren. ${ }^{89}$ Im Einzelnen können dies Produkte/Leistungen, Methoden oder Prozesse sein. Im Rahmen der Diskussion zum Leistungsspektrum des Controllers und der diesbezüglichen Evidenz wurde bereits deutlich, dass in vielen Unternehmen das Leistungsangebot des Controlling wenig transparent ist und daher in den Augen der Kunden Evidenzmängel aufweist. ${ }^{900}$ Neben einer kriteriengeleiteten Strukturierung des ControllingLeistungsangebotes gilt es im Rahmen der regelmäßig einen heterogenen Teilnehmerkreis umfassenden Benchmarkingprojekte insbesondere auch einen $\mathrm{Ver}$ ständnisabgleich aller Teilnehmer herbeizuführen. Unterschiedliche organisations- oder leistungsgeprägte Begriffe können dazu fuihren, „dass faktische Unterschiedlichkeiten durch semantische Identitäten überdeckt oder faktische Identitäten durch semantische Unterschiede verborgen bleiben ${ }^{\text {"901 }}$. Auf Grundlage harmonisierter Begriffsverständnisse müssen im Controlling Leistungen bzw. dahinterstehenden Prozesse identifiziert werden, die einer BenchmarkingBetrachtung zugänglich sind und darüber hinaus diese unter wirtschaftlichen Gesichtspunkten lohnenswert erscheinen lassen. Im Sinne einer Vergleichbarkeit der betrachteten Objekte werden regelmäßig standardisierbare Leistungen

\footnotetext{
897 Vgl. Horváth (1998), S. 405.

${ }^{898}$ Bei den ausgewăhlten Projekten handelt es sich um das Projekt des Arbeitskreises Benchmarking im Controlling an der Wissenschaftichen Hochschule für Unternehmensfuihrung (WHU) und das Projekt des Arbeitskreises vom Institut für Unternehmensführung der Wirtschaftsuniversităt Wien (WU). Vgl. Weber/ Weißenberger/Aust (1997) bzw. Weber/Hamprech//Goeldel (1995) sowie Stadler/Weißenberger (1999).

900 Gl. Horváth (1998), S. 402.

901 Weber/Weißenberger/Aust (1997), S. 5.
} 
bzw. Prozesse zum Benchmarking herangezogen. Die Ausführungen zur Leistungsindividualisierung im Controlling haben gezeigt, dass es einerseits zweckmäßig ist, Leistungen individuell am Entscheidungsunterstützungsbedarf des Empfängers auszurichten. ${ }^{902}$ Andererseits wurde deutlich, dass eine effiziente Informationsaufnahme durch standardisierte Berichtselemente begünstigt wird. ${ }^{903}$ Zudem können auch mittels standardisierter Teilelemente kundenindividuelle Lösungen wirtschaftlich erzeugt werden. ${ }^{904}$ Bezüglich des BenchmarkingPotenzials gilt es, alternative Leistungen des Controlling in Hinblick auf Standardisierbarkeit zu klassifizieren. Im Sinne der Wirtschaftlichkeit eines Benchmarkingprojektes ist es darüber hinaus zweckmäßig, sich zunächst auf solche Prozesse zu fokussieren, die einen hinreichend großen Teil der Controllingkapazität beanspruchen. Ein auf diesen Aspekten aufbauendes Analyseschema zur Bewertung des Benchmarking-Potenzials von beispielhaften Controlling-Leistungen zeigt Abbildung 48.

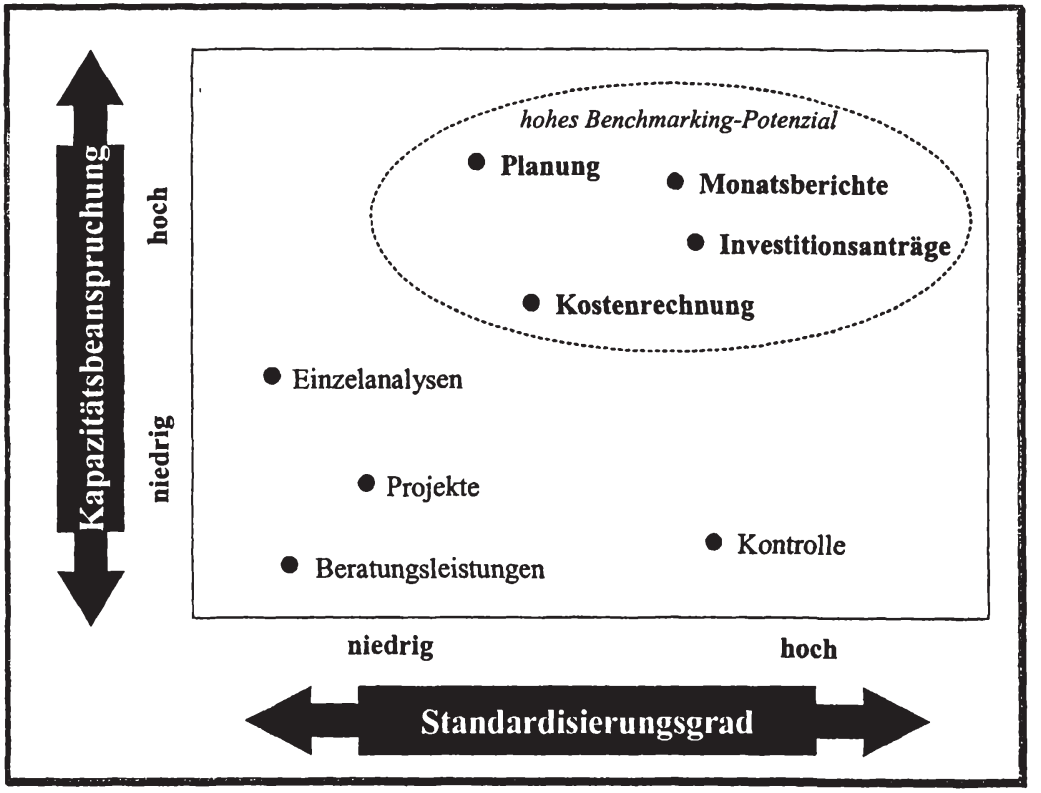

Abbildung 48: Analyseschema zur Bewertung des Benchmarking-Potenzials ${ }^{\text {905 }}$

$902 \mathrm{Vgl}$. 3.4.3.1.

903 Vgl. Neuhäuser-Metternich/Witt (2000), S. 313.

904 Vgl. Reiß/Beck (1995), S. 26.

905 Vgl. die erweiterte Darstellung von Weber/Weißenberger/Aust (1997), S. 6, erstellt auf Grundlage der Abbildung von Weber/Hamprecht/Goeldel (1995), S. 35. 
Der bei Beratungsleistungen, Projekten und Einzelanalysen vorherrschende geringe Standardisierungsgrad führt dazu, dass eine Best Practice nur eingeschränkt - im Sinne einer abstrakten Methodik - abgeleitet werden kann. Kontrollaktivitäten beanspruchen demgegenüber nur einen geringen und - wie in Abbildung 14 dargestellt - sinkenden ${ }^{906}$ Anteil der Controlling-Kapazität, so dass sich das Benchmarking zunächst auf andere Leistungen fokussieren sollte. Die Kostenrechnung, die Investitionsantragsbearbeitung, Planungsaktivitäten sowie Monatsberichte zeichnen sich durch eine hinreichend große Kapazitätsbeanspruchung und eine hohe Vergleichbarkeit aufgrund standardisierbarer Prozesse aus. ${ }^{907}$

Im Folgenden soll der Fokus der Darstellung auf die dem Berichtswesen zuzurechnenden Monatsberichte gelegt werden, ${ }^{908}$ da diese zentral an der Schnittstelle zwischen Manager und Controller stehen und somit dominant den internen Kundennutzen des Controlling in der Wahrnehmung seiner Kunden prägen. ${ }^{909}$

\subsubsection{Benchmarkingprozess}

Im Allgemeinen, so auch im Controlling, bedarf jeder Benchmarkingprozess einer Vorbereitungs-, Analyse- und Umsetzungsphase. ${ }^{910}$ In der Vorbereitungsphase gilt es nach der Bestimmung des Benchmarking-Objektes und der Vergleichsunternehmen aussagekräftige Größen für die Leistungsbeurteilung und die zu ihrer Erhebung notwendigen Informationsquellen zu benennen. Auch wenn es sich bei den hier zu betrachtenden Monatsberichten um standardisierbare Leistungen handelt, ist zu einer Vereinheitlichung der Abgrenzungsmethodik von Berichten ein differenzierter Leitfaden zu erstellen,"11 der die Vergleichbarkeit absichern soll ${ }^{912}$ und damit die Aussagekräftigkeit der Leistungsbeurteilungsgrößen verbessert. Ein idealisierter „Berichts-Steckbrief" kann in diesem Zusammenhang als Muster verwandt werden, um auf dieser Grundlage die

906 Dies zeigt die Entwicklung der Vergangenheit. Vgl. Steinle/Bruch (1999), S. 52, in Anlehnung an die Erhebungen von Peemöller/Schmidt/Meister (1989), S. 295 ff. und Steinle/Bruch/Michels (1998), S. 443 ff.

$907 \mathrm{Vgl}$. Weber/Weißenberger/Aust (1997), S. 6.

908 Hierbei geht es um die Monatsberichterstattung an den Vorstand.

909 Vgl. Stadler/Weißenberger (1999), S. 6 und S. 5.

910 Hier und nachfolgend vgl. Horváth/Herter (1992), S. 4 ff.

911 Ausfuhrlich zur strukturierten Erfassung von Benchmarking-Objekten im Bereich Planung vgl. Hamprecht (1996), S. $296 \mathrm{ff}$.

$912 \mathrm{Zu}$ den einzelnen strukturellen Merkmalen vgl. Kap. 4.2.1.3.1. 
strukturellen Besonderheiten jedes Unternehmens zu erfassen. ${ }^{913}$ Hierzu zählen im Einzelnen: ${ }^{914}$

- der Berichtsinhalt, wie Berichtskreis und Berichtsgegenstand, Datenart und Aggregationsniveau der Daten,

- das Berichtslayout, wie Berichtsmedium, Grad der Standardisierung, Berichtsumfang und Datendichte,

- die Berichtsempfänger, unterschieden in Primär- und Sekundärempfänger sowie

- die legalen Erfordernisse, wie die Anpassung an gesetzliche Vorschriften $^{915}$ und die Unterschriftspflicht.

Im Rahmen der Analysephase des Benchmarkingprozesses können dann die Leistungslücken sowie dafür verantwortliche Ursachen mittels Struktur- und Prozessvergleichen aufgedeckt werden. ${ }^{916} \mathrm{Zu}$ einer vergleichenden Abbildung der Berichtsprozesse mit dem Ziel, Schleifen, Doppelgleisigkeiten und unnötige Liegezeiten aufzudecken, eignen sich Flussdiagramme. ${ }^{917}$ Die letzte Phase des Benchmarkingprozesses bildet die Umsetzungsphase, in der zunächst Ziele und Strategien für die Umsetzung festgelegt werden müssen, um darauf aufbauend konkrete Aktionspläne zu entwickeln und zu implementieren. Da Benchmarking, wie bereits angesprochen, als kontinuierlicher Prozess aufzufassen ist, gilt es bezogen auf die Umsetzungsmaßnahmen Fortschrittskontrollen durchzuführen und auf Basis der damit gesammelten Erkenntnisse das Benchmarking zu wiederholen.

Im Sinne der Kundenorientierung im Controlling sollen nachfolgend zwei Aspekte des Benchmarking differenzierter betrachtet werden. Einerseits ist zu analysieren, wie im Bereich der Leistungsbeurteilungsgrößen Kundenanforderungen angemessen berücksichtigt werden können. Andererseits gilt es anhand der angesprochenen Studien die der Umsetzung zugrundeliegenden Erkenntnisse - im Sinne der Best Practice - vorzustellen.

\footnotetext{
$913 \mathrm{Vgl}$. Weber/Weißenberger/Aust (1997), S. 9.

914 Vgl. Stadler/Weißenberger (1999), S. 6.

$915 \mathrm{Zu}$ jahresabschlussbezogenen Berichtspflichten vgl. Neuhăuser-Metternich/Witt (2000), S. 323-333.

$916 \mathrm{Vgl}$. Horváth/Herter (1992), S. 7 ff.

917 Vgl. Stadler/Weißenberger (1999), S. 6 f.
} 


\subsubsection{Benchmarking Dimensionen im Controlling}

Soweit das Benchmarking-Objekt intersubjektiv definiert ist, stellt sich die Frage nach den relevanten Maßstäben für die Beurteilung der Leistungsfähigkeit alternativer Anbieter. Bezogen auf ein Benchmarking des Berichtswesens ist demnach zu klären, welche einzelnen Charakteristika einer Leistung zu erfassen und zu vergleichen sind. Anknüpfend an die Diskussion zu Beginn des Kap. 3 können im Controlling allgemein zwei Betrachtungsdimensionen der Leistungsbeurteilung unterschieden werden. Zum einen gilt es die Dimension Effizienz, verstanden als ein internes Leistungsmaß für den Controllerbereich, welches das Verhältnis von Output zu Input wiedergibt, ${ }^{918} \mathrm{zu}$ betrachten. ${ }^{19}$ Zum anderen impliziert eine kundenfokussierte Betrachtung des Controlling, die Effektivität als externes Leistungsmaß einzubeziehen, worüber die Befriedigung der Erwartungen und Ansprüche der Controlling-Kunden gemessen werden soll.

Insgesamt bedingen beide Dimensionen die Wirksamkeit des Berichtswesens in den Augen seiner Kunden, da Wettbewerbsvorteile sowohl in effizienten internen Prozessen als auch in der subjektiven Leistungswahrnehmung der Kunden begründet sein können. ${ }^{920}$ Als problematisch erwies sich in der Vergangenheit die oftmals einseitige Fokussierung auf Effizienzgesichtspunkte, da diese sich regelmäßig besser operationalisieren ließen.

\subsection{Effizienz als internes Leistungsmaß}

Die im Rahmen der Effizienzmessung zu betrachtenden Merkmale zeichnen sich dadurch aus, dass sie einer Messung ohne unmittelbare persönliche Einschätzung der Kunden zugänglich sind. Hierzu zählen allgemein strukturelle Merkmale der Berichte sowie prozessuale Merkmale ihrer Erstellung. ${ }^{921}$ Bezüglich der Berichtsstruktur lassen sich im wesentlichen der Berichtsumfang sowie die Darstellungsmethodik (Visualisierung, etc.) vergleichen. ${ }^{922}$ Im Rahmen des Berichtserstellungsprozesses sind sowohl der Bereitstellungstermin als auch die über Flussdiagramme zu analysierende Prozessabfolge relevant. Einzelfallbezogen lassen sich hier

\footnotetext{
918 Ohne den speziellen Bezug zum Controlling vgl. Plinke (1995), S. 85.

919 Speziell zur Effizienzforschung im Controlling vgl. Benz (1998), S. 48 ff. und Sander (1990), S. $115 \mathrm{ff}$.

920 Zum Instrument des Controlling-Audit vgl. umfassend Kreuzer (1995).

921 Vgl. im Folgenden sowohl die Aufzählung von Stadler/Weißenberger (1999), S. 6, als auch die ergänzenden Erläuterungen in der selben Quelle auf S. 7.
} 
- Aktivitäten sowie dafür beanspruchte Zeiten,

- die Anzahl der Prozessträger und Schnittstellen,

- die Anzahl von Rücksprachen,

- Schleifen,

- Liegezeiten und

- Doppelgleisigkeiten

betrachten. ${ }^{923}$ Im Rahmen der Durchführung der Benchmarkingprojekte der WU und WHU konnte bezüglich des Monatsberichtsumfangs eine Spannweite von 7 bis 160 Seiten festgestellt werden. ${ }^{924}$ Als problematisch erweisen sich die teilweise unterschiedlichen Intentionen der Berichte, die teilweise in einer Kurzinformation, teilweise in einem Nachschlagewerk zu sehen sind. Differenziert man zwischen Kurzinformationen/Übersichten und Nachschlagewerken, so weisen die Übersichten in der WHU-Studie einen Umfang zwischen 450 und 2.420 Zeichen und die Nachschlagewerke einen Umfang zwischen 4.250 und 40.500 Zeichen auf. Bezüglich der graphischen Visualisierung in Monatsberichten zeigt die WU-Studie, dass drei der sieben betrachteten Unternehmen keinerlei Graphiken verwenden, demgegenüber weisen die vier anderen Berichte einen graphische aufbereiteten Anteil von mehr als $20 \%$ auf. ${ }^{925}$ Bei den Bereitstellungsterminen konnte eine ebenso große Spannweite festgestellt werden. Die Bereitstellung der Berichte erfolgte in Deutschland und Österreich zwischen 4 und 28 Kalendertage ab Monatsultimo. Erkannt wurde das Problem eines potenziellen Trade-Off zwischen Bereitstellungstermin und Berichtsgenauigkeit, da durch die Verwendung von Schätzwerten Zeit gespart werden kann.

Zur Ergründung der Ursachen alternativer Bereitstellungstermine muss man sich in den Bereich der Prozessanalyse begeben. Hier könnte festgestellt werden, dass Zeitverluste im wesentlichen auf die Datenübermittlung dezentraler Einheiten und manuelle Schnittstellen in der DV-Unterstützung zurückzuführen sind.

Durch die Betrachtung einzelner mittels Benchmarking identifizierbarer Komponenten der Effizienz im Berichtswesen wird deutlich, dass eine interne Analyse des Controlling bereits erste Anhaltspunkte für eine kundenorientierte Aus-

923 Vgl. Stadler/Weißenberger (1999), S. 7.

924 Die im Folgenden dargestellten Ergebnisse beziehen sich für die WHU-Studie auf Weber/Weißenberger/Aust (1997). Zur zusammengefassten Darstellung beider Studien vgl. Stadler/Weißenberger (1999). 
gestaltung von Berichten bereithält. ${ }^{926}$ Insbesondere Berichtsumfang, Berichtsgestaltung und Berichtstermin aber benötigen neben der objektiven Erfassung eine subjektive Bewertung ihrer Bedarfsadäquanz durch die Verwender. Erst die Verbindung von Effizienz- und Effektivitätsmerkmalen erlaubt daher eine umfassend kundenorientierte Gestaltung des Controlling.

\subsection{Effektivität als kundenbezogenes Leistungsmaß}

Vorstehende Ausführungen zur Leistungseffizienz haben gezeigt, dass bspw. über die Verwendung von Schätzwerten Einfluss auf den Bereitstellungszeitpunkt eines Berichtes genommen werden kann. Letztlich kann jedoch erst auf Grundlage der Präferenzen und des Nutzungsverhaltens eines Kunden entschieden werden, inwieweit er bereit ist, Ungenauigkeiten zugunsten eines früheren Berichtstermins zu akzeptieren. ${ }^{927}$ Aufgrund der Notwendigkeit einer Berücksichtigung derart subjektiver Urteile, gilt es im Rahmen der Effektivitätsbetrachtung, Bewertungsmaßstäbe für die Zufriedenheit der Kunden mit dem Berichtswesen $\mathrm{zu}$ bestimmen. ${ }^{928}$ Wie bereits mehrfach angesprochen ist das Ziel einer derartigen Betrachtung nicht zwangsläufig in einer bedingungslosen Ausrichtung am Kunden zu sehen. Vielmehr können über die Ermittlung der internen Kundenzufriedenheit auch wichtige kontroll- und koordinationsrelevante Informationen abgeleitet werden, über die die Wirksamkeit des Controlling gesteigert werden kann..$^{229}$

Der integrative Charakter der Controlling-Leistungen erfordert ein am Prozess der Leistungserstellung ausgerichtetes Verfahren der Zufriedenheitsermittlung. Eine Übersicht der geläufigsten Ansätze, die sich zur prozessualen Messung der Zufriedenheit interner Kunden eignen, zeigt Abbildung 49. Grundsätzlich ist zwischen merkmalsorientierten und ereignisorientierten Verfahren der Zufriedenheitsermittlung zu unterscheiden.

$926 \mathrm{Zu}$ einer umfassenden analytischen und empirischen Ableitung von Merkmalen der Controlling Effizienz vgl. Benz (1998), S. 112 ff. Eine Unterscheidung zwischen Effizienz und Effektivität wird hier nicht vorgenommen.

927 Die Vertreter des WHU-Benchmarkingprojekts haben sich bspw. für einen maximalen Schätzfehler von 5\% für einen früheren Bereitstellungstermin ausgesprochen. Vgl. Stadler/Weißenberger (1999), S. 8. Hierbei handelt es sich aber wahrscheinlich um eine Einschatzung der Controller in ihrer Funktion als Anbieter.

928 Zur Messung der Qualităt interner Dienstleister vgl. Bruhn (1999), S. 539 ff. sowie Reynoso (1999), S. 527 ff. Zum Controlling von Kundenzufriedenheit vgl. Weber (1995), S. $263 \mathrm{ff}$.

929 Zum Einfluss der Wirksamkeit des Controlling auf die Erfullung der Gesamtunternehmensziele vgl. Anderson/O'Reilly (1981), S. 491-501. 
Ereignisorientierte Verfahren fokussieren auf solche Standard- oder Schlüsselerlebnisse eines Dienstleistungsprozesses, die aus Kundensicht als besonders qualitätswahrnehmungsrelevant erachtet werden ${ }^{930}$ und aus Perspektive des Anbieters seine Zielerreichung gefährden können. ${ }^{931} \mathrm{Da}$ derartige Ereignisse im Regelfall in der Interaktion zwischen internem Kunden und Anbieter stattfinden, spricht man bei diesen Messverfahren auch von Kontaktpunktanalysen. ${ }^{932}$ Dies verdeutlicht, dass bezogen auf das Controlling - auch ohne konkrete Messmethodik - die Vergegenwärtigung der Informations- und Interaktionskomponenten einer Leistung erfolgskritisch ist..$^{933}$

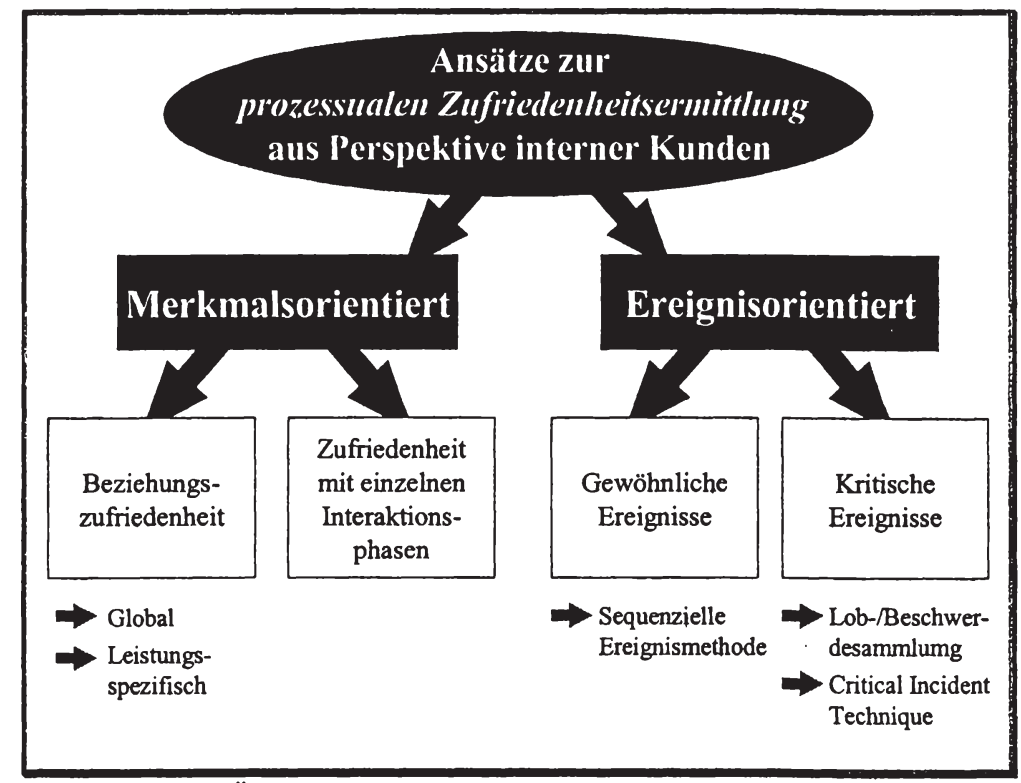

Abbildung 49: Überblick über Ansätze zur prozessualen Zufriedenheitsermittlung ${ }^{934}$

Bei den ereignisorientierten Methoden lassen sich Verfahren unterscheiden, die an kritischen Ereignissen ansetzen und solche, die gewöhnliche Ereignisse fo-

\footnotetext{
$930 \mathrm{Vgl}$. Meffert/Bruhn (2000), S. 229.

931 Zum Controlling vgl. Steinle/Thiem/Rhoden (2000), S. 282.

$932 \mathrm{Vgl}$. hierzu ausführlich Bruhn (1994), S. $11 \mathrm{ff}$.

933 Diese Feststellung hat in Kap. 3.2.1.4 zu der Ableitung einer Informations- und Interaktionsdimension der Controlling-Leistungen gefulhrt.

934 In Anlehnung an Stauss/Seidel (1995), S. 197 ff. sowie Neuhaus (1996), S. 187
} 
kussieren. So werden mit der Sequenziellen Ereignismethode gewöhnliche Ereignisse entlang des Dienstleistungsprozesses identifiziert. Die Kunden müssen bezüglich dieser Ereignisse mittels offener Fragen eine Bewertung ihrer Wahrnehmung vornehmen, worüber eine Analyse ihres Qualitätsempfindens möglich wird. Informationen über die Sequenzintegration von Leistungen des Controlling ${ }^{935}$ und im Rahmen des Benchmarking aufgestellte Flussdiagramme können die Analyse derartiger Prozesse wesentlich erleichtern.

Die Lob- und Beschwerdeanalyse sowie die Critical Incident Technique (CIT) erheben ihre Informationen nicht sequenziell, sondern in Abhängigkeit kritischer Vorkommnisse. ${ }^{936}$ Eine Zuordnung zu einzelnen Prozessphasen ist jedoch anschließend ergänzend möglich. Bei der Critical Incident Technique werden über Interviews Schlüsselereignisse im Erleben des Kunden aufbereitet, d.h. der Kunde wird gezielt zur Artikulation seiner Zufrieden-/ Unzufriedenheit aufgefordert. Demgegenüber werden in der Lob- und Beschwerdeanalyse unaufgefordert artikulierte Empfindungen der Kunden ausgewertet. Wichtig für das Controlling als organisationsinternen Dienstleister ist die Schaffung von Kanälen zur gezielten Artikulation von Unzufriedenheit, ${ }^{937}$ wie dies bspw. durch ,Meckerkästen" geschieht ${ }^{338}$. Vielfach wird der Fehler begangen, nicht artikulierte Unzufriedenheit als Zufriedenheit zu deuten, was überwiegend nicht der Realität entspricht. ${ }^{939} \mathrm{Im}$ Controlling hängt die Artikulation von Unzufriedenheit regelmäßig von der Transparenz des Leistungsangebotes und der Leistungsevidenz $\mathrm{ab}$, denn soweit der Kunde keine konkreten Erwartungen bzgl. der Leistungsqualität und Leistungsnutzung hat, fehlt der Referenzmaßstab für die Wahrnehmung seiner latenten Unzufriedenheit.

Im Gegensatz zu der auf spezielle Kundenkontaktsituationen bezogenen ereignisorientierten Messung der Kundenzufriedenheit fokussiert die merkmalsorientierte Messung auf die Bewertung einzelner Leistungselemente, aus der sich die wahrgenommene Gesamtdienstleistungsqualitä $t^{90}$ ableiten lässt. ${ }^{941}$ Hierbei kann

\footnotetext{
935 Vgl. Kap. 3.3.2.3.

936 Vgl. Stauss/Seidel (1995), S. 201.

937 Allg. zum Management von Kundenzufriedenheit vgl. Günter/Platzek (1992), S. 109 ff.

${ }_{938}$ Speziell zum Controllerbereich vgl. Steinle/Thiem/Rhoden (2000), S. 285.

939 GzUK unterstellt demgegenüber Zufriedenheit, wenn Kunden die Aufgabenerfullung nicht beanstanden. Vgl. Gzuk (1977), S. 235. BERGER nennt explizit furr das Controlling die Anzahl der mulndlichen und schriflichen Rückfragen als einen Erfolgsmaßstab für die Arbeit des Controllers. Vgl. Berger (1989), S. 187.

940 Derartige Verfahren werden gleichermaßen zur Qualitätsmessung wie auch zur Messung von Kundenzufriedenheit eingesetzt.
}

941 Vgl. Meffert/Bruhn (2000), S. 220. 
die Zufriedenheit mittels einzelner Merkmale global (bereichsbezogen) oder leistungsspezifisch für eine Austauschbeziehung ermittelt werden. Ferner können einzelne Interaktionsphasen anhand mehrerer Merkmale bewertet werden, unabhängig von dem Vorfall spezifischer Ereignisse. ${ }^{942}$ Wesentlich bei den merkmalsorientierten Verfahren ist die Vorgehensweise im Sinne des Multiattributansatzes, ${ }^{943}$ da hierdurch konkrete Anhaltspunkte für die Beseitigung von subjektiven Qualitätsmängeln gewonnen werden können. Soweit in Unternehmen derzeit überhaupt Erhebungen zur internen Kundenzufriedenheit im Controlling durchgeführt werden, beschränken sich diese überwiegend auf die Ermittlung einer globalen, bereichsbezogenen Zufriedenheit. Ergänzend lässt bspw. die ERICSSON GMBH halbjährlich für einzelne Controlling-Leistungen Globalzufriedenheiten ermitteln, ohne jedoch leistungsbezogene Attribute zu definieren und zu erheben. ${ }^{944} \mathrm{Im}$ Folgenden soll als Beispiel ein multiattributives Verfahren zur Ermittlung der Kundenzufriedenheit mit dem Berichtswesen des Controlling skizziert werden. Die daraus ableitbaren Erkenntnisse können im Sinne der Effektivitätsdimension in die Best Practice eines Benchmarking einfließen.

In Analogie zu den Ausführungen des Effizienzbenchmarking ist im Rahmen des WU-Benchmarkingprojektes die subjektive Zufriedenheit von ControllingKunden bestimmt worden. ${ }^{945}$ Wurden im Rahmen der Effizienzbetrachtung objektive Merkmale der Berichtspraxis erhoben und verglichen, wird bei der Effektivitätsbetrachtung die subjektive Einschätzung der Kunden gefordert. Hierbei wurde das Augenmerk neben einer statischen Betrachtung der aktuellen $\mathrm{Zu}$ friedenheit auch auf den dynamisch aufzufassenden Änderungsbedarf der Kunden gelegt. Grundlage hierfür war ein Fragebogen, in dem die Kunden des Controlling ihre Zufriedenheit und ihren Änderungsbedarf mittels RatingSkalen artikulieren mussten. Der Fragebogen enthielt einerseits Fragen bzgl. der Berichtsinhalte, wie: ${ }^{946}$

- Enthält der Monatsbericht alle erfolgs- und steuerungsrelevanten Informationen?

- Sind die Berichtsinhalte auf ihre individuellen Anforderungen abgestimmt?

942 Hierin ist auch eine Abgrenzung zur CIT zu sehen.

$943 \mathrm{Vgl}$. Hentschel (1992), S. $111 \mathrm{f}$.

944 Im Einzelnen werden so z.B. der Monatsabschluss inclusive Reporting, Planungsprozesse oder der SAPService bewertet.

945 Im Folgenden zur Vorgehensweise des WU-Benchmarking vgl. Stadler/Weißenberger (1999), S. 8 f.

$946 \mathrm{Zu}$ den nachfolgenden Fragen vgl. Österreichischer Arbeitskreis (BMI), zitiert nach Stadler/Weißenberger (1999), S. 9. 
- Wie oft müssen Sie rückfragen, um Berichtsinhalte zu klären?

- Entsprechen alle Daten Ihren Anforderungen in bezug auf Aktualität?

- Entspricht der Verdichtungsgrad der Daten Ihren Anforderungen?

Andererseits wurde die Zufriedenheit der Kunden mit Aufbau, Gestaltung, Umfang und Bereitstellungsgeschwindigkeit der Berichte über nachfolgende Fragen erhoben:

- Wird der Bericht in bezug auf die Gestaltung nach Ihren Anforderungen erstellt?

- Entspricht die Form (EDV, Papier etc.) in der Sie den Bericht erhalten Thren Anforderungen?

- Sind Sie mit dem Aufbau und der Struktur des Berichts zufrieden?

- Sind Sie mit dem Berichtsumfang zufrieden?

- Liegt der Bericht rechtzeitig, im Sinne von bedarfsgerecht vor?

Bei den hier abgefragten Berichtsmerkmalen wird deutlich, dass eine derartige Befragung gleichsam unabhängig von Benchmarking-Aktivitäten erfolgen kann, da auch ohne Vergleichspartner Informationen über Verbesserungspotenziale gewonnen werden können. Verbesserungspotenzial besteht immer dort, wo die Zufriedenheit der Kunden gering ist oder unabhängig von der Zufriedenheit ein Änderungsbedarf artikuliert wird. ${ }^{947}$ Werden neben einer Bewertung des Änderungsbedarfs in Form von Rating-Skalen offene Fragen zur Sammlung von Anregungen gestellt, ${ }^{948}$ können konkrete Vorschläge der Kunden in die Best Practice einbezogen werden.

Insgesamt konnte im Rahmen des WU-Benchmarking eine verhältnismäßig hohe Zufriedenheit festgestellt werden, die bei den betrachteten Unternehmen zwischen $67 \%$ und $75 \%$ lag. ${ }^{949}$ Gründe für die aktuelle Unzufriedenheit waren im wesentlichen zu oft notwendige Rückfragen aufgrund unklarer Berichtsinhalte, sowie die mangelnde inhaltliche Individualisierung der Berichte. ${ }^{950}$ Die Notwendigkeit einer dynamisch orientierten Analyse im Sinne einer Betrachtung

947 Wie das Verhăltnis von Zufriedenheit und Änderungsbedarf im Einzelnen einzuschătzen ist vgl. Kap. 4.2.1.4 sowie Stauss/Neuhaus (1995b).

948 Konkrete Beispiele zur Anwendung von Rating-Skalen im Rahmen der Bewertung unternehmensinterner Dienstleistungen finden sich bei Neuhaus (1996), S. $186 \mathrm{f}$.

949 Erreichbar waren maximal $100 \%$.

$950 \mathrm{Vgl}$. Stadler/Weißenberger (1999), S. 8. Die inhaltliche Individualisierung ist in diesem Zusammenhang nicht mit der formalen Individualisierung von Berichten zu verwechseln. Vgl. Neuhăuser-Metternich/Witt (2000), S. $311 \mathrm{ff}$. 
des Änderungsbedarfs zeigt sich in den zahlreichen Anregungen der Kunden des Berichtswesens. Im Einzelnen wurde gefordert: ${ }^{951}$

- die Aufnahme mehr nicht-monetärer und externer Größen, sowie strategischer Zielgrößen,

- eine zusätzliche graphische Aufbereitung der Daten,

- eine stärkere Institutionalisierung der Durchsprache von Berichten,

- die Abstimmung von Schlüsselkennzahlen zwischen dezentralem und zentralem Controlling,

- zusätzliche Wettbewerbsinformationen,

- die Aufnahme von Diagnosekennzahlen und

- mehr Vergleichszahlen von anderen Abteilungen bzw. operativen Einheiten.

Auf Grundlage der hier gezielt aus Kundenperspektive artikulierten Änderungsvorschläge und der objektiv erhobenen struktur- und prozessbezogenen Vergleichswerte gilt es im Rahmen der Umsetzungsphase des Benchmarkingprozesses auf Basis einer zu konsolidierenden Best Practice Ziele und Strategien zu entwickeln.

\subsubsection{Best Practice}

Zielsetzung eines Benchmarkingprojektes ist regelmäßig die Identifikation einer Best Practice, die als Referenzmaßstab für die Gestaltung der unternehmensindividuellen Prozesse herangezogen werden kann. ${ }^{952}$ Im Folgenden soll daher übersichtsartig eine Best Practice des Berichtswesens dargestellt werden, bevor auf daraus resultierende Implikationen für die Kundenansprache des Controlling eingegangen wird.

Aufbauend auf die im Rahmen der Effizienzbetrachtung durchgeführten Prozessanalysen und die dort identifizierten Kosten- und Zeittreiber lässt sich ein idealtypischer Berichtsprozess ableiten, der sich durch die in Abbildung 50 dargestellten Merkmale auszeichnet. Zur unternehmensindividuellen Prozessgestaltung sollte nicht eine Best Practice unreflektiert übernommen werden, vielmehr sind die prozessualen Merkmale als Analyseraster zur Bewertung und Gestaltung der eigenen Aufgabenerfüllung zu verstehen. Ergänzend zu dem prozess-

951 Vgl. Stadler/Weißenberger (1999), S. 9.

$952 \mathrm{Zu}$ den Umsetzungsphasen des Benchmarking vgl. Horváth (1998), S. $402 \mathrm{ff}$ 
bezogen Innovationspotenzial können auf Basis der abgefragten Effizienz- und Effektivitätsmerkmale allgemeine Handlungsempfehlungen abgeleitet werden. ${ }^{953}$

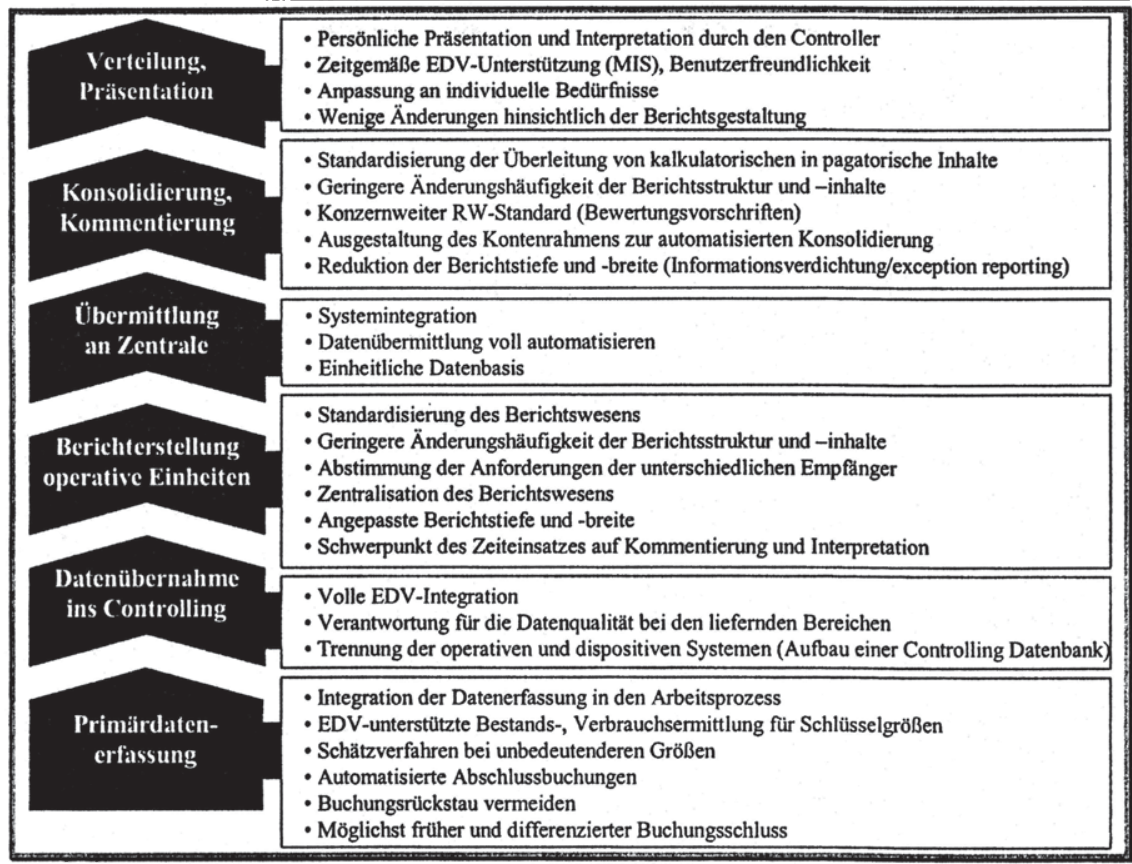

Abbildung 50: Kosten und Zeitmerkmale einer Best Practice im Berichtswe$\operatorname{sen}^{94}$

Bezüglich der Berichtsinhalte ist zunehmend auf die Ausgewogenheit des Berichtswesens, im Sinne einer Balanced Scorecard, ${ }^{955}$ zu achten. Hier gilt es, kundenspezifisch unternehmensinterne und -externe, monetäre und nichtmonetäre Daten zu integrieren und graphisch aufzubereiten. Zur Verkürzung der Berichtserstellungsdauer ${ }^{96}$ müssen Schnittstellen vollständig automatisiert werden, zudem bedarf es einer genauen Fixierung der Mitwirkungspflichten da-

$953 \mathrm{Zu}$ den folgenden Handlungsempfehlungen vgl. Stadler/Weißenberger (1999), S. 9. Vergleichbar auch die Ausfuhrungen von Weber/Weißenberger/Aust (1997), S. $9 \mathrm{ff}$.

954 Osterreichischer Arbeitskreis (BMI), zitiert nach Stadler/Weißenberger (1999), S. 9.

955 Allg. zur Balanced Scorecard vgl. Kaplan/Norten (1996) sowie Kaplan/Norten (1996b), S. 75 ff.

956 Eine Vorlage nach 5 bis 6 Tagen ab Monatsultimo kann als realistisch erachtet werden. Vgl. Weber/Weißenberger/Aust (1997), S. 10. 
tenliefernder Stellen ${ }^{957}$ Die WHU-Studie hat gezeigt, dass über derartige Maßnahmen Bearbeitungs- bzw. Durchlaufzeiten um bis zu 30\% gesenkt werden können. Ferner sind nicht unerhebliche Kosteneinsparungen möglich. ${ }^{958} \mathrm{Im}$ Sinne der Kundenorientierung des Controlling sollte das Augemerk jedoch nicht primär auf dem Kostensenkungspotenzial des Benchmarking liegen. Vielmehr gilt es, die möglichen Einsparungen in die Gestaltung des Leistungsspektrums zu investieren. Bezogen auf das Berichtswesen sollte so die Analyse, Kommentierung und Kommunikation der Berichte forciert werden, um so der Rolle eines internen Beraters künftig gerecht zu werden.

Über die prozessbezogenen und strukturellen Anregungen des Benchmarking können auch strategische Implikationen für die zielgerichtete Ansprache alternativer Kunden abgeleitet werden. Die im Rahmen der Effektivitätsbetrachtung vorgenommene Erhebung von Zufriedenheit und Änderungsbedarf kann im Sinne einer Kundensegmentierung ausgewertet werden. Bezogen auf die in Abbildung 45 dargestellten Kriterien der Marktsegmentierung im Controlling ist dies als eine psychographisch, leistungsspezifische Segmentierung zu bewerten. ${ }^{959}$ Über eine Einordnung alternativer Kunden in ein Portfolio mit den Dimensionen Zufriedenheit und Änderungsbedarf können für die abzugrenzenden Quadranten Strategien ermittelt werden. ${ }^{960}$ Ein derartiges Kundenportfolio zeigt Abbildung 51 .

Ausgehend von einer Betrachtung der eher unzufriedenen Kunden, zeichnen sich fordernd unzufriedene Kunden des Controlling durch eine aktive Artikulation ihres Änderungsbedarfs aus, wodurch dem Controller die Möglichkeit gegeben ist, angemessen zu reagieren. Aus dem Dienstleistungsmarketing ist bekannt, dass es sich bei solchen Kunden um tendenziell abwanderungsbereite Kunden handelt. ${ }^{961}$ Soweit diese Kunden also allein aufgrund des Monopolcharakters einiger Leistungen des Controlling zu den Zwangskunden zählen, ${ }^{962}$ gilt es, ihnen im Rahmen der zunehmenden Deregulierung interner Märkte verstärkt Aufmerksamkeit zu schenken. ${ }^{963}$

\footnotetext{
957 Vgl. hierzu die in Kap. 4.2.4 aufgezeigten Möglichkeit zur Definition von Mitwirkungspflichten im Rahmen von Service Level Agreements.

958 Vgl. Weber/Weißenberger/Aust (1997), S. 11.

959 Vgl. Kap. 4.1.2.1.2 bzw. Freter (1983), S. 46.

960 Für das Berichtswesen vgl. Stadler/Weißenberger (1999), S. 10.

961 Vgl. Meffert/Bruhn (2000), S. 251 bezugnehmend auf Stauss/Neuhaus (1995b).

962 Vgl. hierzu Kap. 3.4.3.4.1.

963 Vgl. hierzu auch die Klassifizierung der Kunden nach Bindungsgraden in Abbildung 46.
} 


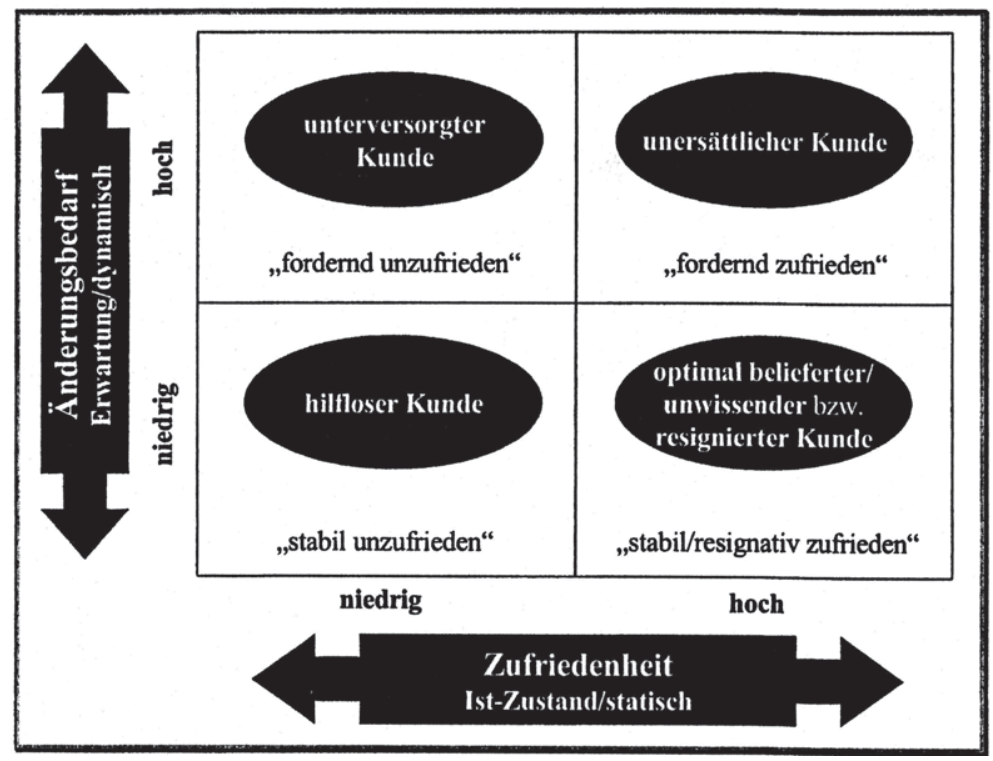

Abbildung 51: Kundenportfolio zur Entwicklung von Informationsversorgungsstrategien im Controlling ${ }^{964}$

Die Tatsache, dass stabil unzufriedene Kunden demgegenüber ein geringes Aktivitätsniveau aufweisen, erschwert deren Zufriedenstellung. Das Controlling muss demnach die Inhalte seines Leistungsangebotes und die mit einzelnen Leistungen verbundenen Nutzungsmöglichkeiten verstärkt kommunizieren, damit den Kunden deutlich wird, wie sie ihren Änderungsbedarf inhaltlich bestimmen können. Ferner gilt es, gezielt Kanäle für die Artikulation des Änderungsbedarfs zu schaffen, da hierüber die Artikulationsschwelle gesenkt werden kann.

Eine hohe Zufriedenheit verbunden, mit einem hohen Änderungsbedarf, charakterisiert den fordernd zufriedenen bzw. unersättlichen Kunden. Fraglich erscheint bei solchen Kunden, ob diese extrem anspruchsvoll sind, ${ }^{965}$ im Sinne dauerhafter Forderungen nach Innovationen. ${ }^{966}$ Demgegenüber ist jedoch denkbar, dass die Controlling-Leistungen, aufgrund ihres Charakters freier Güter, ${ }^{967}$

\footnotetext{
964 Hierbei handelt es sich um eine Kombination des qualitativen Zufriedenheitsmodells von Stauss/Neuhaus (1995b) und des Kundenportfolios von Stadler/Weißenberger (1999), S. 10.

$965 \mathrm{Vgl}$. Stadler/Weißenberger (1999), S. 10.

$966 \mathrm{Zu}$ der Innovationsfunktion des Controlling vgl. Küpper (1997), S. 17 f.

$967 \mathrm{Zu}$ freien Gütern vgl. Hartwig (1992), S. 126.
} 
übermäßig nachgefragt werden. Anreize zu einer ressourcenschonenderen Inanspruchnahme können in solchen Fällen über Plausibilitätskontrollen der Nutzung und insbesondere auch über anreizkonforme Verrechnungspreise implementiert werden. ${ }^{968}$

Vergleichbar differenziert stellt sich die Situation hoher Kundenzufriedenheit verbunden mit einem geringen Änderungsbedarf dar. Hier besteht die Möglichkeit, dass das Management seinen Änderungsbedarf nicht artikuliert, da es sich von den Lösungen des Controlling keine Verbesserung seiner Situation verspricht. Dies wird durch den hohen Anteil an Vertrauenseigenschaften der Controlling-Leistungen verstärkt. Abhilfe kann der Controller in solchen Situationen durch eine gezielte Kommunikation seines Leistungspotenzials bzw. ausgewählter Referenzen schaffen. Ferner kann die unterbleibende Artikulation eines Änderungsbedarfes auch auf den Monopolcharakter des Controlling zurückzuführen sein, da Kunden in Ermangelung eines Alternativanbieters nicht davon ausgehen, dass ihr Änderungsbedarf berücksichtigt wird. Hier gilt es zunächst, die Kooperationsbereitschaft des Controlling deutlich zu machen. Ein weiterer Grund für das Ausbleiben von Änderungswünschen ist die Motivation einzelner Manager, gegenüber dem Controlling nicht aufzufallen ${ }^{969}$ Die scheinbare Zufriedenheit mit dem Controlling soll signalisieren, dass auf Basis der bereitgestellten Informationen sachgerecht gesteuert wird, auch wenn in der Realität Entscheidungen primär intuitiv getroffen werden. Derartige Verhaltensweisen machen eine differenzierte Analyse des Nutzungsverhaltens einzelner Kunden erforderlich, was jedoch durch ihr opportunistisches Verhalten wesentlich erschwert wird. Letztlich kann aber - und dies ist die zunächst naheliegenste Vermutung - der Fall hoher Zufriedenheit und geringen Änderungsbedarfes auch darauf hindeuten, dass Kunden des Controlling schlichtweg zufrieden sind und auch zukünftig problemlösungsadäquate Leistungen des Controlling erwarten.

Insgesamt wurde aus dem Kundenportfolio deutlich, dass eine Analyse der $\mathrm{Zu}$ friedenheit und des Änderungsbedarfes der Kunden ein adäquates Mittel zur Steigerung der Kundenorientierung im Controlling ist. Derartige Analysen können isoliert, oder aber im Rahmen des hier vorgestellten Benchmarking Ansatzes vollzogen werden. Beim Benchmarking kann über eine Bestimmung der Zufriedenheit und des Änderungsbedarfes die Effektivitätsdimension des Con-

968 Zur Verrechnung von Controlling-Leistungen vgl. Kap. 3.3.2.5.

969 Hierzu vgl. Stadler/Weißenberger (1999), S. 10 in Anlehnung an Weißenberger (1997) 
trolling angemessen abgebildet werden. ${ }^{970}$ Demgegenüber vermögen Prozessund Strukturanalysen Aufschlüsse über die Effizienz des Controlling zu geben. Neben dem Berichtswesen eignen sich insbesondere die Kostenrechnung und die Bearbeitung von Investitionsanträgen aufgrund ihres hohen Standardisierungsgrades für eine vergleichende Betrachtung. Demgegenüber bereitet die Analyse von Planungsprozessen aufgrund der schlechten Skalierbarkeit einzelner Merkmale regelmäßig mehr Schwierigkeiten. ${ }^{971}$

Grundlegend bleibt jedoch bei allen Benchmarkingaktivitäten im Controlling über eine konstruktive Selbstkritik die Glaubwürdigkeit gegenüber anderen Unternehmensteilbereichen zu wahren und auch jenseits der Quantifizierbarkeit einzelner Merkmale den Erfahrungsaustausch mit Externen zu suchen. ${ }^{972}$

\subsubsection{Balanced Scorecard für den Controllerbereich}

Die Ausführungen zum Benchmarking des Controllerbereichs haben deutlich gemacht, dass über den Vergleich für die Benchmarking Systematik geeigneter Objekte Aussagen über Effizienz und Effektivität des Controllerbereichs abgeleitet werden können. Deutlich wurde gleichermaßen, dass Teile der Benchmarking Systematik, wie die Kundenzufriedenheitserhebung, ${ }^{973}$ auch ohne direkten Vergleichspartner zu einer regelmäßigen Bewertung der Kundenbeziehungen im Controlling eingesetzt werden können. Insgesamt geht es aber auch im Rahmen eines kundenorientierten Controlling darum, langfristig eine ausgewogene Betrachtung anzustreben, in der Sach-und Formalziele in einem angemessenen Verhältnis zueinander stehen. ${ }^{974}$ Hierzu ist es notwendig, die Controllerbereichsstrategie in operationale und quantifizierbare Zielgrößen zu überführen, über die der Bereich ausgewogen gesteuert werden kann. Die Balanced Scorecard (BSC) erlaubt in diesem Zusammenhang über klassische Kennzahlensysteme hinaus ${ }^{975}$ durch ihre Vier-Perspektiven-Sicht eine anspruchsgruppengerechte Übersetzung von Strategien in Maßnahmen. ${ }^{976}$ Gerade furr organisati-

970 Im Extrem kann sogar über an die Kundenzufriedenheit gekoppelte variable Gehaltbestandteile des Controllers eine anreizkonforme Führung des Controllerbereichs gefördert werden.

971 Vgl. Weber/Weißenberger/Aust (1997), S. 24.

972 Zu den Nutzenmerkmalen des Benchmarking im Controlling vgl. Weber/Hamprecht/Goeldel (1995), S. 16.

973 Ausfuhrlich zur Kundenzufriedenheitsmessung einer Abteilung Personalmanagement vgl. Stauss/ Neuhaus (1995), S. $590 \mathrm{ff}$.

974 Allg. hier und nachfolgend vgl. Gleich (1997), S. 114 ff. und Horváth (1998), S. 566 f.

975 Ein Vergleich klassischer Kennzahlensysteme mit der Balanced Scorecard findet sich bei Klingelbiel (1998), S. 10.

976 Allg. zur Balanced Scorecard vgl. Kaplan/Norton (1996) und Kaplan/Nortgn (1997) 
onsinterne Dienstleister wie das Controlling können so Managementprozesse und deren Wirkungen über eine mehrdimensionale Betrachtung der Bereichsaktivitäten transparent dargestellt werden. Bezogen auf die Kundenbeziehungen des Controlling erlaubt die Kundenperspektive der BSC eine integrierte, regelmäßige Abbildung erfolgskritischer Sachverhalte. Über Einzelmaßnahmen zur Steigerung der Kundenzufriedenheit im Controlling hinaus, kann die BSC als organisatorisches Rahmenkonzept zur Integration bereits vorhandener Ansätze, wie Leitbilderstellung, Kundenbefragungen oder Benchmarking verstanden werden.977 Aus diesem Grund gilt es, nachfolgend zunächst Struktur und Ablauf der Performance Messung mit der BSC zu beschreiben, bevor eine für den Controllerbereich spezifizierte BSC dargestellt wird. ${ }^{978}$

\subsubsection{Struktur und Ablauf des Performance Measurement}

Das Konzept der BSC zählt wie der J. I. Company Approach ${ }^{979}$ und das Quantum Performance-Modell ${ }^{p 80} \mathrm{zu}$ den neueren Ansätzen der Leistungsmessung und -bewertung, die allgemein unter dem Begriff Performance MeasurementKonzepte subsumiert werden. ${ }^{981}$ Die BSC enthält im Sinne eines ausgewogenen Berichtsbogens Kennzahlen unterschiedlicher Perspektiven, die Auskunft über Finanzen, Kunden, Geschäftsprozesse und die Lern- und Wachstumsfähigkeit eines Unternehmen geben sollen. Individuell für jedes Unternehmen gilt es so, unter Mitwirkung des Managements sowohl vergangene Leistungen als auch zukünftige Leistungstreiber abzubilden. ${ }^{982}$ Neben der auf Basis festgelegter Maßgrößen vorzunehmenden Abbildung des Unternehmensgeschehens, im Sinne eines mehrdimensionalen Kennzahlensystems, liegt der Nutzen der BSC insbesondere in ihrer Fähigkeit, die Durchführung und Rückkopplung der Unternehmensstrategie systematisch abzusichern. Ausgehend von einer festzulegenden Vision und Strategie eines Unternehmens gilt es, diese intern zu kommunizieren und in Form von Leistungskennzahlen zu operationalisieren. ${ }^{983} \mathrm{Im}$ Rahmen der Planung der Ressourcenverteilung sind strategische Maßnahmen aufeinander abzustimmen und Meilensteine für ihre Umsetzung zu definieren. Über

\footnotetext{
977 Vgl. Gleich/Haindl (1996), S. 263.

978 Zur Aufgabe der Balanced Scorecard im Rahmen der Neuausrichtung des Controlling vgl. Weber/Schäffer (2000), S. $111 \mathrm{ff}$.

979 Vgl. Sellenheim (1991), S. 50 ff.

980 Vgl. Hronec (1993), $219 \mathrm{f}$.

981 Zum Begriff des Performance Measurement vgl. Eccles (1991), S. 14 ff.

982 Hier und nachfolgend vgl. Horváth (1998), S. 567 f. und S. 570.

983 Nachfolgend vgl. Kaplan/Norten (1996), S. 10 ff.
} 
ein institutionalisiertes Feedback soll das strategische Lernen sichergestellt werden.

Zentral im Rahmen des Managementanspruchs der BSC ist die Absicht, Unternehmensziele und -strategien in Zielvorgaben für Abteilungen, Teams und Mitarbeiter zu transformieren, um daran anknüpfend Anreizsysteme zu implementieren. ${ }^{984}$ Betrachtet man den Controllerbereich als Teil der Gesamtorganisation und unterstellt man, dass die Performance des Controlling - mittelbar über seine Kunden - maßgeblichen Einfluss auf die Erreichung der Unternehmensziele hat, ${ }^{985}$ stellt sich sowohl die Frage nach der Übertragbarkeit des BSC-Konzeptes auf den Controllerbereich als auch die nach seinem potenziellen Beitrag zur Verbesserung der Kundenorientierung im Controlling.

\subsubsection{Spezifikation einer Balanced Scorecard für den Controllerbe- reich}

Im Sinne des Leistungsspektrums eines Controllerbereichs zählt die BSC zunächst zu den innovativen Produkten des Controlling, d.h. es liegt regelmäßig im Verantwortungsbereich des Controllers, unternehmensumfassend die Implementierung der BSC voranzutreiben. ${ }^{986}$ In dem Maße aber, wie Kunden des Controlling die BSC als zentralen organisatorischen Rahmen für ihre Managementprozesse übernehmen, ${ }^{987}$ gilt es für die Controller selbst, ,in den Dimensionen „ihrer" BSC zu denken" ${ }^{\text {"988 }}$. Konkret heißt dies, alle Determinanten der Controller-Performance zu benennen und einer regelmäßigen Messung zu unterziehen. ${ }^{989}$ Bevor jedoch Maßgrößen der Controller-Performance zu bestimmen sind, gilt es zunächst das vollständige Leistungsspektrum des Controllerdienstes abzubilden. Im Gegensatz zum Benchmarking-Ansatz, der sich nur für bestimmte Leistungen eignet, ${ }^{900}$ müssen bei der BSC alle ControllingLeistungen einbezogen werden. Die an späterer Stelle noch zu thematisierenden Service Level Definitions können in diesem Zusammenhang als ein geeignetes Instrument angesehen werden, das Leistungsangebot des Controlling zweckmä-

\footnotetext{
984 Zu den diesbezulglichen Defiziten anderer Kennzahlensysteme vgl. Weber (1999), S. 225 f.

985 ANDERSON/O'REILly zeigen, dass eine derartige Annahme begrindet scheint. Vgl. Anderson/O'Reilly (1981), S. 491-501.

986 Zur Konkurrenz bei derartigen Produkten vgl. Weber/Schäfer (2000), S. 79 sowie Kap. 3.4.2.

987 Vgl. Horváth (1998), S. 569.

988 Weber/Schäffer (2000), S. 111.

989 Allg. zu Einflussgrößen auf die Controller Performance vgl. Witt/Witt (1991), S. 44 ff.

990 Zur Abgrenzungssystematik von Benchmarking-Objekten vgl. Kap. 4.2.1.l g78-3-631-75321-7
} 
Big zu strukturieren und gegenüber seinen Kunden zu dokumentieren. ${ }^{991}$ In Abhängigkeit des Umfangs und der Komplexität des Leistungsangebotes kann es sinnvoll sein, Leistungen zu Leistungsgruppen zu verdichten bzw. zu clustern. ${ }^{992}$ Soweit hierdurch keine unangemessene Einschränkung der Auswertungsmöglichkeiten hervorgerufen wird, ist eine derartige Vorgehensweise insbesondere im Sinne der Wirtschaftlichkeit der regelmäßig anfallenden Systempflege zu bevorzugen.

Analog zu den Ausführungen zum Benchmarking müssen im Rahmen der Ausgestaltung der BSC für jede zuvor abgegrenzte Leistung spezifische, die Performance determinierende Maßgrößen abgeleitet werden. Wurde im Benchmarking zwischen Effizienz- und Effektivitätsmerkmalen differenziert, ${ }^{993}$ erfolgt eine Darstellung der BSC-Indikatoren entlang der vier gewählten Dimensionen. Hierbei können die üblichen Dimensionen der $\mathrm{BSC}^{994}$ als auch für das Controlling tragfähig angesehen werden. Abbildung 52 zeigt den BSC-Berichtsbogen der Controlling-Leistungsgruppe Informationsmanagement eines Unternehmens der chemischen Industrie. ${ }^{995}$

Vorausgegangen ist dem dargestellten Berichtsbogen zunächst eine aus den Unternehmens- und Bereichszielen abgeleitete Festlegung der allgemeinen Performanceziele für das Controlling, ${ }^{996}$ die sich letztlich in den Maßgrößen und den diesen zugeordneten Planansätzen manifestieren. Darauf aufbauend gilt es die aktuelle Performance auf Basis zuvor festgelegter Erhebungsmethoden (z.B. Checklisten, Fragebögen, Selbsteinschätzungen, Planungs- und Budgetierungsdaten) zeitgenau zu ermitteln. ${ }^{997} \mathrm{Im}$ Vergleich mit den zuvor fixierten Planwerten für jede einzelne Maßgröße kann der Stand der Planabweichung in Form von Score-Werten - hier die Werte 1, 2 und 3 - dokumentiert werden (Performance-Stufe 1) ${ }^{998}$. Zur Visualisierung der Score-Werte eignen sich Ampelkarten, ${ }^{999}$ wobei Grün keinen Handlungsbedarf (Scorewert 3), Gelb latenten Hand-

\footnotetext{
991 Vgl. Kap. 4.2 .4 sowie allg. Bölscher (2000), S. 355 ff.

$992 \mathrm{Vgl}$. Gleich/Haindl (1996), S. 265.

$993 \mathrm{Vgl}$. Stadler/Weißenberger (1999), S. 8.

994 Vgl. hierzu die Abbildung von Kaplan/Norten (1996), S. 9.

$995 \mathrm{Vgl}$. Gleich/Haindl (1996), S. 263 ff.

996 Zu einzelnen Performanceindikatoren des Controllers vgl. Witt/Witt (1991), S. 46.

997 Hier und nachfolgend Gleich/Haindl (1996), S. 265.

998 Die hier und nachfolgend vorgenommene Abgrenzung der Performance-Stufen orientiert sich an dem in Abbildung 53 dargestellten Aggregationsprozess.
}

999 In Abbildung 52 sind die jeweiligen Farben der Ampelkarten schriflich vermerkt 
lungsbedarf (Scorewert 2) und Rot akuten Handlungsbedarf (Scorewert 1) signalisiert.

\begin{tabular}{|c|c|c|c|c|c|}
\hline \multicolumn{6}{|c|}{ Berichtsbogen zur Performance Messung } \\
\hline \multicolumn{3}{|c|}{ Bereich: Dezentrales Controlling } & \multicolumn{3}{|c|}{ Leistungsgruppe: Informationsmanagement } \\
\hline \multicolumn{3}{|c|}{ Finanzielle Perspektive } & \multicolumn{3}{|c|}{ Kunden Perspektive } \\
\hline Indikatoren & Hilfsmittel & Score & Indikatoren & Hilfsmittel & Score \\
\hline \multirow{2}{*}{$\begin{array}{l}\text { Kosten des Info- } \\
\text { managements }\end{array}$} & \multirow{2}{*}{$\begin{array}{c}\text { Kostenschătzung } \\
\text { in Euro durch } \\
\text { dezentrales Controlling }\end{array}$} & \multirow[t]{2}{*}{ Rot (1) 0,25} & Darstellung & \multirow{3}{*}{$\begin{array}{l}\text { Checkliste von } \\
\text { zentralem Controlling, } \\
\text { Richtlineneinhaltung } \\
\text { (ja/nein/Note) }\end{array}$} & Gelb (2) 0,125 \\
\hline & & & einheitliche Sprache & & Grün (3) 0,125 \\
\hline & & & $\begin{array}{l}\text { keine Methoden- } \\
\text { schwankungen }\end{array}$ & & Gelb (2) 0,25 \\
\hline & & & Akzeptanz & $\begin{array}{l}\text { Management- } \\
\text { fragebogen }\end{array}$ & Rot (1) 0,5 \\
\hline \multicolumn{2}{|c|}{ PERSPEKTIVENPERFORMANCE } & Rot (1) 0.25 & \multicolumn{2}{|c|}{ PERSPEKTIVENPERFORNANCE: } & Gelb 2$) 0,5$ \\
\hline \multicolumn{3}{|c|}{ Know how Perspektive } & \multicolumn{3}{|c|}{ Interne Ablauf Perspektive } \\
\hline Indikatoren & Hilfsmittel & Score & Indikatoren & Hilfsmittel & Score \\
\hline Mitarbeiter-Schulung & \multirow{3}{*}{$\begin{array}{c}\text { Checkliste von } \\
\text { zentralem Controlling, } \\
\text { Richtlineneinhaltung } \\
\text { (ja/nein/Note) }\end{array}$} & Grin (3) 0,5 & \multirow{2}{*}{$\begin{array}{l}\text { Aktualităt der Info- } \\
\text { Bereitstellung } \\
\text { (centerbezogen) }\end{array}$} & \multirow{2}{*}{$\begin{array}{c}\text { Management-Fragebogen } \\
\text { und Beurteilung durch } \\
\text { zentrales Controlling }\end{array}$} & \multirow[t]{2}{*}{ Grün (3) 0,5} \\
\hline \multirow{2}{*}{$\begin{array}{l}\text { Kennzahlenkenntnise } \\
\text { (z. B. ROACE) }\end{array}$} & & \multirow{2}{*}{ Rot (1) 0,5} & & & \\
\hline & & & \multirow{2}{*}{$\begin{array}{l}\text { Existenz von Schnitt- } \\
\text { stellenproblemen } \\
\text { (unternehmensbezogen) }\end{array}$} & \multirow{2}{*}{$\begin{array}{c}\text { Management-Fragebogen } \\
\text { und Selbsteinschätzung } \\
\text { durch dezentrales Contr. }\end{array}$} & \multirow{2}{*}{ Grün (3) 0,5} \\
\hline & & & & & \\
\hline \multicolumn{3}{|c|}{ PERSPEKTIVENPERFORMANCE Gelb (2) 0,125} & \multicolumn{2}{|c|}{ PERSPEKTIVENPERFORNANC'E } & Griin (3) 0.125 \\
\hline
\end{tabular}

Abbildung 52: Berichtsbogen zur Leistungsgruppe Informationsmanagement ${ }^{1000}$

Neben einer Bewertung der Einzelperformance von Maßgrößen kann über eine Aggregation der Maßgrößen einer Perspektive die Perspektivenperformance abgeleitet werden. Hierzu bedarf es einer mit allen Beteiligten abzustimmenden Gewichtung der Score-Werte ${ }^{1001}$ in Abhängigkeit ihres Einflusses auf die Perspektivenperformance (Performance-Stufe 2). Analog dazu können die vier gewichteten Perspektivenperformance-Werte zu einem bereichsbezogene Leistungsgruppenperformance-Wert aggregiert werden (Performance-Stufe 3). Wie Abbildung 53 zeigt, kann so in einem Performance-Ergebnisbogen der Performance-Wert eines Controllerbereichs bezogen auf eine Leistungsgruppe abgebildet werden.

${ }^{1000}$ In Anlehnung an das Beispiel von Gleich/Haindl (1996), S. 268. Im Folgenden soll dieses Beispiel und die dahinterstehende Systematik weiter verfolgt werden. Vgl. Gleich/Haindl (1996), S. 262-271 sowie Gleich (1997b), S. $553 \mathrm{ff}$.

${ }^{1001}$ In Abbildung 52 sind die Gewichte rechts neben dem im Kreis befindlichen Score-Wert eingetragen. Der Gesamtwert der Perspektive ergibt sich aus dem arithmetischen Mittel der gewichteten Einzel-\$ocore-Werte. 


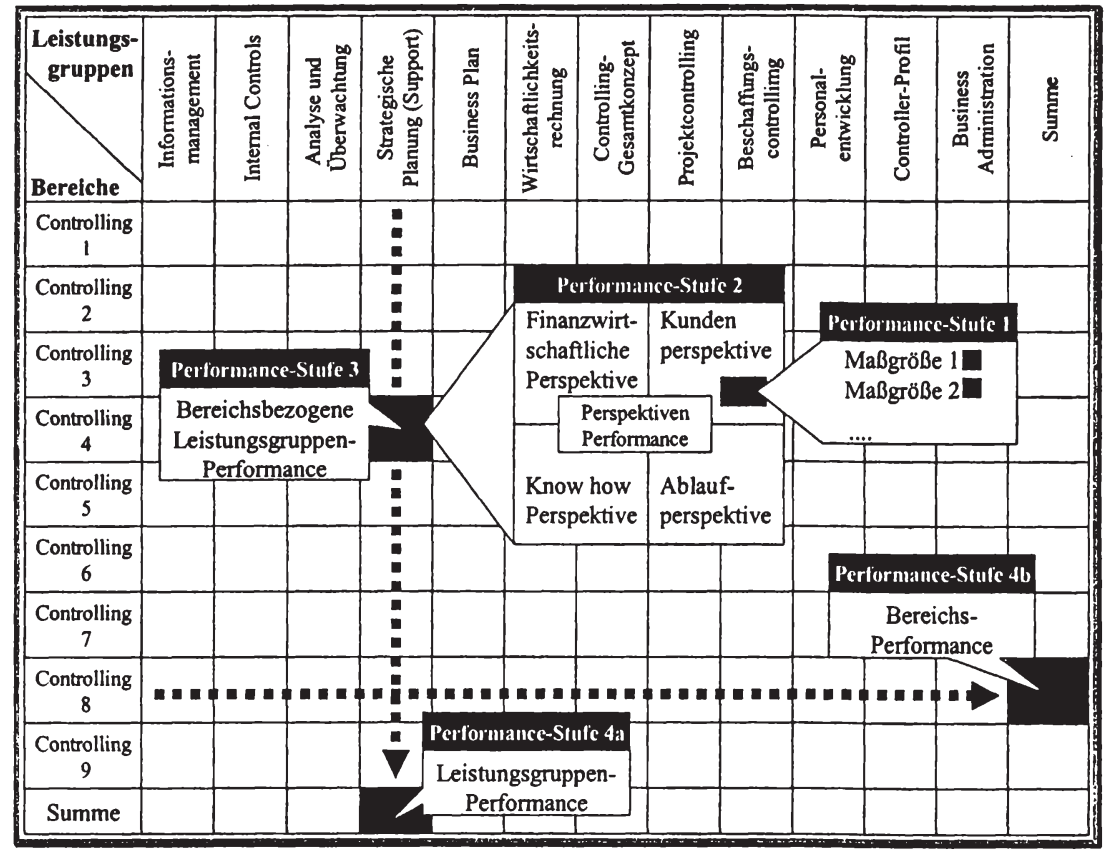

Abbildung 53: Performance-Ergebnisbogen und Performance-Stufen ${ }^{1002}$

Ein derartiger Performance-Ergebnisbogen erlaubt darüber hinaus, soweit ein Unternehmen z.B. verschiedene dezentrale Controllerbereiche hat, die Ableitung einer alle Bereiche betreffenden Leistungsgruppen-Peformance (Performance-Stufe 4a) bzw. einer über alle Leistungsgruppen aggregierte BereichsPerformance (Performance-Stufe 4b). Für die Funktionsfähigkeit eines derartig umfassenden Systems müssen zunächst detaillierte Fragebögen und Checklisten abgestimmt werden, sowie die Zuständigkeiten und Rhythmen für Datenerfassung und -auswertung festgelegt werden. ${ }^{1003}$ Elementar für die Funktionsweise und Akzeptanz der BSC im Controlling sind jedoch die Maßgrößen, sowie die hierfür festzulegenden Planwerte und Toleranzgrenzen.

Bezüglich einer Absicherung der Kundenorientierung im Controlling sind zunächst die Maßgrößen der Kundenperspektive der BSC relevant. In Anlehnung

${ }^{1002}$ Modifiziert nach Gleich/Haindl (1996), S. 266 (Ergebnisbogen) und S. 265 (Leistungsgruppen).

${ }^{1003}$ Zum Implementierungsvorschlag von KAPLAN/NORTEN vgl. Kaplap/Norten (1997), S. 298 
an die Benchmarkingbetrachtung des Controllerbereichs ${ }^{1004}$ sind hier primär $E f$ fektivitätsmaßstäbe gefragt, darüber hinaus können aber auch objektiv ermittelbare Effizienzmerkmale Anhaltspunkte für eine stärkere Kundenfokussierung geben. In dem in Abbildung 52 vorgestellten Beispiel eines Controllerbereichs in der chemischen Industrie wurden für die Kundenperspektive der Leistungsgruppe Informationsmanagement nachfolgende Maßgrößen und Erhebungsmethoden gewählt: ${ }^{100}$

- Darstellungsart der Berichtsleistungen, überprüfbar anhand von Checklisten und der Einhaltung von Unternehmensrichtlinien,

- Einheitlichkeit der Sprache in den Berichten, nachvollziehbar anhand der erbrachten Informations-Leistungen und Checklisten, ${ }^{1006}$

- Ausmaß der Methodenschwankungen, erhoben anhand der Berichtsinhalte und mittels Checklisten,

- Akzeptanz der erbrachten Leistungen, gemessen über fragebogengestützte Kundenerhebungen.

Die Erhebungsmethoden zeigen, dass hinter den Maßgrößen im Einzelnen noch wesentlich differenziertere Fragen stehen, die mit denen der Benchmarkingbetrachtung potenziell vergleichbar sein können. ${ }^{1007}$ Hierbei darf die Komplexität des Instrumentes BSC und die mit seinem regelmäßigen Betrieb verbundenen Aufwendungen nicht unterschätzt werden. Insbesondere auch die Akzep$\operatorname{tanz}$ im Controllerbereich hängt maßgeblich von der Handhabungsfähigkeit des Instrumentes $a b .{ }^{1008}$ Aus diesem Grund kann es insbesondere für weniger differenzierte Controlling-Organisationen zweckmäßig sein, auf eine leistungs- bzw. leistungsgruppenbezogene Abbildung zu verzichten. Im Ergebnis wird dann für den gesamten Controllerbereich eine einzige BSC verwandt, in der folglich alle Perspektiven, so auch die Kundenperspektive, unabhängig von einzelnen Leistungen betrachtet werden. Insbesondere auch das unmittelbare Herunterbrechen von Strategien, im Sinne des Managementanspruchs der BSC, kann hierdurch im Einzelfall erleichtert werden. ${ }^{1009}$ Derartige, für den gesamten Control-

\footnotetext{
${ }^{1004}$ Vgl. Stadler/Weißenberger (1999) sowie Weber/Weißenberger/Aust (1997). Vgl. insbesondere auch Kap. 4.2.1.3.

${ }^{1005} \mathrm{Vgl}$. Gleich/Haindl (1996), S. 267

${ }^{1006}$ Vgl. hierzu und zum folgenden Punkt die Ausfuhrungen zu den formalen Reportingstandards von NeuhäuserMetternich/Witt (2000), S. 313.

${ }^{1007} \mathrm{Vgl}$. hierzu insbesondere die dargestellten Fragen zur Bewertung der Effektivităt des Berichtswesens in Kap. 4.2.1.2.2.

${ }^{1008} \mathrm{Vgl}$. Kaplan/Norten (1997), S. $290 \mathrm{ff}$.

${ }^{1009} \mathrm{Vgl}$. allg. Horváth (1998), S. 569 f.
} 
lerbereich relevante Maßgrößen für die Kunden Perspektive einer BSC könnten beispielhaft

- das Verhältnis der Budgetanteile zwischen Controllern und Consultants, im Sinne von Marktanteilen,

- der Anteil der Bearbeitungszeit von Controller-Aufgaben, die in den letzten zwei Jahren neu eingeführt wurden, an der Gesamtzeit, im Sinne einer Innovationsrate,

- der erreichte Wert eines Manager Satisfaction Index, im Sinne statischer und dynamischer Zufriedenheit sowie

- die Anzahl von Controlling-Schulungen für Nicht-Controller, im Sinne einer Steigerung der Leistungstransparenz und Kundenbindung

sein. ${ }^{1010}$ Der umfassende Charakter der BSC bedingt letztlich insbesondere über die Ausgewogenheit der vier Perspektiven, eine adäquate Absicherung der Kundeninteressen sicherzustellen, denn im Ergebnis können Ursachen für Wettbewerbsvorteile in allen Dimensionen begründet liegen. ${ }^{1011}$ So können über die im Rahmen des Benchmarking getätigten Ausführungen zur Prozessoptimierung im Controlling ${ }^{1012}$ hinaus gerade Maßgrößen im Bereich der Lern- und Entwicklungsperspektive zu einer Intensivierung des Wissensmanagements auffordern. Im Controllerbereich ist hier insbesondere eine Messung die Fortbildungstage der Controller, der Aktualität des Controllerhandbuchs, des Anteils innovativer Projekte oder der interpersonellen Wissensweitergabe in Form von Vorträgen vorstellbar. ${ }^{1013}$ Über die Lern- und Entwicklungsperspektive kann somit langfristig das Leistungspotenzial des Controllerbereichs gemanaget werden, dessen Kommunikation gerade in Abhängigkeit unterschiedlicher informationsökonomischer Leistungseigenschaften eine hohe Wahrnehmungsrelevanz für Kunden hat.

Die bei unternehmensinternen Dienstleistern oftmals eher rudimentär behandelte Finanzperspektive bietet gerade in Verbindung mit einer kundenseitigen $\mathrm{Be}$ urteilung einzelner Leistungen differenzierte Anhaltspunkte für ein adressatenorientiertes Leistungsmangement. Neben der Budgeteinhaltung oder einer Re-

\footnotetext{
${ }^{1010} \mathrm{Vgl}$. Weber/Schäffer (2000), S. 176.

1011 Dies korrespondiert mit den üblichen Dimensionen des Wettbewerbsvorteils. Allg. vgl. Backhaus (1999), S. $26 \mathrm{ff}$.

1012 Derartige Analysen können eine Grundlage für die Ableitung von Maßgrößen der Prozessperspektive der BSC sein.

${ }^{1013}$ Vgl. hierzu Weber/Schäffer (2000), S. 225. 
duktion der Gesamtkosten des Controllerbereichs ist insbesondere auf eine den Kundenpräferenzen entsprechende Allokation der zur Verfügung stehenden Ressourcen zu achten. Ausgehend von diesen Überlegungen soll im Folgenden das Instrument des Target Costing vorgestellt werden.

\subsubsection{Target Costing für Controlling-Leistungen}

Das Instrument des Target Costing ist ein umfassendes Kostenplanungs-, Kostensteuerungs- und Kostenkontrollverfahren, über das gezielt Markt- bzw. Kundenanforderungen in die Phase der Produktentstehung und -gestaltung integriert werden sollen. ${ }^{1014}$ Der Anwendungsschwerpunkt des Target Costing lag in der Vergangenheit zunächst bei industriellen Gütern. ${ }^{1015}$ Die zunehmende Ausweitung auf absatzmarktbezogene Dienstleistungen wirft die Frage nach dem potenziellen Beitrag des Target Costing zu einer Effektivitäts- und Effizienzsteigerung des internen Dienstleisters Controlling auf. Die partielle Monopolstellung des Controlling führt oftmals dazu, dass der Qualitäts- und Kostendruck nur unzureichend ist, ${ }^{1016}$ so dass die Gefahr, gegenüber marktvergleichbaren Leistungen ins Hintertreffen zu geraten, regelmäßig besteht. Daher soll nachfolgend dargestellt werden, wie das Verfahren des Target Costing Kundenanforderungen im Prozess der Leistungsgestaltung abzudecken vermag und wie, korrespondierend damit, eine kundenverträgliche Preisgestaltung zu erfolgen hat. ${ }^{1017}$

\subsubsection{Target Costing-Methodik}

Ein wesentliches Charakteristikum des Target Costing ist der Anspruch, den Zielpreis (Target Price) speziell auf Basis konkreter Kundenanforderungen oder allgemein auf Grundlage der Markt- und Wettbewerbssituation abzuleiten. ${ }^{1018}$ Bezogen auf das Controlling stellt sich in diesem Zusammenhang zunächst die Frage nach dem Bezugsobjekt der Zielkostenermittlung. In Abhängigkeit der angestrebten Differenzierung können Zielkosten für den gesamten Controllerbereich, die Leistungsgruppen oder aber auch für einzelne Leistungen des Con-

\footnotetext{
${ }^{1014}$ Vgl. Horváth (1999), S. 522 sowie allg. Seidenschwarz (2000). Zur Rolle im Rahmen des MarketingControlling vgl. Meffert (2000), S. $1137 \mathrm{f}$.

${ }^{1015}$ Zur Breite der heutigen Anwendung des Target Costing vgl. Hoffjan (1994), S. 24 ff.

${ }^{1016}$ Allg. für interne Dienstleister vgl. Reiß/Schuster (1998), S. 1301.

${ }^{1017}$ In diesem Zusammenhang spricht man auch von Zielkostenfindung und Zielkostenspaltung.

${ }^{1018} \mathrm{Vgl}$. Hummel (1999), S. 492. Die folgenden Ausführungen orientieren sich an dem Market into CompanyVerfahren, welches zu den Subtraktionsmethoden des Target Costing zählt. Vgl. Buggert/Wielpütz (1995), S. $78 \mathrm{f}$.
} 
trolling bestimmt werden. Im Ergebnis handelt es sich dann um den Preis, den das Management für gewisse Controlling-Leistungen oder aber auch das gesamte Unternehmenscontrolling zu zahlen bereit ist. ${ }^{1019}$ In Abhängigkeit der ökonomischen Ausrichtung des Controllerbereichs ist festzulegen, ${ }^{1020}$ ob der kundenseitig zu entrichtende Preis um eine angestrebte Gewinnspanne (Target Profit) zu korrigieren ist, damit als Differenzbetrag die maximal möglichen Kosten (Allowable Costs) bestimmt werden können. Bei der Mehrzahl der Controllerbereiche werden Gewinne zunächst noch keine Berücksichtigung finden. ${ }^{1021} \mathrm{Die} \mathrm{Al}$ lowable Costs stellen die unter Berücksichtigung der Kundenpräferenzen maximal möglichen Kosten eines Bezugsobjektes dar. Subtrahiert man diese Kosten von den Standardkosten unter Beibehaltung der gegenwärtigen Leistungserstellungsmodalitäten im Controlling (Drifting Costs), gelangt man zu der Zielkostenlücke (Target Costs Gap), ${ }^{1022}$ d.h. zu dem kundeninduzierten Kostensenkungspotenzial. Die oftmals mangelnde Transparenz bei unternehmensinternen Dienstleistungen, insbesondere bei solchen des Controlling, macht eine differenzierte Bewertung der bestimmten Zielkostenlücke erforderlich, um daraufhin beurteilen zu können, inwieweit eine Schließung möglich erscheint. Im Ergebnis gilt es, von Seiten der Controllerbereichsführung unter Berücksichtigung von Motivationsaspekten ein realistisches Kostenziel (Target Costs) vorzugeben, um daraufhin Maßnahmen zu dessen Erreichung einleiten zu können. ${ }^{1023}$ Im Controllerbereich ist zu einer Priorisierung der Einsparungen insbesondere das Ausmaß der Wettbewerbsgefährdung heranzuziehen, da eine hohe Wettbewerbsintensität preisinduzierte Abwanderungen begünstigt.

Neben der Zielkostenfindung begründet die Zielkostenspaltung den strengen Marktbezug des Target Costing, ${ }^{1024}$ woraus gleichsam die besondere Relevanz für eine Steigerung der Kundenorientierung im Controlling resultiert. In Abhängigkeit des für den Controllerbereich gewählten Target Costing-Objektes (Gesamtbereich, Leitungsgruppen oder Leistungen) soll mittels der Zielkostenspaltung dieses in einzelne Funktionen, die letztlich den Kundennutzen begründen, zerlegt werden. Darauf aufbauend gilt es die gesamten Zielkosten entsprechend den noch zu ermittelnden Kundenpräferenzen auf diese einzelnen Funktionen aufzuteilen. Auf Basis einer derart differenzierten Kostenvorgabe ist zu

\footnotetext{
1019 Dieser Preis ist im Sinne einer Preisobergrenze zu verstehen.

${ }^{1020} \mathrm{Zu}$ den alternativen Formen einer ökonomischen Ausrichtung des Controllerbereichs vgl. Kap. 3.3.1.2.

${ }^{1021}$ Zur ökonomischen Ausrichtung der Kostenrechnung vgl. Währisch (1998), S. 337 f.

${ }^{1022}$ Vgl. Kavandi (1998), S. 60.

${ }^{1023}$ Allg. vgl. Buggert/Wielpütz (1995), S. 81.

${ }^{1024}$ Vgl. Horváth/Seidenschwarz (1992), S. 145.
} 
überprüfen, mittels welcher Leistungsgruppen, bzw. Leistungskomponenten Zielkosten-Nutzen-Relation optimiert werden können. ${ }^{1025}$ Diese Optimierung erfordert implizit die Bewertung des Beitrages einzelner Komponenten zur Funktionserfüllung des Betrachtungsobjektes.

Im Ergebnis können mit dem Instrument Target Costing Kosteneinsparungen bzw. allgemein die Ressourcenallokation so vollzogen werden, dass in der Wahrnehmung der Kunden Leistungswertschätzung und Leistungsnutzen harmonieren. Bezogen auf das Controlling können so Kostensenkungen bzw. Umverteilungen systematisch erfolgen ${ }^{1026}$ und Anhaltspunkte für eine markt- und kundenorientierte Verrechnungspreisgestaltung gewonnen werden. Darüber hinaus eröffnet eine derart strukturierte Analyse des Leistungsangebotes, insbesondere auch über die Einbeziehung der Kundenpräferenzen, inhaltliche Optimierungsmöglichkeiten. Im Folgenden soll eine beispielhafte Anwendung des Target Costing auf den Controllerbereich dargestellt werden.

\subsubsection{Target Costing-Prozess im Controlling}

Aufbauend auf den Ausführungen zur Methodik des Target Costing, gilt es Spezifika seiner Anwendung im Controlling zu betrachten. Hierbei soll besonderes Augenmerk auf die Praktikabilität der Umsetzung gelegt werden, da die mit der Durchfuihrung des Target Costing verbundenen - nicht unerheblichen Aufwendungen in einem angemessenen Verhältnis zu dem potenziellen Nutzen stehen müssen. Diesbezüglich stellt sich in einem ersten Schritt ${ }^{1027}$ des Target Costing im Controlling zunächst die Frage nach dem Objekt der Zielkostenbetrachtung. Einerseits können - im Sinne der Produkte des Controlling - unmittelbar konkurrenzorientiert Zielkosten für Leistungen oder Leistungsgruppen bestimmt werden. Andererseits können aber auch zunächst für den gesamten Controllerbereich Zielkosten festgelegt werden, da über die dann notwendige Betrachtung der Funktionen bzw. Leistungskomponenten ohnehin eine weitere Differenzierung erforderlich wird. Bei dem hier verfolgten Beispiel ${ }^{1028}$ sollen im Sinne der Praktikabilität zunächst die Zielkosten für den gesamten Controllerbereich bestimmt werden, um dann die auf dieser Grundlage vorzunehmende Ressourcenverteilung auf Leistungsgruppen zu betrachten. Im Sinne einer

\footnotetext{
${ }^{1025}$ Diese Relation kann uber einen Zielkostenindex operationalisiert werden. Die Festlegung von Toleranzbereichen kann über Zielkostenzonen erfolgen. Zu diesen Prozessschritten des Target Costing vgl. Berens/Hoffjan (1998), S. 686 f. und Hoffjan (1994), S. 32 ff.

${ }^{1026} \mathrm{Vgl}$. Steinle/Thiem/Rohden (2000), S. 284.

${ }^{1027}$ Die aufgezählten Schritte beziehen sich auf den in Abbildung 54 dargestellten Prozess.

${ }^{1028}$ Das nachfolgend vorzustellende Beispiel findet sich bei Gleich/Brokemper (1998), S. $148 \mathrm{ff}$.
}

Thomas Mosiek - 978-3-631-75321-7 
markt- bzw. wettbewerbsorientierten Festlegung der Zielkosten für das Unternehmenscontrolling bietet sich, analog zum Benchmarking, eine vergleichende Betrachtung mit anderen Unternehmen an. Über eine Relativierung der Kosten des Controllerbereichs zum Umsatz können für Unternehmen ähnlicher Branchen Vergleichsmaßstäbe gewonnen werden. IBACH beziffert in einer empirischen Erhebung in der amerikanischen Industrie die durchschnittlichen Kosten für den Controller- und Finanzbereich im Jahr 1996 auf ca. 1,4\% vom Umsatz. ${ }^{1029}$ Auf dieser Basis können beispielhaft, abhängig vom Unternehmensumsatz, die Zielkosten für alternative Controllerbereiche bestimmt werden. Wesentlich für die Aussagekraft derartiger Vergleichswerte ist eine weitgehende Übereinstimmung der einem Controllerbereich zugeordneten Aufgaben. ${ }^{1030} \mathrm{Die}$ Aufgabenzuordnung ${ }^{1031}$ beeinflusst auch maßgeblich das Leistungsspektrum des Controllerbereiches, welches in Schritt 2 des in Abbildung 54 dargestellten Target Costing-Prozesses abzugrenzen ist.

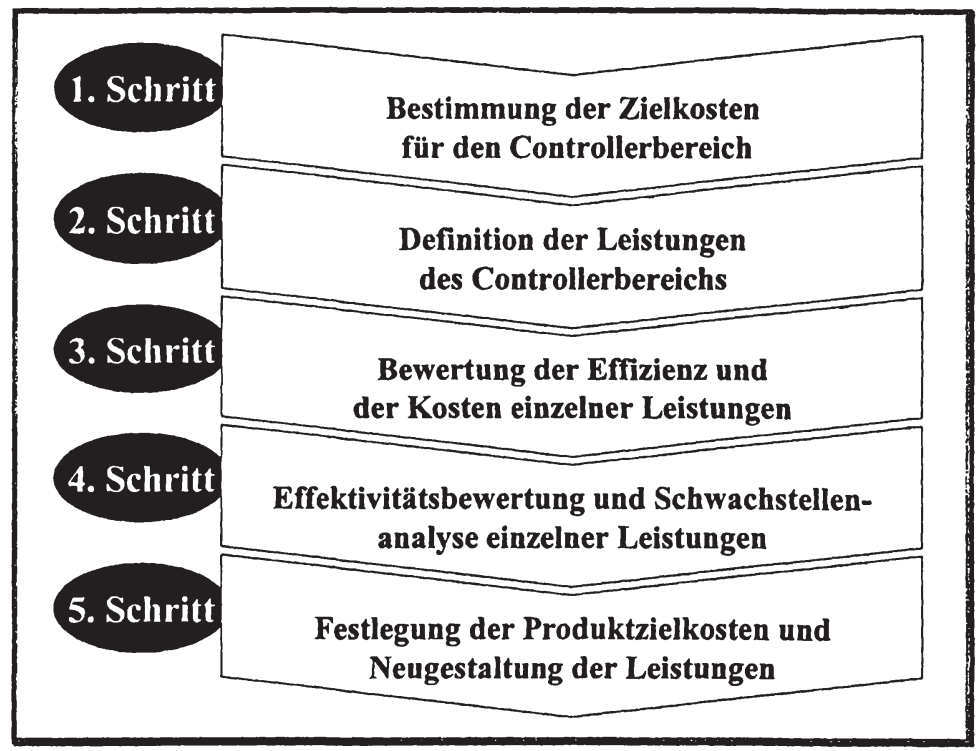

Abbildung 54: Prozess des Target Costing im Controlling ${ }^{1032}$

\footnotetext{
${ }^{1029}$ Vgl. Ibach (1997), S. 4. und allgemein zur Entwicklung der Kosten des Controllerbereichs Horváth (2001), S. 33.

${ }^{1030}$ Dies korrespondiert mit den Ausführungen zu den Strukturmerkmalen der im Rahmen des Benchmarking zu vergleichenden Objekte. Ausfiihrlich vgl. Hamprecht (1996), S. 296 ff.

${ }^{1031}$ Ausfuhrlich zur methodischen Fundierung der Aufgabenzuordnung im Controlling vgl. Kap. 3.2.1.1 sowie Herzog (1999).

1032 Verkürzt nach Gleich/Brokemper (1998), S. $149 \mathrm{ff}$.
} 
Eine klare Definition der Leistungen des Controllerbereichs erlaubt im weiteren Verlauf ein sachgerechte Spaltung der Zielkosten. Im Sinne eines pragmatischen Vorgehens erscheint es ratsam, zunächst Leistungsgruppen, d.h. inhaltlich zusammenhängende Leistungen abzugrenzen, da dies aufgrund der überschaubareren Anzahl regelmäßig weniger Schwierigkeiten bereitet und eine kontinuierliche Leistungsbeurteilung auch unter Aufwandsgesichtspunkten zulässt. ${ }^{1033}$ Sind die Leistungsgruppen objektiv definiert, gilt es in Schritt 3 die derzeitige Ressourcenverteilung im Controllerbereich mittels einer Prozesskostenrechnung zu quantifizieren. ${ }^{1034}$ Diese erfordert ein analytisches Herunterbrechen der Leistungsgruppen in Leistungen/Aktivitäten. So lässt sich beispielsweise die Leistungsgruppe Reporting in die einzelnen Leistungen

- Erstellen und Prüfen der Berichte (60\% der Kapazitäten),

- BAB-Erstellung (20\% der Kapazitäten),

- Präsentation der Berichte (6\% der Kapazitäten),

- Informationsmanagement Berichtswesen ( $8 \%$ der Kapazitäten) und

- externe Berichte erstellen (6\% der Kapazitäten)

zerlegen. ${ }^{1035}$ Auf Basis einer genauen Erhebung von Zeiten, Mengen und Kosten, sowie einer darauf aufbauenden Abbildung der aktuellen Ressourcenverteilung im Controllerbereich können bereits erste Anhaltspunkte für die Effizienzbeurteilung einzelner Leistungen und Leistungsgruppen gewonnen werden. Darüber hinaus müssen analog zu der Effizienzbetrachtung beim Benchmarking, Prozess- und Strukturanalysen durchgeführt werden, ${ }^{1036}$ um festzustellen, wie wirtschaftlich im Controlling Leistungen erstellt werden. ${ }^{1037}$ Neben der internen Betrachtung des Controlling erfordert das Instrument Target Costing in einem 4. Schritt die differenzierte Bewertung der Effektivität einzelner Leistungsgruppen des Controlling. Über die statische und dynamische Operationalisierung der Effektivität in Form von Kundenzufriedenheit und Änderungsbedarf $f^{1038}$ hinaus, kann eine Bewertung der Wichtigkeit einzelner Leistungsgruppen des Controlling vorgenommen werden, ${ }^{1039}$ die sich aus einer Abfrage der kundenseitig wahrgenommen Bedeutung einzelner Leistungsgruppen ergibt.

\footnotetext{
${ }^{1033}$ Vgl. hierzu auch die Abgrenzung der Leistungsgnuppen in Abbildung 53 sowie die Ausfulhrungen hierzu bei Gleich/Haindl (1996), S. 265.

${ }^{1034}$ Grundlegend zur Prozesskostenrechnung vgl. Horváth (1998), S. 532 ff. oder Hummel (1999), S. 498.

${ }^{1035}$ Ausfuhrlich anhand eines umfassenden Beispiels vgl. Gleich/Brokemper (1998), S. 151.

${ }^{1036}$ Vgl. Kap. 4.2.1.3.1 sowie Stadler/Weißenberger (1999), S. 6 ff.

${ }^{1037}$ Für den Marketing- und Vertriebsbereich vgl. Feind-Just (1999), S. $188 \mathrm{ff}$.

${ }^{1038}$ Für das Berichtswesen vgl. Stadler/Weißenberger (1999), S. 8 ff.

${ }^{1039}$ Hier und im Folgenden vgl. Gleich/Brokemper (1998), S. 152. 
Im Ergebnis ist es möglich, mittels der Prozesskostenrechnung für jede Leistungsgruppe den relativen Anteil der eingesetzten Ressourcen und über die Frage nach der Wichtigkeit, die relative Bedeutung einzelner Leistungsgruppen zu bestimmen. Ferner kann mittels eines Fragenkataloges und der Zuordnung entsprechender Scoring-Werte die Zufriedenheit der Kunden quantifiziert werden. Trägt man diese Werte in das in Abbildung 55 dargestellte Portfolio ein, lassen sich Kosten-/Nutzen-Relationen unter ergänzender Angabe der Kundenzufriedenheit beispielhaft visualisieren und gleichsam Schwachstellen aufzeigen.

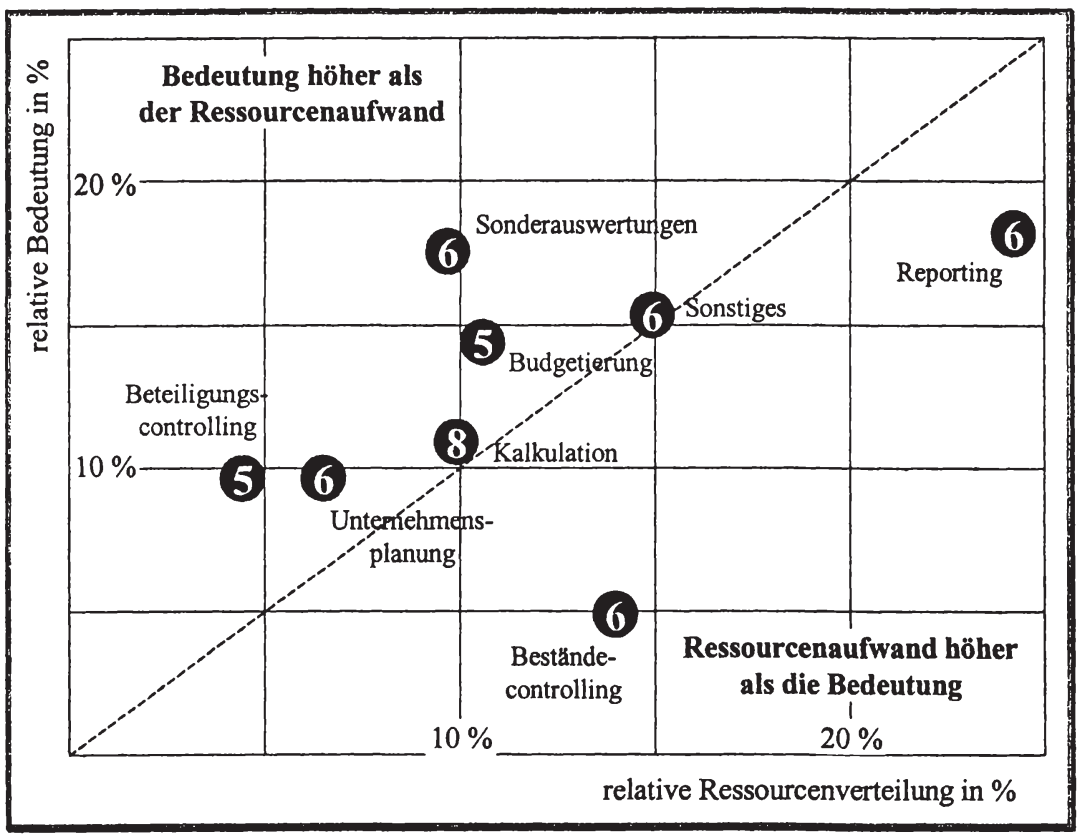

Abbildung 55: Leistungsprofil eines Controllerbereichs ${ }^{1040}$

Bei dem hier zugrundegelegten Beispiel wird deutlich, dass die Leistungsgruppen Reporting und Beständecontrolling deutlich mehr Ressourcen beanspruchen, als dies ihre Bedeutung in den Augen der Kunden rechtfertigt. Zielsetzung sollte demnach eine Positionierung der Leistungsgruppen entlang der gestrichelten Gleichgewichtslinie sein, da diese ausgewogene Kombinationen von

${ }^{1040}$ Gleich/Brokemper (1998), S. 153. Die in den Kreisen abgebildeten Ziffern geben die Zufriedenheit der Kunden mit den Leistungsgruppen wieder. Es erfolgt eine Abstufung zwischen $10=\operatorname{sehr}$ zufrieden und $1=$ völlig unzufrieden. 
Bedeutung und Ressourcenbeanspruchung repräsentiert. In Abhängigkeit der jeweiligen Position einer Leistung lassen sich Handlungsempfehlungen ableiten. Darüber hinaus erlaubt die ergänzende Angabe der Kundenzufriedenheit, aufgrund des ihr zugrundeliegenden Fragenkataloges, eine differenzierte Einschätzung der Ursachen von Leistungsdefiziten. Allgemein kann gerade bei Leistungen mit in Relation zu ihrer Bedeutung zu geringen Ressourcenanteilen davon ausgegangen werden, dass die Kundenzufriedenheit über eine Zuordnung weiterer personeller und sächlicher Kapazitäten gesteigert werden kann. ${ }^{1041}$ Demgegenüber gilt es Leistungsgruppen mit überproportional hoher Ressourcenbeanspruchung auf Kosteneinsparungspotenziale hin $\mathrm{zu}$ untersuchen, um den Gleichgewichtszustand wieder herzustellen. Anhaltspunkte hierfür können mittels Benchmarking oder über die im Rahmen der Prozesskostenrechnung durchgeführten Prozessanalysen gewonnen werden. ${ }^{1042}$ In diesem Zusammenhang ist es von grundlegender Bedeutung sicherzustellen, dass derartige Verschlankungsmaßnahmen nicht zu einer Beeinträchtigung des Kundennutzens führen.

Im Ergebnis geht es in Schritt 5 des Target Costing-Prozesses darum, Zielkosten für alle Leistungsgruppen auf Grundlage der fixierten Zielkosten des gesamten Controllerbereichs festzulegen. Hierbei hat sich die Ressourcenumverteilung bzw. -einsparung, wie dargestellt, konsequent an den Kosten-/Nutzenrelationen der Kunden zu orientieren. Die Erreichung der Zielkosten kann in der Folge mitunter eine Neugestaltung einzelner Leistungsgruppen erforderlich machen.

Grundlegende Voraussetzung für die Anwendbarkeit des Target Costing im Controlling ist das Bestehen einer ausreichenden Leistungstradition ${ }^{1043}$, d.h. die zu bewertenden Leistungen müssen vom Kunden bereits mehrmals in Anspruch genommen worden sein, ${ }^{1044}$ so dass er sich ein konkretes Urteil über seine diesbezüglichen Präferenzen machen konnte. ${ }^{1045}$ Darüber hinaus spielt die Integrativität der Leistungserstellung im Controlling eine nicht zu vernachlässigende Rolle. ${ }^{1046}$ In Abhängigkeit der Intensität, mit der der Kunde des Controllers in den Leistungserstellungsprozess einbezogen wird, steigt die Unsicherheit be-

\footnotetext{
1041 Gerade die für die Zufriedenheit der Kunden wesentliche Individualisierung der Ansprache sowie eine Institutionalisierung der Durchsprache von Berichten bindet regelmäBig personelle Kapazitäten. Vgl. Stadler/Weißenberger (1999), S. 9.

$1042 \mathrm{Vgl}$. Gleich/Haindl (1998), S. 152.

${ }^{1043}$ Vgl. Kap. 3.3.2.4.

${ }^{1044} \mathrm{Zu}$ alternativen Motiven der Leistungsinanspruchnahme vgl. Kap. 3.3.2.1.

1045 Allg. zu den Voraussetzungen des Target Costing vgl. Weber (1999), S. 91.

${ }^{1046}$ Zum Leistungserstellungsprozess vgl. Kap. 3.2.2.1.3
} 
züglich der Kostenplanung. Allgemein bekannt sind sowohl kostensteigernde, kostensenkende oder auch kostenneutrale Wirkungen der Kundenintegration..$^{1047}$ Analog hierzu führt die Leistungsindividualisierung, wie bspw. bei Projektleistungen, zu Prognoseproblemen, ${ }^{1048}$ da Leistungsinhalt und -umfang regelmäßig variieren und daher nur eine methodische Optimierung zulassen. $\mathrm{Zu}-$ sätzlich spricht der regelmäßig notwendige Einsatz der Prozesskostenrechnung für bessere Ergebnisse des Target Costing bei Standardleistungen.

Aufbauend auf den Ausführungen zur finanziellen Perspektive der Balanced Scorecard für den Controllerbereich wurde Target Costing als ein Instrument zur Steigerung der Kostentransparenz vorgestellt. Die Darstellung der Methodik hat gezeigt, dass der Nutzen des Target Costing neben der Kostentransparenz, die primär über die instrumentelle Unterstützung der Prozesskostenrechnung abgesichert wird, insbesondere in der Integration der Nachfragerperspektive zu sehen ist. Bedenkt man darüber hinaus, dass im Rahmen der Zielkostenspaltung und Leistungsneugestaltung Prozess- und Potenzialanalysen Aufschluss über Optimierungspotenziale geben sollen, wird deutlich, dass eine Integration des Target Costing in ein umfassendes Performance MeasurementSystem zweckmäßig erscheint. ${ }^{1049}$ Gerade der Anspruch des Target CostingProzesses, kontinuierlich Kosten-/Nutzenrelationen einzelner Leistungen in Einklang zu bringen, erfordert eine wirtschaftliche Integration der zu erhebenden Größen in ein System. Verwendet man hierfür, wie in den vorangegangenen Ausführungen dargestellt, die Balanced Scorecard, gilt es im Rahmen der Finanziellen Perspektive regelmäßig die Prozesskosten einzelner Leistungsgruppen zu erheben. Ferner kann auf bereits bestehende Fragenkataloge der Kunden Perspektive zur Ermittlung der Kundenzufriedenheit zurückgegriffen werden. Darüber hinaus kann die Bedeutung einzelner Leistungsgruppen als Kennzahl der Kundenperspektive verstanden werden, da es sich hierbei um eine subjektive Einschätzung der Nachfrager handelt, welche wesentlich durch das individuelle Nutzungsverhalten einzelner Kunden geprägt ist. ${ }^{1050}$ Der Prozess der Performancebestimmung kann dann analog zu den Ausführungen in Kap. 4.2.2.2 erfolgen. Das Instrument des Target Costing ist dann in diesem Gesamtzusammenhang als ein spezielles Auswertungs- und Restrukturierungsinstrument zur Absicherung der Kundenorientierung im Controlling zu verstehen.

\footnotetext{
${ }^{1047}$ Allg. zu den Auswirkungen der Integrativität auf Preisbildung und Kostenmanagement vgl. Paul/Reckenfelderbäumer (1995), S. $226 \mathrm{ff}$.

1048 Zur Leistungsindividualisienung im Controlling vgl. Kap. 3.4.3.1.

${ }^{1049}$ Zur Verbindung des Target Costing mit einem Performance Measurement-System bei HEWLETT PACKARD vgl. Gleich (1997b), S. 358 ff.

${ }^{1050}$ Vgl. Kap. 3.4.3.3.
} 
Ein weiterer Nutzen des Target Costing kann in der Fundierung kundenverträglicher Verrechnungspreise gesehen werden. ${ }^{1051}$ Wie bereits hinsichtlich des Controlling diskutiert wurde, ${ }^{1052}$ können kostenorientierte Verrechnungspreise, markt- bzw. konkurrenzorientierte Verrechnungspreise sowie nutzen-bzw. nachfragerorientierte Verrechnungspreise unterschieden werden. ${ }^{1053}$ Der Prozess des Target Costing hat in diesem Zusammenhang deutlich gemacht, dass es Möglichkeiten gibt, einzelne Elemente dieser Verfahren zu kombinieren. So können die für Leistungsgruppen oder Einzelleistungen bestimmten Target Costs als Basis für eine kostenorientierte Verrechnung herangezogen werden, soweit die ökonomische Ausrichtung des Controlling keine Gewinnaufschläge erforderlich macht. ${ }^{1054}$ Nutzen- bzw. nachfragerorientiert sind diese Verrechnungspreise insoweit, als dass die verrechneten Kosten in einem angemessenen Verhältnis $\mathrm{zu}$ den nachfragerseitig artikulierten Leistungspräferenzen stehen. Unterstellt man darüber hinaus, dass die Zielkostenvorgabe für den gesamten Controllerbereich auf Grundlage einer Markt- bzw. Konkurrenzbetrachtung erfolgte, wird deutlich, dass auch ein Effizienzvergleich mit externen Anbietern möglich erscheint.

Insgesamt wird deutlich, dass der Einbezug von Kundenpräferenzen und Wettbewerbsbenchmarks maßgeblich für die Steigerung der Wirksamkeit des Controllings ist. Benchmarking, die Balanced Scorecard und Target Costing können in diesem Zusammenhang erheblich zur kontinuierlichen Leistungsplanung und -messung beitragen. Grundlegend für die Wirksamkeit dieser Instrumente sind - wie bereits mehrfach angedeutet - ein strukturiertes und transparentes Leistungsangebot des Controllerbereichs sowie die Bereitschaft zur Institutionalisierung unternehmensinterner Märkte. Welchen Beitrag dazu Service Level Definitions und Service Level Agreements leisten können, soll nachfolgend gezeigt werden.

\subsubsection{Service Level Definitions und Service Level Agreements im Controlling}

Die kundenorientierte Leistungsgestaltung im Controlling wird wesentlich durch das Ausmaß der Wirksamkeit unternehmensinterner Marktverhältnisse

\footnotetext{
${ }^{1051} \mathrm{Zu}$ unterschiedlichen preispolitischen Strategien vgl. Meffert (2000), S. $482 \mathrm{ff}$. sowie S. $505 \mathrm{ff}$.

${ }^{1052} \mathrm{Vgl}$. Kap. 3.3.2.5.

${ }^{1053} \mathrm{Vgl}$. Meffert (2000), S. $506 \mathrm{ff}$ und Wagenhofer (1998), S. $23 \mathrm{ff}$.

${ }^{1054}$ Aufgrund der Vernachlässigung von Gewinnaufschlägen und Kapitalkosten müssen daher Controlling-

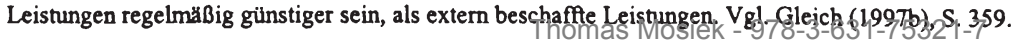


geprägt. Die Etablierung unternehmensinterner Märkte erscheint allgemein immer dann gerechtfertigt, soweit Unternehmensteilbereichen weitgefasste Aufgaben und Verantwortungskomplexe zur eigenverantwortlichen Bearbeitung in einem vorher bestimmten Rahmen anvertraut werden. ${ }^{1055}$ Wesentlich für das $\mathrm{Zu}$ sammentreffen von unternehmensinternem Angebot und Nachfrage, als konstituierendem Merkmal für einen Markt, ${ }^{1056}$ ist die Entscheidung der Unternehmensführung, den Markt als Kontroll-, Anreiz- und Lenkungsmechanismus zuzulassen. Eine wirksame Allokation kann darüber hinaus nur dann erfolgen, wenn unternehmensintern strukturelle Rahmenbedingungen geschaffen werden, die den Leistungsaustausch kultivieren. Im Einzelfall heißt dies für das Controlling, dass zunächst sein Anbieterstatus auf unternehmensinternen Märkten durch die Unternehmensführung festzulegen ist. ${ }^{1057}$ Darauf aufbauend gilt es für den Controllerdienst, die Transparenz seines Leistungsangebotes, im Sinne einer Konkretisierung des Serviceversprechens sicherzustellen, ${ }^{1058}$ um auf dieser Grundlage interne Verpflichtungen gegenüber Kunden eingehen zu können. Hierzu bedarf die Leistungsinanspruchnahme zur Unsicherheitsreduktion beider Transaktionspartner einer vertragsähnlichen Fixierung von Leistung und Gegenleistung. Service Level Definitions (SLD's) und Service Level Agreements $(S L A$ 's) stellen in diesem Zusammenhang geeignete Instrumente zur Etablierung interner Marktstrukturen dar.

Auch wenn im Controlling keine idealtypischen Voraussetzungen für eine uneingeschränkte Vermarktung seiner Leistungen vorliegen, ${ }^{1059}$ müssen die positiven Anreizeffekte der internen Marktorientierung stets den suboptimalen Effekten marktferner Allokationsverfahren gegenübergestellt werden. Die Einführung von SLA's und SLD's vermag oftmals erstmalig Rahmenbedingungen zu schaffen, die auch für scheinbar marktferne Unternehmensteilbereiche wie das Controlling ein marktgerechtes Verhalten von Anbieter und Nachfrager zulassen. Daher sollen im Folgenden zunächst abstrakt die für das Controlling relevante Strukturmerkmale von SLD's und SLA's dargestellt werden, bevor ein Anwendungsbeispiel geschildert wird.

\footnotetext{
${ }^{1055}$ Ludwig (1995), S. 127 ff. sowie Kap. 3.3.2.5.

${ }^{1056} \mathrm{Vgl}$. Siebke (1992), S. 64

${ }^{1057}$ Dies kann im Einzelfall z.B. die Abnahmeverpflichtung betreffen. Vgl. hierzu die Ausführungen zu obligatorischen und fakultativen Dienstleistungen in Kap. 3.3.2.1.

${ }^{1058}$ Zum Problem unscharfer Serviceversprechen interner Dienstleister vgl. Schütz (1993), S. 196 f.

${ }^{1059} \mathrm{Vgl}$. hierzu ausfuihrlich Kap. 3.3.1.2, Kap. 3.3.2.5 und Kap. 3.3.3.4 


\subsubsection{Struktur von Service Level Definitions und Service Level Agreements}

Bei Service Level Agreements handelt es sich um Leistungsvereinbarungen, „welche die Beziehungen zwischen einem Dienstleistungserbringer und einem Dienstleistungsbezüger auf vertraglicher Basis regeln" ${ }^{1060}$. Grundlage hierfür ist die Formulierung von Service Level Definitions, welche die Inhalte einzelner Leistungen strukturiert wiedergeben und so in der Summe das Leistungsportfolio eines Anbieters darzustellen vermögen. SLD's und SLA's zielen damit über eine Transparenzsteigerung und über die Festlegung von Verhandlungsmodalitäten auf die bilaterale Implementierung eines Service-Denkens ab. ${ }^{1061}$ Optimierungspotenziale für das Controlling können in

- der Erhöhung der Leistungserbringung und Leistungsqualität,

- einer verstärkten Kundenausrichtung,

- Anreizen zu Produktivitätsverbesserungen,

- der verursachungsgerechten und transparenten Kostenzuordnung,

- einer Reduktion der Nachfrage nach nicht unbedingt notwendigen Leistungen und allgemein in

- einer Harmonisierung von konträren Zielsetzungen in verschiedenen Unternehmensbereichen

gesehen werden. ${ }^{1062}$ Zentral bei der Gestaltung von SLA's ist ein insbesondere auch aus Kundenperspektive als angemessen empfundenes Verhältnis von Leistung und Gegenleistung herzustellen. Betrachtet man diesbezüglich die in Abbildung 27 dargestellten Voraussetzungen für die Einzelverrechnung von Dienstleistungen, ${ }^{1063}$ die wesentlich deren Marktfähigkeit determinieren, so wird deutlich, dass über SLA's und SLD's gerade eine Erfüllung dieser Voraussetzungen erleichtert wird. ${ }^{1064}$

Bevor SLA's ausgehandelt werden können, müssen die Leistungen in Form von SLD's intersubjektiv beschrieben werden. Dies korrespondiert mit der im Rah-

\footnotetext{
1060 Winkelmann-Ackermann/Bundi (1999), S. 38.

$1061 \mathrm{Vgl}$. Steinle/Thiem/Rohden (2000), S. 285 sowie Arbeitskreis „Leistungsvereinbarungen“ (1998), S. $10 \mathrm{ff}$.

1062 Die hier aufgezăhlten Zielsetzungen beziehen sich einerseits auf Bölscher (2000), S. 355 f. sowie andererseits auf Winkelmann-Ackermann/Bundi (1999), S. 36.

${ }^{1063}$ Für den IT Bereich vgl. Kargl (1998), S. 40 ff.

${ }^{1064}$ SCHERZ kommt im Rahmen einer empirischen Untersuchung zu dem Ergebnis, dass insbesondere die Abgrenzbarkeit der Dienstleistung, die Messbarkeit der Dienstleistung, die Erstellung auf Veranlassung des Empfängers und die Erstellung im Interesses des Empfangers wesentlich fur deren Einzelverrechnung sind. Vgl. Scherz (1998), S. 113 und Abbildung 27. 
men des Benchmarking vorzunehmenden Leistungsabgrenzung, ${ }^{1065}$ so dass auf derartige Erkenntnisse - soweit bspw. eine unternehmensspezifische Best Practice entwickelt wurde - zurückgegriffen werden kann. Ferner sollten SLD's auch die Grundlage für Leistungsgruppenabgrenzungen der Balanced Scorecard oder des Target Costing sein, so dass stets auf ein einheitliches Leistungsportfolio im Rahmen der Leistungsmessung und -planung zurückgegriffen werden kann. ${ }^{1066}$ Insgesamt ist im Sinne der Praktikabilität darauf zu achten, dass zunächst nur die Kernprodukte des Controllerbereichs Niederschlag in SLD's finden, da dies unter wirtschaftlichen Gesichtspunkten sinnvoll erscheint, die Akzeptanz des Systems regelmäßig fördert und somit nicht den Anschein eines übertriebenen Formalismus aufkommen lässt. Deutlich wird, dass derartig strukturierten Produktdefinitionen besonders im Controllerbereich zusätzlich eine Kommunikations- und damit Evidenzsteigerungsfunktion zukommt, da hierüber intransparenzbedingte Inanspruchnahmemängel beseitigt werden können. Die auf Basis der SLD's vorzunehmende Gestaltung und Aushandlung von SLA's erfordert die Berücksichtigung aller aus Kundensicht relevanten Abrufmodalitäten und Leistungscharakteristika. ${ }^{1067} \mathrm{Im}$ Einzelnen stellen sich für das Controlling folgende wesentliche Fragen: ${ }^{1068}$

- Welche Produkte und Dienstleistungen sollen einem bestimmten Kunden in Abhängigkeit seiner Ziele erbracht werden?

- Mit welchen Standardmengen kann der Controller kalkulieren?

- Welches sind die definierten Parameter, um die Qualitätsbedürfnisse des Kunden zu erfüllen (Quality Levels)?

- Welche Prioritäten und Dringlichkeitsstufen sind bei der Leistungserstellung festzulegen?

- Welche Leistungsindikatoren können wirtschaftlich erhoben werden?

- In welchem Rhythmus sind die SLA's zu überprüfen oder anzupassen?

- Ist ein Eskalationsvorgehen bei Problemen definiert?

- Wie werden die Preise und die Verrechnungsart festgelegt?

- Gibt es Vorschriften zu Geheimhaltung, Datenschutz und Sicherheit?

- Welches sind die Rechte und Pflichten des Leistungsbeziehenden bzw. des Leistungserbringers?

\footnotetext{
1065 Vgl. Weber/Weißenberger/Aust (1997), S. 6 ff.

1066 Allgemein hängt es damit von der Chronologie der eingesetzten Instrumente ab, welches die Grundlage für ein nachfolgendes bildet.

${ }^{1067}$ Wesentliche Tatbestände der Leistungsinanspruchnahme wurden im Rahmen der Abrufmodalitäten in Kap. 3.3.2.2 genannt.

${ }^{1068}$ In Anlehnung an die genannten Verhandlungselemente yon Winkelmann-Ackermann/Bundi (1999), S. 39.
} 
Auf Grundlage dieser Fragen können die wesentlichen Bestandteile eines internen Leistungsvertrages abgeleitet werden. Insbesondere die letzte Frage deutet darauf hin, dass es organisatorische Rahmenbedingungen bezüglich des Leistungsaustausches geben muss, ${ }^{1069}$ insbesondere für solche Fälle, in denen obligatorische Leistungen dem Verhandlungsspielraum partiell entzogen werden müssen. ${ }^{1070}$ Dadurch, dass die Leistungserstellung aber im wesentlichen auf $\mathrm{Ver}$ anlassung des Nachfragers erfolgen sollte, gilt es die Ausgestaltung der SLA's (Lieferzeiten, Abnahmerhythmen, Gültigkeitsdauern, etc.) konsequent im Rahmen von Verhandlungen mit der Kapazitätsplanung im Controlling abzustimmen, damit man dort seinen Kapazitätsvorhaltepflichten effizient nachkommen kann. ${ }^{1071}$ Eine auf Basis von SLA's mögliche langfristige Kapazitätsplanung erlaubt darüber hinaus den zuverlässigen Einsatz plankostenbasierter, präferenzadäquater Verrechnungspreise, soweit keine Marktäquivalente bestehen.$^{1072}$ Hierbei kann sowohl eine Grundversorgung berechnet werden (z.B. regelmäßige Kostenstellenberichte in festem Rhythmus) oder aber auch eine vorgangsabhängige Fakturierung erfolgen (z.B. Einzelbericht). Für Leistungen, deren Inanspruchnahme stark variiert, wie z.B. interne Beratungsprojekte, kann eine einzelfallbezogene, aufwandsabhängige Verrechnung erfolgen. ${ }^{1073}$ Zum Zwecke einer Forcierung des Services im Controlling erscheint darüber hinaus die Integration dynamischer Elemente in Verrechnungspreise denkbar, wie z.B. eine prozentuale Senkung der Verrechnungspreise im Zeitablauf. ${ }^{1074}$

Insgesamt gilt es, beim Einsatz von Leistungsvereinbarungen im Sinne der Motivation und Akzeptanz der Kunden darauf zu achten, dass das definierte Leistungsspektrum überschaubar bleibt und die Ausgestaltungsmerkmale der Leistungsvereinbarungen einer wirtschaftlichen Planung und Messung zugänglich sind. Hierdurch kann der notwendige Formalismus gesenkt werden. ${ }^{1075}$ Soweit die SLA's nicht verinnerlicht werden, besteht darüber hinaus die Gefahr, dass ehemals gute informelle Kunden-Lieferanten-Beziehungen belastet werden. ${ }^{1076}$ Bei einer sachgerechten Handhabung bieten SLA's und SLD's für den Controllerbereich zahlreiche Anhaltspunkte, zu einer Kommunikation des Leistungsan-

\footnotetext{
1069 Vgl. allg. Arbeitskreis „Leistungsvereinbarungen“" (1998), S. 10 ff.

${ }^{1070} \mathrm{Zu}$ obligatorischen Leistungen des Controlling vgl. Kap. 3.3.2.1.1.

1071 Allg. für interne Dienstleister vgl. Witt (1985), S. 163.

1072 Zur Kostenplanung im Controlling vgl. Weber (1990), S. 206 ff.

1073 Allg. zur Verrechnung im Rahmen von Leistungsvereinbarungen vgl. Bölscher (2000), S. 357 ff.

${ }^{1074}$ Hierzu vgl. Wahrisch (1998), S. 340.

${ }^{1075}$ Zum Formalismusempfinden vgl. auch Witt (1988), S. 670.

${ }^{1076} \mathrm{Vgl}$. Winkelmann-Ackermann/Bundi (1999), S. 39.
} 
gebotes beizutragen, die Leistungs- und Qualitätsplanung zu unterstützen sowie das Leistungsangebot inhaltlich, zeitlich und quantitativ an den Bedürfnissen der Kunden auszurichten. Wie dies beispielhaft erfolgen kann, soll nachfolgend gezeigt werden.

\subsubsection{Anwendung von Service Level Agreements und Service Level Defintions im Controlling}

Die Anwendung von SLA's und SLD's im Controlling unterliegt jeweils kontextspezifischen Rahmenbedingungen. Daher soll im Folgenden ein Anwendungsbeispiel geschildert werden, um die Struktur von SLA's mit konkreten Inhalten zu füllen und Besonderheiten der unternehmensspezifischen Ausgestaltung zu erläutern. Bei dem zu betrachtenden Controllerbereich handelt es sich um das zentrale Controlling der ERICSSON GMBH.

Zunächst befasste sich das schwedische Mutterunternehmen TELEFONAKTIEBOLAGET LARS MAGNUS ERICSSON mit Reparaturarbeiten an Telegrafenanlagen, später dann begann man mit der Produktion von Telefonen. Der noch heute geltende zentrale Anspruch des Unternehmens ist, offene Telekommunikationslösungen aller Art zu entwickeln, da Kommunikation als ein menschliches Grundbedürfnis verstanden wird. ${ }^{1077}$ In Deutschland ist die ERICSSON GMBH als Tochter der LM ERICSSON seit den Fünfziger Jahren tätig. Neben dem operativen Bereich, liegt ein Schwerpunkt in Forschung und Entwicklung. Mit ca. 2300 Mitarbeitern erzielte ERICSSON in Deutschland einen Umsatz von 832 Millionen Euro. ${ }^{1078}$ Das Leistungsspektrum der ERICSSON GMBH umfasst hochentwickelte Systeme und Produkte für drahtgebundene und mobile Kommunikation in öffentlichen und privaten Netzen. ERICSSON unterhält neben der Hauptverwaltung in Düsseldorf noch zahlreiche Niederlassungen im Bundesgebiet, um die erforderliche Kundennähe sicherzustellen.

Die angestrebte technologische Führerschaft, verbunden mit dem Druck, dauerhaft Innovationen schaffen zu müssen, führt dazu, dass ausgehend vom Forschungs- und Entwicklungsbereich auf allen Ebenen unternehmerisches Denken und Handeln gefördert werden muss. Diese Maxime gilt nicht nur für Unternehmensteilbereiche mit einem unmittelbaren Marktzugang, sondern auch für solche, die unternehmensinterne Dienstleistungen erbringen. Im Folgenden soll dargestellt werden, welche Auswirkungen derartige Tendenzen auf die Organi-

\footnotetext{
1077 Vgl. Ericsson (2000), S. 5.

${ }^{1078}$ Dies sind Werte des Jahrs 1999.
} 
sation und das Leistungserstellungsverhalten des zentralen Controlling haben und inwieweit es gelingt, den Bedürfnissen der Leistungsadressaten über SLD's und SLA's gerecht zu werden.

Die Organisation der hier betrachteten ERICSSON GMBH zeichnet sich durch ihre spartenbezogene Gliederung aus, d.h. Produkte, Produktgruppen oder Geschäftsfelder werden als vorherrschende Gliederungsmerkmale verwandt. ${ }^{1079}$ Das Unternehmen weist vier Geschäftsfelder mit unterschiedlichen inhaltlichen Ausrichtungen auf. Die Organisation des Controlling ist durch ein zentrales Controlling in Form einer Stabsstelle und dezentrale Controllerstellen in der Linie der jeweiligen Geschäftsbereiche/Business Units (BU) gekennzeichnet.

Die Unterstellung des dezentralen Controllings erfolgt dabei nach dem ,dottedline-Prinzip“, d.h. fachlich ist das Controlling der Geschäftsbereiche der zentralen Stabsstelle unterstellt, disziplinarisch hingegen dem jeweiligen Geschäftsbereich. ${ }^{1080}$ Das zentrale Controlling ist Bestandteil der Stabsstelle „Finance, Controlling and Information Systems (IS)/Information Technologie (IT)“ (EDD/F). Neben dieser Stabsstelle gibt es fünf weitere, die sich mit den Bereichen Recht, Logistik, Qualität, Information und Personal beschäftigen. Der derzeitige Stand der Organisation ist das Ergebnis einer Entwicklung, zu deren Beginn die heutigen Geschäftsbereiche ehemals eigene Unternehmen darstellten. Im Rahmen der Zusammenfassung wurden Aufgaben zentralisiert, zu denen auch Teile der Controlling-Aufgaben zählten. Neben rein sachlichen Argumenten, die für eine teilweise zentrale Wahrnehmung gewisser ControllingAufgaben sprachen (Economies of Scale, etc.), fürte die Leistungstradition, verbunden mit spezifischen Präferenzen der Führung zu einen Verbleib gewisser Aufgaben in den einzelnen Geschäftsbereichen.

Im Rahmen darüber hinausgehender Überlegungen zu Etablierung überregionaler Shared Service Center wurde die Diskussion über die Notwendigkeit von Leistungsvereinbarungen angestoßen. ${ }^{1081}$ Über Shared Service Center hinaus scheint es zukünftig denkbar, dass nationale Controlling-Bereiche international um ehemals fest zugeordnete Kunden/Geschäftsbereiche im Konzernverbund konkurrieren. Ferner wird diskutiert, inwieweit Dienstleistungen des Control-

${ }^{1079}$ Der Begriff der Spartenorganisation wird auch synonym mit den Begriffen Divisional- bzw. Geschăftsfeldorganisation verwandt. Vgl. Weber (1999), S. $253 \mathrm{f}$.

${ }^{1080}$ Vgl. Horváth (1998), S. 811 ff. sowie Küpper (1997), S. 457 ff. Vgl. auch Kap. 3.3.1.1.

${ }^{1081}$ Zum Begriff Shared Service Center vgl. Schuurmans/Stoller (1998), S. 37 ff. sowie zur Anwendbarkeit auf das Controlling Kap. 3.3.1.1.2. 
ling auch zukünftig noch für Management Buy Out's, die rechtlich nicht mehr zum Konzernverbund gehören, erbracht werden können. Hiermit würde das Controlling gleichsam auf externen Märkten agieren.

All diese Entwicklungen deuten darauf hin, dass die Leistungserstellung des Controlling einem konkreten Kosten-, Qualitäts- und Wettbewerbsdruck ausgesetzt sind, so dass eine marktvergleichbare Präsentation und Fakturierung von Leistungen erforderlich wird.

Um diesen Ansprüchen gerecht zu werden wurden auf Basis einer differenzierten Analyse der Geschäftsprozesse Leistungen abgegrenzt und beschrieben. Ergänzend hierzu konnten Erkenntnisse aus der regelmäßigen Teilnahme an einem unternehmensübergreifenden Financial Management Benchmarking Programm einbezogen werden. ${ }^{1082}$ In diesem Programm werden für Leistungsgruppen wie bspw. „General Accounting and Reporting“ oder „Profitability and Cost Management"

- Cost Performance Measures,

- Cycle Time Measures sowie

- Efficiency and Quality Performance Measures

im Vergleich zu anderen Unternehmen erhoben. Auf Basis der über diese Messwerte möglichen Leistungsanalyse und -kritik lassen sich die in den SLD's beschriebenen Leistungen und Prozesse im Sinne einer unternehmensspezifischen Best Practice auffassen. In den Service Definitions werden Leistungsgruppen mit den dazugehörigen, vom Controlling zu erbringenden Einzelleistungen, dargestellt. Hierbei sind die Einzelleistungen im Sinne von Prozessschritten aufzufassen.

Abbildung 56 stellt die SLD's für die Leistungsgruppe „Durchführung des Forecasting" dar. Hierbei handelt es sich um den Planungsprozess für die Business Units.

${ }^{1082}$ Hierbei handelt es sich um ein zentral von der Unternehmensberatung PRICE WATERHOUSE COOPERS durchgeführtes Benchmarking Programm. 


\begin{tabular}{|c|c|}
\hline Finance Services / Controlling & \\
\hline \multicolumn{2}{|l|}{ Service Definition } \\
\hline Controlling & $\begin{array}{l}\text { Milwirkungs- } \\
\text { palichten des } \\
\text { Kunden }\end{array}$ \\
\hline Durchführung des Forecasting & \\
\hline $\begin{array}{l}\text { Entwicklung und Sicherstellung der Planungs / Forecasting Grundsätze } \\
\text { und Verfahrensweisen }\end{array}$ & \\
\hline EDD/F erstellt bis Ende Januar eine Grobplanung für den Jahres forecast. & \\
\hline $\begin{array}{l}\text { 1.1.2 EDD/F abemimmt die Vorbereitung eines integrierten Zeitplans für den } \\
\text { Forecastingprozess auf Quartalsbasis. Es erfolgt die Festschreibung allgemeiner } \\
\text { Verfahrensweisen und Forecastinggrundsătze (zB. Planungsstruktur, Planungsprozess, } \\
\text { Planungsschritte, Verantwortlichkeiten, Planparameter, Reporting Formate) bis spătestens } 4 \\
\text { Wochen vor Abgabetermin. }\end{array}$ & \\
\hline \multicolumn{2}{|l|}{$\begin{array}{l}\text { I.1.3 Die Erstellung des Forecas ting erfolgt nach den spezifischen Richtlinien für die } \\
\text { Business Units (BU) und gemäB den Anforderungen der Konzernmutter, soweit erforderlich. }\end{array}$} \\
\hline Durchfuhrung des Forecasting Prozesses (quartal sweise) & \\
\hline $\begin{array}{l}\text { 1.2.1 Nach der Festlegung der Planstruktur (z.B. Projekte, Kostenstellen, Kunden) werden } \\
\text { die Planungsmerkmale von EDD/F festgelegt (z.B. Zahlungsziele, Durchlaufzeiten, Work in } \\
\text { Progress, Anzahlungen). }\end{array}$ & $\begin{array}{l}\text { E.2.3.1.2 } \\
\text { E.2.3.1.3 }\end{array}$ \\
\hline $\begin{array}{l}\text { 1.2.2 Für die Aufstellung einer Planbas is erfolgt die Emittlung der Kosten furr Business } \\
\text { Support Functions-Aktivităten (z.B. Finance, HR, etc.), die Festlegung der Planparameter } \\
\text { (Fremdwăhrungskurse, Zinssătze etc.) und einer Plan-Daten-Bilanz bei EDD/F. }\end{array}$ & E.2.3.1.4 \\
\hline $\begin{array}{l}\text { 1.2.3 Die Stammdaten werden in Anlehnung an die vom Kunden kommunizierten } \\
\text { Notwendigkeiten angelegt, bei Bedarf aktualis iert und die Tools entsprechend angepasst. }\end{array}$ & E2.3.1.6 \\
\hline Die vom Kunden bereitges teltten Ist-Daten werden in den Input-Dateien engănzt. & E.2.3.1.3 \\
\hline Die Freigabe der Input-Dateien erfolgt bei EDD/F. & \\
\hline $\begin{array}{l}\text { 1.2.6 Die Festlegung der Run Rates (Hochrechnung der Kosten auf Bas is Ist-Daten) für } \\
\text { Gemeinkosten und Herstellkosten des Umsatzes (Cost of Sales) pro Planungsmerkmal für die } \\
\text { veschiedenen Aggregationsstufen (Marge pro Produkt, Vertriebsgemeinkosten pro } \\
\text { Verkaufsbüro etc.) erfolgt auf Basis der vom Kunden mitgeteilten Ist-Daten. }\end{array}$ & E.2.3.1.3 \\
\hline $\begin{array}{l}\text { 1.2.7 EDD/F erstellt eine Vorkalkulierung der Bilanz auf Basis der Ist- und Plan-Daten (Plan- } \\
\text { Daten fur Zahlungsziele, Durchlaufzeiten, Investitionen, Anzahlungen etc. als Input vom } \\
\text { Kunden) und fuhrt eine grobe Extrapolation (Umsatze, unkorrigierte Marge, Aufwendungen) } \\
\text { durch. In AnschluB an das Review durch den Kunden wird der Rolling Forecast vervollstăndigt. }\end{array}$ & E.2.3.1.3 \\
\hline 1.2.8 Die Konsolidienung der Forecast Daten erfolgt durch EDD/F. & \\
\hline $\begin{array}{l}\text { 1.2.9 EDD/F diskutiert die Forecastingdaten (Abweichungsanalyse) gemeinsam mit dem } \\
\text { Controlling des Kunden und erfaßt die nach der Diskussion der Planungsdaten eventuell } \\
\text { notwendigen Änderungen. Im Falle notwendiger Ånderungen aus dem Controllermeeting } \\
\text { erfolgt die emeute Konsolidierung. }\end{array}$ & \\
\hline $\begin{array}{l}\text { 1.2.10 Auf Bas is der Ergebnisse aus dem Controllermeeting erfolgt die Ergebnisprăsentation } \\
\text { und -diskussion gemeinsam von EDD/F und dem Management des Kunden. }\end{array}$ & \\
\hline $\begin{array}{l}\text { 1.2.11 Nach der Ergebnisprăsentation werden notwendige Korrekturen / Management } \\
\text { Adjustments erfaBt. }\end{array}$ & \\
\hline 1.2.12 Die angepaßten Forecast-Daten werden in das Management Reporting integriert. & \\
\hline $\begin{array}{l}\text { 1.2.13 Die fur das Forecasting zu erstellenden Financial Reporting-Reports werden im,SLA } \\
\text { Hauptbuchhaltung' aufgefuhrt. }\end{array}$ & \\
\hline
\end{tabular}

\section{Abbildung 56: SLD „Durchführung des Forecasting “1083}

In den SLD's finden sich neben der Leistungsdefinition Verweise auf konkrete Mitwirkungspflichten der Kunden. Die vertragliche Fixierung derartiger Pflichten ist insbesondere bei sequenzintegrierten Leistungen des Controlling, ${ }^{1084}$ wie Planungsaktivitäten, unabdingbar, da sonst im Ergebnis die vom Kunden ange-

${ }^{1083} \mathrm{Vgl}$. Ericsson (2000b), S. 51.

${ }^{1084} \mathrm{Zu}$ sequenzintegrierten Leistungen des Controlling vgl. Kap. 3.3.2.3,1. 
strebte Planungssicherheit nicht erreicht werden kann. Mitwirkungspflichten dienen gerade auch im Bereich der in Abbildung 57 dargestellten Margen- und Abweichungsanalysen einer Förderung des Informationsflusses und einer Verbesserung des Schnittstellenmangements.

\begin{tabular}{|c|c|}
\hline Finance Services / Controlling & \\
\hline Service Definition & . \\
\hline Controlling & $\begin{array}{l}\text { Mitwirkungs - } \\
\text { pflichten des } \\
\text { Kunden } \\
\end{array}$ \\
\hline Kostenrechnung und Controlling & \\
\hline Stammdatenpfiege Im Controlling & \\
\hline$(\ldots)$ & \\
\hline Durchführung der Inventar-Buchführung & \\
\hline$(\ldots)$ & \\
\hline $\begin{array}{ll}2.3 & \begin{array}{l}\text { Durchfuhrung von Margenanalyse und Abweichungsanalyse, } \\
\text { Inkl. Iokale Ergebnisermittlung }\end{array} \\
\end{array}$ & \\
\hline $\begin{array}{l}\text { 2.3.1 EDD/F prüft die Profit Center-Ergebnisse pro Bereich (Basis: Umlage, Kundenauftrăge } \\
\text { in SAP-CO, Umlage Gemeinkosten) durch technische Prüfung der SAP Zahlen (Abgleich } \\
\text { zwischen den verschiedenen SAP-Modulen) und korrigiert die Zahlen bei Bedarf. }\end{array}$ & \\
\hline $\begin{array}{l}\text { 2.3.2 EDD/F übernimmt den Download der relevanten SAP-Berichte und die strukturelle } \\
\text { Aufbereitung fur Analysezwecke. }\end{array}$ & \\
\hline $\begin{array}{l}\text { 2.3.3 Die Zusammensetzung von automatischen Rückstellungen und Work in Progress für } \\
\text { Kundenaufträge wird monatlich auf Basis der Daten aus SAP-CO analysiert. }\end{array}$ & \\
\hline $\begin{array}{l}\text { 2.3.4 EDD/F diskutiert ggf. die Werte für die Auflösung und Bildung der Rückstellungen } \\
\text { und die Abwertung von Work in Progress mit dem Kunden. }\end{array}$ & E.2.3.1.10 \\
\hline $\begin{array}{l}\text { 2.3.5 In Ausnahmesituationen werden die Ist-Daten korrigiert, d.h. es wird ein } \\
\text { Buchungsbeleg furr EDD/F erstellt und die Umlage der Gemeinkosten wird erneut gestartet. }\end{array}$ & E.2.3.1.8 \\
\hline $\begin{array}{l}\text { 2.3.6 EDD/F verfolgt eine kontinuierliche Qualitätsverbesserung durch Probelauf der } \\
\text { Ergebnisermittlung im Monat und rechtzeitige Mitteilung zu notwendigen Korrekturen der } \\
\text { Kundenaufträge. Bei technischen Fehlem in SAP übernimmt EDD/F die Fehlermeldung an } \\
\text { Information Systems (IS) und die Begleitung der Call-Bearbeitung durch IS (Auftragssicherung). }\end{array}$ & E.2.3.1.8 \\
\hline $\begin{array}{l}2.3 .7 \quad \text { Ca. } 50 \% \text { des Auftragsvolumens der fakturierten Aufträge im Monat werden mittels } \\
\text { Ausreißeranalyse bzw. statistischer Margenanalyse untersucht. Die statistisch ausgewerteten } \\
\text { Daten werden an den Kunden weitergeleitet. }\end{array}$ & E.2.3.1.8 \\
\hline $\begin{array}{l}\text { 2.3.8 EDD/F erzeugt SAP Downloads für die Erstellung von gemeinsam definierten } \\
\text { Standardreports für den Kunden (Gesantübers icht, Ergebnis des Verantwortungsbereichs, } \\
\text { Produktergebnis). }\end{array}$ & E.2.3.1.9 \\
\hline $\begin{array}{l}\text { 2.3.9 EDD/F übernimmt die termingerechte Soll-/Ist-Analyse und Kommentierung des } \\
\text { lokalen Monatsergebnisses sowie deren Kommunikation. }\end{array}$ & E.2.3.1.10 \\
\hline $\begin{array}{l}\text { 2.3.10 EDD/F präsentiert die Monatsergebnisse für den Bereich und prüft die Standardreports } \\
\text { mit dem Management des Kunden. }\end{array}$ & \\
\hline $\begin{array}{l}2.3 .11 \mathrm{EDD} / \mathrm{F} \text { steht für die Bearbeitung von Rückfragen und Begleitung von } \\
\text { Fehlerbehebungen zur Verfügung. }\end{array}$ & \\
\hline Profitabilitäts- Bericht & \\
\hline$(\ldots)$ & \\
\hline
\end{tabular}

Abbildung 57: SLD „Margen- und Abweichungsanalyse“"1085

${ }^{1085}$ Vgl. Ericsson (2000b), S. 52 und S. 53. 
Die Mitwirkungspflichten sind als Ausdruck der Integrativität der Leistungserstellung im Controlling aufzufassen. ${ }^{1086}$ Was im Einzelnen unter den Mitwirkungspflichten zu verstehen ist, zeigt - bezogen auf das Beispiel - Abbildung 58 ausschnittsweise.

\begin{tabular}{|c|c|c|}
\hline \multicolumn{3}{|c|}{ 2.3 Mitwirkungspflichten } \\
\hline \multirow{2}{*}{\begin{tabular}{|l|} 
E2.3.1 \\
E2.3.1.1 \\
\end{tabular}} & \multicolumn{2}{|c|}{ Mitwirkungs leistungen des Kunden } \\
\hline & Strategie & $\begin{array}{l}\text { Ånderungen in der Strategie spiegeln sich in der IS-Strategie wider (zB. zu } \\
\text { Releasewechsel): s. SLA IS. }\end{array}$ \\
\hline E2.3.1.2 & Struktur Forecast & $\begin{array}{l}\text { Der Kunde teilt Ānderungs bedarf der Strukturmerkmale (Produkte, Verkaufsbüros, } \\
\text { Kunden) für die Erstellung der Forecaststruktur rechtzeitig mit. }\end{array}$ \\
\hline E2.3.1.3 & $\begin{array}{l}\text { Planungsmerkmale } \\
\text { und -parameter }\end{array}$ & $\begin{array}{l}\text { Der Kunde ist fur die rechtzeitige Bereitstellung und ggf. Óberprufung der } \\
\text { Planungsmerkmale (Zahlungsziele, Durchlaufzeiten, Anzahlungen) und } \\
\text { Planungsparameter (zB. Fremdwăhrungskurse, Zinssătze) gemäB dem } \\
\text { Forecastingzeitplan verantwortlich. }\end{array}$ \\
\hline E.2.3.1.4 & Plan-Bilanz & $\begin{array}{l}\text { Die fur die Erstellung der Plan-Bilanz relevanten Daten werden EDD/F rechtzeitig zur } \\
\text { Verfugung gestellt. }\end{array}$ \\
\hline E.2.3.1.5 & Wertberichtigung & $\begin{array}{l}\text { Der Kunde ubemimmt die sorgfaltige Prufung der von EDD/F generierten } \\
\text { Vorschlags listen für Einzel- und Pauschahwertberichtigungen (EWB, PWB) und die } \\
\text { Bewertung gemæB Niederstwertprinzip (NWP). }\end{array}$ \\
\hline \multirow[t]{3}{*}{ E2.3.1.6 } & Stammdatenpflege & $\begin{array}{l}\text { Das von EDD/F bereitgestellte Formblatt für Kostenstellen / Profit Center wird fur die } \\
\text { Anlage, Änderung oder Sperrung von Kostenstellen / Profit Centers vollständig und } \\
\text { fehlerfrei ausgefullt. }\end{array}$ \\
\hline & & $\begin{array}{l}\text { Bereitstellung der Daten für die Zuordnung der Kostenarten zur Standard- bzw. } \\
\text { Altemativhierarchie. } \\
\text { Der Kunde stellt die fur die Anlage und A ktualis ierung der Leistungsarten benðtigten } \\
\text { Tarife rechtzeitig bereit. }\end{array}$ \\
\hline & & \begin{tabular}{|l} 
Die für die Variantenpflege von SAP-Berichten benotigten Informationen fur \\
Variantenănderungen werden vollstăndig und termingerecht zur Verfügung gestellt.
\end{tabular} \\
\hline E2.3.1.7 & Materialwirtschaft & $\begin{array}{l}\text { Der Kunde abemimmt die Pflege der Preisliste, Produkthierarchien, Materialstammdaten } \\
\text { und die Bestellung aber SAP und liefert Daten zu Verschrottungswerten und Beträgen } \\
\text { fur Abwertung. }\end{array}$ \\
\hline \multirow[t]{3}{*}{ E.2.3.1.8 } & Kundenauftrăge & $\begin{array}{l}\text { Es erfolgt eine weitestgehend fehlerfreie Erfassung der Kundenaufträge gemå Zeitplan } \\
\text { fur den Monatsabschluß und ggf. werden Korrekturen durch das Vertragsmanagement } \\
\text { des Kunden durchgefuhrt. }\end{array}$ \\
\hline & & $\begin{array}{l}\text { Der Kunde prüt die Plan-/Ist-Werte fur ,Product Codes ' und Kunden. Ex ubernimmt } \\
\text { eine Plaus ibilitătsprufung der Plandaten pro Kundenauftrag und abermittelt } \\
\text { notwendigen Ånderungsbedarf. }\end{array}$ \\
\hline & & $\begin{array}{l}\text { Der Kunde stellt Informationen fur notwendige Anpassungen aufgrund statistischer } \\
\text { Auswertungen für EDD/F bereit. }\end{array}$ \\
\hline E.2.3.1.9 & Standardreports & \begin{tabular}{|l} 
Fur zu erstellende Standardreports (zB. Gesamtabers icht, Niederlassungsergebnis, \\
Produktergebnis) sind die Struktur und Berichts inhalte gemeinsam zu definieren.
\end{tabular} \\
\hline E2.3.1.10 & Soll-/lst-Analyse & $\begin{array}{l}\text { Für die Analyse sind die Soll-Gro Ben (zB. Forecast, Vorjahreszahlen, Ziehwerte) zu } \\
\text { definieren und rechtzeitig an Controlling zu kommunizieren. }\end{array}$ \\
\hline E2.3.1.11 & $\begin{array}{l}\text { Neue Produkte und } \\
\text { Produkt listen }\end{array}$ & $\begin{array}{l}\text { Freigabeplăne von Produkten und neue Produktlisten werden spătestens I Woche vor } \\
\text { Beginn des Forecast bereitgestellt. }\end{array}$ \\
\hline E2.3.1.12 & Preisănderungen & $\begin{array}{l}\text { Neue Preise bzw. Vereinbarungen zu neuen oder angepaßten Trans ferpreisen werden } \\
\text { aufgrund ihrer Auswirkungen auf die Margenplanung spattestens } 1 \text { Woche vor Beginn } \\
\text { des Forecast bereitgestellt. }\end{array}$ \\
\hline E2.3.1.13 & Forecast & $\begin{array}{l}\text { Der Kunde liefert weitestgehend fehlerfreie Informationen für das Forecasting. Es wird } \\
\text { ein schneller Abstimmungsproze } B \text { zwischen EDD/F und dem Kunden angestrebt. }\end{array}$ \\
\hline$(\ldots)$ & & $(\ldots)$ \\
\hline
\end{tabular}

Abbildung 58: Ausgewählte Mitwirkungspflichten im Rahmen von SLA's ${ }^{1087}$

${ }^{1086} \mathrm{Vgl}$. Kap. 3.2.2.1.

${ }^{1087}$ Vgl. Ericsson (2000b), S. 55. 
Betrachtet man die Abgrenzung von SLD's zu SLA's sind Mitwirkungspflichten zunächst Bestandteil der Leistungsvereinbarung. Der Bezug zu einzelnen Prozessschritten des Controlling erfordert darüber hinaus eine Zuordnung im Rahmen der SLD's. Welche weiteren Elemente Bestandteil der SLA's bei ERICSSON sind zeigt Abbildung 59.

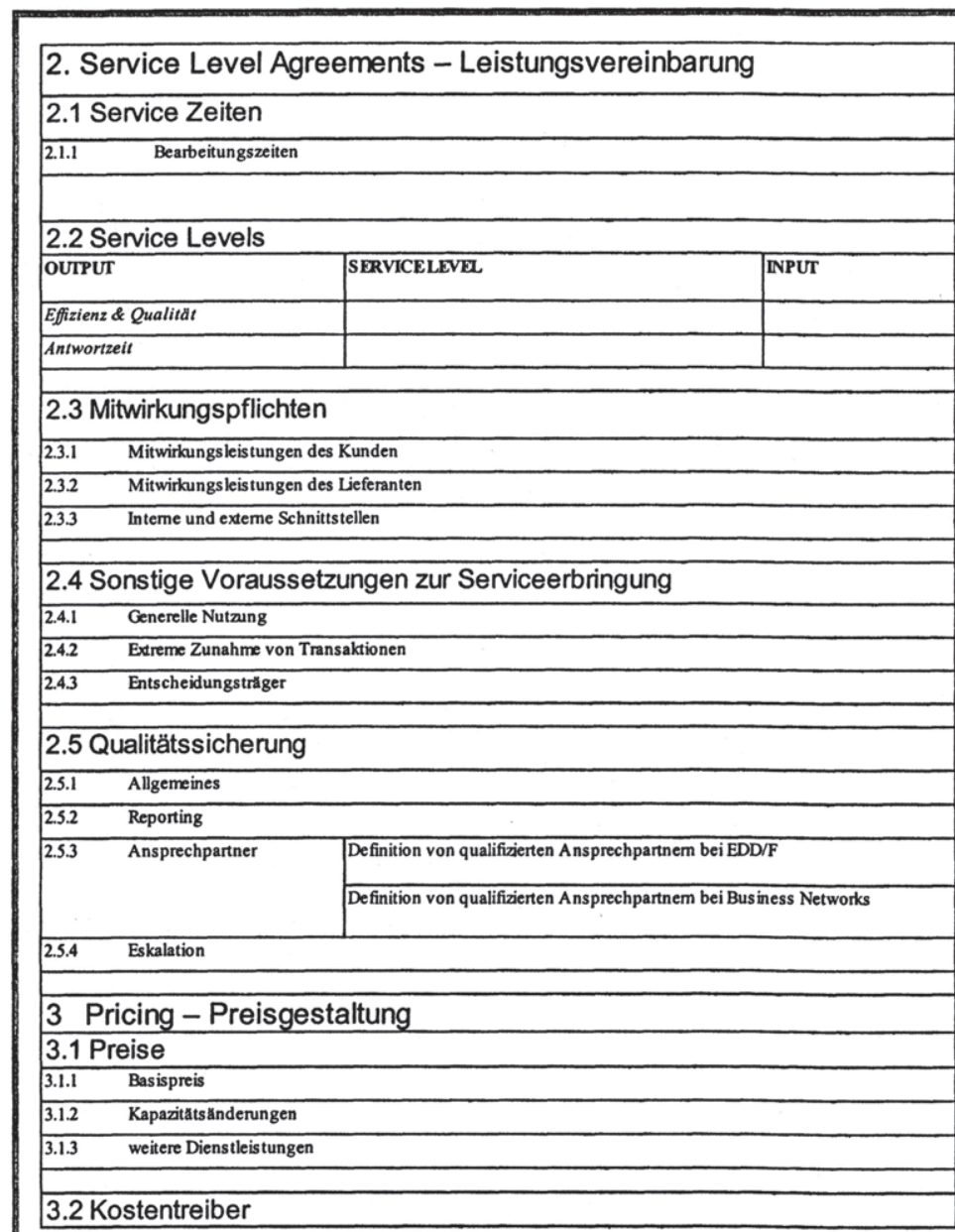

Abbildung 59: SLA-Struktur ${ }^{1088}$

${ }^{1088}$ Vgl. Ericsson (2000b), S. 54, S. 56 und S. 57. 
Ein derartiges SLA wird fur jede Leistungsgruppe und jeden Kunden spezifisch abgeschlossen. Der Übersichtlichkeit halber wurden hier in die einzelnen Kategorien des SLA keine konkreten Inhalte eingetragen. Vielmehr sollen deren Spezifikationen nachfolgend erläutert werden.

Im Einzelnen verbergen sicht hinter den Service Zeiten (2.1) die Bearbeitungszeiten, in denen das Controlling seinen Kunden zur Verfügung steht. ${ }^{1089}$ Die Service Zeiten werden über die Kernbearbeitungszeiten hinaus um Bereitschaftszeiten erweitert, ${ }^{1090}$ in denen zumindest ein Ansprechpartner im Controlling für Kunden zur Verfügung steht.

Ein Bestandteil der Service Levels (2.2) ist der inhaltliche Output des Controlling, der auf den leistungsgruppespezifischen SLD's basiert. Bezogen auf diesen werden Effizienz-, Qualitäts- und Terminanforderungen spezifiziert und ausgehandelt, ${ }^{1091}$ denen dass Controlling nachkommen muss, um die Zufriedenheit der Kunden sicherzustellen und die vertragliche Verpflichtung zu erfüllen. ${ }^{1092}$

Der Leistungsinput entspricht den bereits genannten, aber leistungsspezifisch zuzuordnenden Mitwirkungspflichten der Kunden (2.3.1). ${ }^{1093}$ Die Aufführung an dieser Stelle des SLA's erscheint unmittelbar notwendig, da Leistungsindikatoren, wie bspw. die Antwortzeit, zu ihrer Operationalisierung sowohl Leistungsinput wie -output benötigen. So gilt bspw. der Forecastingprozess als termingerecht fertiggestellt, wenn die Zeit zwischen dem Erhalt der ersten Informationen für den Forecast vom Kunden bis zur Fertigstellung des Forecasts dem ausgehandelten Zeitraum entspricht. ${ }^{1094}$ Unter den Mitwirkungsleistungen des Lieferanten (2.3.2) sind die im Rahmen der SLD's spezifizierten Einzelleistungen/Prozessschritte zu verstehen. Das unter Effizienzgesichtspunkten wesentliche Schnittstellenmanagement ${ }^{1095}(2.3 .3)$ zu internen und externen Kooperationspartnern wie Steuerberatern, Wirtschaftsprüfern, Lieferanten oder anderen

\footnotetext{
${ }^{1089}$ Bei ERICSsON entspricht dies Montag bis Donnerstag, 9-16 Uhr und Freitags 9-13 Uhr. Zugrundegelegt werden die Feiertage der BRD.

${ }^{1090}$ Dies ist Werktags von 8-18 Uhr der Fall.

1091 Die in den SLA's verwandten Begriffe geben insofern nicht die dieser Arbeit zugrundegelegte Abgrenzung von Effizienz und Effektivität wieder. Vgl. dazu Kap. 3.

1092 Die Operationalisierung von Effizienz und Effektivität erfolgt hierbei vergleichbar zu den getätigten Ausführungen zum Benchmarking und zur Balanced Scorecard.

${ }^{1093} \mathrm{Vgl}$. Abbildung 58.

1094 Vgl. Ericsson (2000), S. 54.

${ }^{1095} \mathrm{Zu}$ der Relevanz fitr das Controlling vgl. Stadler/Weißenberger (1999) S. 8 ff
} 
Dienstleistern bedarf ebenfalls einer Regelung im Rahmen der SLA's, wobei es hier zunächst Aufgabe des Kunden ist, relevante Schnittstellen zu benennen. Unter den sonstigen Voraussetzungen zur Service Erbringung ist bezüglich der generellen Nutzung (2.4.1) geregelt, dass das Controlling den Kunden frühzeitig auf Ressourcenengpässe, die nicht proportional zum Anstieg des Transaktionsvolumens stehen, hinzuweisen hat. Demgegenüber gelten die betroffenen Service Level bei einer extremen Zunahme eines bestimmten Transaktionsvolumens als ausgesetzt, bis die Normalauslastung wieder hergestellt ist (2.4.2). ${ }^{1096}$ Allgemein gilt es, dem Kunden einen entscheidungsbefugten Ansprechpartner für Eskalationszwecke zur Verfügung zu stellen (2.4.3).

Im Rahmen der Regelungen zur Qualitätssicherung der SLA's wird im allgemeinen Teil (2.5.1) die Verbindlichkeit der Einhaltung der Grundsätze ordnungsgemäßer Buchführung, der Datenschutz und Datensicherheitsgesetze sowie der Grundsätze ordnungsgemäßer Datenverarbeitung geregelt. Ferner gibt es bezüglich der SLA's ein eigenes Reporting, auf dessen Basis regelmäßig Abstimmungsgespräche über die Entwicklung der Leistungsvereinbarungen stattfinden, um eine kontinuierliche Qualitätssteigerung der SLA's zu ermöglichen. ${ }^{1097}$ Bei den unter 2.5.4. aufgefuihrten Eskalationsregelungen gilt es, in Abhängigkeit der Eskalationsursachen Ansprechpartner zu benennen, die zu einer Bereinigung der Situation beitragen können. Diese decken sich regelmäßig mit den unter 2.5.3 kunden- und anbieterseitig zu benennenden Ansprechpartnern. Differenziert wird bei den Ursachen der Eskalation zwischen dem nicht Einhalten der vereinbarten Service Levels aufgrund

- von Fehlleistungen des Controlling,

- extrem starker und kurzfristig gestiegener Transaktionsvolumina und

- vereinbarter aber nicht erbrachter Vorleistungen durch den Kunden.

Neben Verhandlungspositionen zu Leistungsinhalten und Abrufmodalitäten ist die Preisfixierung eine der wesentlichen Aufgaben der SLA's, da erst auf dieser Basis ein anreizkonformer Leistungsaustausch erfolgen kann. Bei ERICSSON hat man sich bezogen auf Standardleistungen zunächst für die Fakturierung einer Grundversorgung pro Periode, ${ }^{1098}$ im Sinne eines Basispreises (3.1.1) entschieden. Über die Benennung leistungsgruppenspezifischer Kostentreiber (3.2)

\footnotetext{
${ }^{1096}$ Das Aussetzen der betroffenen SLA's ist hierbei auf maximal drei Monate beschränkt.

${ }^{1097}$ Vgl. Ericsson (2000b), S. 57.

${ }^{1098} \mathrm{Zu}$ alternativen Verfahren der Verrechnung im Rahmen von Leistungsvereinbarungen vgl. Boblscher (2000), S. 357.
} 
kann beurteilt werden, inwieweit die Basispreise einer Überprüfung bedürfen. So werden bspw. für die Durchführung des Forecasting die Anzahl der Planobjekte (Produkt/Kunde/Niederlassung), ihre mengenmäßige und strukturelle Veränderung sowie die Anzahl der Veränderung der Planfaktoren (Durchlaufzeiten/Zahlungsverhalten/Umlagen) als Kostentreiber benannt. Tritt in bezug auf diese eine signifikante Änderung ein, gilt es, die Basispreise anzupassen. So werden in Abhängigkeit des kundenspezifischen Transaktionsvolumen bspw. folgende Positionen im Bereich Budgets/Forecast verrechnet: ${ }^{1099}$

- Koordinierung

- Erstellen

- Interne Analysen

- Comments

- SAP-Key User Beratung für Module FI und CO

- Umsetzung der regelmäßigen StrukturAnpassungen und Umlageveränderungen

- Cost Center Accounting
4.500 EUR/Jahr

19.200 EUR/Jahr

6.000 EUR/Jahr

$1.600 \mathrm{EUR} / \mathrm{Jahr}$

15.000 EUR/Jahr

20.000 EUR/Jahr

$35.000 \mathrm{EUR} / \mathrm{Jahr}$

Ändern sich mehr als drei der oben genannten Kostentreiber um mehr als 10\%, sind die hier dargestellten Basispreise neu zu vereinbaren. Bei Kapazitätsänderungen können die in den SLD's beschriebenen Dienstleistungen unter Berücksichtigung der verfügbaren Kapazitäten und evtl. zusätzlich zu vereinbarender Mitwirkungspflichten in angemessenem Rahmen ausgeweitet bzw. reduziert werden. Projektleistungen (3.1.3.) werden grundsätzlich auf Basis einer Aufwandskalkulation verrechnet, die zwischen Controlling und Kunde abgestimmt wird. ${ }^{.100}$

Auf Grundlage der hier für die zentralen Controlling-Leistungen der ERICSSON GMBH vorgestellten SLA's wird deutlich, dass der Nutzen insbesondere in der Schaffung marktvergleichbarer Rahmenbedingungen zu sehen ist, was regelmäßig im Sinne der Kunden ist. Analog zu den Marktverhältnissen muss über den Verhandlungsprozess ein Interessensausgleich herbeigefuihrt werden, was eine Intensivierung des Informationsaustausches erfordert. Hierdurch kann im Controlling eine gezieltere Berücksichtigung der Bedürfnisses alternativer Kunden erfolgen, bei gleichzeitiger Verbesserung der Kapazitätsplanung im

\footnotetext{
${ }^{1099} \mathrm{Vgl}$. Ericsson (2000b), S. 58.

${ }^{1100}$ Die starke Variation der Inanspruchnahme und die damit verbundene mangelnde Planbarkeit erfordert ein solches Vorgehen. Vgl. Bölscher (2000), S. 357.
} 
Controlling. Die Allokationsfunktion interner Märkte kann langfristig bei der Existenz von zentralem und dezentralem Controlling organisatorisch dazu führen, dass der Zentralisationsgrad der intern erbrachten Controlling-Leistungen Ergebnis der Auseinandersetzung interner Wettbewerbsteilnehmer wird. ${ }^{101}$

Insgesamt kann über den Einsatz von SLA's die Wahrnehmung des Controlling als Marktleistung, d.h. die Existenz eines transparenten Angebotes, potenzieller Wettbewerber und alternativer Kunden, deutlich verbessert werden. Über eine konsequente Orientierung an den Bedürfnissen der Kunden kann bei diesen dass Bewusstsein gefördert werden, dass Controlling-Leistungen zwar verfügbar, aber auch knapp und begehrt sind. ${ }^{1102}$

${ }^{1101}$ Vgl. Währisch (1998), S. 337.

${ }^{1102}$ Die Knappheit, Verfügbarkeit und Begehrtheit werden regelmäBig als Charakteristika zur Abgrenzung von Wirtschaftsgütern und freien Gutern verwandt. Vgl. Woll (2000), S 48 ff. 


\section{Resumee}

Da sich Controller mit der Forderung nach mehr interner Kundenorientierung immer öfter konfrontiert sehen, erscheint die Aufarbeitung dieses Problemkomplexes, sowohl aus theoretischer als auch aus praktischer Sicht erstrebenswert. Demgegenüber vermögen weder induktive Ausführungen der Unternehmenspraxis, noch deduktive Theorieansätze der Wissenschaft derzeit eine strukturiertes Bild über gestaltungsrelevante Zusammenhänge und einen abgestimmten Instrumenteneinsatz zur Absicherung der internen Kundenorientierung im Controlling zu geben. Zwar existieren einzelfallbezogene Darstellungen mit beschreibendem Charakter, eine systematische Integration von Erkenntnissen, der sich mit dem Phänomen Kundenorientierung originär beschäftigenden Marketingtheorie, erfolgt indes nicht angemessen.

Das Ziel dieser Arbeit ist daher, die Kundenorientierung im Controlling einer strukturierten Analyse mittels diesbezüglich relevanter Denkstrukturen des Marketing zu unterziehen. Um praxeologische, entscheidungstechnisch verwertbare Aussagen abzuleiten, werden auf Basis der gesammelten Erkenntnisse die Ausgestaltung und der Einsatz von Marketing-Instrumenten sowie Instrumenten des marktorientierten Controllings diskutiert.

Im Ergebnis wird der Controller in die Lage versetzt, Leistungen, Qualitäten und Preise systematisch auf die Bedürfnisse seiner Kunden auszurichten. Über die Generierung und Dokumentation eines spezifischen Nutzens für den Kunden, wird so mittelbar der Beitrag des Controlling zur Erreichung der Unternehmensziele abgesichert. Darüber hinaus kann über die Intensivierung der Inanspruchnahme und Nutzung problemlösungsadäquater Leistungen die Wahrnehmung und Wirksamkeit eines institutionalisierten Controllerbereichs verbessert werden. Mittels einer anreizkonformen Allokation von Leistung und Gegenleistung kann die Ressourcensituation im Controlling gestaltbar gemacht werden. Ferner kann über einen sachgerechten Instrumenteneinsatz sichergestellt werden, dass die verfügbaren Ressourcen effizient auf die Kundenpräferenzen ausgerichtet werden. Insgesamt kann so zu einer Stärkung der Wettbewerbsposition gegenüber externen und internen Anbietern beigetragen werden, wobei die potenziellen Wettbewerbsbeziehungen positiv im Sinne einer standzuhaltenden Innovationskonkurrenz zu interpretieren sind.

$\mathrm{Zu}$ diesem Zweck werden in Kap. 1 zunächst die Problemstellung und Zielsetzung spezifiziert, bevor eine problemlösungsadäquate Methodik und eine daraus abzuleitende Vorgehensweise vorgestellt werden. Auf dieser Basis wird in 
Kap. 2 die der Arbeit zugrundeliegende Controlling-Konzeption beschrieben sowie auf alternative, regelmäßig durch die institutionelle Ausgestaltung des Controlling geprägte Verständnisse eingegangen. Diese Betrachtung ist erforderlich, da die Wahrnehmung des Controlling durch Kunden im wesentlichen über die jeweils kontextspezifische Institution und deren Mitglieder bestimmt wird. Die diese Institutionen betreffenden Entwicklungstendenzen, deuten auf eine zunehmende Notwendigkeit der internen Kundenorientierung im Controlling hin. Im Rahmen der Sondierung diesbezüglicher Problemlösungsansätze, wird nach einer Darstellung der Grundlagen des Marketing auf dessen Entwicklungstendenzen Dienstleistungsmarketing und internes Marketing eingegangen. Neben der aus dem Marketing ableitbaren Anforderung, Kundenorientierung im Sinne einer übergeordneten Denkhaltung abzusichern, vermag das abteilungs- bzw. funktionsorientierte interne Marketing auf Grundlage des TQM-Ansatzes gezielte Strukturierungshinweise für interne KundenLieferanten-Beziehungen zu geben. Zum Abschluss des Kapitels 2 wird aufbauend auf einer Integration von Problemstellung und potenziellen Problemlösungen die weitere Vorgehensweise abgeleitet.

In Kap. 3 wird zunächst auf Basis einer Spezifikation der Ziele des kundenorientierten Controllings verdeutlicht, wie die damit verbundenen Ansprüche im Führungsgesamtsystem eines Controllerbereichs zu verankern sind. Auf Grundlage einer mittels des Marketing Dreiecks abgeleiteten Struktur, werden nachfolgend, die für eine kundenorientierte Ausrichtung des Controlling relevanten Rahmenbedingungen und Gestaltungsparameter analysiert. Die Struktur sieht hierbei eine Differenzierung nach Leistungs-, Organisations-, sowie Kundenund Wettbewerbsbezug vor.

Im Rahmen der Betrachtung von Leistungen wird aufbauend auf die Diskussion zu Konzept und Verständnis des Controlling, die im Rahmen der Institutionalisierung vorzunehmende Aufgabenzuordnung diskutiert, da über die Zuordnung von Aufgaben das Leistungsspektrum des Controlling determiniert wird. Darauf aufbauend wird ein für die Marketingbetrachtung abstrahiertes Leistungsspektrum des Controllers vorgestellt, welches im Sinne eines operativen Instrumentariums zur kundenorientierten Aufgabenerfüllung aufzufassen ist. Bezogen auf die Leistungen werden die Beurteilungsdimensionen als differenziert qualitätswahrnehmungsrelevant aufgefasst und in Hinblick auf ihre Gestaltbarkeit bewertet. Darüber hinaus bieten die informationsökonomischen Leistungseigenschaften Anhaltpunkte, die Beurteilbarkeit einzelner Leistungen des Controlling aus Perspektive der Nachfrager zu klassifizieren. Von besonderer Bedeutung ist 
ferner die Betrachtung der Inhaltscharakteristika einzelner Leistungen, da die kundenorientierte Ausgestaltung des Controlling oftmals zunächst nur auf Unterstützungsleistungen beschränkt wird. Kontrollleistungen, als Leistungen bei denen die Person des Kontrollierten mit der des Kunden identisch ist, werden stets als Bestandteil eines umfassenden Entscheidungsunterstützungsprozesses aufgefasst. Derartige Prozesse sind die Grundlage einer dauerhaften, partnerschaftlich ausgerichteten Kunden-Lieferanten-Beziehung, in der es gerade im Sinne des Kunden ist, kurzfristig auf kontrollinduzierte Defizite hingewiesen zu werden, soweit hierdurch seine langfristige Zielerreichung abgesichert werden kann. So verstanden können auch Kontrollleistungen kundenorientiert erbracht werden, wenn nicht explizit von einem vorsätzlich opportunistischen Verhalten des Kunden ausgegangen wird.

Im Rahmen der Analyse organisationaler Rahmenbedingungen des Controlling werden alternative Formen der organisatorischen Einbindung und unterschiedliche Ansätze einer ökonomischen Ausrichtung von Controllerbereichen diskutiert. Die so charakterisierte Einordnung des Controlling in die Organisation erfordert im Sinne des Marketing eine Strukturierung des Leistungsaustauschs mit anderen Organisationsmitgliedern. Diesbezüglich wird im Rahmen der Leistungsinanspruchnahme diskutiert, inwieweit obligatorische Leistungen eine marktgerechte Allokation zulassen, bzw. über Maßnahmen der Evidenzsteigerung in fakultative Leistungen transformiert werden können. Abrufmodalitäten und Ablaufscharakteristika widmen sich demgegenüber den formalen Rahmenbedingungen eines marktgerechten Leistungsaustauschs und der zeitlichen Integration von Controlling-Leistungen in die Prozesse seiner Kunden. Neben der formalen Ausgestaltung von Transaktionen des Controlling spielen weiterhin informelle Kontakte sowie die Leistungstradition in Unternehmen bei internen Dienstleistungen eine grundlegende Rolle. Als Ausdruck der ökonomischen Ausrichtung wird die Leistungsverrechnung des Controlling am Ende der organisationsbezogenen Gestaltungsparameter diskutiert, um Potenziale einer anreizkonformen internen Leistungsallokation zu ergründen.

Die Betrachtung der für das Controlling maßgeblichen kunden- und wettbewerbsbezogenen Gestaltungsparameter erfolgt auf Basis einer differenzierten Abgrenzung interner und externer Marktteilnehmer. Der Kundenkreis des Controlling wird im Rahmen der Arbeit auf interne Kunden beschränkt, da nur diese eine unmittelbar kundenorientierte Ansprache zulassen. Als Wettbewerber werden sowohl interne wie externe Anbieter von aus Nachfragersicht wahrgenommenen Leistungssubstituten angesehen. Im Sinne der Kunden erweisen sich ins- 
besondere die Leistungsindividualisierung sowie die Leistungsevidenz als gestaltungsrelevant, da sie die Bedarfsgerechtigkeit der Leistungen sowie deren Wahrnehmung maßgeblich beeinflussen. Da im Rahmen der kundenorientierten Betrachtung des Controlling nicht die Leistungserbringung, sondern vielmehr die Leistungsnutzung als Ziel einer Transaktion angesehen werden muss, kommt der Ergründung und Gestaltung des Nutzungsverhaltens gleichsam hohe Bedeutung zu. Auf Grundlage der Wettbewerbsbetrachtung des Controlling wird abschließend zwischen Monopol- und Wettbewerbsdienstleistungen differenziert, da die Konkurrenzsituation nicht pauschal betrachtet werden kann, sondern jeweils einen konkreten Leistungsbezug aufweist. Hieraus ergeben sich unterschiedliche Implikationen in Hinblick auf die notwendige Vermarktungsintensität einzelner Leistungen.

Die Betrachtung der Gestaltungsparameter erlaubt dem Controller, strukturiert wesentliche - für seine Leistungstransaktionen erfolgskritische - Sachverhalte gedanklich zu durchdringen. Auf dieser Grundlage werden in Kap. 4 strategische und operative Instrumente dargestellt, die eine Institutionalisierung der Kundenorientierung im Controlling ermöglichen. Die Kenntnis der Gestaltungsparameter erlaubt an dieser Stelle, die bisher nur auf andere Betrachtungsobjekte angewandten Instrumente sachgerecht den Anforderungen des Controlling anzupassen. Im Einzelnen werden Instrumente zur strategischen Controllerbereichsplanung dargestellt, über die die grundsätzliche interne Ausrichtung des Controllerbereichs und seiner Leistungen systematisch analysiert und geplant werden kann. Im Rahmen der strategischen Marketingplanung gilt es für den Controller, langfristige Handlungsoptionen bezüglich seiner Kunden und Wettbewerber zu bewerten. Eine sachgerechte kundenbezogene Planung kann über die Analyse alternativer Informationsbedürfnisse erfolgen, die gleichsam als Segmentierungskriterien zu verstehen sind. Weitere operationale abgrenzungs- und anspracherelevante Kriterien werden im Rahmen der internen Kundenstrukturanalyse diskutiert. Die darauf folgende Betrachtung konkurrenzorientierter Strategien dient im wesentlichen dazu, die aus einer potenziellen Wettbewerbsituation erwachsenden Handlungsnotwendigkeiten und Optimierungspotenziale zu erkennen.

Der Fokus der im Rahmen der Leistungsanalyse und -planung im Controlling vorgestellten Instrumente liegt im wesentlichen auf deren Beitrag zur strukturierten Absicherung von Kundenanforderungen im Leistungsspektrum des Controllers. So vermag Benchmarking vergleichsbasiert, auf Grundlage abgegrenzter Leistungen und diesbezüglich spezifizierter Messgrößen, differenzierte An- 
haltspunkte zu einer kundengerechteren Leistungsgestaltung zu geben. Da der vom Controller angestrebte Kundenutzen vielfältige Entstehungsursachen haben kann, wird die Eignung der Balanced Scorecard zur Analyse der Performance im Controlling diskutiert. Neben einer expliziten Betrachtung der Kundenperspektive, ist im Rahmen der Performance-Planung und -Messung sicherzustellen, dass auch die Konfiguration der übrigen Perspektiven ein kundengerechtes Verhalten zulässt. Die hierdurch angestrebte Ausgewogenheit ist gleichsam als ein Ziel des Target Costing im Controlling anzusehen, da über eine Verbindung von objektiver Kostenbestimmung und subjektiver Kundenpräferenzbewertung ein Ausgleich der kundenseitig wahrgenommen Kosten-NutzenRelation verfolgt wird. Ferner kann über eine wettbewerbsorientierte Festlegung der Target Costs die Marktorientierung des Controlling gefördert werden. Eine institutionelle Absicherung die Kundenorientierung fördernder interner Marktverhältnisse ist gleichsam der zentrale Anspruch von Service Level Agreements und Service Level Definitions. Gerade die Intransparenz des Leistungsangebotes und des Leistungserstellungsverhaltens im Controlling kann über Service Level Definitions vielfach beseitigt werden. Der Einsatz verhandelter Service Level Agreements begünstigt darüber hinaus die bilaterale Implementierung des Service-Gedankens, was sowohl der Zielerreichung auf Kundenseite, als auch der Aufgabenerfüllung auf Seite des Controlling dienlich ist. Die Ausführungen zur internen Kundenorientierung des Controlling enden mit einem Resumee in Kap. 5.

Insgesamt vermag die Arbeit - im Sinne eines Controlling-Marketing - systematisch Gestaltungshinweise für ein kundengerechtes und unternehmenszielkonformes Controlling zu geben. Aufgrund der Vielschichtigkeit der abgeleiteten Gestaltungsparameter erscheint eine auf empirischer Basis durchzufuihrende Clusterung der Merkmalsausprägungen zweckmäßig, um für diese idealtypische Handlungsempfehlungen vorgeben zu können. Insbesondere auch die beispielhafte Auswahl der Instrumente des kundenorientierten Controlling lässt bezogen auf die Problemstellung weiteren Forschungsbedarf erkennen. Es ist davon auszugehen, dass die Übertragung weiterer, eventuell spezialisierten Teildisziplinen des Marketing entstammender Instrumente auf das Controlling zweckmäBig ist. So weisen mutmaßlich Ansätze des Business-to-Business Marketing, aufgrund der diesen zugrundeliegenden dauerhaften, partnerschaftlichen und daher bindungsintensiven Kunden-Lieferanten-Beziehungen, ein vergleichsweise großes Übertragungspotenzial auf. Ferner deutet der Informationscharakter vieler Controlling-Leistungen darauf hin, dass das derzeit noch wenig entwickelte Marketing für Informationsgüter und -anbieter zukünftig verwertbare 
Erkenntnisse bereithalten könnte. Insbesondere Analysen der Diffusionsprozesse von Informationsleistungen in Unternehmen und Ansätze einer bedeutungsinhalts- bzw. nutzungsartenabhängigen Segmentierung von Kunden sind in diesem Zusammenhang beachtenswert. Aufbauend auf die Ausführungen dieser Arbeit, erscheint eine strukturierte Integration einzelner Instrumente des kundenorientierten Controllings in Form eines Programms erstrebenswert, da hierdurch der Erhebungsaufwand mehreren Instrumenten zugrundeliegender Messwerte, wie bspw. der Kundenzufriedenheit, erheblich reduziert werden könnte.

Insgesamt gilt es für den Controller, sich zukünftig als Manager des unternehmensweiten Controllings zu verstehen, der seine Produkte wirtschaftlich und kundengerecht vermarkten muss. Bei der eigenen Leistungserstellung oder der Integration externer Controlling-Leistungen in die Controlling-Konzeption des Unternehmens muss er stets in den Dimensionen seiner kundenbezogenen Gestaltungsparameter denken. Der Nutzen der Anwendung darauf aufbauender Instrumente steigt erfahrungsgemäß mit einer zunehmenden Komplexität der das Controlling umgebenden Organisationsstrukturen und Leistungsverflechtungen, so dass die Wirtschaftlichkeit des Instrumenteneinsatzes jeweils kontextabhängig zu bewerten ist. Wesentlich für alle Controller ist hingegen die dahinterstehende Denkfigur, die unabhängig von der Institutionalisierung eines derartigen Controller-Marketings begreifen hilft, dass letztlich nur über eine konsequente und systematische Ausrichtung an den Kundenbedürfnissen die Controlling-Ziele erreichbar sind. Wirksamkeit erlangt die interne Kundenorientierung des Controlling jedoch nur dann, wenn beide Transaktionspartner Controller und Manager - eine derartige Denkhaltung ihrem Rollenverständnis zugrundelegen. 


\section{Literaturverzeichnis:}

Abell, D. E. (1980): Defining the Business. The Starting Point of Strategic Planing, Englewood Cliffs/New York 1980.

Adam, D. (1998): Produktionsmanagement, 9. Aufl., Wiesbaden 1998.

Adler, J. (1994): Informationsökonomische Fundierung von Austauschprozessen im Marketing, in: Arbeitspapier zur Marketingtheorie Nr. 3, Weiber, R. (Hrsg.), Trier 1994.

Agthe, K. (1960): Der Controller in der Organisation amerikanischer Unternehmungen, in: ZfO, Nr. 29/1960, S. 48-54.

Ahlert, D. (1994): Warenwirtschaftsmanagement und Controlling in der Konsumgüterdistribution - Betriebswirtschaftliche Grundlegung und praktische Herausforderungen aus Perspektive von Handel und Industrie, in: Integrierte Warenwirtschaftssysteme und Handelscontrolling, Ahlert, D./Olbrich, R. (Hrsg.), 3. Aufl., Stuttgart 1994, S. 3-112.

Albers, S./Eggert, K. (1988): Kundennähe, Strategie oder Schlagwort?, in: Marketing - Zeitschrift für Forschung und Praxis, Nr. 10/1988, S. 5-6.

Amshoff, B. (1993): Controlling in deutschen Unternehmungen: Realtypen, Kontext und Effizienz, 2. Aufl., Diss., Wiesbaden 1993.

Amshoff, B. (1996): Organisation des Controlling, in: Lexikon des Controlling, Schult, C. (Hrsg.), München/Wien 1996, S. 546-555.

Anderson, J. C./O'Reilly, C. A. III. (1981): Effects of an Organizational Control System on Managerial Satisfaction and Performance, in: Human Relations, Nr. 34/1981, S. 491-501.

Ansoff, I. (1966): Management-Strategie, München 1966.

Ansoff, I. (1976): Managing Surprise and Discontinuity - Strategic Response to Weak Signals, in: zfbf, Nr. 28/1976, S. 129-152. 
Anthony, R. N. (1970): Management Accounting Principles, Homewood I11 1970.

Arbeitskreis „Leistungsvereinbarungen” (1998): Leistungsvereinbarungen - Ein Instrument zur Steuerung von Dienstleistungen, in: Betriebswirtschaft und Finanzen, Schriftenreihe des Betriebswirtschaftlichen Ausschusses und des Finanzausschusses, Nr. 24, Verband der chemischen Industrie e.V. (Hrsg.), Mainz 1998.

Arrow, K. J. (1974): The Limits of Organization, New York 1974.

Aust, R. (1999): Kostenrechnung als unternehmensinterne Dienstleistung, Diss., Wiesbaden 1999.

Backhaus, K. (1990): Betriebswirtschaftliche Beratung von Unternehmen durch Wirtschaftsprüfer, in: Die Wirtschaftsprüfung, Nr. 23-24/1990, S. 680688.

Backhaus, K. (1997): Relationship Marketing - Ein neues Paradigma im Marketing?, in: Marktorientierte Unternehmensführung. Reflexionen - Denkanstöße - Perspektiven, Bruhn, M./Steffenhagen, H. (Hrsg.), Wiesbaden 1997, S. 19-35.

Backhaus, K. (1999): Industriegütermarketing, 6. Aufl., München 1999.

Backhaus, K./Aufderheide, D./Späth, G.-M. (1994): Marketing für Systemtechnologien, Stuttgart 1994.

Backhaus, K./Büschken, J./Voeth, M. (2000): Internationales Marketing, 3. Aufl., Stuttgart 2000.

Backhaus, K./Späth, G.-M. (1992): Unternehmensberatung durch Wirtschaftsprüfer: eine sortimentspolitische Grundsatzentscheidung, in: DBW, Nr. 6/1992, S. 761-776.

Bässler, T./Knust, P./Schindera, F. (1999): Operative Planung der internen Dienstleistungen bei der Hewlett-Packard $\mathrm{GmbH}$, in: Controlling, Nr. 6/1999, S. 267-275. 
Baetge, J. (1996): Lästige Pflichtübung, in: Manager Magazin, Nr. 10/1996, S. 169.

Baetge, J. (1997): Gesellschafterorientierung als Voraussetzung für Kundenund Marktorientierung, in: Marktorientierte Unternehmensführung, Festschrift für Meffert, H., Bruhn, M. u.a. (Hrsg.), Wiesbaden 1997, S. 103117.

Baetge, J.(1997b): Aktuelle Entwicklungen in der Rechnungslegung und Wirtschaftsprüfung, Reformbedarf - Perspektiven - Internationale Einflüsse, Düsseldorf 1997.

Baetge, J./Kirsch, H.-J./Thiele, S. (2001): Bilanzen, 5. Aufl., Düsseldorf 2001.

Baetge, J./Roß, P./Uthoff, C. (1994): Ziele und Aufgaben der internen Revision in Kreditinstituten, in: Aktuelle Schwerpunktaufgaben der Revisionstätigkeit (Wissenschaft für die Praxis, Abt. 3: Analysen, Band 8), Wissenschaftsförderung der Sparkassenorganisationen e.V.(Hrsg.), Bonn/Stuttgart 1994, S. 9-47.

Bauer, H. H. (1989): Marktabgrenzung, Berlin 1989.

Benz, K. (1998): Effizienz des Controlling - Analytische und empirische Ableitung von Merkmalen der Controlling-Effizienz, Diss., Bamberg 1998.

Berens, W. (1992): Beurteilung von Heuristiken: Neuorientierung und Vertiefung am Beispiel logistischer Probleme, Habil., Wiesbaden 1992.

Berens, W./Darius, P./Schmitting, W. (1997): Die Verwendung von Graphiken in Geschäftsberichten - Eine empirische Studie, in: Düsseldorfer Schriften zum Controlling, Berens, W. (Hrsg.), Band 2, Aachen 1997.

Berens, W./Hoffjan, A. (1998): Target Costing, in: Lexikon des Rechnungswesens, Handbuch der Bilanzierung und Prüfung, der Erlös-, Finanz-, Investitions- und Kostenrechnung, Busse von Colbe, W./Pellens, B. (Hrsg.), 4. Aufl., München/ Wien 1998, S. 685-687.

Berens, W./Hoffjan, A./Schmitting, W. (1999): Controlling in Fallstudien - Von Erbsenzählern und Zahlenzauberinnen, Stuttgart 1999. 
Berens, W./Hoffjan, A./Strack, M. (1995): Ökologiebezogenes Controlling umweltorientierte Koordination in kommunalen Versorgungsunternehmen, in: ZögU, Nr. 2/1995, S. 143-160.

Berg, H. (1992): Wettbewerbspolitik, in: Vahlens Kompendium der Wirtschaftstheorie und Wirtschaftspolitik, Bender et al. (Hrsg.), Band 2, 5. Aufl., München 1992, S. 239-300.

Berger, H. J. (1989): Controllers persönliches Marketing, in: Controller Magazin, Nr. 4/1989, S. 183-187.

Berger-Vogel, W. (2000): Zwei Drittel des Weges vom Zahlenknecht zum Coach sind zurück gelegt, in: is report, Nr. 9/2000, S. 6-9.

Berry, L. L./Parasuraman, A. (1999): Dienstleistungsmarketing fängt beim Mitarbeiter an, in: Internes Marketing. Integration der Kunden und Mitarbeiterorientierung. Grundlagen, Implementierung, Praxisbeispiele, Bruhn, M. (Hrsg.), 2. Aufl., Wiesbaden 1999. S. 87-110.

Berthel, J. (1992): Informationsbedarf, in: Handwörterbuch der Organisation, Frese, E. (Hrsg.), 3. Aufl., Stuttgart 1992, Sp. 872-886.

Bidlingmaier J. (1973): Marketing I, Reinbek bei Hamburg 1973.

Biel, A. (1988): Motivation und Controlling, in: Controller Magazin, Nr. 1/1988, S. 35-41.

Biel, A. (1996): Aufbau und Gestaltung eines Lean Controlling, in: Controlling, Nr. 1/1996, S. 50-57.

Blazek, A./Deyhle, A. (1986): Treasurer- und Controller-Aufgaben, in: FinanzControlling, Jehle, K./Blazek, A./Deyhle, A. (Hrsg.), 3. Aufl., München 1986.

Bleicher, K./Meyer, E. (1976): Führung in der Unternehmung - Formen und Modelle, Reinbek bei Hamburg 1976. 
Bölscher, A. (2000): Dienstleistungsvereinbarungen zur Steuerung der innerbetrieblichen Leistungserstellung und -verrechnung, in: Controlling, Nr. 7/2000, S. 355-359.

Böni, T./Britzelmeyer, B./Schlegel, M (1999): Ein IT-Produktkatalog als Basis für die Verrechnung von IV-Leistungen im Client-Server-Umfeld, in: Controller Magazin, Nr. 6/1999, S. 485-492.

Bouncken, R. B. (1995): Probleme der psychologischen Kundensegmentierung durch die Verkaufförderung, Marburg 1995.

Brenner, W./Horisberger, P. (1998): Von der input- zur outputorientierten Führung, in: io-Management, Nr. 12/1998, S. 18-26.

Brown, S. (1985): AMA Approves New Marketing Definition, in: Marketing News, Nr. 5/1985, S. 1.

Bruhn, M. (1990): Marketing: Grundlagen für Studium und Praxis, Wiesbaden 1990.

Bruhn, M (1994): Messung der Anforderungen an die Dienstleistungsqualität, in: Qualitätsmanagement im Unternehmen. Grundlagen, Methoden und Werkzeuge, Praxisbeispiele, Hansen, W./Jansen, H. H./Kamiske, G. F. (Hrsg.), Loseblattsammlung, Kapitel 09.02, Berlin 1994, S. 1-32.

Bruhn, M (1999): Verfahren zur Messung der Qualität interner Dienstleister, in: Internes Marketing. Integration der Kunden und Mitarbeiterorientierung, Grundlagen, Implementierung, Praxisbeispiele, Bruhn, M. (Hrsg.), 2. Aufl., Wiesbaden 1999, S. 539-575.

Buggert, W./Wielpütz, A. (1995): Target Costing: Grundlagen und Umsetzung des Zielkostenmanagements, München 1995.

Busch, V./Nölken, D./Tesch, R. (1999): Flexibilisierung von Unternehmensstrukturen auf der Basis von Center-Konzeptionen, in: Controlling, Nr. 10/1999, S. 477-486.

Camp, R. C. (1989): Benchmarking: The Search for Industry Best Practices that Lead to Superior Performance, New York 1989. 
Coenenberg, A. (1973): Verrechnungspreise zur Steuerung divisionalisierter Unternehmen, in: WiSt, Nr. 2/1973, S. 373-382.

Collins, B./Payne, A. (1991): Internal Marketing, in: European Management Journal, 1991, S. 261-270.

Controller Verein e.V. (o.J.): Controller Statements, Controller Verein e.V. (Hrsg.), Loseblattsammlung, Gauting o.J.

Corsten, H. (1985): Die Produktion von Dienstleistungen - Grundzüge einer Produktionswirtschaftslehre des tertiären Sektors, Berlin 1985.

Cramer, B. (1987): Marketing für innerbetriebliche Weiterbildungsleistungen, Diss., Frankfurt 1987.

Darby, M. R./Karni, E. (1973): Free Competition and the Optimal Amount of Fraud, in: Journal of Law and Economics, Nr. 16/1973, S. 67-88.

Davis, T. R. V. (1992): Internal Service Operations: Strategies for Increasing Their Effectiveness and Controlling Their Costs, in: Organizational Dynamics, Nr. 20/1992, Autumn, S. 5-22.

Deyhle, A. (1989): Quo vadis Controller?, in: Controller Magazin, Nr. 4/89, S. 180-181.

Deyhle, A. (1990): Controller Handbuch, Band I-IV, 3. Aufl., Gauting 1990.

Deyhle, A./Conrad, G. (1997): „How to train a Controller“, in: Die Kunst des Controlling, Gleich, R./Seidenschwarz, W. (Hrsg.), München 1997, S. 207-419.

Deyhle, A./Steigmeier, B. (1993): Controller und Controlling, Bern/Stuttgart/ Wien 1993.

Dietl, H. (1993): Institutionen und Zeit, Diss., Tübingen 1993.

Dyllick, T. (1992): Management der Umweltbeziehungen, Öffentliche Auseinandersetzung als Herausforderung, Wiesbaden 1992. 
Eccles, R. C. (1991): Wider das Primat der Zahlen - die neuen SteuerungsgröBen, in: Harvard Manager, Nr. 4/1991, S. 14-22.

Eichler, S. (1999): Trends im Controlling, in: krp, Nr. 5/1999, S. 263-264.

Engelhardt, W. H./Günter, B. (1983): Investitionsgüter-Marketing, 2. Aufl., Stuttgart/Köln/Berlin/Mainz 1983.

Engelhardt, W. H./Kleinaltenkamp, M./Reckenfelderbäumer, M. (1992): Dienstleistungen als Absatzobjekt, Arbeitsbericht Nr. 52 des Instituts für Unternehmensführung und Unternehmensforschung der Ruhr-Universität Bochum, Mai 1992.

Engelhardt, W. H./Kleinaltenkamp, M./Reckenfelderbäumer, M. (1993): Leistungsbündel als Absatzobjekte. Ein Ansatz zur Überwindung der Dichotomie von Sach- und Dienstleistungen, in: zfbf, Nr. 5/1993, S. 395-426.

Engelhardt, W. H./Schwab, W. (1982): Die Beschaffung von investiven Dienstleistungen, in: DBW, Nr. 4/1982, S. 503-513.

Epelman, M. S. (1994): Internal Service Guarantees: Putting the Idea of Internal Customers to Work, in: Proceedings of the $3^{\text {rd }}$ International Seminar on Service Management \& Marketing, Aix en Provence 1994, S. 1-13.

Ericsson (2000): Die Ericsson-Story - Mobilfunktechnik auf dem Weg zur universellen Informationsversorgung, Düssseldorf 2000.

Ericsson (2000b): Service Level Agreement - EDD/F \& Ericsson Business Networks, interner Entwurf, Februar 2000.

Eschenbach, R. (1997): Controlling als Führungsfunktion - Tendenzen und Kontext, in: Controllers Zukunft. Auftrag, Verantwortung, Rollenbild und Selbstverständnis der Controller, Rieder, L. (Hrsg.), Zürich 1997, S. 15 32.

Etzioni, A. (1964): Modern Organizations, Englewood Cliffs 1964.

Eversheim, W./Güthenke, G. (1999): Defizite von Center Konzeptionen für das produzierende Unternehmen, in: io Management, Nr. 9/1999, S. 18-23. 
Ewert, R./Wagenhofer, A. (2000): Interne Unternehmensrechnung, 4. Aufl., Berlin 2000.

Feind-Just, C. (1999): Das interne Benchmarking - ein strategisches Instrument im Marketing-Controlling einer Konzernzentrale mit Beteiligungsgesellschaften, in: Controller Magazin, Nr. 3/1999, S. 188-196.

Financial Executives Institute (FEI) (1962): Controllership and Treasurership Functions Defined by FEI, in: The Controller, Nr. 30/1962, S. 289.

Fischer, J. (1996): Prozessorientiertes Controlling - ein notwendiger Paradigmenwechsel?, in: Controlling, Nr. 8/1996, S. 222-231.

Fließ, S. (1995): Industrielles Kaufverhalten, in: Technischer Vertrieb, Kleinaltenkamp, M./Plinke, W. (Hrsg.), Heidelberg 1995, S. 287-395.

Flipo, J.-P. (1986): Service Firms: Independence of External and Internal Marketing Strategies, in: European Journal of Marketing, Nr. 8/1986, S. 5-14.

Franz, K.-P. (1993): Target Costing - Konzept und kritische Bereiche, in: Controlling, Nr. 3/1993, S. 124-130.

Franz, K.-P. (1997): Kostenmanagement in Deutschland - Ergebnisse einer empirischen Untersuchung in deutschen Großunternehmen, in: Kostenmangement, Wettbewerbsvorteile durch systematische Kostensteuerung, Franz, K.-P./Kajüter, P. (Hrsg.), Stuttgart 1997, S. 481-502.

Frese, E. (1993): Grundlagen der Organisation: Konzepte - Prinzipien - Strukturen, 5. Aufl., Wiesbaden 1996.

Freter, H. (1983): Marktsegmentierung, Stuttgart 1983.

Gabler (2000): Gablers Wirtschaftslexikon, 15. Aufl., Band 3, Wiesbaden 2000.

Gaitanides, M. (1993): Aufbau- und Ablauforganisation, in: HWB, 5. Aufl., Stuttgart 1993, Sp. 190-204. 
Galgenmüller, F./Gleich, R/Pfohl, M. (1999): Anwendungserfahrungen mit einem Performance Measurement-Konzept einer Logistikcontrollingeinheit der Daimler-Chrysler AG, in: Controlling, Nr. 11/1999, S. 535-541.

Gallager, C. A. (1974): Perceptions of the Value of a Management Information System, in: Academy of Management Review, Nr. 1/1974, S. 46-55.

Garbe, H. (1975): Informationsbedarf, in: HWB, Teilband 2, Grochla, E./Wittmann, W. (Hrsg.), 4. Aufl., Stuttgart 1975, Sp. 1873-1882.

Gaulhofer, M. (1987): Strategische Planung beim Controller?, in: ZfB, Nr. 11/1987, S. 1121-1127.

Gaulhofer, M. (1989): Controlling und menschliches Verhalten - Ein Plädoyer für die Einbeziehung verhaltenswissenschaftlicher Erkenntnisse in die Controlling-Diskussion, in: ZfB, Nr. 2/1989, S. 141-154.

George, S./Weimerskirch, A. (1994): Total Quality Management. Strategies and Techniques Proven at Todays Most Successful Companies, New York 1994.

Gleich, R. (1997): Performance Measurement, in: DBW, Nr. 1/1997, S. 114117.

Gleich, R. (1997b): Performance Measurement im Controlling, in: Die Kunst des Controlling, Gleich, R./Seidenschwarz, W. (Hrsg.), München 1997, S. 343-365.

Gleich, R./Brokemper, A. (1998): Kunden- und Marktorientierung im Controllerbereich schaffen - 7 Schritte zur kontinuierlichen Leistungsplanung und -steuerung, in: Controller Magazin, Nr. 2/1999, S. 148-156.

Gleich, R/Haindl, M. (1996): Performance Measurement im Controlling am Beispiel eines Unternehmens der chemischen Industrie, in: Controlling, Nr. 4/1996, S. 262-271.

Gmünden, H. G. (1993): Information: Bedarf, Analyse und Verhalten, in: Enzyklopädie der Betriebswirtschaftslehre, Wittmann, W. (Hrsg.), Band 2, Teilband 2. I-Q., 5. Aufl., München 1993, Sp. 1725 - 1735. 
Grob, H. L. (1995): Investitionsrechnung mit vollständigen Finanzplänen, 2. Aufl., München 1995.

Grochla, E. (1978): Einführung in die Organisationstheorie, Stuttgart 1978.

Grochla, E. (1980): Handwörterbuch Organisation, 2. Aufl., Stuttgart 1980.

Günter, B. (1997): Wettbewerbsvorteile, mehrstufige Kundenanalyse und Kunden-Feedback im Business-to-Business-Marketing, in: Marktleistung und Wettbewerb, Backhaus, K./Günter, B./Kleinaltenkamp, M./Plinke, W./Raffeé, H. (Hrsg.), Wiesbaden 1997, S. 213-231.

Günter, B./Kuhl, M. (1995): Beschaffungspolitik industrieller Nachfrager, in: Technischer Vertrieb, Kleinaltenkamp, M./Plinke, W. (Hrsg.), Berlin/Heidelberg 1995, S. 399-463.

Günter, B./Platzek, T. (1992): Management von Kundenzufriedenheit - Zur Gestaltung des After-Sales-Netzwerkes, in: Marktforschung und Management, Nr. 36/1992, S. 109-114.

Günther, J. (1999): Verrechnung von internen Controlling-Leistungen, in: Vortragsdokumentation der Konferenz "Der Controller als interner Berater", 10.-12.11.1999, Frankfurt am Main 1999.

Gummesson, E. (1996): Toward a Theoretical Framework of Relationship Marketing, in: International Conference on Relationship Marketing: Development, Management and Governance of Relationships, Sheth, J. N./Söllner, A. (Hrsg.), Berlin 1996, S. 5-18.

Gunn, R. W./Carberry, D. P./Frigo, R./Behrens, S. (1993): Shared Services Major companies are reengineering their accounting functions, in: Management Accounting, Nr. 11/1993, S. 22-28.

Gutenberg, E. (1984): Grundlagen der Betriebswirtschaftslehre, Band II: Der Absatz, 17. Aufl., Berlin 1984.

Gzuk, R. (1977): Messung der Effizienz von Entscheidungen, in: Wirtschaftliche Messprobleme, Pfohl, H. C./Rürup, B. (Hrsg.), Band 2, Köln 1977, S. 37-57. 
Hahn, D. (1991): Planungs- und Kontrollrechnung - PuK, 3. Aufl., Wiesbaden 1991.

Hahn, D. (1997): Controlling in Deutschland - State of the Art, in: Die Kunst des Controlling, Gleich, R./Seidenschwarz, W. (Hrsg.), München 1997, S. $13-46$.

Hahn, D./Hungenberg, H./Kaufmann, L. (1994): Optimale Make-or-buyEntscheidung, Entscheidungsmodell zur ganzheitlichen Beurteilung von Bezugsalternativen, in: Controlling, Nr. 2/1994, S. 74- 82.

Hahn, D./Laßmann, G. (1993): Produktionswirtschaft - Controlling industrieller Produktion, Band 3, 2. Teilband, Informationssystem, Heidelberg 1993.

Hamprecht, M.: Controlling von Konzernplanungssystemen, Diss., Wiesbaden 1996.

Hanan, M./Karp, P. (1991): Customer Satisfaction, How to Maximize, Measure an Market Your Company's „Ultimate Product”, New York 1991.

Harbert, L. (1982): Controlling-Begriffe und Controlling-Konzeptionen. Eine kritische Betrachtung des Entwicklungsstandes des Controlling und Möglichkeiten seiner Fortentwicklung, Diss., Bochum 1982.

Hartmann-Wendels, T./von Hinten, P. (1989): Marktwert von Vorzugsaktien, in: zfbf, Nr. 4/1989, S. 263-293.

Hartwig, K. H. (1992): Umweltökonomie, in: Vahlens Kompendium der Wirtschaftstheorie und Wirtschaftspolitik, Bender et al. (Hrsg.), Band 2, 5. Aufl., München 1992, S. 125-163.

Hayek, F. A. (1968): Der Wettbewerb als Entdeckungsverfahren, in: Kieler Vorträge, Nr. 56, Kiel 1968, S. 249-265.

Heinen, E. (1985): Betriebswirtschaftliche Kostenlehre: Kostentheorie u. Kostenentscheidungen, 6. Aufl., Wiesbaden 1985.

Hellmich, R. (1970): Entwicklung eines Planungssystems für Unternehmungen - Versuch einer allgemeinen Grundlegung, Winterthur 1970. 
Hentschel, B. (1992): Dienstleistungsqualität aus Kundensicht. Vom merkmalszum ereignisorientierten Ansatz, Wiesbaden 1992.

Herter, R. N. (1994): Strategische Erfolgsbeurteilung von dezentralen Organisationseinheiten auf Basis der Wertsteigerungsanalyse, München 1994.

Herzog, A. (1999): Gestaltung von Controllership - Die Zuordnung von Aufgaben zu Controllern, Diss., Wiesbaden 1999.

Heuck, J. (2000): Interne Unternehmensberatung am Beispiel der BASF, in: Internes Consulting, Niedereichholz, C. (Hrsg.), München/Wien 2000, S. 89-90.

Hill, W./Fehlbaum, R./Ulrich, P. (1989): Organisationslehre. Ziele, Instrumente und Bedingungen der Organisation sozialer Systeme, 4. Aufl., Bern/ Stuttgart 1989 und 1992.

Höffken, E. (1976): Die Nachfrage nach Kosteninformationen in Unternehmen, in: Schriften zur Unternehmensführung, Band 21, Wiesbaden 1976, S. 8596.

Höffken, E. (1991): Beiträge zur Betriebswirtschaft des Anlagenbaus, in: zfbf, Sonderheft Nr. 28.

Höltl, S. (1999): Implementierung des Management von Kundenzufriedenheit im Controllingsystem als Basis für Kundenorientierung und Prozessmanagement am Beispiel eines mittelständischen Unternehmens, Münster/Hamburg/London 1999.

Hoffjan, A. (1994): Strategisches Zielkostenmanagement für öffentliche Investitionen, in: Zeitschrift für öffentliche und gemeinwirtschaftliche Unternehmen, Nr. 1/1994, S. 24-38.

Hoffjan, A. (1997): Entwicklung einer verhaltensorientierten ControllingKonzeption für die Arbeitsverwaltung, Diss., 2. Aufl., Wiesbaden 1997.

Hoffmann, F. (1968): Der Controller im deutschen Industriebetrieb, in: DB, Nr. 21/1968, S. 2181-2185. 
Homburg, C. (1999): Marktorientierung der Kostenrechnung, in: krp, Nr. 1/1999, S. 7.

Homburg, C./Weber, J. (1999): Kundenorientiertes Rechnungswesen als Beispiel für interne Kunden-Lieferanten-Beziehungen, in: Internes Marketing, Bruhn, M. (Hrsg.), 2. Aufl., Wiesbaden 1999, S. 579-599.

Homburg, C./Weber, J./Aust, R./Karlshaus, J. T.(1998): Interne Kundenorientierung der Kostenrechnung, Schriftenreihe Advanced Controlling, Band 7, Vallendar 1998.

Hopp, F. W. (1992): Kriterien für ein effizientes Controlling - Gedanken eines Versicherers, in: Effektives und schlankes Controlling, Horváth, P. (Hrsg.), Stuttgart 1992, S. 87-288.

Horváth, P. (1978): Aufgaben und Stellung des Controllers, in: BFuP, Nr. 2/1978, S. 129-141.

Horváth, P. (1995): Der Kunde ist König - auch für das Controlling, Editorial, in: Controlling, Nr. 4/1995, S. 183.

Horváth, P. (1995b): Selbstorganisation und Controlling, in: Führungskräfte und Führungserfolg, Krystec, U./Link, F. (Hrsg.), Wiesbaden 1995, S. 255-267.

Horváth, P. (1998): Controlling, 7. Aufl., München 1998.

Horváth, P. (2001): Der Controller: Navigator der Führung, in: Frankfurter Allgemeine Zeitung, Nr. 122/2001, S. 33.

Horváth, P./Herter, R. N. (1992): Benchmarking - Vergleich mit den Besten der Besten, in: Controlling, Nr. 4/1992, S. 4-11.

Horváth, P./Seidenschwarz, W. (1992): Zielkostenmanagement, in: Controlling, Nr. 4/1992, S. 142-150.

Hoyer, H. (2000): Internes Consulting in Deutschland - Ergebnisse einer Marktuntersuchung, in: Internes Consulting, Niedereichholz, C. (Hrsg.), München/Wien 2000, S. 55-81. 
Hronec, S. M. (1993): Vital Signs: Using Quality, Time and Cost Performance Measurements to Chart your Company's Future, New York 1993.

Hummel, F. E. (1954): Market Potential in the Machine Tool Industry, in: Journal of Marketing, Nr. 3/1954, S. 34-41.

Hummel, R. T. (1999): Jüngere Entwicklungen im Kostenmanagement - Target Costing und Prozesskostenrechnung, in: Controlling - Kompendium für Controller/innen und deren Ausbildung, Steinle, C./Bruch, H. (Hrsg.), 2. Aufl., Stuttgart 1999, S. 492-504.

Ibach, M. (1997): Wettbewerbsfit auch in Finanzen und Controlling, in: TreasureyLog, Nr. 3/1997, S. 4-6.

Jakob, F. (1995): Produktindividualisierung. Ein Ansatz zur innovativen Leistungsgestaltung im Business-to-Business-Bereich, Wiesbaden 1995.

Junga, C. (2000): Entwicklung einer Controlling-Konzeption für Staatsanwaltschaften, Diss., Aachen 2000.

Kaas, K. P. (1992): Kontraktgütermarketing als Kooperation von Prinzipalen und Agenten, in: zfbf, Nr. 10/1992, S. 884-901.

Kaldewei, R. (1998): Profitcenterstrukturen und innerbetriebliche Verrechnung, in: krp, Sonderheft Nr. 1/1998, S. 31-34.

Kant, I. (1783): Prolegomena zu einer jeden künftigen Metaphysik, die als Wissenschaft wird auftreten können, Riga 1783.

Kaplan, R. S./Norton, D. P. (1996): The Balanced Scorecard: Translating Strategie into Action, Boston 1996.

Kaplan, R. S./Norton, D. P. (1996b): Using the Balanced Scorecard as a Strategic Management System, in: Harvard Business Review, Nr. 74/1996, S. 75-85.

Kaplan, R. S./Norton, D. P. (1997): Balanced Scorecard - Strategien erfolgreich umsetzen, Stuttgart 1997. 
Kargl, H. (1998): Der Wandel von der DV-Abteilung zum IT-Profitcenter, in: Controlling, Nr. 1/1998, S. 40-46.

Karlowitsch, M. (1997): Entwicklung einer Konzeption des verhaltenorientierten Controlling, in: Düsseldorfer Schriften zum Controlling, Berens W. (Hrsg.), Band 3, Düsseldorf 1997.

Karlowitsch, M. (1999): Controlling unter verhaltensorientierten Aspekten, in: Controlling in Fallstudien - Von Erbsenzählern und Zahlenzauberinnen, Berens, W./ Schmitting, W./Hoffjan, A. (Hrsg.), Stuttgart 1999, S. 9-32.

Kavandi, S. (1998): Ziel- und Prozesskostenmanagement als Controllinginstrumente zur Kostensteuerung unter Berücksichtigung neuer Marktentwicklungen, Diss., Wiesbaden 1998.

Kleinaltenkamp, M. (1995): Einführung in das Business-to-BusinessMarketing, in: Technischer Vertrieb, Kleinaltenkamp, M./Plinke, W. (Hrsg.), Heidelberg 1995, 135-191.

Kleinaltenkamp, M. (1997): Integrativität als Kern einer umfassenden Leistungslehre, in: Marktleistung und Wettbewerb, Backhaus, K./Günter, B./Kleinaltenkamp, M./Plinke, W./Raffeé, H. (Hrsg.), Wiesbaden 1997, S. 83-114.

Kleinaltenkamp, M. (1997b): Kundenintegration, in: WiSt, Nr. 6/1997, S. 350354.

Kleinaltenkamp, M. (2000): Wettbewerbsstrategie, in: Strategisches Businessto-Business Marketing, Heidelberg 2000, S. 57-192

Kleinaltenkamp, M./Fließ, S. (1995): Entwicklung einer strategischen Marketing-Konzeption, in: Technischer Vertrieb, Kleinaltenkamp, M./Plinke, W. (Hrsg.), Heidelberg 1995. S 947-1019.

Klingelbiel, N. (1998): Performance Management - Performance Measurement, in: Zeitschrift für Planung, Nr. 1/1998, S. 1-15.

Knauber, N./Kuloglu, H. C./Podrazil, U. M. (1997): Kundenorientierung durch Marktsegmentierung, in: Die Bank, Nr. 5/1997, S. 300-304. 
Koch, R. (1994): Betriebliches Berichtswesen als Informations- und Steuerungsinstrument, Diss., Frankfurt 1994.

Kosiol, E. (1980): Aufbauorganisation, in: HWO, 2. Aufl., Stuttgart 1980, Sp. 179-187.

Kossbiel, H. (1974): Arbeitsteilung, betriebliche, in: HWB, 4. Aufl., Berlin 1974, Sp. 256-262.

Kotler, P. (1967): Marketing-Management. Analysis, Planing and Control, Englewood Cliffs/New York 1967.

Kotler, P. (1999): Marketing Management, 9. Aufl., Stuttgart 1999.

Kotler, P./Bliemel, F. (1999): Marketing-Management, 9. Aufl., Stuttgart 1999.

Kraus, F. (2001): Pu der Bär, der Hundert-Sechzig-Morgen-Wald und das Controlling - eine Controlling-,Entschlüsselung“ aus „Winnie-the-Pooh“, in: Controller Magazin, Nr. 3/2001, S. 304-308.

Kreuter, A. (1999): Verrechnungspreise in Profit-Center-Organisationen, 2. Aufl., München 1999.

Kreuzer, C. (1995): Controlling-Audit, Ansätze zur Lösung eines Bewertungsproblems, Diss., Wien 1995.

Kühn, R. (1991): Methodische Überlegungen zum Umgang mit der Kundenorientierung im Marketing-Management, in: Marketing- Zeitschrift für Forschung und Praxis, Nr. 13/1991, S. 97-107.

Künzel, H. (1999): Management interner Kunden-Lieferanten-Beziehungen, Diss., Wiesbaden (1999)

Küpper, H.-U. (1997): Controlling - Konzeption, Aufgaben und Instrumente, 2. Aufl., Stuttgart, 1997.

Küpper, H.-U./Weber, J./Zünd, A.(1990): Zum Verständnis und Selbstverständnis des Controlling - Thesen zur Konsensbildung, in: ZfB, Nr. 3/1990, S. 281-293. 
Küpper, H.-U./Winckler, B./Zhang, S. (1990): Planungsverfahren und Planungsinformationen als Instrumente des Controlling, in: DBW, Nr. 4/1990, S. 435-458.

Lanter, N. (1996): Beziehungsdynamik im Controlling, Diss., Bern/Stuttgart/ Wien 1996.

Lehner, U. (1989): Organisation des Controller-Bereichs, in: Controller Magazin, Nr. 4/1989, S. 189-194.

Lube, M.-M. (1997): Kundenmanagement - Die Kundenbeziehung als neue Bezugsgröße des Controlling, in: Controller Magazin, Nr. 3/1997, S. 183189.

Ludwig, W. F. (1995): Mehr Mitarbeiter- und Kundenzufriedenheit durch internes Unternehmertum, in: Kundenzufriedenheit, Simon, H./Homburg, C. (Hrsg.), Wiesbaden 1995, S. 127-141.

Mann, R. (o.J.): Die Praxis des Controlling, Instrumente - Einführung - Konflikte, München o.J.

McCarthy, J. (1964): Basic Marketing: A Managerial Approach, Homewood/I11 1964.

Meffert, H. (1982): 16 Meffert-Thesen zu Marketing und Controlling, in: Absatzwirtschaft, Nr. 9/1982, S. 100-107.

Meffert, H. (1993): Marktorientierte Führung von Dienstleistungsunternehmen. Neuere Entwicklungen in Theorie und Praxis, in: Arbeitspapier Nr. 78 der Wissenschaftlichen Gesellschaft für Marketing und Unternehmensführung e.V., Meffert, H./Wagner, H. (Hrsg.), Münster 1993.

Meffert, H. (1994): Marktorientierte Führung von Dienstleistungsunternehmen neuere Entwicklungen in Theorie und Praxis, in: DBW, Nr. 4/1994, S. 519-541.

Meffert, H. (1999): Marktorientierte Unternehmensführung im Umbruch, in: Marktorientierte Unternehmensfuihrung im Wandel, Meffert, H. (Hrsg.), Wiesbaden 1999, S. 3-33. 
Meffert, H. (2000): Marketing, Grundlagen marktorientierter Unternehmensführung, 9. Aufl., Wiesbaden 2000.

Meffert, H./Bruhn, M. (1995): Dienstleistungsmarketing, Wiesbaden 1995.

Meffert, H./Bruhn, M. (2000): Dienstleistungsmarketing, 3. Aufl., Wiesbaden 2000.

Menon, A./Varadarajan, R. (1992): A Model of Marketing Knowledge Use Within Firms, in: Journal of Marketing, Oct./1992, S. 53-71.

Mertes, M. (2000): Controlling in der Kirche, Diss., Gütersloh 2000.

Miethe, C. (2000): Leistung und Vermarktung unterschiedlicher Formen der Unternehmensberatung, Diss, Wiesbaden 2000.

Müller, W. (1974): Die Koordination von Informationsbedarf und Informationsbeschaffung als zentrale Aufgabe des Controlling, in: zfbf, Nr. 26/1974, S. 683-693.

Müller, W./Fickel, T. (1997): Outsourcing von Controllingaufgaben, in: Controller Magazin, Nr. 2/1997, S. 109-111.

Müßig, M. (1993): Informationsbedarfsanalyse, in: Vahlens Controlling Lexikon, Horváth, P./Reichmann, T. (Hrsg.), München 1993.

Nelson, P. (1970): Information and Consumer Behaviour, in: Journal of Political Economie, Vol. 78, S. 311-329.

Nelson, P. (1974): Advertising as Information, in: Journal of Political Economie, Vol. 82, S. 729-754.

Neuhäuser-Metternich, S./Witt, F.-J. (2000): Kommunikation und Berichtswesen, 2. Aufl., München 2000.

Neuhaus, P. (1996): Interne Kunden-Lieferantenbeziehungen, Diss., Wiesbaden 1996. 
Newman, W. H. (1963): Administrative Actions - The Techniques of Organization and Management, Englewood Cliffs N. J. 1960.

Niedereichholz, C. (1999): Der Controller als interner Berater, Vortragsdokumentation der Euroforum-Konferenz „Der Controller als interner Berater - vom Kostenexperten zum internen Dienstleister", Frankfurt 10.12.11.1999.

Niedereichholz, C. (1999b): Der Interne Consultant - Herausforderungen für die Betriebsorganisation, in: Versicherungswirtschaft, Nr. 24/1999, S. 18331835.

Niedereichholz, C. (2000): Business Plan zur Positionierung einer internen Beratungseinheit, in: Internes Consulting, Niedereichholz, C. (Hrsg.), München/Wien 2000, S. 13-54.

NIST - National Institute of Standards and Technology (1993): Award Criteria 1994, Gaithersburg 1993.

O'Reilly, C. (1989): Corporations, Culture and Commitment: Motivation and Social Control in Organizations, in: CMR, Nr. 4/1989, S. 9-25.

Otto, A. (1999): Return on Controlling - Attraktivität des Controlling von Logistikdienstleistungen, in: krp, Nr. 2/1999, S. 99-107.

Paul, M./Reckenfelderbäumer, M. (1995): Preisbildung und Kostenmanagement unter Berücksichtigung von Immaterialität und Integrativität, in: Dienstleistungsmarketing: Konzeption und Anwendungen, Kleinaltenkamp, M. (Hrsg.), Wiesbaden 1995, S. 226-259.

Peemöller, V.-H./Schmid, R./Meister, U. (1989): Das Berufsbild des Controllers - Eine Auswertung von Stellenanzeigen der Jahre 1985 bis 1987, in: Controller Magazin, Nr. 6/1989, S. 295-301.

Peemöller, V.-H./Schmid, R./Meister, U. (1989b): Anforderungsprofil von Controllern und Internen Revisoren, in: DBW, Nr. 2/1989, S. 227-231.

Pepels, W. (1998): Marketing, 2. Aufl., München 1998. 
Perridon, L./Steiner, M. (1999): Finanzwirtschaft der Unternehmung, 10. Aufl., München 1999.

Pfeiffer, W./Metze, G./Schneider, D./Amler, R. (1991): Technologie Portfolio zum Management strategischer Zukunftsgeschäftsfelder, 6. Aufl., Göttingen 1991.

Pfohl, H. C./ Zettelmeyer, B.(1987): Strategisches Controlling?, in: ZfB, Nr. 2/1987, S. 145-175.

Plinke, W. (1995): Grundlagen des Marktprozesses, in: Technischer Vertrieb, Kleinaltenkamp, M./Plinke, W. (Hrsg.), Berlin/Heidelberg 1995, S. 3-95.

Plötner, O. (1993): Risikohandhabung und Vertrauen des Kunden, in: Arbeitspapier Nr. 2 der Berliner Reihe „Business to Business Marketing“, Kleinaltenkamp, M. (Hrsg.), Berlin 1993.

Pohle, K. (1993): Controlling und Organisation, in: HWB, Wittmann, W. (Hrsg.), Teilband 1, 5. Aufl., Stuttgart 1993, Sp. 661-669.

Preißler, P. R. (2000): Controlling, 12. Aufl., München 2000.

Rafig, M./Ahmend, P. K. (1992): The Limits of Internal Marketing, in: Proceedings, $2^{\text {nd }}$ Workshop on Quality Management in Services, Maastricht 1992, S. 184-198.

Reichmann, T. (1996): Management und Controlling: Gleiche Ziele - unterschiedliche Wege und Instrumente, in: ZfB, Nr. 5/1996, S. 559-585.

Reiß, M. (1992): Spezialisierung: in HWO, 3. Aufl., Berlin 1992, Sp. 22872297.

Reiß, M. (1997): Organisatorische Verankerung des Controlling in den Unternehmensstrukturen des 21. Jahrhunderts, in: Die Kunst des Controlling, Gleich, R./Seidenschwarz, W. (Hrsg.), München 1997, S. 367-380.

Reiß, M./Beck, T. C. (1995): Mass Customization: Kostenverträglichen Service anbieten, in: Gablers Magazin, Nr. 1/1995, S. 24-27. 
Reiß, M./Höge, R. (1995): Controlling-Unterstützung für Center-Organisation und Marktkoordination, in: DB, Nr. 35/1995, S. 1721-1725.

Reiß, M./Schuster, H. (1998): Kunden- und Kostenorientierung interner Service-Bereiche - Aus Zentralbereichen werden Dienstleister, in: Dienstleistungs-Marketing, Meyer, A. (Hrsg.), Band 2, Stuttgart 1998, S. 1300-1320.

Reynoso, J. (1999): Methodological Implications in Measuring Internal Service Quality, in: Internes Marketing, Bruhn, M. (Hrsg.), 2. Aufl., Wiesbaden 1999, S. 527-535.

Rieper, B/Witte, T./Berens, W. (1996): Betriebswirtschaftliches Controlling: Planung - Entscheidung - Organisation; Festschrift für Univ.-Prof. Dr. Dietrich Adam zum 60. Geburtstag, Wiesbaden 1996.

Ringle, G. (1982): Das Zielsystem für die Gestaltung eines innerbetrieblichen Kommunikationssystems, in: Der Betriebswirt, Nr. 2/1982, S. 3-8.

Rockart, J. F. (1979): Chief Executives Define Their Own Data Needs, in: Harvard Business Review, Nr. 59/1979, S. 81-91.

Roth, U. (2000): Der Controller als Berater des Managements, in: Internes Consulting, Niedereichholz, C. (Hrsg.), München/Wien 2000, S. 129-138.

Sander, S. (1990): Der Controllerdienst als Funktionsbereich: Neue Wege für seine Effizienzsteigerung, Diss., Bamberg 1990.

Sathe, V. (1982): Controller Involvement in Management, Englewood Cliffs/I11 1982.

Schade, C. (1997): Marketing für Unternehmensberatung - ein institutionenökonmischer Ansatz, Diss., 2. Aufl., Frankfurt 1997.

Schäffer, U./Weber, J. (1999): Controller können von Consultants lernen, in: Harvard Business Manager, Nr. 1/1999, S. 21-28. 
Scharnagl, J. K./Schubert, G. (1999): Erfolgsfaktoren der Controller-Arbeit bei der BSH Bosch und Siemens Hausgeräte GmbH, in: krp, Nr. 5/1999, S. 310-314.

Scherz, E. (1998): Verrechnungspreise für unternehmensinterne Dienstleistungen, Diss., Wiesbaden 1998.

Scheuch, F. (1993): Marketing, 4. Aufl., München 1993.

Schildknecht, R. (1992): Total Quality Management - Konzeption und State of the Art, Frankfurt/New York 1992.

Schmitz, G. (1997): Marketing für professionelle Dienstleistungen: Bedeutung und Dynamik der Geschäftsbeziehungen, dargestellt am Beispiel der Wirtschaftsprüfung, Diss., Wiesbaden 1997.

Schneider, D. (1983): Marketing als Wirtschaftswissenschaft oder Geburt einer Marketingwissenschaft aus dem Geiste des Unternehmerversagens?, in: zfbf, Nr. 3/1983, S. 197-223.

Schneider, D. (1994): Betriebswirtschaftslehre, Bd. 2: Rechnungswesen, 2. Aufl., München/Wien 1994.

Schneider, D. (1995): Betriebswirtschaftslehre, Bd. 1: Grundlagen, 2. Aufl., München/Wien 1995.

Schneider, D. (1997): Marketing Wissenschaft als Lehre marktorientierter Unternehmensführung und betriebswirtschaftliche Strukturmerkmale für Wettbewerbsfähigkeit, in: Marktleistung und Wettbewerb, Backhaus, K./Günter, B./Kleinaltenkamp, M/Plinke, W./Raffée, H. (Hrsg.), Wiesbaden 1997, S. 13-32.

Schoch, D. (1993): Strategisches Controlling. Konzept und Möglichkeiten der EDV-Unterstützung, Diss., St. Gallen 1993.

Schrädler, J. (1996): Unternehmensberatung aus organisationstheoretischer Sicht, Diss., Wiesbaden 1996. 
Schrijvers, A. (1999): New Public Management - Konzeption, Elemente und praktische Erfahrungen, in: Umsetzung neuer Rechnungs- und Informationssysteme in innovativen Verwaltungen, Budäus, D./Gronbach, P. (Hrsg.), Freiburg 1999, S. 243-256.

Schubert, U. (1972): Der Management Kreis, in: Management für alle Führungskräfte in Wirtschaft und Verwaltung, Band 1, Stuttgart 1972.

Schüller, S. (1984): Organisation von Controllingsystemen in Kreditinstituten, Diss., Münster 1984.

Schütz, P. (1993): Marketing interner Dienstleister - Marketing ohne Markt?, in: Dienstleistungsmarketing: eine Bestandsaufnahme, Tagungsband zum 2. Workshop für Dienstleistungsmarketing, Thelen, E. M./Mairamhof, G. B. (Hrsg.), Frankfurt 1993, S. 193-201.

Schulz, R. (1999): Das Zusammenspiel zwischen internem und externem Marketing - am Beispiel von Henkel, in: Internes Marketing, Bruhn, M. (Hrsg.), 2. Aufl., Wiesbaden 1999, S. 623-641.

Schulze, H. S. (1992): Internes Marketing von Dienstleistungsunternehmen: Fundierungsmöglichkeiten mittels ausgewählter Konzepte der Transaktionsanalyse, Diss., Berlin 1992.

Schumpeter, J. A. (1942): Capitalism, Socialism and Democracy, New York 1942; deutsche Übersetzung: Kapitalismus, Sozialismus und Demokratie, 3. Aufl., München 1982.

Schuurmans, L./Stoller, C. (1998): Der Shared Service Center Trend, in: io Management, Nr. 6/1998, S. 37-41.

Schweitzer, M. (1992): Profit-Center, in: Handwörterbuch der Organisation, Frese, E. (Hrsg.), 3. Aufl., Stuttgart 1992, Sp. 2078-2089.

Schweitzer, M./Küpper, H.-U. (1986): Systeme der Kostenrechnung, 4. Aufl., Landsberg 1986.

Seidenschwarz, W. (2000): Target Costing. Marktorientiertes Zielkostenmanagement, 2. Aufl., München 2000. 
Sellenheim, M. (1991): J. I. Case Company - Performance Measurement, in: Management Accounting, Nr. 9/1991, S. 50-53.

Serfling, K. (1992): Controlling, 2. Aufl., Stuttgart/Berlin/Köln 1992.

Siebke, J. (1992): Preispolitik, in: Vahlens Kompendium der Wirtschaftstheorie und Wirtschaftspolitik, Bender et al. (Hrsg.), Band 2, 5. Aufl., München 1992, S. 62-122.

Siegel, G./Sorensen, J. E. (1999): Counting More, Counting Less - Transformations in the Management Accounting Profession, The 1999 Practice Analysis of Management Accounting, Montvale 1999.

Simon, H./Homburg, C. (1998): Kundenzufriedenheit als strategischer Erfolgsfaktor - Einführende Überlegungen, in: Kundenzufriedenheit, Konzepte Methoden - Erfahrungen, Simon, H./Homburg, C. (Hrsg.), 3. Aufl., Wiesbaden 1998, S. 17-32.

Spremann, K. (1988): Reputation, Garantie, Information, in: ZfB, Nr. 6/1990, S. 561-586.

Stadler, S. M./Weißenberger, B. E. (1999): Benchmarking des Berichtswesens Mehr Effizienz und Kundenorientierung im Controlling, in: Controlling, Nr. 1/1999, S. 5-11.

Stamm, M. (1991): Erfolgsfaktoren für's Controlling, Gauting 1991.

Stamm, M. (1992): Controlling „Verkaufen“: Motivation, Manipulation, Zwang, in: Controller Magazin, Nr. 5/1992, S. 258-263.

Stauss, B. (1991): Internes Marketing als personalorientierte Qualitätspolitik, in: Dienstleistungsqualität, Bruhn, M./Stauss, B. (Hrsg.), Wiesbaden 1991, S. 227-246.

Stauss, B. (1995): Lernen vom Dienstleistungsmarketing, in: Absatzwirtschaft, Sondernummer Okt./1995, S. 25.

Stauss, B., (1995b): Internes Marketing, in: Handwörterbuch des Marketing, 2. Aufl., Sp. 1045-1056, Stuttgart 1995. 
Stauss, B./Neuhaus, P. (1995): Interne Kundenzufriedenheit als Zielgröße das Total Quality Management: Dargestellt am Beispiel einer Abteilung Personalmanagement, in: Internes Marketing: Integration der Kunden- und Mitarbeiterorientierung, Bruhn, M. (Hrsg.), Wiesbaden 1995, S. 575-609.

Stauss, B./Neuhaus, P. (1995b): Das Qualitative Zufriedenheitsmodell, in: Diskussionsbeiträge der Wirtschaftswissenschaftlichen Fakultät Ingolstadt, Nr. 66, Ingolstadt 1995.

Stauss, B./Schulze, H. S. (1990): Internes Marketing, in: Marketing - Zeitschrift für Forschung und Praxis, Nr. 3/1990, S. 149-158.

Stauss, B./Seidel, W. (1995): Prozessuale Zufriedenheitsermittlung und Zufriedenheitsdynamik bei Dienstleistungen, in: Kundenzufriedenheit, Konzepte - Methoden - Erfahrungen, Simon, H./Homburg, C. (Hrsg.), Wiesbaden 1995, S. 179-203.

Steinle, C. (1999): Organisation des Controlling, in: Controlling - Kompendium für Controller/innen und deren Ausbildung, Steinle, C./Bruch, H. (Hrsg.), 2. Aufl., Stuttgart 1999, S. 29-47.

Steinle, C. (1999b): Systeme, Objekte und Bestandteile des Controlling, in: Controlling - Kompendium für Controller/innen und deren Ausbildung, Steinle, C./Bruch, H. (Hrsg.), 2. Aufl., Stuttgart 1999, S. 279-340.

Steinle, C./Bruch, H. (1999): Controllerrollen - fachliche und persönliche Anforderungsprofile, Selbstverständnis und Fremdwahrnehmung, in: Controlling - Kompendium für Controller/innen und deren Ausbildung, 2. Aufl., Stuttgart 1999, S. 48-61.

Steinle, C./Bruch, H./Michels, T. (1998): Controller-Rollen: Anforderungsprofile, Persönlichkeit und Selbstverständnis - Ein empirisches Schlaglicht, in: Zukunftsgerichtetes Controlling, Steinle, C./Eggers, B./Lawa, D. (Hrsg.), 3. Aufl., Wiesbaden 1998, S. 443-468.

Steinle, C./Thiem, H./Rohden, H. (2000): Controlling als interne Serviceleistung, in: Controlling, Nr. 6/2000, S. 281-287. 
Stoffel, K. (1995): Controllership im internationalen Vergleich, Wiesbaden 1995.

Strobel, W. (1979): Controlling und Unternehmensführung, in: Controlling und Finanzplanung, Schriften zur Unternehmensführung, Jakob, H. (Hrsg.), Band 26, Wiesbaden 1979, S. 5-40.

Thieme, H. J. (1992): Wirtschaftssysteme, in: Vahlens Kompendium der Wirtschaftstheorie und Wirtschaftspolitik, Bender, D. et al., (Hrsg.), Band 1, 5. Aufl., München 1992, S. 1-48.

Töpfer, A. (1984): Innovationsmanagement, in: Handbuch Strategisches Marketing, Wieselhuber, N./Töpfer, A. (Hrsg.), Landsberg am Lech 1984, S. 391-407.

Trilse, I. (2000): Controlling als Service Center, in: Vortragsdokumentation des Congresses „Der Controller im Dialog 2000”, 3.-4.5.2000 Bad Homburg.

Trilse, I. (2001): Weiterentwicklung von Controlling-Bereichen - ein Erfahrungsbericht aus der Bankenpraxis, in: krp, Nr. 2/2001, S. 105-109.

Tylkowski, O./Guth, S. H./Spieker, M. (2001): Qualitätsprüfung in der Kostenrechnung - Notwendigkeit oder verschwendete Energie?, in: krp, Nr. 2/2001, S. 95-103.

Ulrich, H. (1968): Die Unternehmung als produktives soziales System, Bern/ Stuttgart 1968.

Ulrich, H. (1978): Unternehmenspolitik, Bern/Stuttgart 1978.

Ulrich, H. (1985): Controlling als Managementaufgabe, in: Controlling und Unternehmensführung, Probst, G. J. B./Schmitz-Dräger, R. (Hrsg.), Bern 1985, S. 15-27.

Vahs, D. (1994): Situative Einflußgrößen des industriellen Controlling. Ergebnisse einer empirischen Studie, in: Journal für Betriebswirtschaft, Nr. 2/1994, S. 77-90. 
Währisch, M. (1998): Organisation der Kostenrechnung als internes Dienstleistungszentrum, in: krp, Nr. 6/1998, S. 331-342.

Währisch, M. (1998b): Kostenrechnungspraxis in der deutschen Industrie, Diss., Wiesbaden 1998.

Währisch, M./Henselek, H. (1997): Betriebliche Einsatzfelder von Informationen der Kosten- und Erlösrechnung, in: WiSt, Nr. 4/1997, S. 330-340.

Wagenhofer, A. (1998): Ermittlung von Verrechnungspreisen für Profit Center, in: krp, Sonderheft 1/1998, S. 23-30.

Wagner, H. (1983): Gibt es die ,ideale Controller-Persönlichkeit"?, in: Controller Magazin, Nr. 3/1983, S. 95-104.

Watzlawick, P./Beavin, J. H./Jackson, D. D. (1990): Menschliche Kommunikation - Formen, Störungen, Paradoxien, 8. Aufl., Bern/Stuttgart/Toronto 1990.

Weber, J. (1990): Controlling der Kostenrechnung - Zur Notwendigkeit des Einsatzes von Controlling-Instrumenten zur strategischen und operativen Ausrichtung der Kostenrechnung, in: krp, Nr. 4/1990, S. 203-208.

Weber, J. (1990b): Change Management für die Kostenrechnung, in: Controlling, Nr. 3/1999, S. 120-126.

Weber, J. (1994): Controller sind Navigatoren, Co-Piloten, Hofnarren - oder gar Pfarrer?, in: Controller Magazin, Nr. 5/1994, S. 267-270.

Weber, J. (1995): Controlling von Kundenzufriedenheit, in: Kundenzufriedenheit Konzepte - Methoden - Erfahrungen, Simon, H./Homburg, C. (Hrsg.), 3. Aufl., Wiesbaden 1995.

Weber, J. (1997): Marktorientiertes Controlling, Schriftenreihe Advanced Controlling, Band 4, Vallendar 1997.

Weber, J. (1997b): Controllership vs. Controlling, in: Controlling, Nr. 3/1997, S. $180-182$. 
Weber, J. (1999): Einführung in das Controlling, 8. Aufl., Stuttgart 1999.

Weber, J./Hamprecht, M./Goeldel, H. (1995): Benchmarking des Controlling, Ein Ansatz zur Effizienzsteigerung betrieblicher Controllingbereiche, in: krp, Nr. 1/1995, S. 15-19.

Weber, J./Schäffer, U. (1999): Re-Inventing Controlling, Schriftenreihe Advanced Controlling, Band 9, Vallendar 1999.

Weber, J./Schäffer, U. (1999b): Controller auf dem Weg zum internen Berater, in: krp, Nr. 1/1999, S. 8-10.

Weber, J./Schäffer, U. (2000): Balanced Scorecard \& Controlling, 2. Aufl., Wiesbaden 2000.

Weber, J./Weißenberger, B./Aust, R.(1997): Benchmarking des Controllerbereichs. Ein Erfahrungsbericht, WHU-Forschungspapier Nr. 43, März 1997.

Weber, J./Weißenberger, B./Aust, R.(1998): Benchmarking des Controllerbereichs. Ein Erfahrungsbericht, in: BFuP, Nr. 4/1998, S. 381-401.

Webster, F. E. Jr./Wind, Y. (1972): Organizational Buying Behaviour, Englewood Cliffs/New York 1972.

Weiber, R./Späth, M. (1998): Vermarktung von Telekommunikationsdiensten im Geschäftskundenbereich: Herausforderung an das Investitionsgütermarketing, in: Entwicklungen des Investitionsgütermarketing, Büschken, J./Meyer, M./Weiber, R. (Hrsg.), Wiesbaden 1998, S. 229-301.

Weilenmann, P. (1989): Dezentrale Führung: Leistungsbeurteilung und Verrechnungspreise, in: ZfB, Nr. 9/1989, S. 932-956.

Weißenberger, B. (1997): Die Informationsbeziehung zwischen Management und Rechnungswesen, Diss., Wiesbaden 1997.

Welge, M. K. (1975): Profit-Center-Organisation, Wiesbaden 1975. 
Welge, M. K. (1988): Unternehmensführung, Band 3, Controlling, Stuttgart 1988.

Williamson, O. E. (1975): Markets and Hierarchies: Analysis and Antitrust Implication, New York 1975.

Williamson, O. E. (1985): The Economic Institutions of Capitalism. Firms, Markets, Relational Contracting, New York 1985.

Winkelmann-Ackermann, S./Bundi, M. (1999): Service Level Agreements gezielt einsetzen, in: io Management, Nr. 3/1999, S. 36-40.

Witt, F.-J. (1985): Marketing für innerbetriebliche Leistungen, in: BFuP, Nr. 2/1985, S. 162-175.

Witt, F.-J. (1986): Controlling von Unternehmensberatungsleistungen, in: Controller Magazin, Nr. 3/1986, S. 129-132.

Witt, F.-J. (1988): Die Typologisierung unternehmensinterner Leistungen, in: ZfB, Nr. 7/1988, S. 660-682.

Witt, F.-J. (1988b): Unternehmensinterne Make-Or-Buy-Entscheidungen, in: Controller Magazin, Nr. 2/1988, S. 77-82.

Witt, F.-J. (1989): Portfolios für unternehmensinterne Leistungen, in: Controller Magazin, Nr. 3/1989, S. 156-162.

Witt, F.-J. (1992): Handelscontrolling, München 1992.

Witt, F.-J./Witt, K. (1991): Controller-Performance, in: Controller Magazin, Nr. 1/1991, S. 44-46.

Witt, F.-J./Witt, K. (1996): Controlling für Mittel- und Kleinbetriebe, 2. Aufl., München 1996.

Witte, E. (1973): Organisation und Innovationsentscheidungen, Göttingen 1973.

Wöhe, G. (2000): Einführung in die Allgemeine Betriebswirtschaftslehre, 20. Aufl., München 2000. 
Woll, A. (2000): Allgemeine Volkswirtschaftslehre, 13. Aufl. München 2000.

Woratschek, H. (1996): Die Typologie von Dienstleistungen aus informationsökonomischer Sicht, in: Der Markt, Nr. 1/1996, S. 59-71.

Ziegenbein, K. (1992): Controlling, 4. Aufl., Kiehl 1992.

Ziener, M. (1985): Controlling in multinationalen Unternehmen, Landsberg am Lech 1985.

Zink, K. J. (1996): TQM, in: Handwörterbuch der Produktionswirtschaft, Kern, W./ Schröder, H. H./Weber, J. (Hrsg.), 2. Aufl., München 1996, Sp. 2072 2088.

Zünd, A. (1985): Der Controller Bereich (Controllership), Randbemerkungen zur Institutionalisierung der Controller Funktion, in: Controlling und Unternehmensführung, Probst, G. J. B./Schmitz-Dräger R. (Hrsg.), Bern 1985, S. 28-40.

ZVEI (1993): Unternehmenscontrolling, ZVEI (Hrsg.), Frankfurt 1993. 


\section{Beltrăge zum Controlling}

Herausgegeben von Wolfgang Berens

Band 1 Wolfgang Berens / Joachim Strauch: Due Diligence bei Unternehmensakquisitionen - eine empirische Untersuchung. Unter Mitarbeit von Thorsten Behrens und Julia Lescher. 2002.

Band 2 Andreas Slemes: Marktorientierte Kreditrisikobewertung. Eine empirische Untersuchung mittels Künstlicher Neuronaler Netze. 2002.

Band 3 Kart Christoph Heinen: Die Berücksichtigung von Kosten in der Konkurrenzanalyse. 2002.

Band 4 Thomas Mosiek: Interne Kundenorientierung des Controlling. 2002. 


\section{Innovationscontrolling}

\section{Eine Controlling-Konzeption zur effektiven und effizienten Gestaltung innovativer Prozesse in Unternehmen}

FrankfurtM., Berlin, Bern, Bruxelles, New York, Oxford, Wien, 2001. XV, 272 S., zahlr. Graf.

Europäische Hochschulschriften: Reihe 5, Volks- und Betriebswirtschaft. Bd. 2703

ISBN 3-631-37459-3 · br. € 45.50*

Die effektive und effiziente "Produktion“ von Innovationen stellt eine der größten Herausforderungen an das Management von Unternehmen dar. Einen wesentlichen Baustein der erfolgreichen innovativen Tätigkeiten bildet dabei die Gestaltung der Innovationsprozesse. Das Management sieht sich jedoch bei der Wahrnehmung der Gestaltungsaufgabe von Innovationsprozessen mit einer zunehmenden Komplexität und Dynamik der Unternehmensum- und -innenwelt konfrontiert. Dies schlägt sich u.a. darin nieder, daß es dem Innovationsmanagement mittels des klassischen Managementinstrumentariums oftmals nicht gelingt, die richtigen Entscheidungen über Inhalte und Gestaltungsalternativen von Innovationsprozessen in der erforderlichen Zeit zu treffen.

Im Mittelpunkt des Interesses steht bei dieser Arbeit die Beratung des Innovationsmanagements bei der Wahrnehmung seiner Gestaltungsaufgabe mit einer geeigneten Controlling-Konzeption und einem ausgewählten Kennzahlensystem.

FrankfurtM - Berlin - Bern - Bruxelles - New York · Oxford · Wien

Auslieferung: Verlag Peter Lang AG

Jupiterstr. 15, CH-3000 Bern 15

Telefax (004131) 9402131

*inklusive der in Deutschland gültigen Mehrwertsteuer

Preisănderungen vorbehalten

Homepage http://wuw.peterlang.de 